\title{
A Summary of the Models Used for the Mechanical Response of Disposal Rooms in the Waste Isolation Pilot Plant with Regard to Compliance with 40 CFR 191, Subpart B
}

B. M. Butcher, F. T. Mendenhall

Prepared by

Sandla National Laboratories

Albuquerque, New Mexico 87185 and Livermore, Callfornia 94550

for the United States Department of Energy

under Contract DE-ACO4-76DPO0789 
Issued by Sandia National Laboratories, operated for the United States Department of Energy by Sandia Corporation.

NOTICE: This report was prepared as an account of work sponsored by an agency of the United States Government. Neither the United States Government nor any agency thereof, nor any of their employees, nor any of their contractors, subcontractors, or their employees, makes any warranty, express or implied, or assumes any legal liability or responsibility for the accuracy, completeness, or usefulness of any information, apparatus, product, or process disclosed, or represents that its use would not infringe privately owned rights. Reference herein to any specific commercial product, process, or service by trade name, trademark, manufacturer, or otherwise, does not necessarily constitute or imply its endorsement, recommendation, or favoring by the United States Government, any agency thereof or any of their contractors or subcontractors. The views and opinions expressed herein do not necessarily state or reflect those of the United States Government, any agency thereof or any of their contractors.

Printed in the United States of America. This report has been reproduced directly from the best available copy.

Available to DOE and DOE contractors from

Office of Scientific and Technical Information

PO Box 62

Oak Ridge, TN 37831

Prices available from (615) $576-8401$, FTS $626-8401$

Available to the public from

National Technical Information Service

US Department of Commerce

5285 Port Royal Rd

Springfield, VA 22161

NTIS price codes

Printed copy: A17

Microfiche copy: A01 
SAND92-0427

Distribution

Unlimited Release

Category UC-721

Printed August 1993

\title{
A Summary of the Models Used for the Mechanical Response of Disposal Rooms in the Waste Isolation Pilot Plant with Regard to Compliance with 40 CFR 191, Subpart B
}

\author{
B.M. Butcher and F.T. Mendenhall \\ Sandia National Laboratories \\ Albuquerque, New Mexico 87185
}

\begin{abstract}
A summary is presented of the results of a number of studies conducted prior to March 1992 that have led to a conceptual model describing how the porosity (and therefore the permeability) of waste and backfill in a Waste Isolation Pilot Plant disposal room changes with time and also describes how results from calculations involving mathematical models of these processes are used to provide input into performance assessment of the repository. Included in the report are descriptions of essential material response or constitutive models that include the influence of gas generation and the response of simple gas-pressurized cracks and fractures in salt, marker beds, and clay seams. Two-dimensional versus three-dimensional disposal room configurations and descriptions of the differences between numerical codes are also discussed.
\end{abstract}

Calculational results using the mathematical models for disposal room response are described, beginning with closure of empty rooms and becoming progressively more complex. More recent results address some of the effects of gas generation in a room containing waste and backfill and intersected by a gas permeable marker bed. Developments currently in progress to improve the evaluation of the disposal room performance are addressing the coupling between brine flow and closure and the two-dimensional capability for analyzing a complete panel of rooms. Next, a method is described for including disposal room closure results into performance assessment analyses that determine if the repository is in compliance with regulatory standards. The coupling is accomplished using closure surfaces that describe the relationship among porosity, total amount of gas in the repository, and time. A number of conclusions about room response and recommendations for further work are included throughout the report.

\section{MASTER}




\section{CONTENTS}

1.0 INTRODUCTION

$1-1$

1.1 The ․ssposal Room Closure Process ................................................................... $1-1$

1.2 Disposal Room Modeling Objectives.......................................................... $1-3$

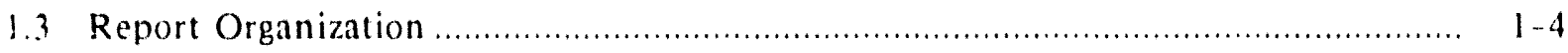

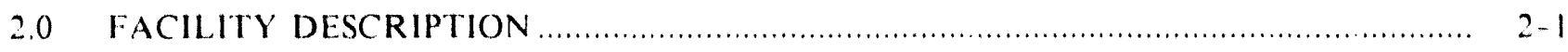

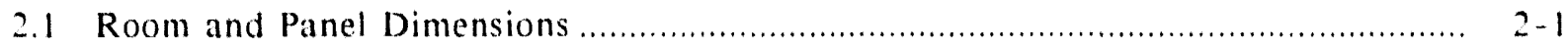

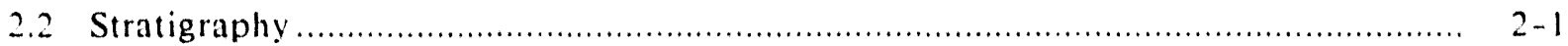

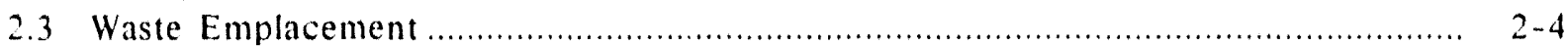

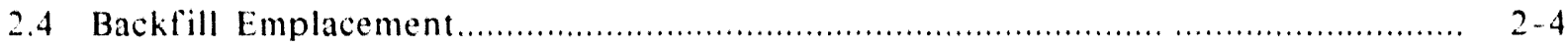

3.0 DISPOSAL ROOM MATHEMATICAL. MODELS.................................................. $3-1$

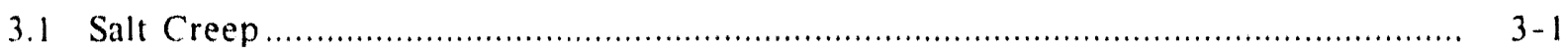

3.2 Backfill Creep-Consolidation Model .............................................................. $3-3$

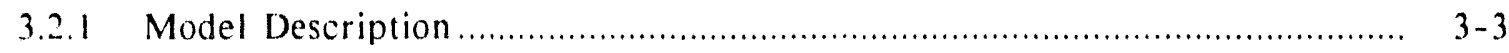

3.2.2 Discussion of the Model ...................................................................... $3-4$

3.2.3 An Alternate Form of the Consolidation Relation ................................... 3-7

3.2.3.1 Crushed Salt Backfill Mixtures................................................ 3-9

3.2.4 Backfill Constitutive Equations................................................................ $3-10$

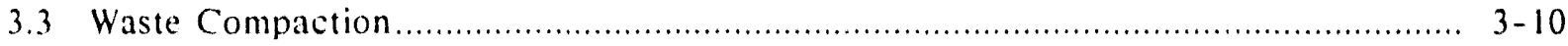

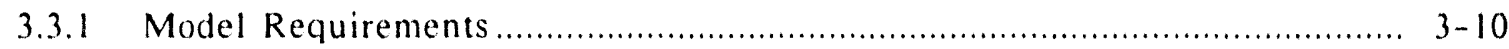

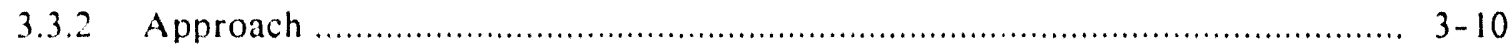

3.3.3 Method of Estimating Composite Compaction Curves ............................... 3-11

3.3.4 Compaction Models ............................................................................. $3-15$

3.4 Disturbed Rock Zone Effects ....................................................................... 3-17

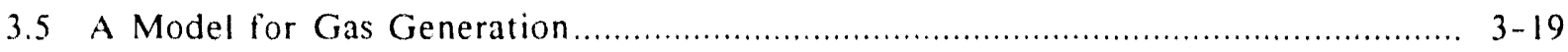

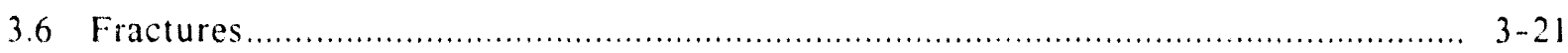

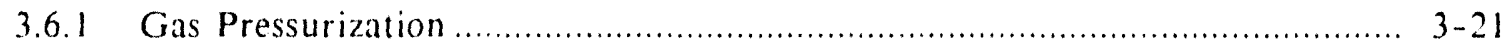

3.6.1.1 Regulatory Implications of Gas Generation ............................. 3-21

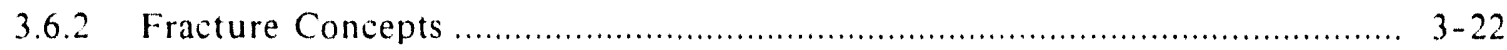

3.6.3 Fracture Constitutive Models .............................................................. 3-23

3.6.3.1 Impermeable Fractures ....................................................... 3-23

3.6.3.2 Permeable Fractures .............................................................. $3-26$

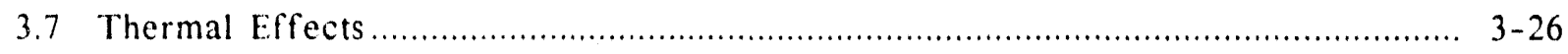

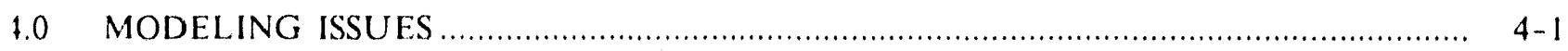

4.1 Two-Dimensional Versus Three-Dimensional Modeling ................................... $4-1$

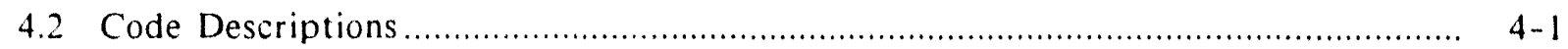

4.2.1 SPECTROM-32 Model Descriptions.......................................................... 4-2

4.2.2 SANCHO Model Descriptions ............................................................. $4-3$

4.3 Array Rooms - Initial and Boundary Conditions............................................... 4-3

4.3.1 Half-Room Configurations ............................................................ $4-3$

4.3.2 Quarter-Room Configurations .......................................................... 4-5

4.3 .3 Other Assumptions .......................................................................... $4-7$

4.4 Isolated Room - Initial and Boundary Conditions ........................................... 4-7 


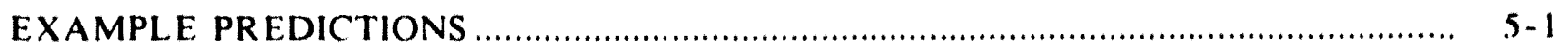

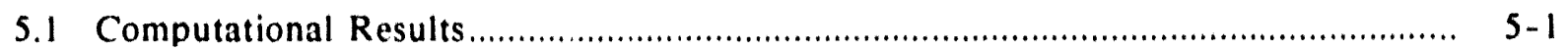

5.1.1 Empty Room Calculations ................................................................. $5-1$

5.1.2 Backfilled Room Calculations ............................................................ $5-5$

5.1.3 Rooms Filled with Waste and Backfill .................................................... 5-7

5.1.4 Mechanical Closure of Rooms Containing Waste, Backfill, and Gas............ 5-14

5.1.4.1 Early Gas Pressurization Estimates Assuming a Perfectly Sealed Room............................................................................. 5-14

5.1.4.2 Recent Closure Analyses of a Perfectly Sealed Room................... 5-16

5.1.4.2.1 Void Fraction Results for Varying Amounts of Gas ...... 5-17

5.1.4.2.2 Gas Pressure Histories for Varying Amounts of Gas...... 5-20

5.1.4.2.3 Calculations for an Altered Gas Generation Rate.......... 5-20

5.1.4.2.4 Safety Valve Calculations ............................................ 5-23

5.2.1 Results of Calculations Examining the Role of Fractures on Room

Pressurization............................................................................. 5-23

5.2.1.1 Impermeable Fractures (Case 1) ............................................ 5-23

5.2.1.2 Permeable Fractures (Case 2) ................................................... 5-27

6.0 ADDITIONAL MODEL DEVELOPMENTS ........................................................ $6-1$

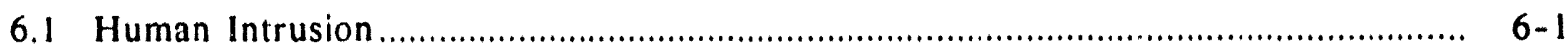

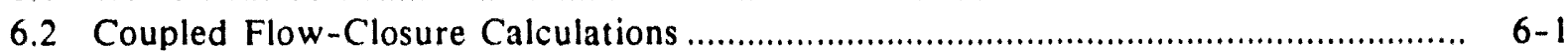

6.2.1 Coupled Single Fluid Phase/Geomechanical Code...................................... 6-2

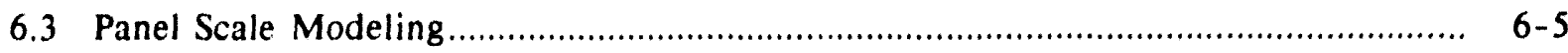

6.4 Engineered Alternatives Task Force (E.ATF) Benchmark Problems....................... 6-11

7.0 APPLICATION TO PERFORMANCE ASSESSMENT WITH REGARD TO 40 CFR 191

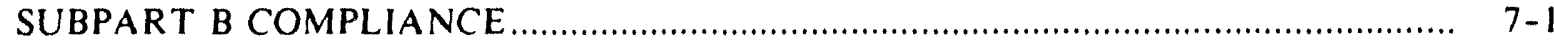

8.0 CONCLUSIONS AND RECOMMENDATIONS........................................................ 8

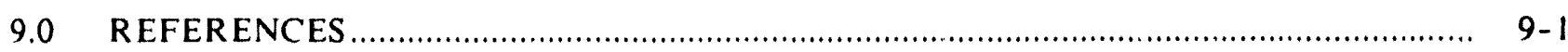

APPENDIX A: MEMORANDA REGARDING DISPOSAL ROOM MODEL ................ A-1

APPENDIX B: CONSTITUTIVE EQUATIONS FOR BACK FILL CONSOLIDATION IN SANCHO AND IN SPECTROM-32 .................................... B-1

APPENDIX C: SANCHO AND RE/SPEC WASTE MODELS ..................................... C-1

APPENDIX D: DISPOSAL ROOM CALCULATIONS …......................................... D-1 


\section{Figures}

1-1 Proposed WIPP Repository showing both TRU-waste disposal areas and experimental areas

2-1 Plan view of the WIPP underground storage area showing the panels, disposal rooms, and haulage ways.

2-2 Stratigraphy at the WIPP repository horizon

2-3 September 1983 reference stratigraphy

2-4 Local stratigraphy around Room D in the WIPP repository experimental area .........

3-1 The relationship between plots of the $\log _{10}$ volumetric strain rate versus fractional density for WIPP test sample CS-8 containing $70 \mathrm{wt} \%$ salt $/ 30 \mathrm{wt} \%$ bentonite and predictions using the Sjaardema and Krieg (1987) model with parameters determined by Brodsky and Pfeifle

3-2 A comparison between the calculated compaction curve for all the waste in the WIPP repository and the recommended compaction curves for combustible, metallic, and simulated sludge wastes.

3-3 Repository waste compaction relationships used by Sandia and RE/SPEC for disposal room closure analyses

3-4 Generated data and fits for composite waste models used in SPECTROM-32 disposal room closure analyses.....

3-5 Schematic illustration of the constitutive model for the gas permeable and gas impermeable segments of the fracture layer

4-1 Finite-element mesh used to analyze a half-room representation of an empty disposal room

4-2 Finite-element mesh used to analyze a quarter-room representation of an empty disposal room

5-1 Closure of an empty disposal room

5-2 Closure of a disposal room completely filled with crushed salt backfill

5-3 Closure of a disposal room completely filled with crushed salt and bentonite backfill.

5-4 SANCHO results for a disposal room filled with crushed salt backfill and TRU waste

5-5 SPECTROM-32 results for a disposal room filled with crushed salt backfill and TRU waste for the waste consolidation assumption that $\sigma_{\mathrm{a}}=3 \sigma_{\mathrm{m}}$ 
5-6 SPECTROM-32 results for a disposal room filled with crushed salt backfill and TRU waste for the waste consolidation assumption $\sigma_{\mathrm{a}}=\sigma_{\mathrm{m}}$

5-7 SANCHO results for a disposal room filled with crushed salt/bentonite backfill and TRU waste

5-8 SPECTROM-32 results for a disposal room filled with crushed salt/bentonite backfill and TRU waste

5-9 The effects of gas generation on disposal room closure

$5-18$

5-10 The effects of gas generation on disposal room pressurization

5-11 The effects of altered rates of gas-production generation on disposal-room closure

5-12 The effects of altered rates of gas-production generation on disposal-room pressurization

5-13 The effect on disposal-room closure of allowing unlimited gas leakage at room gas pressures above $15 \mathrm{MPa}$

5-14 The pressure history corresponding to the closure shown in Figure 5-13

5-15 Finite-element mesh used to analyze a quarter room intersected at its mid height by a fracture.

5-16 Gas pressurization of a room intersected at its mid height by an impermeable fracture.

5-17 The compressive vertical stress history in the first element of an impermeable fracture, the element adjacent to the DRZ, during closure

5-18 Opening of a permeable fracture by gas pressurization

5-19 Gas-pressure/void-volume history in a disposal room intersected by a permeable fracture.....

6-1 Predictions of brine accumulation within empty disposal rooms assuming (1) no change in volume because of closure (reference curve); (2) uncoupledbrine flow into a closing room (geometry); and (3) brine flow into a closing room, coupling the surrounding stress field with the fluid flow

6-2 Plan view of the WIPP storage area showing the vertical symmetry planes assumed for a 2-D calculation of the closure of a panel

6-3a The finite-element mesh used to analyze closure of a complete 2-D panel. $6-7$ 


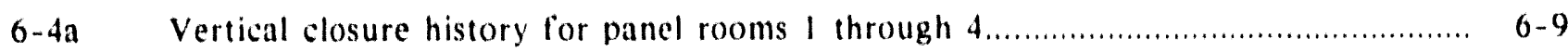

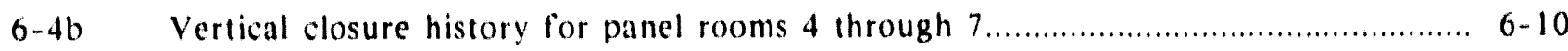

6-5 A comparison of the closure history for an empty TRU storage room, used by IT (US DOE, 1991) in their evaluation of the performance of various engineered alternatives waste forms with more mechanistically based SANCHO results

6-6 A comparison of the closure history for a perfectly sealed disposal room experiencing gas pressurization, used by IT (US DOE, 1991) in their evaluation of the performance of various engineered alternatives waste forms with more mechanistically based SANCHO results

7-1 The relationship of elements of the disposal-room model to the system used by the WIPP Performance Assessment Group at Sandia to demonstrate compliance of the WIPP repository with federal and state regulations

7-2. The unprocessed TRU waste closure surface used for the 1992 comparison of predicted WIPP performance with 40 CFR Part 191. Subpart B

7-3 A contour plot of a typical gas-filled porosity surface for closure of a disposal room containing untreated waste

7-4 The revised gas-filled porosity contour plot taken from Figure $7-3$, reflecting a human intrusion at 1000 years. The calculation is restarted from the conditions indicated by point " $b$ ".

\section{Tables}

3-1 Backfill Consolidation Model Parameter Values

3-2 TRU Simulated Waste Compaction Curves.....

5-1 Disposal Room Calculations

7-1 Two-Phase Flow Nomenclature

C-1 Assumed relationship between the mean stress and the volume strain for $\mathrm{CH}$ TRU waste used in SANCHO disposal room closure analyses (new SANCHO compaction curve)

C-2 Composite CH TRU waste consolidation model constants used in the SPECTROM-32 disposal room closure analyses 


\subsection{INTRODUCTION}

The Waste Isolation Pilot Plant (WIPP) is a research and development facility to demonstrate the safe management, storage, and eventual disposal of transuranic (TRU) waste generated by Department of Energy (DOE) defense programs. The WIPP is located in southeastern New Mexico at a depth of approximately $655 \mathrm{~m}(2150 \mathrm{ft})$ in bedded halites within the Salado Formation (Bechtel, 1986). The repository is a series of underground drifts, panels, and disposal rooms as shown in Figure 1-1. Each disposal room is about 4 by 10 by $91 \mathrm{~m}(13 \times 33 \times 300 \mathrm{ft})$ and will be filled with 55-gallon drums or other containers of TRU waste. After the containers are placed in the room, crushed salt or some other type of backfill will be placed over, around, and between the containers to fill much of the remaining room void space. After backfilling, the room will be closed to further access and its contents allowed to consolidate with time.

The objectives of this report are (1) to summarize the results of studies that have led to development of a conceptual model for describing how the porosity and permeability of waste and backfill change with time and (2) to show how results from calculations involving mathematical models of these processes are used 10 provide input into performance-assessment predictions that determine if the repository is in compliance with regulatory standards. Specifically, the majority of work reported in this document refers to post closure 40 CFR $191 B$ performance assessment. Exceptions are those areas regarding RCRA, which are clearly identified. The contents represent progress in development over a period of approximately four years, ending March 1992. Before discussing these objectives further, a brief discussion of the waste environment after a room is filled is necessary.

\subsection{The Disposal Room Closure Process}

The disposal room closure process is a complex and interdependent series of events starting after a disposal room is filled with waste. Three processes are important: (1) the volume of the excavation decreases as the salt surrounding the room creeps in response to overburden loads; (2) brine migrates towards the room because fluid pressure in and adjacent to the room is lower than the equilibrium fluid pressure that existed in the salt prior to excavation (the far-field fluid pressure far away from the excavation); and (3) decomposition, corrosion, and radiolysis processes within the waste may generate gas. The presence of gas within the disposal room is important not only because gas pressurization may retard both the closure process and fluid flow, but also because it may include entrained hazardous volatile organic compounds (VOCs), whose migration must be limited. All three processes continue with time until the forces causing closure become equal to opposite forces caused by brine and gas pore pressurization and backstress exerted by the waste and backfill. Closure in this report is defined as the decrease in volume of a mine excavation in salt with time, as the surrounding salt deforms into it. The ability of salt to deform with time, to eliminate voids, and to create an impermeable salt barrier around the waste was one of the principal reasons for locating the WIPP repository in a bedded salt formation.

In the absence of any disruption of the natural barrier surrounding the repository (e.g., the absence of an inadvertent human intrusion), continued gas generation may eventually reduce the closure process to the point where it stops. After a minimum void or pore volume is reached, the void volume within the room may then begin to increase, if leakage of the gas away from the waste is not sufficient to relieve pressurization. Void volume increase will continue until gas generation ceases and pressure equilibrium is eventually established with the sur- 


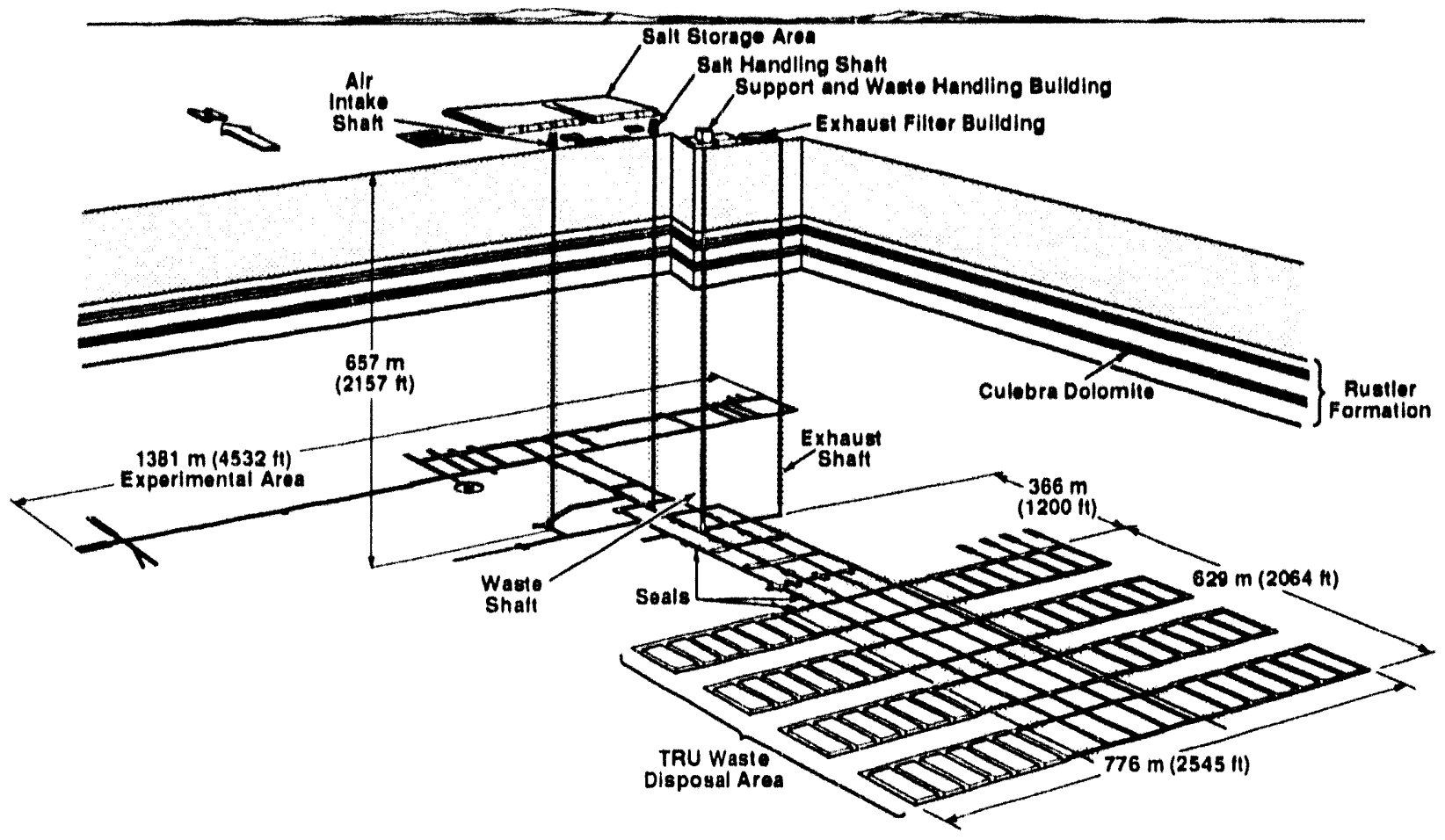

TAI.6346.59.10

Figure 1-1. Proposed WIPP Repository showing both TRU-waste disposal areas and experimental areas (after Waste Management Technology Dept., 1987). The disposal areas are composed of haulage ways, access drifts, and eight panels, each containing seven disposal rooms. 
rounding formation. For the purposes of post-closure RCRA, gas leakage meshanisms must he defined to demonstrate that VOC gases do not escape to the accessible environment in excess of the regulated amounts.

Eventually, changes in the room volume will begin 10 equilibrate as the stress within the room approaches lithostatic stresses (14.8 MPa) after long periods of time. While subsequent decreases in room porosity, the void space in the room, may occur because of slow consolidation of the waste, very long-term chemical phase alteration of the waste, or reduction of pore pressure by fluid transport away from the room. these changes are expected to have little effect on the t11 CFR $19 / B$ post-closure performance of the repository. Firr the purposes of the report. the final

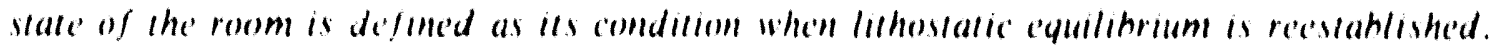

While the steps in normal closure of the repository are hundreds to thousands of years in duration, they can be interrupted at any time by events that disrupt the state of the repository. For example, inadvertent human intrusion into the repository, such as drilling into $\mathrm{it}$, could have a major effect, bypassing natural barriers and releasing pressurized gas and brine, which in turn may alier or restart various parts of the closure process (WIPP Performance Assessment Division, 1991a, Vol. I, Section 2.1.2).

\subsection{Disposal Room Modeling Objectives}

The various stages of closure described in Section 1.1 indicate that the mechanical and physical states of the repository are continually changing with time. Models are required 10 predict these changes to evaluate the performance of the repository and demonstrate compliance with waste storage regulations. Two types of models will be described. The conceptual models for the disposal room are qualitative and identify all of the various processes that contribute to mechanical and, to some extent, fluid flow changes within the room. The words "disposal room model" in this report refer to the combined conceptual models. Each conceptual model must then be described by the second type of model, a mathematical model, for quantitative prediction of the response of a room. These mathematical models, in turn, must be integrated inio the very broad computational process that eventually is used to demonstrate quantitative compliance of the repository with federal and state regulations.

The principal objective in construction of the disposal room model for investigation of repository conditions is to predict the porosity of the waste and backfill at any time. The need for porosities of the waste is twofold. First, transport of soluble radionuclides in brine is one of the principal ways that radionuclides can escape from the repository.' The void volume remaining in the room at any given time, as computed from porosity, determines how much radioactive brine could be present if all the voids filled with brine.

Second, the porosity of the waste or backfill is one of the most important factors determining permeability. In reverse, while the permeabilities of the waste and backfill actually determine the rate of transport of soluble radionuclides in brine out of the repository, their direct coupling with porosity permits the use of the porosities as indirect indicators of flow magnitudes. Furthermore, if porosities are used, a translation from porosity to permeability is possible: given

1 Cultings removal from drilling penetration of the repository during an inadvertent human intrusion is the other principal mechanism by which radioactive materials can reach the surface of the earth. 
the porosity of a waste malerial at a given time, its permeability on be estimated from relationships derived from laborators data le . Hutcher el al. 1991a. I uker el al, 1991) Therefore. the disposal room model can be cast in terms of the porosils of the waste and backfill as a function of time. Computational results desstibed lated in this teport will thow that consolidation of crushed salt backfill, of a minture of etushed sall and hentonite baskrill w relatively impermeable states, is much more tapid than the soneolidation of the wate

Another objective of the room modeling effort is to provide computational methods for solving room response problems. Devolopment of the computational methods for addreving closure has been a parallel approach involving two diffecent numetical codes this procese was considered necessary because calculations in many sases proved to be much more difficult than anticipated, and problems with the numerical techniques frequently meant that resulis could not be obtained in time to satisfy progranmatic milestones. For a given problem, the two codes usualls did not encounter the same difficulties. and therefore, if one tailed to solve the problem. results sould almost always be obtained from the other. The two numelisal sodes used are the rinile. element, finite-strain code SANCHO (Stone et al., 1985) and the finite element, small clrain cude SPECTROM-32 (Callahan et al. 1990). Although the codes appear at first examination to he almost identical in the manner that problems are solved. they are actually quite different in several respects (Butcher et al., 1991a), as will become evident in Sestion 10 of this report

\subsection{Report Organization}

Because the disposal room model described in this report must lanslate into a computational method for acquiring quantitative closure information. The repont is organied acsording to the way that a salculation would be performed In Chapter 2 some of the information for establishing the initial conditions and configuration of the problem is described this information includes a description of the repository and the initial sontiguration of a disposal room. The foum dimensions, the stratigraphy of the surrounding salt, and hou the waste is emplaced

Chapter 3 describes the material response of constitutice models needed for the calculation These include mathematical descriptions for:

- creep of intact salt.

- creep consolidation of salt-based backlill.

- consolidation of the naste,

- deformation of the disturbed cone of rock surrounding the room.

- gas pressurization, and

- the response of large scale cracks and frantutes in salf and discontinuous features in the stratigraphy such as marker beds and clay seams.

Some additional factors that must be considered for slosure calculations are examined in Chapter 4. These include the use of two-dimensional (2 D) versus three dimensional (1) (D) disposal room configurations, descriptions of the differences between the iwo numerical sodes, and discussion of the special assumptions used in some of the calculations

Example results are described in Chapter 5 These begin with resulls for the simplest bye of calculation, slosure of an emply room, and become progressively mote somples More recent results address the effects of gas generation in a room containing wask and hathill and intersected by a gas permeable marher bed. 
Chapter $o$ is a brief description of some of the development work in progress to improve the model lor example, the consequences of an inadvertent human intrusion have yet to be addressed. although the methods for completing this step are already in place. Incorporation of the eftect of brine flow on gas generation and closure by coupled-flow analysis is also being addressed. as woll as the leasibility of various panel-seale modeling schemes.

A major objective of this report, a proposal of the way the disposal room model can be coupled into assessment of repository performance, is in Chapter 7. The mechanism of information transfer is currently specification of (closure) surfaces desc ibing the relationship of three lariables, potosity, total amount of gas in the repository, and time, for various assumed sequences of events.

Vinally, Chapter 8 is a summary of the contents of the report and recommendations for further development. 


\subsection{FACILITY DES'SRIPTION}

\subsection{Room and Panel Dimensions}

The WIPP repository is located in southeastern New Mexico at a depth of approximately 655 $\mathrm{m}(2150 \mathrm{ft})$ in bedded halites within the Salado Formation (Bechtel, 1986). Present plans call for mining eight panels of seven rooms as shown in Figure 2-1. Backfill will be placed around the waste as each panel is filled. Waste will also be placed in the drifts between the panels, which then may be backfilled, to utilize space corresponding to approximately two additional panel volumes. An experimental region exists at the north end of the repository that will be decommissioned and backfilled as the underground experiments are completed. No waste will be stored in the experimental area.

All underground openings in the waste panels have rectangular cross sections. Rooms are nominally $4 \mathrm{~m}(13 \mathrm{ft})$ high by $10 \mathrm{~m}(33 \mathrm{ft})$ wide by $91.4 \mathrm{~m}(300 \mathrm{ft})$ long, ${ }^{1}$ and drifts are generally $3.96 \mathrm{~m}(13 \mathrm{ft})$ high by $4.3 \mathrm{~m}(14 \mathrm{ft})$ wide (WIPP Performance Assessment Division, 1991a, Section 3.1.1). The width of the pillars between the rooms is $30.5 \mathrm{~m}(100 \mathrm{ft})$; the distance between the centerline of a room and the centerline of a pillar, the basic unit of symmetry, in the interior of a panel, is $20.27 \mathrm{~m}(66.5 \mathrm{ft})$.

The configuration for closure analysis is generally a single disposal room. Because of computational limitations, most past numerical closure calculations have been limited to $2-D$ plane strain analyses that examine closure of the 4-m-by-10-m cross section of an infinitely long room. These rooms, in turn, either are assumed symmetric, with vertical symmetry planes at the center of each pillar and each room, as would be valid for an infinite series of rooms, or modeled as a single, isolated room. Extensive calculations of 3-D effects at intersections of the rooms with the panel access drifts have not been completed largely because of restraints imposed by the numerical methods and computation time. However, some results are available from calculations by Argüello et al. (1989) and Argüello (1990). Additional discussion of 3-D effects is in Section 4.1.

\subsection{Stratigraphy}

Closure analyses of disposal rooms are limited to the stratigraphy of the Salado Formation because this geological unit is of sufficient extent to contain far-field computational boundaries. The detail of the stratigraphy is usually limited to that within approximately $\pm 55 \mathrm{~m}$ vertical distance from the horizon of the repository because stratigraphical features farther away have little influence on closure (Munson et al., 1989a). The Salado in the vicinity of the repository is composed mainly of five geological materials: halite, argillaceous halite, anhydrite, polyhalite, and clay in clay seams, as shown in Figure 2-2. Marker Bed 139 is the principal interbed in the vicinity of the repository horizon and is an anhydrite layer approximately $1 \mathrm{~m}$ thick located several meters below the repository floor.

1 Exact dimensions are $3.96 \mathrm{~m}$ high by $10.06 \mathrm{~m}$ wide by $91.44 \mathrm{~m}$ long. 


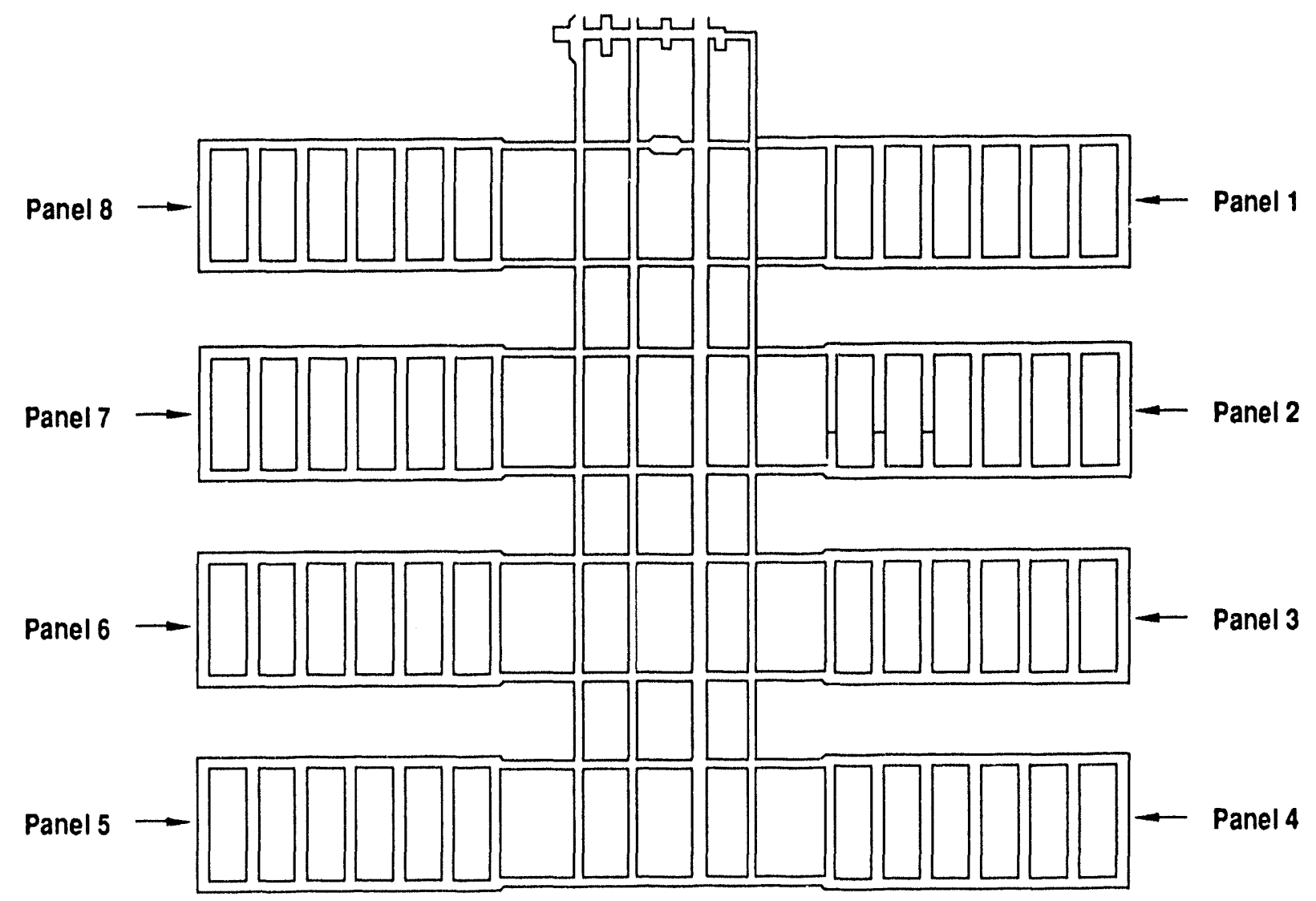

TRI-6334-206-3

Figure 2-1. Plan view of the WIPP underground storage area showing the panels, disposal rooms, and haulage ways (after Bechtel, 1986). 


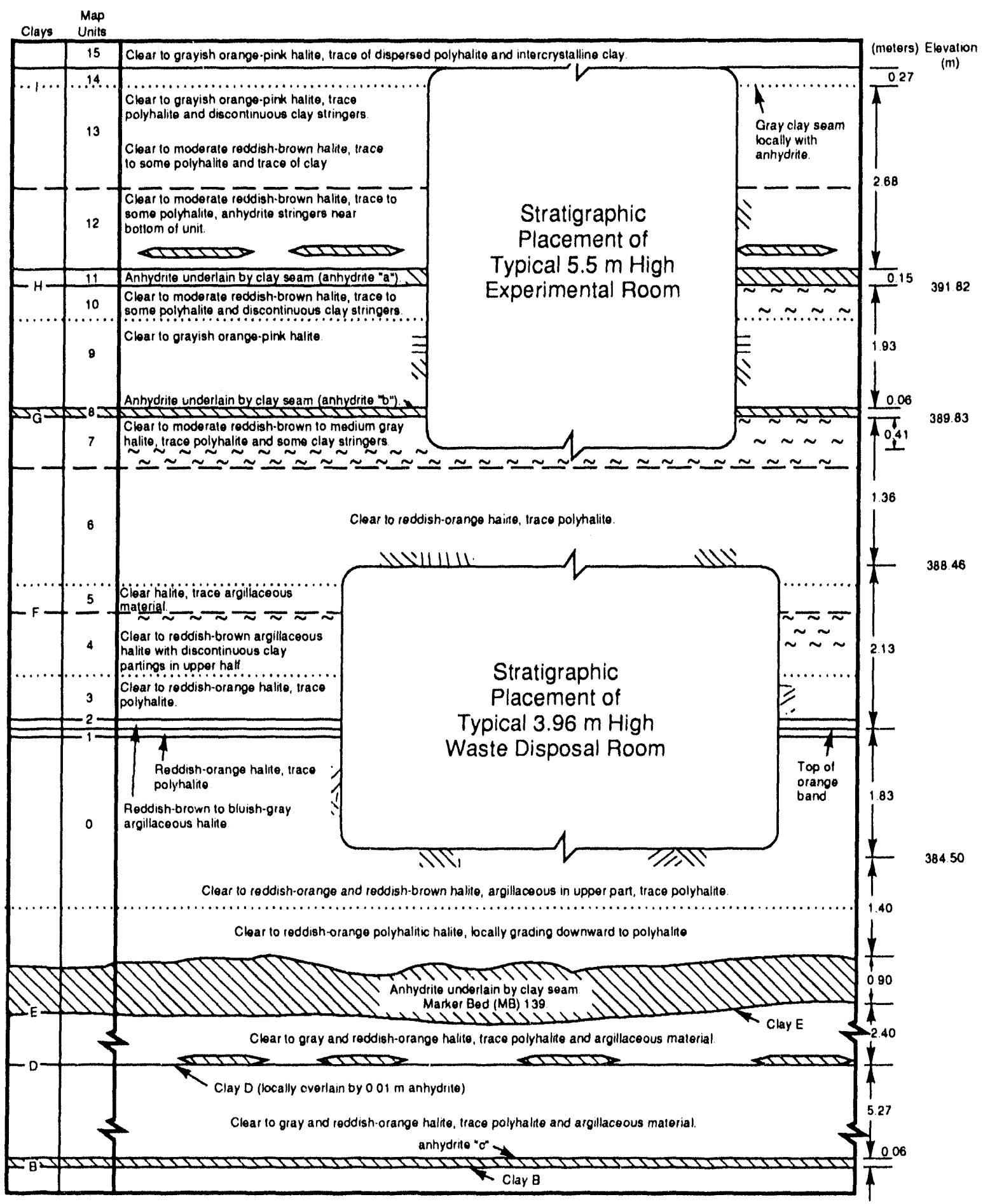

TRI-6334-257-2

Figure 2-2. Stratigraphy at the WIPP repository horizon (after Bechtel, 1986, Figures 6-2 and 6-3; Lappin et al., 1989, Figure 4-12). Units in the disposal area dip slightly to the south, but disposal excavations are always centered about the orange marked band (reddish-orange halite) (taken from WIPP Performance Assessment Division, 1991a, Figure 2.2-3). The stratigraphy above and below the limits of this figure is mostly halite. 
Two principal abstractions of the repository stratigraphy have been used for closure analyses. In one, much of the rock surrounding the disposal room is classified as halite (Krieg, 1984); in the other, it is described as argillaceous halite (Munson et al., 1989a). The first, shown in Figure 2-3, is the reference stratigraphy defined by Krieg (1984). This stratigraphy has been used for calculations performed with the finite-element computer code SANCHO (described in Section 4.2.2), although most SANCHO analyses have assumed that the stratigraphy surrounding a disposal room is composed entirely of halite. The reasons leading to this representative stratigraphy are described in Section 5.1.1. This assumption was based on comparison calculations that showed little difference between results obtained using the reference stratigraphy and results using pure halite (Morgan, December 9, 1987 memo in Appendix A).

The second abstraction of the WIPP stratigraphy for closure calculations is shown in Figure 2-4 (Munson et al., 1989a). Although this stratigraphy was originally developed for analyses of the closure of the in situ Room D experiment at the WIPP, its use has been extended to closure analyses for other drifts and rooms performed with the numerical code SPECTROM-32. It has also been used for empty disposal room estimates with SPECTROM-32. To make comparisons with SANCHO results easier, the most recent SPECTROM-32 disposal room closure calculations have also been for a stratigraphy surrounding a disposal room composed entirely of halite.

\subsection{Waste Emplacement}

The WIPP's current design capacity is approximately $175,000 \mathrm{~m}^{3}$ and will contain a current inventory estimate of about 532,500 55-gallon drums and 33,500 boxes of contact-handled, transuranic (CH TRU) waste (WIPP Performance Assessment Division, 1991a, Section 3.4). Approximately $60 \%$ of this waste may be co-contaminated with waste considered hazardous under the Resource Conseriation and Recovery Act (RCRA) (US EPA, 1987). A small portion of the waste must be remotely handled $(\mathrm{RH})$ and will be contained in canisters emplaced horizontally in the walls of the rooms.

Ideal packing of a total of 680455 -gallon containers, the most that can physically be placed in a disposal room, has been assumed as the amount of waste in a single room (WIPP Performance Assessment Division, 1991a, Sec. 3.16) for most closure analyses. This configuration is based on placement of the drums in groups of seven hexagonal-packed units, or 7-packs, 54 units long by 6 units wide. Seven-packs would be stacked three high, with the top units covered by up to $2 \mathrm{ft}$ of backfill.

The remaining space above the backfill is a ventilation space, approximately $2 \mathrm{ft}$ high (Bechtel, 1986). The ventilation space dimension is included in the storage room reference design dimensions recommended by Bechtel (1986), which state that the level of salt should be maintained at $9.75 \mathrm{~m}(10 \mathrm{ft}, 8 \mathrm{in})$ above the floor of the room, and a ventilation space of $0.4 \mathrm{~m}(1 \mathrm{ft}, 4 \mathrm{in})$ minimum should be maintained until the room is filled and plugged.

\subsection{Backfill Emplacement}

Layers of backfill will surround the waste (on top of it and between it and the walls of the room) to minimize void volume (e.g., Butcher et al., 199la). Backfill eliminates as much void space as possible. Principal backfill materials under consideration are pure crushed salt, crushed 

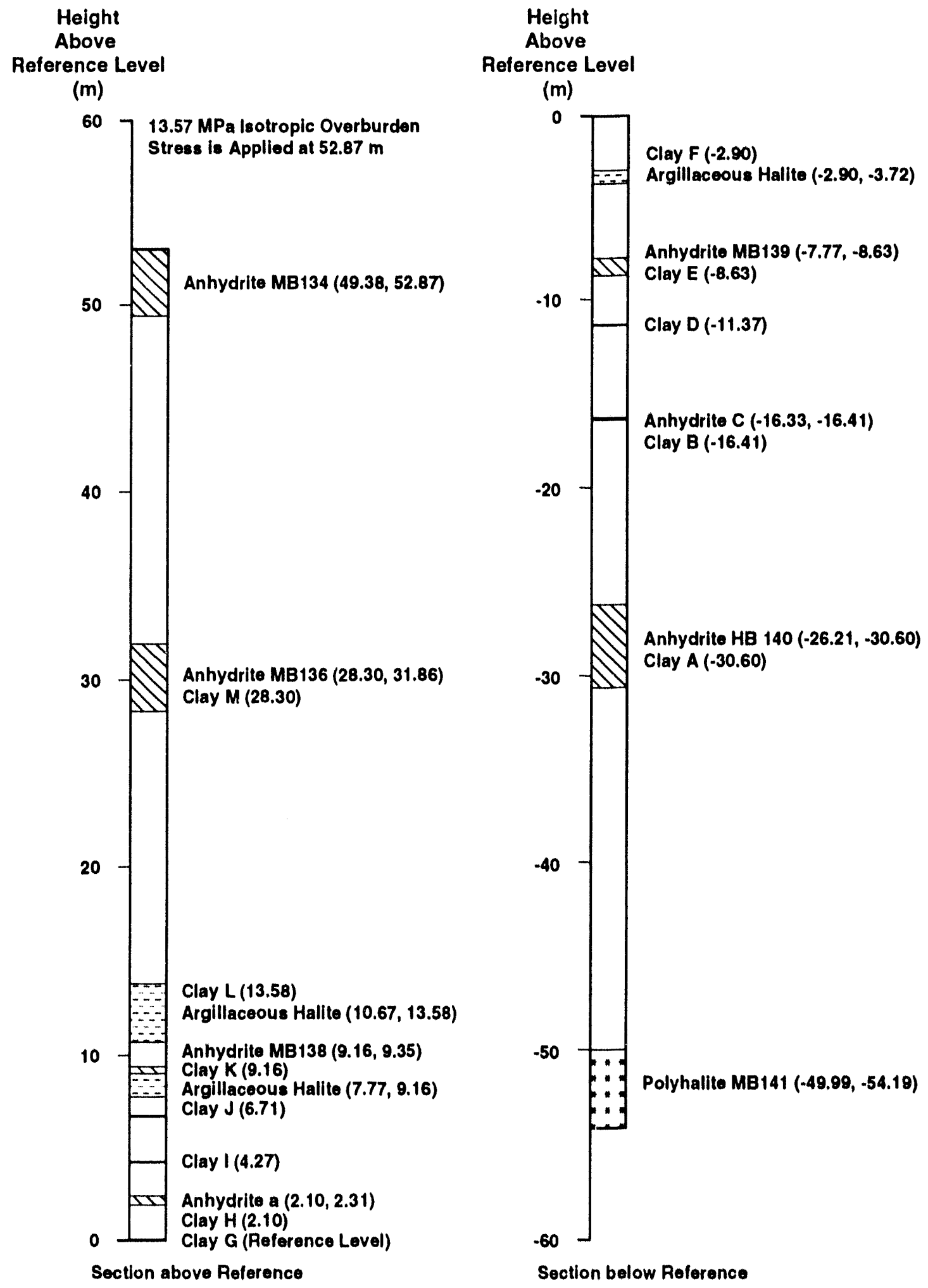

TRI.6345-130-0

Figure 2-3. September 1983 reference stratigraphy (after Krieg, 1984). Anhydrite b should not be used in a structural model. It is only included for reference purposes. 


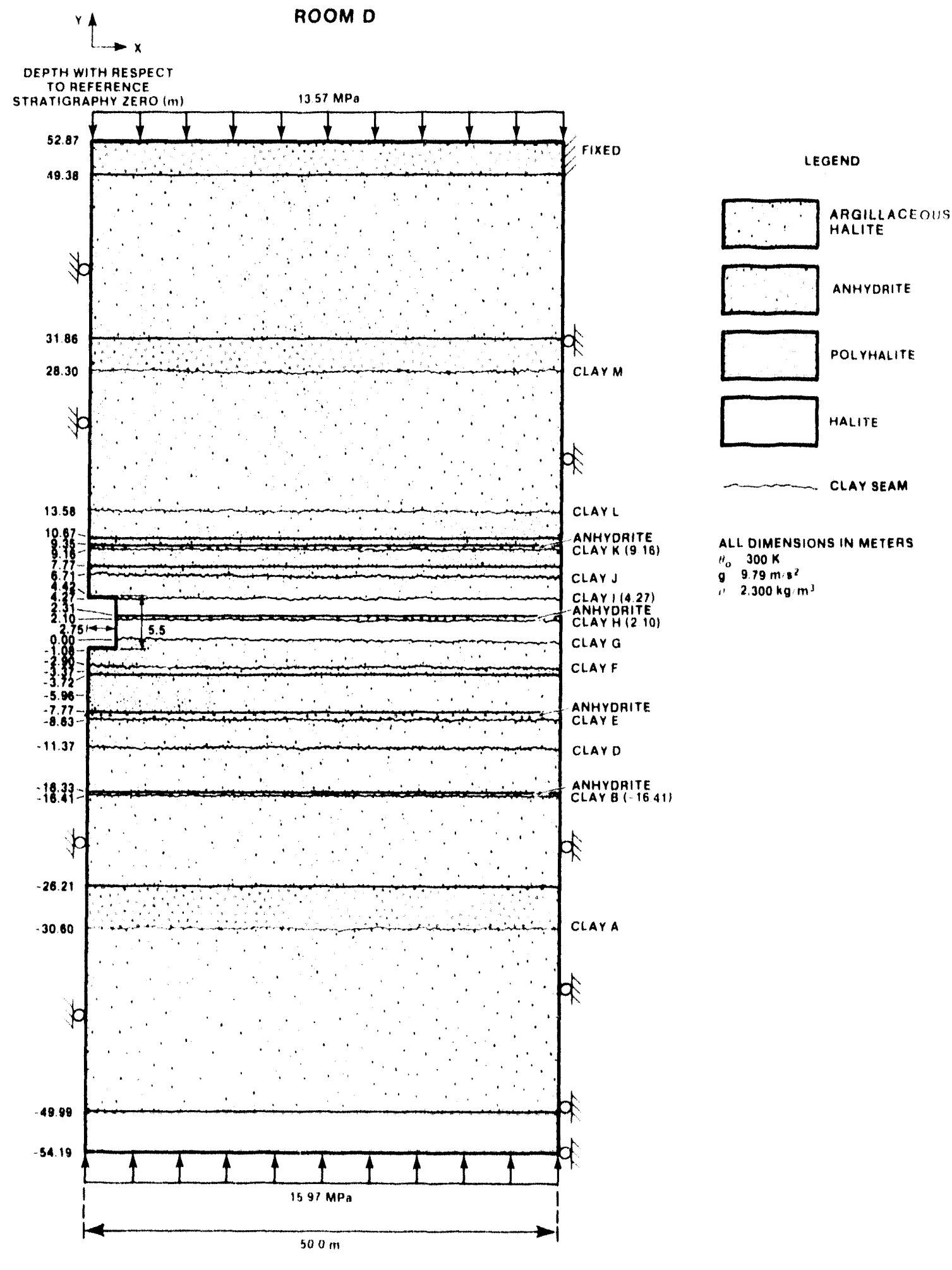

IIII 6.436 1310

Figure 2-4. Local stratigraphy around Room D in the WIPP repository experimental area (after Munson et al., 1989a). 
salt mixed with bentonite or other additives, or grout. Backfill emplacement is anticipated to be straightforward because a wide range of mechanical and pneumatic methods has been developed by the mining industry for backfilling various types of excavations. To promote higher density and uniformity and to facilitate emplacement of salt-based backfill, all salt particles larger than about $12.5 \mathrm{~mm}$ ( 1 in) will probably be removed from mine-run crushed salt and recrushed (Butcher et al., 1991a). 


\subsection{DISPOSAL ROOM MATHEMATICAL MODELS}

\subsection{Salt Creep}

Halite (salt) has the interesting characteristic of being able to deform with time under low shear stresses. This mechanical property causes mined cavities or voids in bedded or domal salt formations to decrease in volume with time until (1) either they are reduced to very small openings or (2) brine and gas pressure within the pores increases sufficiently to counteract stresses causing closure. After the waste is emplaced in the repository, the salt will deform around it, reduce any void volume that could eventually fill with brine, and surround the waste with a tight, impermeable barrier.

Because published results of experiments and modeling of the creep consolidation of salt are so extensive, a summary of such information is beyond the scope of this report. Instead, attention is focused on two milestones in constitutive modeling of the salt surrounding the repository. The first, by Krieg (1984), was construction of a reference steady-state creep law based on a comprehensive examination of all data relevant to WIPP salt prior to 1984. An elastic/secondary creep relationship was defined. The rationale for limiting the description to secondary, steadystate creep, rather than including a primary or transient creep function, was that long enough periods of time were under consideration to render transient effects of lesser importance. This conclusion was based on the expectation that any transition to steady state would occur quickly, and transient strain would be limited in magnitude in relation to the large strains accompanying long-term closure. The assumption was made, therefore, that because the preponderance of the deformation was expected to be from steady-state creep, total strains predicted from steady-state creep rates would be only slightly in error.

A reference stratigraphy for the region surrounding the disposal rooms was also recommended by Krieg (1984), as were reference mechanical properties for dominant nonhalite features such as anhydrite and polyhalite marker beds and clay seams. This information was assumed for calculations addressing comparisons with early closure data from the first underground experimental tests initiated at the WIPP.

Comparison of closure estimates with early closure data almost immediately indicated that mined openings in the WIPP were closing approximately three times faster than was predicted with the reference creep law. As a consequence, simple fixes to the reference creep law were instituted. Major changes involved dividing the elastic moduli values of the reference creep law by a factor of 12.5 , and greatly simplifying the stratigraphy of the disposal room model by changing it to a uniform formation of $100 \%$ halite. A mechanistic justification for reduction of the moduli was not apparent at that time; the stratigraphy was simplified because of major uncertainty in modeling features. With the modulus and stratigraphy changes, closure estimates using the modified reference creep law and stratigraphy were found to be in agreement with closure data, and they have been incorporated in all subsequent SANCHO calculations. Specification of the parameter values used in the SANCHO model will be deferred until the next section on backfill consolidation because they are also part of the backfill model. 
The second milestone in constitutive modeling of salt was provided by Munson et al. (1989a; 1989b) after examination of a number of possible explanations for the inadequacy of the original reference creep law. This model also changed the original reference creep law and stratigraphy recommendations in several ways. First, Munson proposed that a Tresca flow criterion be used instead of the von Mises flow criterion implicit in Krieg's reference creep law. Second, based on examination of core from boreholes drilled $15.2 \mathrm{~m}(50 \mathrm{ft})$ above and below the rooms, Munson constructed a different stratigraphy composed almost entirely of argillaceous halite for the rock surrounding the repository (Figure 2-4) and proposed a different value for the coefficient of friction controlling slippage along clay seams. The steady-state creep rate for argillaceous halite was adjusted to be slightly greater than the rate for clean salt. The final change was to include a description of primary (transient) creep in the constitutive model to represent initial deformations during and after underground mining activities. Closure estimates using the new creep model were shown to be in agreement with a much larger portfolio of subsequent closure data, avoiding the need for adjustment of elastic constant values. Munson's model has been the basis of much of the more recent SPECTROM-32 analyses. Specification of the parameter values used in the SPECTROM-32 model will be deferred until the next section on backfill consolidation because they are also part of the backfill model.

Since Munson's model appears to overcome the limitations of Krieg's reference creep law, its inclusion in SANCHO calculations would be useful, particulally with regard to benchmarking comparisons of results from the two different codes. Incorporation into SANCHO has been difficult, however, and central processing unit (CPU) times for calculations using this model have increased to the point where routine calculations with it are prohibitive. This situation is complicated by the fact that even with the simpler E/12.5 approximation, the computer time required for complex SANCHO calculations involving waste, backfill, and gas has increased to the point where the feasibility of any more than several calculations for each type of problem has become questionable. Typical CPU times for detailed disposal room closure problems are several hours or more of CRAY computer time (Stone and Argüello, December 17, 1991, memo in Appendix A). Thus, while the E/ 12.5 approximation is an oversimplification of salt creep response, it has been widely used. Justification for its use is that it appears to provide predictions that are conservative in the sense that they produce less rapid closure than the closure histories obtained from SPECTROM-32 with Munson's constitutive relation. Less rapid closure means higher permeability, and if demonstration of compliance is possible under these circumstances, then refinement of the model will imply even lower permeabilities, assuring even better compliance.

The procedure of reducing the value of an elastic modulus or increasing the initial elastic strain of a constitutive model to acquire better agreement with creep closure data from underground openings in salt has been reported several times in the literature (Munson, September 19, 1990, memo in Appendix A). Because of its critical influence on WIPP closure results, the implications of the E/12.5 reduction have been examined by both Morgan and Krieg (1990) and Munson. For example, Morgan and Krieg (1990) concluded from a series of calculations of closure of a vertical shaft the success of the E/12.5 reduction could not be explained by the hypothesis that it was more representative of the elastic response of the damaged or microcracked rock adjacent to the surfaces of WIPP excavations. Nor was a second hypothesis that the degraded modulus increased the elastic strains in the salt mass remote from the room surfaces to the extent 
that the integrated effect produced increased closures valid. Morgan and Krieg's study did reveal, however, quite different unloading behavior around openings in rock salt than was observed in calculations without the reduction.

Munson observes that the E/12.5 assumption in closure analyses has the effect of representing the complex transient-strain portion of the salt creep response with a large, instantaneous step function elastic strain at time zero; i.e., all of the transient strain appears instantly upon application of the stress, rather than gradually with time. The reader is referred to Munson's memo (Appendix A) for a detailed analysis of other impacts of this assumption on creep closure predictions, from which the information in this section is derived. According to Munson:

The difference between using an artificially large elastic strain to represent the transient creep strain and using the true transient strain function for a problem with changing stress fields is that the deformations are forced to earlier times with the artificially large elastic strain representation. For the same incremental change in stress the true transient creep strain model will develop strain more sluwly than for the assumed instantaneous elastic strain model. Consequently closures calculated using the instantaneous elastic step function to represent the transient strain will have a greater amount of early curvature than those using the true transient response representation.

In contrast, for later times, the overprediction would decrease with time until the step function response and the correct transient strain function become equal as steady-state creep is achieved. For very late times, assuming that the choice of the modulus reduction factor $(E / 12.5)$ is correct, the calculated total strains should be about the same for either the step function or the correct transient creep strain representations. An exception to this equivalency would be if, after initial stress loading, the stress is reduced, in which case all of the step function elastic strain will "come back out" of the calculation. For the present, however, the expediency of using the E/12.5 approximation is considered to cutweigh the computer time constraints that the more scientifically based model entails, and thus it remains the basis of disposal room analyses with SANCHO.

\subsection{Backfill Creep-Consolidation Model}

\subsubsection{Model Description}

A mathematical model that defines consolidation of two backfill materials, (1) pure crushed salt and (2) $70 \%$ by weight salt and $30 \%$ by weight bentonite, is described in this section. These two materials were the principal backfills proposed for the WIPP prior to 1990 . Based on a comparative study of the performance of both backfills, salt/bentonite has been proposed as the most desirable material for use in WIPP disposal rooms (Butcher et al., 193/a). Information relative to the usefulness of other, alternate backfills considered by the Engineered Alternatives Task Force (EATF) (US DOE, 1991) is not included because the exact nature of these candidates has yet to be established. The model for only one backfill model is described, because with suitable redefinition of material constants it can be applied to both salt-based backfill materials.

Like pure solid salt, crushed salt continues to deform under stress with time. Unlike the deformation of solid salt, which usually is a shearing process that does not alter its density, the deformation of crushed salt is principally volumetric in nature: the density of granular salt usually increases during consolidation as salt grains deform to reduce void volume. The essential requirement for a relationship for backfill consolidation is that the rate of consolidation is dependent on both the stress in the backfill and its porosity, both of which change with time. 
The model currently used for predicting consolidation of backfill surrounding waste in a disposal area was based on data from creep consolidation tests by Holeomb and Shields (1987) on WIPP salt with water added. 1 For a constant applied stress, volume histories from these tests were represented by an empirical equation of the form:

$$
e_{v}=a \cdot \log _{10}(t)+c .
$$

The parameter $e_{v}$ is the volume strain, $t$ is time, and a and $c$ are constants. The volume strain is defined in terms of density as:

$$
e_{v}=1-\rho_{0} / \rho
$$

where $\rho_{0}$ is the initial density of the material to be consolidated, $\rho$ is its density at time $t$, and $e_{v}$ is considered positive for decreasing volume. ${ }^{2}$ While Equation 3.2 .1 is an accurate representation of salt creep consolidation, a consolidation relatiunship that (1) does not explicitly refer to time; and (2) is based on the absolute quantity of density rather than volume strain, which refers to an arbitrary zero state, was considered more desirable for generalized finite-element analysis. Accordingly, Sjaardema and Krieg (1987) replaced Equation (3.2.1) with the differential equation:

$$
\left.d \rho / d t=B_{0} \bullet \exp \left(B_{1} P\right)-1\right] \exp (A \rho),
$$

where $\mathrm{d} \rho / \mathrm{dt}$ is the derivative of density with respect to time, $\mathrm{P}$ is defined as $--1 / 3 \sigma_{k k}$, where $\sigma_{k k}$ is the trace of the crushed salt stress sensor, and $A, B_{0}$, and $B_{1}$ are constants. When gas is present $P$ represents the stress in the skeleton. The value of one is subtracted from the exponential term in this equation to assure a correct limit of zero consolidation rate when the pressure becomes zero. Sjaardema and Krieg applied linear regression procedures to the available data on WIPP salt, calculated constants for Equation (3.2.3), and found that the coefficients of correlation for each of the regression analyses were greater than 0.999 . This observation suggested that the new equation 1 as an accurate approximation of Equation (3.2.1). If the density in Equation (3.2.3) becomes equal to the theoretical solid density for salt, all voids are eliminated, and the backfill is assunied to continue to deform according to the salt-creep model described in Section 3.1.

Equation (3.2.3) is the volumetric creep consolidation model used in both SANCHO and SPECTROM-32. Values for $B_{0}, B_{1}$, and $A$ for pure crushed salt and salt/bentonite are listed in Table 3-1 (Pfeifle, 1991; Pfeifle and Brodsky, 1991) and apply to any backfill with a grain size distribution approximately the same as mine-run WIPP salt (Pfeifle, 1987a; Pfeifle, 1987b). MX80 Granular Volclay bentonite produced by the American Colloid Company was the bentonite used in the salt/bentonite consolidation tests (Pfeifle, 1987a: Pfeifle, 1987b).

\subsubsection{Discussion of the Model}

Because Equation 3.2.3 is based entirely on hydrostatic consolidation data, it does not include the combined effects of hydrostatic and irreversible shear deformation. Consolidation by

1 Water added is defined as salt with at least 0.5 weight percent water.

2 In SPECTROM-32 the volume strain is defined as $e_{v}=\rho_{0} / \rho-1$. 
Table 3-1. Backfill Consolidation Model Parameter Values

Volumetric Creep Consolidation Parameters"

SANCHOI

\begin{tabular}{cc}
\hline Pure Crushed & Salt $/$ \\
Salt & Bentonite
\end{tabular}

SPECTROM-322

\begin{tabular}{cc}
\hline $\begin{array}{c}\text { Pure Crushed } \\
\text { Salt }\end{array}$ & Salt/ \\
Bentonite
\end{tabular}

\begin{tabular}{lllll}
\hline$A\left(\mathrm{~m}^{3} \mathrm{~kg}^{-1}\right)$ & -0.0173 & -0.0345 & -0.0173 & -0.0345 \\
$\mathrm{~B}_{0}\left(\mathrm{~kg}^{\bullet} \mathrm{m}^{-3} \mathrm{~s}^{-1}\right)$ & $1.3 \cdot 10^{8}$ & $1 \cdot 10^{21}$ & $1.3 \bullet 10^{8}$ & $1 \cdot 10^{21}$ \\
$\mathrm{~B}_{1}\left(\mathrm{MPa}^{-1}\right)$ & 0.82 & 0.6 & 0.82 & 0.6 \\
$\rho_{0}\left(\mathrm{~kg} / \mathrm{m}^{3}\right)^{\mathrm{b}}$ & 1400 & 1480 & 1400 & 1478
\end{tabular}

Elastic Constantsc

SANCHO

$\begin{array}{lc}\begin{array}{c}\text { Pure Crushed } \\ \text { Salt }\end{array} & \text { Salt/ } \\ \text { Bentonite }\end{array}$

SPECTROM-32

\begin{tabular}{cc}
\hline $\begin{array}{c}\text { Pure Crushed } \\
\text { Salt }\end{array}$ & Salt/ \\
Bentonite
\end{tabular}

\begin{tabular}{lccll}
\hline$K_{0}(\mathrm{~Pa})$ & $1408^{\mathrm{d}}$ & $1408^{\mathrm{d}}$ & 17600 & 17600 \\
$K_{1}\left(\mathrm{~m}^{3} \bullet \mathrm{kg}^{-1}\right)$ & 0.00653 & 0.00653 & 0.00653 & 0.00945 \\
$G_{0}(\mathrm{~Pa})$ & $864^{\mathrm{e}}$ & $848^{\mathrm{e}}$ & 10600 & 10600 \\
$G_{1}\left(\mathrm{~m}^{3} \cdot \mathrm{Kg}^{-1}\right)$ & 0.00653 & 0.00653 & 0.00653 & 0.00945
\end{tabular}

Deviatoric Creep Consolidation Parameters for Solid Halite

SANCHO1

Pure Crushed

Salt

\section{SPECTROM-322}

Pure Crushed

Salt

\begin{tabular}{lcc}
\hline$A_{c}\left(\mathrm{~Pa}^{4} \cdot 1.9 / \mathrm{s}\right)$ & $5.79 \cdot 10^{-36}$ & $\uparrow$ \\
$\mathrm{n}$ & 4.9 & Munson-Dawson creep parameters \\
$\mathrm{Q} / \mathrm{RT}$ & 20.13 & $\downarrow$
\end{tabular}

a. $d \rho / d t=B_{0}\left[\exp \left(B_{1} P\right)-1\right] \exp (A \rho)$.

b. the solid density of halite was $2141 \mathrm{~kg} / \mathrm{m}^{3}$; the solid density of bentonite was $2700\left(\mathrm{~kg} / \mathrm{m}^{3}\right)$.

c. $K=K_{0} \exp \left(K_{1} \bullet \rho\right), G=G_{0} \exp \left(G_{1} \bullet \rho\right)$.

d. These values are Sjaardema and Krieg's values divided by 12.5 .

e. Sjaardema and Krieg's shear value divided by 12.5 is 848 . The source of the 864 value is unknown.

1. References: Sjaardema and Krieg, 1987; Weatherby, May 17, 1989, memo in Appendix A; Weatherby et al., 1991a.

2. Reference: Callahan and DeVries, 1991.

3. Reference: Ibid. Table 2-1, and assuming a Tresca flow condition. 


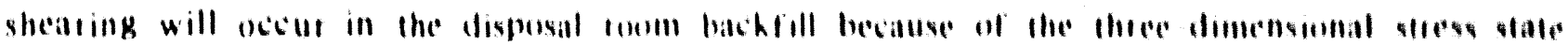

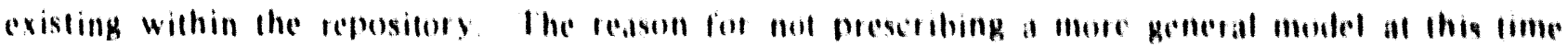

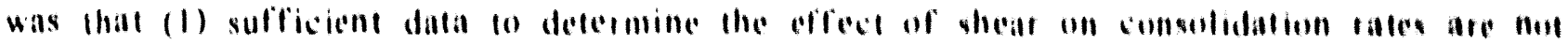

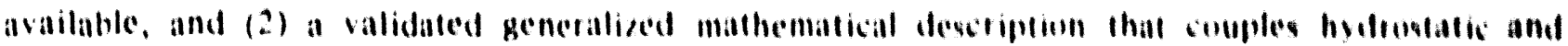

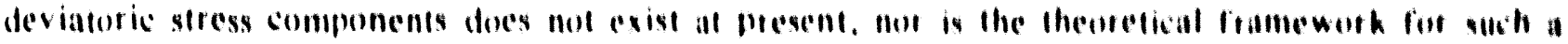
generalized model likely to be available within the ment liew years

However, a justificaloon for ignoring shearing sless in the backfill consolidation mostel can be made from experimental observalions. Consolidation data for many granular materials, as reported in the soil mechanies liferature, have established that combinations of deviatoric and hydrostalic stress canse more rapid consolidation than when consolidation is prescribed as putely

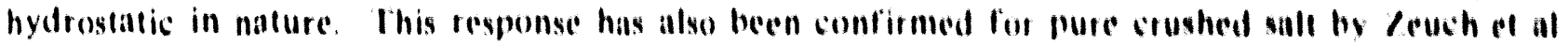
(1991). Zeuch's shear consolidation tests at constant mean stress on WIPl' sall show mone tapid consolidation than under hydrostatic loading. Thus, the omission of the influence of shear sleses

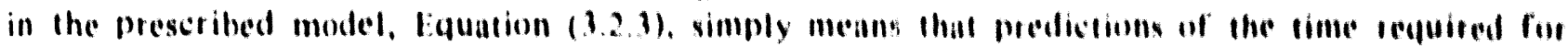
consolidation of salt backfill to a prescribed porosify will always be greilfes than would actually be observed in three-dimensional tests in whish all three princibal stresses were difterent lligher

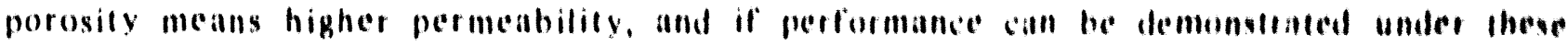
conditions, actual performance is concluded fo be even beller

An interesting contradiction to this discussion is that, although the affects of shear stress have heen cireumvented in development of the backlill consolidation model, the deviatorte response of the backlill must be preseribed in both SANCIIO and SPIC "IROM 12 I0 conform fo their computational method and get them lo run. Thus, while sulficient data lo support the deviatorie part of the consolidation model are not available, assumptions were necessary lo obtain solutions. Quite different deviatoric responses were prescribed for ench code, as destribed in Appendix B. In support of this procedure, computational results, described later in the repont,

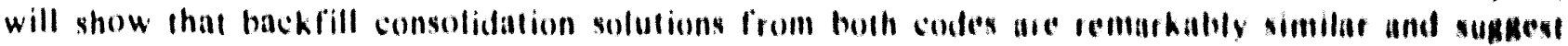
that slosure is very fapid. This observation implies that the different assumplions used for

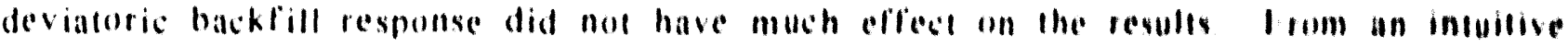
perspective, the immediate conseguence of using only hydrostatle dita lo sonstrust the mescribed WIPP backlill model for salt is that the predicted time for slosure to a specified poressity is expected 10 be an upper bound to actual response. Therefore. the implieation is that if sompliance can be demonserated using this simple model, the actual performance of the bachlill is likely le be aven betler.

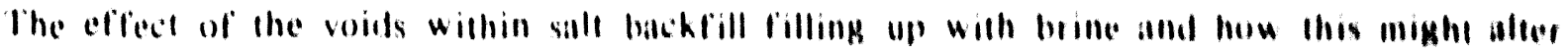

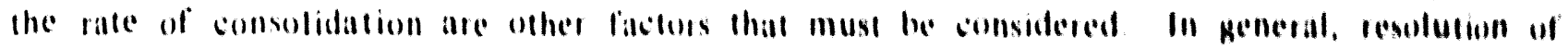

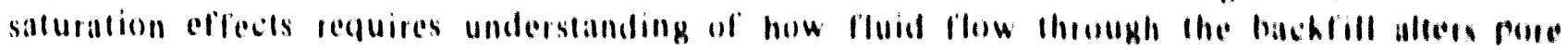

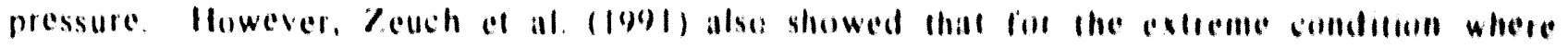

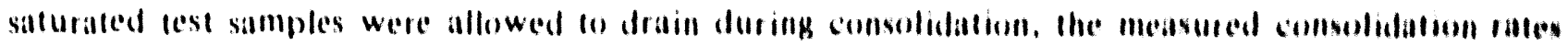
were more rapid than for equivalent unsaturated conditions. lhus, the hvolrostatic model af

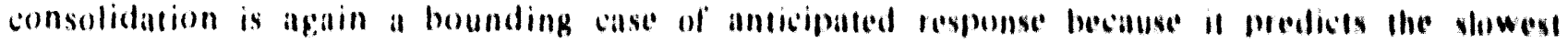
response. Further, the actual allount of water present in lle hachlill cian be assumed lo have litlle affect on consolidation, once corrections for bore pressure using the concept of equivalent sless have been anplied

A rinal comment about the model is that theoredically a granulat salt mixture will eventually consolidate to solid density, given enough time. However, the mathemalleal consolidation mentel deseribed in this section is not considered valid when the porosity of the sald falls below sto

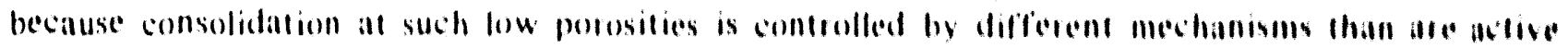


at higher porosities. This limitation is not of concern because at porosities less than $5 \%$ the permeability of the salt backfill is near the permeability of intact salt and therefore is low enough to prevent substantial brine flow through the backfill.

\subsubsection{An Alternate Form of the Consolidation Relation}

Although the relationship between density and pressure in Equation (3.2.3) is the primary relationship used to describe the creep consolidation of backfill materials in SANCHO and SPECTROM-32, Zeuch et al. (1985) have shown that the physical interpretation of volumetric creep is clearer by rewriting the equation in terms of fractional densities and volumetric strain rates. This representation will be used to show how well the model represents experimental results. The current fractional density of the consolidating salt is defined as its current dry density divided by the density it would have if all voids were eliminated (i.e., the crystalline density of the dry mixture). ${ }^{3}$

$$
f=\rho / \rho_{\text {solid }} \text {. }
$$

The definition of $f$ is on a dry mixture basis, even though water or gas may exist within the voids. This convention is adopted because fluids within the voids can eventually be reduced as additional consolidation causes fluid flow out of the WIPP disposal rooms. In contrast, any definition that includes the presence of the fluids would also require the implicit assumption that no fluid motion would occur in order for a unique mathematical solution to exist under prevailing constitutive model assumptions. Another useful simplification in computing values for $f$ is the assumption that the crystalline density is independent of mean stress and therefore can be represented by the density of the unstressed backfill material. This assumption is reasonable because the solid phases in the backfill are so incompressible relative to the large changes in volume that occur during consolidation.

Using this transformation, Equation (3.2.3) becomes an approximately linear relationship between $\log _{10}\left(e_{v}\right)$ and $f$ that can be used to examine the consistency of the empirical model with the data: the data are plotted in a semi-logarithmic plot to see how closely they define a straight line as predicted by the model (Figure 3-1) (Brodsky and Pfeifle, 1992).

To show this relationship, Sjaardema and Krieg's model (1987),

$$
\mathrm{de}_{\mathrm{v}} / \mathrm{dt}=\rho_{0} / \rho^{2} \bullet\{\mathrm{d} \rho / \mathrm{dt}\}=\rho_{0} / \rho^{2} \bullet \mathrm{B}_{0} \bullet\left[\exp \left(\mathrm{B}_{1} \mathrm{P}\right)-1\right] \exp (\mathrm{A} \bullet \rho)
$$

is used to obtain

$$
\mathrm{de}_{\mathrm{v}} / \mathrm{dt}=\left(\mathrm{B}_{0} \bullet \rho_{0}\right) /\left(f \bullet \rho_{\text {solid }}\right)^{2}\left[\exp \left(B_{1} \bullet P\right)-1\right] \exp \left(A \bullet f \bullet \rho_{\text {solid }}\right) .
$$

Taking the logarithm to the base 10 of both sides (base 10 is used for plotting convenience), the expression for the volumetric strain rate is then

$$
\log _{10}\left(d e_{v} / d t\right)=C_{1}+C_{2} \bullet f,
$$

3 The dry crystalline density of pure crushed salt is assumed to be $2140 \mathrm{~kg} / \mathrm{m}^{3}$ (Holcomb and Shields, 1987); the dry crystalline density of a mixture of $30 \%$ by weight bentonite and $70 \%$ by weight pure crushed salt is $2280 \mathrm{~kg} / \mathrm{m}^{3}$, using a value of $2700 \mathrm{~kg} / \mathrm{m}^{3}$ for the theoretical solid density of bentonite (Pusch, 1980). 


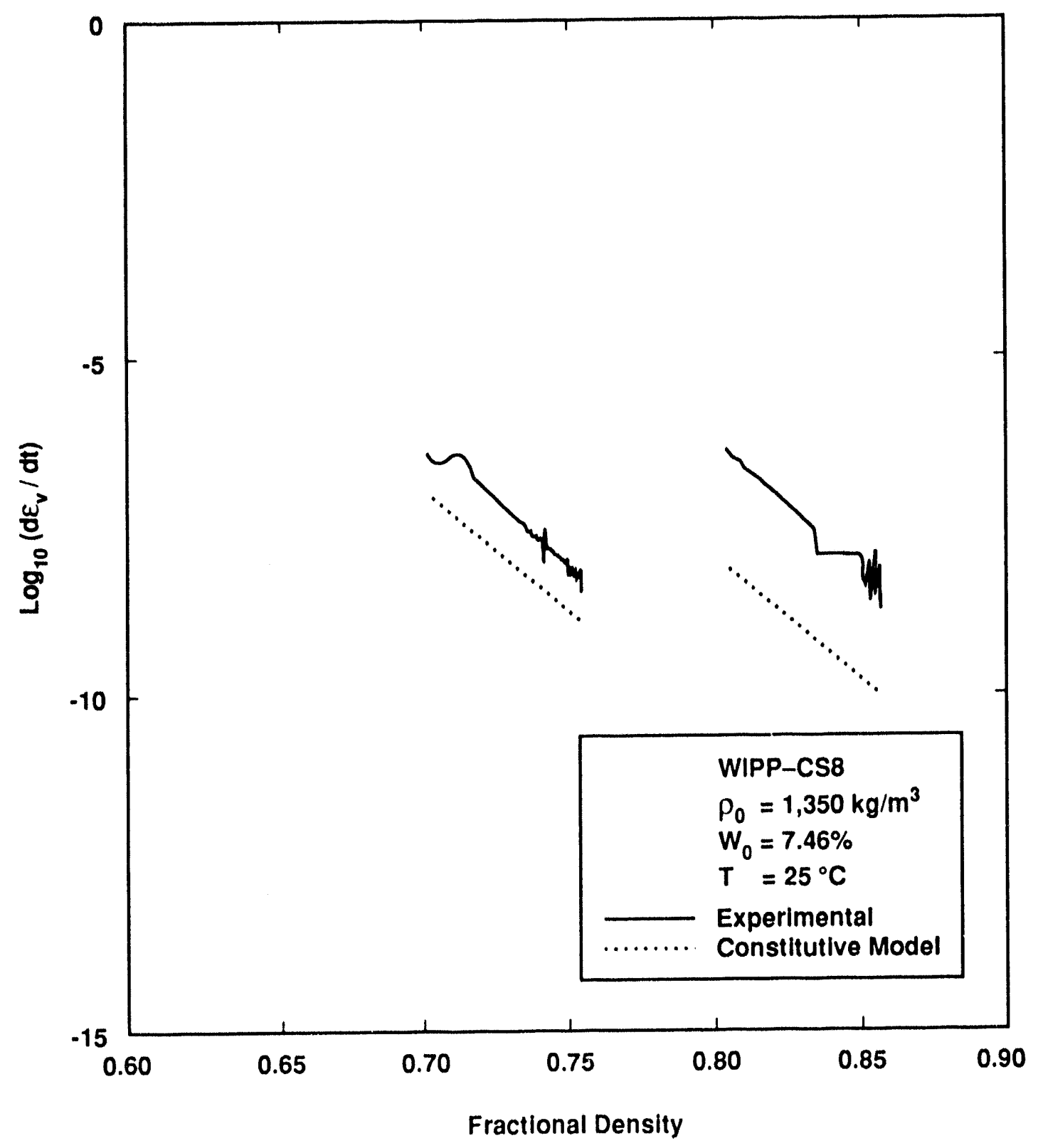

TคI.6345.147.0

Figure 3-1. The relationship between plots of the $\log _{10}$ volumetric strain rate versus fractional density for WIPP test sample CS-8 containing $70 \mathrm{wt} \%$ salt/30 wt $\%$ bentonite and predictions using the Sjaardema and Krieg (1987) model with parameters determined by Brodsky and Pfeifle (after Brodsky and Pfeifle, 1992). The fact that the constitutive model predictions lie below the experimental data in this figure is fortuitous and should not be interpreted as evidence of conservatism of the model. Other examples exist where the model predictions lie above the data. 
with

$$
C_{1}=\log _{10}\left(\left(B_{0} \bullet \rho_{0}\right) /\left(f \bullet \rho_{\text {solid }}\right)^{2} \bullet\left[\exp \left(B_{1} \bullet P\right)-1\right]\right)
$$

and

$$
C_{2}=A \bullet \rho_{\text {solid }} / \log _{e}(10)
$$

While $C_{2}$ is a constant, the term $C_{1}$ varies with $f$ because it contains the factor $f^{2}$. However, in comparison to the changes in magnitude of the term $C_{2} \bullet f$, the variation in value of $C_{1}$ over the usual range of $f,(0.6<f<1)$, is small enough that for many conditions its change can be ignored, and the linear relationship is preserved.

The two typical experimental histories for the consolidation data for salt/bentonite backfill under constant pressure (Brodsky and Pfeifle, 1992) plotted in Figure 3-1 illustrate the linearity that is expected in salt/bentonite backfill consolidation data according to Equation (3.2.7). The observation that the experimental curve for an applied pressure of $3.45 \mathrm{MPa}$ does not superimpose the model prediction is attributed to uncertainty in the stress dependence for consolidation, the term $\exp \left(B_{1} \bullet P\right)$ in Equation (3.2.6). While these comparisons do not include all the data that are available, they show that, on the average, the model is reasonable, at least in terms of its linearity with regard to $f$ at constant stress.

Comparisons such as are illustrated in Figure $3-1$ are made using the original model constants, even if their representation of the data is imperfect. The reason for retention of the original constants is that frequent alteration of the model constants would make comparison of the results of ongoing calculations exceedingly difficult. Updating the material constants is planned only if new data differ significantly from the model, which has not been the case so far, or if a more accurate description is required for performance assessment, which currently does not appear to be necessary.

\subsubsection{CRUSHED SALT BACKFILL MIXTURES}

Equation (3.2.5) has also been used to examine the consolidation of dilute mixtures of nondeforming materials. The objective of this task was to determine how additives to the backfill might change its time-dependent consolidation response. Additives could be (1) materials to limit the amount of gas in the disposal room or (2) shredded metal waste mixed with crushed salt to reduce void volume. Shredding hard-to-deform geometrical shapes of metal waste and embedding the pieces in a deformable medium such as salt is of interest because consolidation would be much more complete than for unprocessed metal waste.

For two non-interacting components, Equation (3.2.5) becomes (Callahan and DeVries, 1991)

$$
\left.\mathrm{de}_{\mathrm{v}} / \mathrm{dt}=\rho_{0} \mathrm{~m}_{\mathrm{B}} / \rho^{2} \cdot \mathrm{B}_{0} \bullet \exp \left(\mathrm{B}_{1} \mathrm{P}\right)-1\right] \exp (A \bullet \rho)
$$

where $\rho_{0}$ is now the initial density of the mixture, $\mathrm{de}_{\mathrm{v}} / \mathrm{dt}$ is the volume strain or compaction rate, and $m_{s} i$ i: the mass fraction of the crushed salt. Olsson (November 7, 1991, memo in Appendix A) recently compared estimates using this equation with experimental results for a mixture of steel disks mixed "rith crushed salt. Test results showed that for mass fractions of steel of 0.44 (solid volume fraction of 0.18 ), the linearity of the variation of the compaction rates with fractional density was fairly represented. However, the observed volume strain rates at any given fractional 
density were two orders of magnitude less than the predicted rates at equivalent pressures. Thus, mixture theory, as applied here, is inadequate for mixes containing nearly $20 \%$ by volume of an incompressible additive, but this conclusion does not rule out its application to more dilute mixtures.

\subsubsection{Backfill Constitutive Equations}

The elastic response of the consolidating medium and how it creeps under deviatoric stresses must also be specified to complete the mechanical constitutive equation. The full set of constitutive equations used in SANCHO for backfill consolidation is described in Appendix B, as is some of the basis for the constitutive equations for SPECTROM-32. Material constants for the respective constitutive equations are summarized in Table 3-1. Studies have shown that the exact method of specification of the elastic response of the various types of backfills and the values used have little influence on compaction predictions (Weatherby and Brown, April 30, 1990, memo in Appendix A; Weatherby et al., 1991a). Therefore, the reader is referred to the documentation of the various models (Callahan and DeVries, 1991; Sjaardema and Krieg, 1987; Weatherby et al., 199/a) for more detail about how elastic constant values were estimated.

\subsection{Waste Compaction}

\subsubsection{Model Requirements}

Mathematical models of the consolidation of unprocessed $\mathrm{CH}$ TRU waste are described in this section. These models were developed by acquiring experimental data from tests in which simulated TRU waste samples were compacted under axial compressive stress within rigid steel dies. Test results were then used to define the composite compaction response of each of the three major types of waste: combustibles, metals, and sludges. Further combination in accordance with the relative amounts of each type of waste in the repository inventory produced an estimated composite compaction curve for the repository.

Compaction curves for the waste are required because the waste compacts with time as the room volume decreases and the waste containers collapse. Most of the unprocessed waste materials have high initial porosities (or void volumes). As compaction proceeds, the reduced porosity of the waste is required to determine decreases in permeability of the waste. In turn, these factors help determine rates of brine transport throughout the disposal room. The porosity of the waste at a given time also determines the amount of soluble radionuclides contained in brine-filled voids within the waste.

\subsubsection{Approach}

The approach used in compaction model development was based on several considerations. First, simulated waste was used because of the difficulties inherent in working with real (radioactive) waste. This approach was justified because the mechanical response of the waste depends entirely on its nonradioactive constituents, such as plastics, cloth, sorbents, etc. The presence of trace radioactive elements has no effect on compaction. 
Second, assembly of samples that exactly duplicated (are "representative" of) real waste was considered impossible because of the compositional variability of TRU waste. The alternative was to adopt the composition of real waste (Butcher, 1989) based on an earlier study by Clements and Kudera (1985) of CH TRU waste drums from the Idaho National Engineering Laboratory (INEL), determine the compaction characteristics of simulations of eacu of these individual components, and then compute their combined response using simple mixture theory (i.e., the assumption is that, given the composition of a waste form such as combus,ible waste, its compaction as a mixture can be defined from knowledge of the compactibility of its components). An additional advantage of the synthesis approach since much of the waste has yet to be generated, is that future changes in waste composition can be accommodated by simple recalculation. This iterative process is applicable not only for the individual types of waste, but also for all the waste in the repository, and can be used to correct for changes caused by updated inventories.

Compaction curves for different waste types were derived from the curves of the individual waste components (Butcher et al., 199/b). In this process, the initial components for testing were defined from limited knowledge of the waste. Materials such as polyethylene and polyvinyl chloride plastics, wood cubes, mixtures of wood cubes and rags, Oil-Dri, vermiculite, portland cement, and mixtures of steel, copper, lead, and aluminum scrap were included. The information from these tests was then used to construct compaction relationships for five dominant waste components:

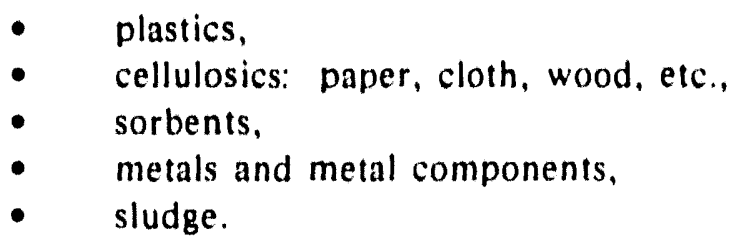

Tests conducted on actual mixtures of each type of waste were found to compare favorably with the estimated consolidation derived from combination of the consolidation properties of individual components.

An additional step in the testing program was to axially compact full-sized 55-gallon drums of simulated combustible, metallic, and sludge waste mixtures (Butcher et al., 199/b). These results served to provide (1) data that could be directly applied to room consolidation and (2) a check of the composite relationships obtained using the properties of individual waste components in a given waste category. Upon reconciliation of these results, compaction relations for combustible, metallic, and sludge waste were constructed, and these in turn combined to estimate an inventory average waste response. These curves are shown in Figure 3-2 and are tabulated in Table 3-2 (Butcher et al., 199/b). An assumption in acquiring data for these calculations was that waste in the form of hard building materials such as bricks or cinder blocks represented a very small portion of the inventory. Additional work will be required if the type of waste to be stored in the repository includes increased amounts of contaminated building materials and scrap metal produced from decommissioning and facility dismantling programs.

\subsubsection{Method of Estimating Composite Compaction Curves}

The state of compaction of the waste at a given stress is obtained by computing the total volumes and void volumes of the individual waste components and adding them together (Butcher et al., 199/b). Assuming that $w_{1}, w_{2}, w_{1}(i=110 \mathrm{n})$ are the weight fractions of the $n$ waste components in a given waste category, and $\rho_{1}, \rho_{2}, \rho_{i}(i=110 \mathrm{n})$ are the respective densities of the 


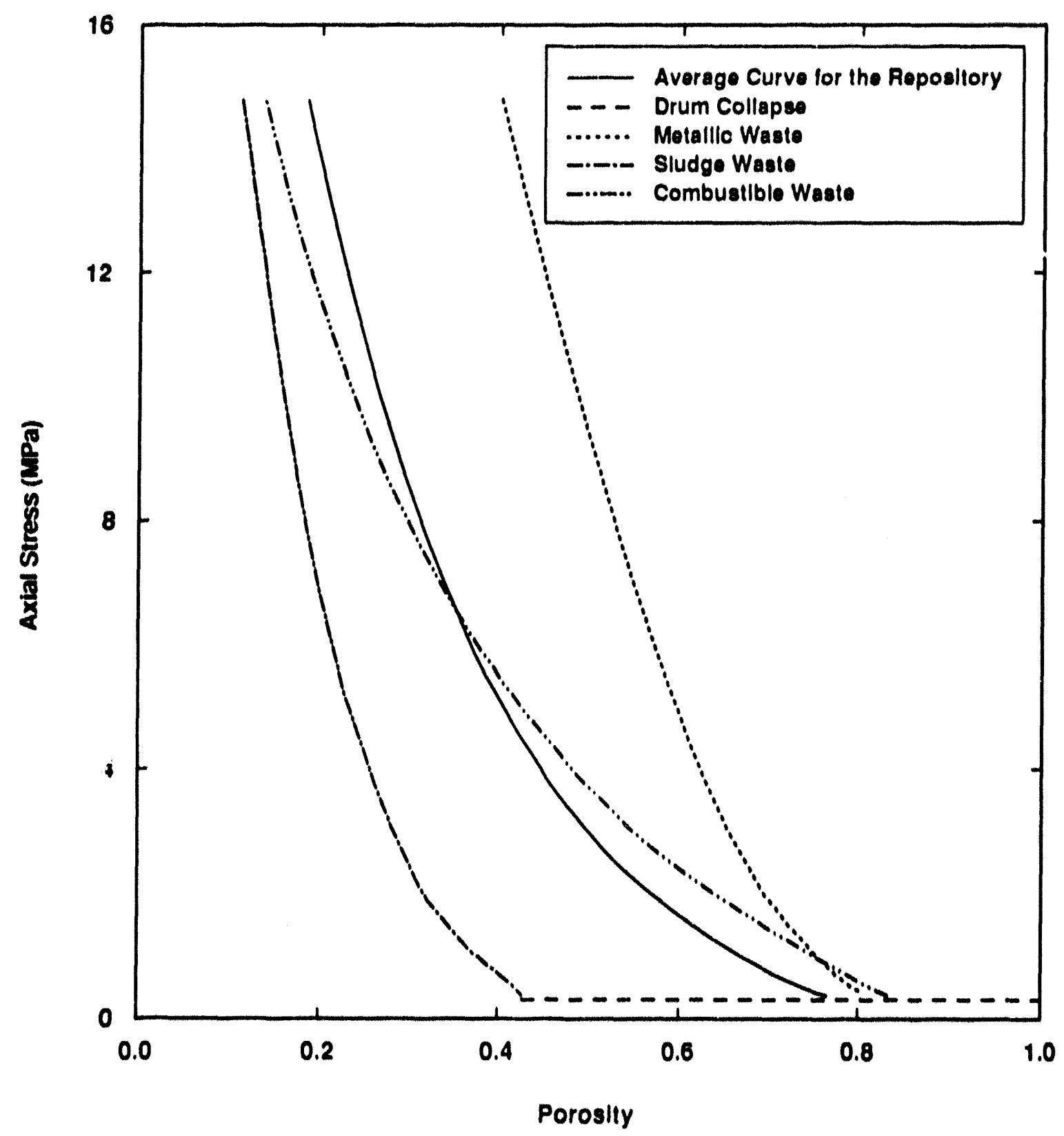

IIII 6345442

Figure 3-2. A comparison between the calculated compaction curve for all the waste in the WIPP repository and the recommended compaction curves for combustible, metallic, and sludge wastes (after Butcher et al., 199/b). 
Table 3-2. TRU Simulated Waste Compaction Curves (Butcher et al., 199/b)

\begin{tabular}{|c|c|c|c|c|}
\hline $\begin{array}{l}\text { Stress } \\
(\mathrm{MPa})\end{array}$ & $\begin{array}{c}\text { Average } \\
\text { Repository } \\
\text { Porosity }\end{array}$ & $\begin{array}{c}\text { Metallic } \\
\text { Waste } \\
\text { Porosity }\end{array}$ & $\begin{array}{c}\text { Combustible } \\
\text { Waste } \\
\text { Porosity }\end{array}$ & $\begin{array}{c}\text { Sludge } \\
\text { Waste } \\
\text { Porosity }\end{array}$ \\
\hline $\begin{array}{l}0.4 \\
0.6 \\
0.8 \\
1.0 \\
1.2 \\
1.4 \\
1.6 \\
1.8 \\
2.0 \\
2.2 \\
2.4 \\
2.6 \\
2.8 \\
3.0 \\
3.2 \\
3.4 \\
3.6 \\
3.8 \\
4.0 \\
4.2 \\
4.4 \\
4.6 \\
4.8 \\
5.0 \\
5.2 \\
5.4 \\
5.6 \\
5.8 \\
6.0 \\
6.2 \\
6.4 \\
6.6 \\
6.8 \\
7.0 \\
7.2 \\
7.4 \\
7.6 \\
7.8 \\
8.0\end{array}$ & $\begin{array}{l}0.765 \\
0.722 \\
0.696 \\
0.672 \\
0.649 \\
0.628 \\
0.607 \\
0.588 \\
0.570 \\
0.553 \\
0.538 \\
0.525 \\
0.512 \\
0.499 \\
0.488 \\
0.477 \\
0.467 \\
0.457 \\
0.447 \\
0.438 \\
0.429 \\
0.421 \\
0.412 \\
0.404 \\
0.397 \\
0.390 \\
0.383 \\
0.376 \\
0.370 \\
0.363 \\
0.357 \\
0.351 \\
0.345 \\
0.339 \\
0.334 \\
0.328 \\
0.323 \\
0.318 \\
0.313\end{array}$ & $\begin{array}{l}0.805 \\
0.783 \\
0.768 \\
0.755 \\
0.741 \\
0.729 \\
0.717 \\
0.706 \\
0.696 \\
0.687 \\
0.678 \\
0.670 \\
0.662 \\
0.655 \\
0.649 \\
0.642 \\
0.636 \\
0.630 \\
0.624 \\
0.618 \\
0.612 \\
0.607 \\
0.602 \\
0.596 \\
0.591 \\
0.586 \\
0.581 \\
0.576 \\
0.572 \\
0.567 \\
0.562 \\
0.558 \\
0.553 \\
0.549 \\
0.544 \\
0.540 \\
0.535 \\
0.531 \\
0.527\end{array}$ & $\begin{array}{l}0.830 \\
0.801 \\
0.776 \\
0.752 \\
0.729 \\
0.706 \\
0.683 \\
0.661 \\
0.640 \\
0.620 \\
0.602 \\
0.584 \\
0.567 \\
0.552 \\
0.536 \\
0.522 \\
0.508 \\
0.494 \\
0.482 \\
0.469 \\
0.457 \\
0.446 \\
0.435 \\
0.424 \\
0.414 \\
0.404 \\
0.394 \\
0.385 \\
0.376 \\
0.367 \\
0.358 \\
0.350 \\
0.342 \\
0.334 \\
0.326 \\
0.319 \\
0.319 \\
0.305 \\
0.248\end{array}$ & $\begin{array}{l}0.549 \\
0.410 \\
0.394 \\
0.377 \\
0.363 \\
0.350 \\
0.338 \\
0.328 \\
0.318 \\
0.310 \\
0.302 \\
0.296 \\
0.289 \\
0.284 \\
0.278 \\
0.273 \\
0.268 \\
0.263 \\
0.258 \\
0.253 \\
0.247 \\
0.243 \\
0.237 \\
0.231 \\
0.228 \\
0.224 \\
0.221 \\
0.217 \\
0.214 \\
0.211 \\
0.208 \\
0.204 \\
0.201 \\
0.198 \\
0.195 \\
0.192 \\
0.190 \\
0.187 \\
0.184\end{array}$ \\
\hline
\end{tabular}


Table 3-2. TRU Simulated Waste Compaction Curves (continued)

\begin{tabular}{|c|c|c|c|c|}
\hline $\begin{array}{l}\text { Stress } \\
\text { (MPa) }\end{array}$ & $\begin{array}{l}\text { Average } \\
\text { Repository } \\
\text { Porosity }\end{array}$ & $\begin{array}{c}\text { Metallic } \\
\text { Waste } \\
\text { Porosity }\end{array}$ & $\begin{array}{c}\text { Combustible } \\
\text { Waste } \\
\text { Porosity }\end{array}$ & $\begin{array}{l}\text { Sludge } \\
\text { Waste } \\
\text { Porosity }\end{array}$ \\
\hline $\begin{array}{r}8.2 \\
8.4 \\
8.6 \\
8.8 \\
9.0 \\
9.2 \\
9.4 \\
9.6 \\
9.8 \\
10.0 \\
10.2 \\
10.4 \\
10.6 \\
10.8 \\
11.0 \\
11.2 \\
11.4 \\
11.6 \\
11.8 \\
12.0 \\
12.2 \\
12.4 \\
12.6 \\
12.8 \\
13.0 \\
13.2 \\
13.4 \\
13.6 \\
13.8 \\
14.0 \\
14.2 \\
14.4 \\
14.6 \\
14.8\end{array}$ & $\begin{array}{l}0.308 \\
0.303 \\
0.298 \\
0.293 \\
0.289 \\
0.284 \\
0.280 \\
0.276 \\
0.272 \\
0.267 \\
0.263 \\
0.259 \\
0.255 \\
0.292 \\
0.248 \\
0.244 \\
0.241 \\
0.237 \\
0.233 \\
0.230 \\
0.226 \\
0.223 \\
0.220 \\
0.216 \\
0.213 \\
0.210 \\
0.207 \\
0.204 \\
0.201 \\
0.198 \\
0.195 \\
0.192 \\
0.189 \\
0.186\end{array}$ & $\begin{array}{l}0.523 \\
0.518 \\
0.514 \\
0.510 \\
0.506 \\
0.502 \\
0.498 \\
0.494 \\
0.490 \\
0.486 \\
0.482 \\
0.478 \\
0.475 \\
0.471 \\
0.467 \\
0.463 \\
0.459 \\
0.456 \\
0.452 \\
0.448 \\
0.445 \\
0.441 \\
0.438 \\
0.434 \\
0.430 \\
0.427 \\
0.423 \\
0.420 \\
0.417 \\
0.413 \\
0.410 \\
0.406 \\
0.40 .3 \\
0.199\end{array}$ & $\begin{array}{l}0.291 \\
0.285 \\
0.278 \\
0.272 \\
0.266 \\
0.260 \\
0.254 \\
0.249 \\
0.243 \\
0.238 \\
0.232 \\
0.227 \\
0.222 \\
0.217 \\
0.212 \\
0.208 \\
0.203 \\
0.198 \\
0.194 \\
0.190 \\
0.185 \\
0.181 \\
0.179 \\
0.173 \\
0.169 \\
0.165 \\
0.162 \\
0.158 \\
0.194 \\
0.151 \\
0.147 \\
0.144 \\
0.140 \\
0.117\end{array}$ & $\begin{array}{l}0.181 \\
0.179 \\
0.176 \\
0.174 \\
0.171 \\
0.169 \\
0.167 \\
0.164 \\
0.162 \\
0.160 \\
0.157 \\
0.155 \\
0.153 \\
0.151 \\
0.149 \\
0.147 \\
0.145 \\
0.143 \\
0.141 \\
0.139 \\
0.137 \\
0.135 \\
0.133 \\
0.131 \\
0.129 \\
0.128 \\
0.126 \\
0.124 \\
0.122 \\
0.120 \\
0.118 \\
0.117 \\
0.119 \\
0.113\end{array}$ \\
\hline
\end{tabular}


waste at the assumed stress, the volume of each component per unit weight of the mixture is its weight fraction divided by its density, $\rho_{i}$ :

$$
V_{i}=w_{i} / p_{i}
$$

Weight fractions were 0.28 for combustible waste, 0.28 for metallic waste, and 0.44 for sludge (Butcher et al., 1991). The total volume of all the components per unit weight of the mixture is

$$
v-\sum_{1-1}^{n} v_{1}-\sum_{1}^{n} w_{1} / \rho_{1}
$$

and the average density of the mixture is $p=1 / \mathrm{V}$. The porosity of the mixture is $\left(1-\rho / \rho_{0}\right)$, where $\rho_{a}$ is the theoretical solid density of the mixture. (This procedure was also used to estimate $\rho_{a}$. the composite solid density of a mixture from the theoretical solid densities of its components.)

\subsubsection{Compaction Models}

The compaction behavior of waste is represented in SANCHO by a volumetric plasticity model with a plecewise linear function defining the relationship between the mean stress, $4,0_{m}$, and the volume strain ev (Weatherby et al., 199/a). This relationship, described further in Appendix $C$, is related to the repository compaction axial stress $\sigma_{n}$ curve shown in Figure 3-3, according to $\sigma_{m}-\sigma_{\mathrm{n}} 3$. Although the devintoric response of the waste has not been characterized, the compaction experiments on 55 -gallon drums of simulated combustible, metallic, and sludge waste showed that the drums do not undergo significant lateral expansion until most of the void space inside the drums has been eliminated (Butcher of al., 199/b). i.e., the thin steel walls of the drums have sufficient strength to elastically confine the waste. The constants in the volumetric plasticity model were defined to sapture this anticipated characteristic. The deviatoric yield function $F$ has the rorm

$$
1=a_{d}=3 a_{m}=0
$$

where $a_{1}=\left(1 / 2 s_{1}, s_{1 j}\right)^{1 / 2}$, with $s_{1 j}$ the deviatoric stress components. The deviatoric response was assumed to be clastic-perfectly plastic. Values assumed for the elastic shear modulus and elastic bulk modulus were 222 and $313 \mathrm{MPa}$, which were much larger (stiffer) than would be compuled from the instantaneous slopes of the curve shown in Figure $3-3$.

the stress-strain behavior of the waste is represented in SPLCTROM-32 by a nonlinear elastic model, also based on the assumption that $o_{m}-0_{0} 3$ (Callahan and DeVries, 1991). The nonlinear representation of waste consolidation is described in Appendix C IRE/SPEC Waate Model). The mean stress assumption in SPLCTROM-32, like the SANCHO assumption, is also related to observations of the lateral expansion of 59 -gallon drums of simulated combustible and metallic waste that occurred during compaction. In contrast, if we assume that the waste during compaction was actually under a hydrostatic state of stress, then $o_{r}$ and $o_{\theta}$ would equal $o_{n}$, and $o_{*}$

4 For principal stresses $a_{1}$, $a_{2}$, and $a_{3}$. or $a_{1}$, $a_{g}$, and $a_{n}$, the mean stress $a_{m}-\left(o_{1}+a_{2}+a_{3}\right) / 3$ or $\left(a_{1}+a_{\theta}+0_{2}\right)$ 


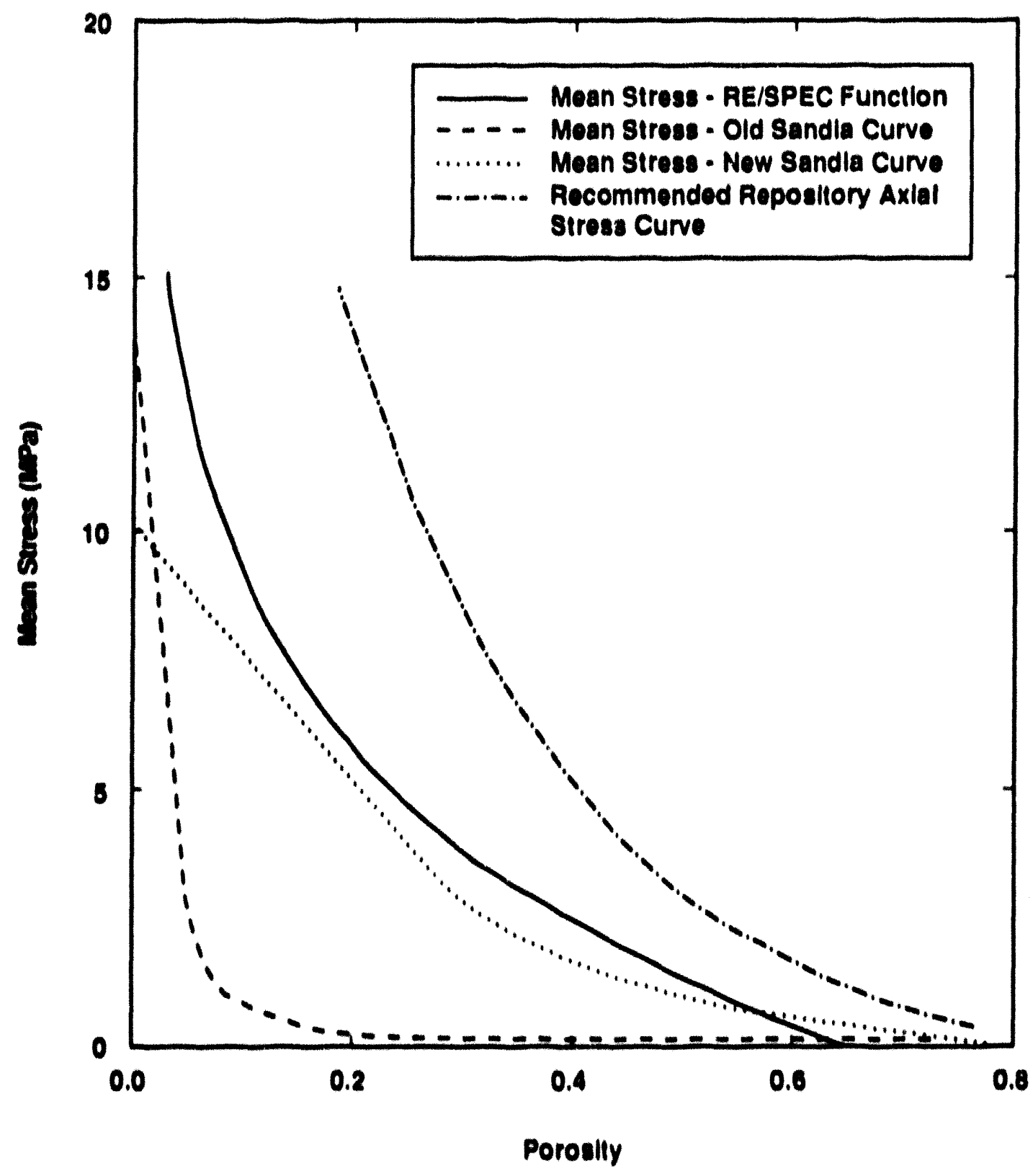

III aMs iss 0

Figure 3:3. Repository waste compaction relationships used by Sandia and RI:SPEC for disposal room slosure analyses (Butcher el al. 199/b; Callahan and DeVries, 1991: Weatherby of al. 199/a). The Sandia mean stress curves are defined in a manner that allows the wasle fo reach 0 porosity at a finile value of the mean stress. The recommended repository axial stress curve is also included in this figure 10 show its relationship Io the mean stress curves. Ideally the mean stress should be one third the axial stress all a given value of the porosily 
- $\sigma_{m}$. Callahan and DeVries recognized that neither assumption was correct and chose the $o_{n}=$ $3 \sigma_{m}$ assumption because it provided the less stiff representation of the TRU waste (Callahan and DeVries, 1991). They felt that the less stiff representation was more conservative because it provided less resistance to room closure and lower back pressure on the surrounding backfill and increased the time required to obtain lower porosities in the backfill surrounding the waste. However, upon additional review, this assumption was not considered valid because the slowest, not the fastest, closure is of interest for evaluating disposal room performance. More recently, the influence of the $\sigma_{\mathrm{a}}=3 \sigma_{\mathrm{m}}$ assumption became evident during efforts 10 reconcile the SPECTROM32 predictions for closure of a disposal room containing waste and crushed-salt backfill consolidation with SANCHO predictions. Accordingly. closure calculations were repeated using the $\sigma_{\mathrm{n}}=\sigma_{\mathrm{m}}$ assumption. These results, described in Section 5.1.3, were found to be much closer to the expected response.

The functional form actually used for describing compaction was

$$
\sigma_{n}=\ln \left(\phi / \phi_{0}\right) / k_{1}
$$

where $\phi$ is the porosity, defined as $\phi=1-\rho / \rho_{\rho}$, with $\rho$ the current density and $\rho_{f}$ the final or theoretical solid density. $\phi_{0}$ the initial porosity, and $\alpha$ a material parameter. Elastic constants were derived directly from this relationship. The compaction curves used in SPECTROM-32 (Callahan and DeVries, 1991) are shown in Figure 3-4. The curve labeled "series," representing elements of the various waste components in series (the same consolidation load acting on each component), appeared intuitively to be more representative of actual compaction conditions and was selected for use. The parallel curve represents elements in parallel (with varying loads applied to each waste component according 10 its portion of the inventory).

\subsection{Disfurbed Rock Zone Effects}

The presence of a disturbed rock zone (DRZ) has been reported near and around WIPP excavations. As mining of the Salado proceeds, halite near the excavation cracks and dilates (increases in cotal volume) during deformation into the excavated cavities. Much of the dilation is altributed to grain boundary loosening acompanied by the formation of microfractures. As deformations continue, however, larger fractures can form at stratigraphic interface separations. The dilation produced by these processes creates a region of enhanced porosity, permeability, and interconnectivity that decreases with distance from the excavation (Stormont, 1990).

A mechanical fluid-flow model of the DRZ has been reported by Stormont $(1990)$ but has not been included as part of disposal room closure analyses. From experimental studies, the following relationships for describing the permeability and the porosity inside the DRZ of an approximately circular excavation have been proposed:

$$
K-K \operatorname{eexp}(-D(Z-r)) \text {. }
$$

where

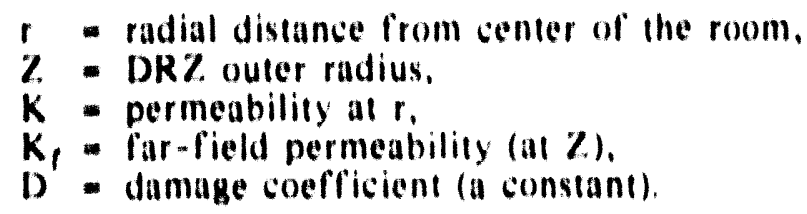




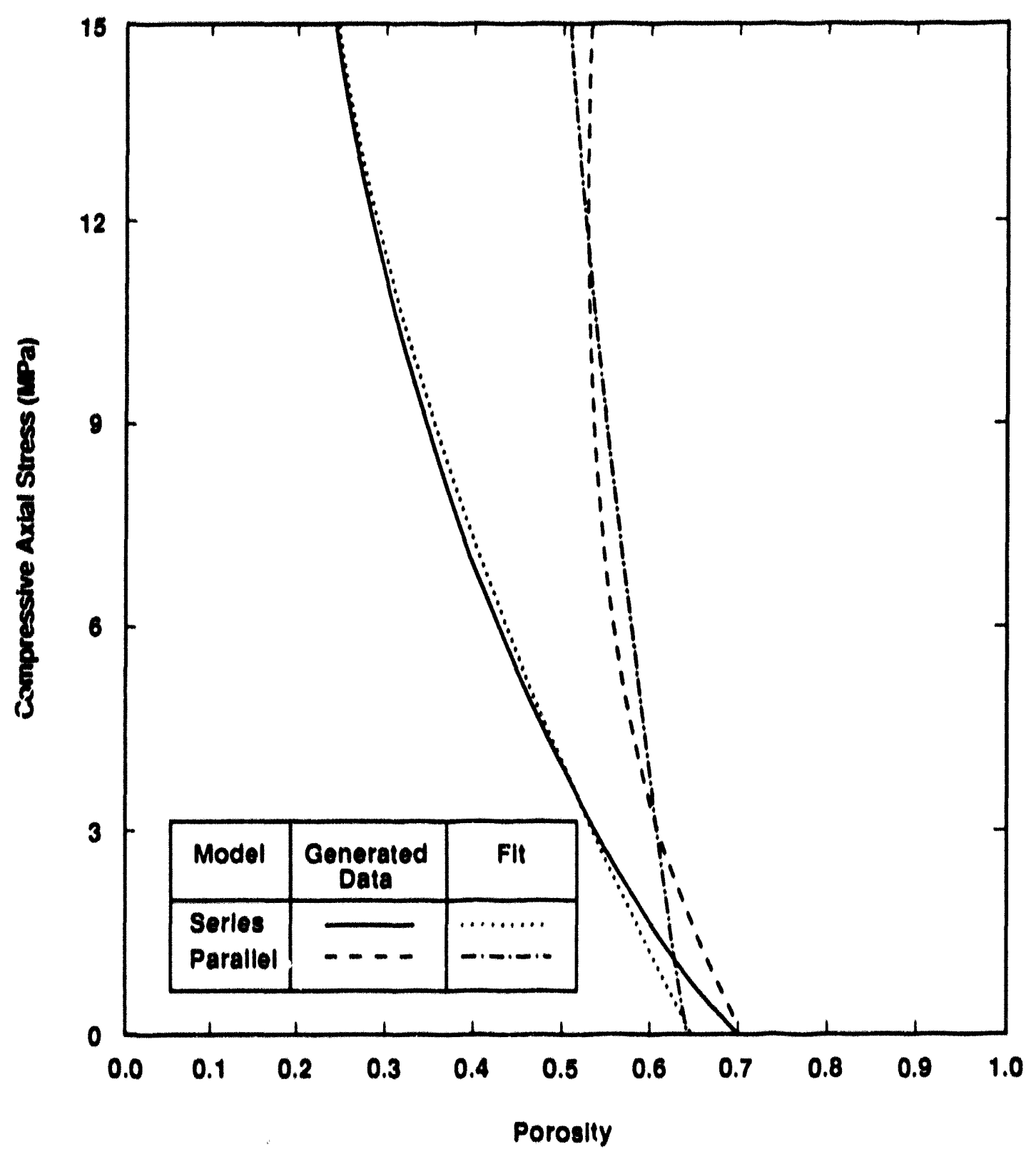

Tค1.6345. 137.0

Figure 3-4. Generated data and fits for composite waste models used in SPECTROM-32 disposal room closure analyses (after Callahan and DeVries, 1991). 
At a given time, the damage coefficient $D$ in Equation (3.4.1) is determined from the permeability at the surface of the circular excavation where $r=r_{0}$, the extent of the $D R Z(Z)$, and the permeability of the far field. The porosity distribution in the DRZ is determined from the variation in permeability:

$$
\phi=\phi_{l} \cdot\left(K / K_{f}\right)^{1 / 3}
$$

where

$$
\begin{aligned}
& \phi=\text { porosity } \\
& \phi_{f}=\text { far-field porosity at } Z \text {. }
\end{aligned}
$$

The DRZ issues of relevance for the disposal room models are related to brine inflow, gas storage, fluid permeability, and room consolidation. To date, the geomechanical models used in room closure analyses do not specifically include any of the DRZ structure because of the expectation that the cracks existing in the DRZ will rapidly close and heal as consolidation proceeds, returning the zone to its original state. This assumption is supported by microcrack healing studies on the WIPP salt (Brodsky, 1990). The rapid healing of the DRZ is also supported by observation that the DRZ is not likely to be any more porous or permeable than crushed salt backfill. Crushed salt backfill consolidates rapidly, as discussed in Section 4.1.2, and in fact, Equation (3.4.2) could be coupled with the salt backfill model (Section 3.2) to provide first approximations of early healing as a function of time. Whether the salt totally or only partially heals is important with regard to gas migration. If only partially healed, the DRZ may allow the pressurization of pre-existing closed fractures in nonhalite interbeds and marker beds within the DRZ, as discussed in Section 3.7.

Another DRZ issue is whether its extent is independent of time, once formed, or whether it is dynamic and expanding in thickness. If it is expanding, then both $K, Z$, and $D$ in Equation (3.4.1) would indirectly become functions of time. Equation (3.4.2) then becomes a much more complicated description of how the rate of growth alters healing processes, and how, in turn, both these processes are related to the presence of either brine or gas as a function of time. These issues also relate to questions of (1) whether brine inflow into the room is a consequence of dewatering occurring within the DRZ, (2) how much gas can be stored in the DRZ, (3) the extent that a component of fluid flow through the repository may eventually occur in the DRZ, and (4) what effect the DRZ presence has on seals placed to limit such flow.

To summarize, past analyses of closure assumed that the DRZ is static and heals rapidly. On this basis, the DRZ is expected to have at most a transient impact on the disposal room behavior, which can be ignored.

\subsection{A Model for Gas Generation}

Gas produced by decomposition of cellulosic waste, corrosion of metals, or radiolysis of TRU waste has always been a subject of interest to the WIPP Project (Lappin et al., 1989, Sec. 4.2). The presence of gas is beneficial in the sense that gas occupies void volume that would otherwise be eventually filled with brine. However, in the opposite sense, gas pressurization could force flow of radioactive brine out of the repository, it could inhibit closure, it could open preexisting fractures and provide paths of easy transport away from the repository, and VOCs could also become entrained in gas and escape from the repository. 
Not including dissolved gas in the formation brine and gas already present in the waste. the three major sources of gas within the repository are:

1. From anoxic metallic corrosion of drums, metal boxes, and metallic constifuents of the waste. These reactions require water and produce large amounts of hydrogen gas. Water availability in the form of brine (brine availability) determines whether these reactions can occur and their rates. Laboratory losts show. for example, that the rate of corrosion of metal waste immersed in brine is orders of magnitude faster than the rate of corrosion of metals exposed to water vapor (WIPp Performance Assessment Division, 1991a, Sec. 1.31.

2. From microbial activity, either aerobic or anaerobic, halophilic of nonhalophilic, consuming cellulosic and other waste materials. Reactions produce tarbon dioxide. and perhaps hydrogen sulfide, methane and nitrogen. Whether these reactions product or consume water is not presently established.

3. From radiolysis, which produces oxygen and hydrogen. Gas production from radiolysis is expected to be negligible, in comparison to the potential amount of gas produced from decomposition and corrosion.

Gas generation is important because it can inhibit closure and therefore increase the permeability of the backfill, waste, and near-field Salado Formation.

Estimation of the types and amounts of gases produced within the repository is extremely complex. Gas generation depends on (1) the type of waste, (2) the amount of brine in contact with the waste, including capillarity/wicking effects, (3) the amount of waste exposed to humid gas, and (4) the composition of the gas already in the room. Both the SANCHO and SPECTROM= 32 codes contain modules used to compute gas generation for a prescribed distribution of hrine. but they lack the capability to directly couple gas pressurization to brine availability (l.e. the balance between brine flow into the repository and brine consumption by the chemical reactions): Further discussion of closure-brine flow-gas pressurization coupling will be deferred until section 6.2 .

Gas pressure in the SANCHO and SPI:C TROM-12 modules is delermined by coupling ans generation description to the ideal gas law. Thus, by defining how much gas has been produced. the gas pressure at any time can be estimated by

$$
P=n R T / V \text {. }
$$

where

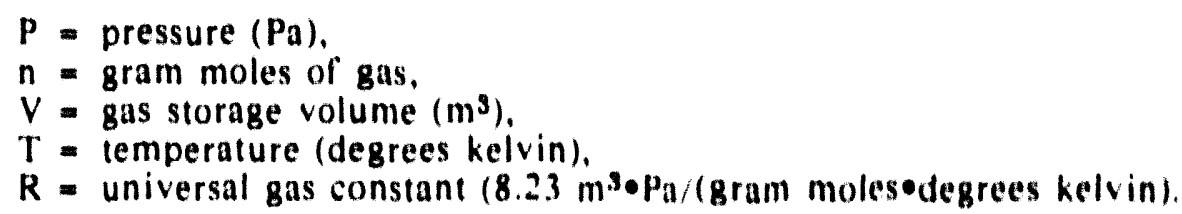

The gas storage volume is defined as the void volume within the room, DRZ, and interbeds, which is easily accessible to the gas, or the void volumes of each of these regions considered independently. Gas-generation rates are embedded in $n$, which represents the amount of gas contained in the void volume region:

$$
n=f \cdot d \cdot \int_{0}^{t} d n / d t d t
$$


where:

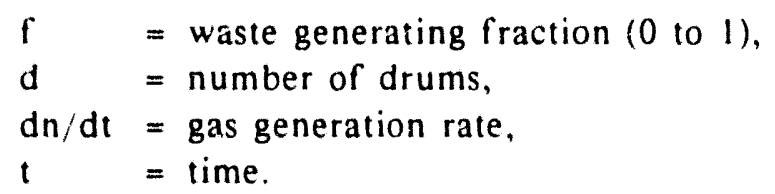

The scaling fraction $f$ is included in Equation (3.5.2) for convenience in varying gas generation rates from the maximum values expected for completely inundated (submerged with brine) conditions. Maximum rates for most of the cases studied were the values suggested in Lappin et. al. (1989) of $2.66 \mathrm{~g}$-moles/drum/year for the first 500 years and $0.86 \mathrm{~g}$ moles/drum/year for 500-700 years. After 700 years, all gas production is assumed to cease. These rates are based on the assumption that the supply of brine in intimate contact with the waste is unlimited, a condition that will be discussed in more detail in Section 6.2. A value of $f=$ $i$ indicates that the maximum rates apply: lesser rates or leakage over the period of gas production are simulated by choosing $f<1$. The duration of gas production is controlled by varying the value of $t$ and altering the values assumed for $\mathrm{dn} / \mathrm{dt}$. The model presented in this section is simple in comparison to the complexity of the gas generation problem, but it is considered to capture the essential features of the effects of gas generation on closure. Further discussion of this topic is deferred to Section 6.2.

\subsection{Fractures}

\subsubsection{Gas Pressurization}

The rate of closure of a disposal room will decrease when the combined backstress exerted by waste and pore pressure becomes large enough to oppose stresses causing salt creep. As the gas pressure in the room rises, the pressure of the gas may actually increase above lithostatic. If pressures above lithostatic do occur, additional gas storage volume will eventually be created.

Calculatic is of the effect of gas on room closure (e.g., Section 5.1.4), for the assumption of a perfectly sealed room, show that the onset of generation of additional volume by expansion of the room actually begins at pressures well below lithostatic. For example, for the case of maximum gas potential (e.g., Section 3.5), the curve in Figure 5-9 that represents the void fraction history of a perfectly sealed room shows that the void fraction decreases to a minimum value (closure stops and the void volume within the room begins to increase) at about 150 years. The gas pressure corresponding to this reversal is approximately $10 \mathrm{MPa}$, well below lithostatic stress of 14.8 $\mathrm{MPa}$.

\subsubsection{REGULATORY IMPLICATIONS OF GAS GENERATION}

The conceptual model of void volume creation or equivalently the question of "where does the gas go?" is of great interest in regard to the performance of the repository (e.g., Lappin et al., 1991). In terms of the radioactive standard (40 CFR 191, US EPA, 1985), the issue can be redefined to whether gas escaping from the repository can accelerate or retard flow of radioactive brine from the disposal rooms. Gas, by opening fractures or rendering marker beds more permeable, could create short circuits through which radioactive brine flows. Three factors tend to moderate this concern: 
1. A gaseous environment may prevent contact of the waste with brine and thus eliminate the principal mechanism for radionuclide migration: transport of soluble radionuclides in brine. In fact, the initial presence of gas is beneficial because flow of radioactive brine is delayed, and any process that postpones brine flow is beneficial to the performance of the repository.

2. Although gas pressurization may open fractures or enhance porosity and permeability of interbeds and other features of the stratigraphy, these conduits will eventually close and be greatly reduced in flow effectiveness if gas leaks away, pressures drop, and brine flow is re-established. Without the presence of gas, the fractures are expected to close until mechanical equilibrium is re-established. This process may even impede future brine flow by partially restoring the formation to its previous state of impermeability. Recovery is expected to be particularly effective in salt because of its time-dependent deformation. While the argument is sometimes made that brine trapped within fractures could hold them open and thus contribute to enhanced flow, this possibility is discounted because the brine cannot go anywhere if the formation is in mechanical and fluid flow equilibrium and therefore enhanced migration of radioactive brine is not possible.

3. Any fracture enhancement of brine migration is likely to occur horizontally rather than vertically because of the orientation of the stratigraphy and is expected to reduce the impact the gas may have on the migration of radioactive brine upwards to the Culebra Formation. Furthermore, horizontal motion of radioactive brine is of lesser concern than vertical motion, which is the most direct path to the surface of the earth.

While void volume creation to accommodate gas is expected to have little impact on repository performance with regard to $40 C F R 191 B$, it is more important with regard to migration of hazardous waste, as defined by the RCRA (specifically 40 CFR 268, US EPA, 1987). Compliance with this standard is necessary because many of the $\mathrm{CH}$ TRU wastes planned for disposal in the WIPP repository are expected to contain small amounts of hazardous components and therefore are classified as mixed waste. The migration of VOCs is of particular concern. The nature of pressure induced volume change is important because VOCs are expected to become entrained in corrosion and/or decompositional gas. Therefore, the burden of performance assessment with regard to the RCRA is to demonstrate that the rates of release of soluble or gaseous VOCs beyond the WIPP boundaries are acceptable, and this, in turn, depends on how much gas moves away from the repository. Thus, compliance with the RCRA requires a credible answer to the question, "where does the gas go?"

The effects of gas mir,ration must also be considered when addressing compliance with the National Environmental Policy Act of 1970 (NEPA). NEPA documentation does not focus on specific regulatory guidelines but involves the use of "best estimate" and "degraded property" sets of input parameters to investigate repository safety. Safety is defined in terms of health risks. Gas migration is important to the NEPA to the extent that gas is a vehicle for transporting VOCs and the small amounts of radon produced by nuclide decay processes.

\subsubsection{Fracture Concepts}

The discussion in the previous section suggests that investigation of gas-driven fracture enhancement of the transport of radioactive nuclides and hazardous gases out of the WIPP repository is necessary to assess compliance with regulatory requirements, especially with regard to the RCRA. Several mechanics issues apply: (1) Is pressurization sufficient to cause fractures to initiate in the host halite? (2) Is pressurization sufficient to cause fractures to initiate in the 
nonhalite interbed material in the Salado? (3) Will pre-existing fractures, assuming they exist, dilate and propagate in either halite or nonhalite materials to relieve pressurization?

A simple fracture model has been developed to explore the behavior of fractures in the Salado and has been applied to two types of response. Case 1 represents an existing (impermeable) fracture in halite or an interbed extending beyond the DRZ "at can open, given sufficient tensile stress, but is not penetrated by gas in its closed state. Because the portion of the crack beyond the $D R Z$ is not penetrated by gas, the pore pressure in this region is zero. Thus, the term "impermeable" is used in the sense that the fracture beyond the DRZ does not transmit gas and create a gas pore pressure unless stress conditions cause the crack to open. This case is, in fact, a test of whether new fractures will initiate in the salt, as discussed further in Section 5.1.4.1.

Case 2 represents a (permeable) fracture in a nonhalite interbed or clay seam, or a discontinuous interface between an interbed or clay seam and halite, where gas can penetrate even when closed. Diffusion of the gas is defined by relationships derived from two-phase flow simulation results (e.g., Section 5.2). The term "permeable" is used in the sense that gas can diffuse along the closed fracture beyond the DRZ.

For the impermeable fracture, gas pressurization does not oceur beyond the first one meter of the fracture layer from the room boundary (the assumed thickness typical of the DRZ) unless the fracture opens. Fracture opening occurs when stress normal to the fracture layer becomes tensile. Gas can then enter. For the permeable fracture model, gas can diffuse into the fracture prior to its opening and, like the impermeable fracture, it too opens if tensile stress is present. However, the increased pore pressure makes opening of the permeable fracture much easier. Once the fracture opens, the open part of the fracture is assumed to be at the same pressure as the gas pressure in the room. This assumption is justified from results of two-phase flow calculations that show that the pore pressure gradients behind the gas penetration front are small near the room (Davies, June 26, and October 4, 1990, memos in Appendix A; e.8., Section 5.1.4.2).

Both horizontally oriented and vertically oriented fractures have been considered for the impermeable fracture analyses; whereas only the horizontal fracture orientation has been considered in the permeable fracture problem. In addition, the impermeable fracture model has been applied using the initial conditions and boundary conditions for a single room in an infinite array of rooms; whereas the permeable fracture model has been applind to both a single room in an infinite array and an isolated room boundary.

\subsubsection{Fracture Constitutive Models}

\subsubsection{IMPERMEABLE FRACTURES}

A simple fracture layer model was developed to simulate the loading introduced by gas pressure acting on the face of a pre-existing fracture or boundaries of a narrow strip of fracture material along the centerlines of a cross section of a disposal room (Argüello et al., 1992). The fractured material was assumed to be unable to support tensile stresses normal to the plane of the fracture. The model is uniaxial in nature and, therefore, only suited for analyzing problems involving layers that run along symmetry planes. Its use is restricted to fractures that run either in the horizontal direction or in the vertical direction. 
In this simulation, the fracture layer is divided into two segments: one segment, within the DRZ, is hypothetically connected to the disposal room and permeable to gas flow; the other segment is initially impermeable to the gas. Within the region that is connected to the disposal room and permeable to the gas flow. the stress normal to the fracture plane is computed from the following constitutive relations:

$$
\begin{array}{ll}
\sigma_{n}=\sigma^{*}-p_{k} & \text { for } \sigma^{*}<0, \\
\sigma_{n}^{*}=-p_{k} & \text { for } \sigma^{*} \geq 0,
\end{array}
$$

where

$$
\begin{aligned}
& \sigma^{*}=\sigma_{n}^{0}+E \bullet c_{n}, \\
& \sigma^{*}=\text { element average of } \sigma^{*} \text { at last time step, } \\
& \sigma_{n}=\text { stress normal to fracture layer, } \\
& \sigma_{n}^{0}=\text { initial normal stress, } \\
& E=\text { Young's modulus, } \\
& c_{n}=\text { normal strain across crack plane, } \\
& p_{n}=\text { disposal room pressure. }
\end{aligned}
$$

The sign convention for this analysis was that tensile stresses were positive and the disposal room pressure was negative. For the fracture segment that is impermeable to the gas, the stress normal to the fracture plane is computed from

$$
\begin{array}{ll}
\sigma_{n}=\sigma^{*} & \text { for } \sigma^{*}<0 \\
\sigma_{n}=-p_{g} & \text { for } \sigma^{*} \geq 0 .
\end{array}
$$

Figure 3-5 illustrates schematically how the constitutive model is defined in both segments of the fracture layer. The fracture layer model is used in conjunction with a quadrilateral element integrated with four-point Gauss quadrature, and hence the average stresses in the above equations refer to the average taken at the four integration points. The stress-strain relations for the axial stress that acts in the plane of the fracture layer and the shear stress that acts across the fracture layer were constructed so that the fracture layer had no axial stiffness, with the consequence that shear distortions of the elements in the fracture layer were minimized. The axial stiffness of the layer was set to zero so that the layer would not limit the flow of salt toward the storage room. The specific relationships used to compute the axial stress and shear stress in the permeable and impermeable regions were

$$
\begin{aligned}
& \sigma_{a}=-p_{s}, \\
& \tau_{\text {an }}=G \cdot \gamma_{\text {an }},
\end{aligned}
$$

where

$$
\begin{aligned}
& \sigma_{\Delta}=\text { axial stress, } \\
& \tau_{\text {sn }}=\text { shear stress across the fracture layer, }
\end{aligned}
$$




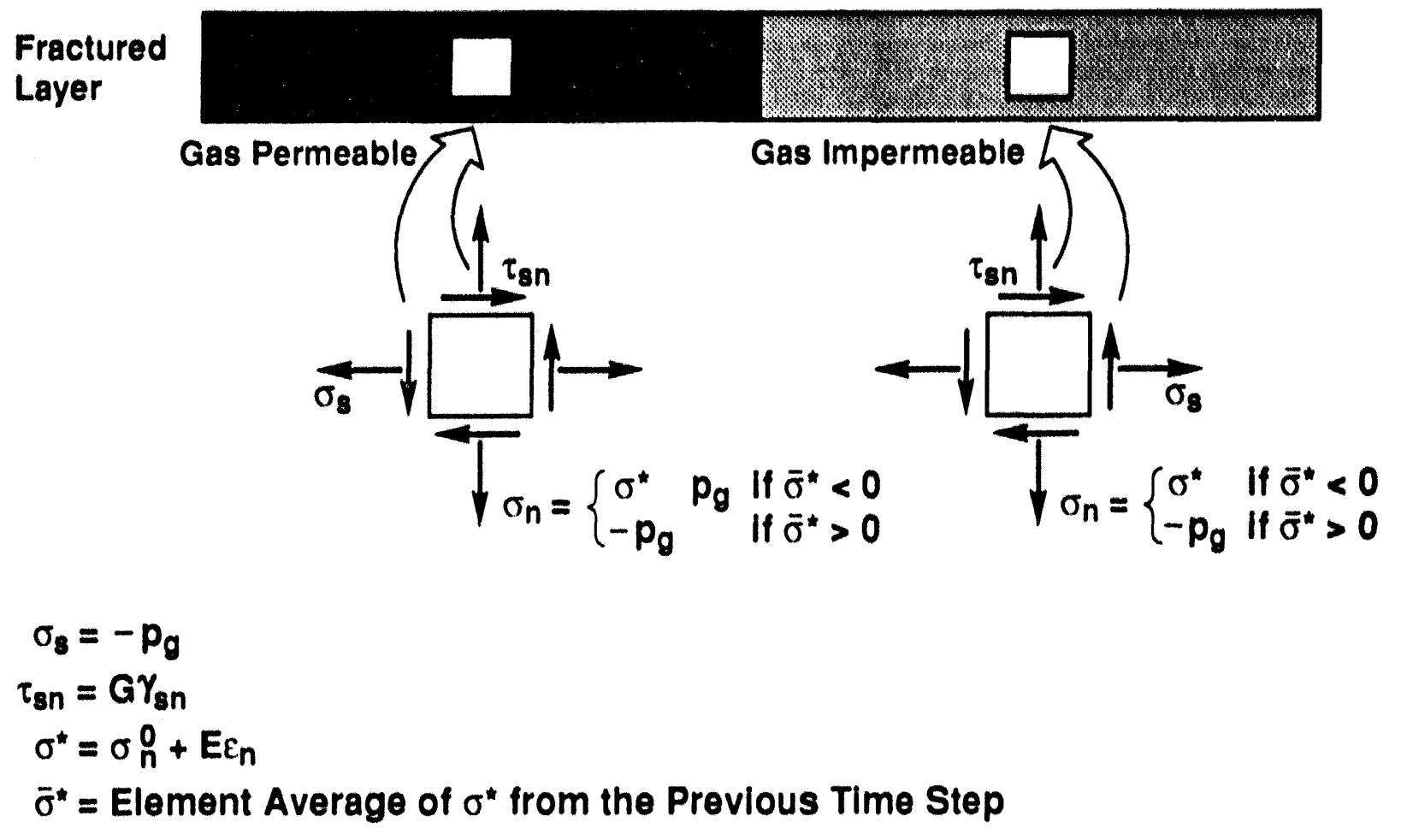

พค1.6340-200.1

Figure 3-5. Schematic illustration of the constitutive model for the gas permeable and gas impermeable segments of the fracture layer (Argüello et al., 1992). 
$\mathrm{G}$ - shear modulus,

$\gamma_{\text {en }}=$ shear strain across the fracture layer.

This model was incorporated into the SANCHO finite-element code.

\subsubsection{PERMEABLE FRACTURES}

The permeable fracture model is a simple extension of the impermeable fracture model. The same relations are used within the region that is connected to the disposal room and permeable to the gas flow, but instead of this region being limited to the extent of the DRZ, its length varies with time. The gas permeable distance is given by the power law

$$
x(t)=1.14 \times 10^{-6.13 .22}
$$

where the distance $x$ is measured in meters from the wall face (Figure 3-5) and $t$ is the time in years. This equation is a fit to the results from an analysis by Davies (1990 memos in Appendix A), where a two-phase flow simulation was performed for an interbed overlaying a waste disposal room, as will be described in Section 5.2. Its applicability is limited to distances less than $600 \mathrm{~m}$ from the disposal room.

\subsection{Thermal Effects}

CH TRU waste does not generate large amounts of heat, and any heat produced is expected to be rapidly dissipated secause of the high thermal conductivity of salt (i.e., localized hot spots within the repository are not expected). Heat loads from $\mathrm{CH}$ TRU waste were not included in the disposal room models because they were considered too small. The thermal loads imposed by RH waste are larger, however. Nevertheless, heat loads from RH waste were not included in the disposal room models because the complexity introduced by inclusion of heat flow in the analysis did not justify the small increase in closure rate caused by elevated temperatures in the salt. This assumption is supported by results from exploratory 3-D thermal stress analyses, by Arguello et al. (1989), which simulated the effects RH TRU canisters emplaced in horizontal unlined boreholes drilled into the walls of disposal room walls would have on closure. One of Arguello's conclusions was that although the temperature of the salt adjacent to the canisters increased by at least $3.5^{\circ} \mathrm{K}$ during the 6-year simulation (2.5-year thermal load duration), changes in closure results caused by thermal effects were almost imperceptible. While a much greater impact of thermal load might have been observed had the calculations been continued in time, the net result is always an increase rather than a decrease in the rate of closure. Furthermore, more rapid closure is considered beneficial to the disposal room performance in terms of 40 CFR $19 / B$ because decreases in permeabilities of the backfill and waste proceed more rapidly. Rapid closure may or may not be a benefit to disposal room performance for RCRA, depending on whether or not it helps to isolate the waste from Grine and therefore minimizes gas production. Omission of RH heating in the disposal room model is justified at present because (1) if adequate performance of the repository can be demonstrated without its consideration, then added complication of including thermal effects in the analyses can be circumvented, and (2) if thermal effects had been included, room performance would be even better than current estimates suggest. 


\subsection{MODELING ISSUES}

\subsection{Two-Dimensional Vereus Three-Dimenelonal Modeling}

The geometrical configurntion of the WIPP is $3-\mathrm{D}$ in nature, ptrticularly with regard to the limited number of rooms in a panel and the intersections of dixposal rooms with access drifis. While a 3-D finite-element structural analysis of the intersection of a typical disposal room and access drift at the WIPP has been performed by Arguello (1990), and a 1-D thermal stress analysis of the effects of heat producing waste on slosure is also available (Arguello et al, 1989), almost all closure analyses for the WIPP must be abstracted $102-\mathrm{D}$ problems. The $2-\mathrm{D}$ anproximation is necessary because 3-D stress analysis codes are not yet available that can (1) utilize the complex salt creep, backfill consolidation, and waste consolidation deformation models needed for closure calculations and (2) still generate solutions in reasonable times.

The equivalence between a 3-D structure and a 2-D model is exnct for iwo special cases:

- In plane stress, the configuration consists of a planar plate of small constant thickness acted un by forces in the plane of the plate only. A state of plane stress paralles to the $x y$ plane is said to exist if

$$
0_{1}=0, t_{x y}=t_{z x}=0, t_{y y}=t_{y y}=0,
$$

where o represents normal stress and, represents sheuring stress.

- In plane strain, corresponding to a constant thickness section of the configuration in which zero strain exists in the direction of its thickness. A state of plane strain parallel to the xy plane is said to exist if

$$
c_{a}=0, r_{x a}=r_{a x}=0, r_{y a}=r_{a y}=0
$$

and in-plane stresses $o_{\mathrm{a}}$ are independent of 2 throughout the thickness. The parameter $a$ represents normal strain and y represents shear strain

The disposal rooms considered in the numerical calculations described in this report are approximately 10 meters by 4 meters by 91 meters. The assumption of plane strain is therefore appropriate for sections of the room perpendicular in: its length and located sufficiently close 10 its midpoint to minimize room end effects fi.e. the rooms are so long that slosure of the cross sectional area of the room at its midpoint is not altered by the 3-1) structure of access ways of drifts at its ends). This configuration corresponds to the plane strain configuration. Case 2 described above. Without fully developed, 1 ) stress analysis codes with fully developed creep and constitutive models, the exact magnitudes of the deviations cnused by ignoring end effects are difficult to estimate. However, the uncertainty caused by ignoring end effects is considered to be less than the uncertainty eaused by other factors influencing closure, such as gas generation.

\subsection{Code Descriptions}

liven with the simplification of two-dimensionality, stress analyses of WIPP disposal room behavior have consistenlly required the most advanced analysis lechniques. As a conseguence. development of new tools. new capabilities, new constitutive equations, and/or new models has been needed for each new WIPP problem. An additional complication is that experimental verification of predictions is unlikely because the calculations extrapolate disposal room response 
thousands of yeats into the ruture

Avourance that computational tesults were teasunable is of utmost concetn

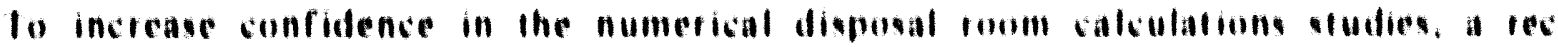

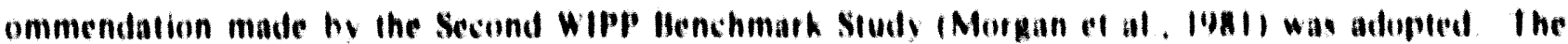
Henchmark Study compated tesulis from the solution ol standati mobleme liv cight dirferent stress analyois codes if estimate code lo code variability the recommendation was that critical design ealculations should be made with mote than one sode therefore, Iwo dirterent contes with different code desctiptons wete used lo obtain sulutions to mans of the problems devetibed in this report. Results for equivalent problems were then sowe chesked and differences used to Iroubleshoot inconsistencies and establish meaningful uncetaints limits for dovure tespense the

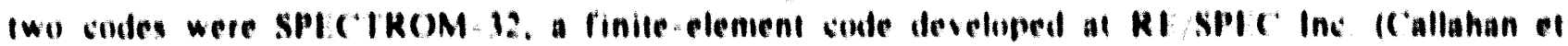
al. 1990 ) and \$ANCIIO, a finile element code develonol at Sandia National I aboratories IStone el al. 19431

\subsubsection{PECTROM-32 Code Doseripllons}

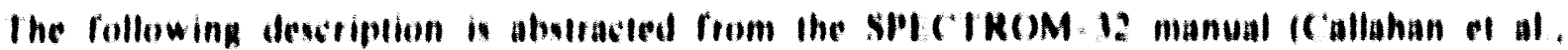

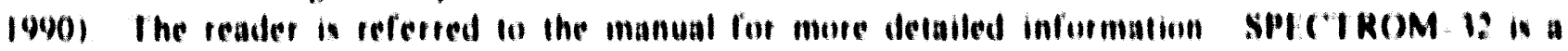

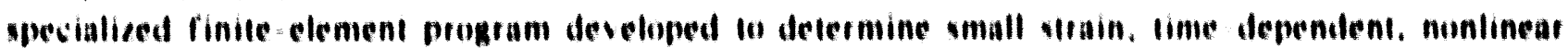

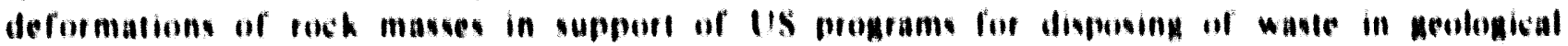
furmations

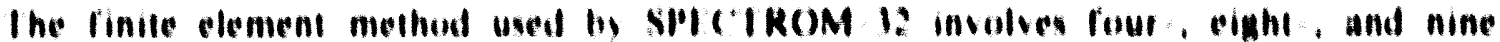
noded inoparametic guadrilateral dements the progem is firmulated uning the direst

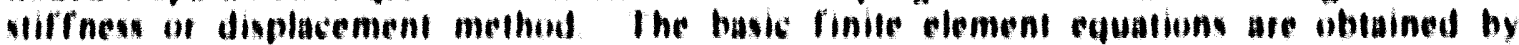

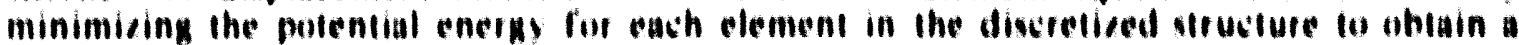
surem of algebrate equations fot each element in lesms of the nodal divplacements for the element and the applied forces acting on it the peinsipal of vitual woth is alou

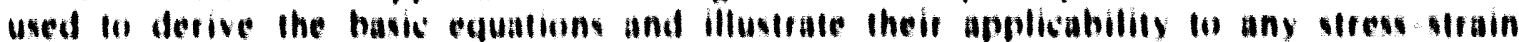

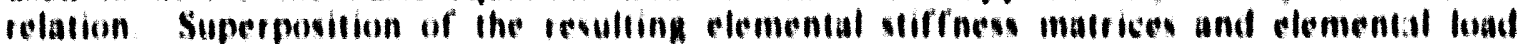

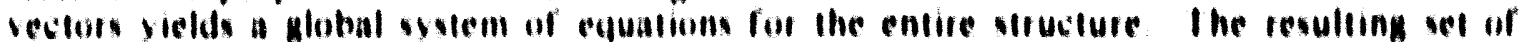

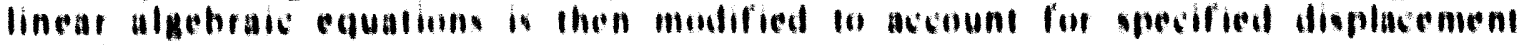
houndary condirions

Ior poblems involving lime dependent deformation. Thowe equations are then whed

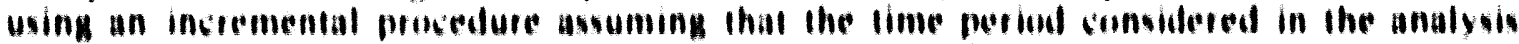

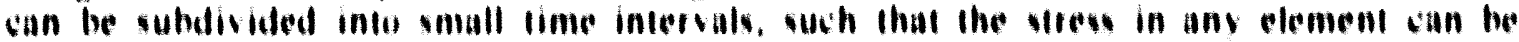

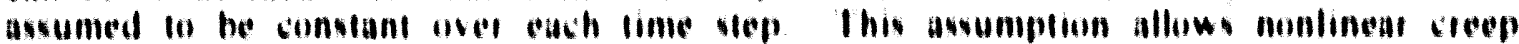

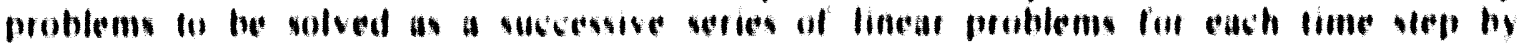

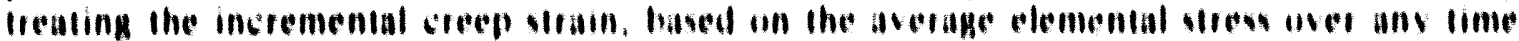

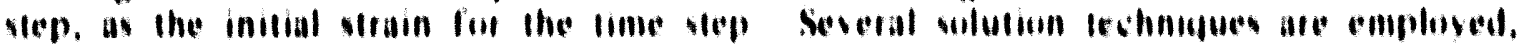

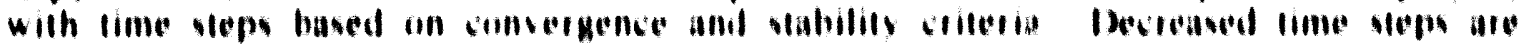
used lis increase the aceutate of the resules

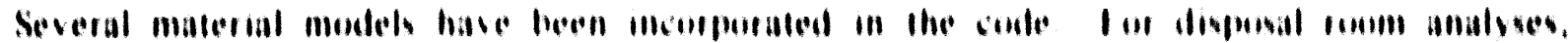

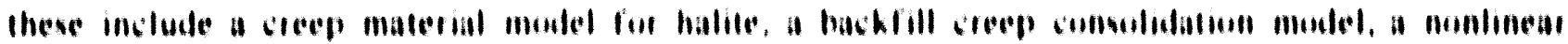

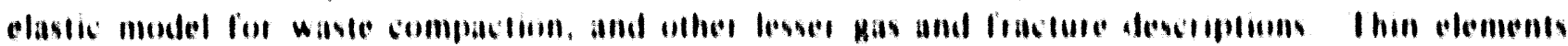

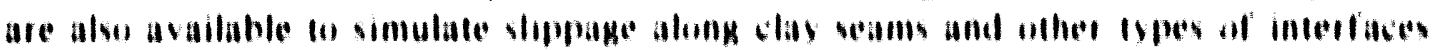

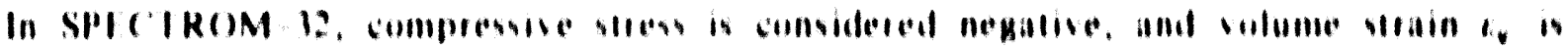
defined as 


$$
c * \omega_{n}+1 \text {. }
$$

where is the current density of a material and on is its inilial density. The current densily is computed from the current volumetric strain, which is definet as the trace of the strain tensor $(i, c, c=6 m)$

\subsubsection{SANCHO Model Doseriptlons}

The following description is Iaken from the SANC110 manual (stone of al. 1985) The reader is referred to the manual for more detailed information

SANCIIO is a special purpose, linite plement program that has been developed to solve the quasistatic. large deformalion (1.e. Iarge train), inelaslic response of iwo dimensional solids. The element library is based on a bilinear isoparameirlic quadrilateral with constani hulk strain. The equilibrium solution slialesy uses an lieralive scheme designed around self adaptive dynamie relasation algotithm the lierative scheme is based on explicil central dirference puedo The code is explicit in nature so that no sliffess matris is formed or factorized, which reduces the amount of sompuler slorage necessary for execution

The SANCIIO code has a standard material model interface that is used with number of material models incorporated wilhin the code For disposal room analyses. these include creep material model for halite, backrill creep consolidation model, a volumeitic plasticity model ifor waste compaction), and other leswer as and fracture descripitons A sliding interface capability is also available to simulate slippage along slay seams and other Iypes of inierfaces In SANCIIO. compressive uress is considered neative and and volume clrain $c_{y}$ is defined as

$$
\text { c. } \log \left(a p_{0}\right) .
$$

where o is the current densily of a material and op is is inilial density

\subsection{Array Aooms $=$ Inilial and Boundary Condillons}

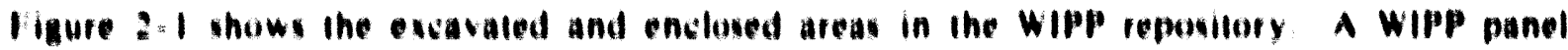

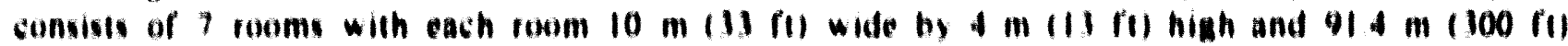
long and separaled by a sall pillar $105 \mathrm{~m} 1100 \mathrm{fll}$ thich Becauce the panel sructure consisis of " ceries of rooms. the usual computarional approash is 10 reduce it 16 a $2: 0$ configuration by considering a disposal room in the center of the panel Plane strain is then ovoked by considering a crose section of the midpoint of a vingle foum in an infinile ariay of identical rooms. oach $10 \mathrm{~m}$ i $m$ ceparated by 10.5 m lhich pillars of sall

\subsubsection{Malf-Room Conflguratione}

The arfay room configuration can be abolracled even further by ovoking symmetoy considerations. Iigure t.l shows a iypical hall foom linile cloment mosh for a 


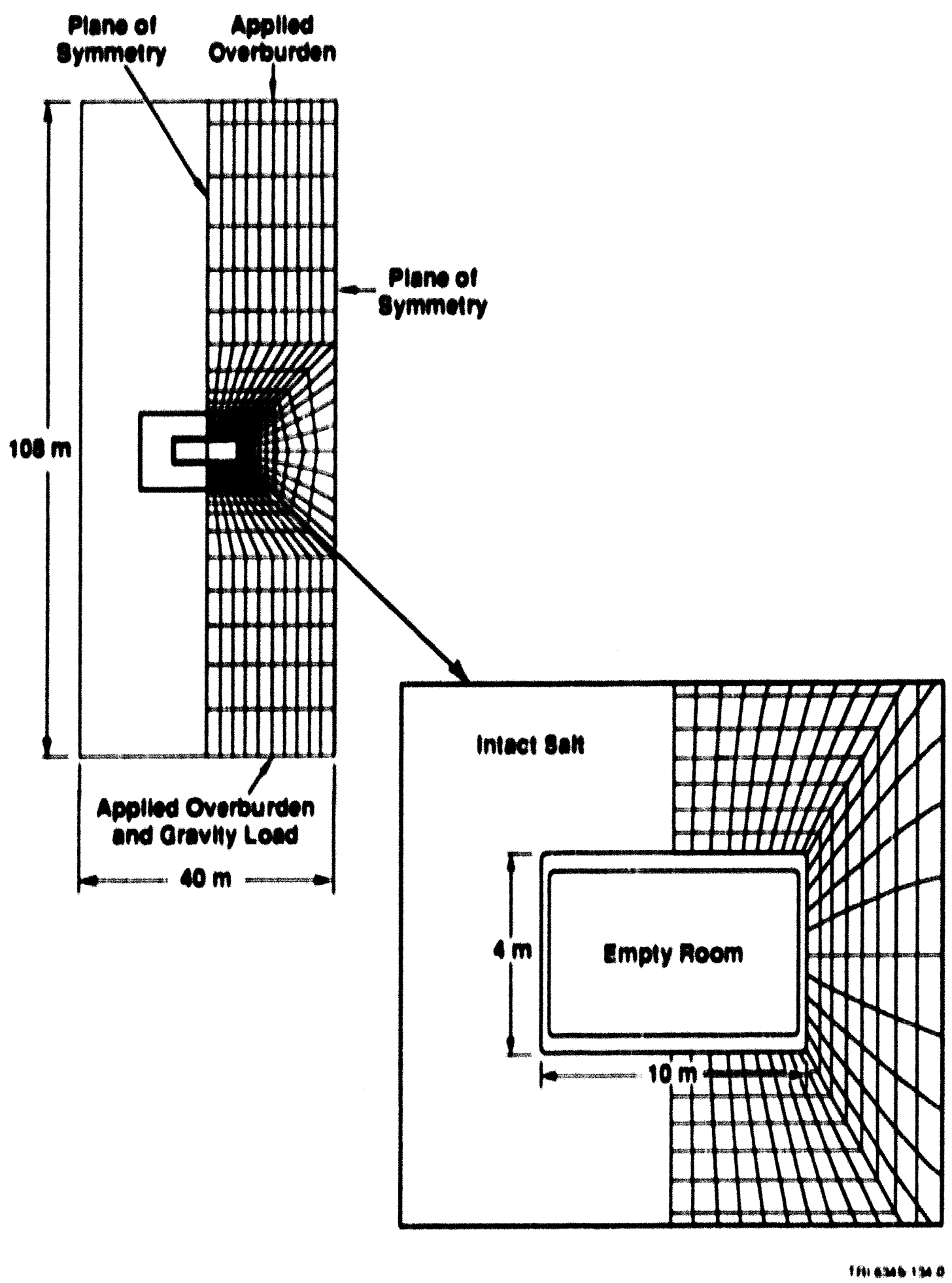

Figure 4 . F. Finite element mosh ured to analyze a half room representation of at emply disposal room (Weatherby, May 17, 1989, memo in Appendix A). 
disposal toom. which mas contain on the order of 1000 elements I In the nast, finile clement problems of this type have required as mush as several hundred minutes of (CPU lime on a CRAY compuler (Stone and Argoello. December 17, 1991, memo in Appendix A). CPU limes of this duration did not permit inclusion of much additional detail in the form of configuration and model refinements to the problems. Ilowever, tecent sode improvements have greatly reduced solution CPU limes to a more manageable duration. The horizontal limits of the half - room finite element mesh are the vertical centerline of the room and the vertical centerline of the adjacent nillar. corresponding to a lotal horieontal length of $20.3 \mathrm{~m}$. The certical planes of symmetry for the half-room define boundary conditions for the nodes along the left and right edges of the mesh. which are lived against horizontal motion.

The vertical limils of the mesh were sel far enough from the room to be unaffected by nonuniform deformations saused by closure. A verlical mesh length of approximalely $50 \mathrm{~m}$ above and below the horizontal centerline of the room was found to be adequate. Pressure boundary conditions wete applied to the top and bottom boundaries of the half-room, with values defined by the somputed lithostatic stress at the respective elevations. Often. the lithostatic pressure was assumed constant throushout the undeformed configuration, selling normal stresses in the horizonial, vertical and out of =plane directions equal to the lithostatic pressure (148 MPa) The inilial stresses for elements inside the disposal room were set equal to zero.

\subsubsection{Quanter-Room Conflgurations}

A quarler - foom is sometimes used inslead of a half = roum to reduce the number of elements even further This additional abstraction requires the assumption that regions containing the waste and hackfill are vellically symmelrle around a horleontal plane passing through the center of room. A lypical quarter foom mesh is shown in ligure 4.2 . While il does not exactly reproduce the actual asymmetric configuration of the room (waste placed on the floor of the roum and covered with backfill). other simplifying approvimations with regard to the room contents? are considered to introduce mote uncertainties into the resulls Quarter * room boundary conditions for the nodes along the left and tight edges of the mesh are that they undergo no horizontal motion. and the bottom row of nodes representing the horizonial line of symmetry through the mid a point of the room is also fixed against motion in the verlical direclion. The pressure boundary condition is applied only to the noder along the top of the mesh

Discusion of how well predictions from 2-D array fooms are representative of end rooms in a veven-room panel are deferred to Section 6.3

I In contrast. a preliminary analyois of a configuration representing an entire panel of empty rooms. described in Section 6.3, may require as many as 23,000 elements (Stone and Arguello, December 17, 1991, memo in Appendix A).

? The terms "lichostatic stress" und "lithostalle pressure" san be used interchangeably in this report because creep deformation of the halise has eliminated all stress gradienis within the undisturbed formation. as cerified by in silu stess determinations (Wawersik and stone. 1989).

3 An example is the assumplion that amount of each lype of waste in the room is exactly proportional to its representation in the total inventory This assumplion is probably incorrect because receipt of waste at the WIPP is not likely to be random, with some rooms containing more of a certain lype of waste than others 


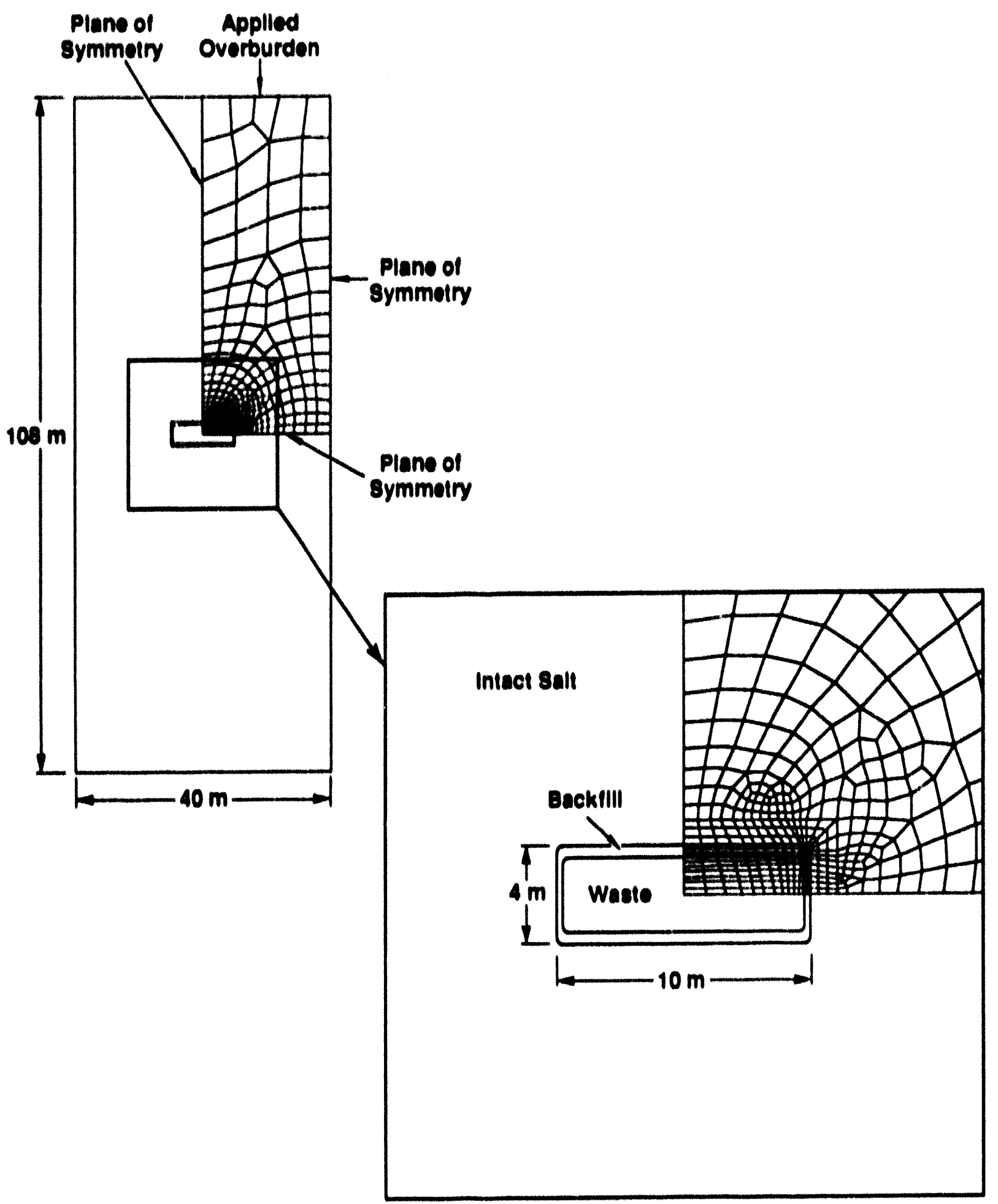

1ค1. 6340114.

Figure 4-2. Finite-element mesh used to analyze a quarter-room representation of an empty disposal room (Weatherby et al., 199/a). 


\subsubsection{Other Assumptions}

An important assumption for simplifying closure calculations was that closure of the disposal room is not greatly affected by the gravitational forces acting on the material in the immediate vicinity of the room. This assumption is supported by early studies showing that the near-field gravitational forces and the local variation in the initial stress field with depth have little effect on closure rates. Thus, the near-field gravitational forces and the variation in the initial stress field were neglected entirely in obtaining the numerical results described in this report.

Another major simplifying assumption for almost all of the calculations was that the stratigraphy surrounding the disposal room could be ignored and the formation considered all salt on the basis of the sensitivity analysis performed by Morgan (December 9, 1987, memo in Appendix A).

\subsection{Isolated Room - Initial and Boundary Conditions}

While the infinite array of rooms is the easiest to model, closure results from such calculations are limited to approximations of the response of the center room of the seven-room panel, where adjacent rooms influence closure. The results are approximations because, while the seven rooms do interact with each other, it is also likely that seven rooms are too few to deform as an infinite array of rooms. In addition, the array approximation does not represent the end rooms of the panel because these rooms have no adjacent rooms on one side of them. The array approximation is even less valid for other drifts and access ways within the panel because they are shorter and have numerous intersections that amplify three-dimensionality.

The opposite to an array room is an isolated room, which has no adjacent openings to influence closure and is a bound to the response of an end room of a panel. In fact, early calculations to investigate the closure of end panel rooms alsu addressed isolated rooms because an isolated-room configuration and an array-room configuration are considered to bracket all possible 2-D closure responses of disposal rooms in a panel.

The 2-D representation of a single isolated room is also a cross section of the room half-way along its length, to avoid end effects (Morgan, December 9, 1987, memo in Appendix A). A typical finite-element mesh configuration is similar to either a half-or quarter-room array configuration. However, in contrast to the array room mesh, the isolated room mesh configurations were larger because there are no vertical symmetry boundaries corresponding to the pillar centerline. Scoping studies have shown that the appropriate distances for the boundaries are 100 meters horizontally and 50 meters vertically, to assure that they do not influence closure during the time period of interest.

The boundary conditions for the isolated room mesh configuration were such that the nodes along the left and right edges of the mesh were fixed against horizontal motion. likewise, the bottom row of nodes was fixed against motion in the vertical direction, and a uniform pressure condition was applied 10 the top to reflect the effect of overburden stress. The lithostatic pressure was assumed constant throughout the undeformed configuration, with normal stresses in the horizontal, vertical, and out-of-plane directions set equal to the lithostatic pressure (14.8 $\mathrm{MPa}$ ).

The assumptions were also made that (1) closure of the isolated disposal room was not greatly affected by the gravitational forces acting on the material in the immediate vicinity of the 
room, (2) that the stratigraphy could also be ignored, and (1) that the rock surrounding the room was all sall (Morgan, Decembet 9, 1987, memo in Appendix 1 )

A discussion of the extent that predictions from a 2-1) isolated roum are representative of end rooms in a seven room panel are deferted to Section 6.3 


\subsection{EXAMPLE PREDICTIONS}

\subsection{Computational Results}

The major objective of numerical predictions of the structural response of a WIPP disposal room is to define the degree of consolidation of the contents with time. This description must include the extent that waste and backfill consolidation inhibits the closure process, as well as the effects of gas generation and/or brine inflow. The products of the model are porosity estimates that are required for performance assessment calculations because (1) the pore volumes, when filled with brine, affect the amount of radionuclides in solution, (2) the porosity defines the permeability of the waste and backfil! at any given time and hence strongly influences the rate of migration of soluble radionuclides to other locations within the repository, and (3) the pore volumes can act as storage volume for gas.

Development of the numerical methods for addressing the closure part of the disposal room model has been accomplished in a series of stages (Argüello et al., 1991) that became increasingly more detailed as better material response models and mathematical methods were available. As mentioned in Section 4.2, a parallel approach involving two different numerical codes was used because calculations in many cases proved to be much more difficult than anticipated, and eccentricities of the numerical techniques frequently meant that results in some cases could not be obtained to satisfy programmatic milestones. The two numerical codes used are the finite-element, finite-strain code SANCHO (Stone et al., 1985) and the finite-element, small-strain code SPECTROM-32 (Callahan et al., 1990). Although the codes initially appear to be similar because they both utilize finite-element techniques to solve stress analysis problems, they are significantly different in several respects (Butcher et al., 1991a), as will become evident in the following discussion.

\subsubsection{Empty Room Calculations}

The simplest closure calculations addressed the creep response of empty rooms in salt over short periods of simulated closure. These results were used for comparisons with early room closure measurements that were becoming available from underground tests at the WIPP Site. As confidence in the numerical predictions increased, calculations were extended to longer times to investigate the performance of the repository (Ehgartner, 1990; Morgan, June 2, 1987, memo in Appendix A; e.g., Appendix D, Calculations $($ to 3 ). Conditions assumed in these calculations are summarized in Table 5-1; results digitized from the original references are shown in Figure $5-1$.

A first estimate for the length of time for room closure was possible from the empty room closure results by assuming that the backfill and waste within the room would offer no resistance to closure and would simply collapse to increasingly denser states as the total volume of the room decreased. Thus, information about the rate that an empty room closed and the amounts and theoretical solid densities of waste and crushed-salt backfill within the room was sufficient to compute void fraction (porosity) variation with time (Lappin et al., 1989). Results for void fractions below about 20\% were ignored because the assumption of zero backstress was clearly questionable at such dense states. Definition of the average void fraction (porosity) of the disposal room contents as a function of time and the relationship between the porosity and permeability were sufficient to estimate an average permeability for the room contents and to infer rates of brine flow. 


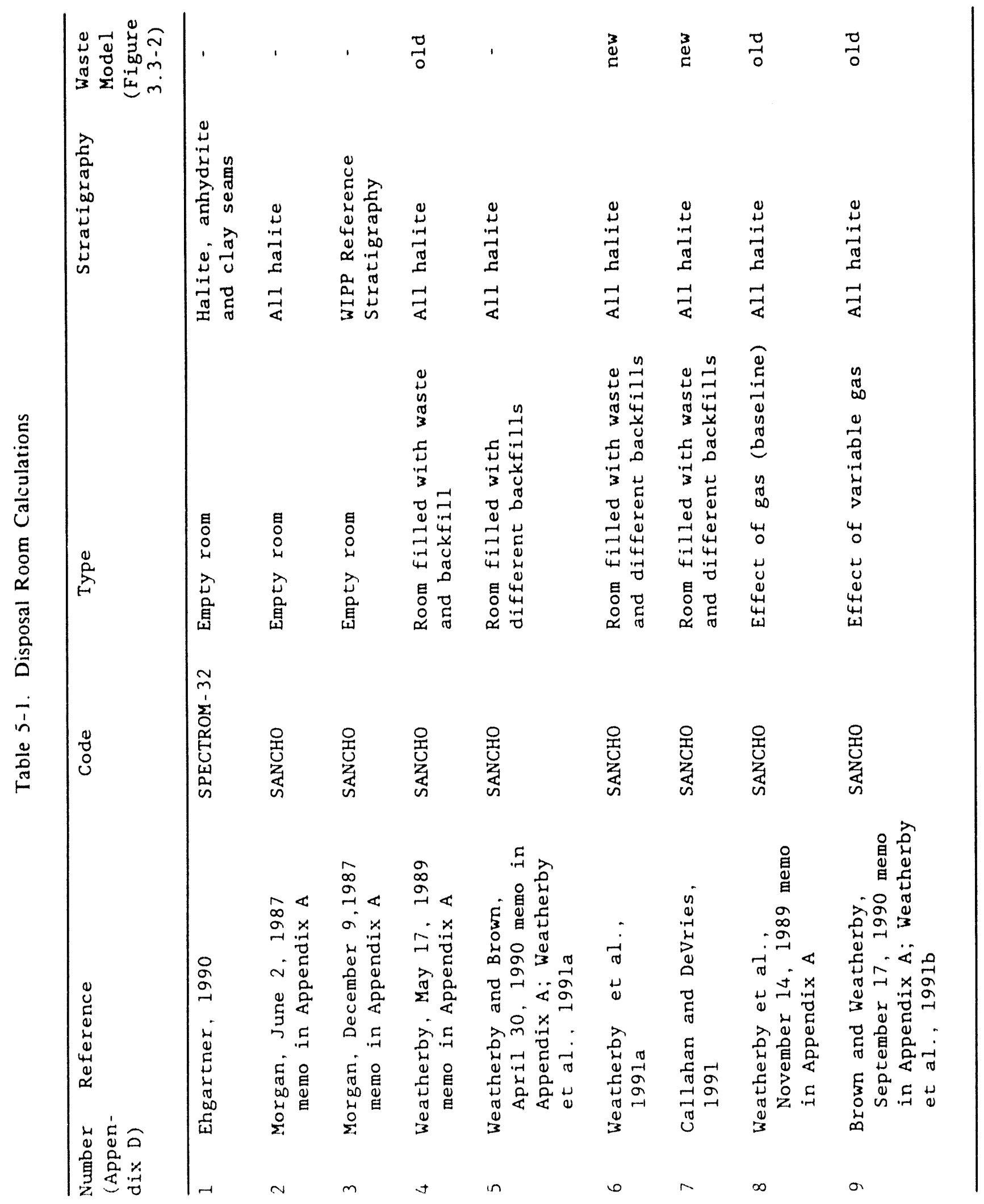




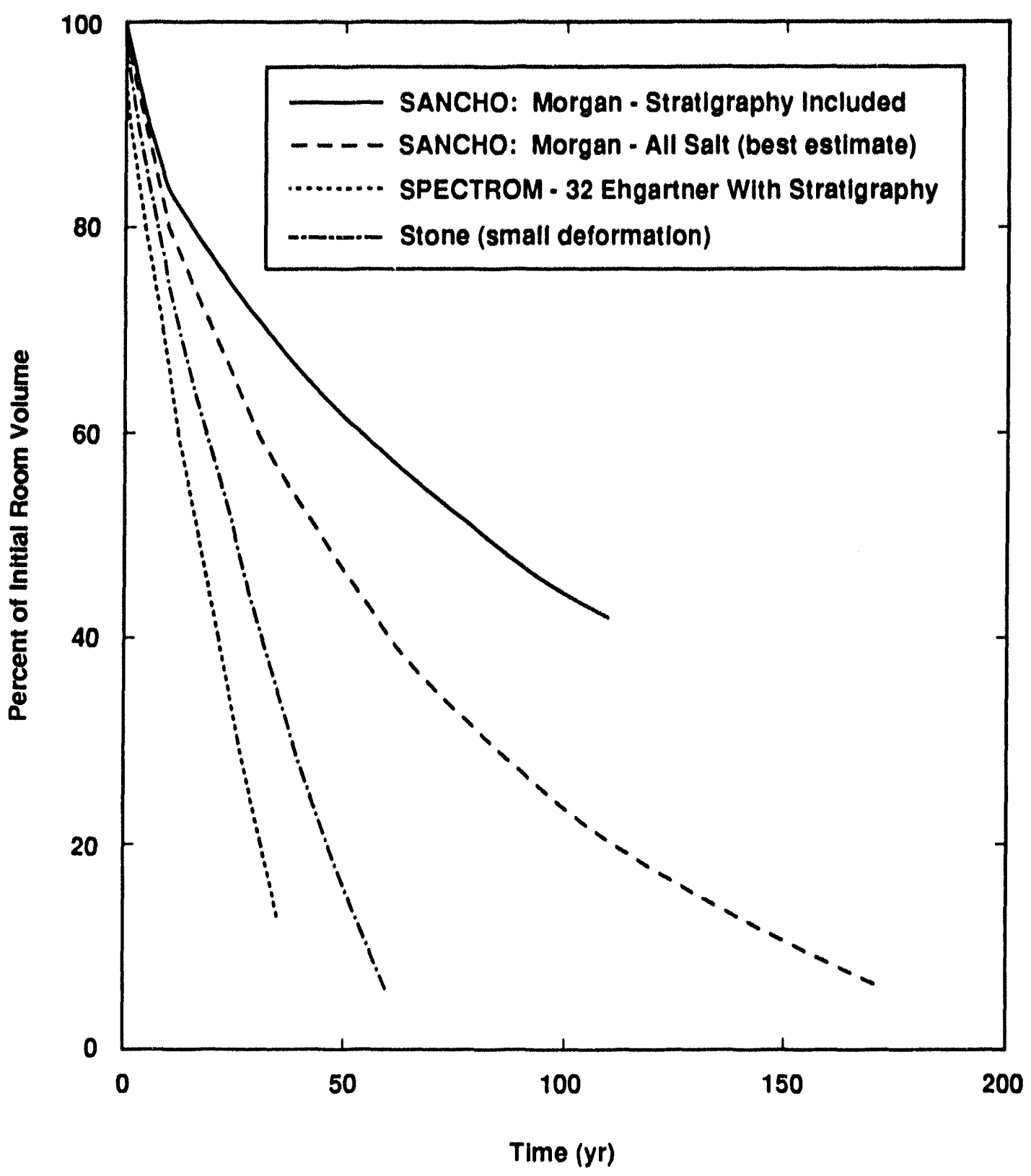

TR1-6346-138-0

Figure 5-1. Closure of an empty disposal room (Morgan, June 2, 1987, memo in Appendix A; Morgan, December 9, 1987, memo in Appendix A; Ehgartner, 1990; Stone and A.rgüello, April 1, 1992, memo in Appendix A). 
The void fractions of the room contents rather than their porosities were used to summarize closure results to emphasize that the values represent averages for entire rooms. Thus, the void fraction is defined as the void volume within a room at a given time divided by its total volume at that time. Void fractions computed in this manner are, in fact, numerically equal to average porosities, but their use serves to emphasize the relatively crude nature of the estimates. Insufficient information existed to compute differences in porosity between the waste and the backfill from early room-closure results.

Examination of the computational results for empty room closure in Figure 5-1 shows that closure, according to the SPECTROM-32 calculations, is more rapid than the SANCHO results. According to the SPECTROM-32 calculations, closure to $10 \%$ of the initial room volume is virtually complete within 40 years; whereas, according to the SANCHO calculations, closure requires over 150 years, or a factor of 4 greater. This factor is greater than expected from the Second WIPP Benchmark Study, which concluded that about a factor of 2 was likely in solutions of standard problems with 8 different codes (Morgan et al., 1981; Munson and Morgan, 1986). However, different constitutions models were used in the current study, whereas the Second WIPP Benchmark Study used identical constitutive models.

The exact source of the discrepancy between empty-room closure predictions is not apparent, but it must reflect some of the differences between SPECTROM-32 and SANCHO. Reasons that come immediately to mind are (1) SPECTROM-32 is based on small deformation theory, which may introduce serious errors as closure progresses to the large strains of interest; (2) the relationship for the creep of solid halite in SANCHO is scaled (E/12.5 assumption) to force correspondence of estimates with experimental closure data, whereas the creep description in SPECTROM-32 is more scientifically based. As mentioned in Section 3.1, the Munson-Dawson model for creep of solid halite is currently not available in SANCHO, and so a direct comparison with the SPECTROM-32 results is not possible.

Additional insight into resolution of the discrepancy has recently been obtained from calculations with a new code called SANTOS by Stone and Arguello (April 1, 1992, memo in Appendix A), which were undertaken to investigate determining the difference between emptyroom closure results computed using small and large deformation formulations. The SANTOS finite-element code was modified for this study so that it could generate both types of solutions. In addition, the effects of incorporating contact surfaces to prevent overlapping of the corner elements of the room configuration were determined. The contact surface feature is important because once overlapping occurs, as illustrated in Stone and Argüello's memo (April 1, 1992, memo in Appendix $A$ ), the computational results no longer have any physical meaning.

The best-estimate empty-room closure curve determined by Stone and Argüello, using the old reference elastic-secondary creep model with a reduced elastic constant E/12.5 and assuming small deformation behavior, is included in Figure $5-1$. The corresponding large deformation curve determined by Stone and Argüello was very similar to the best-estimate curve calculated by Morgan (June 2, 1987, memo in Appendix A) and therefore was not included in the figure. Ehgartner's (1990) SPECTROM-32 curve shown in Figure 5-1 was also obtained using small deformation behavior. A comparison shows that the SANTOS small deformation prediction for the time to closure to about $10 \%$ of the initial rojm volume is only a factor of about 1.25 to 1.3 greater than the SPECTROM-32 solution, rather than the factor of 4 to 5 difference that is observed between the SPECTROM-32 solution and the best-estimate SANCHO solution. Using reverse logic, our conclusion is that had SPECTROM-32 been based on large rather than small deformation behavior, agreement with SANCHO to within a factor of about 1.30 would have been observed. 
From the discussion of the preceding paragraph, it would appear that the principal source of the large discrepancy between the SANCHO and SPECTROM-32 curves for empty-room closure can be attributed to the inadequacies of small deformation theory and that finite-deformation analyses must be used in the future for closure predictions. The fact that many past calculations have been performed with small deformation codes does not make these results without value, however, because they have assisted in conceptual model development.

The factor of 4 to 5 discrepancy in time to close to $10 \%$ of the initial room volume (Figure $5-1$ ) is misleading, however, because the disposal rooms will not be empty but rather will be filled with waste and backfill. In reality, the room volume that must be eliminated is much smaller than the entire initial volume of the room. For example, if $f_{a}$ is the initial volume fraction of solids in the room $\left(f_{a}=V_{a} / V_{0}\right.$, where $V_{a}$ is the initial volume of solids at theoretical solid density in the room and $V_{0}$ is the initial room volume) and $\phi$ is the average porosity of the room after some amount of closure, then the percent of the initial room volume $f_{V}$ corresponding to $\phi$ is

$$
f_{V}=f_{a} /(1-\phi) \cdot 100
$$

Reasonable values for $f_{8}$ are 0.2 to 0.4 , corresponding to initial porosities in the room of between 0.8 and 0.6 . Therefore, if the room collapsed to a completely solid state (no voids), $\phi$ would be 0 , and $f_{\mathrm{V}}$ would have to decrease from 100\% to only 20 to $40 \%$ (Figure $5-1$ ) rather than to the $0 \%$ value required by the empty room calculations to close the room. For $40 \%$ closure, the closure time estimated using SANCHO is more like a factor of 2.8 larger than the time predicted with SPECTROM-32, rather than a factor of 4 to 5 based on complete closure of an empty room. Even this estimate is larger than it should be because perfect consolidation is assumed.

If, on the other hand, actual consolidation of the room contents greater than a final equilibrium state of $\phi=0.3$ never occurs, then $f_{V}$ would have to decrease even less, from $100 \%$ to only 30 to $60 \%$, according to Equation (5.1.1), and the difference between the two code results is a factor of 2.5 (Figure $5-1$ ) for $f_{V}=60 \%$. In addition, if the estimates had been obtained with a large deformation version of SPECTROM-32, simple scaling suggests that the differences would likely have been much less than a factor of 2.5 , and would be considered adequate agreement. Even with the most pessimistic interpretation, empty rooms are predicted to close to less than $10 \%$ of their original volume within 150 years.

\subsubsection{Backfilled Room Calculations}

Both waste and backfill are expected to exert increased backstress as the rooms close. Therefore, backfill consolidation was the next essential feature of closure introduced into the disposal room calculations and was based on the Sjaardema-Krieg constitutive model for backfill (e.g., Section 3.2). The first calculations addressed the closure of cavities completely filled with crushed salt backfill (Weatherby et al., 1991a; Weatherby and Brown, April 30, 1990, memo in Appendix A; Callahan and DeVries, 1991; e.g., Appendix D, Calculations 5 and 7) because compaction data for the waste were not yet available.

The results of pure salt backfill consolidation are shown in Figure 5-2. Although still atypical of the actual disposal room because wastes were not included, these calculations provided scoping information about how regions of the repository, such as access ways to the panels, might close if backfilled. A comparison of the curves from SANCHO and SPECTROM-32 in Figure 5-2 shows that a discrepancy between the two solutions is still present. However, it is not nearly as 


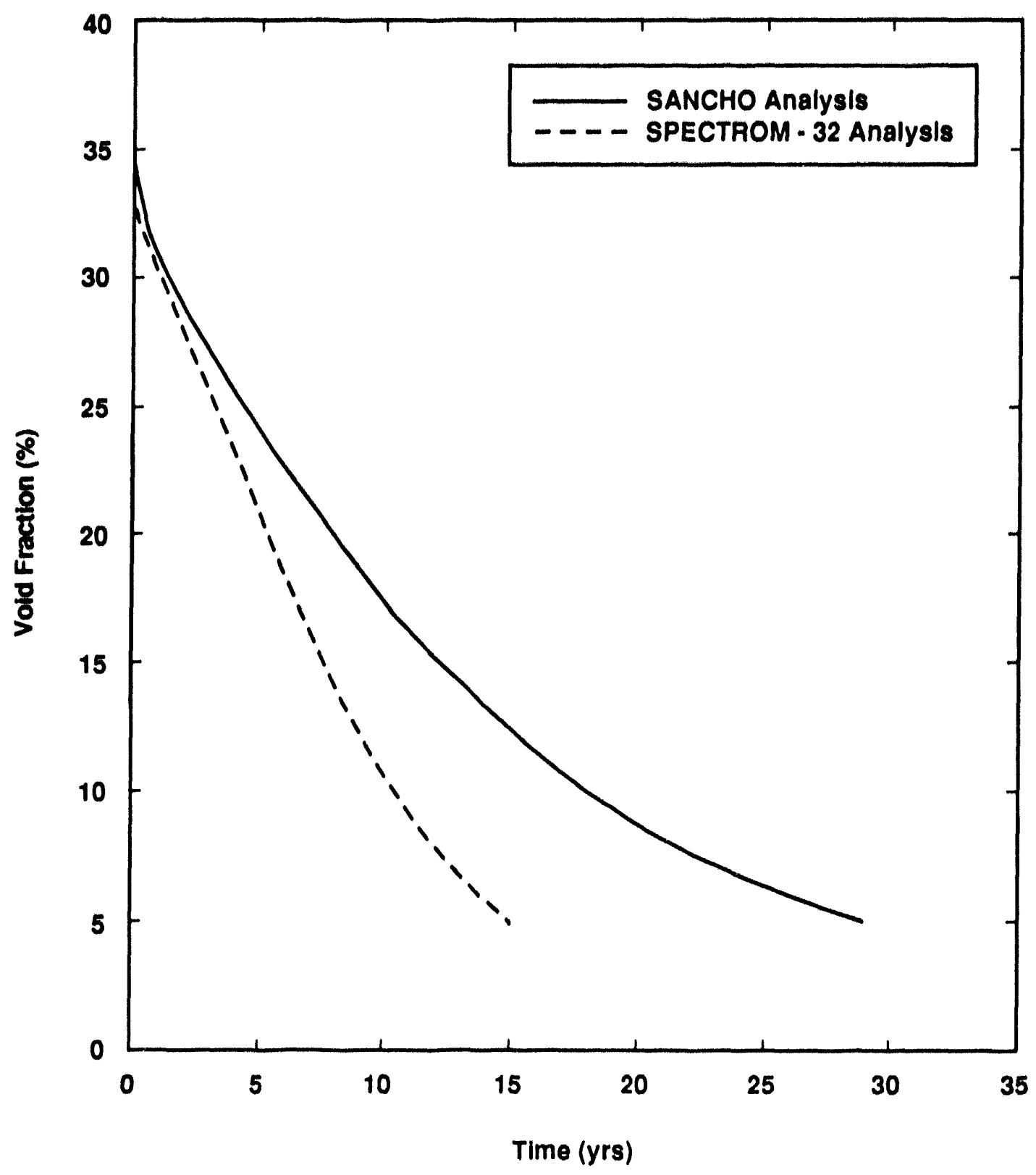

TR1.6345.140.0

Figure 5-2. Closure of a disposal room completely filled with crushed salt backfill (Weatherby et al., 1991a; Weatherby and Brown, April 30, 1990, memo in Appendix A; Callahan and DeVries, 1991). 
pronounced as the discrepancy observed in the empty room results. Another reason for less difference between the infinitesimal-and finite-strain predictions may be that, with backfill within the room, less deformation is required to eliminate the void volume within the room.

Backfill calculations were repented, as data became available, for backfill mixtures of 70 wt $1 / 1$ crushed salt and $30 \mathrm{wt} / \mathrm{m}$ bentonite (Weatherby et al., 1991a; Weatherby and Brown, April 30, 1990, memo in Appendix A; Callahan and DeVries, 1991; e.g., Appendix D. Calculations 5 and 7). The effect of the bentonite backfill additive (Butcher et al., 199/a) was of particular interest. These results in Figure 5-3 show that the difference between the SANCIIO and SPECTROM-32 curves almost disappeared but that time required for consolidation of sall/bentonite backfill has become much longer than for pure crushed salt. Closure of rooms completely filled with backfill occurs on the order of under 40 years for pure crushed-salt backfill, and 4 limes longer for crushed salt/bentonite backfill. In addition, late time void fractions for salt/bentonite are somewhat greater than for pure crushed salt. While the greater long-term void fraction of the salt/bentonite might be perceived as a problem in regard to repository performance. Butcher et al. (199/a) have shown that the decrease in consolidation rates caused by addition of bentonite to the backfill is offset by the fact that less consolidation is required to assure a given state of permeability in salt/bentonite mixtures: although densification proceeds more slowly, a less dense final state is required, and the time to reach a given permeability remains relatively constant.

\subsubsection{Rooms Filled with Waste and Backfill}

The next level of closure analysis detail was to include waste consolidation in the calculations (no air gap was assumed present). Because the expected design waste was known to have a very high initial porosity, construction of the first consolidation model was based on the assumption that the waste exerted little backstress on the surrounding salt until it approached solid density (Weatherby, May 17, 1989, memo in Appendix A; e.8., Appendix D, Calculation 4). This relationship is labeled as the "old" SANCHO consolidation curve in Figure 3-3. Later, as experimental consolidation data for simulated waste became available (Butcher et al., 199/b), it became evident that the original model was "too soft," and it was replaced by the "new" consolidation curve, also shown in Figure 3-3.

All SANCHO calculations after and including the results described by Weatherby et al. (1991a; e.8., Appendix D, Calculation 6), Figures 5-4 and 5-7, were obtained using the new consolidation curve. All SPECTROM-32 results after and including the results described by Callahan and DeVries (1991; e.g. Appendix D. Calculation 7), Figures 5-5, 5-6, and 5-8, were also obtained using a similar curve, although the method of incorporating it in SPECTROM-32 differed from the way in which consolidation data were incorporated in SANCHO (e.g., Section 3.3). Gas generation was assumed to be zero for all calculations.

Several conclusions are evident from the closure estimates for rooms filled with waste and backfill (Figures 5-4 to 5-8). First, it is apparent that the backfill consolidates much more rapidly than the waste and eventually reaches a much lower value of void volume than the waste. Figure 5-4 shows, for example, that pure, crushed-salt backfill, according to the SANCHO prediction, has consolidated to a void fraction of less than $10 \%$ in fewer than 40 years, in agreement with the backfill consolidation results described in Section 5.1.2. Similar SANCHO calculations for crushed-salt/bentonite backfill predict somewhat longer backfill consolidation times. The SANCHO backfill consolidation curves are quite similar to SPECTROM-32 results for similar backfill. 


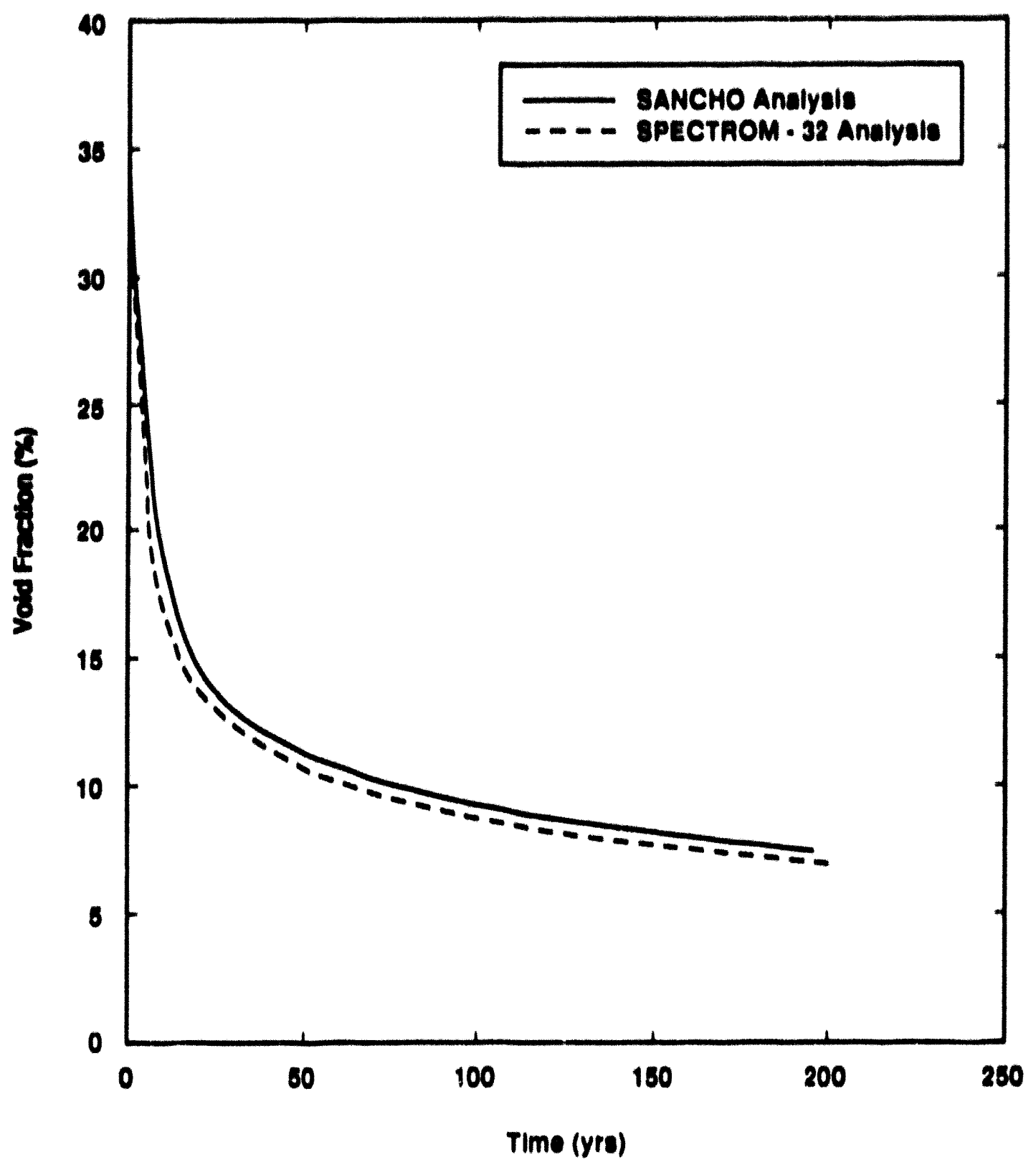

IAI CME 1410

Figure 5-3. Closure of a disposal room completely filled with crushed salt and bentonile backfill (Weatherby el al., 1991n; Weatherby and Brown. April 30, 1990, memo in Appendix A; Callahan and DeVries, 1991). 


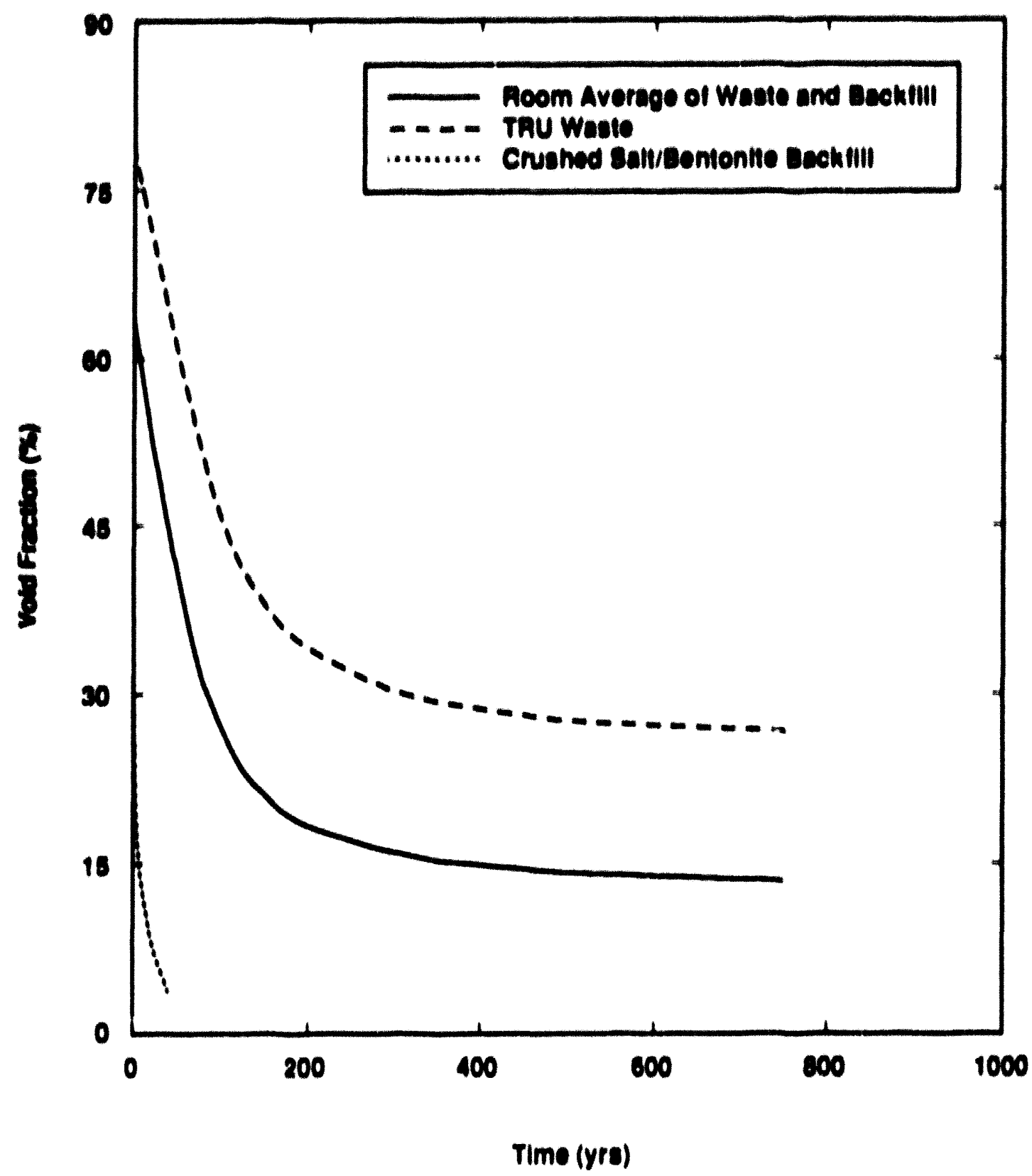

In:ME 1110

Figure 5-4. SANCllO resulls for a disposal room filled with crushed sall backfill and TRU waste (Weatherby of al, 1991a). 


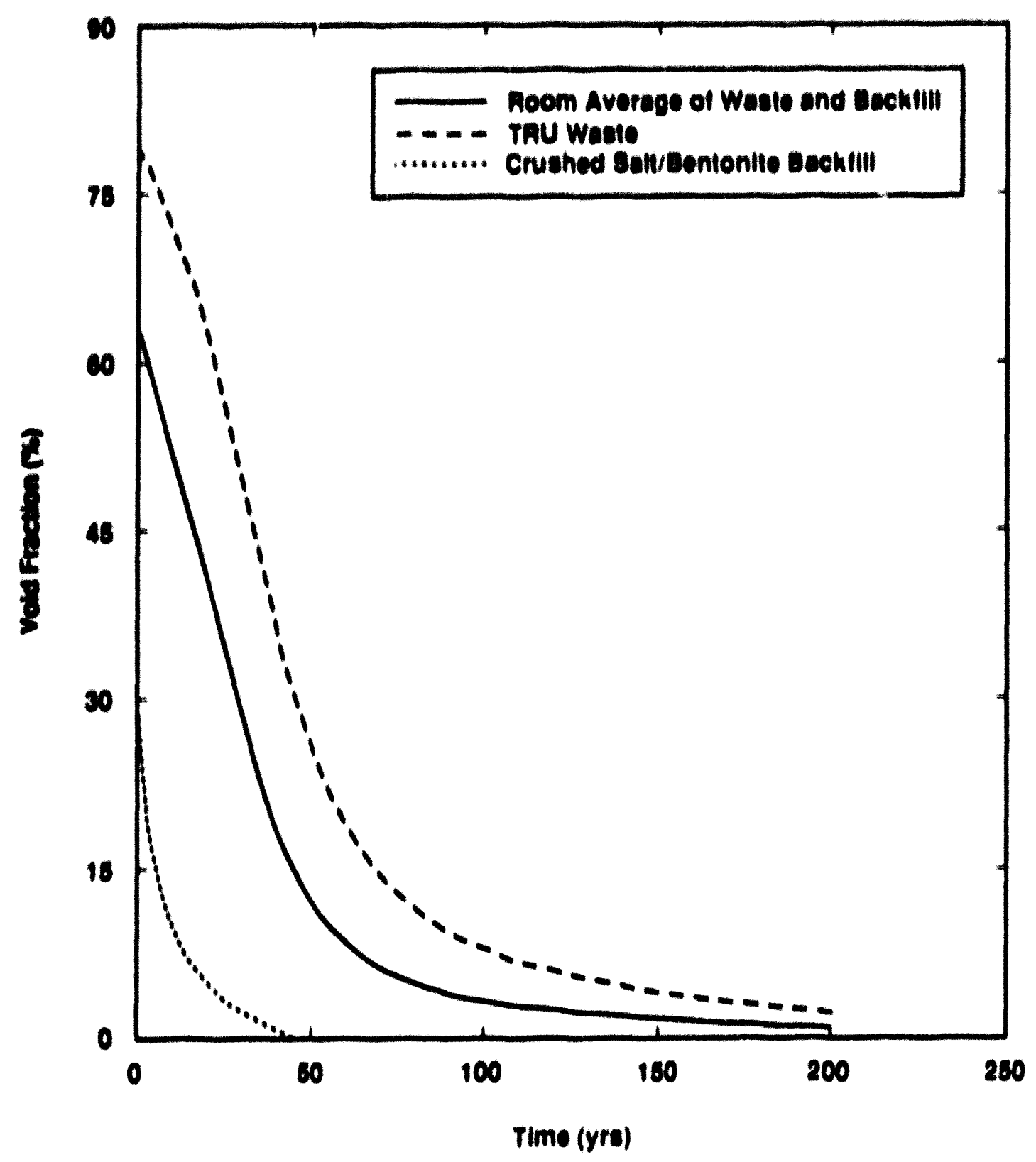

Iin 1400

Vigure 5-5. SPICTROM 12 resules for a disposal room rilled with erushed salt backfill and TRU waste for the waste consolidation assumption that $a_{n}=30_{m}$ CCaltahan, March 13, 1992, memo in Appendix A). 


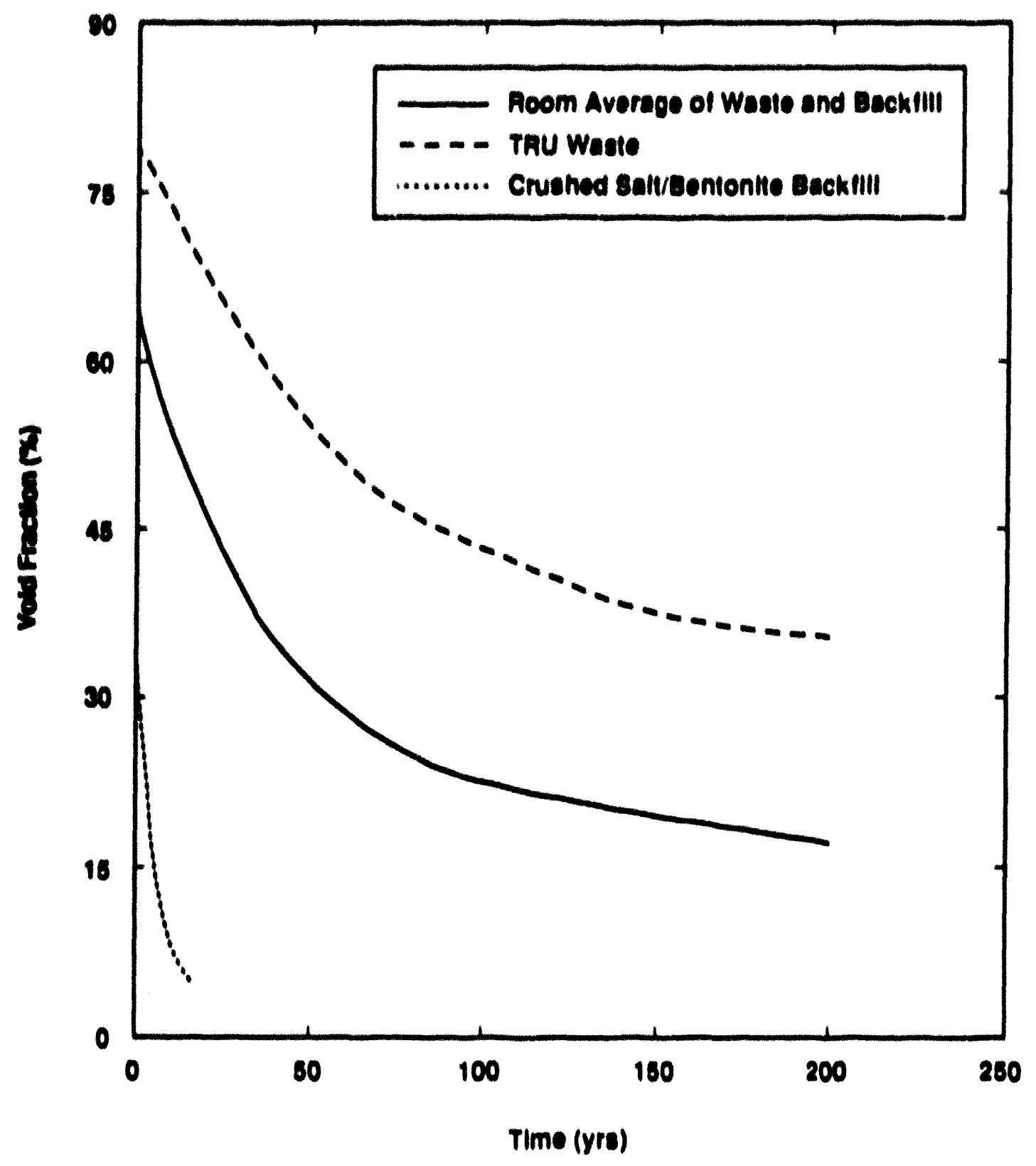

Inisus 1400

Figure 5-6. SPECTROM-32 results for a disposal room filled with crushed sall backfill and TRU waste for the waste consolidation assumption that $\sigma_{n}=\sigma_{m}$ (Callahan, March 13, 1992, momo in Appendix A). 


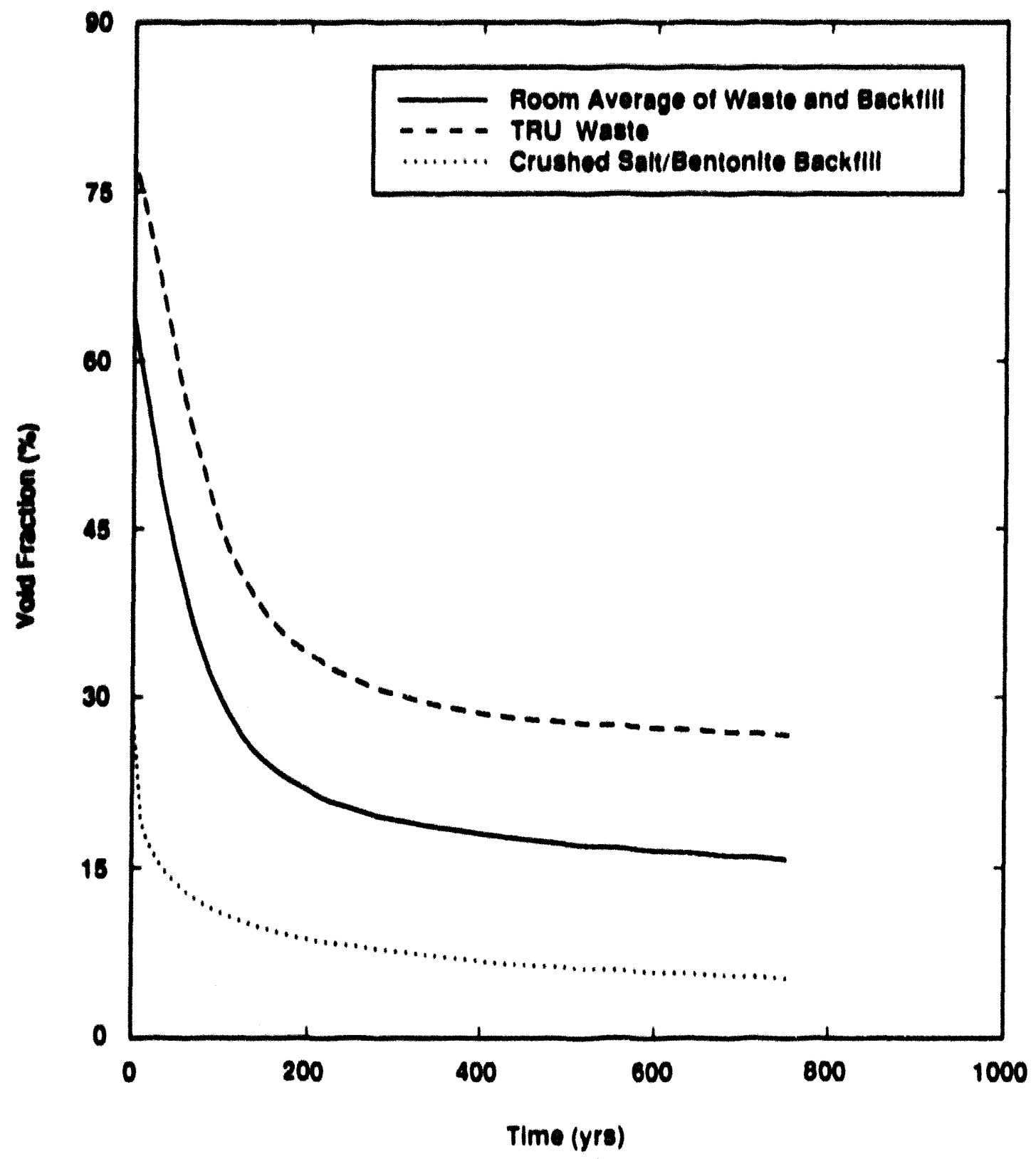

III. 02401460

Figure 5-7. SANCHO results for a disposal room filled with crushed salt/bentonite backfill and TRU waste (Wentherby el al., 1991a). 


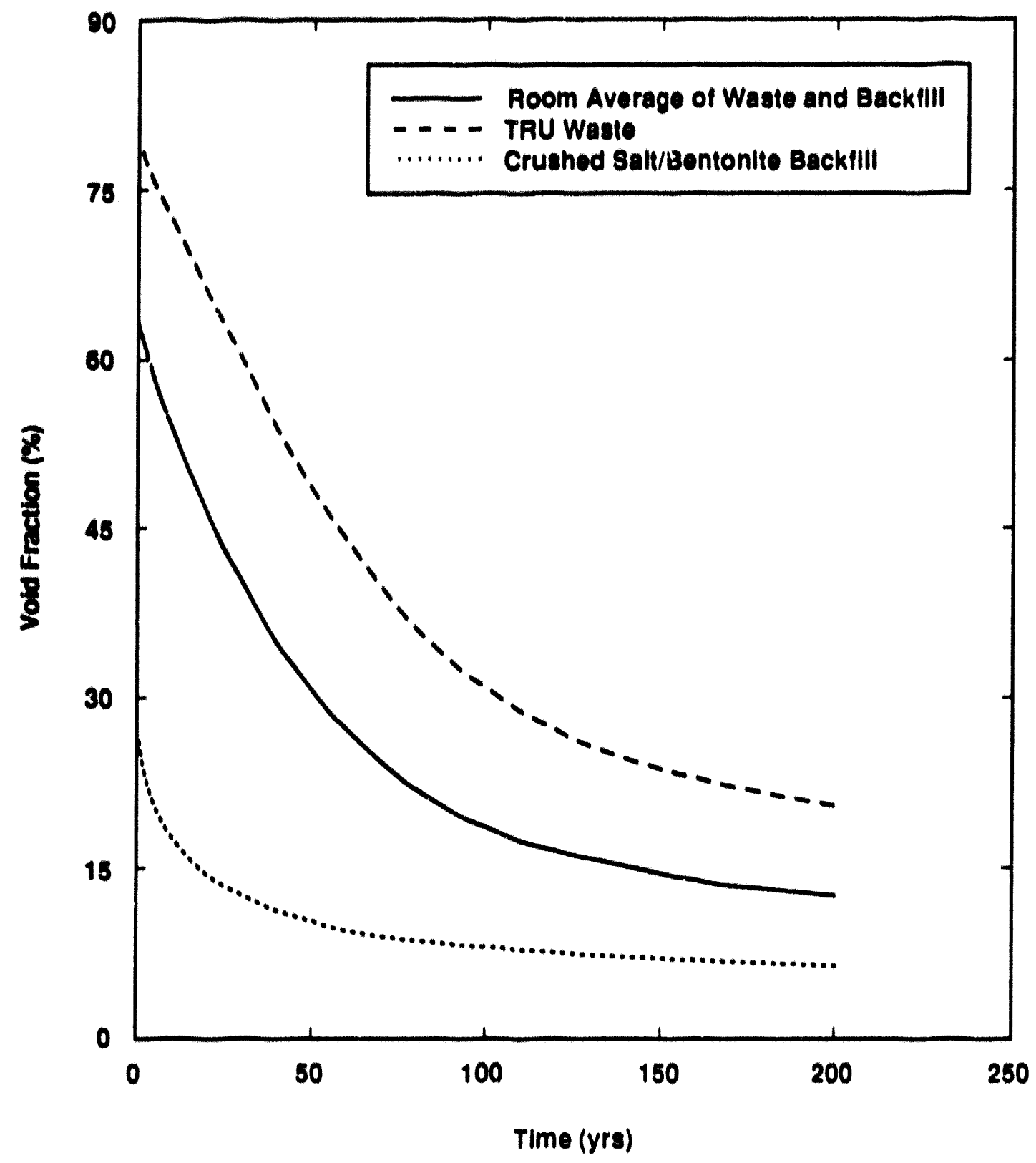

1月1.0345:114.0

Figure 5-8. SPECTROM-32 results for a disposal room filled with crushed salt/bentonite backfill and TRU waste (Callahan and DeVries, 1991). 
Other conclusions are evident from the waste consolidation curves in Figures 5-4 10 5-8 The most apparent conclusion is that SANCHO waste consolidation predictions differ greatly from predictions made with SPECTROM-32. For example, whereas the SANCHO waste consolidation curve in Figure 5-4 shows waste consolidation to no less than about 30' porosity, calculations for an equivalent configuration with SPECTROM-32 show consolidation 10 less than 5 th. The SANCHO estimates of waste consolidation are also seen to be nearly independent of the type of backfill present, as they should be, because of use of the non-dimensional void fraction parameter as the measure of closure. In contrast, the waste consolidation curves computed with SPECTROM-32 appear to differ, depending on the type of backfill.

The difference between the waste consolidation curves compuled with SANCHO and the initial SPECTROM-32 results is considered to be a direct result of the use of different mathematical waste consolidation models (Section 3.3). The SANCHO predictions are consistent with results from tests in which various types of waste were compacted axially while restruined in rigid dies to prevent lateral motion. These tests imply that average waste void volumes less than $18 \%$ at lithostatic stress are not likely to be achieved. They also show that the stiffness of the metal waste was the principal factor preventing consolidation to smaller void fractions (Butcher et al., 199/b). It is difficult to accept the initial SPECTROM-32 estimate of eventual compaction to less than $5 \%$ void fraction in view of the experimental observations. On the other hand, the SANCHO estimate may be pessimistic in the sense that the model used to obtain it underpredicts consolidation and does not account for any salt flow into a waste element. Because of the perceived inconsistency in the predicted state of compaction of the waste after a hundred years, an additional calculation was undertaken. This calculation examined the consequences of the $o_{0}=$ 30 $m$ assumption used for the SPECTROM-32 compaction relation (e.8., Section 3.3.4) by replacing it with the assumption that $\sigma_{\mathrm{g}}=\sigma_{\mathrm{m}}$. Although the results shown in Figure 5-6 indicate even less long-term compaction than the SANCHO results (Figure 5-4), they are considered to be a betler representation of the actual response of the waste. In fact, the SPECTROM-32 results assuming o. - 3o $o_{m}$ (Figure 5-5) and the results assuming $\sigma_{n}=\sigma_{m}$ (Figure 5-6) are considered to define the extremes of possible waste compactability, with the SANCHO result lying between them and more toward the upper bound. The consequences of the $\sigma_{n}-3 \sigma_{\mathrm{m}}$ assumption in SPECTROM-32 on closure of a room filled with salt/bentonite backfill, described in the next section, have yet to be determined.

In view of the results presented in the previous paragraph, the SANCHO predictions are considered to represent a more conservative analysis of repository response, in terms of the estimated permeabilities of the waste, and as such will remain the basis for 10 CFR $10 / B$ performance assessment until model refinement makes a more accurate estimate possible. The results show that consolidation of the waste is not complete, that it is controlled by the incompressibility of the metal waste, and in turn is the principal factor controlling the permeability of the disposal room contents.

\subsubsection{Mechanical Closure of Rooms Containing Waste, Backfill, and Gas}

\subsubsection{EARLY GAS PRESSURIZATION ESTIMATES ASSUMING A PERFECTLY SEALED ROOM}

The first estimates of the effect of gas generation on the closure history of a disposal room. for the Drafi Supplemental Envirommental Impact Stateme'ni (DSEIS) (US DOE, 1989), assumed that cavities that contained gas were perfectly sealed (1.appin et al., 1989, Sec. 4.10.2). This assumption was considered an upper bound representation of the effects of gas pressurization. 
Selection of baseline gas generation potentials (total amount of gas possible) and rates (Lappin et al. 1989. Sec, 4.2) constituted an important accomplishment of the DSEIS effort.

The initial DSEIS study included estimates of pressures that would occur in various sealed, gas-storage volumes correspond ig to a gas generation potential of 589 moles/drum for anoxic microbial decay and $\mathbf{8 9 4}$ moles/drum from anoxic corrosion of metals. In the baseline case, the gas-production rate was assumed at $2.66 \mathrm{~g}$-moles/drum/year for 500 years, after which time corrosion reactants were used up. Sufficient brine was assumed present to allow corrosion reactions to go to completion. After 500 years, a rate of $0.86 \mathrm{~g}$-moles/drum/year, corresponding to microbial decay, was assumed for the next 200 years, after which all gas production ceased. Gas-pressure estimates were computed with the ideal gas law and were demonstrated to be sufficiently accurate using more detailed gaseous equations of state, as will be described later in this section.

Several results from the DSEIS gas-pressurization calculations for a perfectly sealed repository can be used to provide insight for further gas-pressurization studies. First, a total gas pressure of $6.7 \mathrm{MPa}$ was found to exist if zero closure of a waste panel was assumed (Lappin et al., 1989. Sec, 4.10.2. Table 4.8). For tiis estimate, the available storage volume was computed as the excavated volume of the waste panel less the volumes occupied by waste and backfill. This caiculation provides an incuitive measure of the total amount of gas generated. A second calculation assumed that (1) the repository was allowed to close without back pressure from internal gas generation and (2) all of the gas was contained within the unsaturated void volume of 13.000 cubic meters that existed after closure was considered complete. The void volume for this calculation was the "final state" DSEIS value representing the estimated final consolidated state of the waste $(0.18$ final porosity) and backfill (equal or less than 0.05 final porosity), assuming no effects of gas generation. The gas pressure under this condition, using a non-ideal gas law, was predicted to increase to about $240 \mathrm{MPa}$, much greater than a lithostatic pressure of $14.8 \mathrm{MPa}$ at the horizon of the repository. Clearly, pressurization to $240 \mathrm{MPa}$ is not realistic because halite surrounding the repository will expand or interbeds will fracture at much lower pressures to provide pressure relief. Cavity expansion and fracture opening are expected to create more void volume or vent some of the gas to other regions of the formation long before the gas pressure has reached $240 \mathrm{MPa}$. This example does emphasize, however, that the extent of pressurization of a disposal room will depend on the magnitude of the void volume existing in the room at a given lime.

Even when estimates of additional available volume for gas storage, such as the void volume within Marker Bed 139 and the DRZ, were added to the final volume of the repository to obtain a total volume of 105,000 cubic meters, the calculated pressure was $18 \mathrm{MPa}$. This estimate was interpretnd as suggesting that even if all usable volume within the repository were available for gas storage, the resulting pressurization would still be above lithostatic pressure (14.8 MPa). Pressures above lithostatic pressure are generally considered adverse because of the likelihood of fracture openings in the interbeds that could vent the gas to unacceptable repository regions, act as short circuits for brine saturation of the waste before final compaction, and/or act as conduits for migration of radioactive brine out of the repository.

A similar but somewhat more thorough study of the potential for gas pressurization was undertaken by Davies (June 26 and October 4, 1990, memos in Appendix A). The primary objective of these gas-storage pressure calculations was to identify gas-storage scenarios that would produce final gas pressures that were less than lithostatic pressure. An added objective of the study was to estimate lateral gas migration distances in nonhalite interbeds. 
The first part of Davies' study examined differences between pressures estimated using the ideal gas law and those computed using other laws for nonideal gas behavior. Results using the various laws differed only when the gas storage volume was under 100,000 cubic meters and the pressure was well above lithostatic. Above $20 \mathrm{MPa}$, the nonideal gas behavior of hydrogen was found to be increasingly important. As expected, storage volumes comparable to the volumes assumed for the DSEIS calculations yielded approximately the same pressures.

When Davies considered gas storage in interbeds, gas storage pressures for an "intermediate state" of the repository with a porosity of approximately 0.42 were found to be relatively insensitive to the presence of interbeds and to assumptions about interbed porosity. This result assumed creep closure had ceased because of internal gas pressurization. An intermediate state of closure of the room is typical of the strite of consolidation of the waste if gas pressurization partially inhibits compaction, as discussed further in the next section of this report. The insensitivity arose because the volume within the entire repository for this condition was already large enough to reduce the final gas pressure to below lithostatic.

The most significant result from Davies' calculations was the observation that when interbed storage was added to the "final state" volume considered in the DSEIS (full consolidation without any impediment to closure from gas), the final gas pressure was $19 \mathrm{MPa}$. Interbed porosities of 0.01 and an interbed area corresponding to a $400-\mathrm{m}$ buffer zone surrounding the repository were assumed. The gas pressure of $19 \mathrm{MPa}$ was again greater than lithostatic pressure but much less than the DSEIS estimate of pressurization to over $200 \mathrm{MPa}$. Thus, these calculations showed that gas-storage pressures for a "final state," fully consolidated repository were quite sensitive to assumptions about interbed porosity and saturations.

Davies' final calculations determined the size of the interbed area that would be required to make gas-generation pressures less than lithostatic. Various interbed porosities were assumed. For the condition of a 0.01 interbed porosity mentioned in the previous paragraph, gas would have to migrate a distance on the order of $1000 \mathrm{~m}$ to produce the desired reduction in pressure. This distance is approximately one third of the distance between the southern edge of the wasteemplacement panels and the southern boundary of the WIPP Site.

\subsubsection{RECENT CLOSURE ANALYSES OF A PERFECTLY SEALED ROOM}

Since completion of the DSEIS support document (Lappin et al., 1989), a series of calculations has been completed by Weatherby et al. (November 14, 1989, memo in Appendix A), Weatherby et al. (1991b), and Brown and Weatherby (September 17, 1990, memo in Appendix A) (e.g., Appendix D, Calculations 8 and 9) that investigate the behavior of a disposal room when both the total gas potential and gas-generation rates are reduced.

In addition to the base gas-generation case (gas generation at $2.66 \mathrm{~g}$-moles/drum/year for 500 years and $0.86 \mathrm{~g}$-moles/drum/year for an additional 200 years), three other cases were examined in these calculations: (1) What happens to the disposal room as the total amount of gas generated is reduced to 60,40 , and $20 \%$ of the total gas used in the DSEIS report (the gas is assumed to be produced over the same time periods as for the base case)? (2) What happens if the gas-generation rates are reduced to $20 \%$ of the base-case values and the total gas generated is either the same or much less than the base-case assumptions (the time for gas generation is extended)? (3) What happens if the disposal room reaches lithostatic pressure and some sort of safety-valve mechanism bleeds off gas just fast enough to maintain the pressure at a constant 
lithostatic value? In all cases, sufficient brine was assumed present to allow the corrosion reactions to go to completion.

The single room in an infinite-array configuration was used in these studies, as described in Section 2.6, with the gas-generation model described in Section 3.6.

\subsection{Void Fraction Results for Varying Amounts of Gas}

Figures 5-9 and 5-10 show the responses of disposal rooms to variations of total gas production. In Figure 5-9, the baseline gas-generation rate is represented by the curve for $f=1.0$ (e.g., Section 3.5; Weatherby et al., November 14, 1989, memo in Appendix A, summarized in Calculation 8, Appendix D) and compared with lesser rates of gas production defined by $f=0.2$ to 0.6 (Weatherby et al., 199/b; Brown and Weatherby, September 17, 1990, memo in Appendix A, summarized in Calculation 9, Appendix D). Variation of the gas production by reducing $f$ values not only decreases the amount of gas produced but can also be thought of as a crude attempt to correct for leakage during the gas-production period. However, this procedure cannot account for leakage ${ }^{1}$ after gas production ceases. In addition, it cannot account for the fact that gas created by corrosion is dependent on the availability of brine for the chemical reactions, as discussed in Section 6.2.

The void-fraction results (Figure 5-9) indicate that, as the amount of gas produced is reduced, smaller minimum void fractions are achieved. Specifically, with 100, 60, 40, and 20\% gas producing waste fractions (corresponding to values of $f$ equal to $1,0.6,0.4$, and 0.2 in Equation (3.5.2) of Section 3.5), the minimum void fractions become 25, 20, 14, and 9\%. While less gas is required to pressurize the smaller void volumes to lithostatic pressure, it was also noted that the quantity of gas and energy in a disposal room at $25 \%$ void fraction and lithostatic pressure is much greater than the quantity of gas and energy in a room with a $10 \%$ void fraction or less at lithostatic pressure.

After a minimum void fraction is achieved, the curves in Figure 5-9 show that the void fractions begin to increase. They will eventually reach the equilibrium void fraction corresponding to lithostatic pressure, given sufficient time, as calculated from the amount of gas defined by Equation (3.5.2). This increase in void fraction will be referred to as room expansion (i.e., the room begins to creep open again). Just as the room creeps closed in response to the lithostatic stress of the surrounding rock, when the internal pressure exceeds the overburden loads, the net force causes deformation to reverse and produce room expansion or inflation. Inflation is possible, of course, only if hydrofacture of the marker beds does not occur to relieve any gas pressure in excess of lithostatic.

The greatest amount of closure for the case $f=0.2$ in Figure $5-9$, of about $9 \%$ void fraction, is smaller than anticipated because the results predated a waste compaction relationship in SANCHO based on experimental data and used instead the "old" SANCHO consolidation relationship for waste (Figure 3-3). Because the old compaction relationship generated little backstress until low porosities were reached, estimated minimum void fractions from these calculations were considered low, especially when gas generation was minimal. Another difference

1 While gas is being generated, the value of $f$ reflects the net amount of gas existing within the room at a given time, i.e., the amount of gas that has been produced up to that time less the amount of gas that has leaked out of the room. 


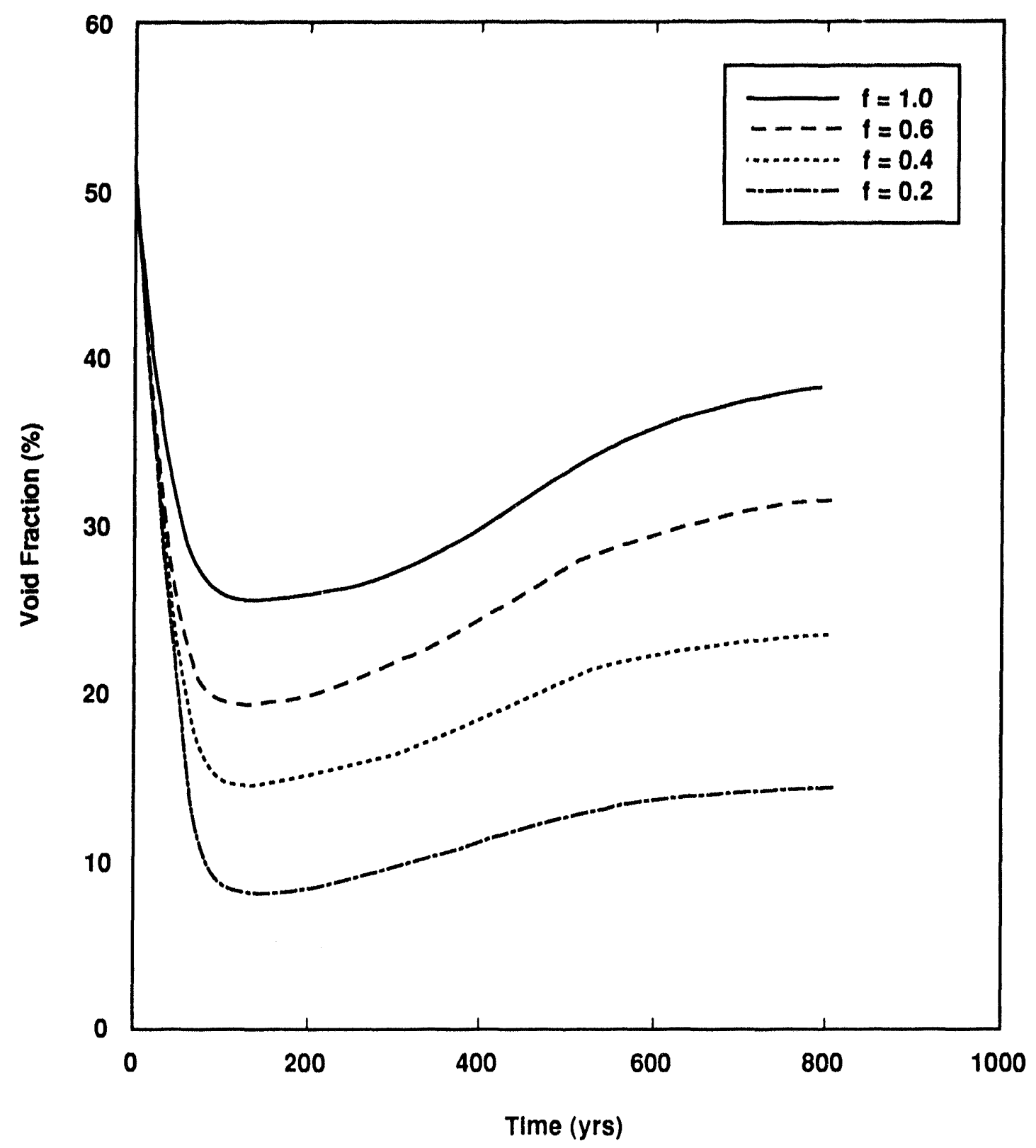

TR1.6346.115.0

Figure 5-9. The effects of gas generation on disposal room closure (Weatherby et al., 199/b; Brown and Weatherby, September 17, 1990, memo in Appendix A). 


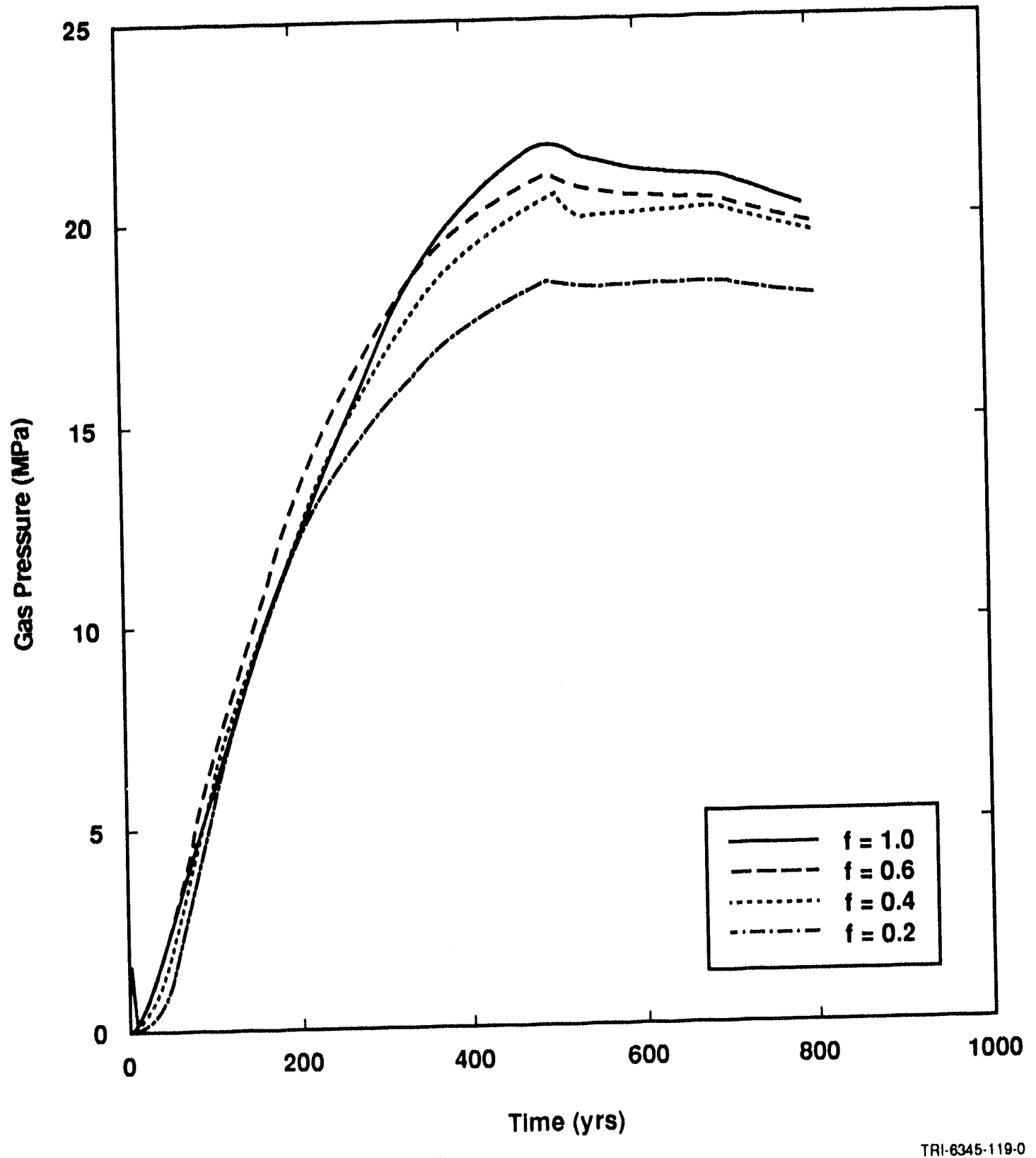

Figure 5-10. The effects of gas generation on disposal room pressurization (Weatherby et al., 1991 b; Brown and Weatherby, September 17, 1990, memo in Appendix A). 
of Brown and Weatherby's results from past calculations (see September 17, 1990, memo in Appendix A; Weatherby et al., 199/b) was that a different value of the solid volume of the waste was used to compute void fractions (e.g., Appendix D, Calculations 8 and 9). Adoption of the new solid volumes required adjustment of the curve for $f=1.0$ by scaling the initial porosity to compare it with the other curves in the figure.

\subsection{Gas Pressure Histories for Varying Amounts of Gas}

The gas pressure histories corresponding to the void fraction results are shown in Figure 5-10. In all cases, pressures eventually rose above and then gradually decayed to lithostatic pressure. In the absence of fracturing, overpressurization of the room during gas-generation is expected because a delay in time exists between the time the gas reaches a given pressure and the time it takes for salt creep to respond. In fact, a general observation is that some degree of overpressurization will eventually occur for all gas-generation rates and porosities in a sealed room whose volume is being reduced by a deforming medium. For example, the void volume in crushed-salt backfill will continue to decrease until it is (1) reduced to a very low value or (2) the pore pressure in the voids increases to an equilibrium value, in most cases lithostatic pressure. We assume that a small value of the porosity does not exist, below which further consolidation is impossible, no matter how much pressure is applied.

Correlation between the void-fraction curves and the corresponding pressure histories shows that, in all cases, the minimum void fraction is reached in about 150 years and corresponds to room pressures of about 9 to $10 \mathrm{MPa}$, after which the void fraction begins to increase. The fact that the minimum void fraction in all the gas-generation cases normally occurred at a room pressure of about 9 to $10 \mathrm{MPa}$ appears to be in conflict with the expectation that room expansion should only commence when the gas pressure exceeds lithostatic pressure. The explanation for this apparent contradiction depends on the existence of the stress gradients in the halite caused by the presence of the excavation (Brown and Weatherby, September 17, 1990, memo in Appendix A; Weatherby et al., 199/b). At early times, i.e., times less than 200 years, the near-room stress is less than lithostatic within the first $4 \mathrm{~m}$ from the room boundary and about $10 \mathrm{MPa}$ at the wall itself. At later times, the external pressure varies from lithostatic in the far field to a maximum value in excess of lithostatic at about $0.25 \mathrm{~m}$ from the room boundary $(0.25 \mathrm{~m}$ deep into the walls) and drops to about $10 \mathrm{MPa}$ at the wall face.

The stress gradient results described in the previous paragraph are interpreted as a stress cage around the room with a peak stress value in excess of lithostatic, similar to the stress cage that was encountered around a borehole in salt during in situ stress measurements (Wawersik and Stone, 1989). Because the stress in the halite adjacent to the room walls is less than lithostatic, all that is necessary is for the gas to exert a back stress greater than this value to reverse the closure process and initiate room expansion. This process is believed to be the reason for the minimums in the void volumes at approximately $10 \mathrm{MPa}$ internal room pressure.

\subsection{Calculations for an Altered Gas Generation Rate}

Time integration intervals were also extended by a factor of 5 for calculations where the rate of gas production was reduced by a factor of $5(0.2$ times a baseline rate of 2.66 (gmoles/drum/year, etc.) (cf Equation 3.5.2) or by a factor of $25(0.04$ times a baseline rate of 2.66 (g-moles/drum/year, etc.). Void-fraction history curves are shown in Figure 5-11 and pressure history curves are shown in Figure 5-12. The void-fraction curves show that the minimum void fractions are $8 \%$ and $2 \%$, respectively. Caution should again be used in interpreting Figures $5-11$ 


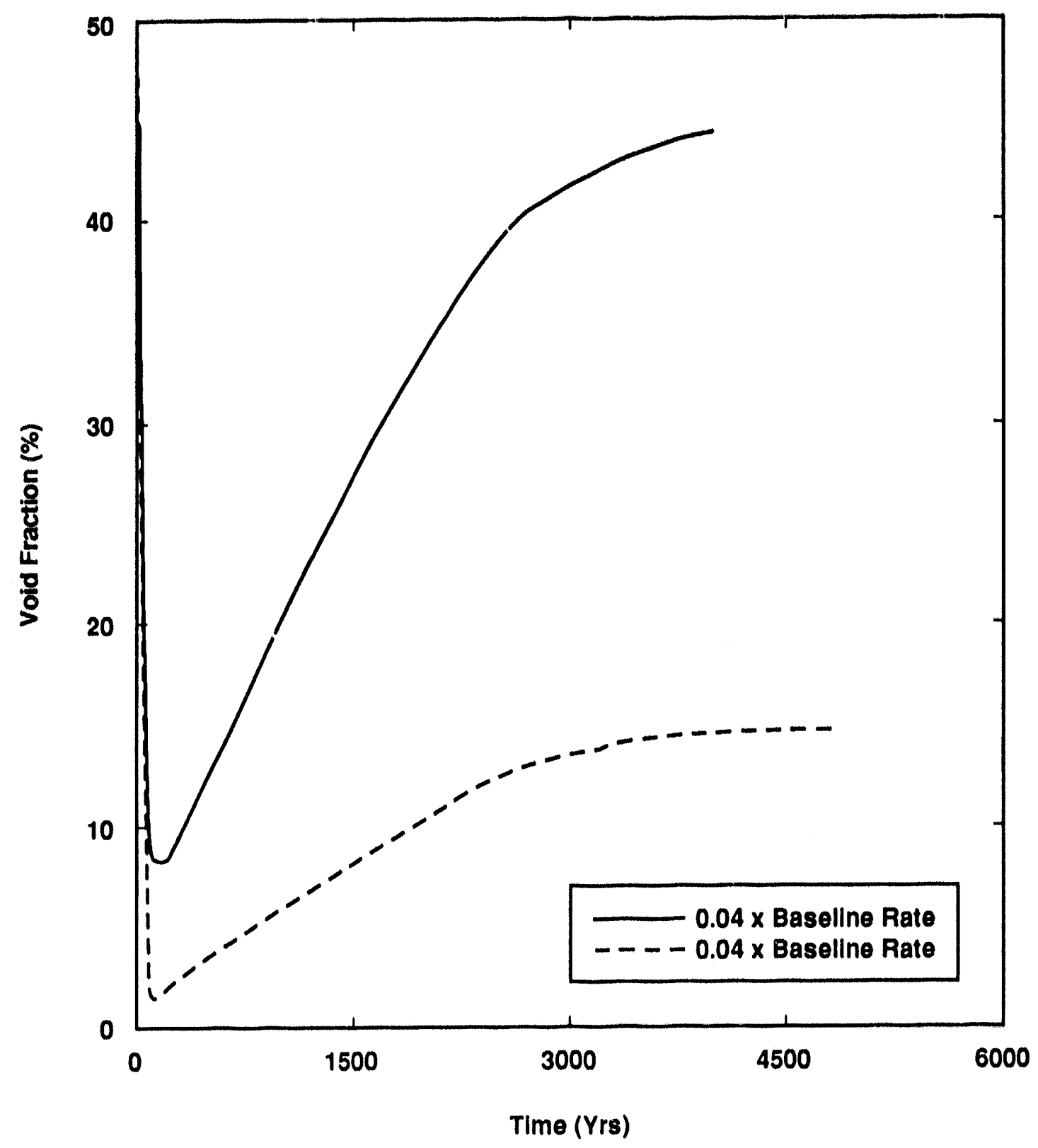

TR1.6345.120.0

Figure 5-11. The effects of altered rates of gas-production generation on disposal-room closure (Bown and Weatherby, September 17, 1990, memo in Appendix A). 


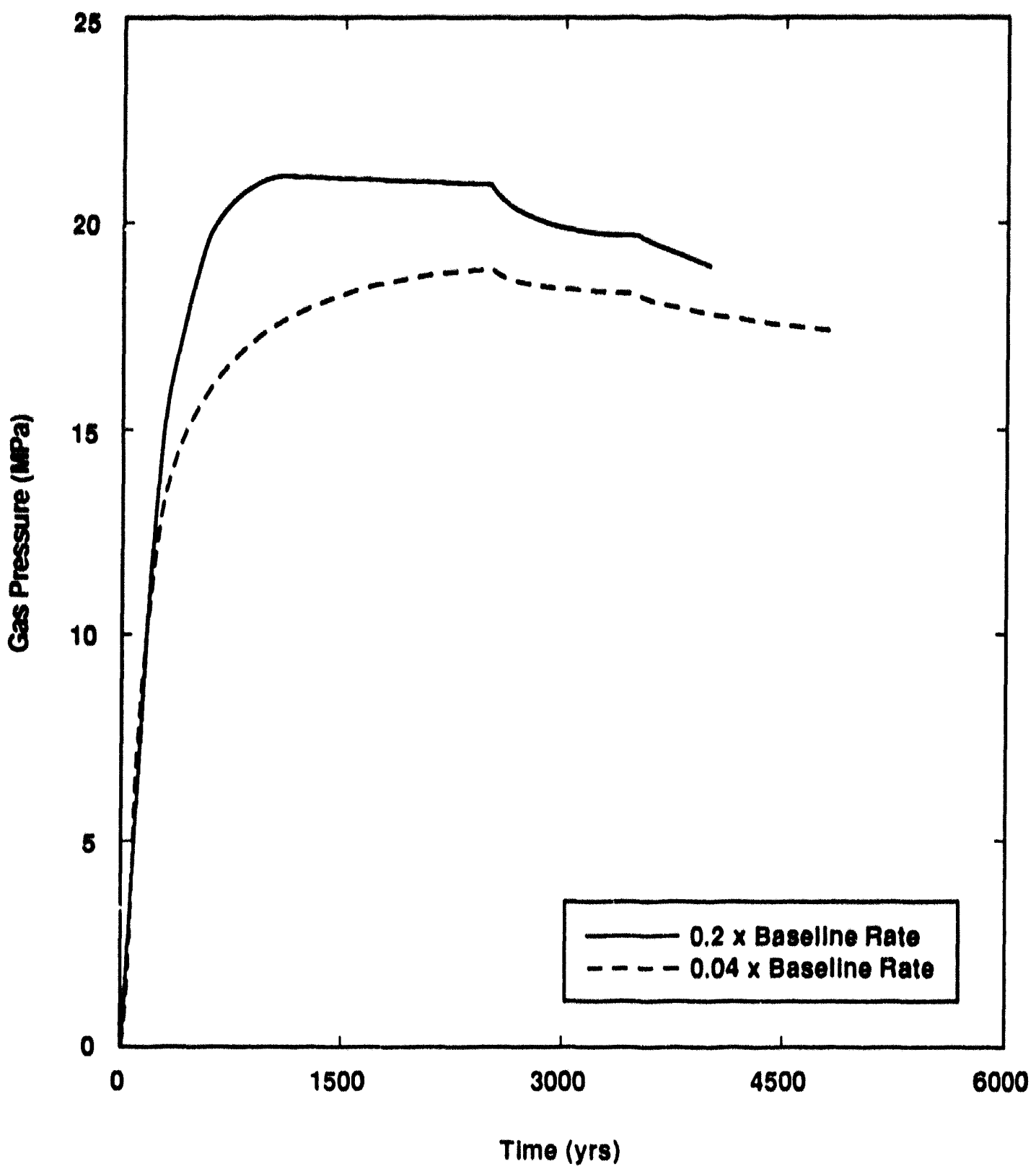

TR1.6345.122.0

Figure 5-12. The effects of altered rates of gas-production generation on disposal-room pressurization (Brown and Weatherby, September 17, 1990, memo in Appendix A). 
and 5-12, however, because the calculations are based on the "old" consolidation relation for the waste. Thus, curves that show void fractions less than 0.18 (the expected average final porosity of unprocessed waste) are considered unrealistic and eventually should be revised to be representative of the appropriate waste and backfill conditions.

The pressure history curves in Figure 3-12 show that reduction of lotal gas production by a factor of 5 decreases the peak pressure by only $18 \%$, from about $21.51018 .5 \mathrm{MPa}$, and is still above lithostatic. As stated in the previous paragraph, the reason for such a small change in the reduction of peak room pressure is the fact that room pressure is determined by the relationship between the amount of gas generated, the void volume available, and the room's natural tendency to arrive at an equilibrium balance between all the forces acting on it.

\subsection{Safety Valve Calculations}

Figures 5-13 and 5-14 show the result of assuming that the internal pressure approached lithostatic and then holding the pressure steady at that value because of some unspecified gas bleed-off mechanism. After reaching a minimum void fraction, the room is seen to expand slighlly. This behavior is expected as the stress cage decays to a uniform state of lithostatic pressure.

\subsubsection{Results of Calculatione Examining the Role of Fractures on Room Pressurization}

As discussed in Section 3.6.2, a fracture model has been developed for the disposal room to explore the behavior of fractures in the Salado (Argluello et al., 1992). Two types of response were considered. Case I represents an existing impermeable fracture in halite or an interbed extending beyond the DRZ that can open, given sufficient tensile stress, but is not penetrated by gas in its closed state. Because a portion of the crack beyond the DRZ is not penetrated by gas, the gas pore pressure in this region is zero. Case 2 represents a permeable fracture in a nonhalite interbed or clay seam, or a discontinuous interface between an interbed or clay seam and halite, that gas can penetrate even when closed. Diffusion of the gas is defined by relationships derived from two-phase flow simulation results (Davies, June 26 and October 4, 1990, memos in Appendix A: Equation (3.6.3)).

The finite-element mesh representation used for both these analyses is shown in Figure 5-15 (Arguello et al., 1992). These calculations predated introduction of a waste compaction relationship in SANCHO based on experimental data and instead used the "old" SANCHO consolidation relationship for waste (Figure 3-3). Because the old compaction relationship assumed little backstress until low porosities were reached, these results, like the gas expansion results described in Section 5.1.4, predict lower minimum void fractions than are currently expected. However, this discrepancy is considered to have little effect on the objective of the calculations, which was to explore two different conceptual models of the role of fractures during disposalroom pressurization.

\subsubsection{IMPERMEABLE FRACTURES (CASE 1)}

For the Case 1 fracture, gas pressurization is not permitted beyond the first one meter of the fracture layer from the room boundary (an assumed thickness typical of the DRZ) unless the 


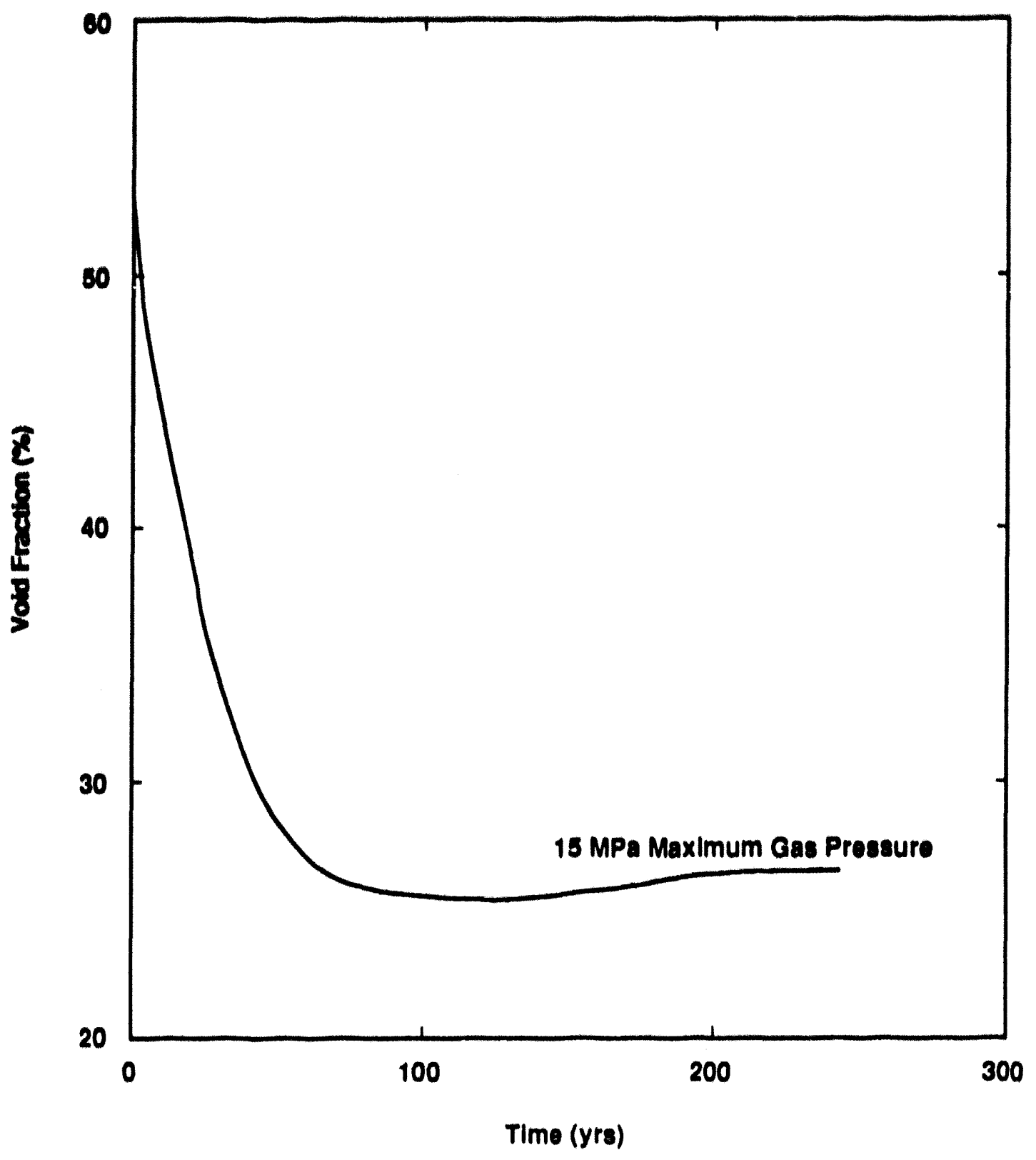

Tค1.6348 123.0

Figure 5-13. The effect on disposal-room closure of allowing unlimited gas leakage at room gas pressures above $15 \mathrm{MPa}$ (Brown and Weatherby, September 17, 1990, memo in Appendix A). 


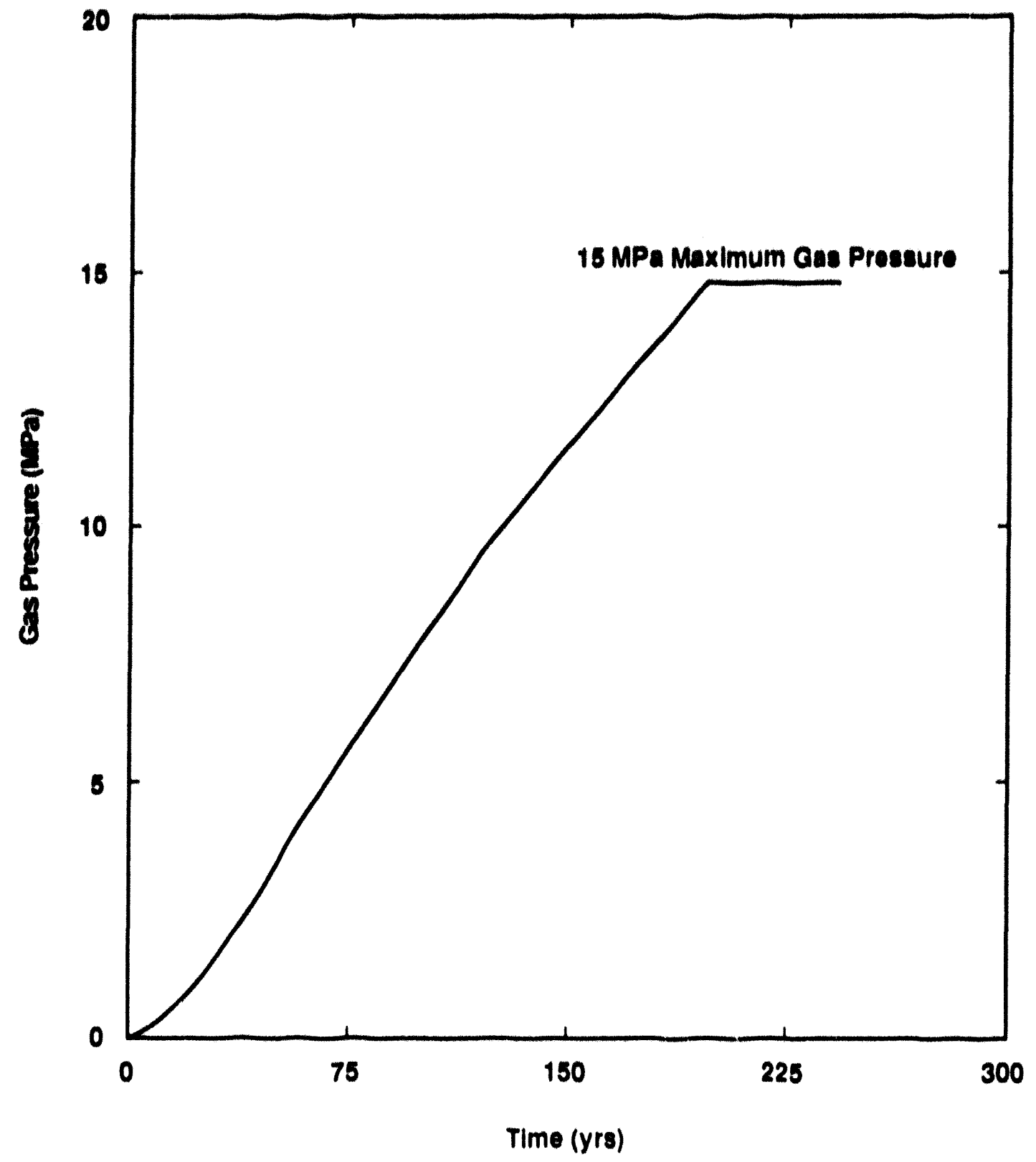

Tค1 635:121:0

Figure 5-14. The pressure history corresponding to the closure shown in Figure 5-13 (Brown and Weatherby, September 17, 1990, memo in Appendix A). 


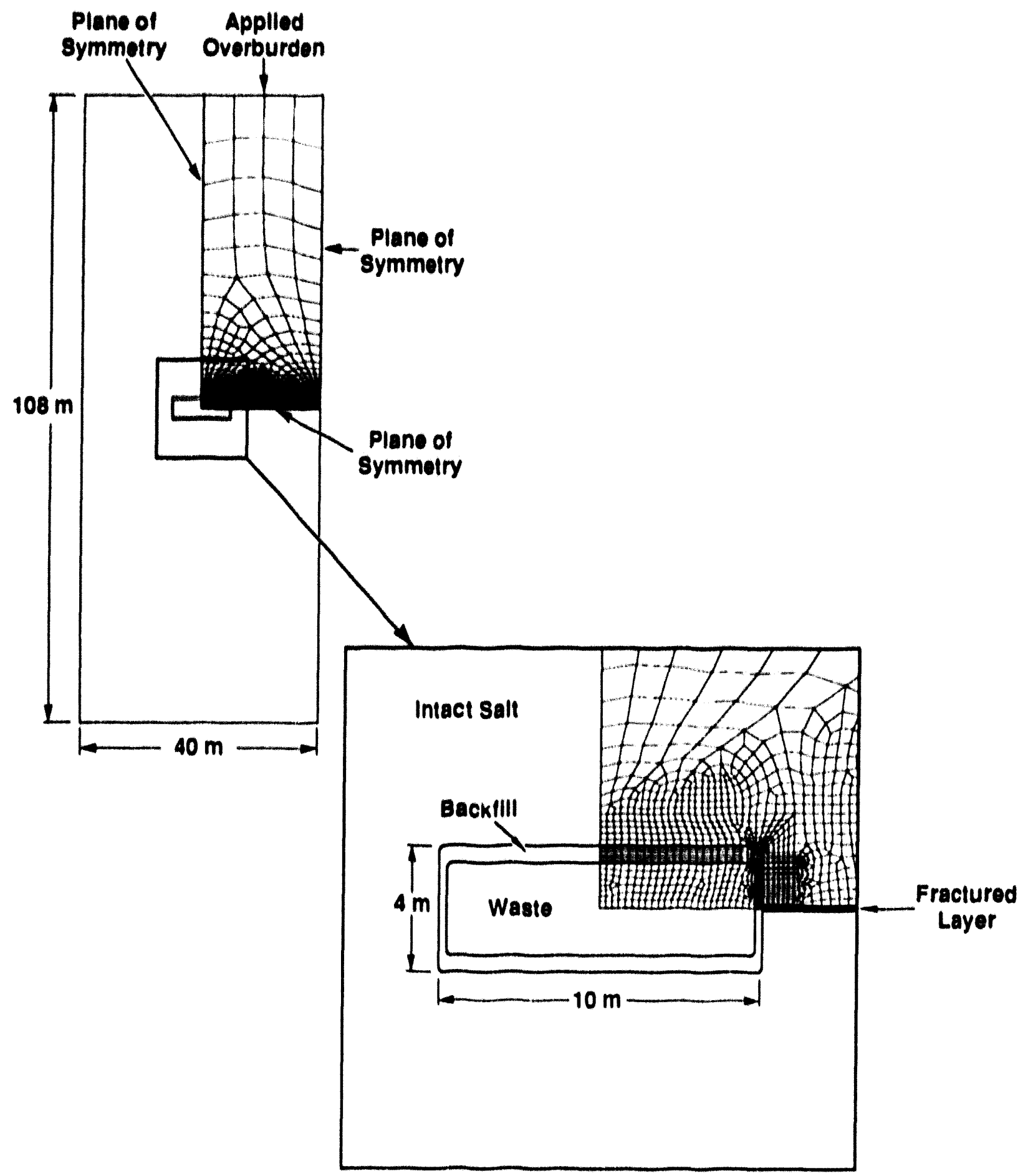

III1 6346 212.1

Figure 5-15. Finite-element mesh used to analyze a quarter room intersected at its mid height by a fracture (Argüello et al., 1992). 
fracture opens. This fracture will be labeled the gas impermeable fracture. It can open if stress normal to the fracture layer becomes tensile, allowing gas to enter. The disposal-room pressure history curves derived from these calculations in Figure 5-16 show that the gas pressurn continues to rise above lithostatic pressure until it reaches a maximum at around $22 \mathrm{MPa}$.

The vertical stress history (the stress across the crack) in the first element ahead of the tip of the crack one meter in from the disposal-room wall controls crack opening. If this stress becomes rensile, then, according to the model, the impermeable portion of the crack begins to open. The vertical stress history in Figure 5-17 shows that the stress in the first element decreases (the negative sign indicales compressive stress) as the room is pressurized but remains compressive throughout the entire simulation time. In fact, the stress never drops below a compressive stress of about $5 \mathrm{MPa}$. Thus, the crack cannot open if the stress is not tensile, and this element cannot become permeable.

Examination of the entire maximum principal stress field along the crack shows, in fact, that all other elements outside the DRZ remain compressive (Argitello et al., 1992), i.e., the entire stress field around the crack remains compressive because the salt is able to creep and increase the volume of the room sufficiently to accommodate the slow gas pressurization. Both horizontally oriented and vertically oriented (impermeable) fractures have been examined in this study and found to exhibit the same response. If the stress field always remains compressive, no new fractures can initiate and propagate to create a conduit for gas flow to other regions of the repository. We conclude from this calculation, therefore, that initiation of new cracks in the halite by gas pressurization is not possible at the gas-generation and deformation rate: expected in the WIPP.

\subsubsection{PERMEABLE FRACTURES (CASE 2)}

The reason for the fracture-related calculation described as Case 2 is that the geological formation surrounding the repository is not a monolithic region of salt but rather is a layered structure of salt, horizontal antiydrite interbeds, and clay seams. The stratigraphy of this formation has already been described in Section 2.2. Because the anhydrite interbeds are discontinuities in the salt formation that have limited cohesion with adjacent halite, and because anhydrite has quite different mechanical properties than salt particularly with regard to stiffness and brittleness, it is not surprising that these layers contain naturally occurring, partially healed fractures (Borns, 1985). Thus, they are more permeable than intact salt. Similar observations are relevant for partings and clay seams, with the exception that these layer materials tend to be more easily deformable.

Whether the fracture systems of these layers are of the nature of long continuous cracks or networks of small, interconnected cracks is unimportant in the Case 2 calculations. Such detail is greater than the structural detail that can be addressed with the codes during a disposal-room claculation. Instead, the structures of interbeds and clay seams are important because they are likely pathways for gas migration either by porous media flow or by flow through fractures (Mendenhall et al., 1991), and as such, they can be approximated as containing simple fractures.

The fracture configuration used for Case 2 represents a permeable fracture in a nonhalite interbed or clay seam, or a discontinuous interface between an interbed or clay seam and halite that gas can penetrate, even when closed. The manner that gas migrates along this type of fracture has been defined by relationships derived from two-phase flow simulation results by Davies (June 26, and October 4, 1990, memos in Appendix A) that specifically addressed this problem. For this calculation, Davies assumed a constant room volume corresponding to the final 


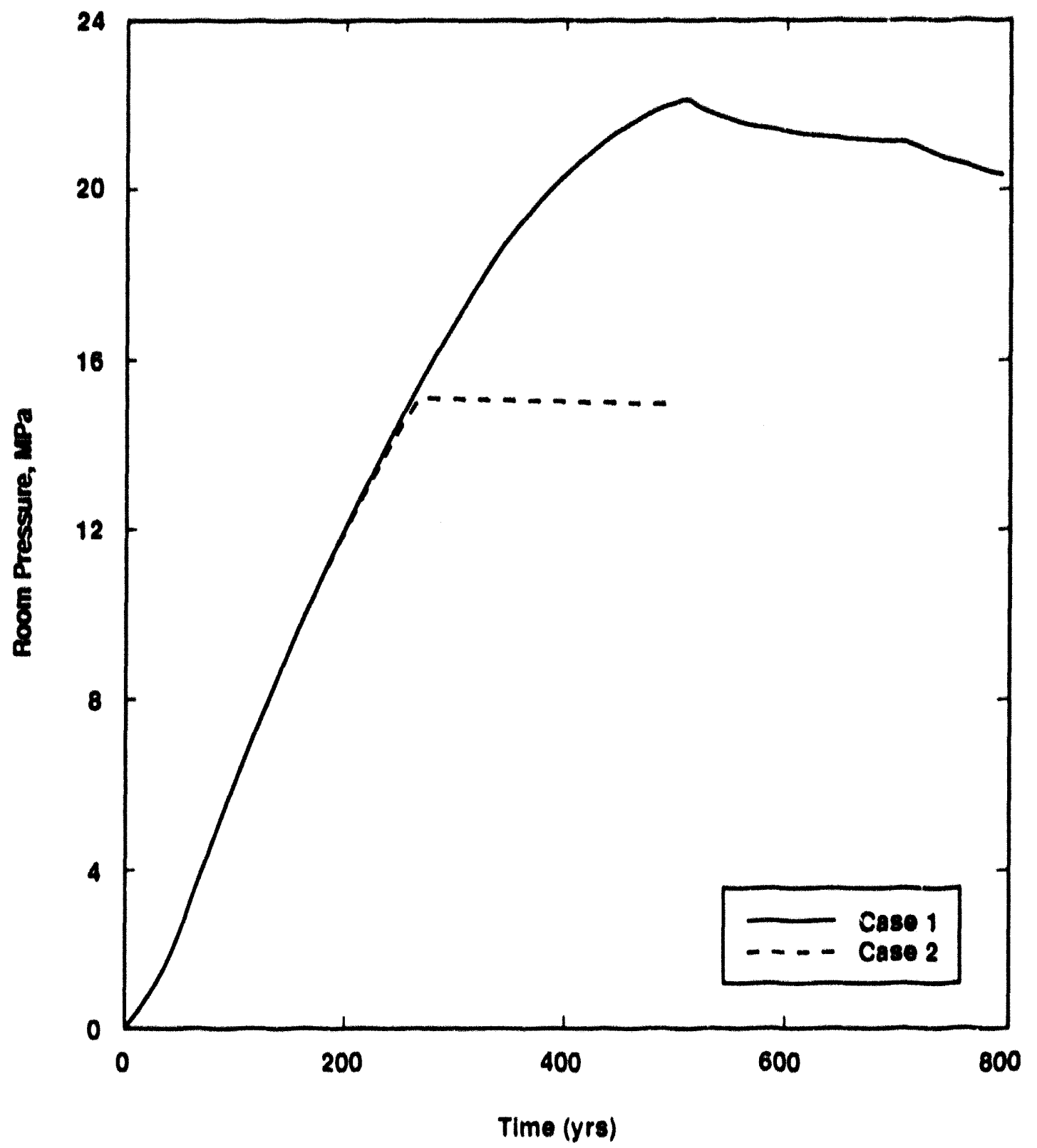

Int.635.1240

Figure 5-16. Gas pressurization of a room intersected at its mid height by an impermeable fracture (Argüello et al., 1992). 


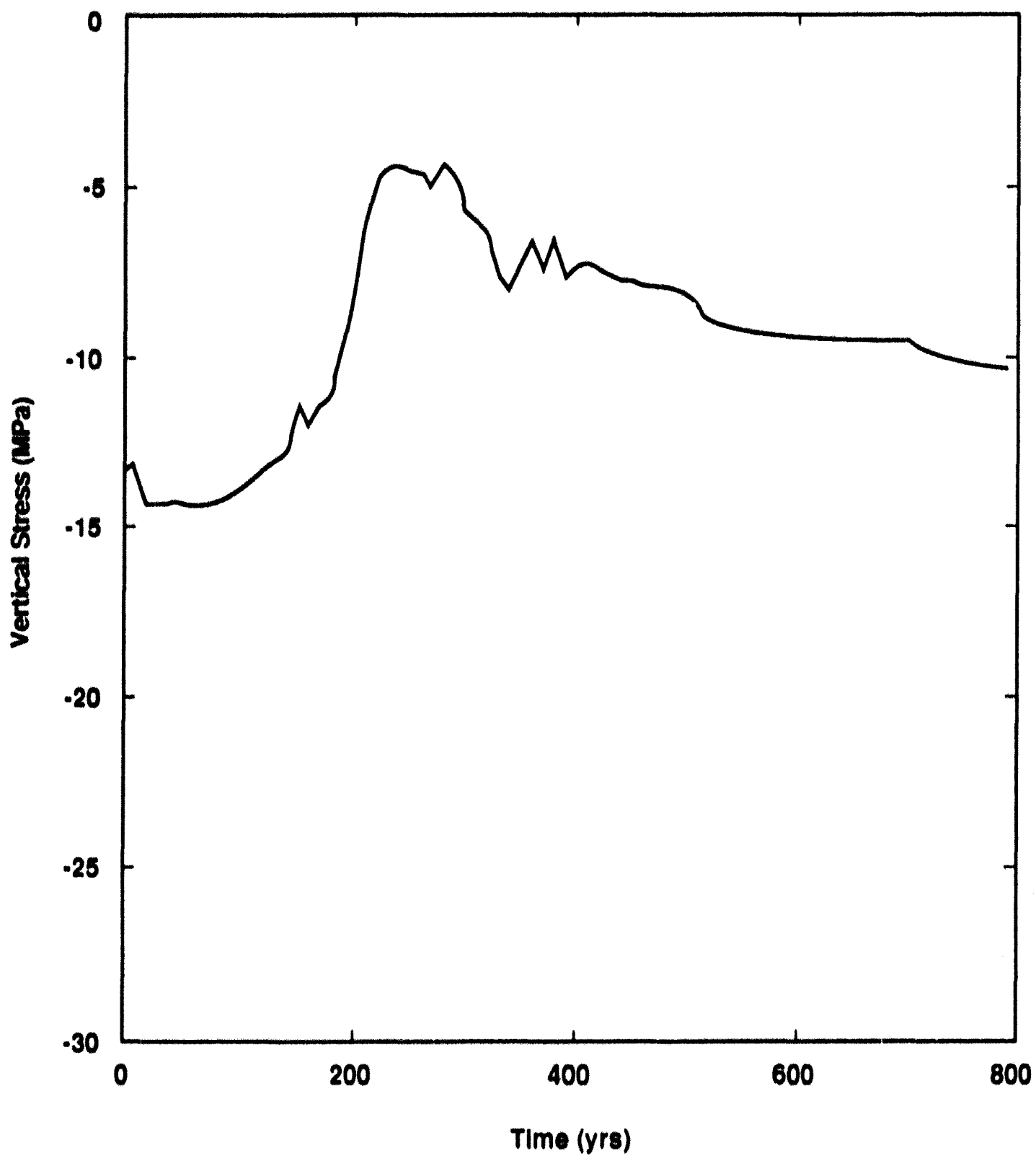

Tค1.6345.125.0

Figure 5-17. The compressive vertical stress history in the first element of an impermeable fracture, the element adjacent to the DRZ, during closure (compressive stress is negative). The curve shows that the stress never drops below a compressive stress of less than about $5 \mathrm{MPa}$. Thus, the crack cannot open if the stress is not tensile, and this element cannot become permeable. (Arguello et al., 1992). 
equilibrium closure state determined for the baseline gas-closure calculation described in Section $5.1 .4(\mathrm{f}=1.0)$. The void volume of this state was $973 \mathrm{~m}^{3}$, and the void fraction was about $40 \%$. Although this volume was held constant with time, it was believed to be an adequate approximation of the disposal room for purposes of conceptual model verification. Other key assumptions were that (1) gas generation rates were approximately the same as the baseline rates in Section 5.1.4, (2) the stratigraphy could be simplified to two overlying and urderlying composite interbeds with permeability of $10^{-19} \mathrm{~m}^{2}$, (3) the composite interbeds were always directly hydraulically connected to the disposal room because of the DRZ, and (4) the initial water saturation within the room was 0.29 .

The principal published results from Davies' two-phase analysis were in the form of gas-saturation curves showing the gas content of the upper interbed (highest gas content) at distances $4.5,10,20,130$, and $600 \mathrm{~m}$ away from the vertical centerline of the room. Curves for each station showed complete brine saturation until gas arrival, followed by an increase in gas content until a maximum was reached. Close in to the disposal room, the gas saturation front was fairly abrupt; whereas, at the $600 \mathrm{~m}$ station, the increase in saturation was over a longer period of time. Interpretation of the results also showed that, for a given depth of penetration of the gas, the difference in gas pressure between the front and the room was small. These conclusions suggested two major simplifications in the observed two-phase response: (1) gas migration within the marker bed could be approximated as a moving boundary, and (2) the gas in back of the moving boundary could be assumed to be at the same pressure as the gas pressure within the disposal room. Thus, gas penetration of the fracture for crack-opening analysis could be specified by a power law that defined the location of the moving boundary as a function of time,

$$
x(t)=1.14 \times 10^{-6} \cdot t^{3.22},
$$

where the distance $x$ is measured in meters from the wall face, and $t$ is the time in years. This relationship was considered an adequate representation of crack pressurization. This relationship is valid for $x<600 \mathrm{~m}$. The error introduced by simplifying the SANCHO mesh configuration even more by locating the fracture interface at the midheight of the room rather than $2.1 \mathrm{~m}$ above the room, as in the two-phase flow calculation, was not considered important.

For the permeable fracture model, gas was then assumed to diffuse into the fracture prior to its opening, and like the impermeable fracture, it too opened if tensile stress was present. Opening was assisted by the increased pore pressure: once the fracture opened, the open part of the fracture was assumed to have the same pressure as the gas pressure in the room. Unlike Case 1 , tensile stress did develop at the fracture interface in this calculation, as implied by the opening profiles at different times after the start of gas pressurization (Figure 5-18).

Figure 5-18 shows, for example, that after 240 years the fracture has opened almost $40 \mathrm{~mm}$ at its point of emergence into the disposal room. The opening then decreases with distance into the interior of the fracture to zero opening about $7 \mathrm{~m}$ from the wall. Although the distance of gas pressurization corresponding to 240 years after initiation of gas pressurization would be $53 \mathrm{~m}$ according to Equation (5.2.1), this value is actually misleading because the problem configuration was for an interior room in an array of rooms. Thus, gas penetration to a distance of half the pillar thickness, or $15.3 \mathrm{~m}$, was sufficient to cause communication through the plane of symmetry to an adjacent room. According to Equation (5.2.1), penetration $15.3 \mathrm{~m}$ away from the room would have taken about 165 years.

The effect of using array symmetry is further emphasized by the opening curve at 260 years in Figure 5-18, which shows that opening is complete throughout the entire pillar and that gas 


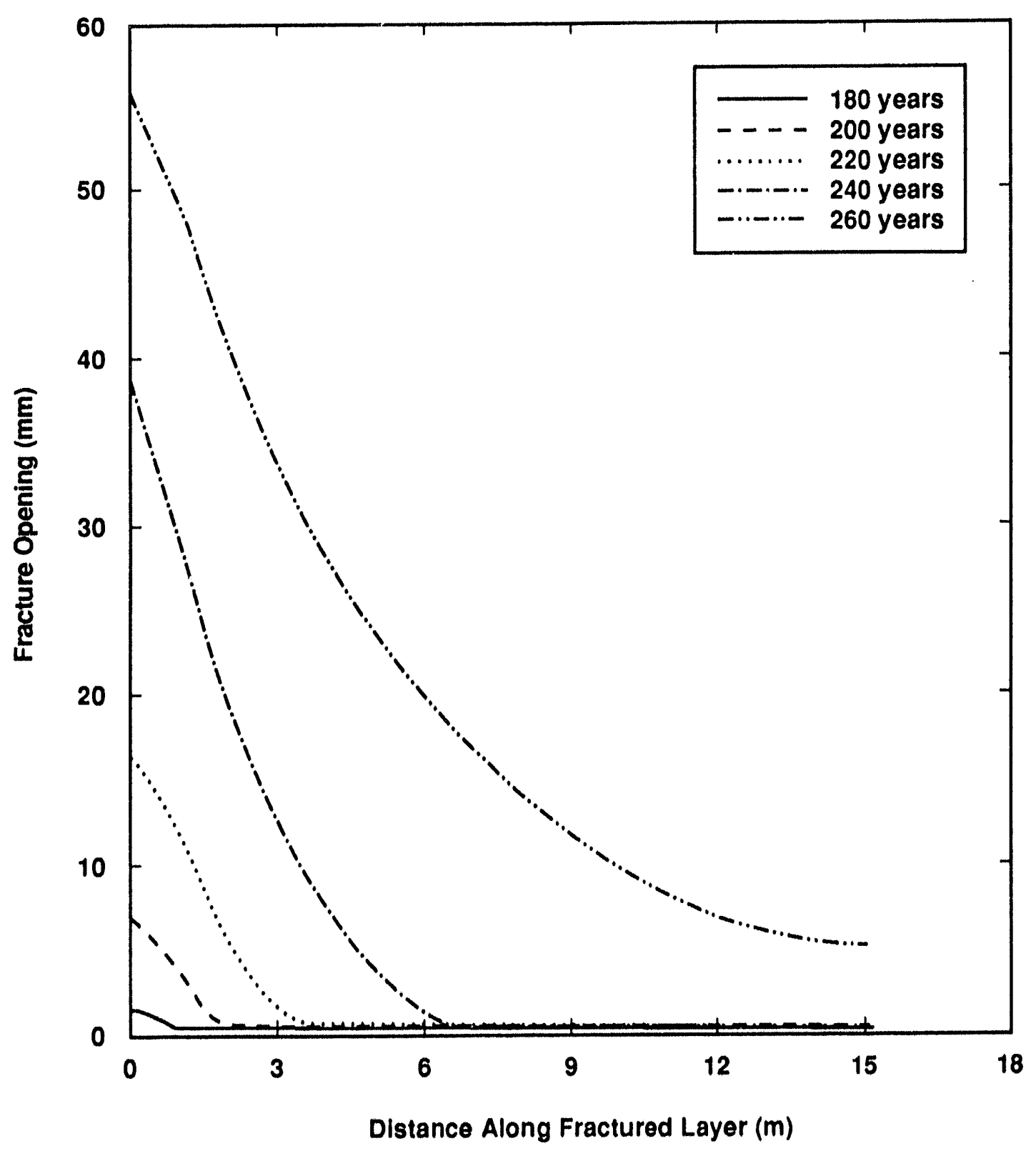

TRI.6345-116.0

Figure 5-18. Opening of a permeable fracture that intersects a disposal room at its midheight by gas pressurization. The configuration assumed for this calculation was a single room within an infinite array of rooms. Opening of the fracture proceeded from the edge of the disposal room to reach the midpoint of the pillar between it and an adjacent room after 240 years. The entire pillar was then lifted upward, forming a gap containing gas at lithostatic pressure (14.8 $\mathrm{MPa}$ ). (Arguello et al., 1992). 
pressurization has now raised the entire pillar upward a distance of about $6 \mathrm{~mm}$ at its centerline. This response suggests that for the gas production assumed, in the absence of other leakage paths, gas communication between different rooms in a panel is inevitable if easy gas migration through fractures in the interbeds can occur.

The gas-pressure history of the room during crack opening is also of interest. The pressure/void-volume relationship in Figure 5-19 shows that the room-closure history is initially typical of the histories observed for perfectly sealed rooms. Closure slows as gas pressure increases as a minimum volume is reached, and then gradual expansion begins. Unlike a perfectly sealed room, however, fracture opening commences when lithostatic pressure is reached, with the consequence that now additional volume for gas is generated by the opening crack, and the pressure remains constant. The implication from this calculation that the room void volume simply increases to maintain a constant lithostatic pressure after lithostatic pressure is reached may be an artifact of the model, as will be discussed in the next paragraph. Nevertheless, the fracture is expected to act somewhat like a safety valve by reducing additional pressurization once a set pressure, in this case lithostatic pressure, is reached.

To provide additional insight about the constraints imposed on fracture opening by the array-room configuration, calculations were also performed for a permeable crack opening from an isolated room. The first difference observed was that pressurization of an isolated room was slower than the pressurization of an array room. This difference will be discussed in Section 6.3. Retarded pressurization is caused by lower mean and deviatoric stresses in the halite surrounding the isolated room as compared to the stress field around an array room. The slower closure rate resulted in higher void volumes within the isolated room, which provided greater gas-storage volumes than for the array room at a given time.

The fracture in the isolated room analysis remained closed for about 270 years and then began to respond to the pressure buildup. Unfortunately, the fracture opening displacement for the isolated room became numerically unstable after about 310 years of simulation time. The cost of running the analysis was excessive, and the problem was terminated.

In summarizing the results of this section, it is important to emphasize that all conclusions in the preceding paragraphs must be verified by additional analysis. A relatively crude model of gas generation was used in the permeable fracture calculation, and a number of very broad simplifying assumptions, including array room symmeiry, was required to obtain a solution. The results confirm, however, that fairly large changes in room performance are to be expected when the constraining assumption of a perfectly sealed room is removed. Examination of these changes will be the thrust of future studies. In addition, supporting evidence may come from planned experimental work to show that the marker beds in the undisturbed region outside the DRZ contain pre-existing fractures, will readily accept fluid pressurization, and will dilate as pressures approach lithostatic. 


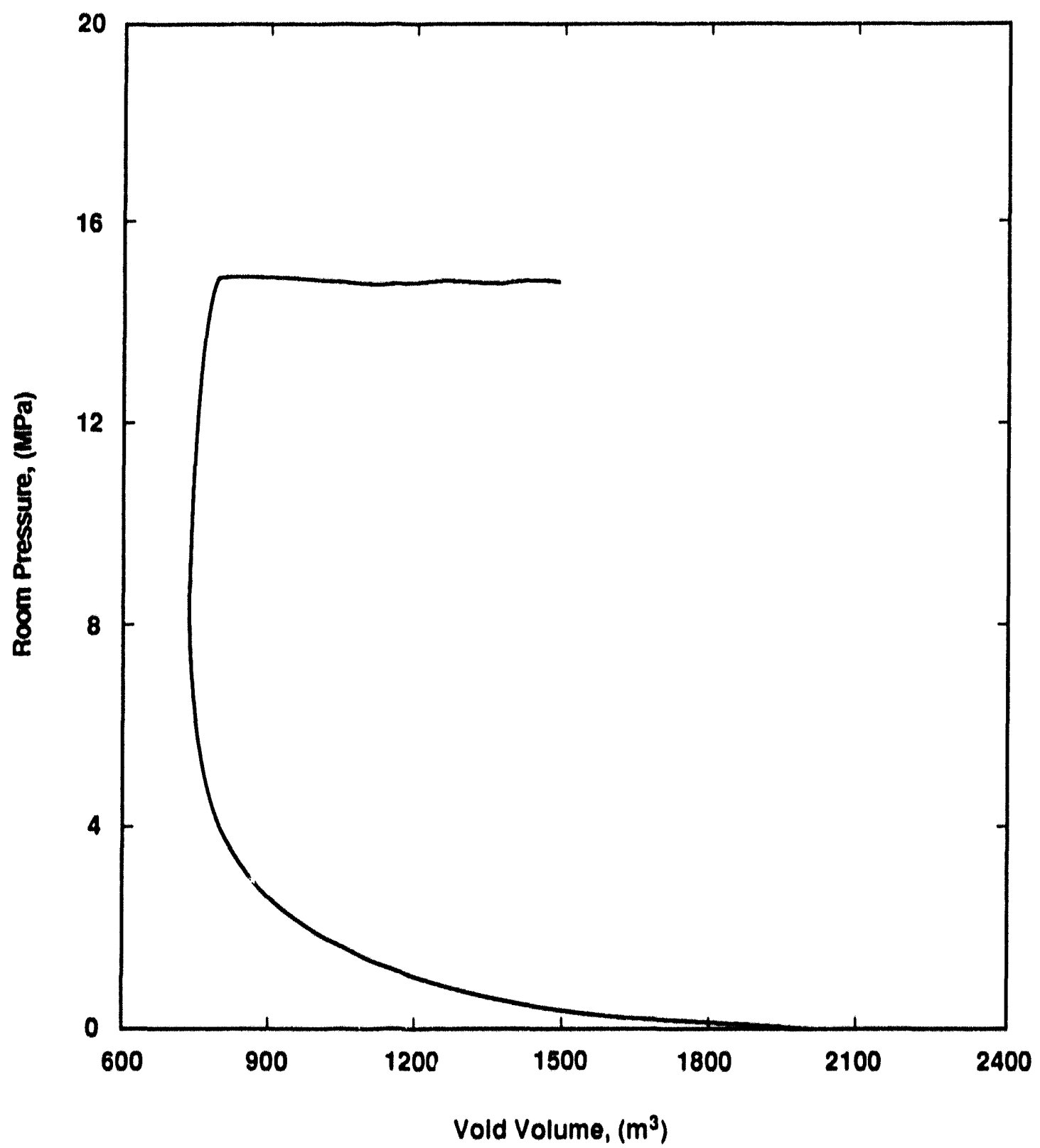

TP1.6345.143.0

Figure 5-19. Gas-pressure/void-volume history in a disposal room intersected by a permeable fracture. The rapid increase in void volume indicated by the horizontal portion of the curve at $14.8 \mathrm{MPa}$ pressure occurred when opening reached the midpoint of the adjacent pillar at around 250 years and began to lift the entire pillar upward. (Arguello et al., 1992). 


\subsection{ADDITIONAL MODEL DEVELOPMENTS}

\subsection{Human Intrusion}

The human intrusion scenarios for the WIPP generally assume that drilling in connection with programs for hydrocarbon exploration will eventually penetrate the repository at some time after it is decommissioned (40 CFR 191, US EPA, 1985; WIPP Performance Assessment Division, 1991b). The assumption is also made in some scenarios that the drilling crew will be unaware that they have drilled into a radioactive waste repository and will continue drilling until the borehole intersects a brine reservoir in the Castile Formation. Drilling will then be suspended, and the site eventually abandoned after plugging the hole. Variations of this scenario are abandonment of the hole after it intersects the repository or multiple penetrations in which abandonment of a first hole will be followed many years later by a second hole penetrating the repository.

The immediate effect of human penetration of the repository will be depressurization of either gas or brine in the room, followed by resumption of transport of brine into the room. The sources of brine can be from the surrounding formation, up from Castile brine pockets, or down the borehole from regions above the repository. Any new brine in contact with the waste will reestablish gas production and, if it is not used up, become radioactive. Depressurization of the room contents will also cause resumption of closure processes.

The changing environment of the room after a human intrusion must be examined to predict the consequences of depressurization on radioactive brine migration. Although consolidation calculations that investigate drilling penetration have not been completed, the computational methods described in the previous sections can be used to predict the nonlinear behavior of the subsequent consolidation process. The procedure appears straightforward. Closure calculations, such as the gas calculations described in Section 5.1.4, are stopped at the time of human intrusion and the amount of gas within the room redefined. This process requires assumptions about how much gas escapes instantaneously up the borehole and what the subsequent borehole-leakage rate is, which can be obtained by independent calculations. The code is then restarted and allowed to continue in a normal fashion. Some approximations of closure after depressurization that can be used in lieu of the availability of actual closure histories are discussed in Chapter 7.0.

\subsection{Coupled Flow-Closure Calculations}

An obvious deficiency of the present room disposal calculations is the absence of a method for relating the dependence of corrosion gas production to the availability of brine. 1 A more refined model would address the following circle of events: (1) as gas is produced and as gas pressure increases, brine inflow into the room would be inhibited and brine may even be expelled from the room and, in turn, (2) if the waste is not wet, corrosion will be much slower and less gas will be produced. Thus, the race is on between brine/waste interaction, gas-pressure buildup, and brine rejection by gas. The outcome of this competition cannot be predicted without including brine flow in the calculations.

1 Any change in the current assumption that microbial decomposition occurs without brine consumption would also influence brine availability. 
Definition of how much brine is actually available at a given time to interact with the wastc would ultimately require coupling of a structural code with a two-phase flow code. However, while two-phased analyses, such as described by Davies (October 4, 1990, memo in Appendix A), offer insight into gas/brine distributions, these codes also lack the capability to directly include empty room closure, much less the suphisticated response associated with backfill consolidation and waste compaction. Similarly, the computer time required for solution of 2-D, flow-independent closure problems involving relatively simple disposal room configurations with structural codes (SANCHO and SPECTROM-32) is about as large as can be tolerated and is expected to be excessive if a complete two-phase flow description were added to them. Given the current state of development of numerical codes in terms of solution techniques and the amounts of computer time required for solutions, it is unlikely that any code will be available in the near future to couple in detail the structural and two-phase flow processes.

Three approaches are in use to compensate for inability to directly relate two-phase gas/brine flow to gas-controlled closure. In the first approach, illustrated by Davies' calculations (June 26, and October 4, 1990, memos in Appendix A) described in Section 5.2, the disposal room volume is assumed fixed at appropriate values determined from mechanical closure calculations. Then the flow analysis is performed assuming that the volume remains constant with time. This approach is appropriate only as long as the volume change associated with subsequent closure remains small, which is unlikely if gas is still being generated. A second approach is to reduce the two-phase flow part of the problem to a simpler description that can be included in either flow or closure calculations. An example that couples single-phase brine flow with the geomechanical codes will be described in the next section. Other methods attempt a simplified description of closure in the flow codes. The third approach evokes the concept of closure surfaces, described in Chapter 7.0 .

\subsubsection{Coupled Single Fluld Phase/Geomechanical Code}

Although implicit coupling between a complete two-phase gas/brine-flow description and the present stress analyses is unrealistic, coupling brine flow with the geomechanical room behavior is possible and is a goal for the WIPP disposal room modeling effort. In this study, a simplified model describing saturated transient fluid flow has been added to SANCHO, according to the following equation (Mendenhall et al 1991):

$$
C_{1} \frac{\partial p_{v}}{\partial t}-C_{2} \nabla^{2} p_{v}=-C_{3} \frac{\partial \sigma}{\partial t}
$$

where

$$
\begin{aligned}
& C_{1}=\frac{\left(1-\phi_{0}\right)}{K}-\frac{1}{K_{1}}+\frac{\gamma_{10}}{\gamma_{f}} B \phi \\
& C_{2}=\frac{k}{v}
\end{aligned}
$$




$$
\begin{aligned}
& C_{3}=\frac{\left(1-\phi_{0}\right)}{K}-\frac{1}{K} \\
& \sigma=\text { mean stress }\left(\sigma_{i i} / 3\right), \text { with compressive stress negative, } \\
& p_{v}=\text { pore pressure, }
\end{aligned}
$$

and

$$
\begin{aligned}
& k=\text { permeability, } \\
& \nu=\text { fluid viscosity, } \\
& \phi \quad=\text { porosity, } \\
& \phi_{0}=\text { reference porosity, } \\
& \gamma_{f}=\text { fluid density, } \\
& \gamma_{f_{0}}=\text { reference fluid density, } \\
& K=\text { drained bulk modulus, } \\
& K_{1}=\text { solids bulk modulus, } \\
& B=\text { compressibility. }
\end{aligned}
$$

The left side of Equation (6.2.1) represents the transient saturated flow equation, and the right side is the coupling with the time varying stress field. If the stress field does not vary with time (or $C_{3}$ is zero), then Equation (6.2.1) simplifies to the standard saturated transient flow equations. Also note that if the mean normal stress increases with time, the right hand term becomes a sink term, and if the mean normal stress decreases with time, it becomes a source term (Mendenhall et al., 1991). This can be thought of as either creating or reducing pore space in the saturated, deformable medium.

Results from application of the coupled brine-flow model to brine flow into an empty disposal room are shown in Figure 6-1. The "reference" curve represents brine flow into a constant room volume, i.e., independent of time. The "geometry" curve represents brine flow into a room closing with time but not coupled to the surrounding stress field. In this case, decreased brine inflow is caused by the reduction of the surface area of the room with time. The "coupled" curve shows the reduction in brine flow caused by both the reduction in surface area of the room and by the increased pore space caused by the normal stress gradient adjacent to the room (as discussed in the previous paragraph), which changes with time.

Figure 6-1 shows that brine accumulations using the coupled saturated-flow model are actually less than estimates assuming that the room volume remains constant at its initial value. These calculations define the brine flux through the room boundary as a function of time. The next step in improving the model would be to assume that any brine entering the room is instantaneously in contact with the waste. The amount of free brine could then be used, along with assumptions about how rapidly it is being consumed by the reaction, to estimate how much gas is generated. Although these refinements of the coupled-flow model are not yet available, they would be a major improvement. In comparison with a full, two- phase flow description, they include the dependence of corrosion on brine availability. In addition, they capture, in part, reduction of brine-flow flux into the room because of gas pressurization. As the gas pressure within the room increases, the gradients driving brine into the room are reduced by the increasing gas pressure at the room boundaries. Flow should eventually cease as the room pressure becomes

2 The simplest conceptual model is that brine forms puddles on the floor of the disposal room, allowing any iron in contact with it to corrode at the corrosion rate measured in the laboratory. 


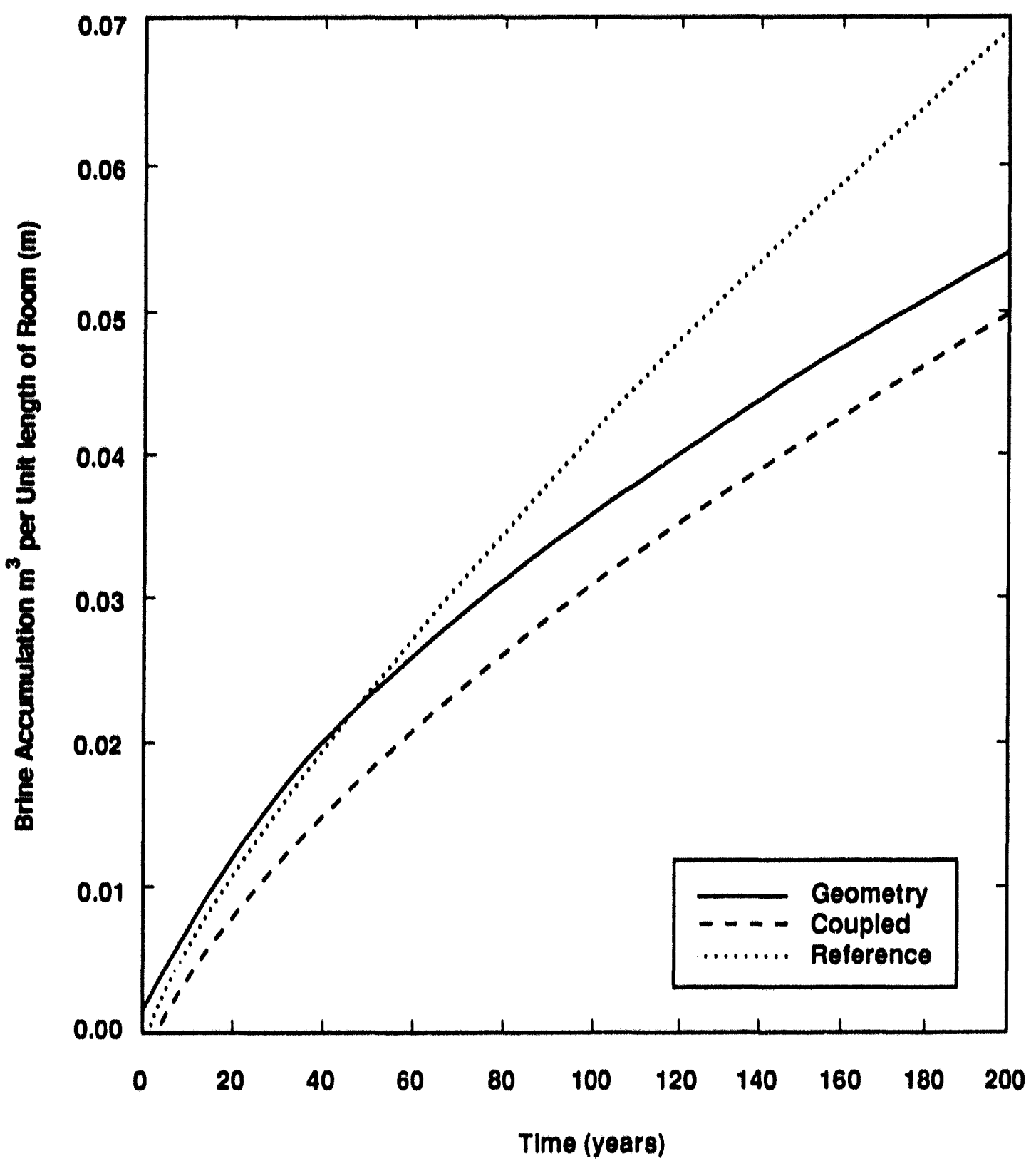

TR1. 6346-46.2

Figure 6-1. Predictions of brine accumulation within empty disposal rooms assuming (1) no change in volume because of closure (reference curve); (2) uncoupled-brine flow into a closing room (geometry); and (3) brine flow into a closing room, coupling the surrounding stress field with the fluid flow. (Mendenhall et al., 1991). 
equal to the far-field pore pressure. The features of flow not described by this model are gas leakage from the room and situations when the gas pressure is sufficient to actually dewater the room by forcing brine away from it out through the interbeds. Such response has already been discussed in Section 5.2. The coupled model is considered bounding because it maximizes gas pressure within the room. Additional refinements would reduce gas generation because even less brine would be available for corrosion or reduce gas pressurization because leakage has not been considered.

\subsection{Panel Scale Modeling}

Closure results for isolated rooms and rooms in an infinite array are different from each other, as discussed in Section 5.1.4. Furthermore, although neither configuration is an exact representation of a panel room, one of these two idealizations had to be assumed for past analyses because of numerical analysis limitations. The questions, remain, however, as to whether either representation provides a satisfactory approximation to closure, and what order of magnitude of uncertainty is introduced by this simplification.

Recently, a new finite-element stress analysis code (SANTOS) has been applied to questions pertaining to the uncertainty introduced by assumed room configurations (Stone and Arglaello, December 17, 1991, memo in Appendix A). To put SANTOS capabilities into perspective, early empty-room closure calculations with SANCHO by Morgan (June 2, 1987, memo in Appendix A) utilized approximately 700 quadrilateral finite elements for the discretization and required 200 minutes of CPU time on the Cray computer for closure solutions. In contrast, the 2-D panel scale calculation with SANTOS contained over 23,000 quadrilateral finite elements but needed only 55 minutes of CPU time on the computer. This enhancement of problem scope and running time permits examination of various aspects of closure that previously were not feasible.

The SANTOS calculation by Stone and Argüello (December 17, 1991, memo in Appendix A) was the first 2-D analysis of the creep closure of a complete WIPP panel. It encompassed a vertical cross-section of the repository intersecting seven rooms in a single panel, at their midpoints, and the two haulage ways that run north/south at the facility to provide access to the panels (Figure 6-2). A plane of symmetry was assumed to exist about a north/south line drawn through the center of the four haulage ways: mesh discretization for the seven room panels, the two haulage ways, and the transition zone between the two types of excavations are shown in Figures 6-3a and 6-3b. The stratigraphy for this calculation was assumed to be all salt, and Young's modulus was divided by 12.5 (e.g., Section 5.1). The reader is referred to Stone and Argüello's memo in Appendix A for further details of the numerical model.

The results of the panel calculation for totally empty rooms in Figures 6-4a and 6-4b show vertical closure for rooms and haulage ways over 200 years. Vertical room closure is defined as the sum of the floor and ceiling displacements at the room centerline. Because the rooms are $4 \mathrm{~m}$ high, a closure of $4 \mathrm{~m}$ signifies that the floor and ceiling touch. Room 4 , which is located at the center of the panel and has been previously modeled as an array room, closes most rapidly. Estimated closure of an array room (Morgan, June 2, 1987, memo in Appendix A) almost exactly duplicates Room 4 closure. Room 1, at the end of the panel away from the haulage ways, exhibits 


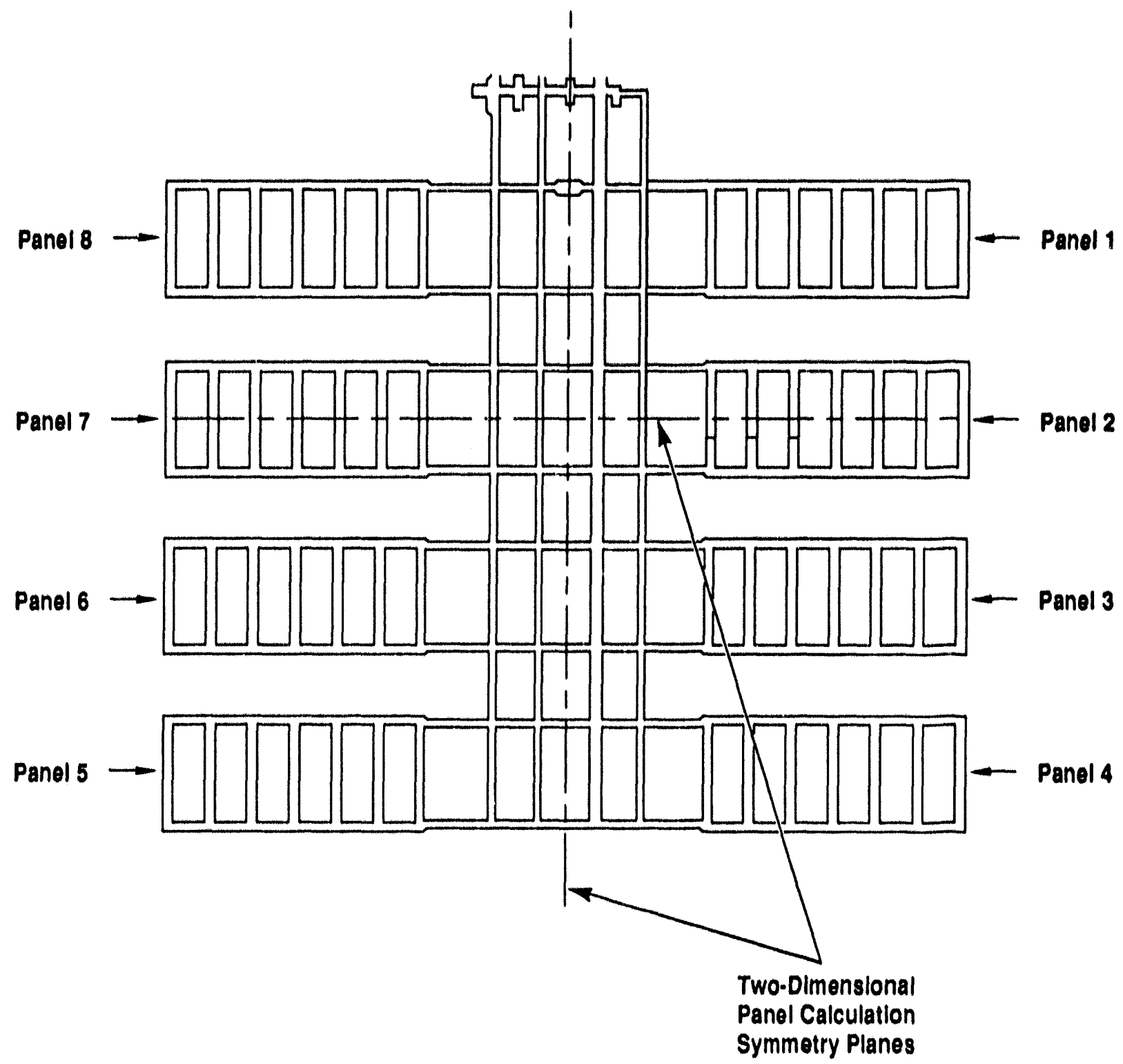

TA1.6334.206.4

Figure 6-2. Plan view of the WIPP storage arca showing the vertical symmetry planes assumed for a 2-D calculation of the closure of a panel (Stone and Arguello, December 17, 1991, memo in Appendix A). 


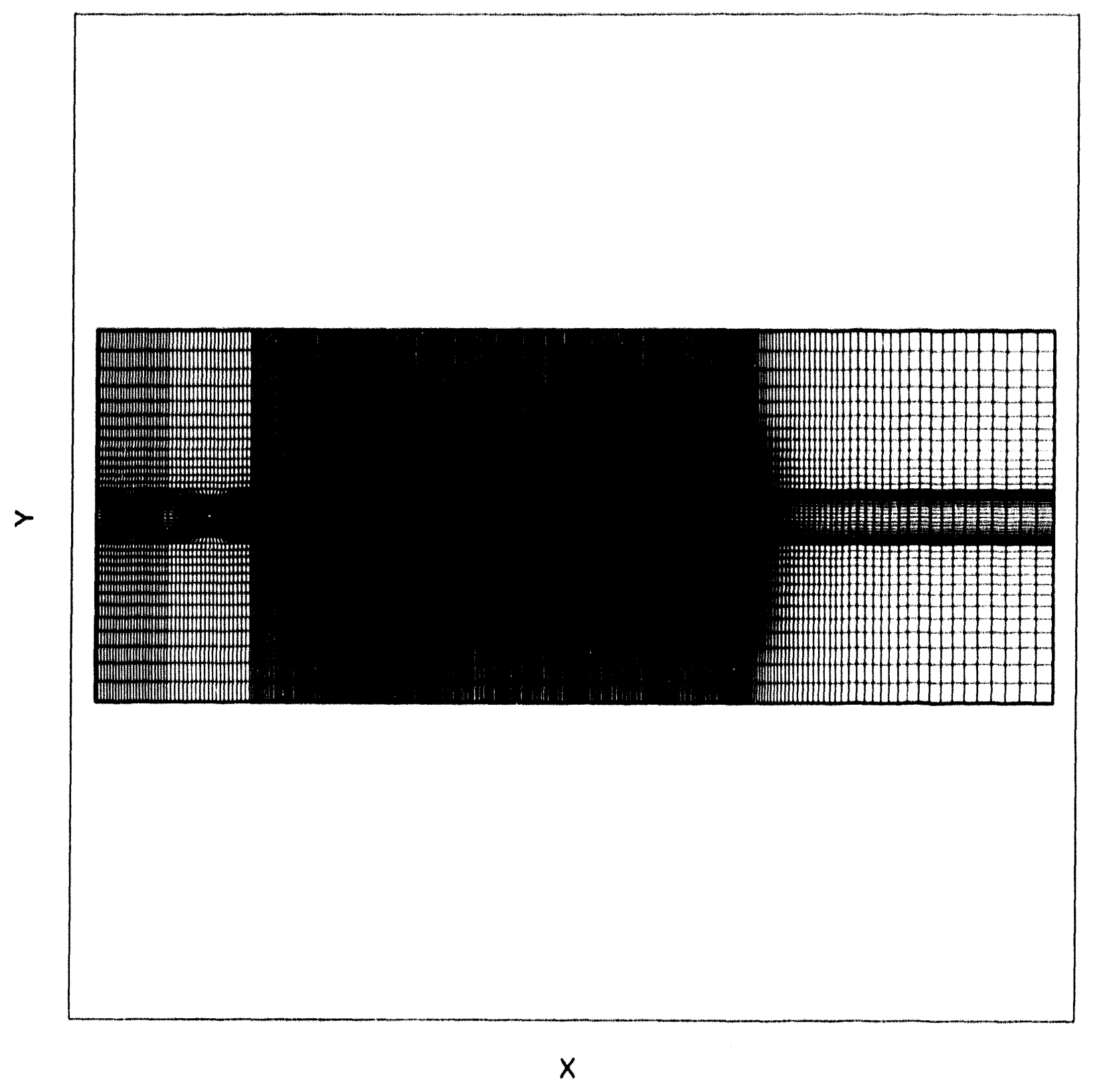

IAI 63451950

Figure 6-3a. The finite-element mesh used to analyze closure of a complete 2-D panel (Stone and Arguello, December 17, 1991, memo in Appendix A). 


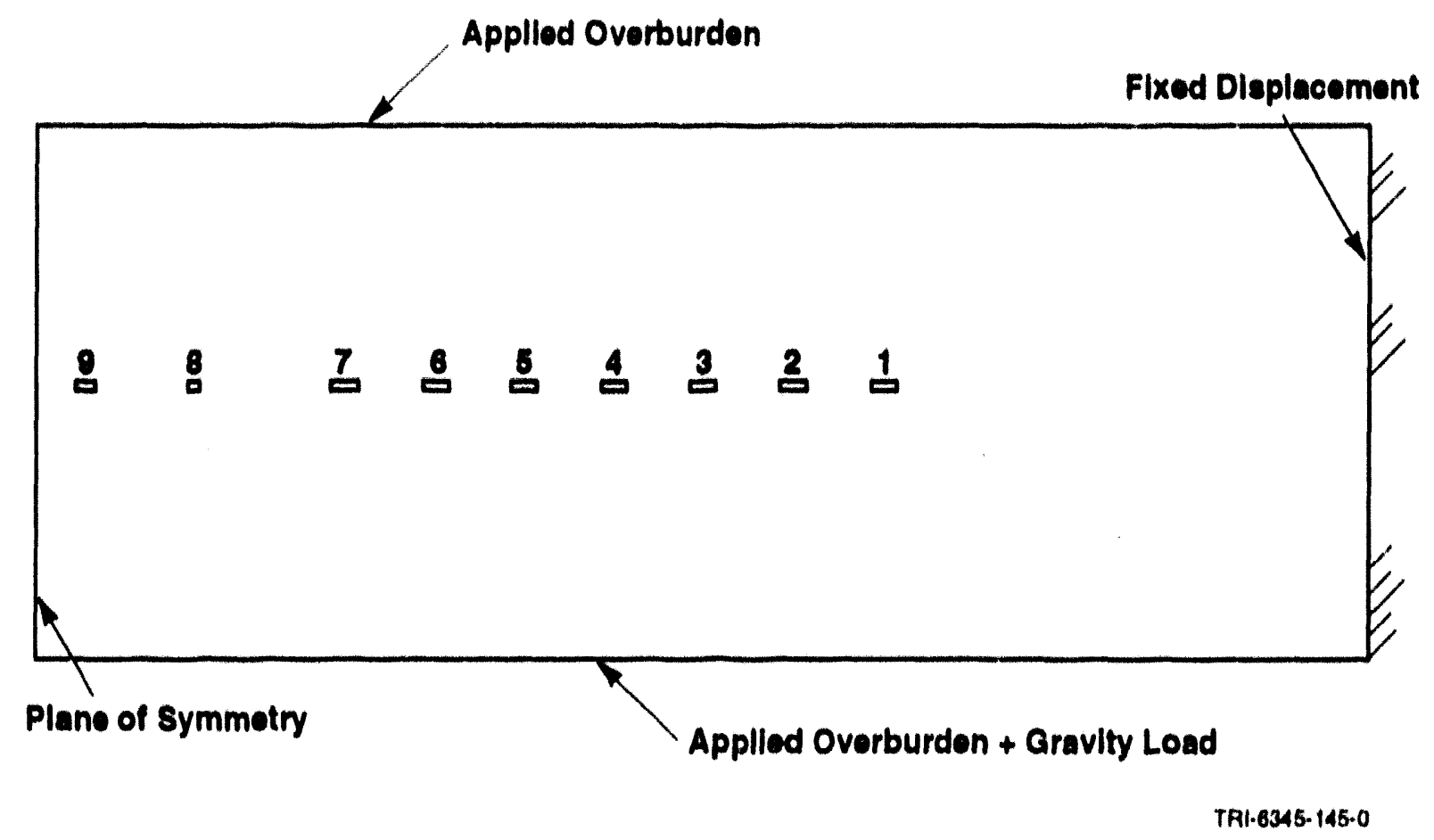

Figure 6-3b. A schematic of the boundary conditions applied to the panel analysis (Stone and Arguello, December 17, 1991, memo in Appendix A). 


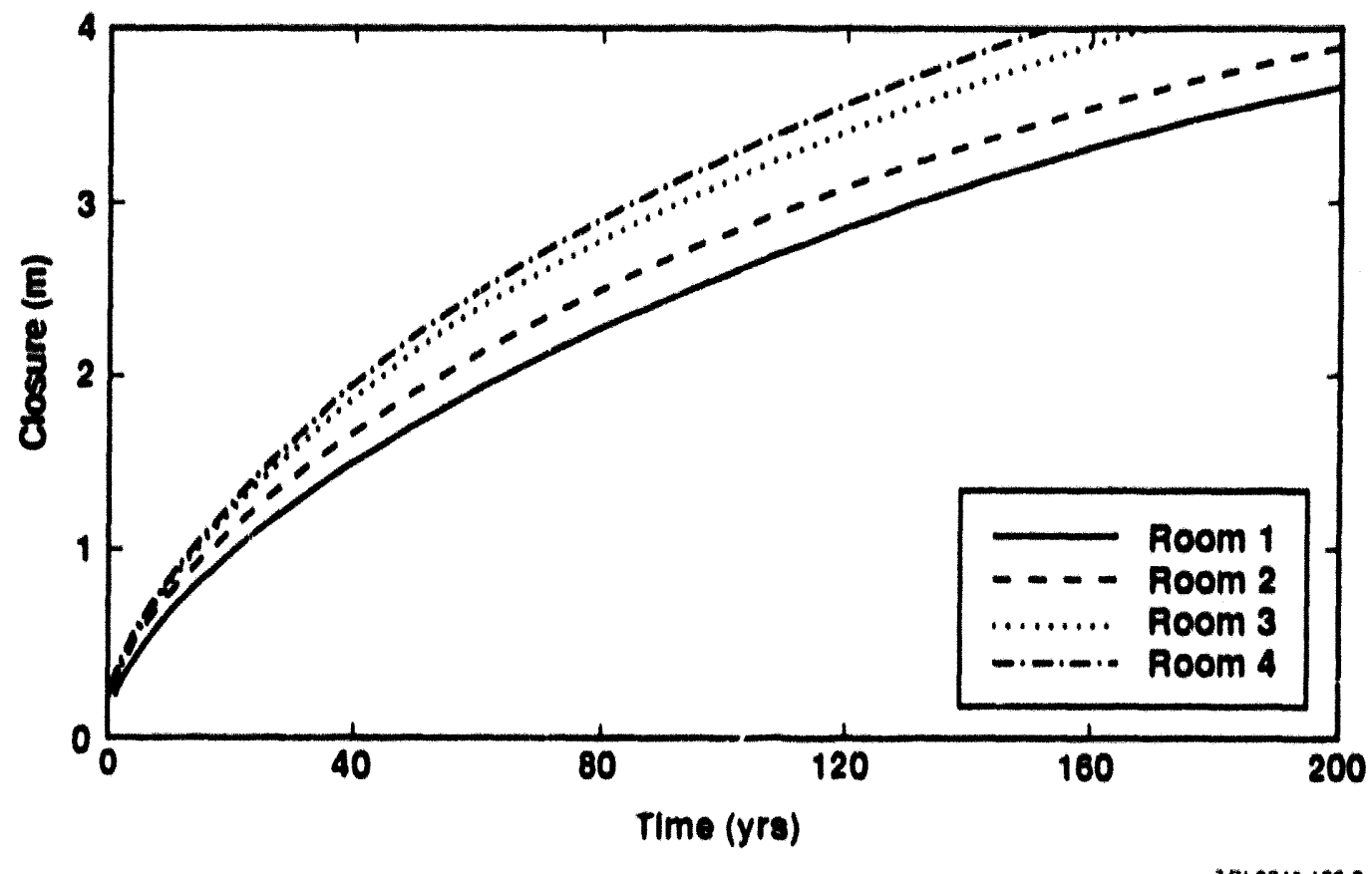

Figure 6-4a. Vertical closure history for panel rooms I through 4 (See Figure 6-3b for room locations) (Stone and Arguello, December 17, 1991, memo in Appendix A). 


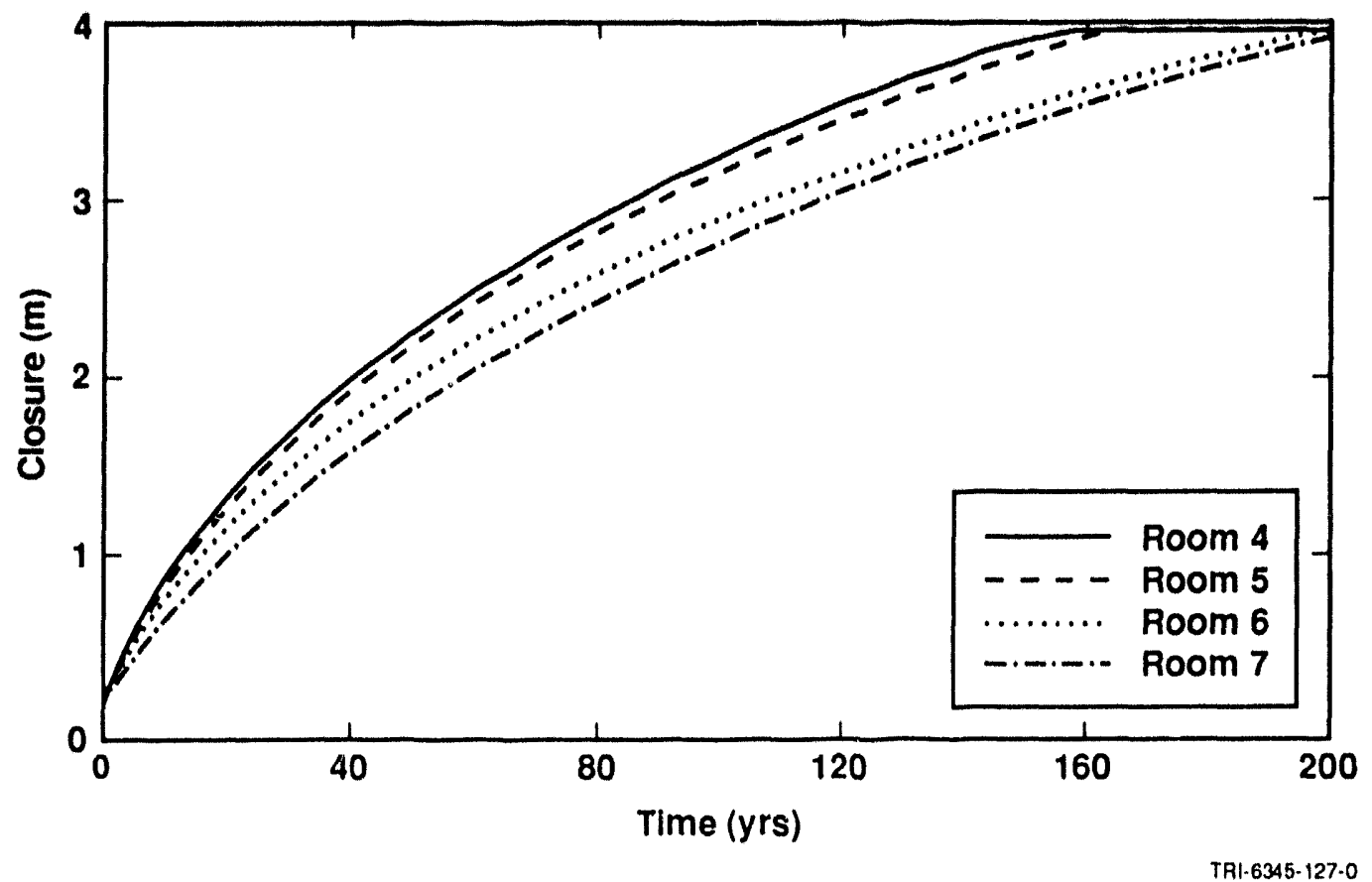

Figure 6-4b. Vertical closure history for panel rooms 4 through 7 (See Figure 6-3b for room locations) (Stone and Argüello, December 17, 1991, memo in Appendix A). 
the slowest closure and is closer in configuration to an isolated room. The difference between the maximum closure (Room 4) and the minimum closure (Room I) at about 160 years is about $0.8 \mathrm{~m}$, or roughly $25 \%$ of the total closure that has occurred in Room 1. The closure of actual disposal rooms is expected to be much less because the rooms would be filled with waste and backfill.

The 2-D panel calculation suggests that the penalty for use of simpler room configurations is an uncertainty of less than $25 \%$ in estimates of how much the room has closed within a given period of time. On a relative basis, array-room closure is too fast, and isolated-room closure is too slow. Errors of this magnitude are considered tolerable for conceptual model studies. The reader is reminded that these conclusions are obtained from 2-D calculations. The question of how well the estimated closures replicate closure at the ends of the room, where the 3-D geometric influence is most pronounced, has yet to be resolved. In terms of other uncertainties associated with the repository performance, such as gas production, these uncertainties are of the order of $25 \%$ and are considered too small to exert much influence on compliance with $40 C F R \quad 19 / B$.

\subsection{Engineered Alternatives Task Force (EATF) Benchmark Problems}

The objective of these calculations was to compare the empirical closure model used by International Technology, Inc. (IT) (US DOE, 1991, Vol. 2, p. B-15) for the EATF analyses with results for room closure from SANCHO, which are based on more scientifically based deformation mechanism theory. The benchmark comparison described in this report was limited to the mechanical aspects of disposal-room closure, although the EATF calculations also included gas and brine flow. The IT closure can be obtained by direct integration of their first-order differential equations. The same initial volumes of backfill and waste as used for the SANCHO calculations were assumed. Integration was performed with an advanced mathematical applications subroutine for solving systems of differential equations in Version 2.5 of the commercially available software package MATHCAD for personal computers.

The equations used by IT for early predictions of the mechanical closure of a unit-long slice of the disposal room are

$$
\begin{array}{ll}
\mathrm{dw} / \mathrm{dt}=-1.042 \cdot 10^{-19} \bullet\left(\left(\sigma_{\infty}-\sigma\right) / 0.0068975\right)^{4.95} \mathrm{w} & \sigma<\sigma_{\infty} \\
\mathrm{dh} / \mathrm{dt}=-4.117 \cdot 10^{-19} \cdot\left(\left(\sigma_{\infty}-\sigma\right) / 0.0068975\right)^{4.95} \mathrm{~h} & \sigma<\sigma_{\infty},
\end{array}
$$

where $t$ is time in years, $w$ is the room width in meters, $h$ is the room height in meters, $\sigma_{\infty}$ is the far-field lithostatic pressure (14.8 MPa), and $\sigma$ is the backstress exerted by waste, backfill, and/or gas within the room. The constants for these equations were obtained by adjustment of their values until agreement was obtained with test measurements of closure rates in situ at the WIPP. The instantaneous average volume of the roorn at a given time $t$ was calculated from

$$
V=w h .
$$

The volume of the room approaches $V=V_{\infty}$ at long times, where $V_{\infty}$ is the asymptotic volume of the room when $\sigma=\sigma_{\infty}$. Gas pressure within the room can continue to increase after this time, but the backstress exerted by the waste and backfill was assumed constant and the room volume also was assumed to remain constant. These conditions apply because the IT model has no provision for expansion of the room volume once lithostatic stress is achieved. In addition, the IT model gives no information about stress distributions in the intact salt adjacent to the room boundary, nor can it be used to predict how porosity is distributed throughout the room. 
Later in the EATF program, the IT equations for mechanical closure were revised to permit closure estimates for different sized rooms. These equations will be called the "refined" equations in the discussion that follows:

$$
\begin{aligned}
& \mathrm{dw} / \mathrm{dt}=-4.594 \cdot 10^{-20} \bullet\left(\left(\sigma_{\infty}-\sigma\right) / 0.0068975\right)^{4.95} h^{1.065} w^{0.63} t^{-0.32} \\
& \text { for } \sigma<\sigma_{\infty} \\
& \mathrm{dh} / \mathrm{dt}=-1.220 \cdot 10^{-20} \bullet\left(\left(\sigma_{\infty}-\sigma\right) / 0.0068975\right)^{4.95} \mathrm{~h}^{1.18} \mathrm{w}^{1.039} \mathrm{t}^{-0.24} \\
& \text { for } \sigma<\sigma_{\infty}
\end{aligned}
$$

where $t$ is time in years, $w$ is the width (now in feet), $h$ is the height (now in feet), $\sigma_{\infty}$ is the lithostatic pressure (14.8 $\mathrm{MPa}$ ), and $\sigma$ is the backstress exerted by waste, backfill, or gas within the room. ${ }^{3}$

Two cases were considered for the comparison: (1) an empty room $(\sigma=0)$ and (2) a room with backstress from gas, backfill, and waste. Mechanical equilibrium requires that the stress in the backfill equals the stress in the waste $\sigma_{b w}$, and the total backstress on the room boundaries is assumed to be the sum of this backstress and the pore pressure caused by gas pressurization $\sigma_{\mathrm{g}}$, that is, $\sigma=\sigma_{\mathrm{bw}}+\sigma_{\mathrm{g}}$ in Equation (6.4.3a) and Equation (6.4.3b).

For the IT equations, the functional relationship for $\sigma_{b w}$ was obtained from the stress, average-room-density data used by IT for the base case (US DOE, 1991, Vol. 1, Table 3) 4 , and the ideal gas law was used to define $\sigma_{g}$ assuming the rate of gas production of 2.66 moles/drum/year for 500 years. Integration time limits for the IT results that follow were limited to 500 years because the reduction of gas generation rates to $0.86 \mathrm{moles} / \mathrm{drum} /$ year from 500 to 700 years simply increased calculated gas pressurization at constant volume without providing additional insight into the predicted state of consolidation of the waste. This insensitivity arose because of the IT constraint prohibiting room expansion.

The results of the comparison are shown in Figures 6-5 and 6-6. Figure 6-5 shows that the empty room results of the IT model using the initial IT equations are a good approximation to the calculations with SANCHO by Morgan (June 2, 1987, memo in Appendix A) that neglected the stratigraphy surrounding the repository. Because the SANCHO calculational result was found to be in agreement with early experimental room-closure data and the IT model was based on the same experimental room-closure data, agreement between the two curves was expected, at least for the early stages of closure. The experimental data used by IT was more complete because it included recent data. Figure 6-5 also shows that the closure history predicted by IT using the refined equations produces somewhat slower closure, but these results are still not as slow as the

3 The constants $4.594 \cdot 10^{-20}$ in Equation (6.4.3a) and $1.220 \cdot 10^{-20}$ in Equation (6.4.3b) were computed by dividing the values $5.523 \cdot 10^{-19}$ and $1.464 \cdot 10^{-19}$ cited in the reference by 12 , to correct for the fact that $\mathrm{dw} / \mathrm{dt}$ and $\mathrm{dh} / \mathrm{dt}$ were assigned dimensions of in/yr and $\mathrm{w}$ and $\mathrm{h}$ were in $\mathrm{ft}$.

4 No attempt was made to correct these results for the slight differences in initial backfill volumes assumed for the SANCHO and IT calculations. The ratio of initial backfill volume to initial waste volume assumed by IT was 0.525 , and the ratio of initial backfill volume to initial waste volume for the SANCHO calculations was 0.502 . These slight variations are caused by different assumptions with regard to the waste volume and how the volume represented by the air gap was included within the calculations. 


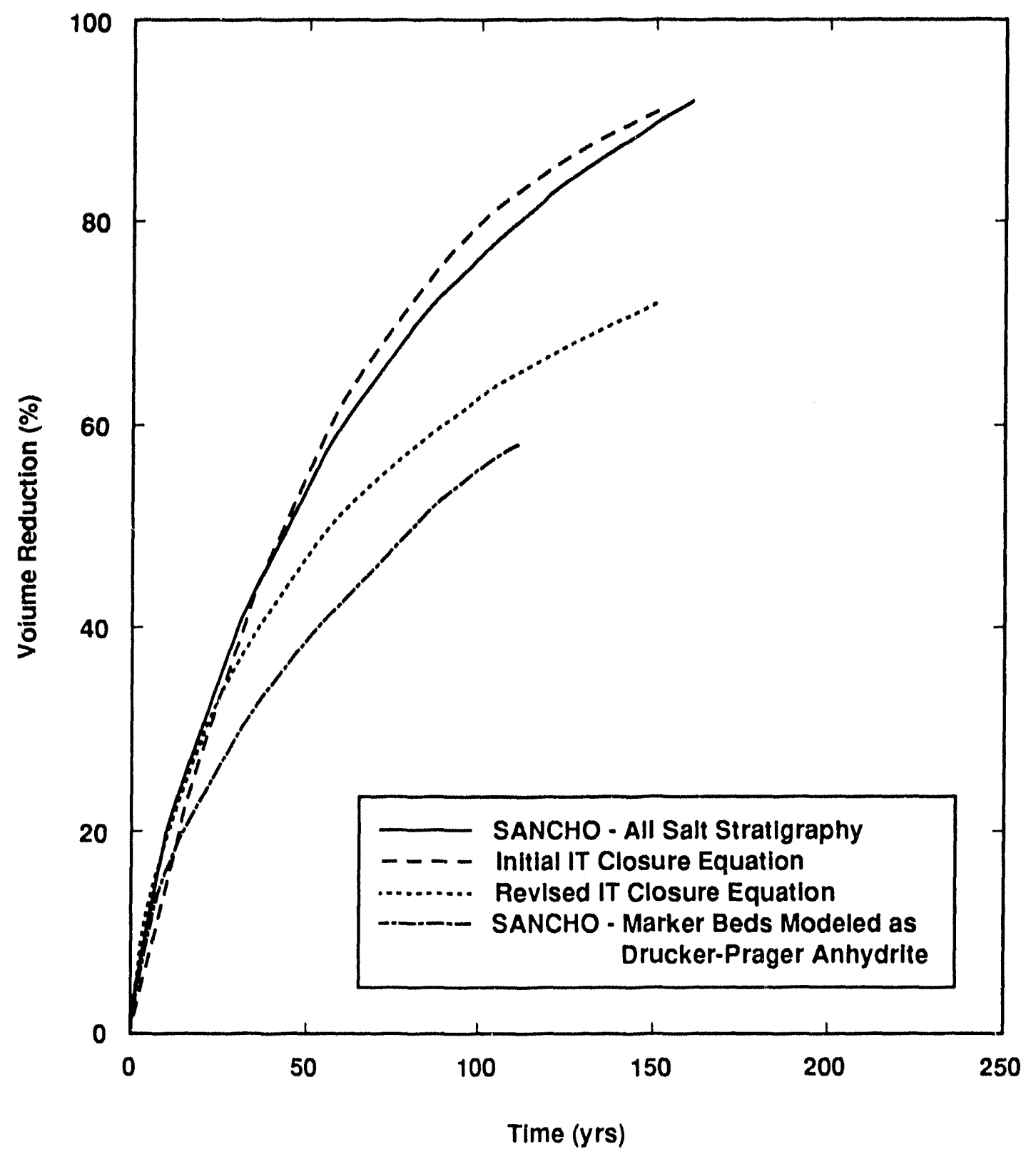

TRI-6345. $128 \cdot 0$

Figure 6-5. A comparison of the closure history for an empty TRU storage room, used by IT (US DOE, 1991) in their evaluation of the performance of various engineered alternatives waste forms with more mechanistically based SANCHO results (Morgan, June 2, 1987, memo in Appendix A). 


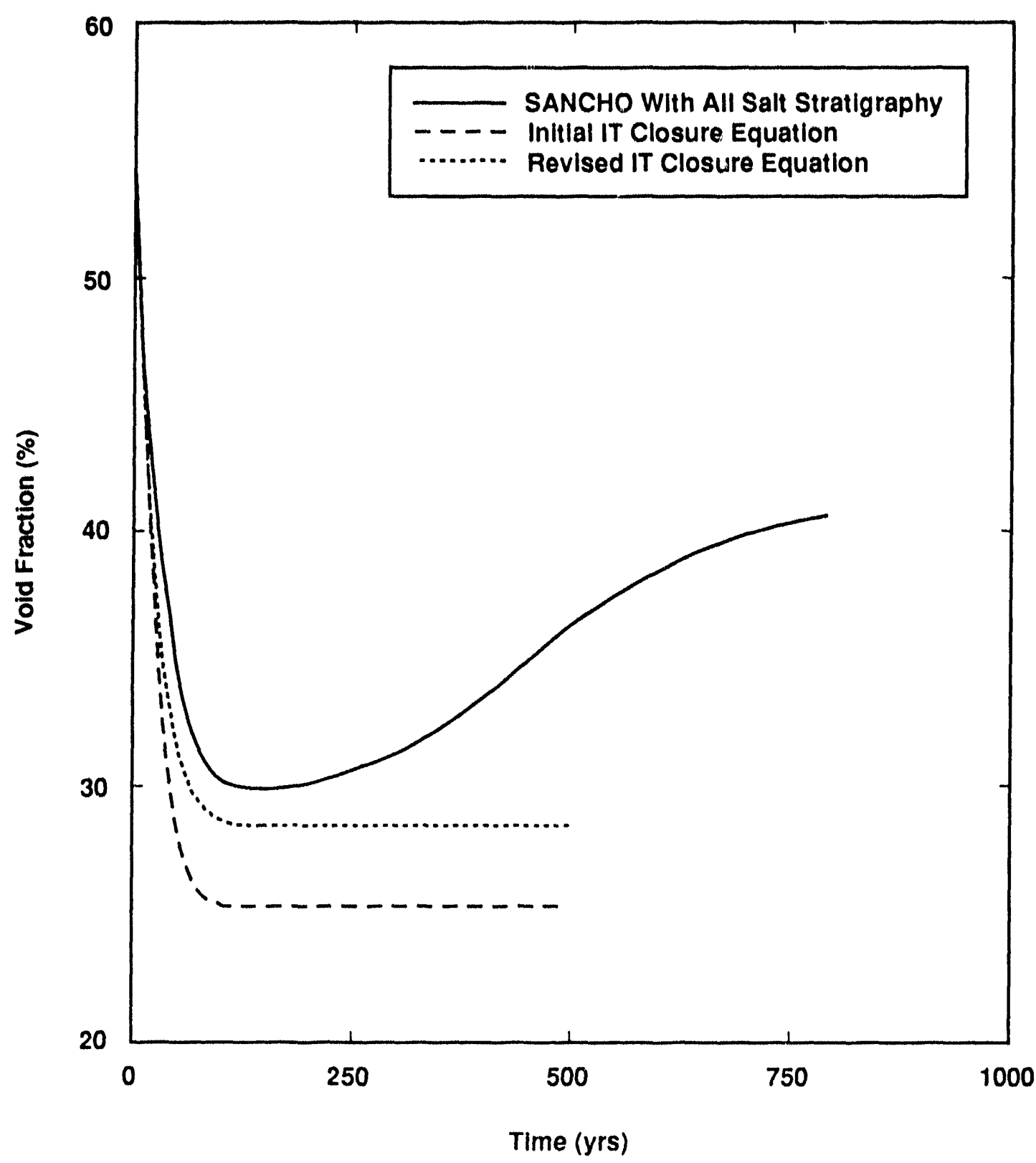

TR1.6345-129.0

Figure 6-6. A comparison of the closure history for a perfectly sealed disposal room experiencing gas pressurization, used by IT (US DOE, 1991) in their evaluation of the performance of various engineered alternatives waste forms with more mechanistically based SANCHO results (Brown and Weatherby, September 17, 1990, memo in Appendix A). 
results of the SANCHO calculation that attempted to model anhydrite marker bed deformation (Morgan, June 2, 1987, memo in Appendix A)

Figure 6-6 shows how gas generation within the disposal room affects its final porosity. Eariy-time agreement between the SANCHO prediction and the IT model appears to be good, although the reader is reminded that small differences in void fraction become more important as the void fraction decreases because they introduce large changes in waste and backfill permeability. The most profound difference between the IT and SANCHO results in Figure 6-6 is the later-time deviation in closure rates caused by the imposed IT constraint of no volume expansion. According to the IT assumption, the void fraction in the room decreases to a minimum, after which it remains constant (the horizontal portion of the curves in Figure 6-6). In the SANCHO results, closure reaches a minimum and then reverses, causing room expansion (e.g., Section 5.1.4). The consequence of the IT assumption is that any additional generation of gas after the constant-volume state is reached causes the gas pressure to increase rapidly to unrealistic values well above lithostatic pressure. This is contrary to physical expectations; room pressurization to several times lithostatic pressure is not likely because the fractures would simply open up and vent the gas to other regions of the Salado until lithostatic pressure was re-established (e.g., Section 5.2).

The observations in the previous paragraph are also a reminder to the reader that extrapolations of non-mechanistic descriptions hold no credibility beyond the regions where their results have been verified by more fundamentally consistent models. Even with codes incorporating mathematical models based on physical mechanisms, extrapolations in time of the extent required for WIPP performance assessment are largely unverifiable. If the models are written correctly, such extrapolations at least have merit in the sense that some degree of physical consistency is maintained. 


\subsection{APPLICATION TO PERFORMANCE ASSESSMENT WITH REGARD TO 40 CFR 191 SUBPART B COMPLIANCE}

Compliance of the WIPP with 40 CFR 191 and other regulations will be established by performance assessment predictions that must demonstrate that transport of radionuclide materials into the accessible environment will not exceed the limits imposed by the standards. A major goal of the disposal-room closure model development effort is to provide a means for including the effects of closure in compliance evaluation. To complete the disposal-room model, closure information must be provided in a form that can be used in the statistical performance assessment methodology. As shown in Figure 7-1, the components of the disposal room model described in this report are part of the Performance Assessment panel system conceptual model (WIPP Performance Assessment Division, 1991c).

A major part of the numerical solution capability for Performance Assessment involves fluid flow because transport of soluble radionuclide materials by fluids constitutes one of the principal mechanisms of release. In these analyses, single-phase or two-phase fluid flow models are required according to whether gas is present as a separate phase. Thus, while single-phase fluid analysis may be appropriate for brine transport in the Culebra upper aquifer regions, a two-phase capability must be used for describing brine flow within and adjacent to the waste panels. The reader is referred to the Preliminary Comparison With 40 CFR Part 191. Subpart B for the Waste Isolation Pilot Plant. December 1991 (WIPP Performance Assessment Division, 1991b, Volume 1, Section 5.2.5; WIPP Performance Assessment Division, 1991c, Volume 2, Chapters 4 and 5) for a comprehensive discussion of this subject.

The observations of the previous paragraph imply that two-phase flow is a principal technical area that must be examined to evaluate the performance of a waste panel. Flow is described by two mass conservation partial differential equations and three constraints comprising the following set of equations (e.g., WIPP Performance Assessment Division, 199/c, Volume 2, Section 5.2.2.4):

Gas Component Conservation:

$$
\begin{aligned}
& \nabla \cdot\left[\frac{\alpha \rho_{n} K k_{r n}}{\mu_{n}}\left(\nabla P_{n}-\rho_{n} g \nabla D\right)+\frac{\alpha C_{N w} \rho_{w} k_{r w}}{\mu_{w}}\left(\nabla P_{w}-\rho_{w g} \nabla D\right]+\alpha q_{n}+\alpha q_{r n}\right. \\
& =\alpha \frac{\partial\left(\phi \rho_{n} S_{n}+\phi C_{N w} \rho_{w} S_{w}\right)}{\partial t}
\end{aligned}
$$

Brine Component Conservation:

$\nabla \cdot\left[\frac{\alpha C_{W w} \rho_{w} k_{r w}}{\mu_{w}}\left(\nabla P_{w}-\rho_{w} g \nabla D\right)\right]+\alpha q_{w}+\alpha q_{r w}=\alpha \frac{\partial\left(\phi C_{W w} \rho_{W} S_{W}\right)}{\partial t}$ 


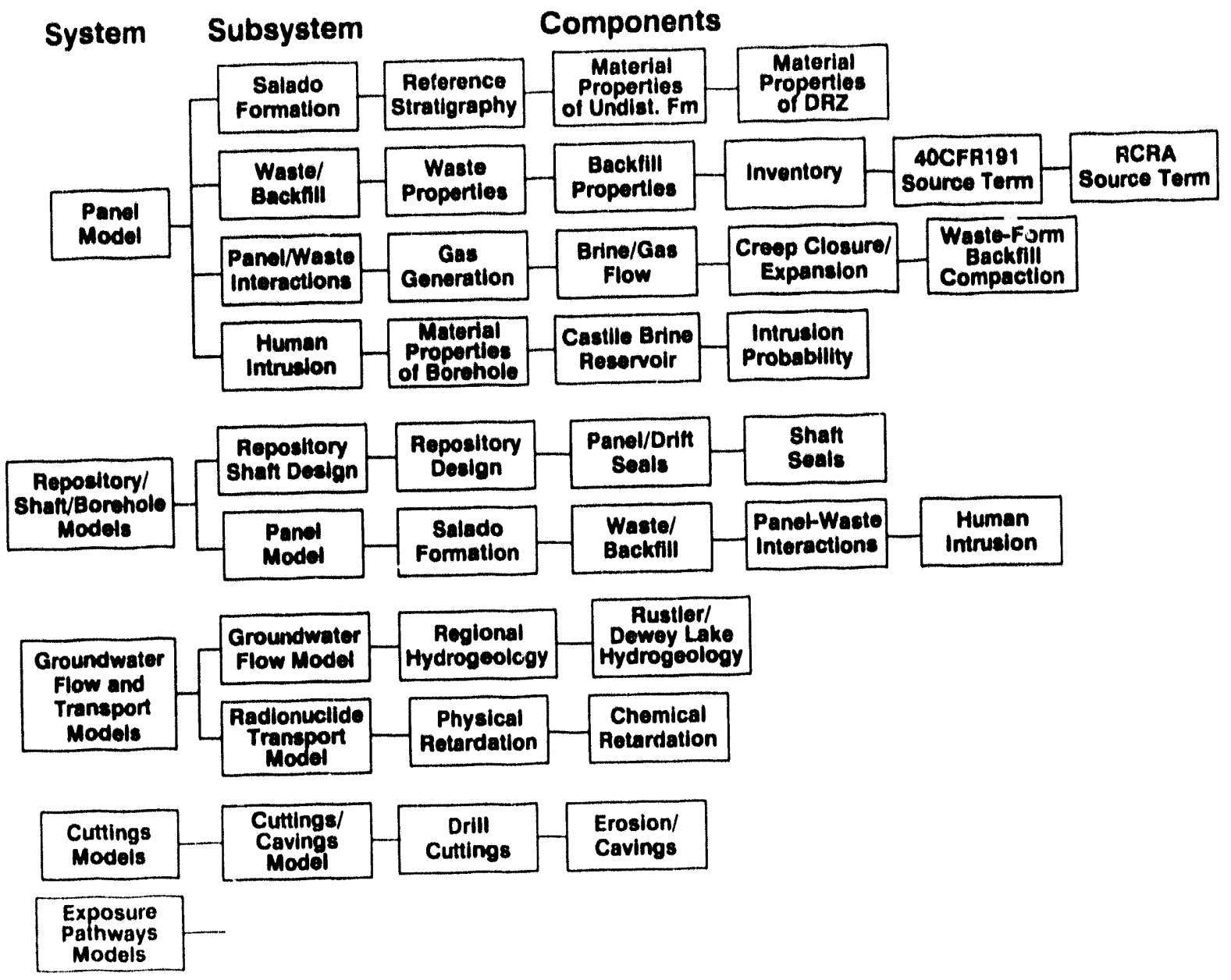

Figure 7-1. The relationship of elements of the disposal-room model to the system used by the WIPP Performance Assessment Group at Sandia to demonstrate compliance of the WIPP repository with government regulations. 
Saturation Constraint:

$S_{n}+S_{w}=1$

Mass Fraction Constraint:

$C_{N w}+C_{W w}=1$

Capillary Pressure Constraint:

$P_{n}-P_{w}=P_{c}$.

In the above equations, uppercase subscripts refer to components; lowercase subscripts refer to phases. The subscript " $n$ " refers to the nonwetting phase or gas phase, and the subscript " $w$ " refers to the wetting phase or water/brine phase. The term $\alpha$ is a geometric factor that allows the same equations to be used regardless of the number of dimensions modeled in the problem (e.g., WIPP Performance Assessment Division, 199/c, Volume 2, 1991, Section 5.2.2.4). The rest of the nomenclature is defined in Table 7-1. Given the necessary initial, boundary, and material parameter information, Equations (7.1) to (7.5) must be solved numerically to determine the flow field through the repusitory, up the shafts or human intrusion boreholes, and horizontally along the Culebra strata to the boundary of the repository as described by Guzowski and Helton (e.g., WIPP Performance Assessment Division, 199/b, Chapter 4).

The two-phase flow equations have been reproduced in this section to remind the reader of their full extent in the non-simplified form. Examination of the number of parameters and the complexity of these equations shows that their solution is far from trivial and would be much more difficult if room closure phenomena were included. In fact, addition of a geomechanical closure model would require coupling the two-phase flow equations with an even more numerous set of equations, leading to even greater solution difficulty, as has already been discussed in Section 6.2. Even if unlimited state-of-the-art computer resources were available to solve the equations, the large number of solutions required by the Performance Assessment methodology would make this task impossible within the time scale needed to meet programmatic milestones.

We are forced to conclude, therefore, that a direct solution of fully coupled equations that include bot: : wo-phase flow and geomechanical closure is out of the question at this time. A simplification is required that captures the approximate nature of closure while at the same time keeps the two-phase flow equations manageable. A secondary requirement is that the method of specifying the extent of closure must be consistent with the performance assessment requirement for addressing uncertainty in input parameters as described by Helton (e.8., WIPP Performance Assessment Division, 199/b, Chapter 3). Rather than having to determine a new room-closure solution for every change of a room-closure parameter, development of a method in which the consequences of parameter variation can be established by interpolating from predetermined solutions would be convenient.

The key to a potential closure simplification is presently assumed to be related to the gasfilled porosity of the material within the room: the part of the parameter $\phi$ in Equations (7.1) and (7.2) that contains gas. Porosity is the principal variable controlling permeability, and it is determined by a number of factors. The simplest situation would be if no gas were generated. The calculations in Section 5.1.3 of this report show that the waste and backfill compact relatively rapidly to a fairly dense state under this condition. The room contents then remain at constant density or would very slowly continue to densify over long periods of time. 
Table 7-1. Two-Phase Flow Nomenclature (WIPP Performance Assessment Division, 199/c, Section 5.2)

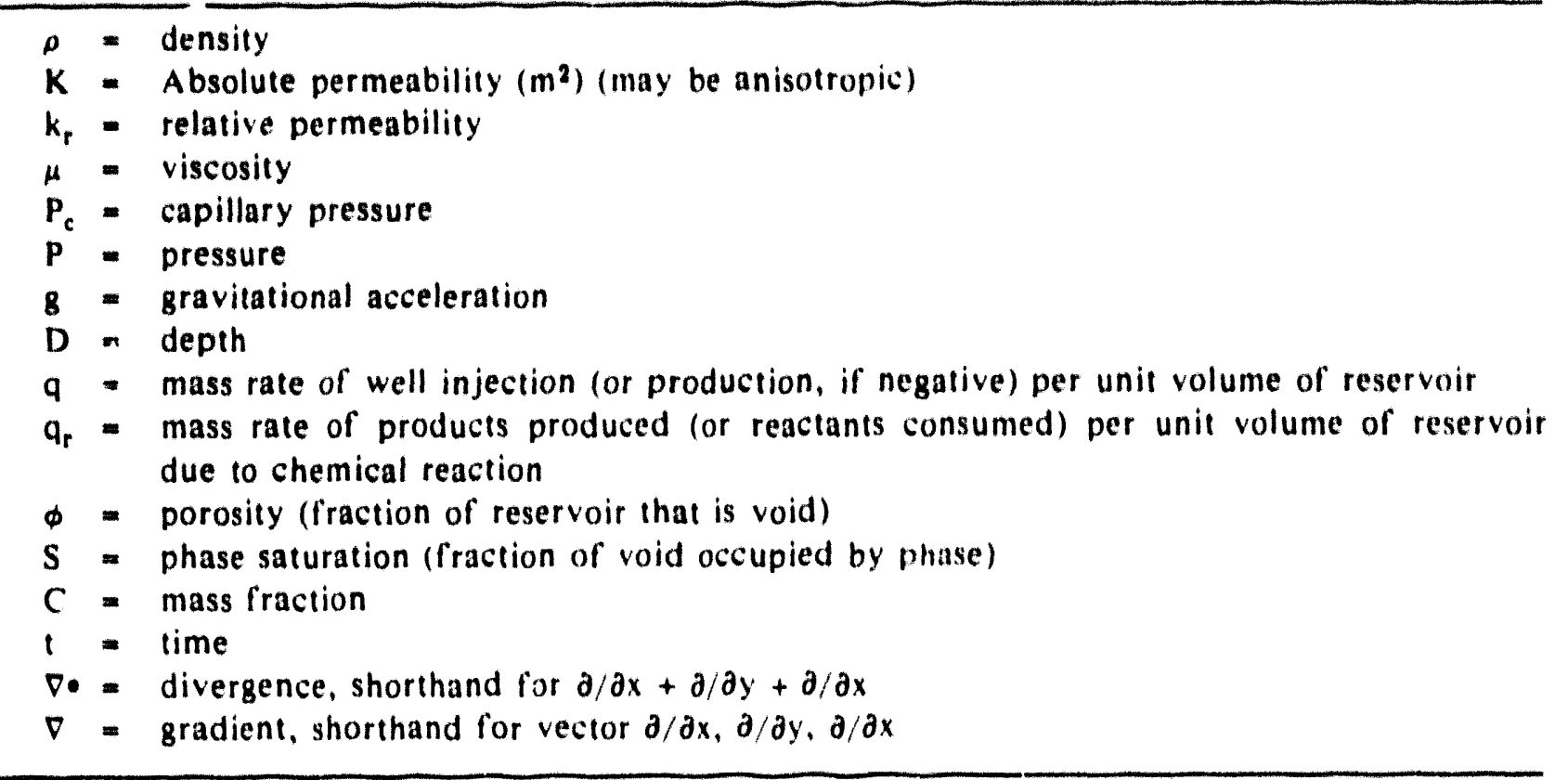

The presence of gas complicates room closure, as illustrated by the results of Section 5.1.4, because gas pressurization of the room may exert sufficient backstress to cause closure to temporarily cease. The word "temporarily" is used because a human intrusion will restart the closure process. Whereas, in the absence of gas, the average porosity of the waste may be on the order of 0.18 , base line gas generation assuming unlimited brine availability may be sufficient to limit porosity reduction within the room to as high as 0.4 . Baseline gas generation suggests a highly permeable state of the room contents. In addition, all intermediate states of consolidation are possible and depend upon the storage volume available for the gas, the amount of brine in contact with the waste, and how much gas leaks away from the room through interbeds, shafts, and boreholes. Room response to these parameters is highly nonlinear and does not fit any standard mathematical function. The problem, therefore, is how to keep track of gas and its very strong inflience on porosity without becoming so badly entangled in detail that a numerical solution becomes impossible.

The method that is under consideration for defining disposal-room closure information is to summerize results in terms of a closure surface, such as the one shown in Figure 7-2. This surface defines the relationship between the primitive variables of gas-filled porosity, the instintaneous amount of gas present in that porosity, and time. The "gas-filled porosity" introduces a complication to our proposal to use this type of surface to include closure into flow calculations, huwever, because the disposal room may be partially or totally saturated with brine. In other words, the gas-filled porosity represents only part of the total porosity $\phi$, one of the variables that must be considered in solution of Equations 7-1 to 7-5. The complication that separation of the porosity into two independent components introduces into solution of the flow equations will be discussed more fully in a subsequent paragraph, but for the present, discussion will focus on the reasons this approach is considered useful. 


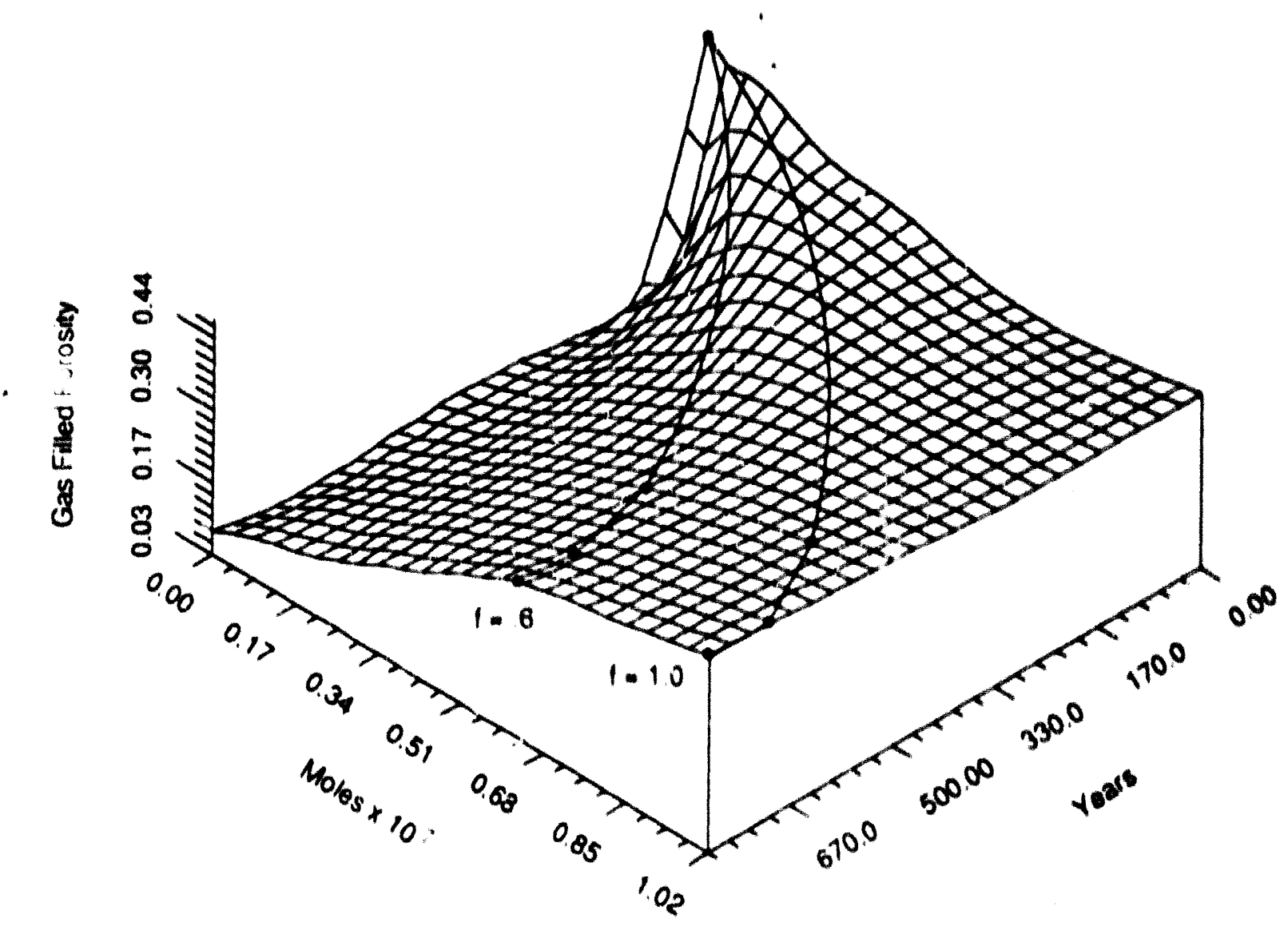

thI 6un 1250

Figure 7-2. The unprocessed TRU waste closure surfiace used for the 1992 comparison of predicted WIPP performance with 10 CFR Parl 101 . Suhpart $B$. 
The motioation for the closure surface approach was suggested by the results of how gas moduction influences closure. Section 5.14, where the curves for different values of $f$ (Figure - 10 suggest the surlace concept. For example, a gas generation result for $f=0.8$ is expected to lall hetween the result for $f=1.0$ and $f=0.0$. Generation of such surfaces requires closure mlutions for a limited number of, but widely varying, gas-generation conditions. The surface in linure z was developed by assuming values for $f$ of $0,0.2,0.4,0.6$, and 1.0 , performing the analusis for each gecoration potential and forming a surface from the results. This surface bounds the condition of no gas generition $(f=0)$ and base-line gas generation $(f=1)$.

We assume for the present that a surface such as the one shown in Figure 7-2 is a suitable approximation of any closure history caused by a prescribed variation of $f$ with time or gaseneration rate with time (i.e., that this curve lies on the surface and can be defined by interpolation). The surface construction then permits the generalization that as long as the surface in defined and bounds possible states of the repository, it can be used to approximately define any closure history evolving from a specified Performance Assessment scenario. While the closure hisfory was defined, for convenience, from calculations for a single room, the results can be eviended to represent the response of a panel, which is one of the basic configurations for Petformance Assessment analyses.

Several factors provide the justification for using closure surfaces or the data used to construil them for Performance Assessment. First, it is almost impossible to keep track of all detailed phenomena related to gas flow in a closure calculation (i.e., what the gas-leakage rate should be, how much brine is a ailable, etc.). However, for a first approximation, the results described in Section 5.1 imply that the instantaneous amount of gas currently residing within the porosity is the dominant parameter that controls closure because it and the storage volume alone determine the gas pressure. It does not really matter whether this gas is the net result of the rate of gas produced less the amount of gas that leaks out, or if it exists in a perfectly sealed room, or whether gas nroduction is intermittent. If each of these conditions caused the same amount of gas in the dispusal room at a given time and the porosity of the room is the same, then the hypothesis 18 that the slosure rates would be identical. In other words, the assertion is that it is not necessary fo know why a certain amount of gas is present at a given time within the disposal room to compute the pressure that it exerts on the room surroundings.

When sast in the sense described in the previous paragraph, utilization of the current amount of gas as one of the primitive independent variables of the surface is seen to avoid a whole host of complexities, any of which could introduce great difficulty into data transfer to Performance Assessment. It follows, therefore, that use of gas-filled porosity as the primitive dependent variable is a means by which closure information can be introduced into flow analyses. Coupling of the closure surface data to two-phase flow is proposed noting that the total porosity $\phi$ is the sum of the gas-filled porosity $\phi_{\mathrm{g}}$ and the brine-filled porosity $\phi_{\mathrm{b}}$, both of which are cunsidered 10 be slowly varying in time. An added assumption is that the pressure of the gas is the same as the brine pressure Nothing in the closure construction gives any information about the current hrine content of the room at any given time, however, so a value for this variable, as well an for the number of moles of gas in the room, must be provided by the flow calculation, either for a nanel, or an individual room or otherwise. Given the amount of gas at a specified lime, the corresponding gas porosity is obtained from the closure surface, and then added to the brine porosity to obtain the total porosity $\phi$. Finally, the pore pressure is calculated from the number of moles of gas and the gas porosity, and the calculation allowed to proceed. Infortunately, whether this method of solution can be incorporated in to the two-phase flow codes is unclear 
The closure surface concept is, in fact, much more general than may be apparent from the previous discussion. Consider the gas depressurization that is likely to occur during a human intrusion. When the borehole penetrates the repository at a certain time, leakage of some of the gas up through the borehole is inevitable. The actual amount of instantaneous release by this mechanism can be prescribed from independent estimates of leakage up the hole, assuming constant gas-storage volume within the room because escape times are short relative to closure rates. Once the amount of release is established, a new value for the amount of gas in the voids can be determined. Furthermore, the total porosity of the disposal room must remain constant because the intrusion is for all practical purposes instantaneous, and the room is not able to respond to rapid changes in the loadings. The new conditions after closure are defined, therefore, by the porosity just prior to the intrusion and by a new gas content, conditions which are represented by a different point on the closure surface. The new state is obtained by proceeding along a constant porosity path on the surface, starting at the amount of gas present just before intrusion at time $t_{a}$ (Figure 7-3) and ending at the amount of gas present immediately after intrusion, which is calculated independently. Figure 7-3 and the process for making this construction are described in the next paragraph. The end point of the constant porosity path will define a new time $t_{b}$. The time-scale of the closure surface time axis must then be shifted so that $t_{b}$ corresponds to the intrusion time. With this time scale shift, the calculation can be continued, using the revised closure surface, starting from the redefined position on the surface immediately after the intrusion.

Figure 7-3 shows a gas-filled porosity contour plot of a recently constructed closure surface. Initially, closure proceeds along some path on the porosity surface defined by the gas-evolution history dictated by the particular choice of input parameters. Assume that this path intersects point "a" on the porosity contour plot at approximately 1000 years, as shown on Figure 7-3. Point "a" falls on the "40" porosity contour, indicating that an average room porosity of about $40 \%$ is expected at 1000 years, and corresponds to approximately $6.4 \cdot 10^{6}$ moles of gas in the room at that time.

Now assume a human intrusion at 1000 years into a disposal room with conditions defined by point "a" in Figure 7-3. If the gas pressure within the room is assumed to drop to the pressure the drilling fluid would have at that elevation, the amount of gas remaining within the room can be calculated from the known gas-filled porosity of the room. Suppose the remaining gas is $3.6 \cdot 10^{6}$ moles. To adjust the closure surface for this intrusion, the $40 \%$ porosity contour is followed until a point on it, point " $b$," corresponds to a gas content of $3.6 \bullet 10^{6}$ moles. Although this point is associated with a time of about 200 years on the original surface, we know that it now represents the state of the disposal room immediately after intrusion at 1000 years. To compensate for the difference in time, it is sufficient to redefine the time corresponding to point "b" as occurring at 1000 years, rather than 200 years, and proceed with the calculation. The old closure surface is shifted in time, so that the calculation can be restarted from point " $b$ " in Figure $7-4$ at a time corresponding to the time of the intrusion (1000 years).

The closure history along the surface then continues to evolve from the new conditions if changes in the amount of gas uccur because of leakage and other processes. We assume for the porosity surface approximation that the extent of closure as reflected by the porosity is not strongly path-dependent, and therefore a single surface is a sufficiently accurate approximation of what is actually estimated to occur within the repository. There is some confirmation of the adequacy of this assumption from the disposal room results described in this report, and additional analyses are required to support tinis conclusion. In particular, the effects of a human intrusion are subject to uncertainty and must be confirmed before the porosity surface representation of closure can be applied extensively to performance assessment compliance determinations. 


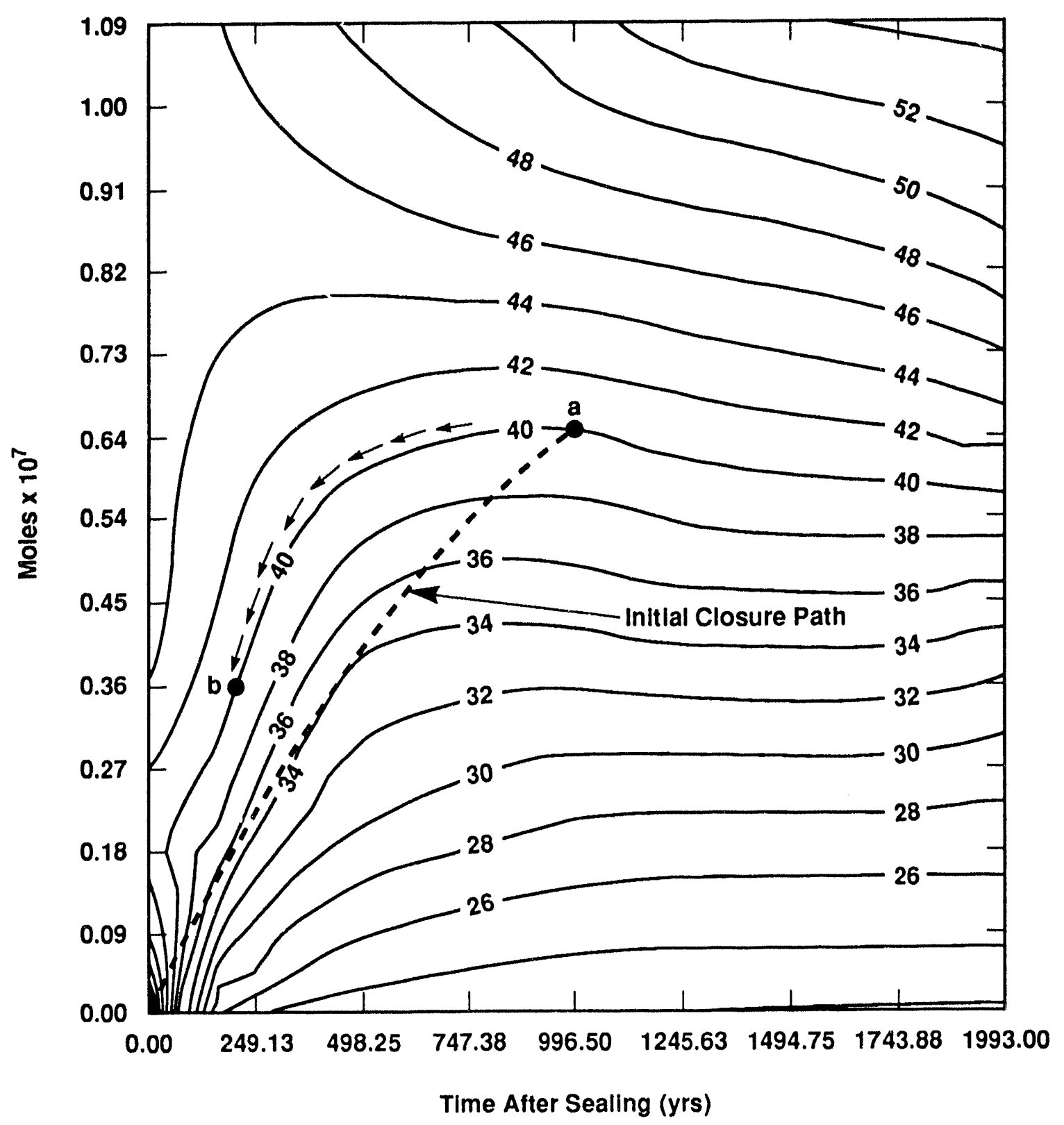

TA1.6345-148.0

Figure 7-3. A contour plot of a typical gas-filled porosity surface for closure of a disposal room containing untreated waste. 


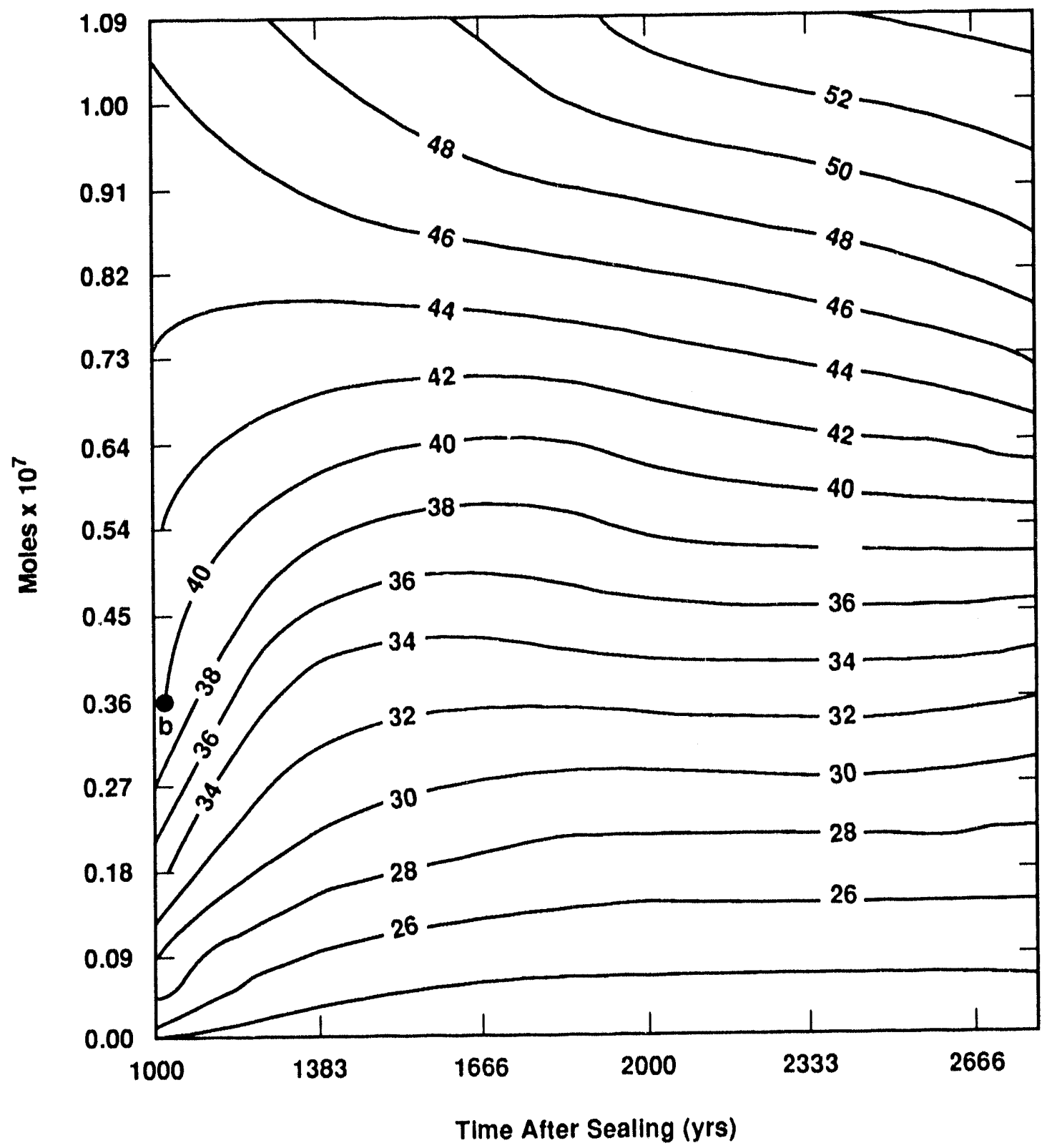

TRI.6345.196.0

Figure 7-4. The revised gas-filled porosity contour plot taken from Figure 7-3, reflecting a human intrusion at 1000 years. The calculation is restarted from the conditions indicated by point "b." 
$7-10$

$7-10$ - 


\subsection{CONCLUSIONS AND RECOMMENDATIONS}

The contents of this report represent the progress in development of a disposal room model over a period of approximately four years, ending about March 1992. Our capability has increased from execution of simple calculations of the closure of empty rooms and preliminary backfill analyses using a consolidation model for pure crushed salt to the refinement of analytic methods and development of mathematical models needed for detailed disposal-room closure calculations. The model development included models for salt/bentonite backfill response, waste compaction, and gas generation within the room, and examination of the consequences of gas pressurization on fracture opening.

Whereas early calculations were limited to symmetric, single-room configurations, 2-D analyses of entire waste panels are now feasible. The refinement of descriptions of brine inflow into the disposal room and improvement of the gas-generation model have been demonstrated by coupling a brine-flow description into the present stress-analyses capability. Finally, after investigation of several options, the present consensus is that closure surfaces offer the best approach to incorporation of complex room-closure response into more global performance assessment numerical analyses.

These conclusions and recommendations will be presented in the order that the information was presented. Most of the observations are in regard to detailed calculations (Chapter 5.0) that were completed to explore various aspects of the conceptual model and relate either to the adequacy of the mathematical closure models or to the extent that various features of the model impact room performance.

1. Continued examination of the difference between empty-room closure histories computed with SANCHO and with SPECTROM-32 is required, even though this discrepancy is not considered to strongly impact disposal room performance (Section 5.1.1).

2. Closure estimates for empty rooms containing no gas suggest that, even with the most pessimistic interpretation, the rooms are predicted to close to less than $10 \%$ of their original volume within 150 years (Section 5.5.1).

3. Backfill consolidation in disposal rooms with no gas is estimated to produce highly dense states within 40 years for pure-crushed salt backfill and takes 4 times as long for crushed salt/bentonite backfill. Backfill consolidation is so rapid that it is not a controlling concern in the demonstration of satisfactory repository performance for 40 CFR $191 B$ (Sections 5.1.2 and 5.1.3).

4. The reason for the exceptionally low final void fractions (porosities) calculated using the SPECTROM-32 nonlinear elastic waste consolidation model must be resolved (Section 5.1.3).

5. Even without the presence of gas, waste-void fractions (porosity) of unprocessed metal waste may remain large as consolidation nears completion, causing this waste type to remain highly perneable. Consolidation of metallic waste is one of the principal mechanical processes controlling the permeability of the disposal-100m contents. Assuming an undisturbed repository and no gas, the observed closure times on the order of 150 years are short enough to assure that consolidation of waste and backfill is largely complete before brine at the expected brine flow rates can saturate the room (Sections 5.1.3 and 3.3). 
6. Assuming sealed disposal rooms and DSEIS gas pressurization, minimum values of the void fraction (porosity) in the room occurred in about 150 years, after which the rooms began to expand. The onset of room expansion occurs at room pressures of about 8 to $10 \mathrm{MPa}$. As gas production continued, the gas pressure exceeded lithostatic pressure. Reduction of total gas production by a factor of 5 decreased the peak pressure by only $18 \%$, from about 21.5 to $18.5 \mathrm{MPa}$, still above lithostatic. These results indicate that when gas leakage from the room is slight, some, albeit small, elevation of gas pressure above lithostatic is inevitable, in the absence of hydrofracturing, for all quantities of potential gas production. The reason for this conclusion is that salt formation surrounding the repository requires time to adjust to changing stresses and reach mechanical equilibrium (lithostatic stress) (Section 5.1.4).

7. Calculations assuming simulated permeable interbed fractures showed that gasstorage pressures for a fully consolidated repository were sensitive to the presence of interbeds and to assumptions about interbed porosity. Results from analysis of a disposal room intersected by a gas-impermeable horizontal fracture suggest that initiation of new cracks in pure halite by gas pressurization is not possible. Unlike impermeable fractures, opening of gas-permeable fractures was predicted to occur when lithostatic pressure is reached. In the simulation, the pressure within the disposal room then remains constant at lithostatic pressure, and additional volume for gas is generated by the opening crack. Additional calculations are required, however, to demonstrate the applicability of these disposal rooms' performance. These results confirm the intuition that changes in room performance are to be expected when the constraining assumption of a perfectly sealed room is removed (Section 5.2).

8. Calculations to determine how a human intrusion alters closure of a gas-filled disposal-room should be a top priority of the disposal-room modeling task in the future (Section 6.1).

9. A coupled flow-closure calculation is described in Section 6.2 that eventually can provide a means for refining the gas-generation model used in closure calculations by including the dependence of corrosion processes on the availability of water. This approach is considered bounding to the extent that it overestimates the amount of water available for corrosion (Section 6.2).

10. A 2-D empty-room-panel calculation suggests that after 160 years, as closure becoines extensive, results for single isolated-room or infinite-array configurations, typical of rooms in the center of a panel, differ by less than $25 \%$ from the closure of nonsymmetric end rooms in a panel. Similar calculations to determine uncertainties attributed to 3-D effects introduced by intersections of rooms with access drifts would be useful (Section 6.3).

11. Closure surfaces, or the data used to construct them, offer a means of incorporating complex room closure response into more global performance assessment numerical analyses (Section 7). 


\subsection{REFERENCES}

Argüello, J.G. 1990. "Analysis of WIPP Disposal Room and Entryway Drift Intersection," Rock Mechanics Contributions and Challenges: Proceedings of the 31st U.S. Symposium, Colorado School of Mines, Golden. CO. June 18-20, 1990. Eds. W.A. Hustrulid and G.A. Johnson. Brookfield, VT: A.A. Balkema. 329-336.

Argüello, J.G., M.A. Molecke, and R. Beraun. 1989. "3D Thermal Stress Analysis of WIPP Room T RH TRU Experiments," Rock Mechanics as a Guide for Efficient Utilization of Natural Resources, Proceedings of the 30th U.S. Rock Mechanics Symposium. West Virginia University. Morgantown. $W V$, June 19-22, 1989. Ed. A.W. Khair. SAND88-2734C. Brookfield, VT: A.A. Balkema. 681-688.

Argüello, J.G., J.R. Weatherby, C.M. Stone, H.S. Morgan, and W.R. Wawersik. 1991. "Room Response Analyses to Investigate Waste Disposal Issues," Proceedings of the 7 th International Congress on Rock Mechanics, Aachen. FRG. September 16-20, 1991. Vol, 3, 1867-1871.

Argüello, J.G., J.R. Weatherby, C.M. Stone, and F.T. Mendenhall. 1992. "Effect of Internal Gas Generation on the Extension of Pre-existing Fractures Around WIPP Disposal Rooms," Rock Mechanics, Proceedings of the 33rd U.S. Symposium. Santa Fe, NM, June 3-5, 1992. Eds. J.R. Tillerson and W.R. Wawersik. Brookfield, VT: A.A. Balkema. 399-408.

Bechtel, Inc. 1986. Waste Isolation Pilot Plant Design Validation Final Report. DOE-WIPP86-010. San Francisco, CA: prepared for the US Department of Energy by Bechtel National, Inc.

Borns, D.J. 1985. Marker Bed 139: Study of Drillcore From A Systematic Array. SAND85-0023. Albuquerque, NM: Sandia National Laboratories.

Brodsky, N.S. 1990. Crack Closure and Healing Sludies in WIPP Sall Using Compressional Wave Velocity and Alteruation Measurements: Test Methods and Results. SAND90-7076. Albuquerque, NM: Sandia National Laboratories.

Brodsky, N.S., and T.W. Pfeifle. 1992. Consolidation of the Waste Isolation Pilot Plant Crushed Salt/Bentonite Mixtures as a Function of Confining Pressure and Moisture Content as Compared With Constitutive Model Predictions. SAND91-7071. Albuquerque, NM: Sandia National Laboratories.

Butcher, B.M. 1989. Waste Isolation Pilot Plant Simulated Waste Compositions and Mechanical Properties. SAND89-0372. Albuquerque, NM: Sandia National Laboratories.

Butcher, B.M., C.F. Novak, and M. Jercinovic. 1991a. The Advantages of a Salt/Bentonite Backfill for Waste Isolation Pilot Plant Disposal Rooms. SAND90-3074. Albuquerque, NM: Sandia National Laboratories. 
Butcher, B.M., T.W. Thompson, R.G. VanBuskirk, and N.C. Patti. 199/b. Mechanical Compaction of Waste Isolation Pilot Plant Simulated Waste. SAND90-1206. Albuquerque, NM: Sandia National Laboratories. See also: Luker, R.S., T.W. Thompson, and B.M. Butcher. 1991. "Compaction and Permeability of Simulated Waste," Rock Mechanics as a Multidisciplinary Science. Proceedings of the 32nd U.S. Symposium. University of Oklahoma, Norman. OK. July 10-12, 1991. Ed. J-C. Roegiers. SAND90-2386C. Brookfield, VT: A. A. Balkema. 693-702.

Callahan, G.D., and K.L. DeVries. 1991: Analyses of Backfilled Transuranic Wastes Disposal Rooms. SAND91-7052. Albuquerque, NM: Sandia National Laboratories.

Callahan, G.D., A.F. Fossum, and D.K. Svalstad. 1990. Documentation of SPECTROM-32: A Finite Element Thermomechanical Stress Analysis Program. DOE/CH/10378-2; RSI-0269. Rapid City, SD: RE/SPEC, Inc. for the U.S. Department of Energy Chicago Operations Office. Vols, 1-2.

Clements, T.L. Jr., and D.E. Kudera. 1985. TRU Waste Sampling Program: Volume I -- Waste Characterization. EGG-WM-6503. Idaho Falls, ID: Idaho National Engineering Laboratory.

Ehgartner, B. 1990. Geomechanical Analyses in Support of the Waste Isolation Pilot Plant (WIPP). SAND90-0285. Albuquerque, NM: Sandia National Laboratories.

Holcomb, D.J., and M. Shields. 1987. Hydrostatic Creep Consolidation of Crushed Salt With Added Water. SAND87-1990. Albuquerque, NM: Sandia National Laboratories.

Krieg, R.D. 1984. Reference Stratigraphy and Rock Properties for the Waste Isolation Pilot Plant (WIPP) Project. SAND83-1908. Albuquerque, NM: Sandia National Laboratories.

Lappin, A.R., R.L. Hunter, D.P. Garber, and P.B. Davies, eds. 1989. Systems Analysis, LongTerm Radionuclide Transport, and Dose Assessments, Waste Isolation Pilot Plant (WIPP), Southeastern New Mexico; March 1989. SAND89-0462. Albuquerque, NM: Sandia National Laboratories.

Lappin, A.R., C.A. Gotway, M.A. Molecke, R.L. Hunter, and E.N. Lorusso. 1991. Rationale for Revised Bin-Scale Gas-Generation Tests with Contact-Handled Transuranic Wastes at the Waste lsolation Pilot Plant. SAND90-2481. Albuquerque, NM: Sandia National Laboratories.

Luker, R.S., T.W. Thompson, and B.M. Butcher. 1991. "Compaction and Permeability of Simulated Waste," Rock Mechanics as a Multidisciplinary Science. Proceedings of the 32nd U.S. Symposium. University of Oklahoma, Norman, OK, July 10-12, 1991. Ed. J-C. Roegiers. SAND90-2386C. Brookfield, VT: A.A. Balkema. 693-702.

Mendenhall, F.T., B.M. Butcher, and P.B. Davies. 1991. "Investigations into the Coupled Fluid Flow and Mechanical Creep Closure Behavior of Waste Disposal Rooms in Bedded Salt," Waste-Generated Gas at the Waste Isolation Pilot Plant: Papers Presented at the Nuclear Energy Agency Workshop on Gas Generation and Release from Radioactive Waste Repositories. Eds. P.B. Davies, L.H. Brush, M.A. Molecke, F.T. Mendenhall, and S.W. Webb. SAND91-2378. Albuquerque, NM: Sandia National Laboratories. 3-1 through 3-15. 
Morgan, H.S., and R.D. Kreig. 1990. "Investigation of an Empirical Creep Law for Rock Salt that Uses Reduced Elastic Moduli," Rock Mechanics Contributions and Challenges, Proceedings of the 31st U.S. Rock Mechanics Symposium. Colorado School of Mines. Golden. CO. June 18-20, 1990. Eds. W.A. Hustrulid and G.A. Johnson. Boston, MA: A.A. Balkema. 965-972.

Morgan, H.S., R.D. Krieg, and R.V. Matalucci. 1981. Comparative Analysis of Nine Structural Codes Used in the Second WIPP Benchmark Problem. SAND81-1389. Albuquerque, NM: Sandia National Laboratories.

Morgan, H.S., C.M. Stone, and R.D. Krieg. 1985. "The Use of Field Data to Evaluate and Improve Drift Response Models for the Waste Isolation Pilot Plant (WIPP)," Research and Engineering Applications in Rock Masses, Proceedings of the 26ih U.S. Symposium on Rock Mechanics. South Dakota School of Mines \& Technology. Rapid City, SD. June 26-28, 1985. Ed. E. Ashworth. SAND84-2126C. Boston, MA: A.A. Balkema. Vol. 2, 769-776.

Morgan, H.S., C.M. Stone, and R.D. Krieg. 1986. An Evaluation of WIPP Structural Modeling Capabilities Based on Comparisons with Soulh Drift Data. SAND85-0323. Albuquerque, NM: Sandia National Laboratories.

Munson, D.E., and H.S. Morgan. 1986. Methodology for Performing Parallel Design Calculations (Nuclear Waste Repository Application). SAND85-0324. Albuquerque, NM: Sandia National Laboratories.

Munson, D.E., A.F. Fossum, and P.E. Senseny. 1989a. Advances in Resolution of Discrepancies Between Predicted and Measured In Silu WIPP Room Closures. SAND88-2948. Albuquerque, NM: Sandia National Laboratories. See also: Munson, D.E., and K.L. DeVries. 1991. "Development and Validation of a Predictive Technology for Creep Closure of Underground Rooms in Salt," Proceedings of the 7th International Congress on Rock Mechanics, Aachen. FRG, September 16-20, 1991. Ed. W. Wittke. SAND90-1147. Vol. 7, 127-134.

Munson, D.E., A.F. Fossum, and P.E. Senseny. 1989b. Approach to First Principles Model Prediction of Measured WIPP In Silu Room Closure in Salt. SAND88-2535. Albuquerque, NM: Sandia National Laboratories.

Pfeifle, T.W. 1987a. Mechanical Properties and Consolidation of Potential DHLW Backsill Materials: Crushed Salt and 70/30 Bentonite/Sand. SAND85-7208; Topical Report RSI0282. Albuquerque, NM: Sandia National Laboratories.

Pfeifle, T.W. 1987b. Backfill Material Specifications and Requirements for the WIPP Simulated DHLW and TRU Waste Technology Experiments. SAND85-7209. Albuquerque, NM: Sandia National Laboratories.

Pfeifle, T.W. 1991. Consolidation. Permeability. and Strength of Crushed Salt/Bentonite Mixtures With Application to the WIPP. SAND90-7009; Topical Report RSI-0357. Albuquerque, NM: Sandia National Laboratories.

Pfeifle, T.W., and N.S. Brodsky. 1991. Swelling Pressure, Water Uptake, and Permeability of 70/30 Crushed Sall/Bentonite. SAND91-7070; Topical Report RSI-0378. Albuquerque, NM: Sandia National Laboratories. 
Pusch, R. 1980. Swelling Pressure of Highly Compacted Bentonite. KBS Project Technical Report 80-13; Lulea 1980-08-20. Stockholm: Suensk Kärnsbrănsleförsörjning.

Sjaardema, G.D., and R.D. Krieg. 1987. A Constitutive Model for the Consolidation of WIPP Crushed Salt and Its Use in Analyses of Backfilled Shaft and Drift Configurations. SAND87-1977. Albuquerque, NM: Sandia National Laboratories.

Stone, C.M., R.D. Krieg, and Z.E. Beisinger. 1985. SANCHO, A Finite Element Computer Program for the Quasistatic, Large Deformation. Inelastic Response of Two-Dimensional Solids. SAND84-2618. Albuquerque, NM: Sandia National Laboratories.

Stormont, J.C. 1990. Summary of 1988 WIPP Facility Horizon Gas Flow Measurements. SAND89-2497. Albuquerque, NM: Sandia National Laboratories.

US DOE (Department of Energy). 1989. Draft Supplement Environmental Impact Statement, Waste Isolation Pilot Plant. DOE/EIS-0026-DS. Washington, DC: US Department of Energy, Office of Environmental Restoration and Waste Management. Vols. 1-2.

US DOE (Department of Energy). 1991. Evaluation of the Effectiveness and Feasibility of the Waste Isolation Pilot Plant Engineered Alternatives: Final Report of the Engineered Alternatives Task Force. DOE/WIPP 91-007, Rev. 0. Carlsbad, NM: Waste Isolation Pilot Plant. Vols. 1-2.

US EPA (Environmental Protection Agency). 1985. "Environmental Standards for the Management and Disposal of Spent Nuclear Fuel, High Level and Transuranic Radioactive Wastes; Final Rule," Federal Register. Vol. 50, no. 182, 38066-38089.

US EPA (Environmental Protection Agency). 1987. "Land Disposal Restrictions," Code of Federal Regulations 40. Part 268. Washington, DC: Superintendent of Documents, US Government Printing Office.

Waste Management Technology Department. 1987. The Scientific Program at the Waste Isolation Pilot Plant. SAND85-1699. Albuquerque, NM: Sandia National Laboratories.

Wawersik, W.R., and C.M. Stone. 1989. "A Characterization of Pressure Records in Inelastic Rock Demonstrated by Hydraulic Fracturing Measurements in Salt," International Journal of Rock Mechanics and Mining Sciences and Geomechanics Ahstracts. Vol. 26, no. 6, 613-627.

Weatherby, J.R., W.T. Brown, and B.M. Butcher, 1991a. "The Closure of WIPP Disposal Rooms Filled with Various Waste and Backfill Combinations," Rock Mechanics as a Mullidisciplinary Science, Proceedings of the 32nd U.S. Symposium. University of Oklahoma. Norman. OK. July 10-12, 1991. Ed. J-C. Roegiers. Brookfield, VT: A.A. Balkema. 919-928.

Weatherby, J.R., J.G Argüello, B.M. Butcher, and C.M. Stone. 199/b. "The Structural Response of a WIPP Disposal Room with Internal Gas Generation," Rock Mechanics as a Multidisciplinary Science. Proceedings of the 32nd U.S. Symposium. University of Oklahoma. Norman. OK, July 10-12, 1991. Ed. J-C. Roegiers. Brookfield, VT: A. A. Balkema. 929-937. 
WIPP Performance Assessment Division. 1991a, Preliminary Comparison with 40 CFR Part 191. Subpart B for the Waste Isolation Pilot Plant. December 1941 - Volume 3: Reference Data. SAND91-0893/3. Albuquerque, NM: Sandia National Laboratories.

WIPP Performance Assessment Division. 1991b. Preliminary Comparison with 40 CFR Part 191. Subpart B for the Waste Isolation Pilot Plant. December 1991 - Volume 1: Methodology and Results. SAND91-0893/1. Albuquerque, NM: Sandia National Laboratories.

WIPP Performance Assessment Division. 1991c. Preliminary Comparison with 40 CFR Part 191, Subpart B for the Waste Isolation Pilot Plant. December 1991 - Volume 2: Probability and Consequence Modeling. SAND91-0893/2. Albuquerque, NM: Sandia National Laboratories.

Zeuch, D.H., D.J. Holcomb, and H.S. Lauson, 1985. Analysis of Consolidation of Granulated Rocksall Using a Plastic Flow Model for Isostatic Hot-Pressing. SAND84-1106. Albuquerque, NM: Sandia National Laboratories.

Zeuch, D.H., D.J. Zimmerer, and M.E.F. Shields. 1991. Interim Report on the Effects of BrineSaturation and Shear Stress on Consolidation of Crushed. Natural Rock Salt from the Waste Isolation Pilot Plani (WIPP). SAND91-0105. Albuquerque, NM: Sandia National Laboratories. 
APPENDIX A: MEMORANDA REGARDING DISPOSAL ROOM MODEL

An 
A - 2 


\section{Appendix A: Memoranda Regarding Disposal Room Model}

\section{Referenced Memoranda}

Brown, W.T., and J.R. Weatherby. 1990. "Influence of Gas Generation Potential and Gas Generation Rate on the Performance of CH-TRU Disposal Rooms," September 17, 1990 memo to B.M. Butcher and F.T. Mendenhall. Albuquerque, NM: Sandia National Laboratories.

Callahan, G.D. 1992. "Further Discussion of the TRU Waste Model," March 13, 1992 RE/SPEC External Memorandum to F.T. Mendenhall. Rapid City, SD: RE/SPEC, Inc.

Davies, P.B. 1990a. "Observations on Interbed Pressurization from Recent 2-Phase, WasteGenerated Gas Simulations," June 26, 1990 memo to B.M. Butcher and F.T. Mendenhall. Albuquerque, NM: Sandia National Laboratories.

Davies, P.B. 1990b. "First Fluid Pressurization Data Set for Interbed/Fracture Dilation Calculations," October 4, 1990 memo to F.T. Mendenhall. Albuquerque, NM: Sandia National Laboratories.

Morgan, H.S. 1987a. "Estimate of the Time Needed for TRU Storage Rooms to Close," June 2, 1987 memo to D.E. Munson. Albuquerque, NM: Sandia National Laboratories.

Morgan, H.S. 1987b. "TRU Storage Room Calculation with Stratigraphy," December 9, 1987 memo to D.E. Munson. Albuquerque, NM: Sandia National Laboratories.

Munson, D.E. 1990. "Why Reduced Modulus and Similar Methods Appear to Work for Calculations of Creep Closure in Salt," September 19, 1990 memo to distribution. Albuquerque, NM: Sandia National Laboratories.

Olsson, W.A. 1991. "Implications of the Experimental Consolidation of Crushed Salt/Metal Mixtures and Proposed Work," November 7 , 1991 memo to A.R. Lappin. Albuquerque, NM: Sandia National Laboratories.

Stone, C.M., and J.G Argüello. 1991. "Panel Scale Calculations for the Waste Isolation Pilot Plant (WIPP)," December 17, 1991 memo to F.T. Mendenhall. Albuquerque, NM: Sandia National Laboratories.

Stone, C.M., and J.G. Argüello. 1992. "Resolution of the Discrepancy in Predicted Closure of an Empty Disposal Room," April I, 1992 memo to B.M. Butcher and F.T. Mendenhall. Albuquerque, NM: Sandia National Laboratories.

Weatherby, J.R. 1989. "Finite Element Analysis of TRU Storage Rooms Filled With Waste and Crushed Salt," May 17, 1989 memo to B.M. Butcher. Albuquerque, NM: Sandia National Laboratories.

Weatherby, J.R., J.G. Argüello, and C.M. Stone. 1989. "The Effect of Gas Generation on the Performance of CH-TRU Disposal Rooms," November 14, 1989 memo to B.M. Butcher and D.E. Munson. Albuquerque, NM: Sandia National Laboratories.

Weatherby, J.R., and W.T. Brown. 1990, "Closure of a Disposal Room Backfilled with a Salt/Bentonite Mix," April 30, 1990 memo to B.M. Butcher. Albuquerque, NM: Sandia National Laboratories. 
A. 4

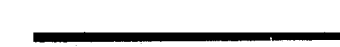$$
A-4
$$

A-4 
Sandia National Laboratories

Albuquerque, New Mexico 87185

date: September 17, 1990

to: B. M. Butcher, 6345 and F. T. Mendenball, 6345

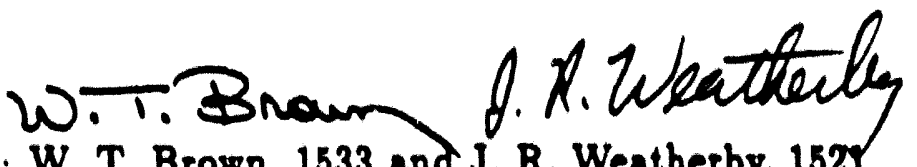

from: W. T. Brown, 1533 and J. R. Weatherby, 1521

subjact: Influence of Gas Generation Potential and Gas Generation Rate on the Performance of CH.TRU Disposal Rooms

\section{INTRODUCTION}

Gases produced by decomposition and corrosion of waste and waste containers may cause a slow build-up of pressure in the Waste Isolation Pilot Plant (WIPP) Contact Handled. Transuranic (CH.TRU) waste disposal rooms. A previous study (1) provided an estimate of the pressure history inside a leak-tight disposal room and the resulting creep response of the surrounding salt formation. This study predicted that room pressures would exceed the lithostatic stress level at the repository horizon, but that the principal stresses at all locations in the salt formation would remain compressive. In other work [2], the effect of gas generation on the extension of preexisting cracks was considered for the case where gas pressure acts on the faces of the initial crack but is not able to permeate into the formation ahead of the crack. Results from this study indicate that, under these conditions, cracks which are one meter in length, or shorter, will not propagate as the result of room pressurization for the rate and amount of gas generation considered.

This memo documents results of recent finite element analyses that consider rates and amounts of gas generation that deviate from the baseline cases considered in [1] and [2]. The current study examines bow changes in the gas potential (i.e., the total amount of gas generated) and gas generation rate might influence the peak pressures and the degree of waste and backfill consolidation inside of the rooms. In addition, the gas loss rate that would prevent the room pressure from exceeding the lithostatic stress level is estimated for the gas generation history in the baseline case.

The boundary conditions and contents of the disposal room are the same as those assurned in the analyses presented in [1] and [2]. A typical disposal room containing 6800 drums of CH.TRU waste is modeled with a two-dimensional, plane strain finite element model. Gas generation is considered from both microbial decomposition and anoxic corrosion of the waste. In the baseline case which is taken from [3], both mechanisms are assumed to be active during the first 300 years, and gas is generated at a rate of $2.66 \mathrm{~g} \cdot \mathrm{mole} / \mathrm{drum} / \mathrm{year}$. From 300 years to 700 yenrs, only microbial decomposition contributes lo gas generation. 
and the gas generation rate drops to $0.86 \mathrm{~g} \cdot \mathrm{mole} / \mathrm{drum} / \mathrm{year}$. At the end of 700 years, gas production is assumed to cease completely.

The current study considers several cases which deviate from the baseline case. First, the number of drums which generate gas was varied. This situation might arise, for example, if corrosive mechanisms (such as liquid brine) act on only a portion of the waste drums. Second, both the number of drums which generate gas and the rate of gas generation per drum were vasied. The rate of gas generation per drum may be reduced by vitrification of some of the waste. Finally, a hypothetical situation was considered in which gas begins to penetrate into permeable layers (e.g., clay seams, parting planes in the salt, anbydrite marker beds) in the salt formation after the room pressure reaches the lithostatic stress level. This condition was simulated by stopping further pressurization when the room pressure reached the lithostatic pressure. The change in room volume was then determined as a function of time, and the amount and rate of gas loss required to maintain the lithostatic pressure was computed from the ideal gas law.

\section{DISPOSAL ROOM ANALYSES}

\subsection{The Finite Element Model}

Figure 1 shows the SANCHO [6] finite element mesh used in the plane strain analysis; it is the same mesh that was used in the original study [1]. Because of symmetry conditions, only a quarter-section of the region shown in Figure 1 was actually included in the finite element model. The waste, crushed-salt backfill, and intact salt were described in the same manner as before. The boundary conditions which were applied in the analysis simulate the conditions which exist around a deeply busied disposal room located near the middle of a long string of equally spaced storage rooms. Both the left and right edges of the mesh were fxed against horizontal motion. The lower edge of the mesh was fixed against vertical motion because it represents a symmetry plane through the midheight of the room. The stratigraphy was assumed to consist entirely of intact salt with no clay seams, parting planes, or anhydrite beds included. The intact salt was given an initial hydrostatic stress field $\left(\sigma_{0}=\sigma_{v}=\sigma_{1}\right)$ of $14.8 \mathrm{MPa}$, corresponding to the atress state which is believed to exist at the repository horizon before excavation.

As in the eurlier studies $[1,2]$, the elastic-secondary creep constitutive model described by Krieg [4] was used for the intact salt. The material models and material constants used for the wate and backfill (crushed salt) were identical to those used in the earlier studies $[1,2]$. The values of the shear and bulk moduli were obtain by dividing the measured values (4) by a factor of 12.5 . The rationale behind reducing the elastic constants is discussed in $[5]$. 


\subsection{Gas Generation Potential and Gas Generation Rate}

In both previous studies [1,2] of gas generation, each storage room was assumed to contain 6800 drums of waste. During the fisst 300 years, microbial decomposition and anoxic corrosion together were assumed to produce gas at a combined rate of $2.66 \mathrm{~g}$. moles/drum/yr. After the first 800 years, the gas production due to corrosion ended, and only the microbial decomposition was asumed to continue at a conatant rate of 0.86 8.moles/drum/yr until 700 years. Finally, at 700 yeass, the ges production was wsumed to cense entirely.

As before, the finite element code SANCBO was modified to compute the room pressure and to apply the resulting forces to nodes on the room boundary (1). The ges pressure was computed from the ideal gas law based on the current "void" volume in the room and the total amount of gas in the room. This takes the form:

$$
P_{0}=\frac{N R T}{V-V_{s}}
$$

where:

$$
\begin{aligned}
P_{s} & =\text { gas pressure in } \mathrm{Pa}_{a} \\
N & =\text { mass of ges in } \mathrm{g} \cdot \text { moles } \\
R & =\text { gas constant }=8.23\left(\mathrm{~m}^{3} \mathrm{~Pa}\right) /(\mathrm{g} \cdot \text { mole } \mathrm{K}) \\
T & =300 \mathrm{~K} \\
V & =\text { cursent volume of storage room in } \mathrm{m}^{3} \\
V_{s} & =\text { volume of solids in the storage room }=1660 \mathrm{~m}^{3}
\end{aligned}
$$

Table 1 shows bow the volume of solids $V_{s}$ was determined; note that the current value is alightly different from that used in the previous studies $[1,2]$. This difference asises because of the air gap which is present at the top of the room in the design configuration. In the present calculations, the gas pressure is computed assuming that the gap is not present, but is inutead filled with crushed ant. In $[1,2]$, the ast gap was treated as void volume for the purpose of calculating the ga preasure.

The mase of gas present in the room at any time will be simply:

$$
N(t)=f \times D \times \int_{0}^{t} \dot{N}_{0} d t
$$

where $D$ is the total number of drums, $f$ is the fraction of drums generating gas, and $\dot{N}_{0}$ is the appropriate gas generation sate per drum. In the baseline case, $\dot{N}_{0}$ was $2.66 \mathrm{~g}$. moles $/ \mathrm{drum} / \mathrm{yr}$ for the first 300 years and was $0.86 \mathrm{~g} \cdot \mathrm{moles} / \mathrm{drum} / \mathrm{yr}$ for the time period from 300 years to 700 years. Table 2 contains a list of values of the constants appearing in Equation 2 for ench analyass case. In all cases, the total number of drums $D$ was assumed to be 6800 . 
In Cases $A, B, C$, and $D$, the total gas generation potential was varied by reducing $f$ from $100 \%$ in Case A (the baseline case) to $20 \%$ in Case D. All other variables appearing in Equation 2 were unchanged from the baseline values. This parameter atudy was motivated by the possibility that only a fraction of the drums in the repository may actually generate gas aince some of the drums may not come into contact with brine.

In Cases $E$ and $F$, the gas generation sate $\dot{N}$, was reduced from the baseline case. Several physical conditions might cause a reduction in the rate at which gas is generated. For example, if some of the wate is vitrified then the rate of gas generation might be reduced substantially. If the waste drume ase in contact with water in its vapor form rather than is its liquid form, the rate of ga production may also be considerably alower. We have considered two hypothetical aituations in which the rate of gas production is reduced. In Case $E$, the fraction of waste drume producing ges $f$ wa asoumed to be unchanged from the baseline case, but the rate at which a aingle drum produced gas $N_{0}$, was reduced to $20 \%$ of its baseline value. This was implemented by reducing $N_{0}$, by $\&$ factor of 3 , and extending, by a factor of $B$, the time period in which gas generation occurs 80 that the total amount of gas generuted was the same as that in the baseline case. Case $F$ models a situation in which the fraction of drums generating gas is reduced to $20 \%$, and the rate of gas generation per drum is also reduced to $20 \%$ of its baseline value. As in Case E, the time period in which gas generation occurs is extended by a factor of 3 so that the total amount of gas generated by each active drum is the unme as in the baseline cise.

Finally, we consider a situation in which the gas pressure does not exceed the magnitude of the lithostatic stresses. This was done by limiting the pressure (Equation 1) to 14.8 MPa. After this pressure level is reached, the void volume of the room can still change because the intact salt and the backfill experience a creep response under the lithostatic load. We assume that a constant pressure level is maintained by loss of gas through flow into permeable layers which intersect the disposd room. This is modeled by assuming that the total amount of gas available is $N=N_{0}-N_{L}$, where $N_{G}$ is the mass of gas generated and $N_{L}$ is the mass of gas which flows out of the room. Using Equation 1, this leads to:

$$
N_{L}=N_{O}-\frac{P V_{L}}{R T}
$$

and,

$$
N_{L}=N_{0}-\frac{P \dot{V}_{V}}{R T} .
$$

Equations 3 and 4 apply after $P$ reaches the conatant bithontatic pressure of $14.8 \mathrm{MPa}$. In both of these expressions, the pressure is held constant at $14.8 \mathrm{MP}$ and $V_{v}=V-V_{s}$ is the void volume. 


\subsection{Results}

\section{Influence of Gas Potential}

Cases $A, B, C$, and $D$ show how the storage room response changes as the number of drums generating gas is varied. In these analyses, the room was assumed to be filled and sealed immediately after excavation. The calculations simulated the response for a period of 800 years. Gas generation was assumed to begin immediately after the room was sealed.

The dependence of the void fraction $\left(V_{0} / V\right)$ on the total gas generation potential is of primary concern. Throughout these andyses, we asumed that both the waste and the backfll could be compacted to full solid density. Bowever, for some waste forma, this may not be achievable under bithostatic atress conditions. The time variation of the void fraction in the room (waste + backfill) is shown in Figure 2 for various values of gas generation potential. In each case, the vold fraction reached a minimum value after approximately 140 years and then began to increase. This, reinflation of the room occurred before the room pressure exceeded the lithostatic atress level. However, as shown in earlier studies [2], the maximum principal otresses in the vicinity of the room walls were less than the lithostatic load, and it was only secessary to exceed the value of these local stresses ( $10 \mathrm{MPa}$ ) to initiate reiaflation of the room. There was a significant variation of the minimum void fraction with change in gas generation potentid. These values are plotted in Figure 3 ar a function of $f$. The "fina" void fraction is defined as the void fraction that will remain when the soom pressure and stresses in the salt formation have all returned to the bithostatic condition. The calculations were not run long enough to achieve this state; however, the final equilibrium void volume can be determined directly from Equation 1 because both the equilibrium value of $P_{0}(14.8 \mathrm{MPa})$ and the total number of moles of gas generated ase known for each case. The equilibrium value of the void fraction is also plotted in Figure $3 \mathrm{at}$ a function of $\mathrm{f}$.

The corresponding pressure variations are shown in Figure 4. For each case, the peak room pressure occursed at approximately 300 years. when the gas generation due to corrosion was complete. The magaitude of the peak pressure varied, as expected, with the gas generation potential. This variation is shown in Figure $s_{;}$note that all of the predicted peak values are greater than the bithostatic pressure (14.8 MPA). This plot implies that the peak pressuse approaches bithostatic only as the gas potential approaches sero. However, the results of this study are conaisteat with previous results in that all atresses remained compressive even when the room pressure was larger than the lithostatic pressure at the depth of the repository. As explained in the earlier aludy (1), the stresses remain compressive because of the relatively alow rates of pressurization.

\section{Influence of Gas Generation Rate}

To investignte the effect of varying the gas generation rate $\left(\dot{N}_{0}\right)$, this value was reduced by a factor of five from the baseline case. Two different cases were evalunted for a simulntion 
time of $\mathbf{4 0 0 0}$ years. In Case E, all drums generated gas, whereas in Case F only $20 \%$ of the drums were assumed to generate gas. Figure 6 shows the void fraction as a function of time for Cases $A, E$, and $F$. In both cases, the void fraction in the room reached a minimum value after approximately 100 years. Since the final equilibrium value of the void fraction in the room is only a function of the total amount of gas that is generated, the equillbrium value of the void fraction for Case $E$ will be the same as that for Case $A$, and the equilibrium value for the void fraction in Case $F$ will be the same as that for Case D.

Pressure bistories for Ceses $A, E$, and $F$ are shown in Figure 7. The values of peak pressure estimated for a given value of gas generation potential did not nasy oignificantly with generation rate. Bowever, the pressure built up at a much slower rate, which was consintent with the slower rate of gas generation.

\section{Gas Loss}

It is not presently possible to model gas diffusion in SANCHO. In all of the simulations which model gas generation, the salt which constitutes the WIPP repository is assumed to be completely impermenile to the flow of gas. In reality, permeable layers intersect the walls of the storage room. Ade quate information is not yet avalable regarding the change in permeability of these reame with pressure; however, we considered a hypothetical situation in which the gas began to penetrate into clay seams, anhydrite beds, and/or parting planes in the salt when the room pressure reached the lithostatic atress level. We then calculated the rate at which gas must flow into these layers to prevent the room pressure from exceeding the lithostatic stress level.

In this simulation, the room pressure was allowed to vary according to Equation I until reaching the bithostatic level (14.8 MPa). Beyond this point, the room pressure was held conatant at $14.8 \mathrm{MPa}$; however, the room volume continued to change as the salt continued to creep. Although the gas pressure in the room was equal to the mean lithostatic atress, the stress distribution in the surrounding oalt was not uniform, and the odt continued to creep, causing the room volume to slowly increase. When the pressure is conatant but the room volume varies, then, according to the ideal gas law, the mass of gas in the room must aloo change. We assume that any change in the gas mass is due to a combiantion of gas generation and gas lose into the clay seams. The total amount of gas loss and the rate of gas loss were calculated uaing Equations 3 and 4.

In this problem, the gas generation history was assumed to be the same as the baseline case (Case A). The calculation was sun out to 1000 years. Figure 8 shows the pressure history which was computed from the current simulation. After the room pressure reached $14.8 \mathrm{MPa}$ at approximately 200 yenrs, the pressure was held constant. The void volume in the room is plotled as a function of time in Figure 9. As seen in the expanded plot (inset), the void volume continued to slowly increase after lithostatic pressure was achieved in the room. 
The predicted mass of gas generated and lost versus time is shown in Figure 10. The amount of gas lost was less than the amount generated at any given time. At 500 and 700 years, the rates of generation and loss changed at approximately the same rates. The rates of generation and loss are displayed in Figure 11, and the ratio of loss rate to generation rate is shown in Figure $12 \mathrm{as}$ a function of time. Prior to a time of about 180 years, there was no gas loss. At the time when gas loss was assumed to begin, the rate of lon wa initially equal to $97 \%$ of the generation rate and slowly increased until the two rates were almost equal. At 300 yeass, both rates dropped, and then the rate of loss slowly increwed. At 700 years, the generation rate dropped to rero; however, because the cavity volume continued to increase alowly, the computed loss rate became alightly negative. This may mean that a amall amount of gal will diffuce back into the cavity after ga generation has censed, or it may indicate that the room preasure will drop slightly below the lithostatic level if the gas generation is abruptly terminated.

\section{SUMMARY}

Resules of these calculations show how reductions in gas generation potential increase the degree of waste and backfill consolidation and reduce the peak gas pressure in the disposel room. Even bigher degrees of waste and backfll consolidation can be achieved by reducing both the gas generation rate and the gas generation potential. Bopefully, decreases in the amount and/or rate of gas generation can be achieved with modifications to the waste or backfill.

A simple analysis was also conducted to determine the rate of gas lose from the disposal room that would prevent the room pressure from exceeding the bithostatic stress level. This analysis showed that the required rate of gas loss from the disposal room (e. g., into clay seams) must be approximately equal to the rate of gas generation to arrest the buildup of pressure. 


\section{REFERENCES}

1. J. R. Weatherby, J. G. Arguello and C. M. Stone, "The Effect of Gas Generation on Performance of CB.TRU Disposal Rooms", memo to B. M. Butcher, 6343, November 14, 1989.

2. J. R. Weatherby and J. G. Arguello, "The Effect of Gas Generation on the Extension of Preexiating Fractures Around WIPP Disposal Rooms", memo to B. M. butcher, 6346, March 28, 1990.

3. "Systems Andyois, Long-Term Radionuclide Traasport, and Dose Assessments, Weate Isolation Pilot Plant, Southeatern New Mexico, March 1989," A. Lappin and R. Buater (editors), SAND89-0462, April 1889.

4. R. D. Krieg, "Reference Stratigraphy and Rock Properties for the Waste Isolation Pilot Plant (WIPP) Project", SAND83-1908, January 1984.

5. B. S. Morgan and R. D. Krieg, "Inventigation of an Empirical Creep Law for Rock Salt That Uses Reduced Elastic Moduli," in Rock Mechanice Contributions and Challenges - Proceedings of the S1ot U.S. Symposium, W. A. Hustrulid and G. A. Johnson (editors), 1900.

6. C. M. Stone, R. D. Krieg, Z. E. Beisinger, "SANCHO, A Finite Element Com. puter Program for the Quasistatic, Large Deformation, Inelastic Response of Two. Dimensional Solida," SAND84-2618, April 1985. 
Table 1. Total Volume and Void Fraction of Each Region in the Storage Room.

\begin{tabular}{|c|c|c|c|}
\hline \multicolumn{3}{|c|}{ Volume by Region } & Void Fraction \\
\hline $\begin{array}{l}\text { Volume of Drums } \\
\text { Volume of Backfill }\end{array}$ & $\begin{array}{l}=1,817 \mathrm{~m}^{3} \\
=1,829 \mathrm{~m}^{3}\end{array}$ & $\begin{array}{l}\left(64,125 \quad t^{3}\right) \\
(65,585 \\
\left.f^{3}\right)\end{array}$ & $\begin{array}{l}0.685 \\
0.40\end{array}$ \\
\hline \multicolumn{4}{|c|}{ Total Solid Volume $\left(V_{s}\right)$ : } \\
\hline
\end{tabular}

Table 2. Parameters From Equation 2 Defining the Gas Generation History.

\begin{tabular}{|c|c|c|c|c|}
\hline Case & $f$ & $D$ & $\begin{array}{c}N_{0} \\
\text { (g-molins/drum/year) }\end{array}$ & TIME PERIOD \\
\hline A & 1.0 & 6800 & $\begin{array}{l}2.66 \\
0.86 \\
0 .\end{array}$ & $\begin{array}{l}0 \text { years }<t<300 \text { years } \\
300 \text { years }<t<700 \text { years } \\
t>700 \text { years }\end{array}$ \\
\hline B & 0.6 & 6800 & $\begin{array}{c}2.66 \\
0.86 \\
0 .\end{array}$ & $\begin{array}{l}0 \text { years }<t<500 \text { years } \\
500 \text { years }<t<700 \text { years } \\
t>700 \text { years }\end{array}$ \\
\hline C & 0.4 & 6800 & $\begin{array}{c}2.66 \\
0.86 \\
0 .\end{array}$ & $\begin{array}{l}0 \text { years }<t<500 \text { years } \\
500 \text { years }<t<700 \text { years } \\
t>700 \text { years }\end{array}$ \\
\hline D & 0.2 & 6800 & $\begin{array}{c}2.66 \\
0.86 \\
0 .\end{array}$ & $\begin{array}{l}0 \text { years }<t<500 \text { years } \\
300 \text { years }<t<700 \text { years } \\
t>700 \text { years }\end{array}$ \\
\hline $\mathbf{E}$ & 1.0 & 6800 & $\begin{array}{c}0.532 \\
0.172 \\
0 .\end{array}$ & $\begin{array}{l}0 \text { years }<t<2500 \text { years } \\
2500 \text { years }<t<3500 \text { years } \\
t>3500 \text { years }\end{array}$ \\
\hline$F$ & 0.2 & 6800 & $\begin{array}{c}0.332 \\
0.172 \\
0 .\end{array}$ & $\begin{array}{l}0 \text { years }<t<2500 \text { years } \\
2500 \text { years }<t<3500 \text { years } \\
t>3500 \text { years }\end{array}$ \\
\hline
\end{tabular}




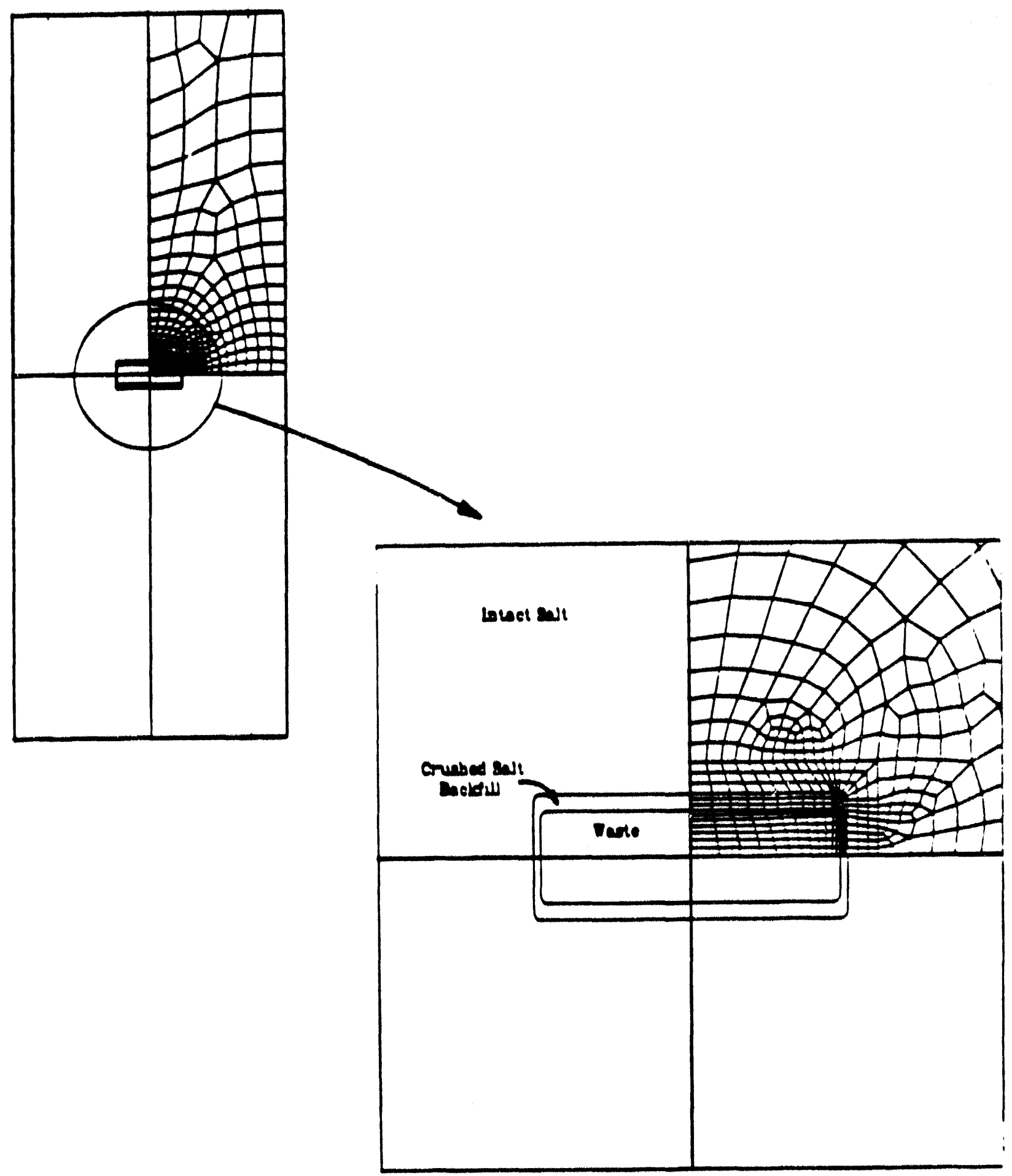

Figure 1. Plane Strain Finite Element Model of a TRI Storage Room 


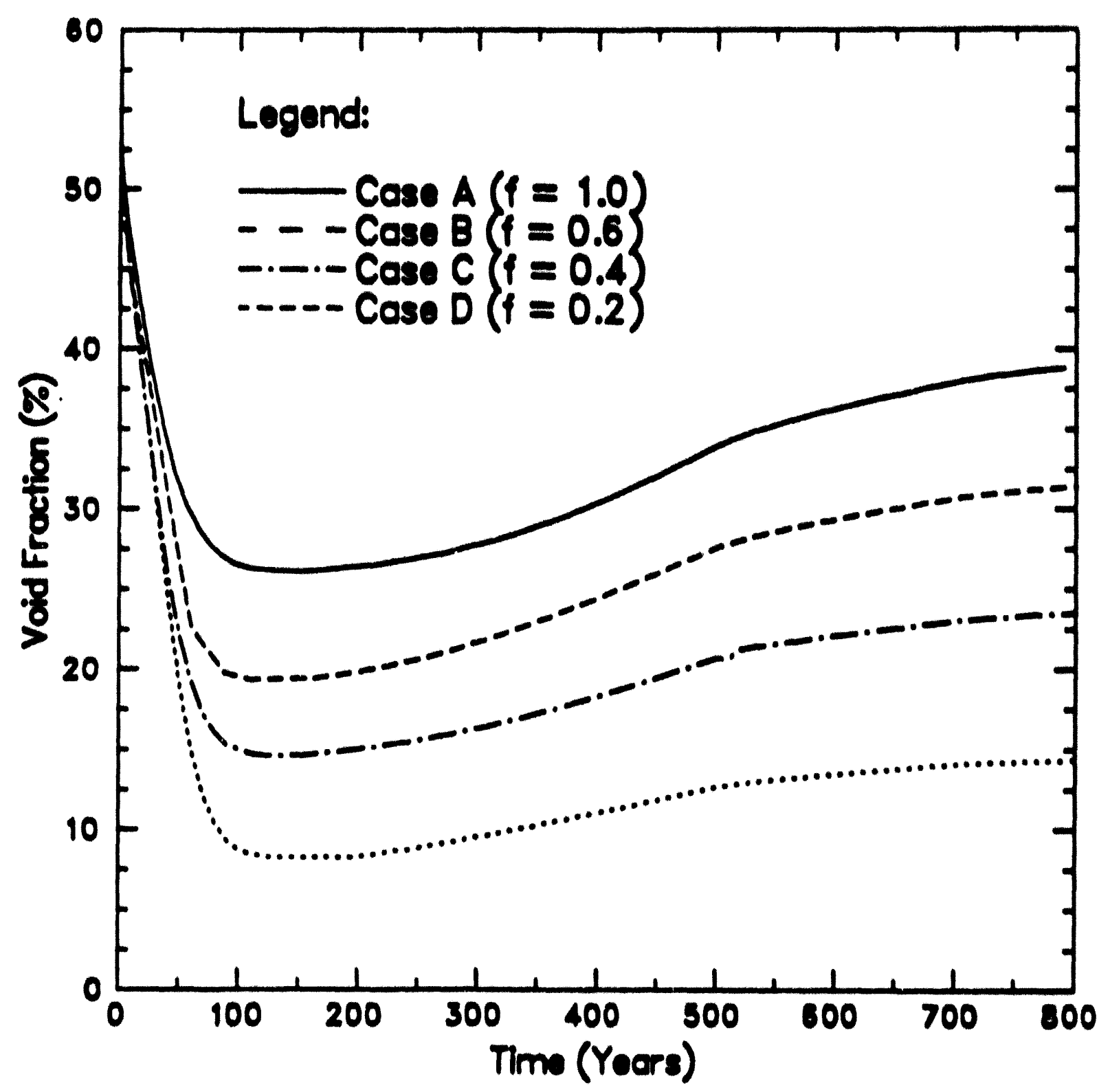

Figure 2. Void fraction in the room as a function of time for Cases $A, B, C$, and D. The rate and amount of gas generation per drum was the same as the beseline value in all four cases. Only the number of drums generating gas was varied between the four cases. 


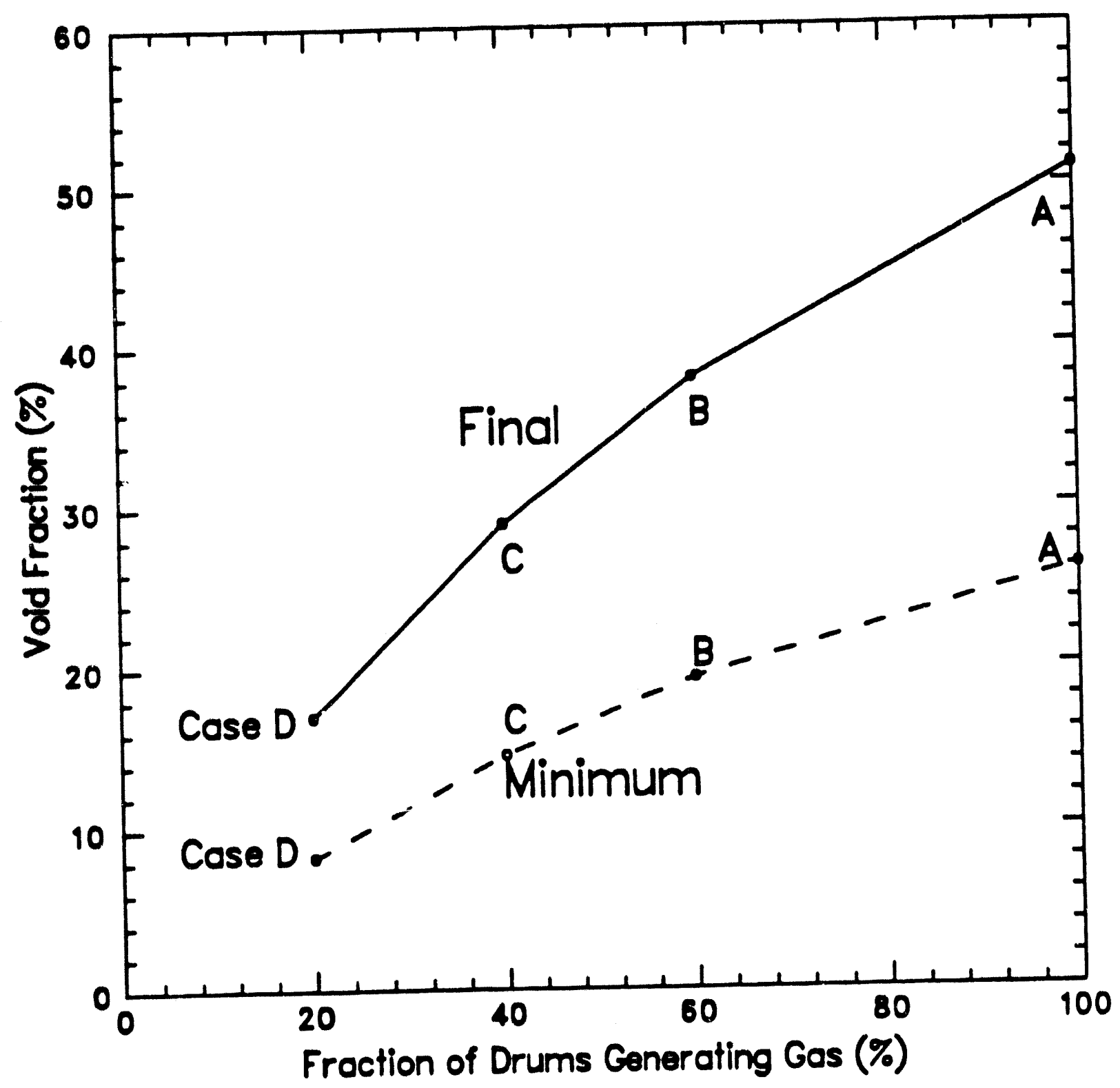

Figure 3. Minimum void fraction and final equilibrium void fraction as a function of the fraction of drums generating gas. The final void fraction is defined as the void fraction that will remain when the room pressure and stresses in the salt formation have all returned to the bthostatic condition. These results were obtained from the finite element analyses of Cases $A, B, C$, and $D$. The rate and amount of gas generation per drum was the same as the baseline value in all four cases. Only the number of drums generating gas was varied between the four cases. 


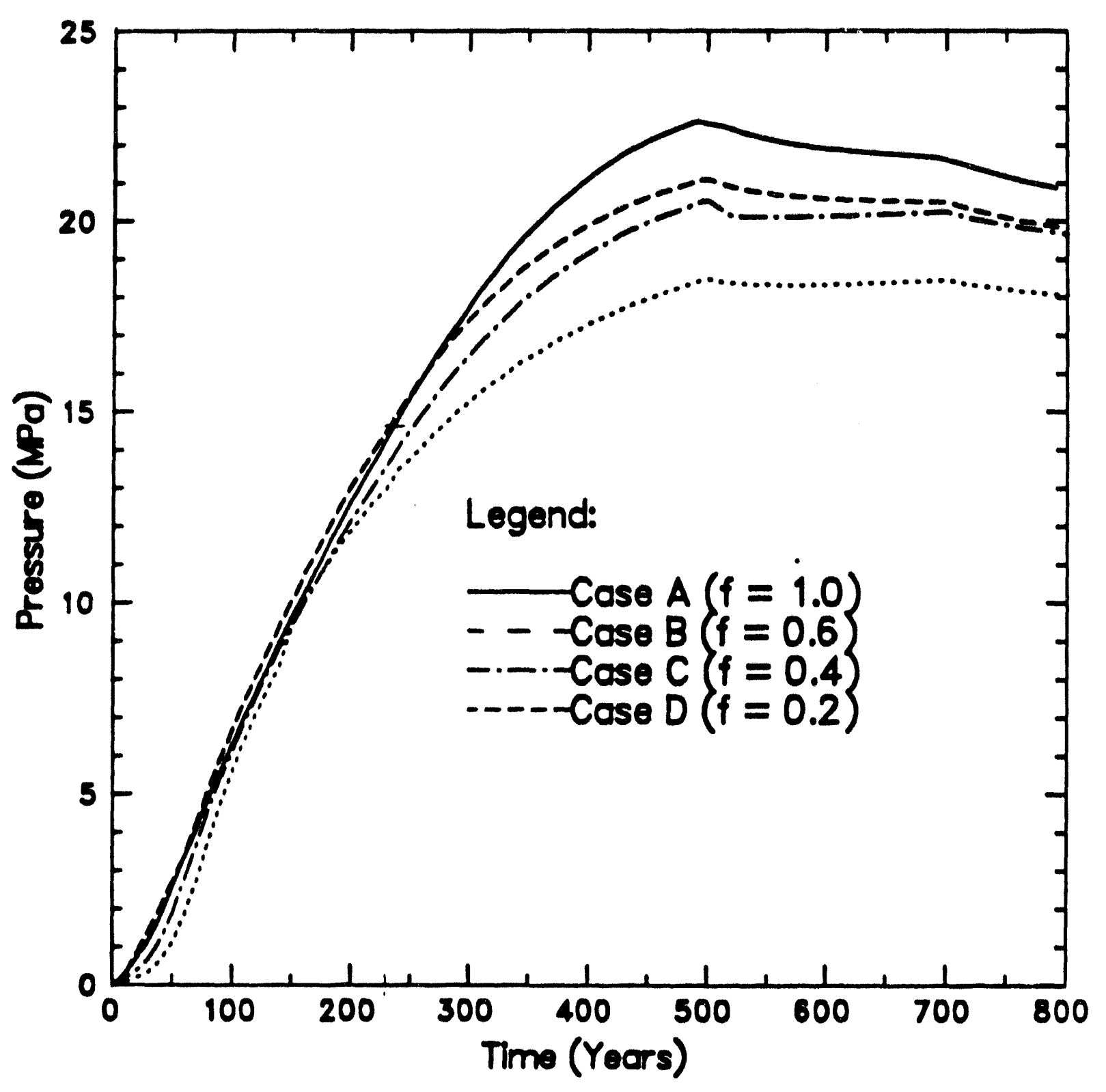

Figure 4. Room pressure as a function of time for Cases A, B, C, and D. The rate and amount of gas generation per drum was the same as the baseline value in all four cases. Only the number of drums generating gas was varied between the four cases. 


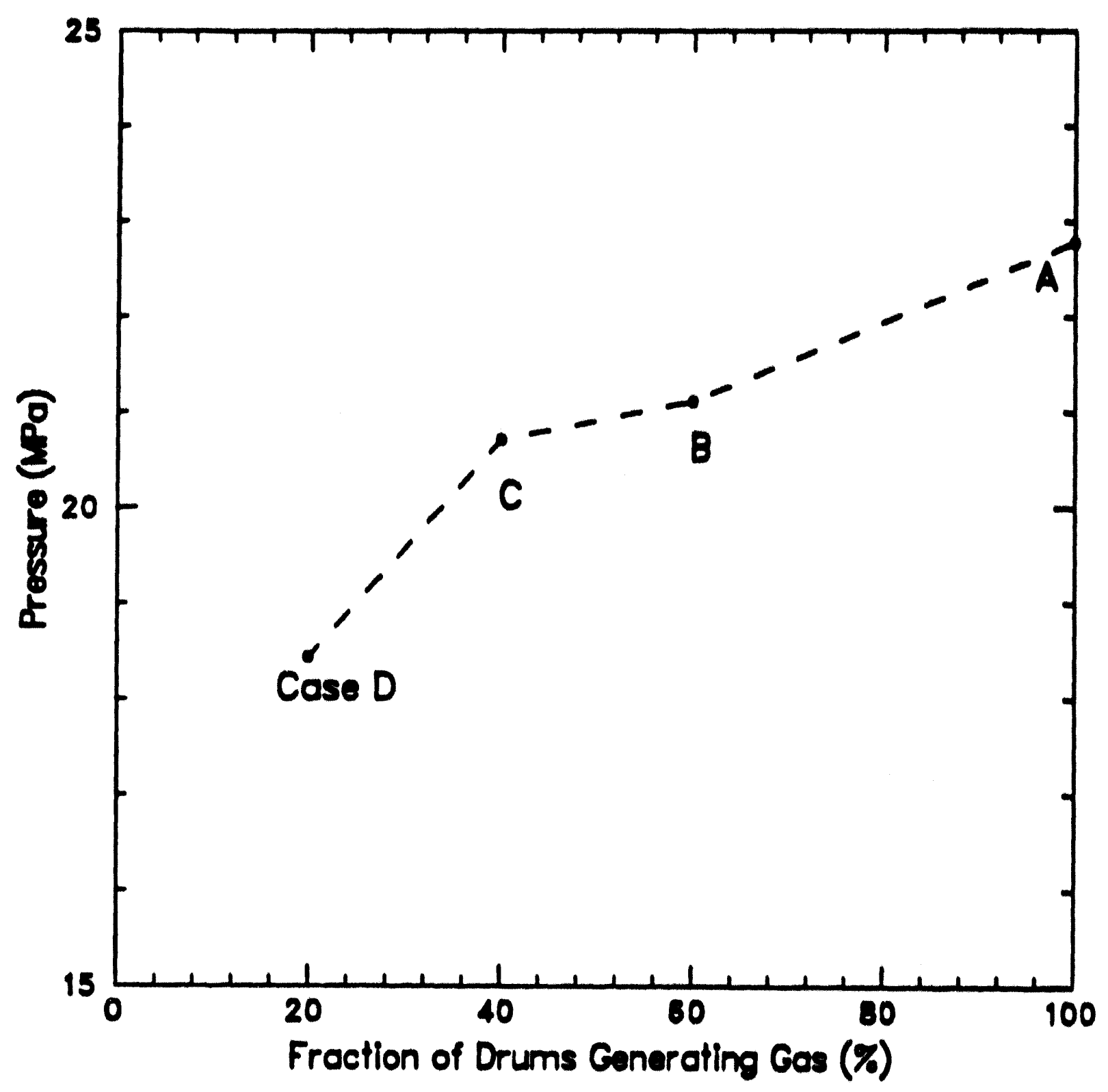

Figure 5. Peak pressure in the disposal room as a function of the fraction of drums generating gas. These results were obtained from the finite element analyses of Cases A, B, C, and D. The rate and amount of gas generation per drum was the same as the baseline value in all four cases. Only the number of drums generating gas was varied between the four cases. 


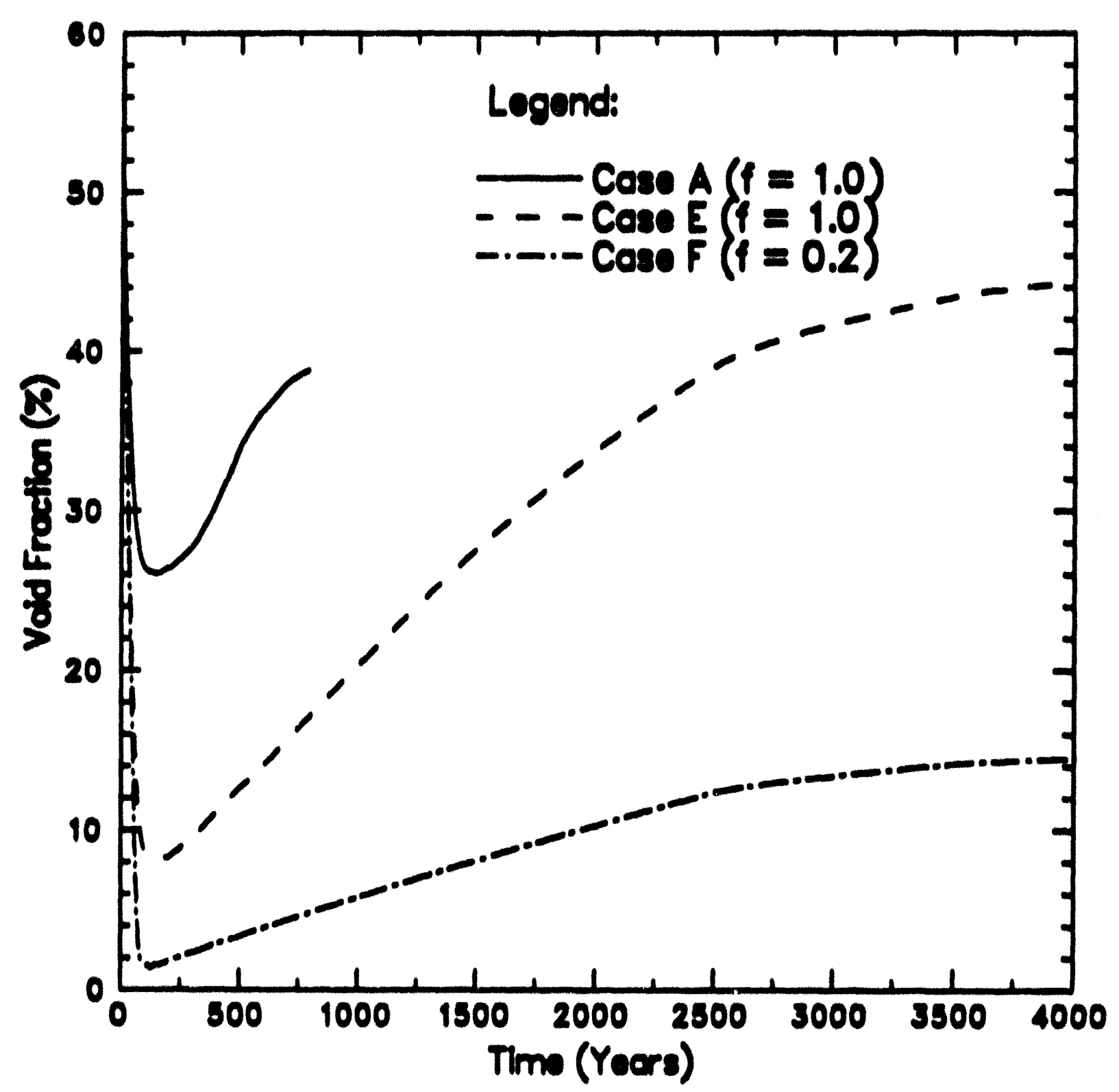

Figure 6. Void fraction in the disposal room as a function of time for Cases A, E, and $F$. In Case $E$, the total amoun' of gas generated is the same as that in the baseline case (Case A); however, the rate of gas generation is $20 \%$ of the baseline value. In Case F, the rate of gas production pes drum is $20 \%$ of that in the baseline case, and the total amount of gas generated is $20 \%$ of the baseline value. 


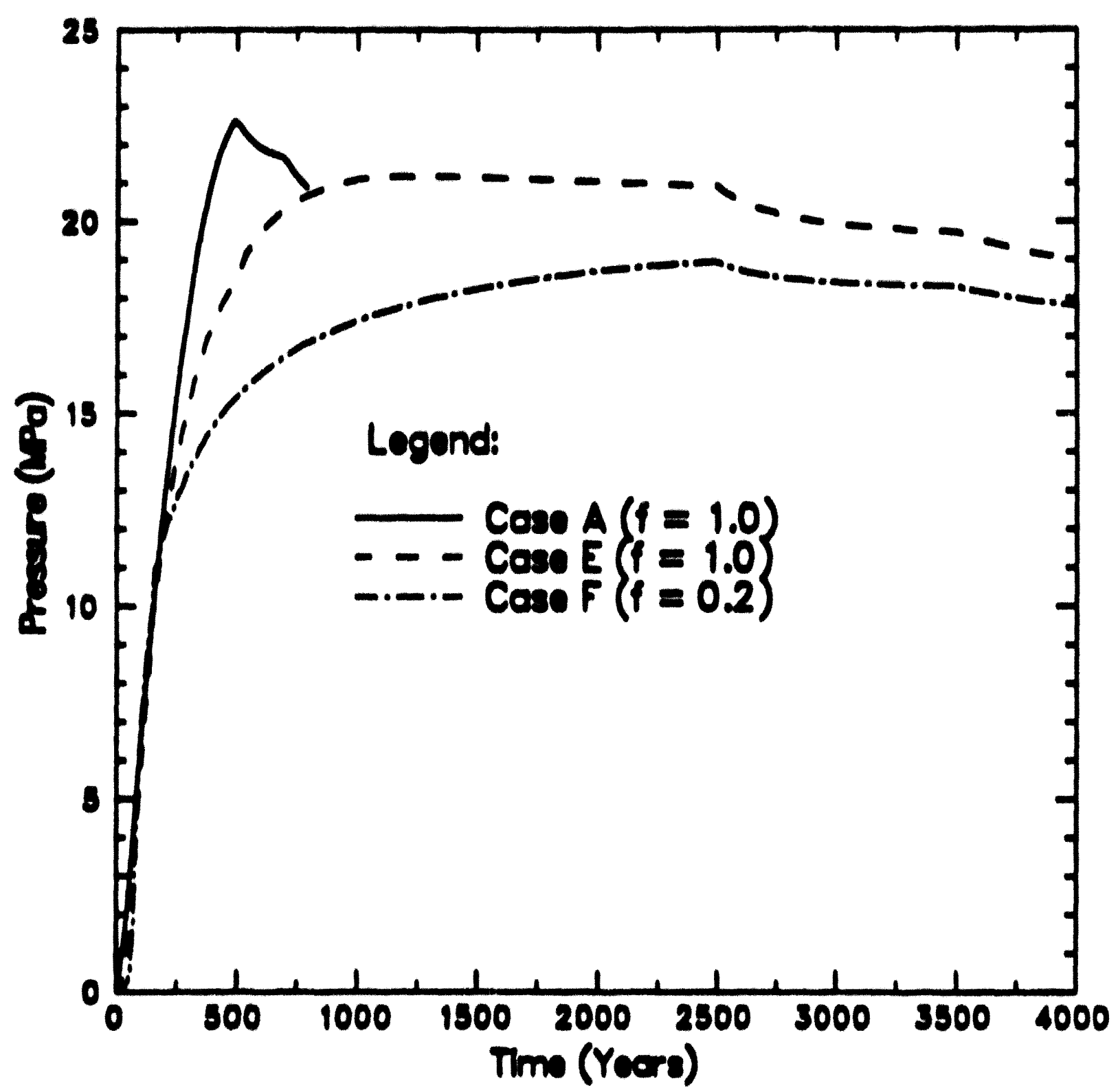

Figure 7. Room pressure as a function of time for Cases $A, E$, and $F$. In Case E, the cold amount of gas generated is the same as that is the baseline case (Case A); however, the rate of gas generation is $20 \%$ of the baseline value. In Cace $F$, the rate of gas production per drum is $20 \%$ of that in the baseline case, and the total amount of gar generated is $20 \%$ of the baseline value. 
B. M. Burches and F. T. Mendenhall -17-

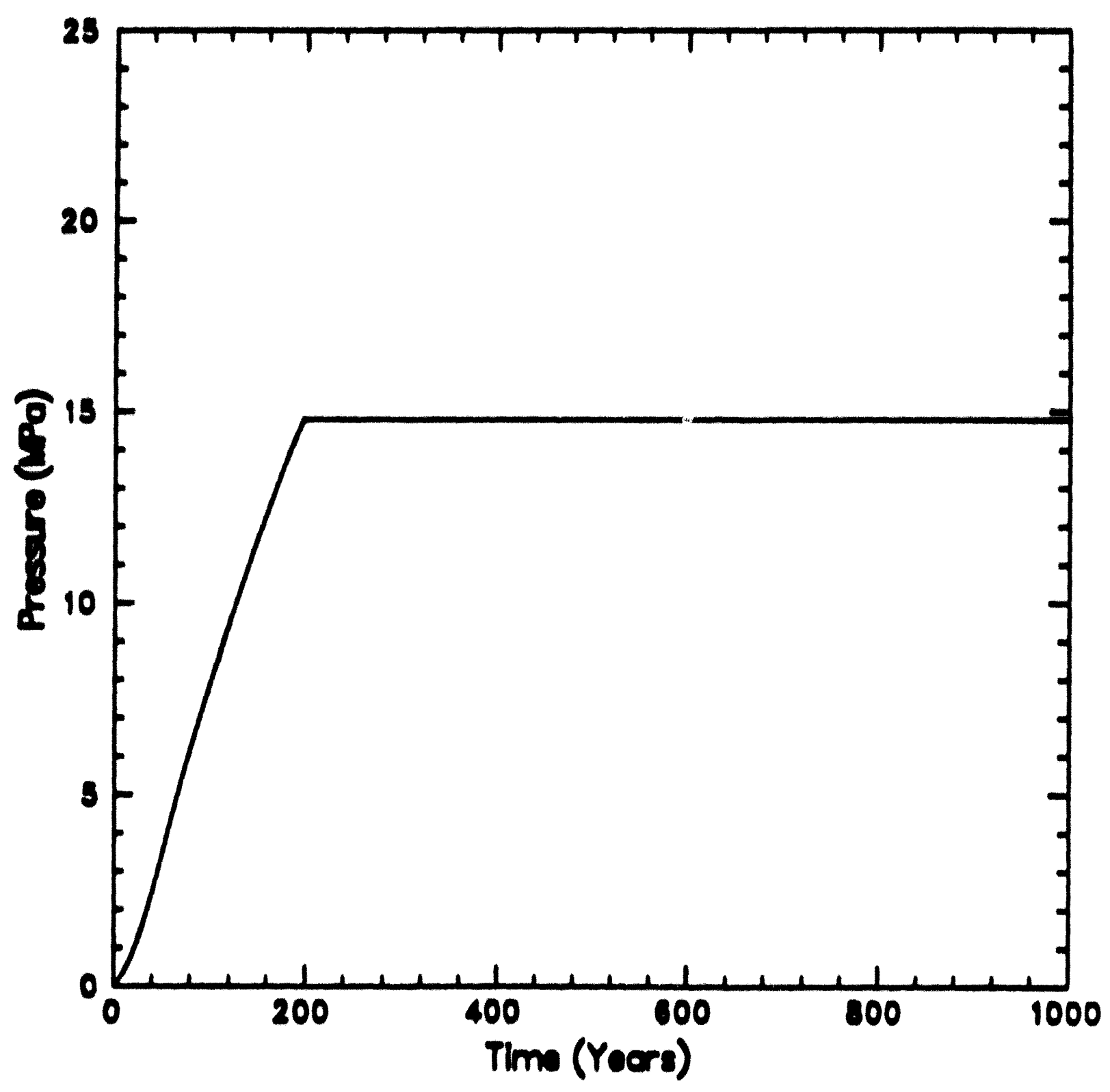

Figure 8. Pressure history for a disposal room in which the internal pressure is cosstraned to remain at, or below the bithostatic stress level. 


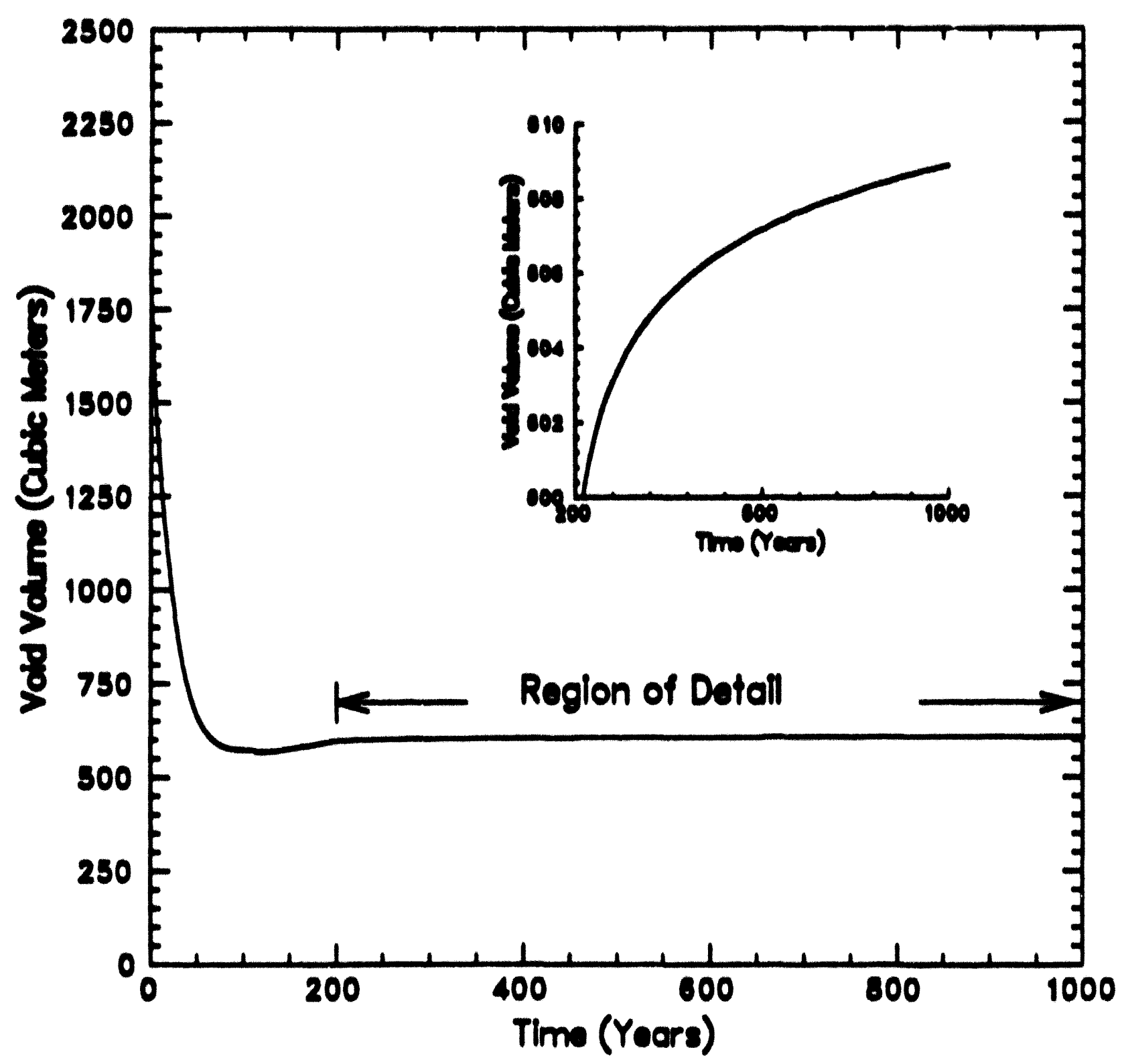

Figure 9. Time variation of the void volume in a disposal room limited to bithostatic pressure. The insert shows an expanded view after the lithostatic level is attained. 


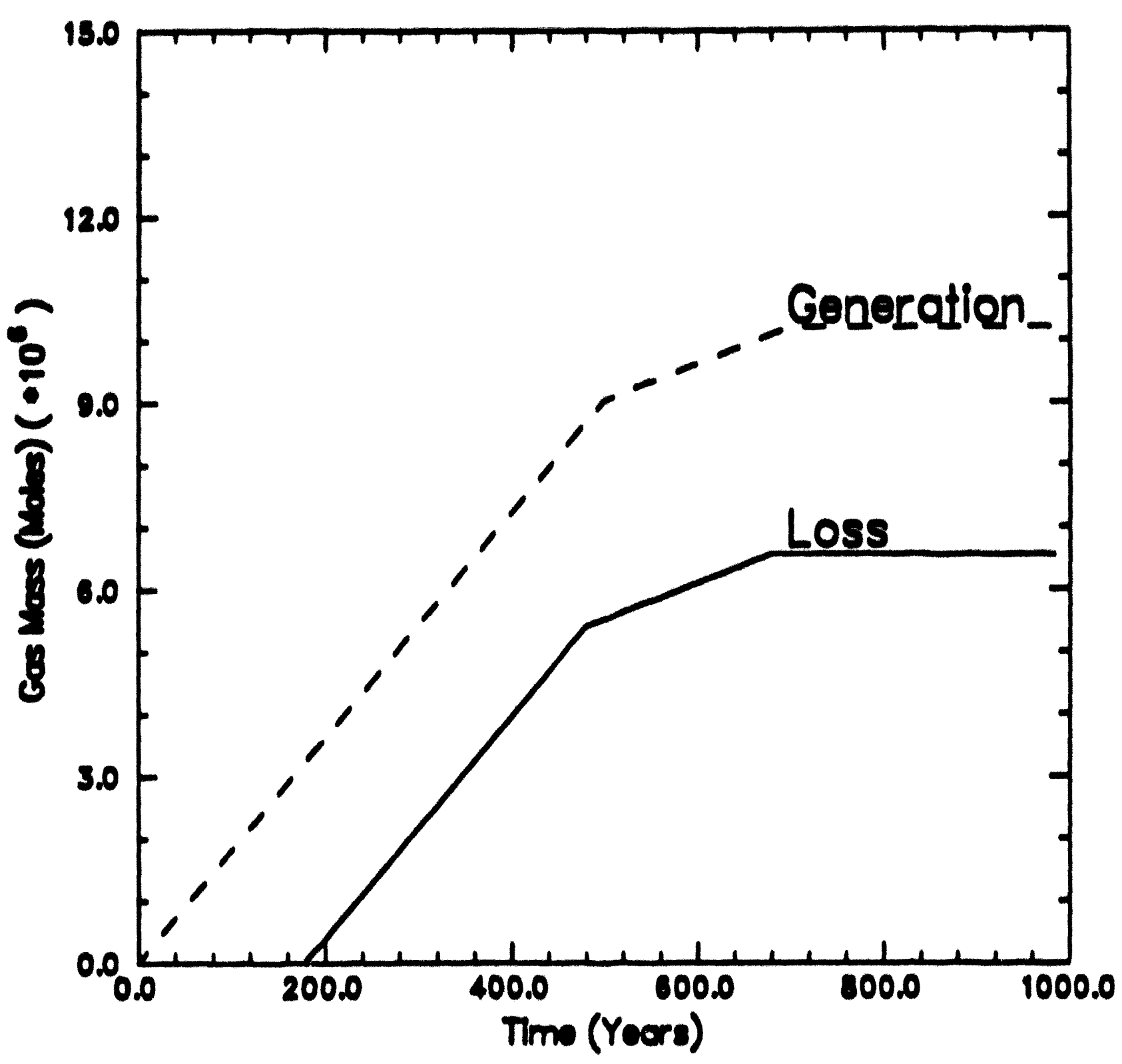

Figure 10. Comparison of total gas generation to total gas loss sequired for constant room pressure $\left(P_{0}=14.8 \mathrm{MPa}\right)$. 


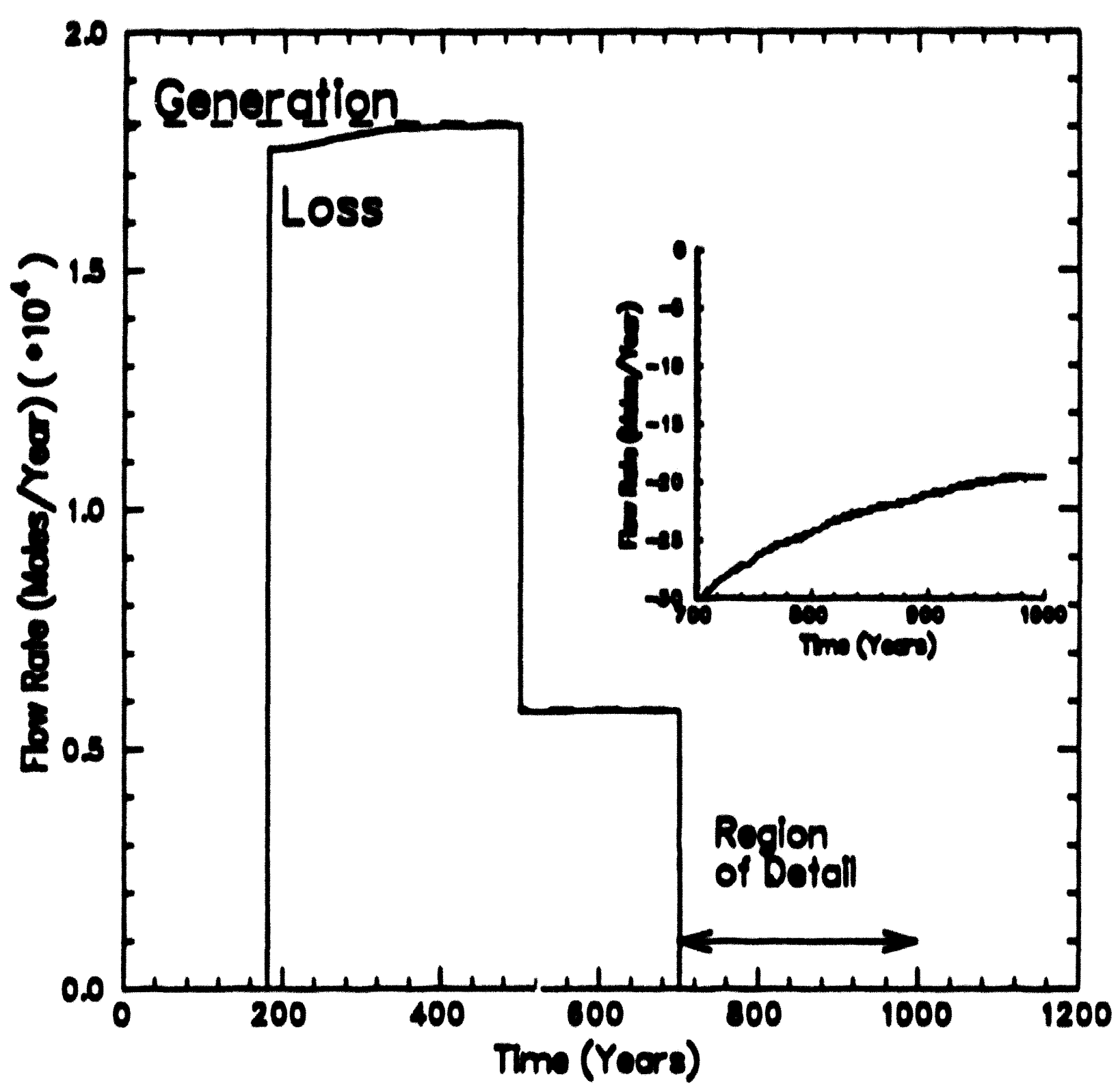

Figure 11. Comparison of the gas generation sate as a function of time 10 the gas loss rate as a function of time required for conslant room pressuse $\left(P_{0}=14.8 \mathrm{MPa}\right)$. After gas generation has ceased (inset), the loss rate becomes negative, indicating a small flow rate of gas back into the disposed room. 


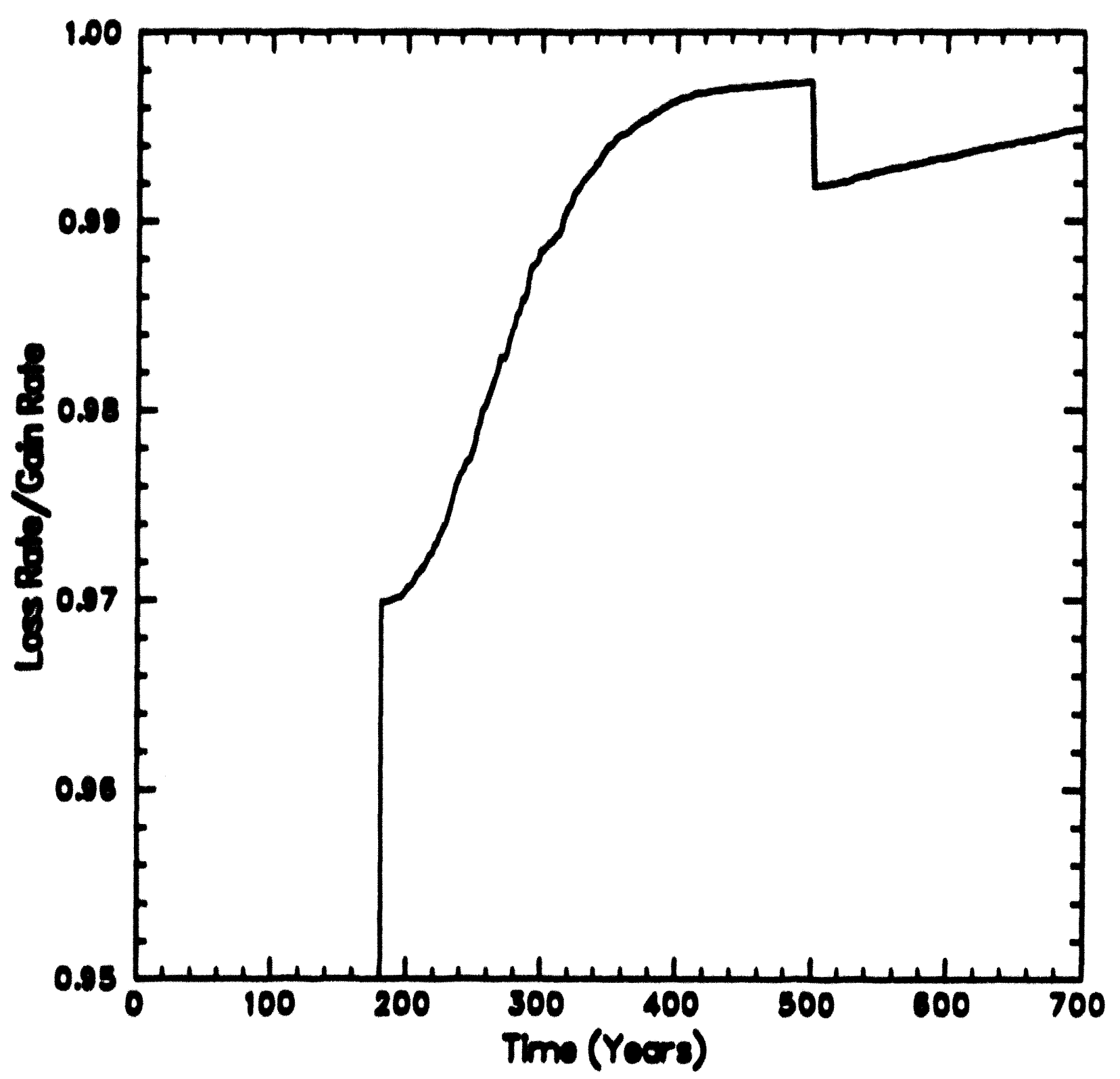

Figure 12. Ratio of the gas loss rate to the gas generation rate required for conctant room pressure $\left(P_{0}=14.8 \mathrm{MPa}\right)$. 


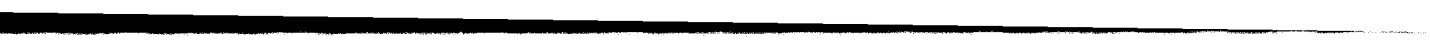

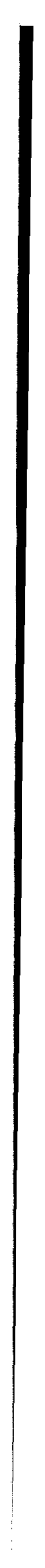

A. 26 


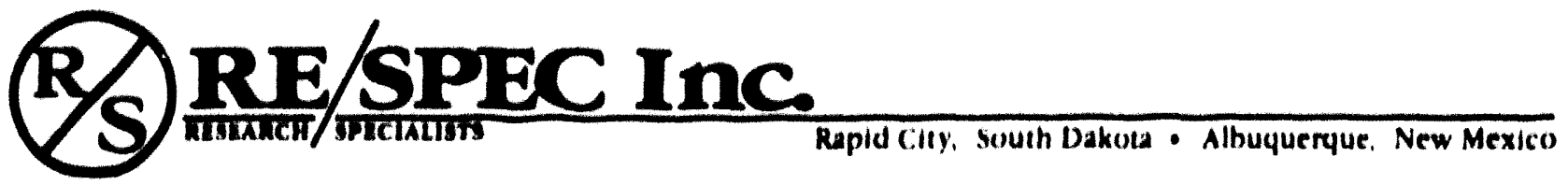

\section{External Memorandum}

108
Dr. Ired T. Mendenhall
Sendia Nationd Laboratorica
Organisation 6348
P.O. Box 8800
Albuquerque, NM 87188-8800
cet Dr. Berry M. Buteher (Bendle Divinlon 6848)
Mr. C. A. Madole (8nadie Divibion 3716)
Mr. Duane A. Labreche (RE/8PEC)
Project Recordo Fllo 217/OR10

Droms

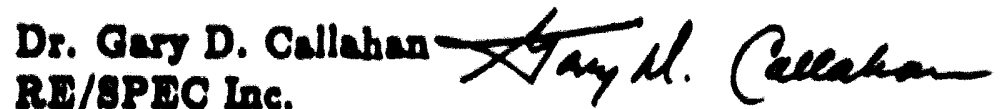

P.0. Bor 798

Rapid CIty, 8D 87700

Datet March 18, 1092

8ubject Durther Discunsion of the TRU Weste Modal

(Bendle Contract No. 78-7829)

\section{Introduction}

The purpowe of thin momorandum it to present edditional dincusion on the TRU wate model dheuned in Callahen and DoVrice [1991] and hopofully clarify some of the inconvinteneics dincuased by Dr. Butcher in hlo memorandum dated March 4, 1092.

\section{TRU Waste Model Discussion}

The besic equation used to deacribe the TRU wate behavior is

$$
\sigma_{0}=\frac{1}{n} \ln \left(\frac{\phi}{\phi_{0}}\right)
$$


Then, the questions are: what is the impact of Assumption (2) on the waste parameters, and what is the impact of these different parameter values on the results?

To anawer these questions, we will firat examine the tangent bulk modulus, which is detined a

$$
K=\frac{d \sigma_{m}}{d \epsilon_{v}}=\frac{d \sigma_{m}}{d \phi} \frac{d \phi}{d \rho} \frac{d \rho}{d \epsilon_{v}}
$$

Mean strese is substituted into Equation 1 for the two different assumptions. Firnt, oubatituting $\sigma_{m}=\sigma_{1} / 3$ and performing the differentiation indicated in Equation 2 on Equation 1 result in

$$
K(\rho)=\frac{\rho^{2}}{3 \kappa \rho_{0}(\rho \rho-\rho)}
$$

For the accond asumption, substituting $\sigma_{m}=\sigma_{a}$ and performing the differentiation indicated in Equation 2 on Equation 1 results in

$$
K(\rho)=\frac{\rho^{2}}{R \rho p(\rho)-\rho)}
$$

With the second aseumption, we see that the TRU waste $b$ three times atifier than that obtained uning the Brat asumption. This is also shown in Figure 1, which in a reproduction of Figure 2-7 in Callahan and DeVries (1091) for the series model. In Figure 1, the ordinate has been changed from axial stress to mean atrese, and the series model representation (squarea) for the second sesumption hes been added. The ordinate was changed to mean atrees to avoid confunion. The confunion is apparent because Dr. Butcher statea in his memorandum that Callahan's Figure 2-7 supports a porosity of 21 percent at lithostatic preasure (18 MPa). A lithostatic preasure of $15 \mathrm{MPa}$ impliee that $\sigma_{n v}=\sigma_{n w}=\sigma_{n}=15 \mathrm{MPa}$. However, the inherent aesumption in Figure 2-7 was that the lateral components were sero. Thus, to achiove a mean otrese of $18 \mathrm{MPa}$ (under Asoumption (1)), the axial atreas would have to be $48 \mathrm{MPa}$. Also, we see from Figure 1 that at a lithoutatic value of 15 MPa, the curve (circles) generated for Assumption (1) yields a porosity value of bout 4 percent.

Although there is a significant difference between the curves obtained uning the two asumptions, the procedure for adopting Asaumption (2) to produce the stlfier TRU waste model is aimple. To obtain TRU atiffnesses according to Assumption (2), material parameter $\alpha$ is divided by 3 . Therefore, the same material model adopted for the TRU waste and included in SPECTROM-32 can be used to represent the otlffer TRU waste and obtain the higher values of poronity. 


\section{New Crushed Salt TRU Waste Model Analysis}

To illustrate the influence of Assumption (2) on the room acale results, the problem representing the room flled with TRU waste and covered with crushed salt as reported by Callahan and DeVries [1991) was run with TRU waste properties dictated by Aesumption (2). The resulta of this analyala (labelled $\sigma_{m}=\sigma_{0}$ ) are compared with the results (labelled $\sigma_{m}=\sigma_{0} / 3$ ) generated vis Aesumption (1) a reported by Callahan and DeVries [1091] in Figurea 2 through 4. Figure 2 compares the vertical and horizontal room closures from the two analysen, and as expected, the atiffer TRU wate model produced the least amount of room closure. Figure 3 compares the mean atress historles at different locations. Figure 4 shown the average void fraction results obtained for the two different TRU wate representa. thons. Figure 4 is comparable to Figure B-6 given in Callahan a id DeVriee (1091). The SANCHO reaults were removed, and the SPECTROM-32 reaults obtalned by replacing material parameter $\kappa$ by $\kappa / 3$ are included. The result obow that the otiffer TRU waste model indeed causee the crushed ealt beckell to convolldate more rapidly, although the change is moderate. The TRU wate exhlbits an average vold fraction of about 36 percent after 200 years for the stlffor model, which b a subatan. thal increase from the previous reeult (about 3 percent). The average vold fraction in the room is about 18 percent after 200 years for the atifior model compared to the previous renult of about 1 percent.

\section{Conclusions}

Two different methods were used to generalize the TRU wate functional form (Equation 1) to three-dimenaional states of atreas. The two methodo produce TRU waste atiffnesses that vary by a factor of 3 . The results produced by these two generalizations can be aubstantially different. The firat generalisation (Aesumption (1)) produces conservative resulte with respect to the beckell material; wherean, the second generalisation (Assumption (2)) produces conservative results with respect to the TRU waste when porosity is the variable being considered lo a typical diaposed room environment.

\section{References}

Butcher, B. M., T. W. Thompuon, R. G. VanBunklrk, and N. C. Pattlo, 1001. Mechanical Compaction of Waste Isolation Pilot Plant Simulated Waste, SAND90-1206, prepared by Sandla National Laboratories, Albuquerque, NM, June.

Callahan, G. D., and K. L. DeVries, 1991. Analyose of Backfilled Tranuranic Waste Storage Rooms, SAND91-7052, prepared by RE/SPEC Inc., Rapid Clty, SD, RSI-384, for Sandia National Laboratories, Albuquerque, NM. 
RS1-217-92-007

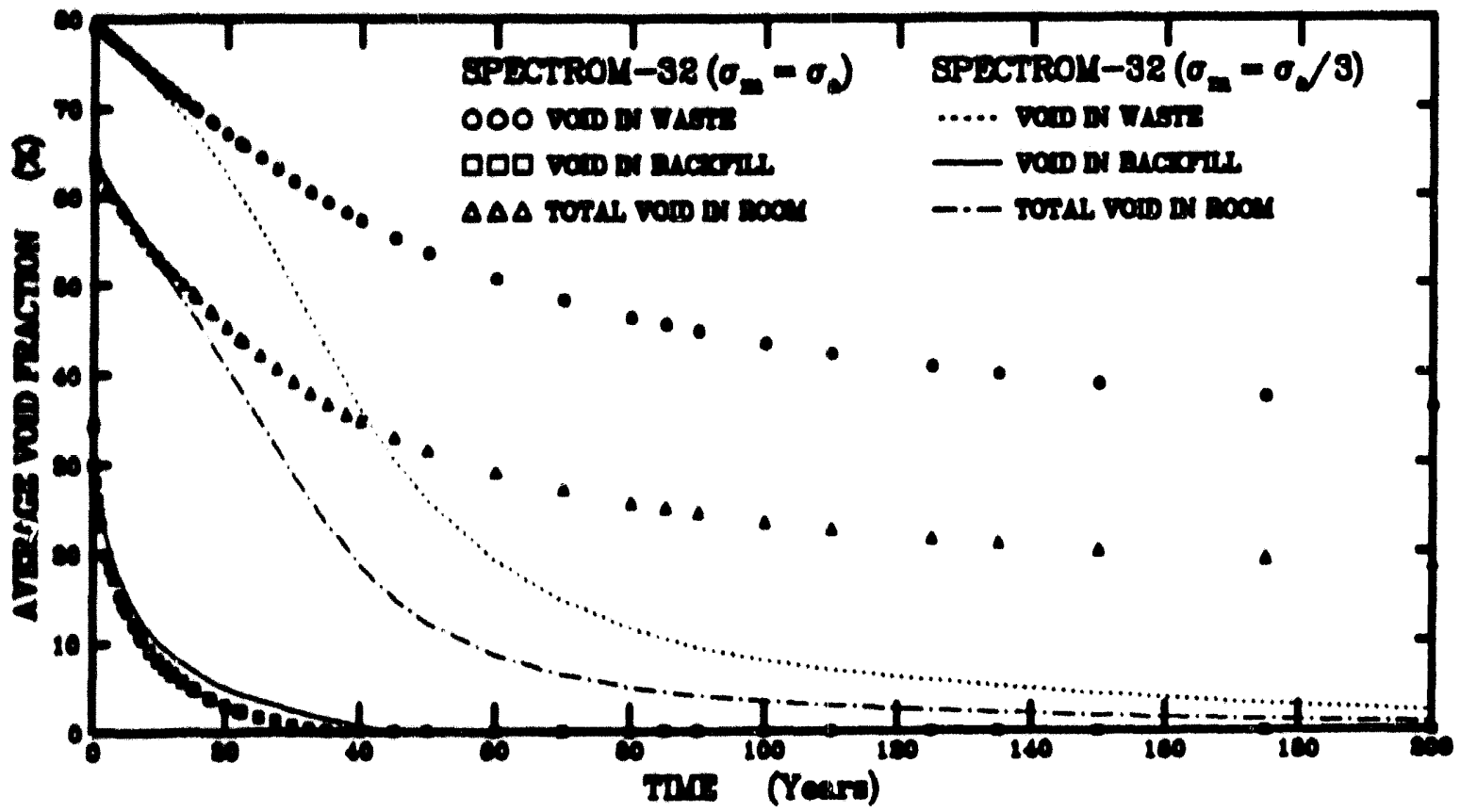

Figure 4. Average Void Fractions in a Room Containing TRU Waste and Crushed Salt (Curre:t Volume). 


\title{
Sandia National laboratories
}

\author{
Albuquerque. New Mexico 87185
}

\begin{abstract}
Date: June 26, 1990
To: $\quad$ Barry Butcher (6345)

Fred Mendenhall (6345)

From:

Peter Davies (6344) Peten Pavies

Subject: $\quad$ Observations on Interbed Pressurization from Recent 2-Phase, Waste-Generated Gas Simulations
\end{abstract}

The purpose of this memo is to provide you with some preliminary, but possibly significant, results from a recently completed, long-term, waste-generated gas simulation that explicitly includes interbeds. This simulation focuses on a disposal room in a panel, i.e. there is a no-flow boundary at the pillar centerline located $-16 \mathrm{~m}$ from the outside edge of the room (Figure 1). The room is surrounded by halite with a permeability of $10^{-20} \mathrm{~m}^{2}$. In addition to the halite, the simulation includes two interbeds, Marker Bed 139 below the room and anhydrite " $b$ " above the room, both with permeabilities of $10^{-19} \mathrm{~m}^{2}$. Threshold pressures are specified at $23 \mathrm{MPa}$ in the salt and $0.3 \mathrm{MPa}$ in the interbeds. The room is configured in an "intermediate" closure state, i.e. it has dimensions and void volume taken from the minimum void volume reached in the coupled gas/closure calculations reported by Weatherby et al. (11-14-89 memo). The two interbeds are connected to the room using a "fracture-like" connection through the DRZ that allows direct hydraulic communication between the room and the interbeds. Gas generation is specified at Larry Brush's estimated values from the SEIS. Fully coupled gas and brine flow is simulated using the ECLIPSE multiphase simulation code.

What I would like to highlight in the simulation results is the nature of gas breakthrough and pressurization in the interbeds. Gas pressure builds in the room until a point (at about 70 years) where gas pressure in the room (7-8 MPa) is sufficient to drive gas upward into anhydrite "b" (Figures 2,3 , and 4). Once this breakthrough occurs, gas propagates relatively rapidly through the interbed and by slightly over 100 years, the interbed has reached the upper limit of gas saturation $(-75 \%$, given the specified capillary pressure curve) (Figure 2). Also note that once gas has entered the interbed, gas pressure within the interbed follows the room pressure quite closely (Figure 3).

Because brine has a much higher density than gas, brine entering the room tends to accumulate in the lower part of the room, which causes Marker Bed 139 to remain isolated from the gas for a much longer period of time (Figures 4 through 8 ). This occurs because the high water saturation in the lower portion of the room keeps the relative permeability to gas very small. Only after the brine in the lowermost part of the room has been driven out and this lower area reaches higher gas saturations does the relative permeability to gas rise to a level that allows gas penetration into Marker Bed 139 (beginning at approximately 200 years) (Figures 5,7 ). Once initial penetration has occurred, gas propagates laterally through the interbed in a fashion similar to the earlier lateral propagation through anhydrite "b" (Figure 7). Also similar to anhydrite " $b$ ", once gas has penetrated the interbed, its pressure within the interbed follows room pressure quite closely (Figure 8).

The primary observation that I would like to draw your attention to is that given our current measurements and estimates of physical properties, gas readily penetrates, flows along, and pressurizes interbeds that are hydraulically connected to the room (in this case connected via the DRZ). Also, once penetration occurs, gas pressure in the interbed tracks gas pressure in the room quite closely. 
This behavior raises a fundamental question about the nature of the coupled hydrologic/mechanical response to high gas pressures. It is likely that the primary source of permeability in the interbeds is features such as the subhorizontal, preexisting, partially healed fractures identified by Borns (1985). Because these interbeds are likely to pressurize over a laterally extensive area and because the gas is likely to be located in discrete fractures, the response to pressures in excess of lithostatic may well be dilatation and possibly interconnection of the existing fractures to whatever degree is necessary to allow gas flow outward in order to relieve gas pressure in the room. In other words, if room pressure were to reach levels in excess of lithostatic, it is not a question of propagating a new fracture of unknown orientation outward from a disposal room, but rather, of dilatation and interconnection of pre-existing fractures, causing a temporary increase in permeability that enhances outward flow of gas.

I think that this scenario is worth exploring further and I would suggest that the coupled gas/closure calculations reported in the Weatherby et al. 3-28-90 memo would be a logical place to start. These calculations focused on a crack extending laterally from the room. However, the crack was only considered permeable to gas over the first meter and was considered to have zere permeability to gas beyond that. As a first cut, it might make sense to work this same problem with the crack that is permeable to aas over its entire lensth. The critical process to monitor in this type of simulation will be the dilatation of the fracture.

One final note of caution. These simulations are preliminary. We have a number of important model components that have not yet been implemented and we have a large amount of sensitivity work to do. Therefore, the results should be viewed from the standpoint of the processes and system behavior that they suggest. These results should not be taken as a quantitative prediction of specific brine and gas flow rates, gas pressures, etc.; nor are they appropriate input for performance assessment modeling. The reason for communicating these results at this relatively early stage is that they may have fundamental, conceptual implications about the nature of interbed pressurization and the nature of gas release under high pressure conditions.

\section{REFERENCES}

Borns, D.J., 1985, Marker Bed 139 - A Study of Drillcore from a Systematic Array: Sandia National Laboratories, SAND85-0023.

Weatherby, J.R., Arguello, J.G., and Stone, C.M., 1989. The Effect of Gas Generation on the Performance of CH-TRU Disposal Rooms: Memorandum to B.M. Butcher and D.E. Munson, November 14, 1989.

Weatherby, J.R., and Arguello, J.G., 1990, The Effect of Gas Generation on the Extension of Preexisting Fractures Around WIPP Disposal Rooms: Memorandum to B.M. Butcher and D.E. Munson, March 28, 1990. 
cc: W.D. Weart (6340)

E.D. Gorham (6344)

A.R. Lappin (6345) 
Figure 1.

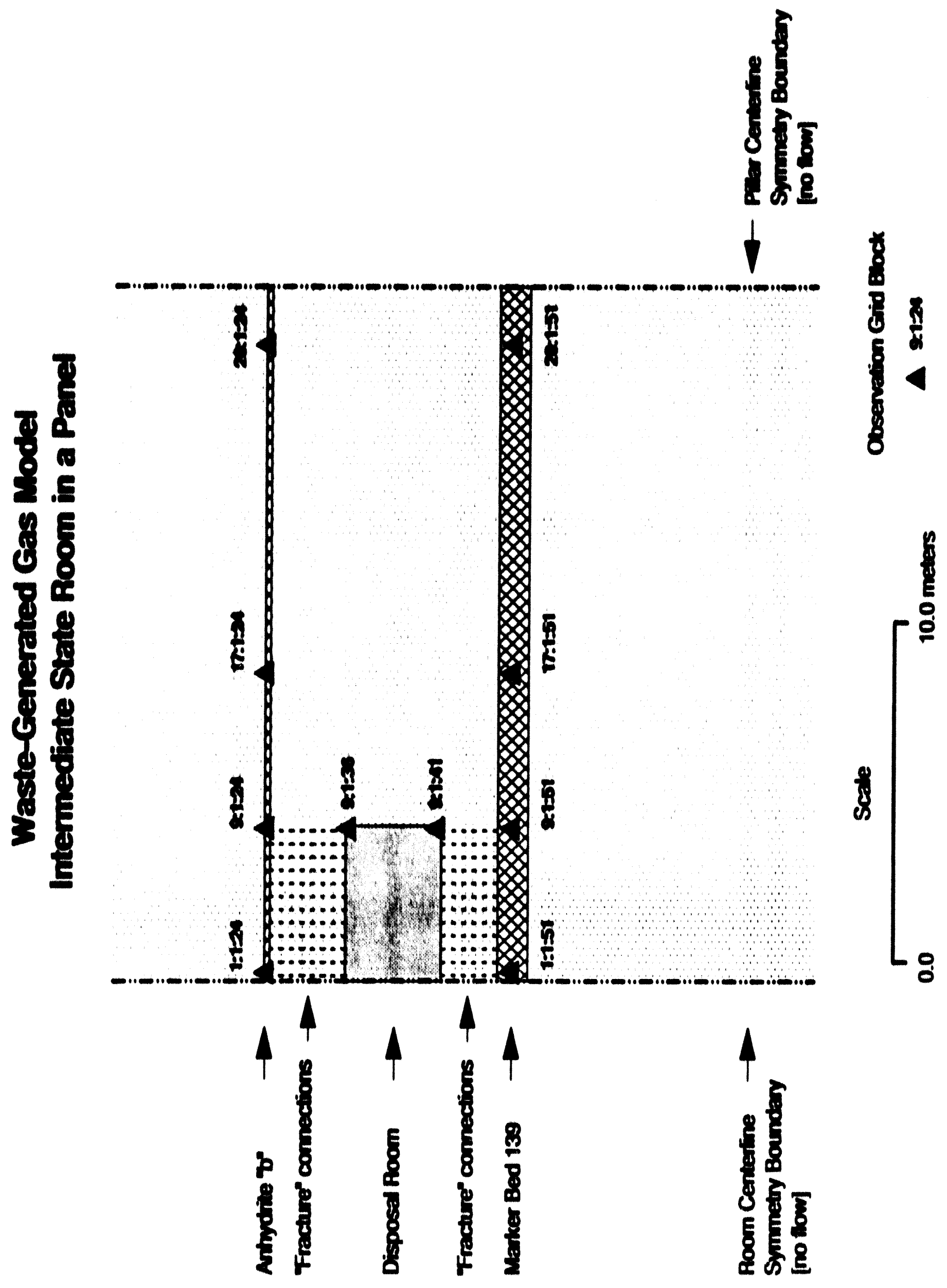


Figure 2.

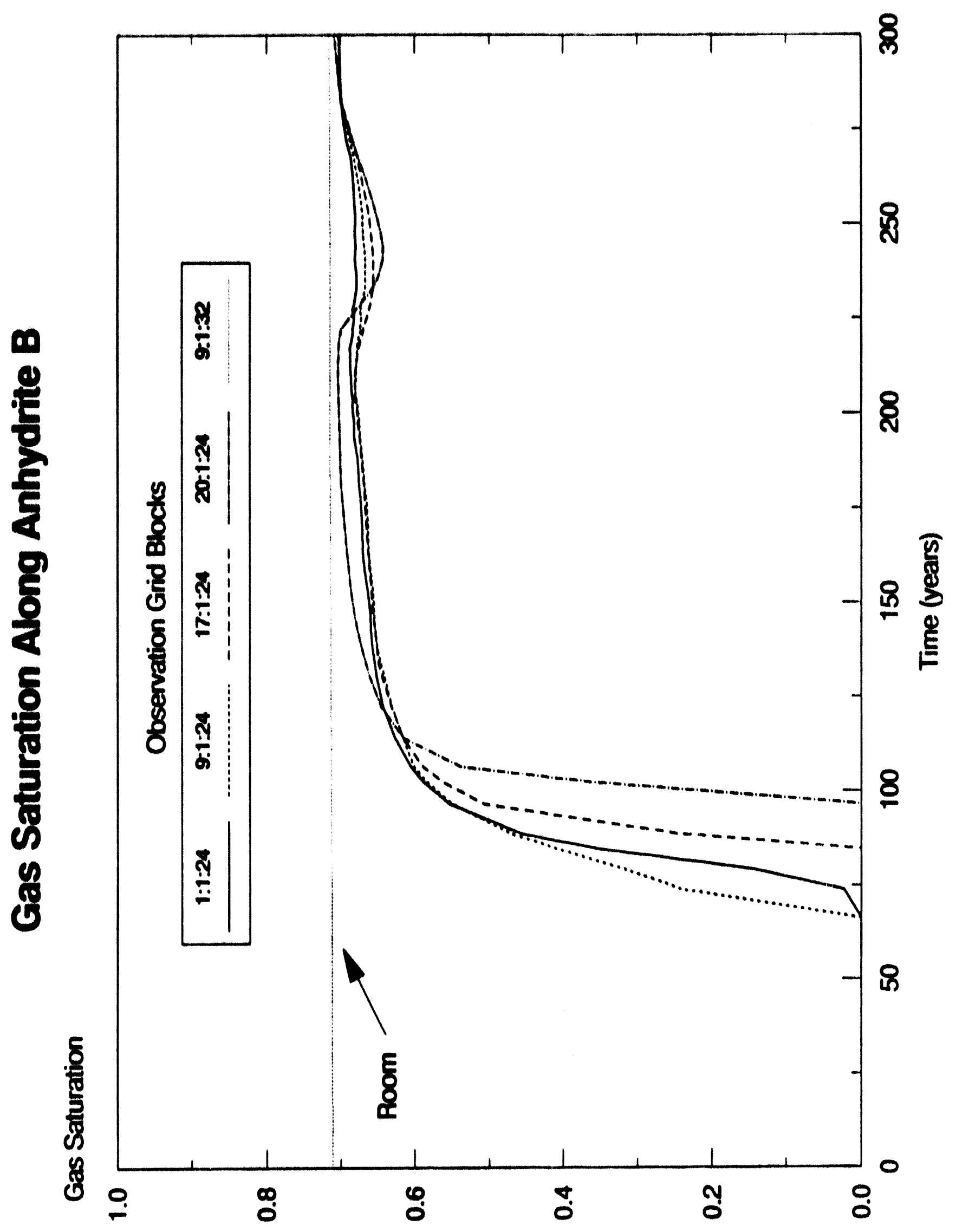


Figure 3.

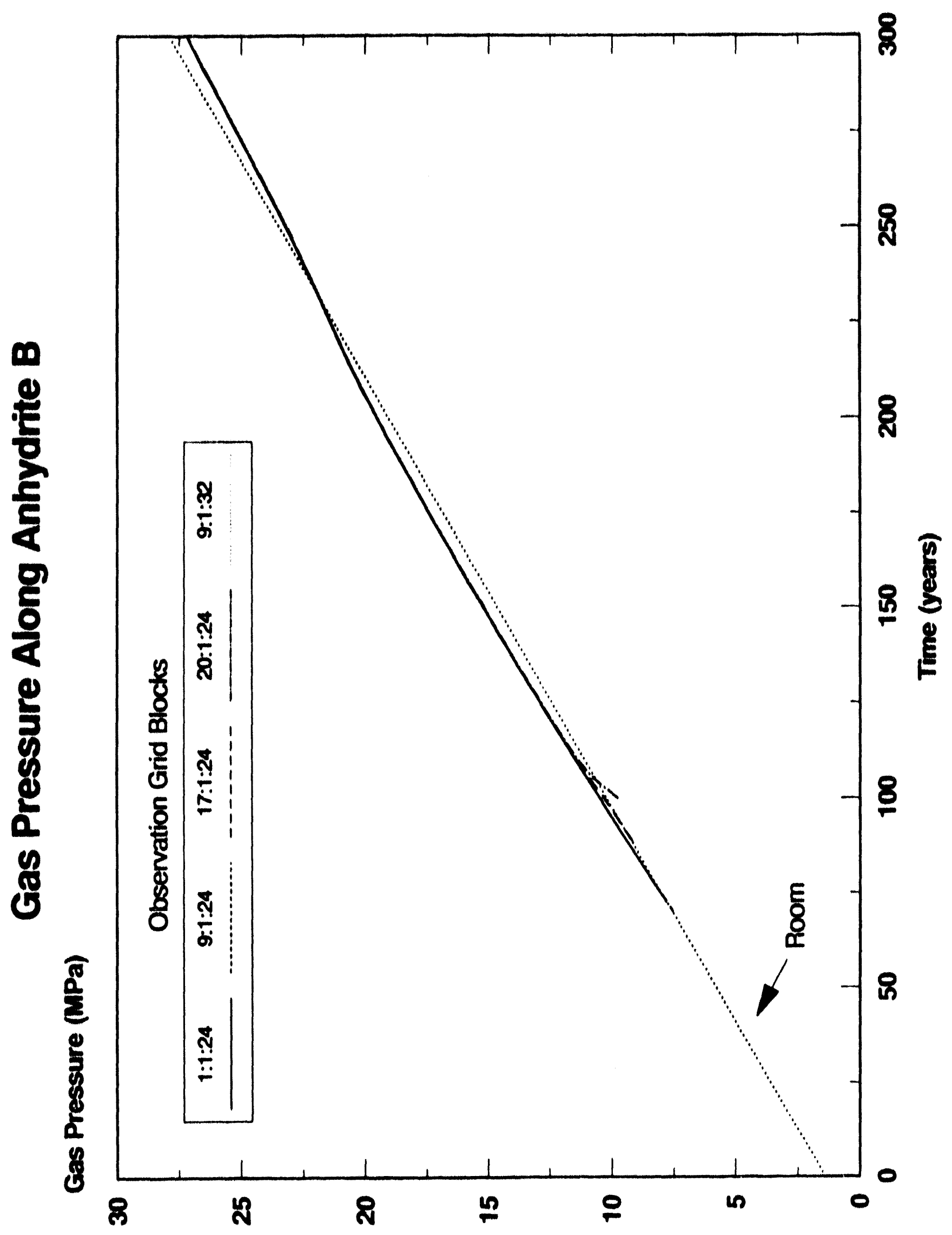




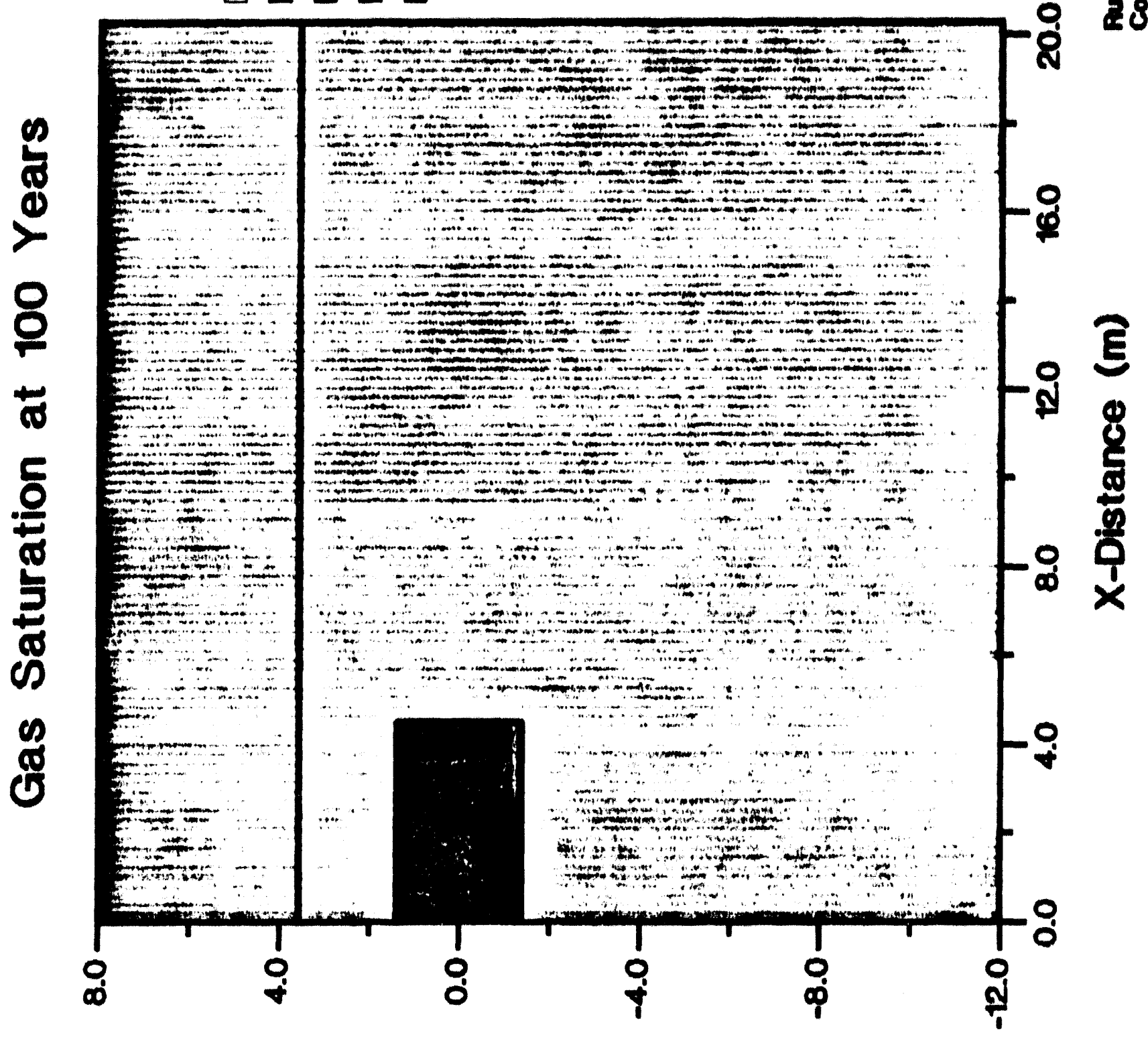

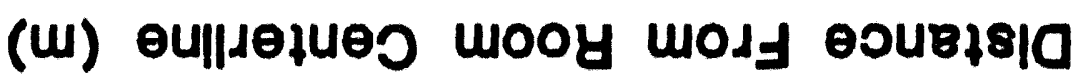




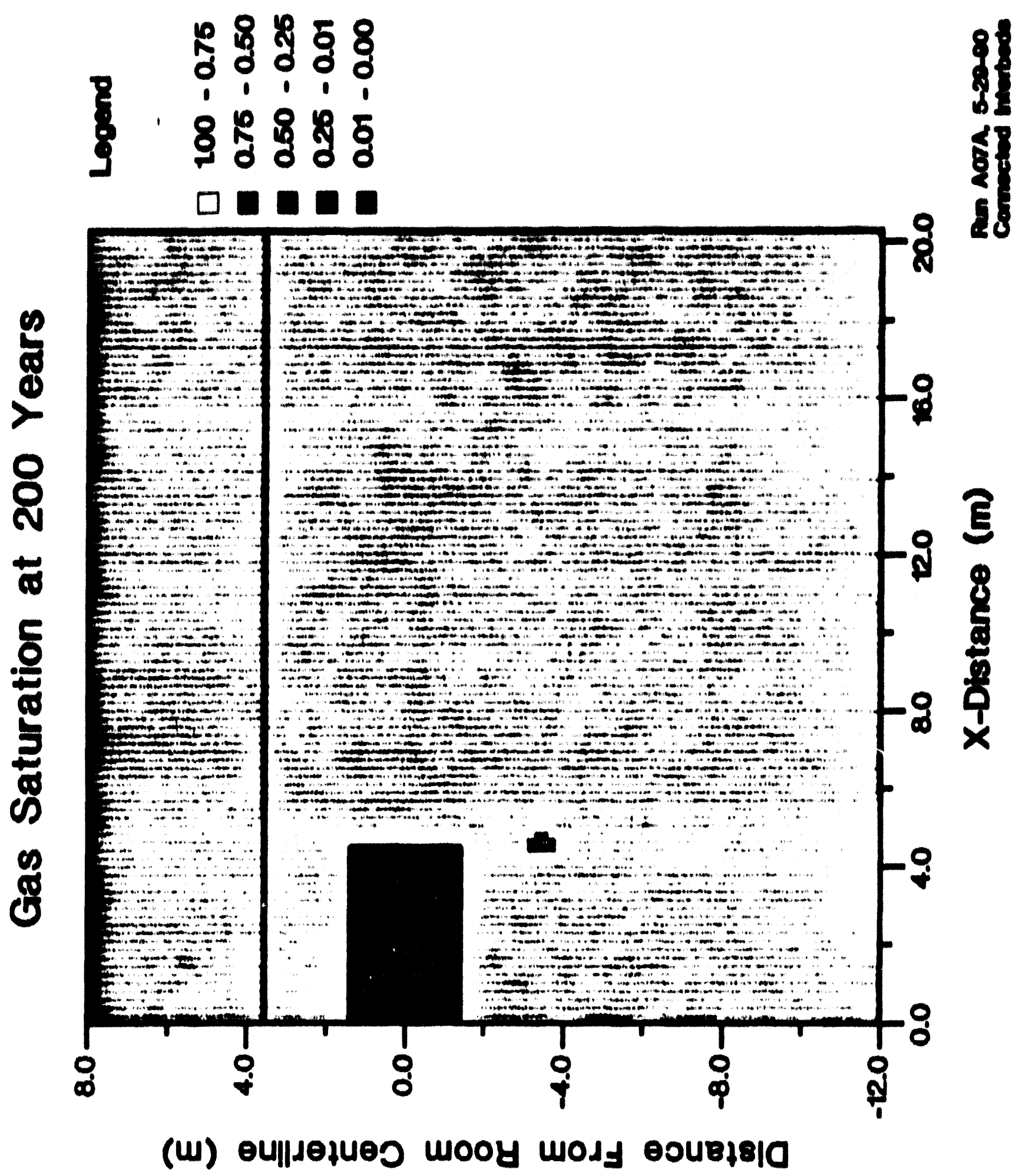




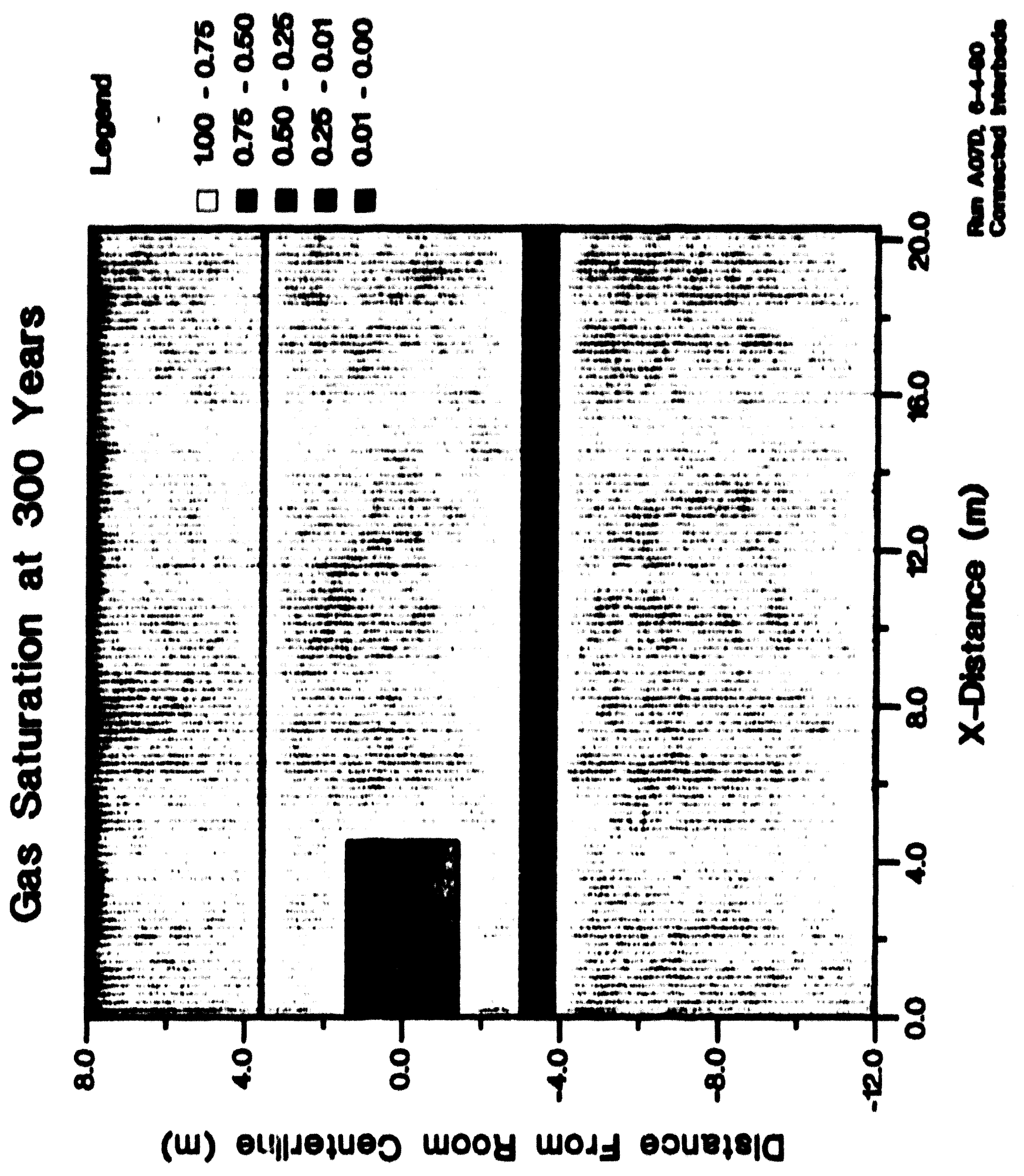


Figure 7.

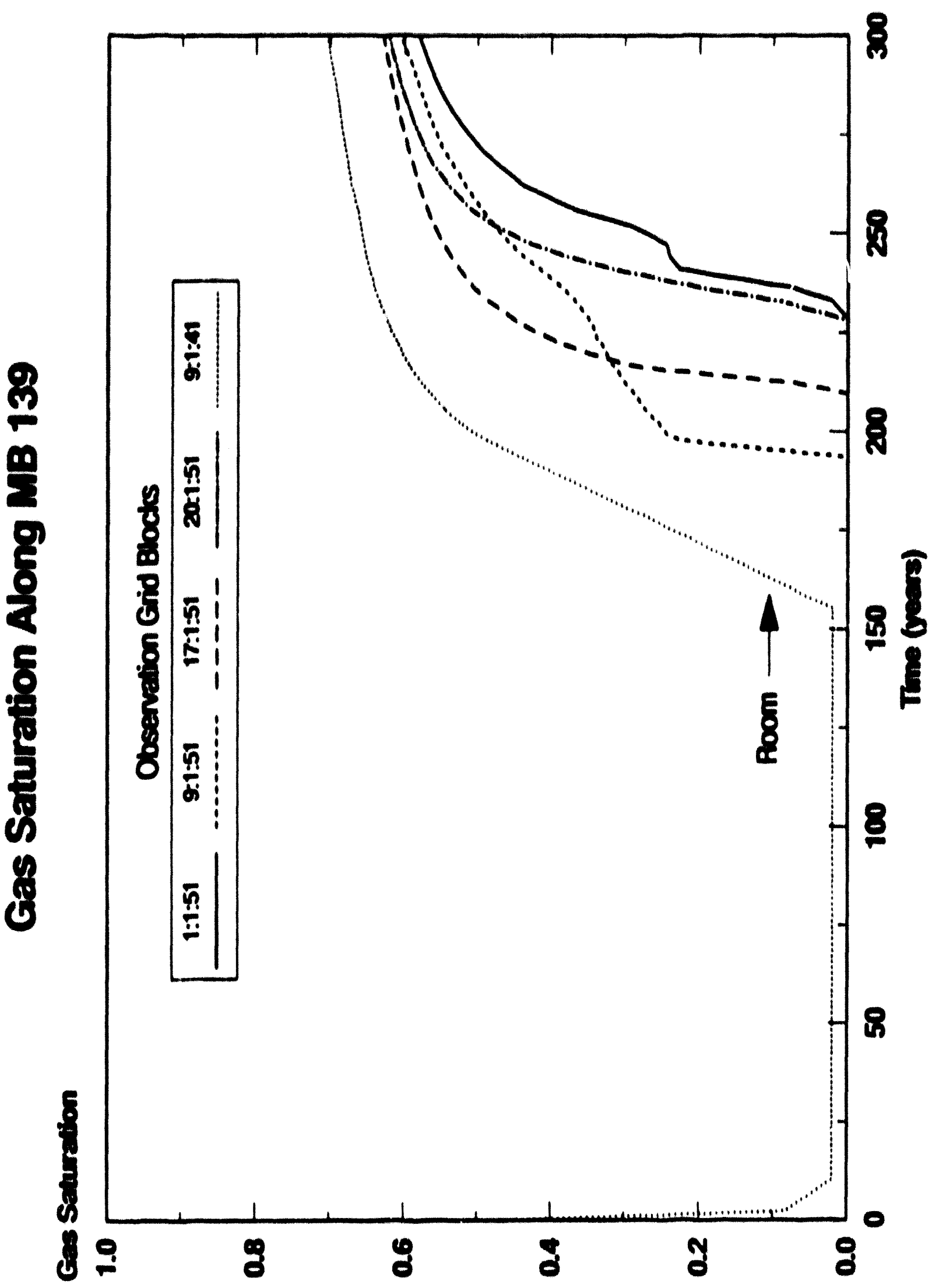


Figure 8.

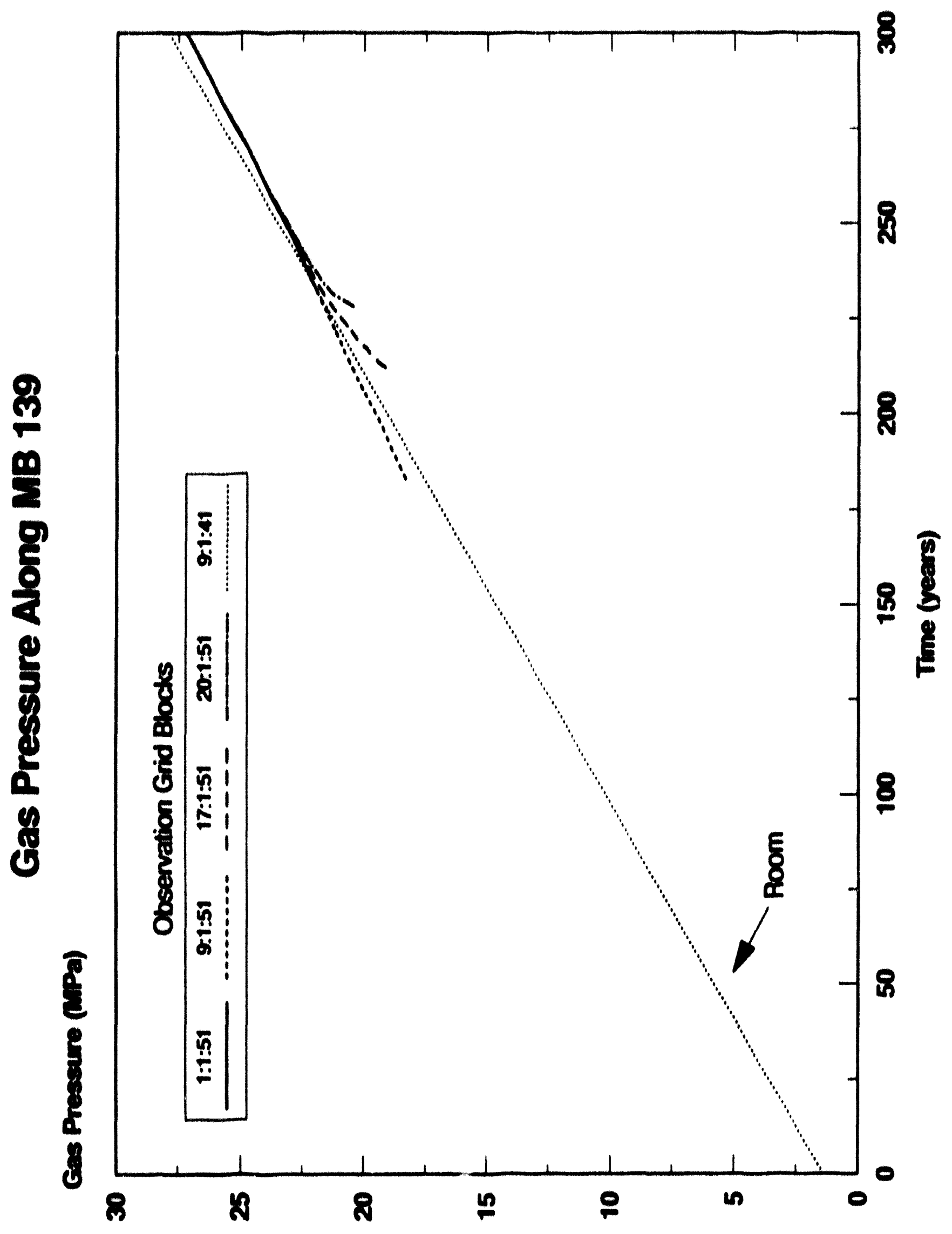


-

$$
\text { A. } 42
$$




\title{
Sandia National Laboratories
}

\author{
Albuquerque New Mencen $8718 \mathrm{~s}$
}

Date: October 4, 1990

To:

Fred Mendenhall (6345)

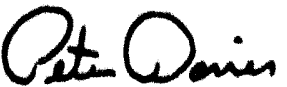

From: Peter Davies (6344)

Subject: Firat Fluid Pressurization Data Set for Interbed/Fracture Dilatation Calculations

Attached is the firat fluid pressurization data set (hard copy and noppy) for input to the preliminary interbed/fracture dilatation calculation. This data set is for an interbed overlyins a weste disposal room assuming fixed "inuadated" gas generation rates, so intermediate room closure state, and IE-19 $m 2$ permenbility in the interbeds. The configuration of this simulation is shown schematically in Figure 1. This calculation was used as the "oxample" simulation in my September 19 th presontation to the NAS WIPP panel and the handout from that presentation contains a sulte of plots showlas the overall 2-phase now behavior. Figures 2 and 3 of this memo show the ges saturation history and the gas pressure history (respectively) for a group of observation srid blocks alons the upper interbed. Table I contains a listing of principle essumptions, information about the location of observation srid blocks, and a large table with sas saturation, ges pressure, and bribe pressure.

There are two potentially important differences between the curreat geometry of the 1520 frncture model and the 2-phuse flow model used to senernte the fluid pressure laformation: 1) In the fracture model, the horizontal frecture/interbed directly intersects the room at its midpoint, whereas in the 2-phase flow model, the interbed of interest is located 2.1 meters above the room. However, in the 2-phase now model, the interbed is connected to the room by high transmissibility coanections that represent disturbed-rock-zone fracturing, rock bolting, and/or vertical boraholes. 2) In the fracture model there is only one fracture/interbed, wheress in the 2-phase Mow model there are two interbeds and gas flow into the lower interbed reduces that amount of gas setting into the overlying interbed.

For future simulations, I think that we will need to use a finer grid in the vicinity of the mignating gas front and that we will need to record the pertinent anturation and pressure information at many more blocks. While this will require more effort than the present simulations, I see no problem in being able to run this more refined grid.

While there are some differences model configuration, I think that this data sot will be sufficient to set the mechenical component of this interbed/frecture dilatation analysis through a preliminary round of calculations. In future iterations, I think that we can configure both the 2-phese flow and seomechanical models to provide a tightly coordinated analysis. 
Table 1

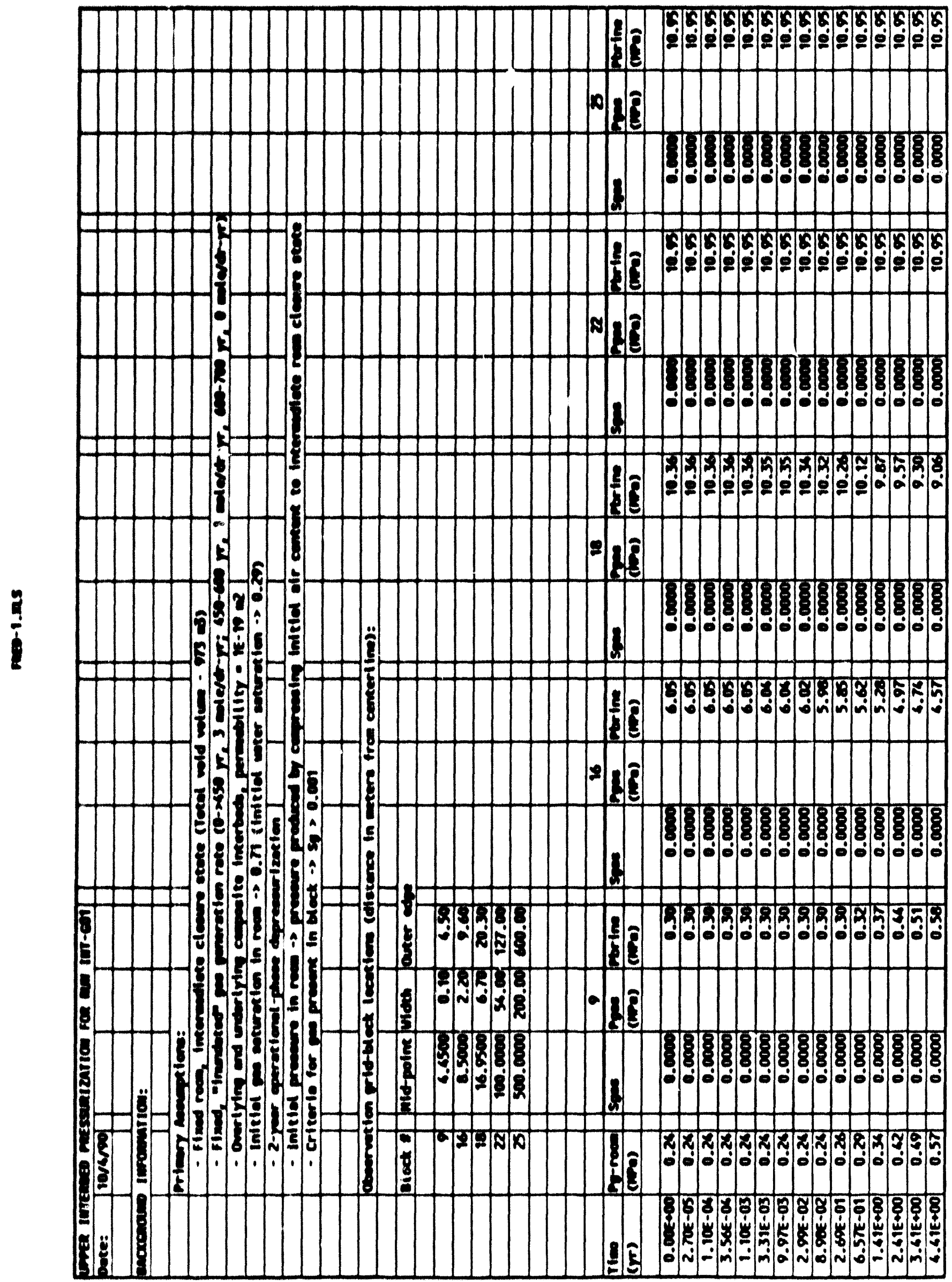




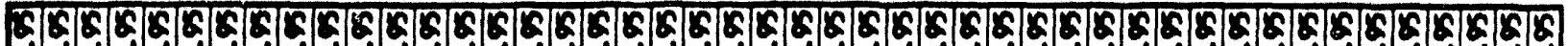

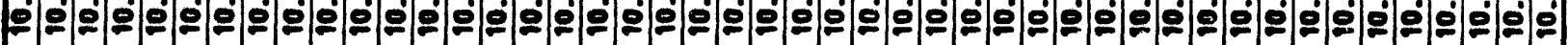

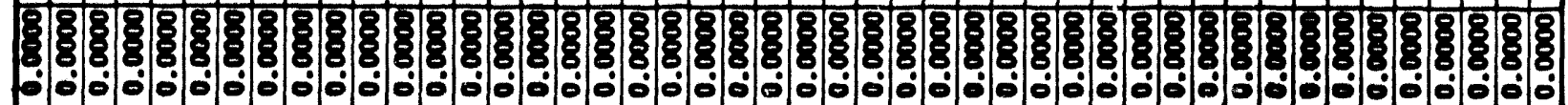

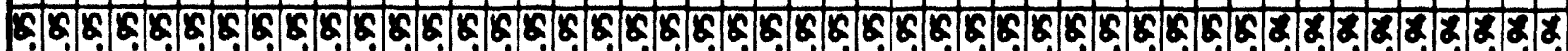

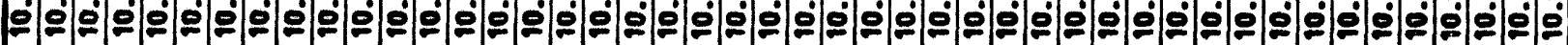

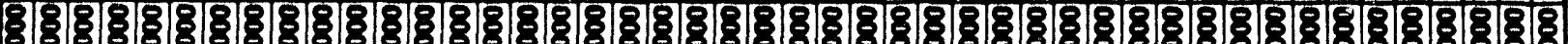

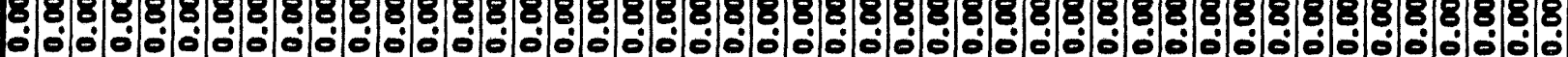

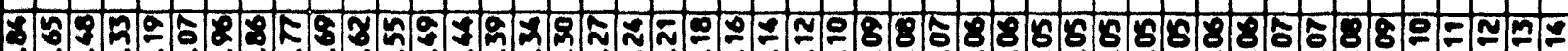

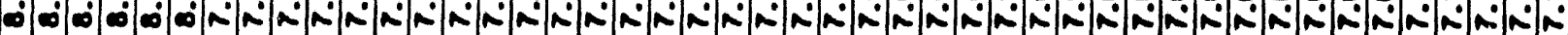

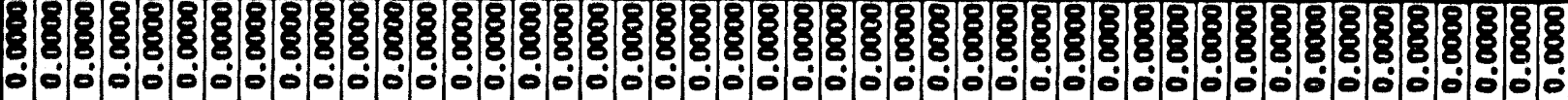

5:ER

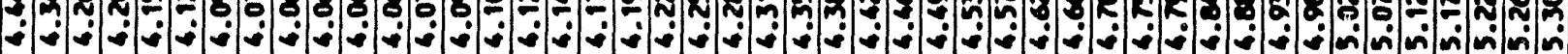

88 ํํำ -

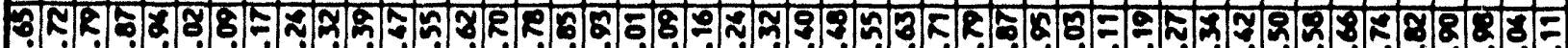

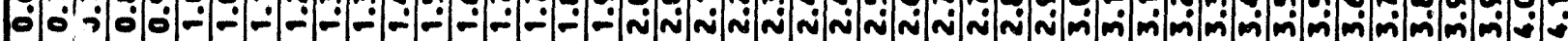

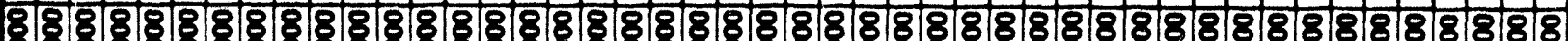

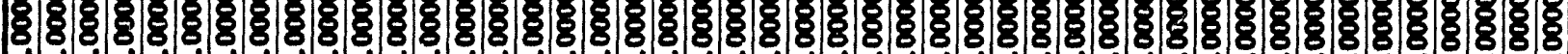
ó

3.

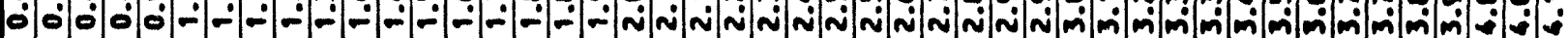

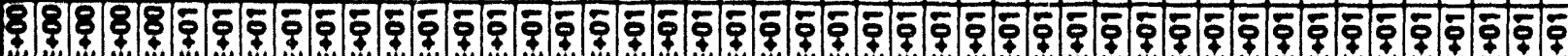

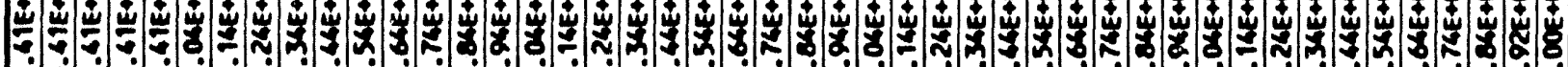

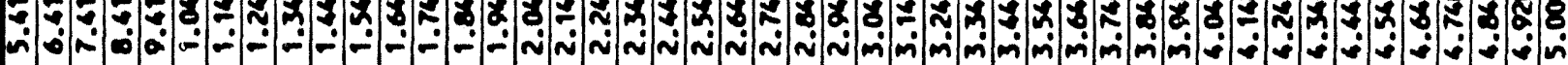




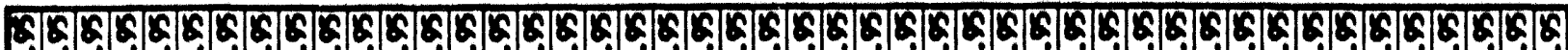

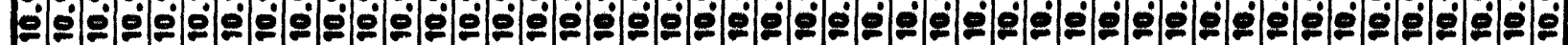

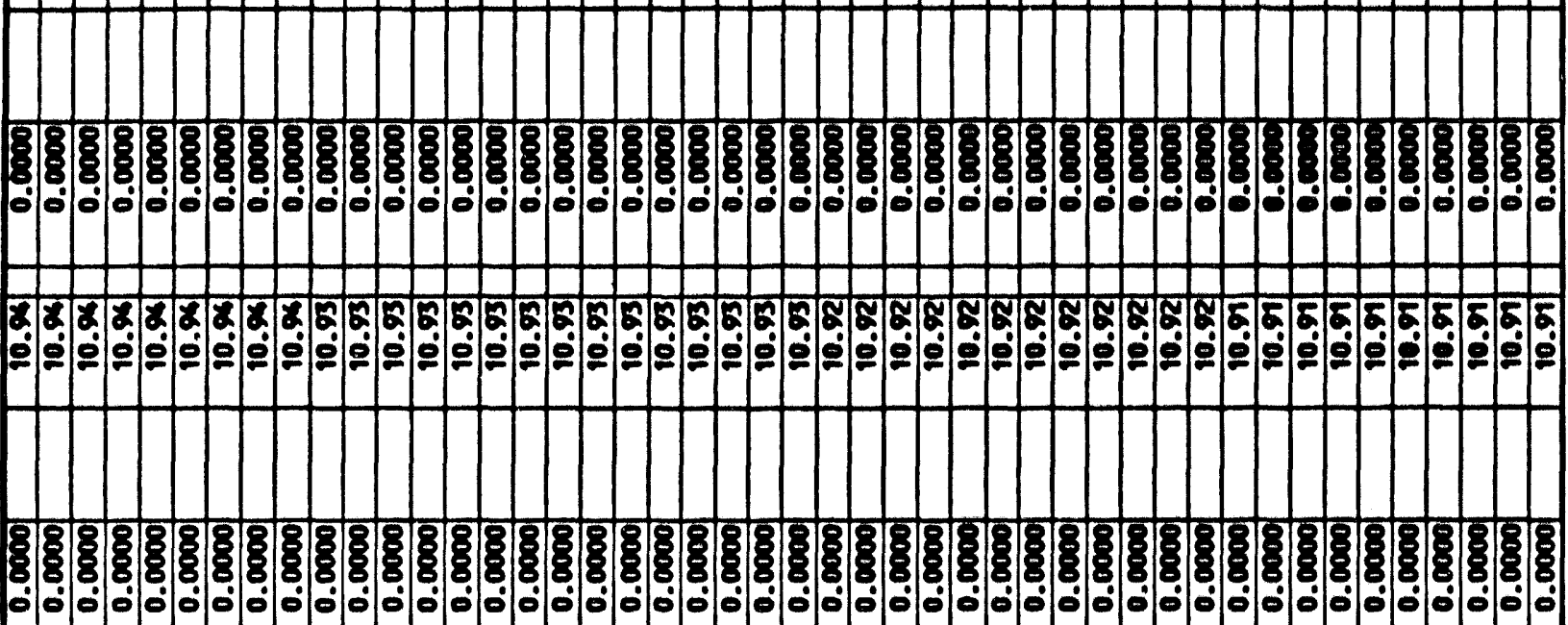

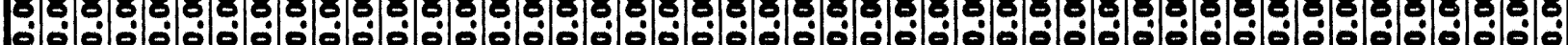

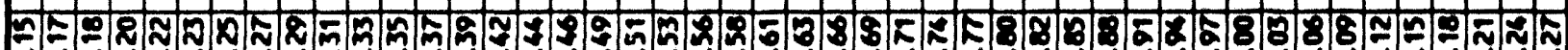

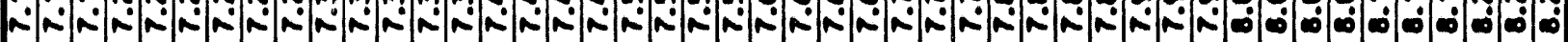



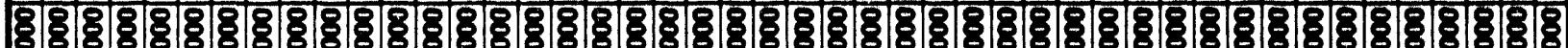

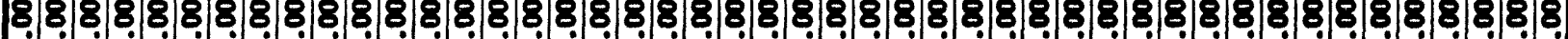

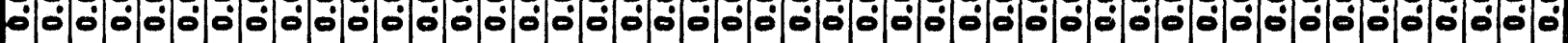

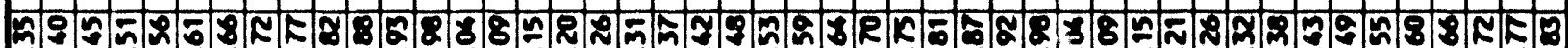

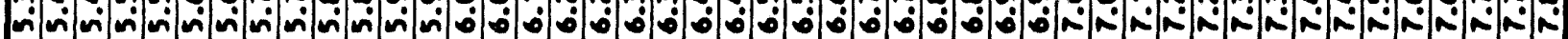

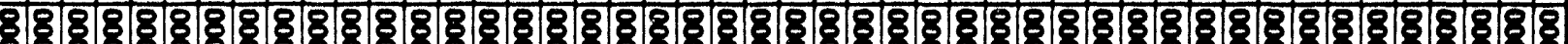
8.8

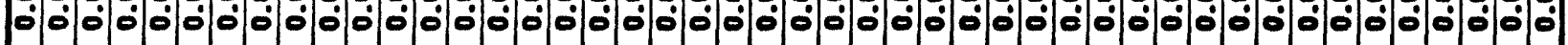

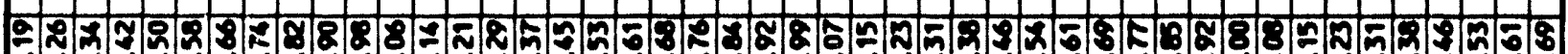

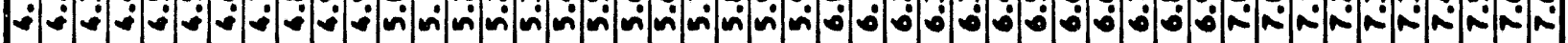

קo

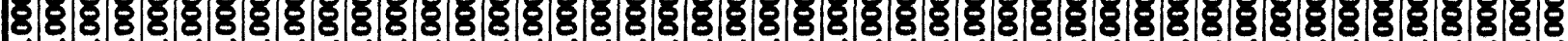
-

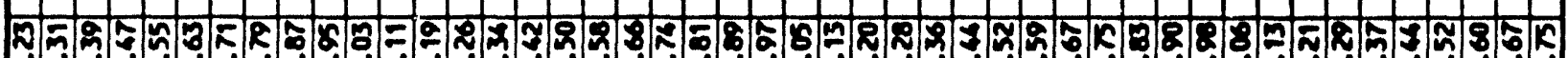

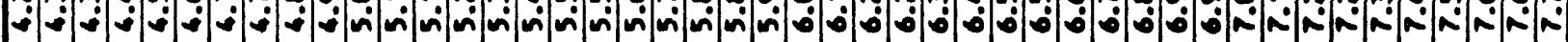

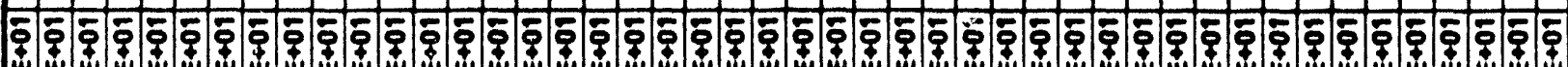

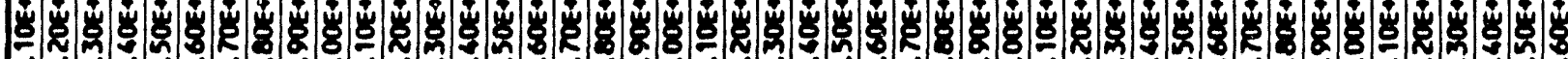

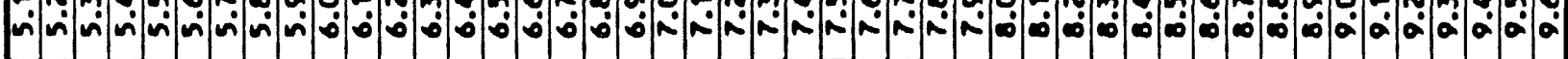




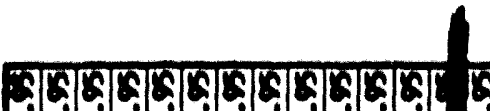
6)

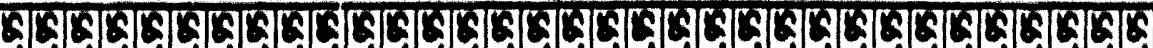
1000

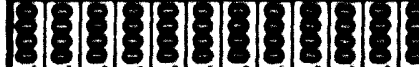

อ่

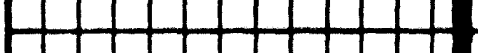

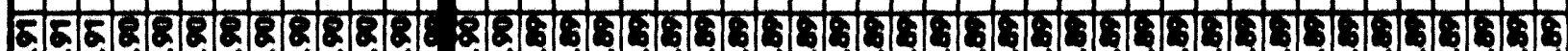

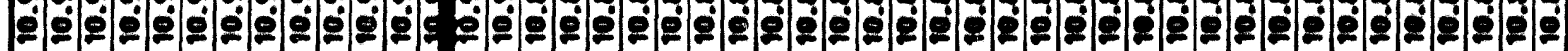

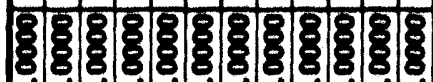
○ी

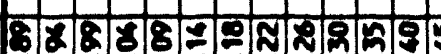

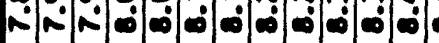

Вहो-

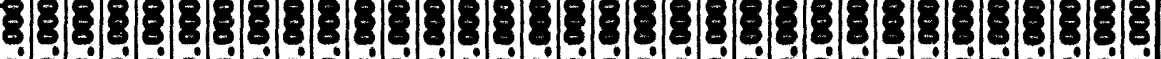

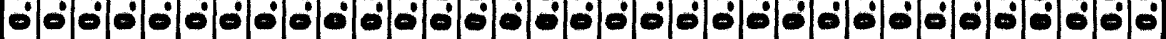

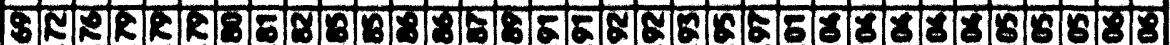

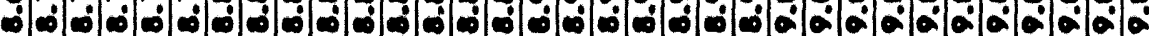

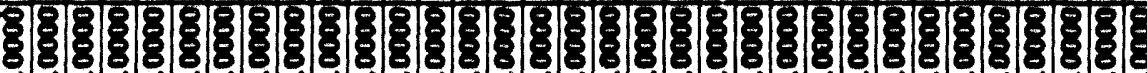 ○}

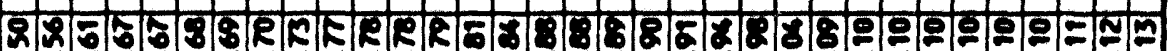

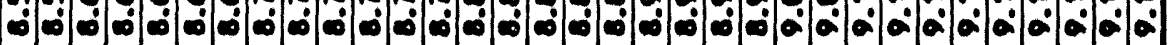

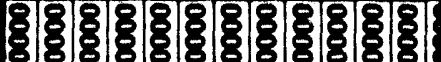
○

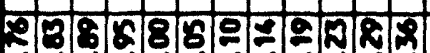

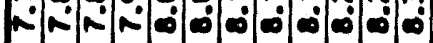

W

8 ○ी

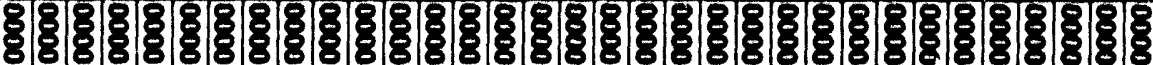

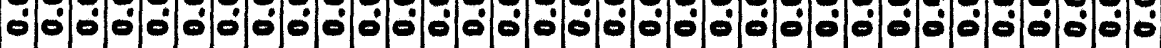

ORER

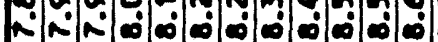

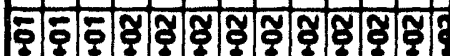

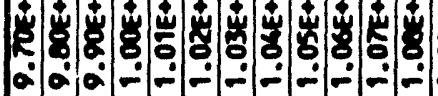

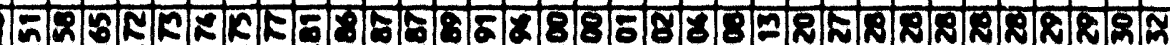

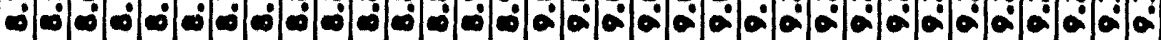

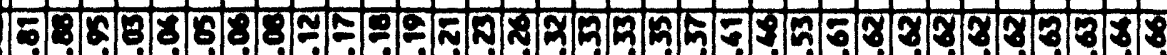

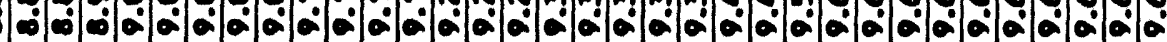

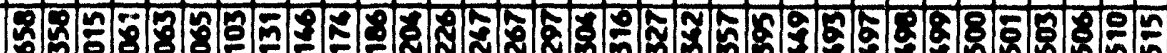

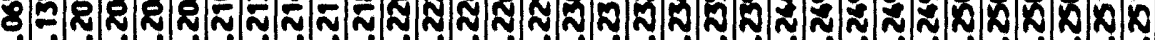

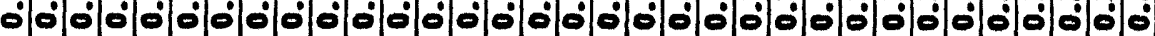

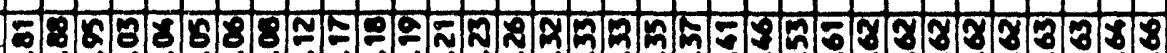

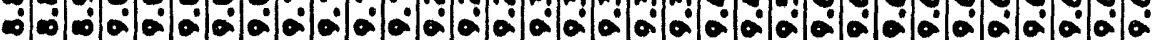

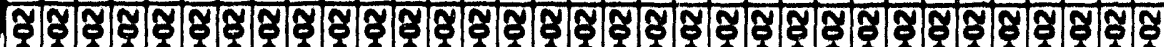

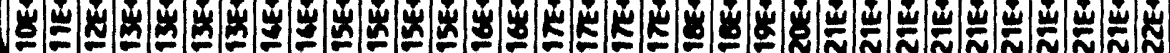
$\div \div \div \div \div \div \div \div=0 \%$ 


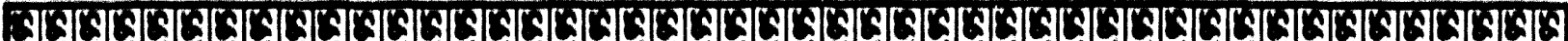
6 id

(2)

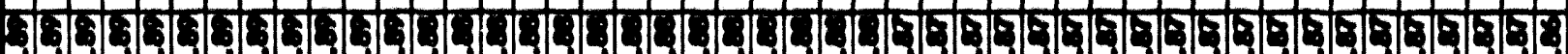

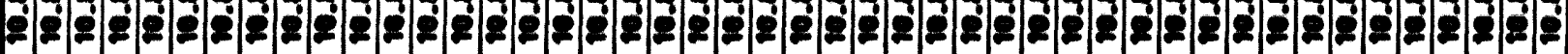

30 (5) S-5)

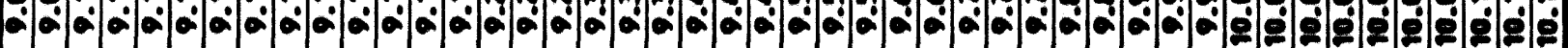

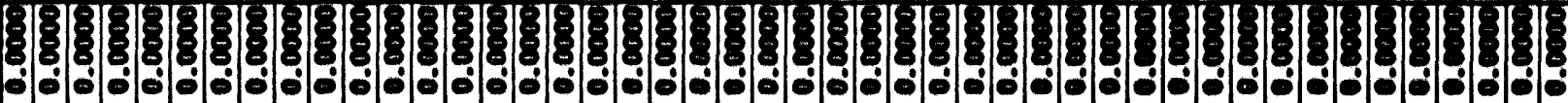

n-

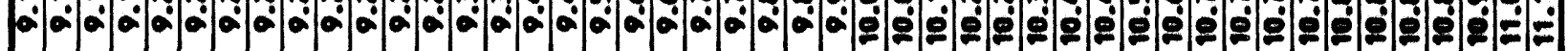
T.

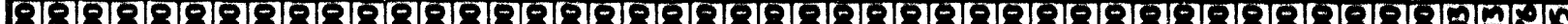

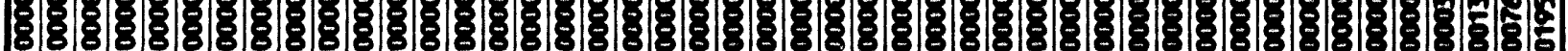

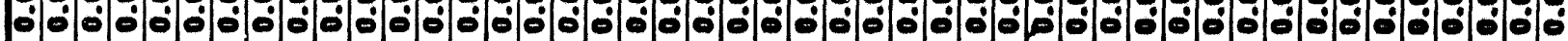

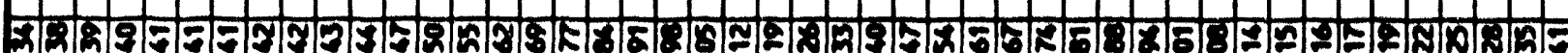

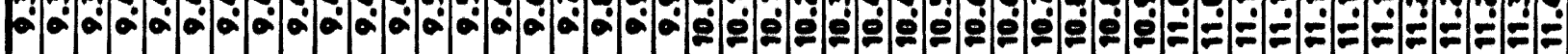

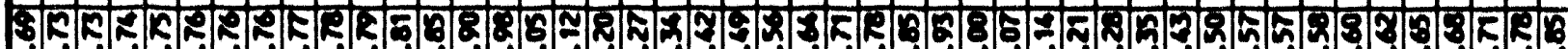

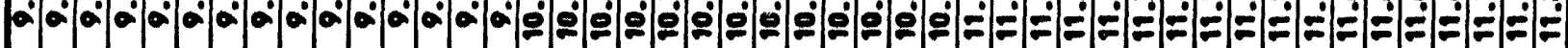

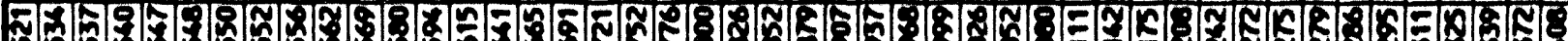

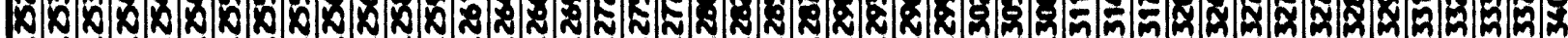

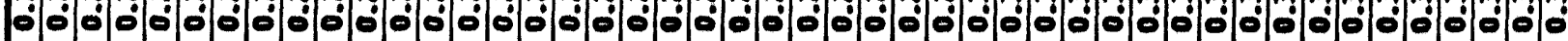

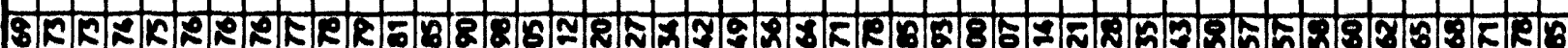

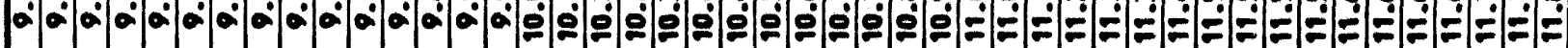

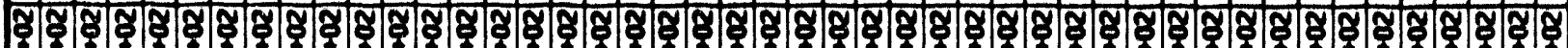

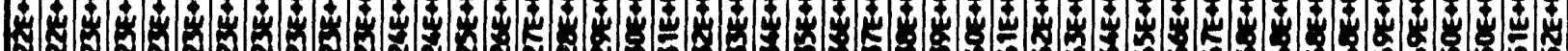
N̦ N 


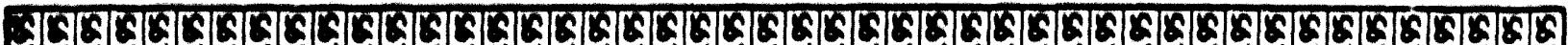

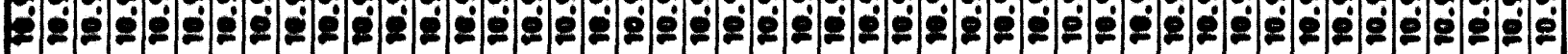

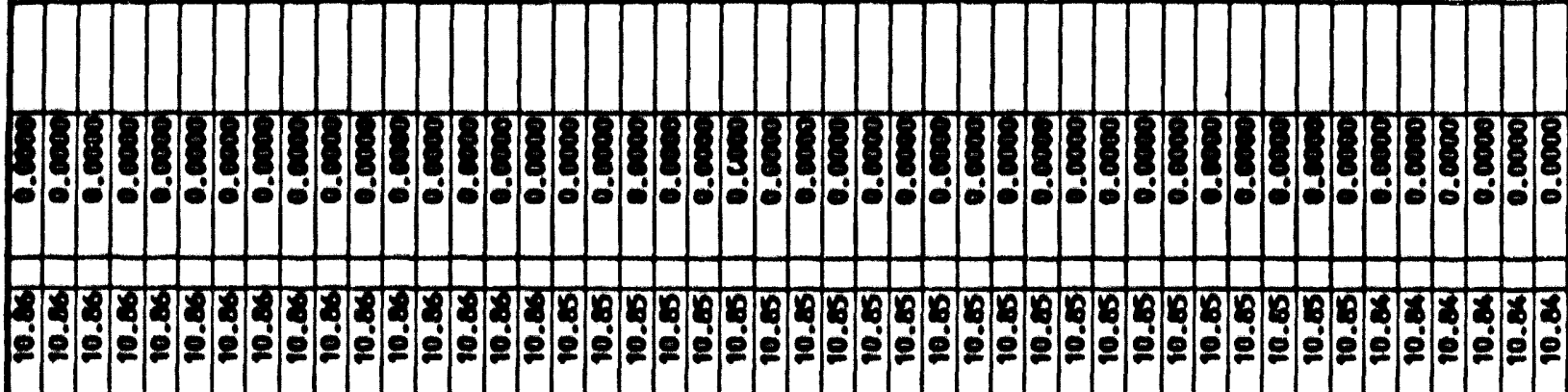

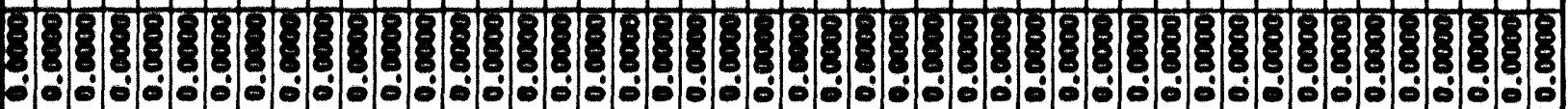

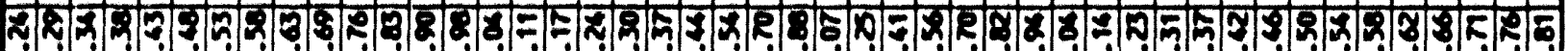

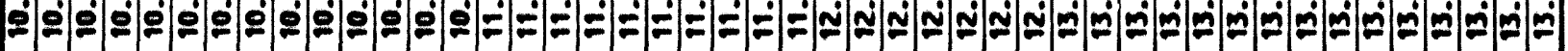

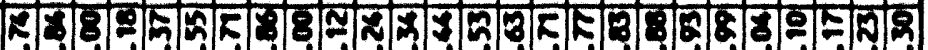

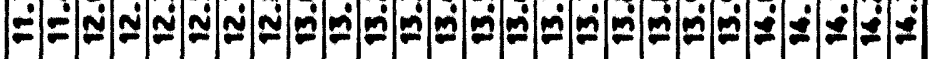

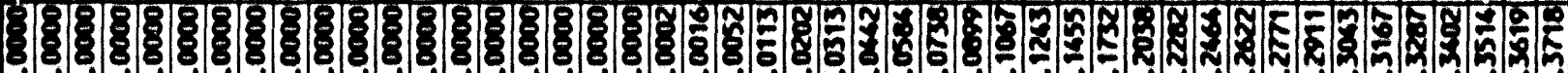

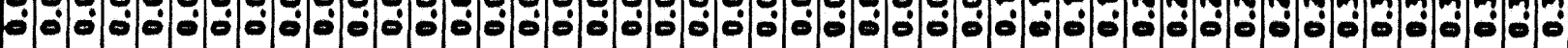

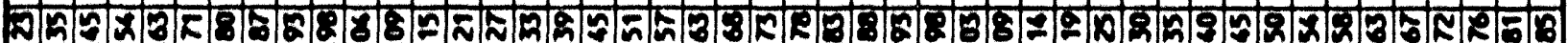

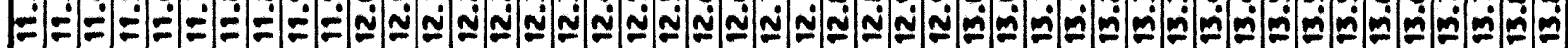

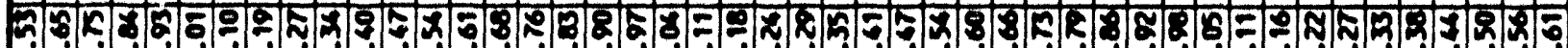

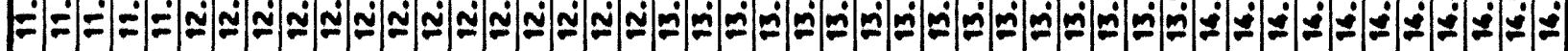

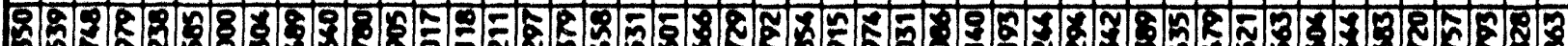

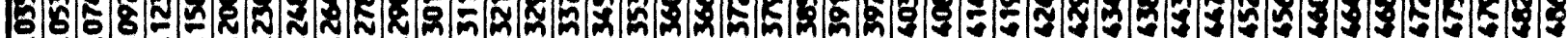

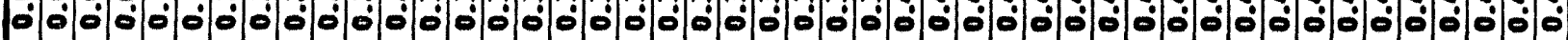

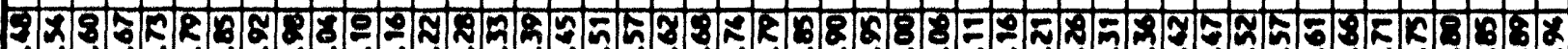

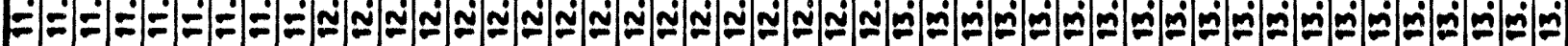

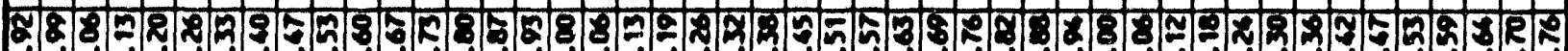

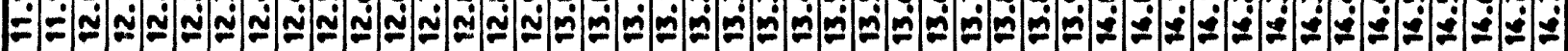

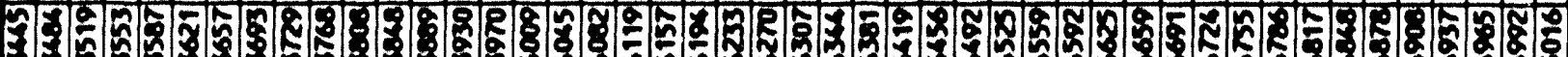

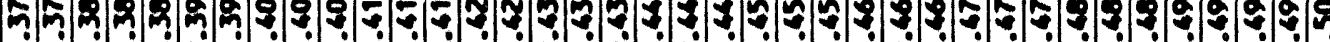

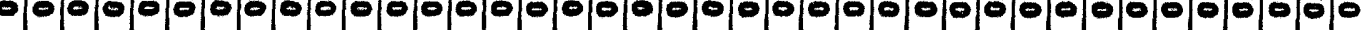

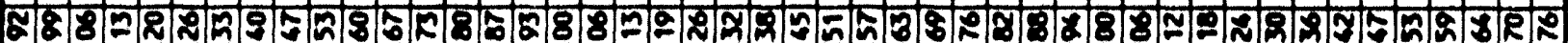

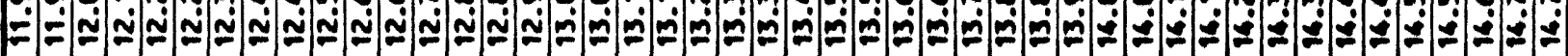

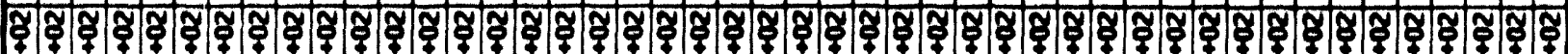

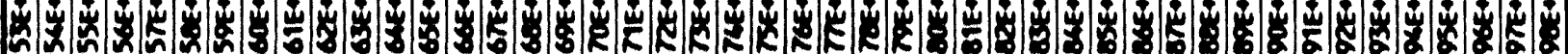

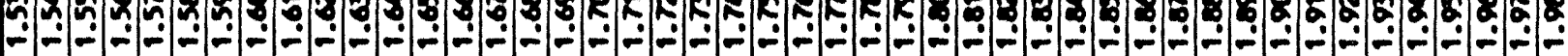




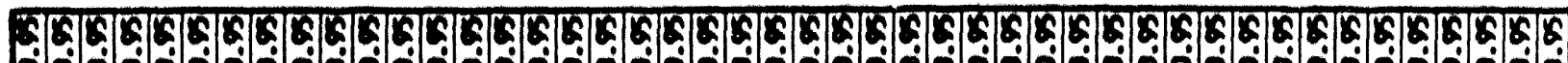

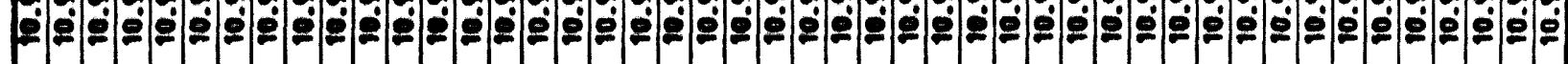

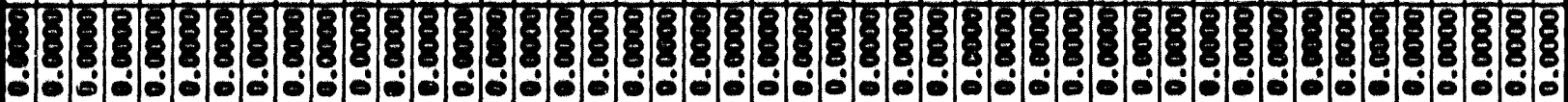

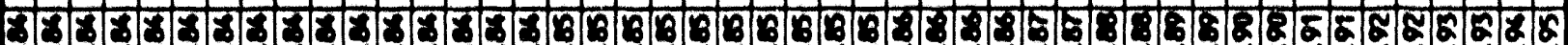

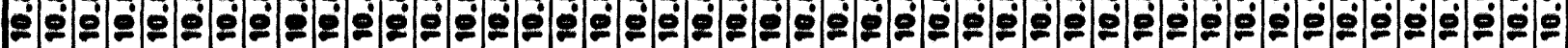

8.8

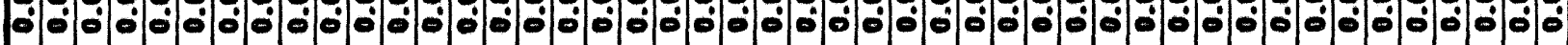

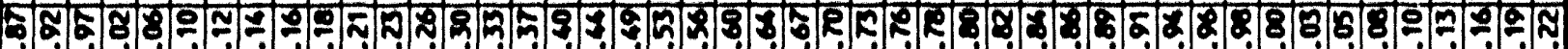

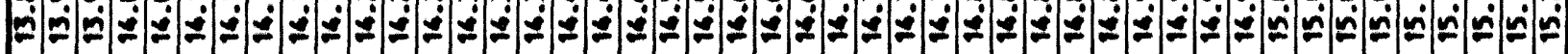

-

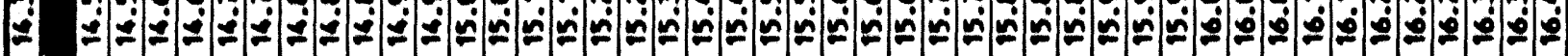

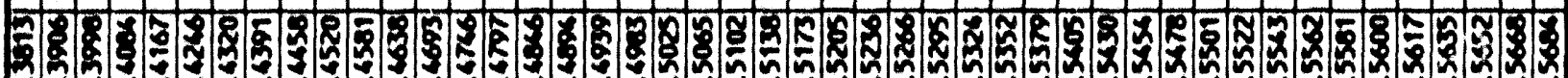

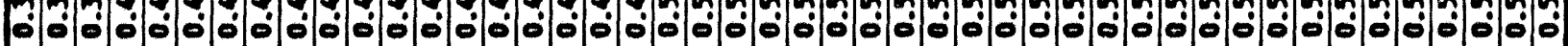

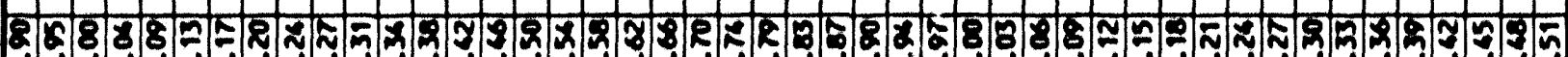

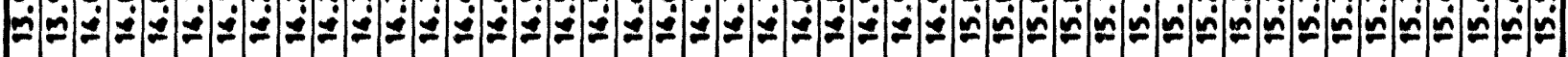

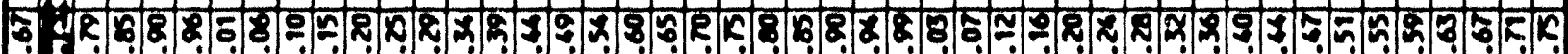

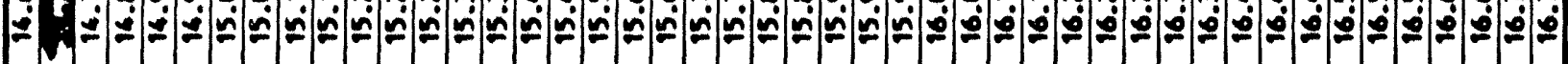

3.

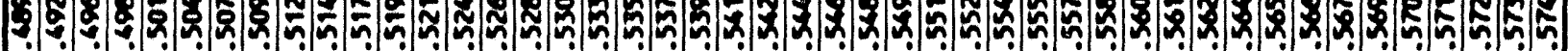

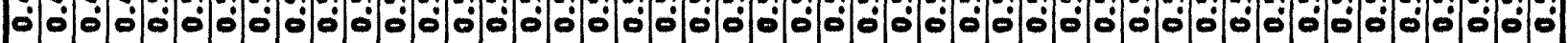

20

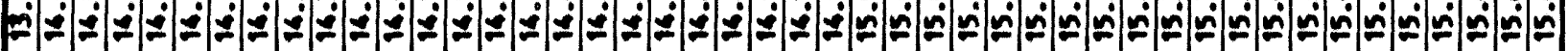

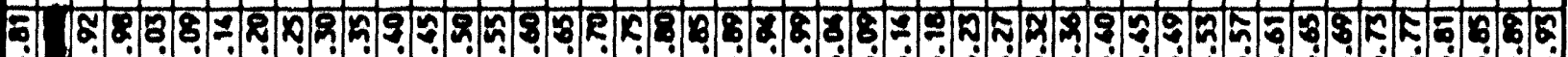

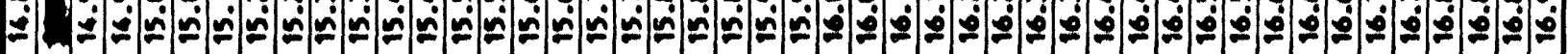

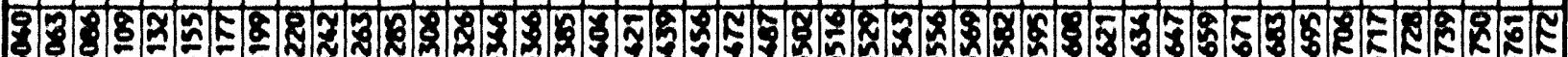

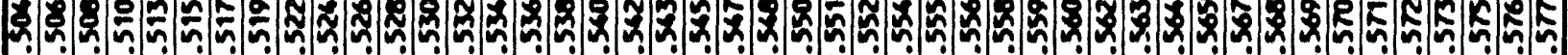

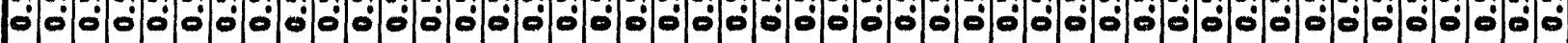

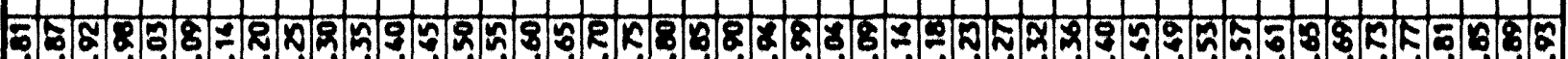

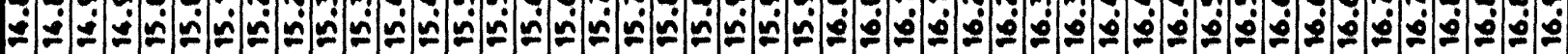

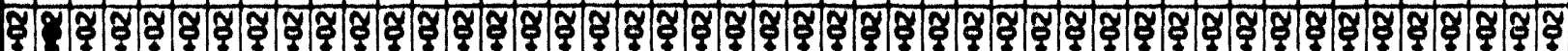
8. 


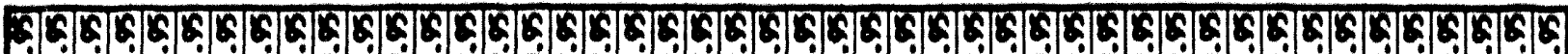

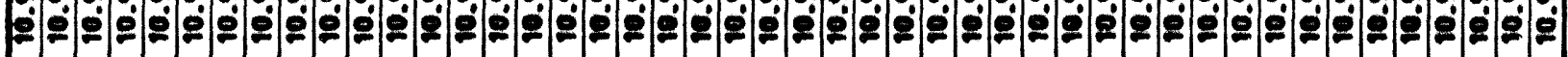

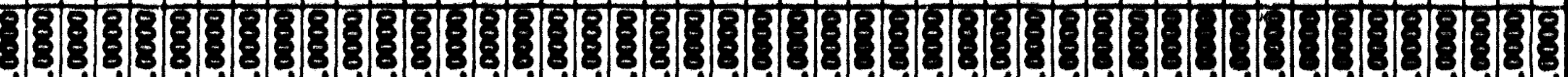

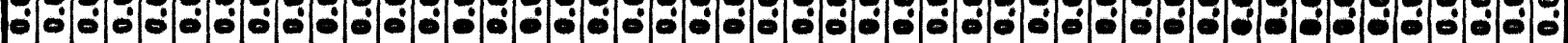

\&20

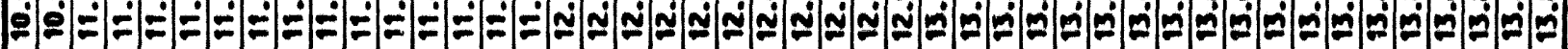

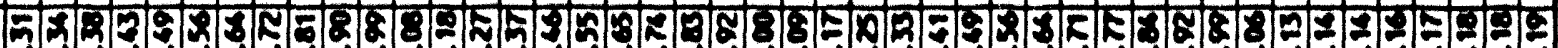

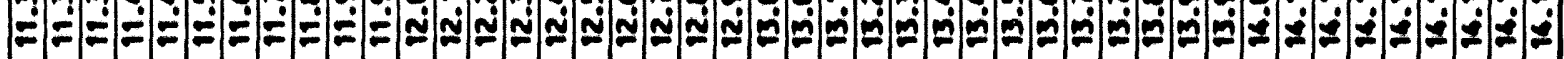

䒹

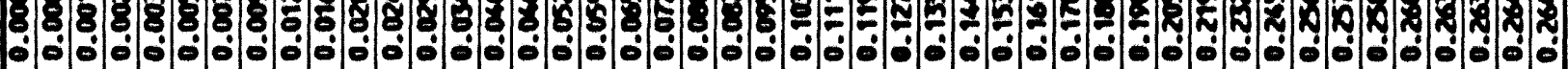

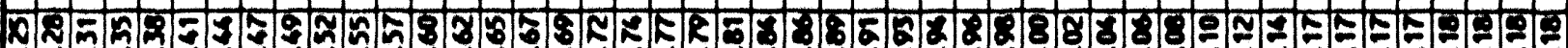

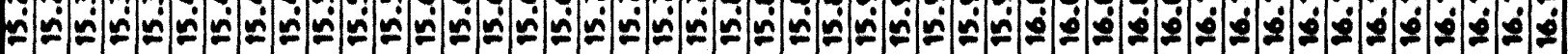

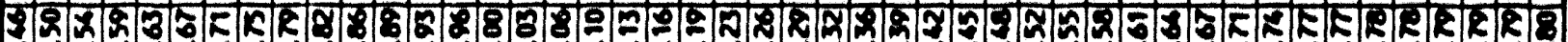

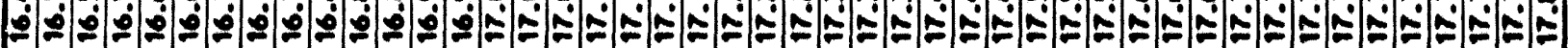

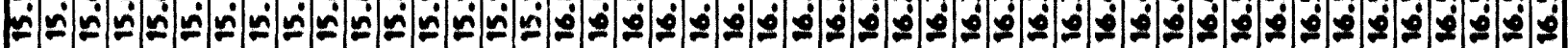

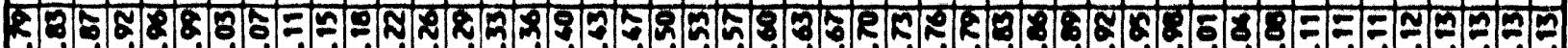

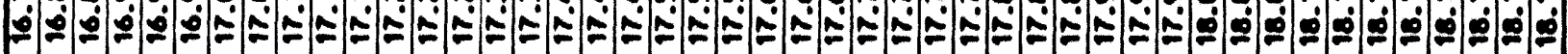

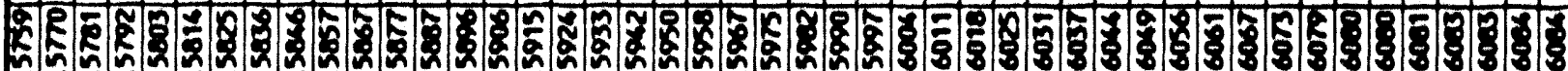

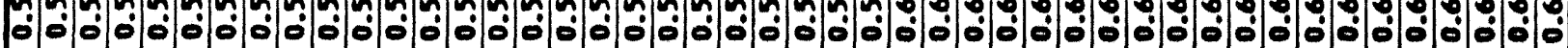

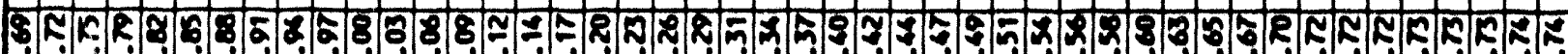

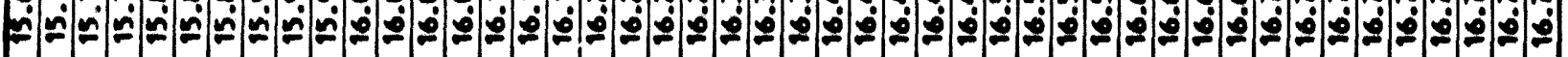

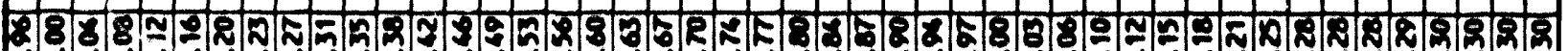

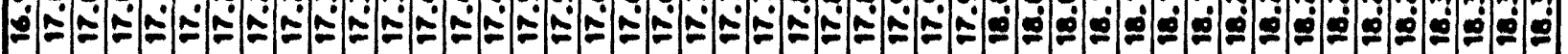
A

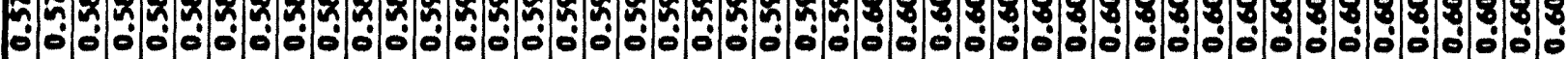

-88 8 -

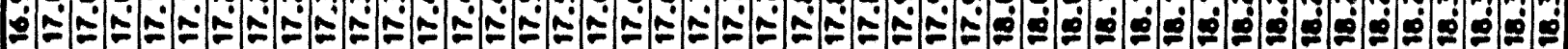

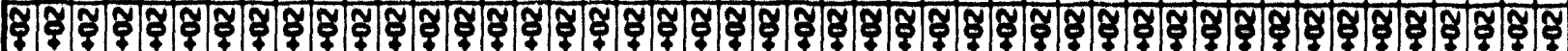

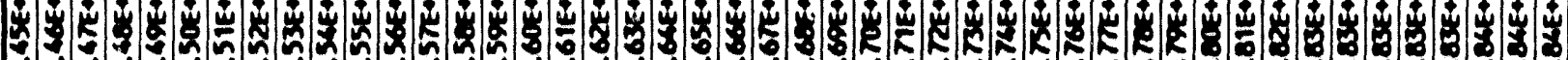

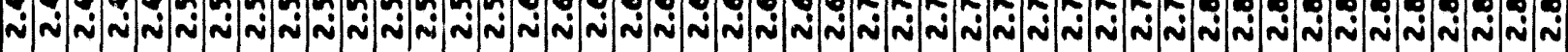




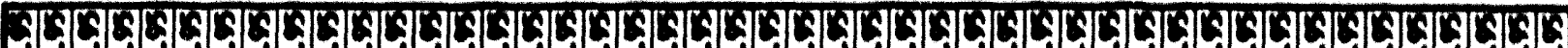

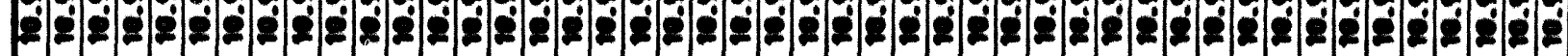

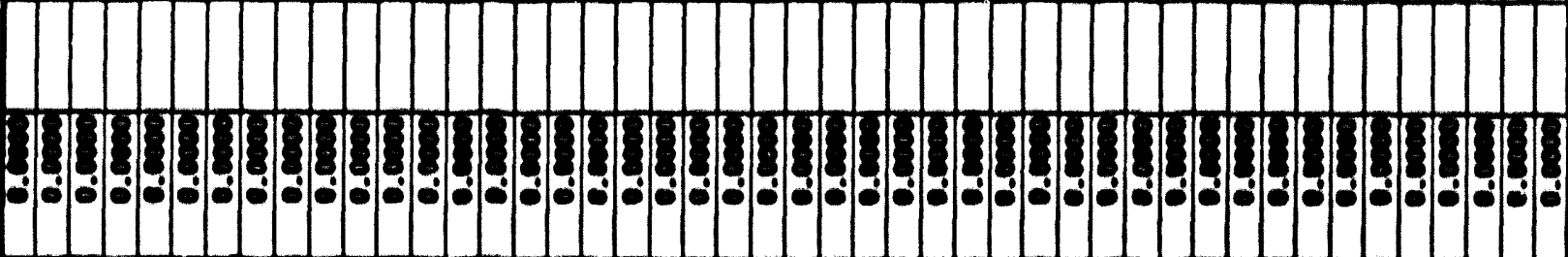

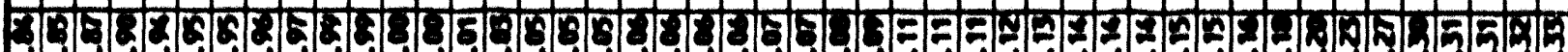

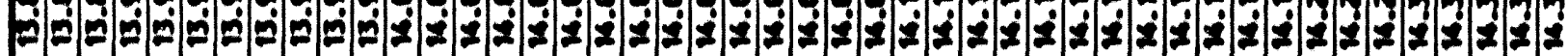

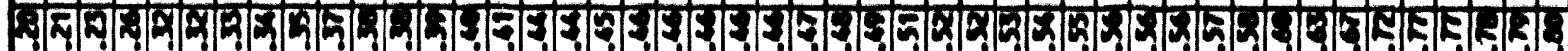

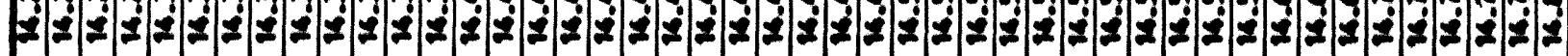

- E E

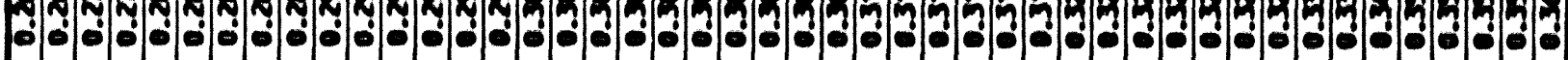

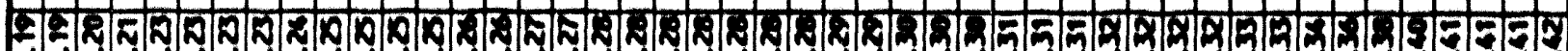

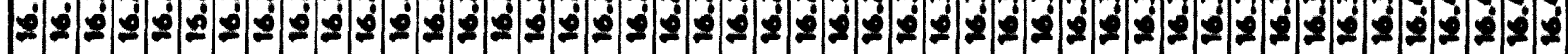

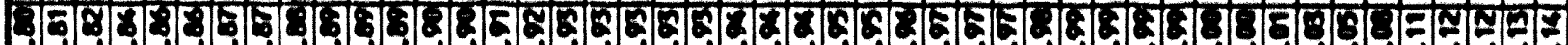

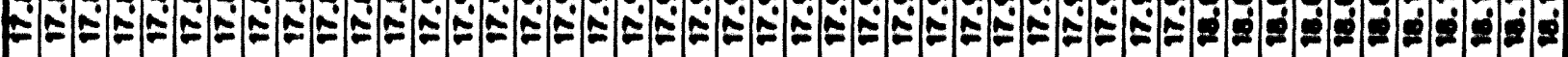

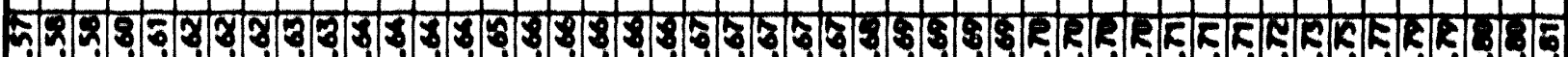

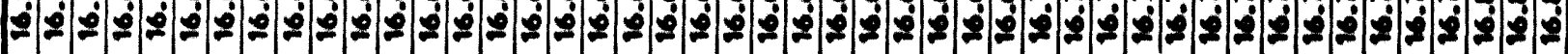

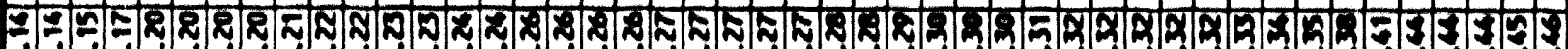

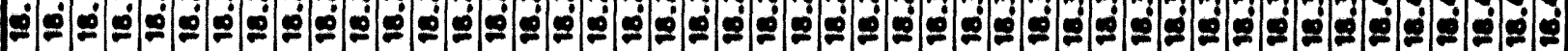

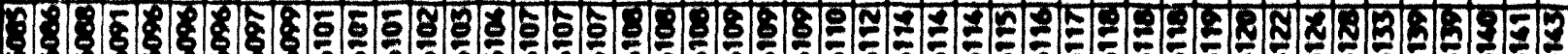

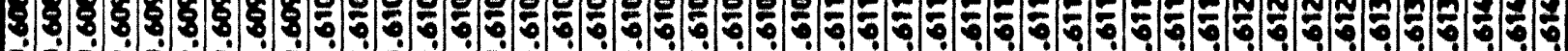

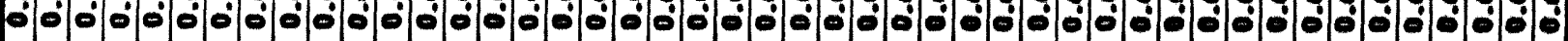

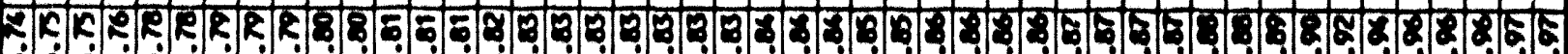

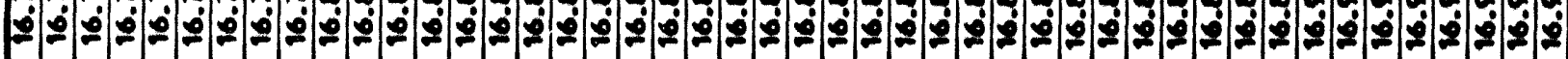

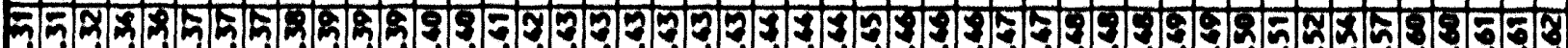

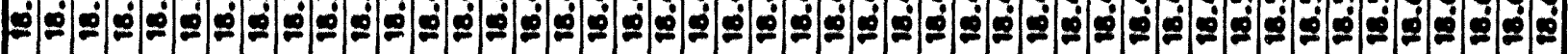

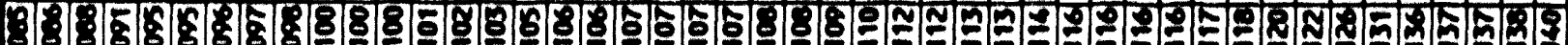

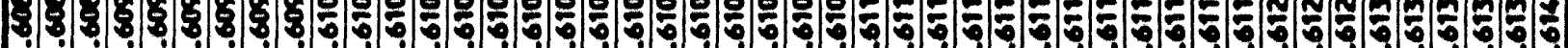

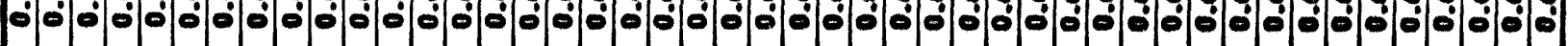

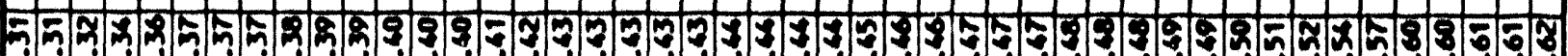

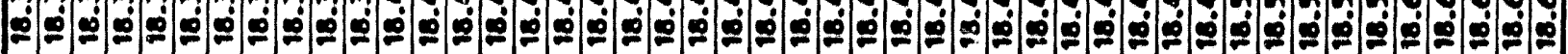

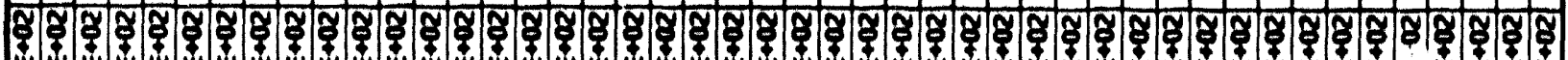

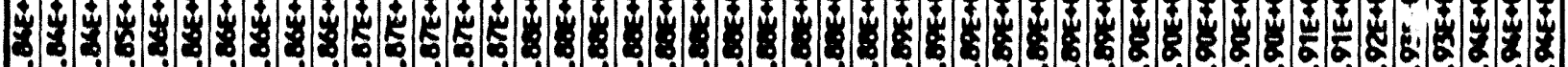

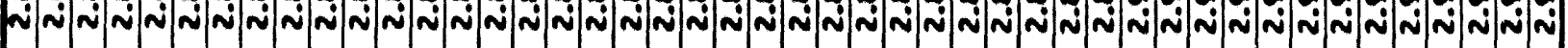




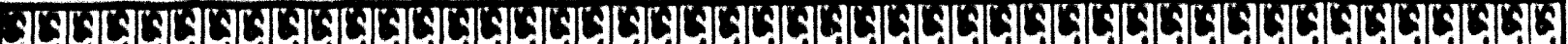

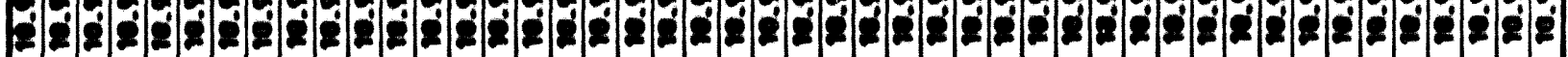

6 :

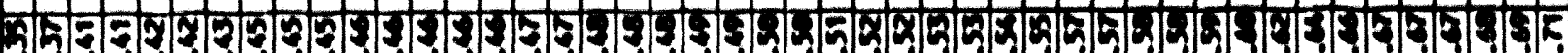

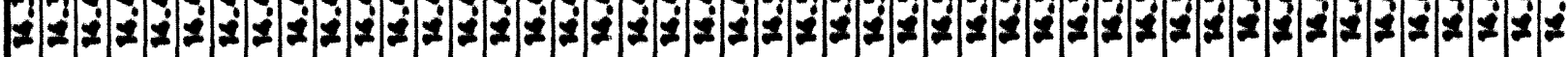

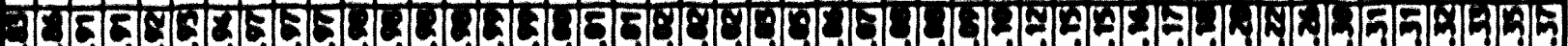

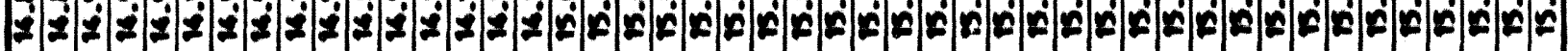

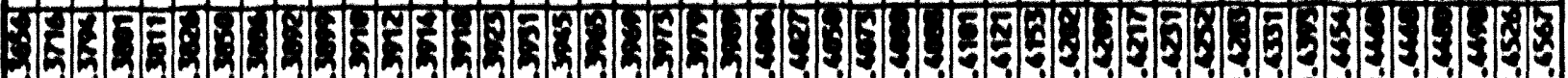

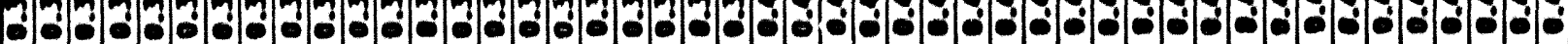

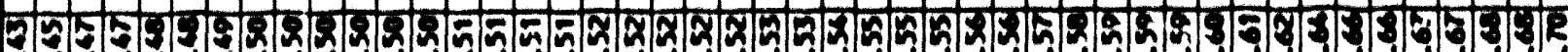

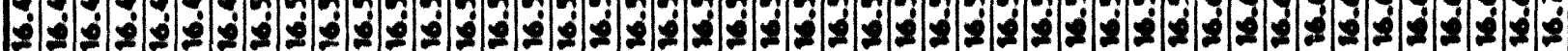

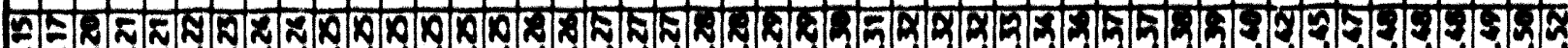

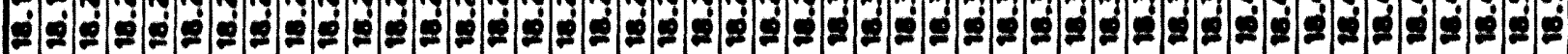

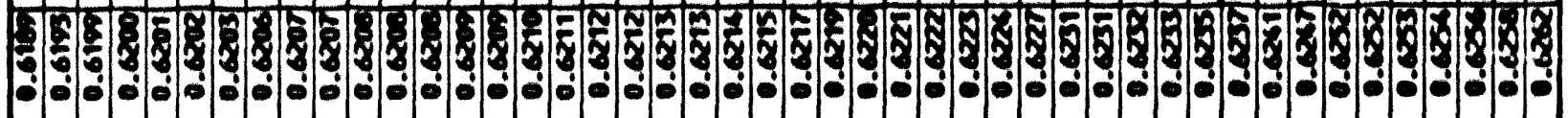

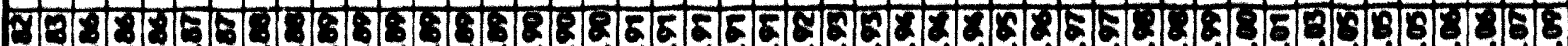

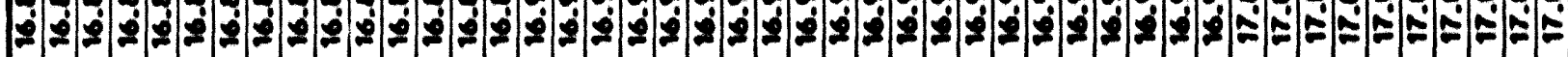
- F N

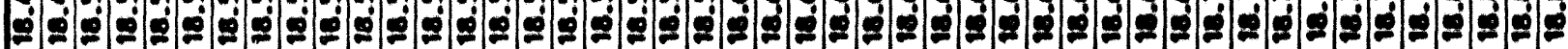

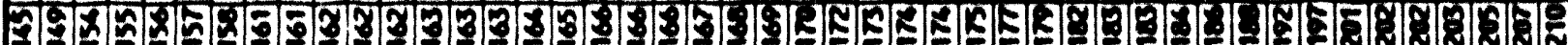
5.

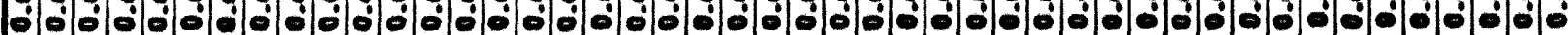

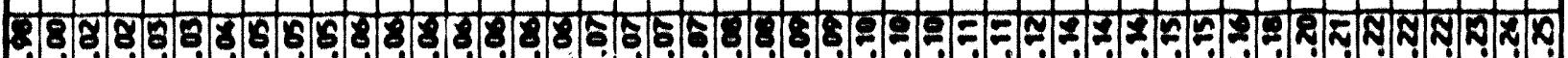

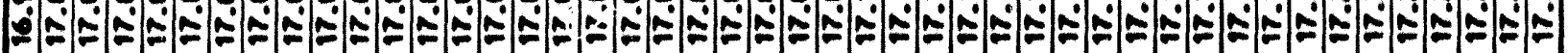

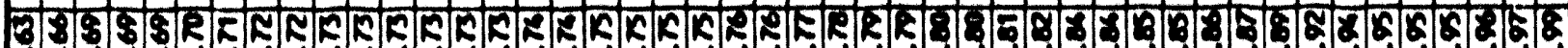

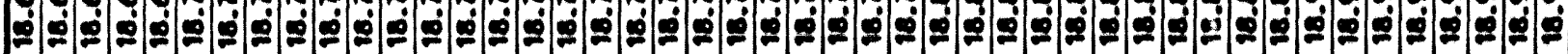

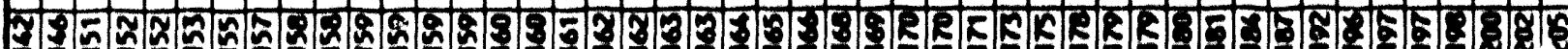

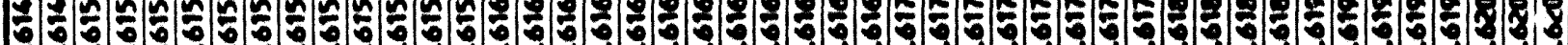

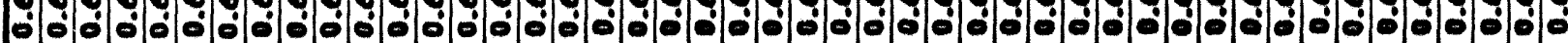

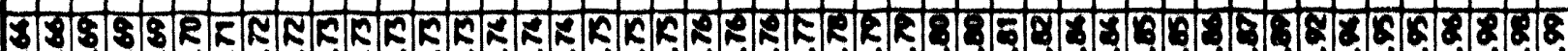

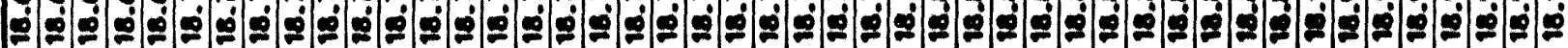

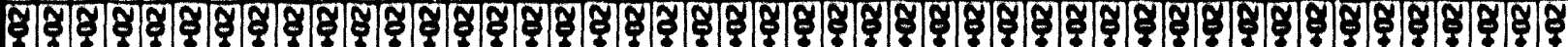

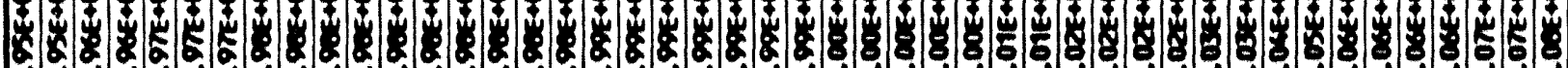

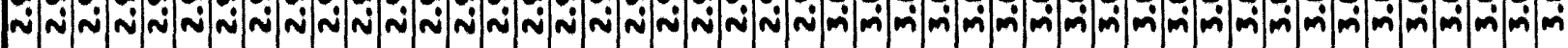




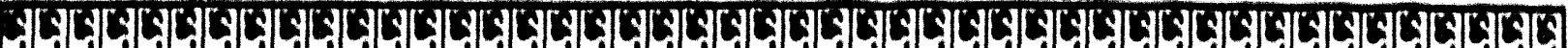

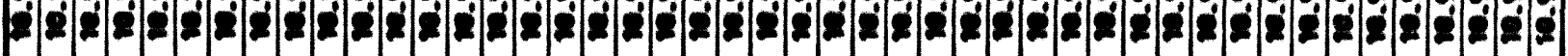

a.

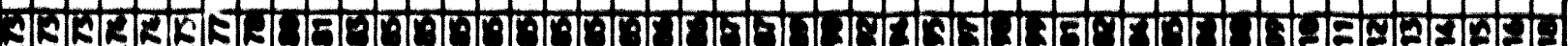

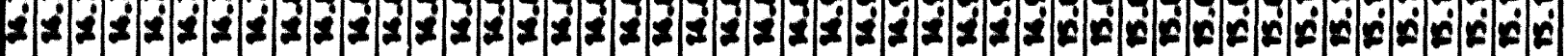

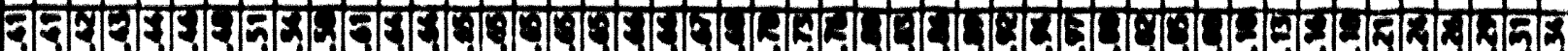

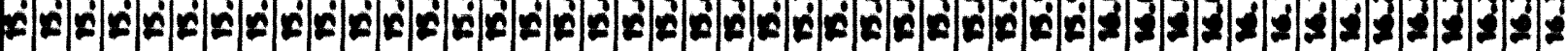

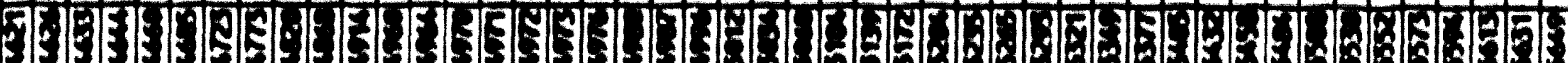

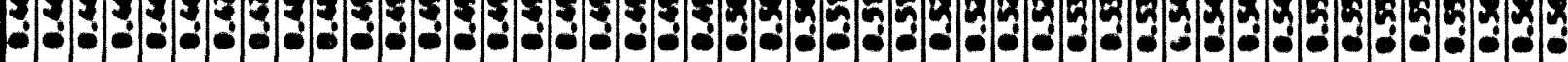

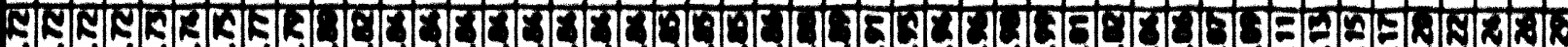

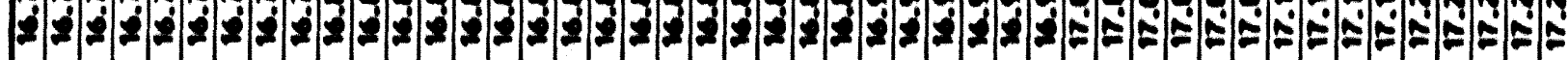

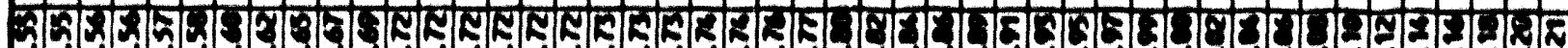

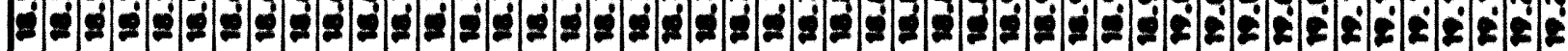

5 (5)

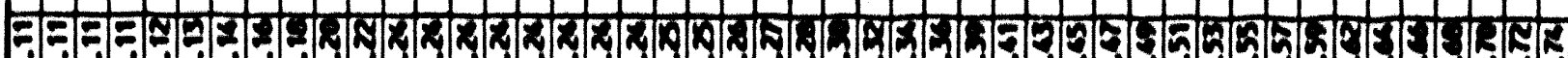

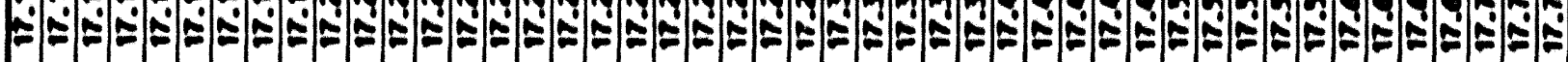
535 E E C E

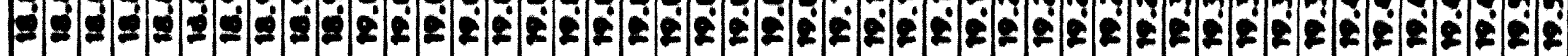

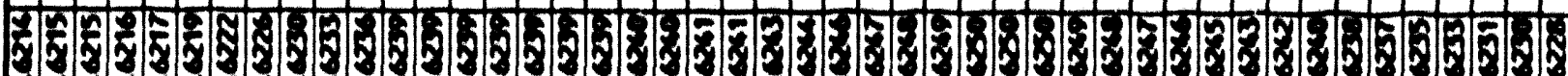
3.

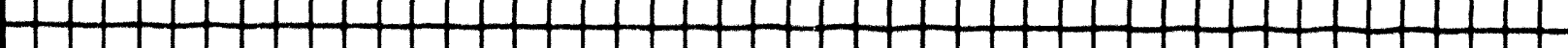

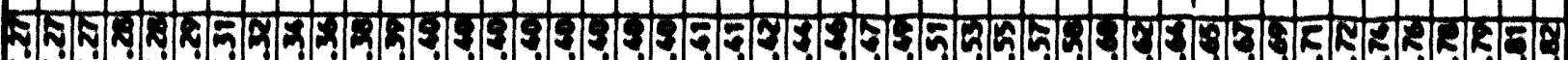

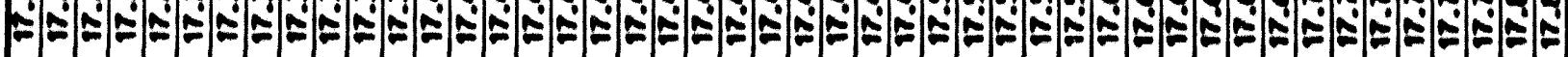

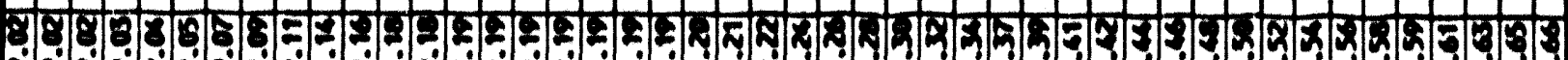

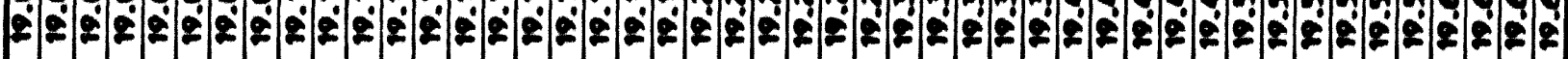

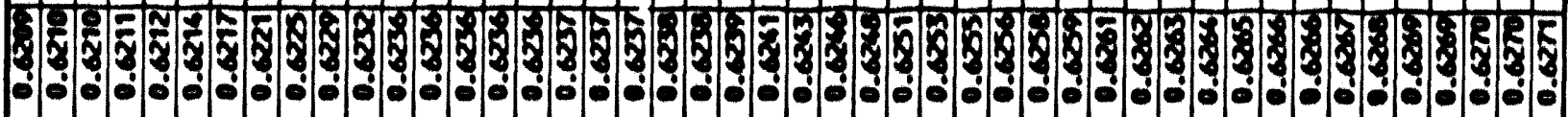

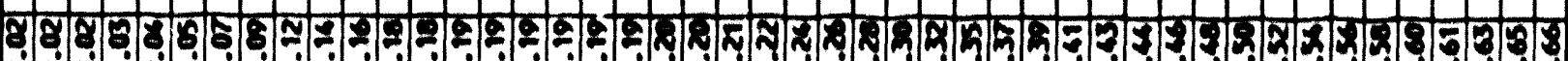

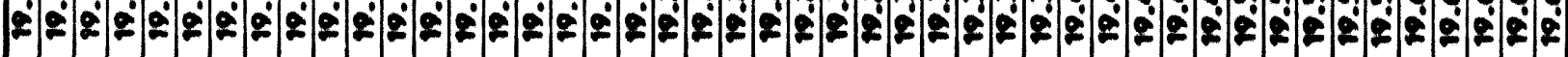

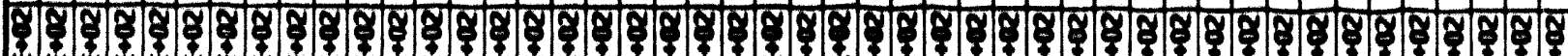

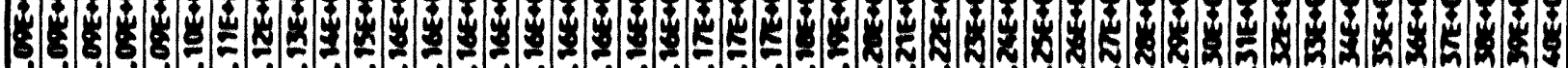

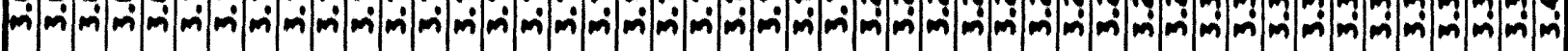




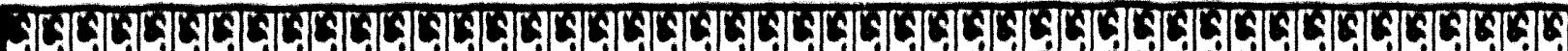

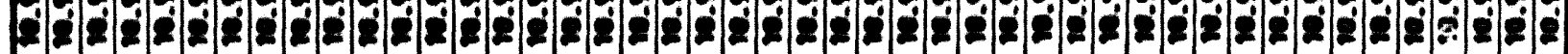

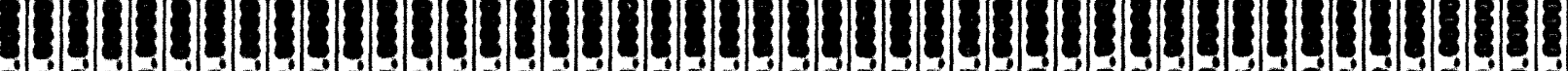
- á.

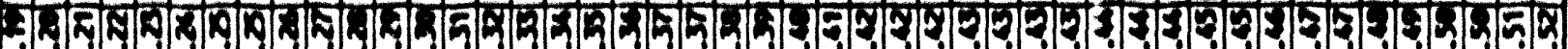

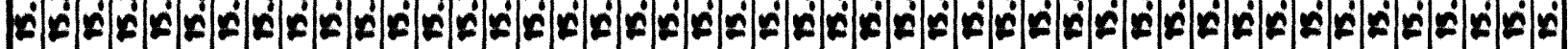

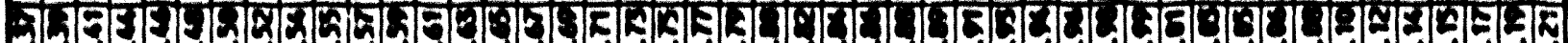

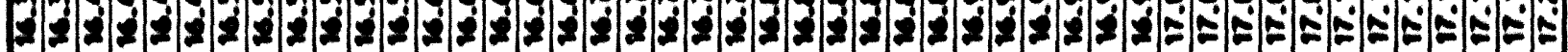
E. 6.

- 2 n

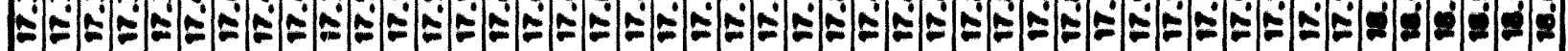

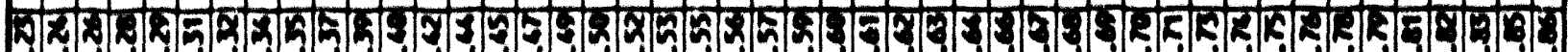

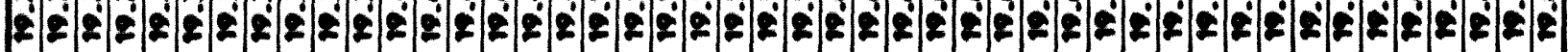
-

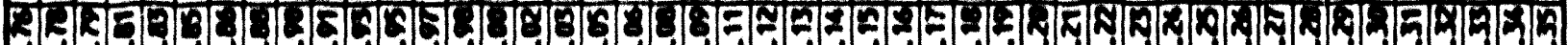

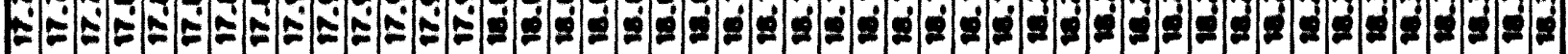

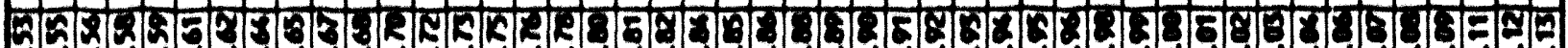

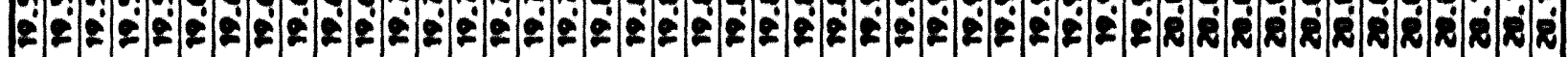

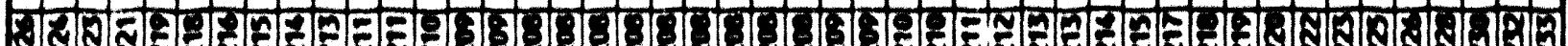

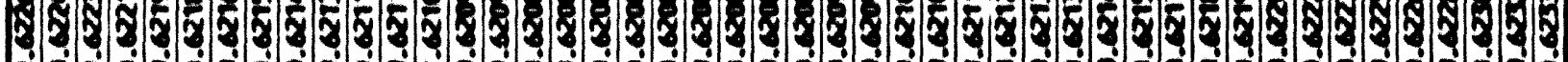

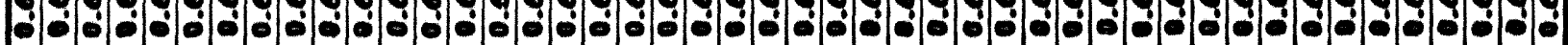

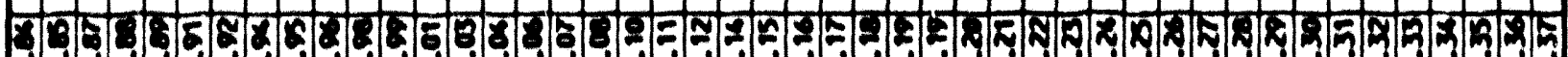

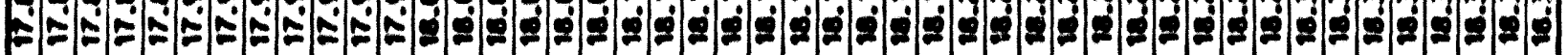

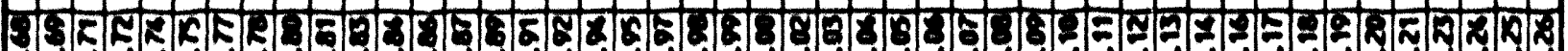

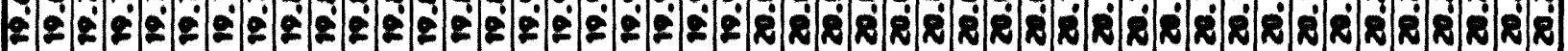

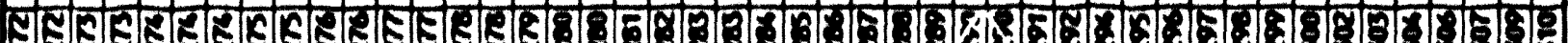
3.5\%

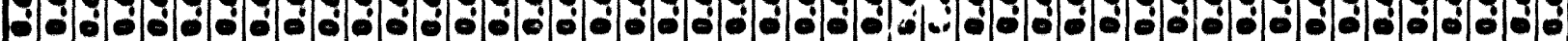

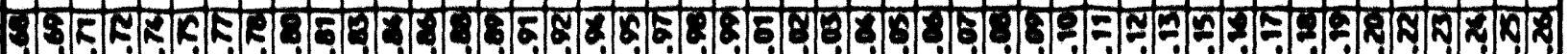

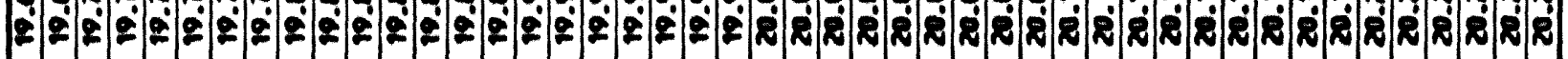

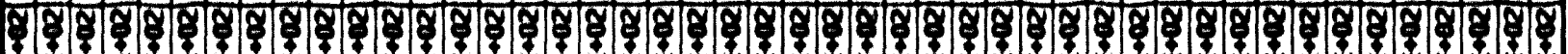

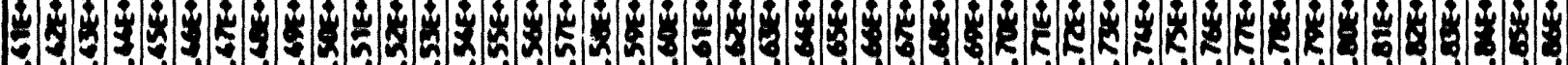

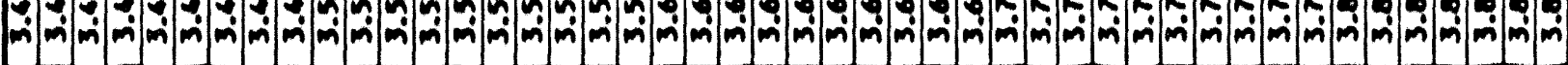




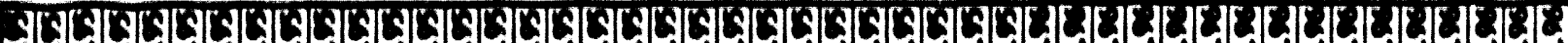
B. (

60

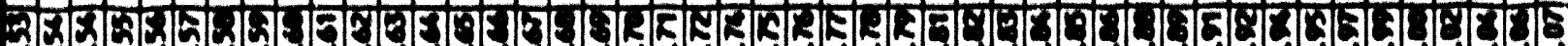

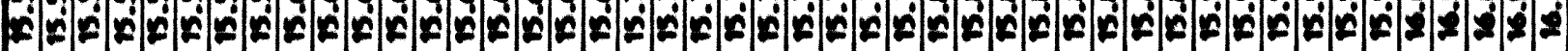

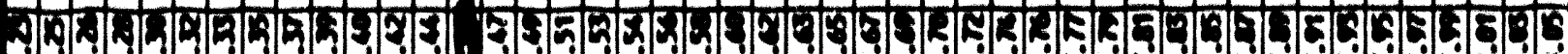

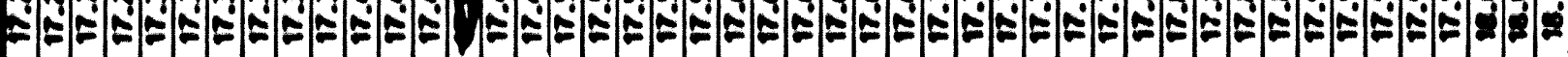

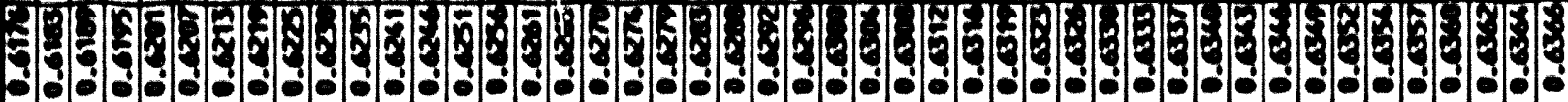

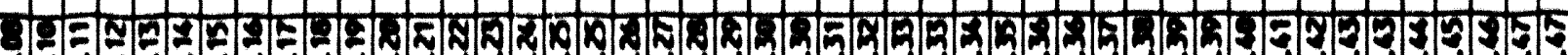

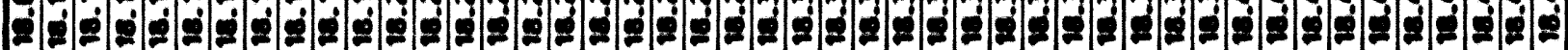

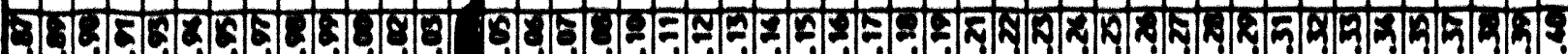

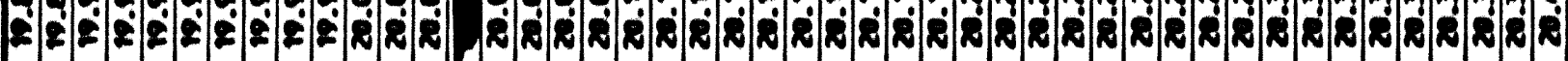

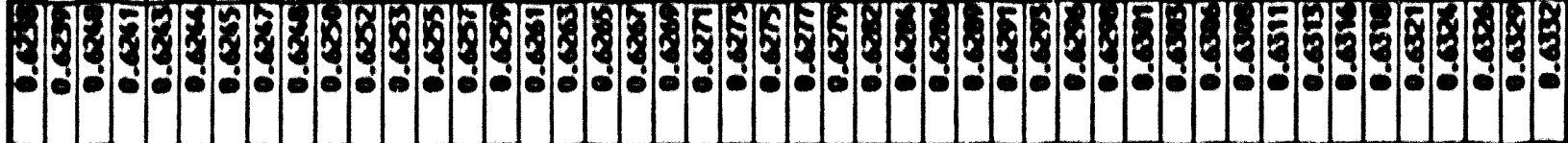

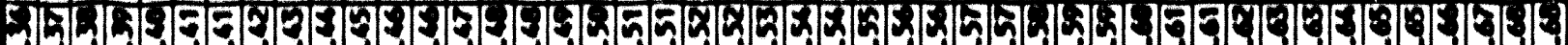

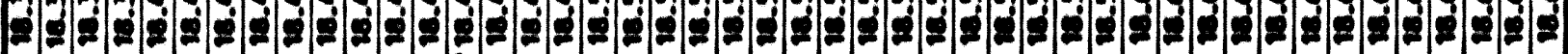

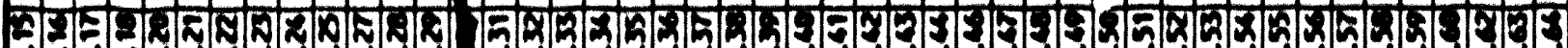
ลี่ (5) 60 -

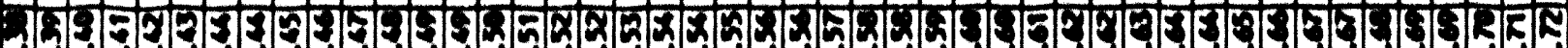

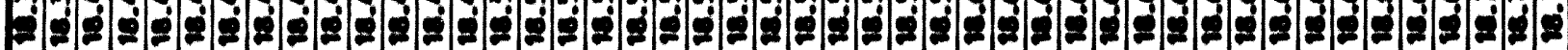

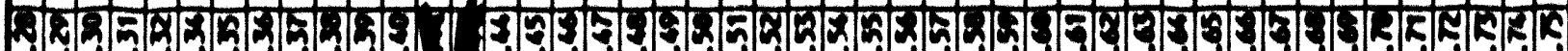

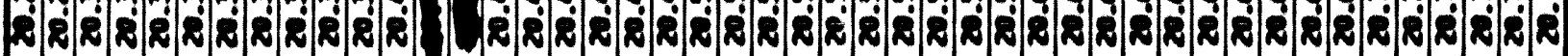

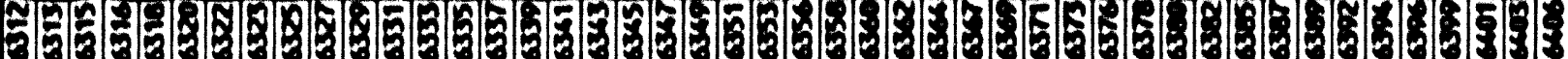
○ o

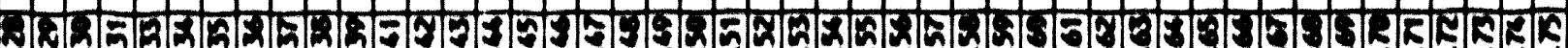

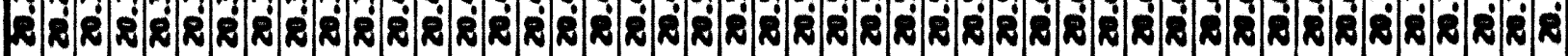

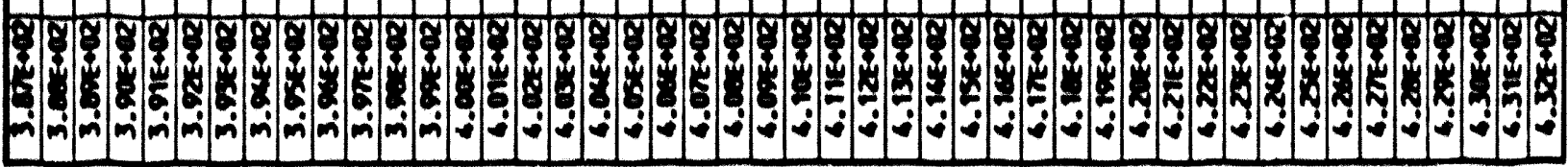




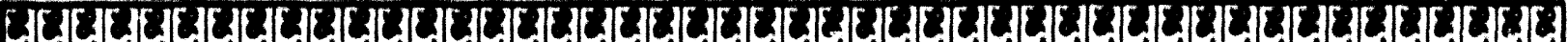

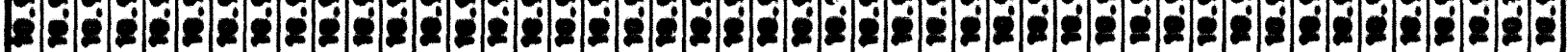

1

b-

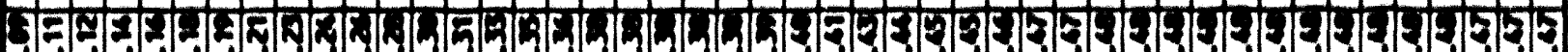

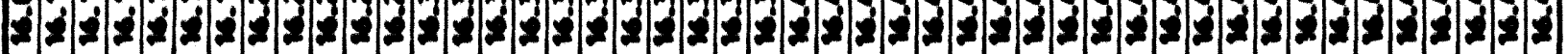

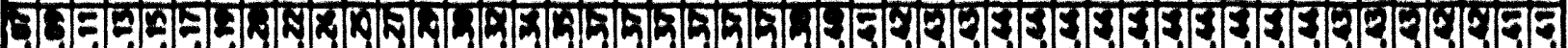

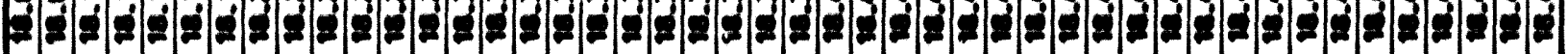

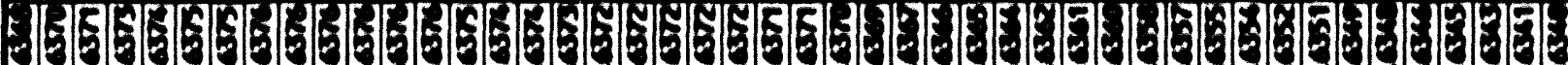

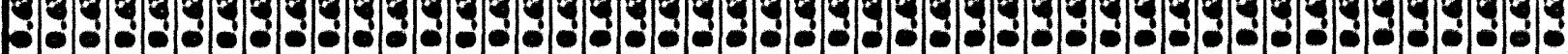

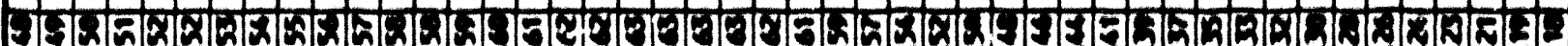

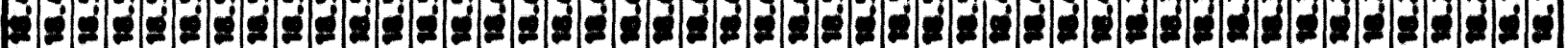

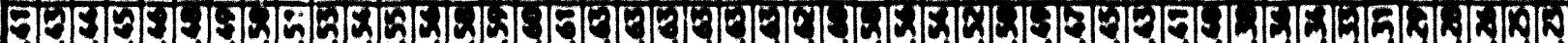

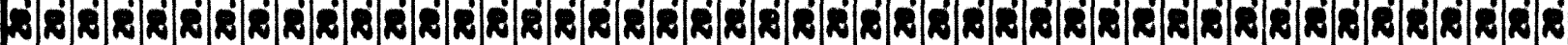

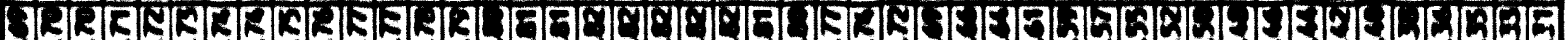

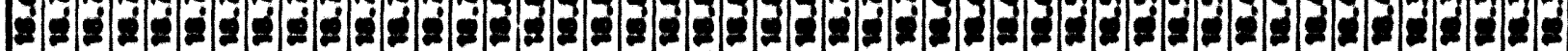

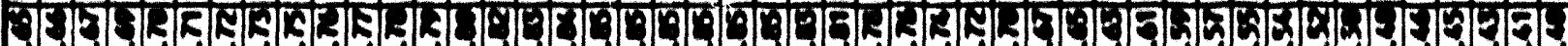

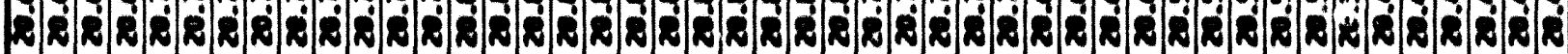

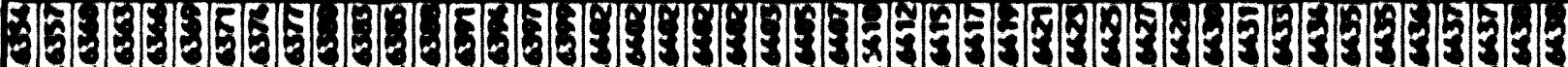

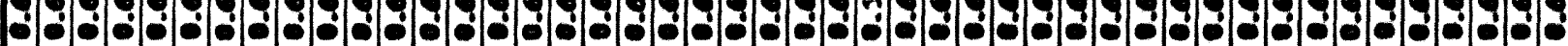

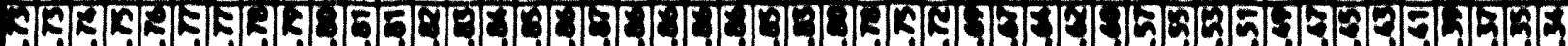

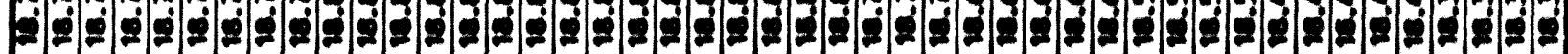

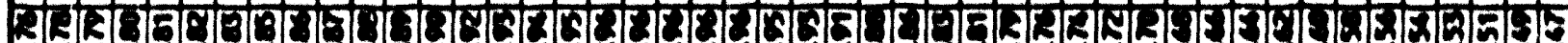

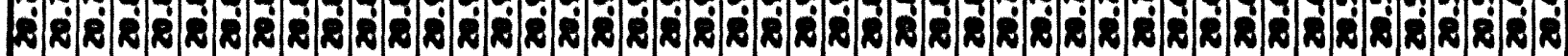

1E-

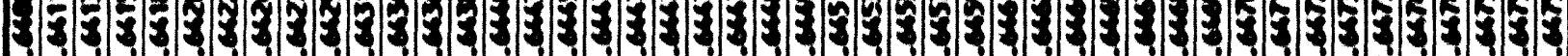

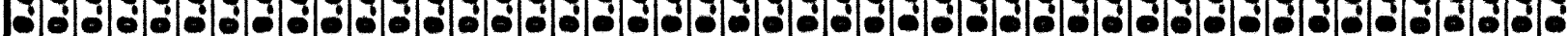

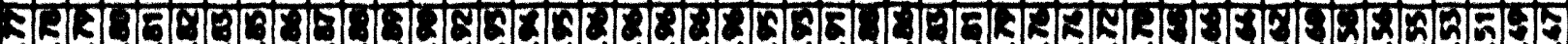

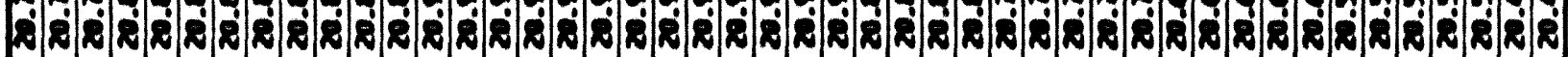

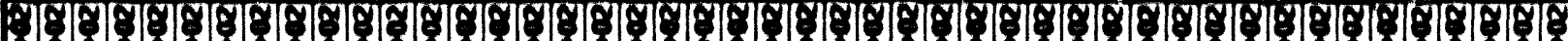
(3) H. n 


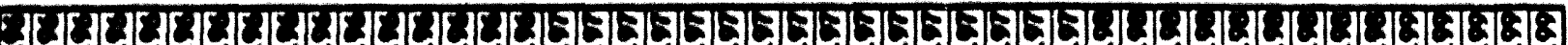
6.

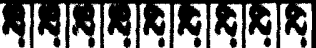

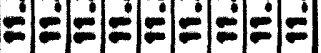
13 ( -

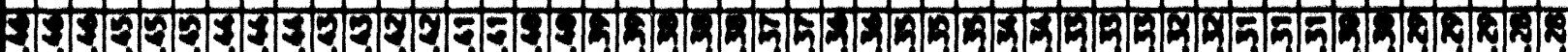

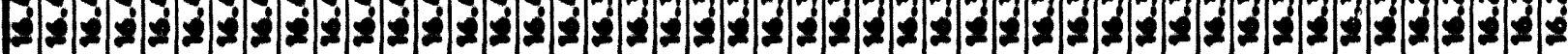

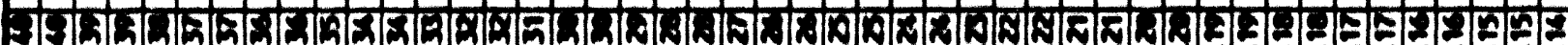

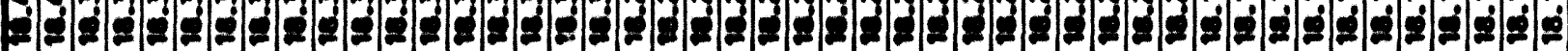

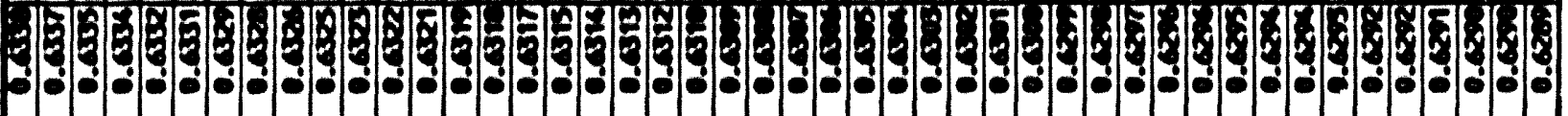

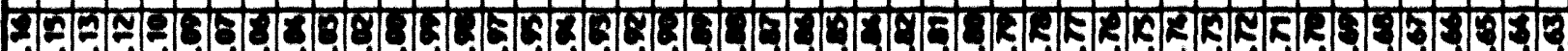

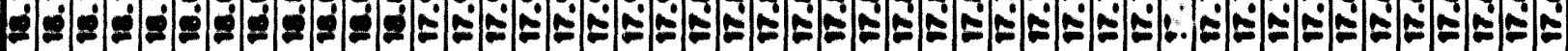

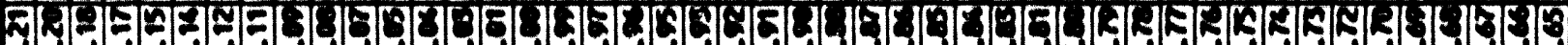

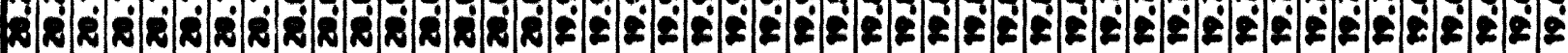

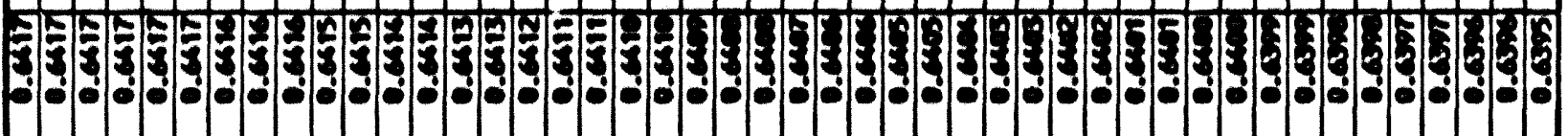

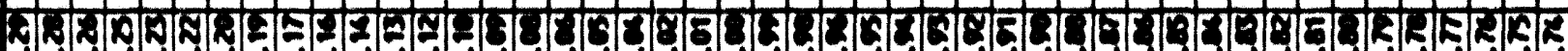

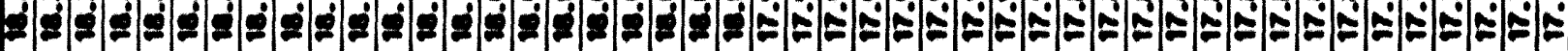

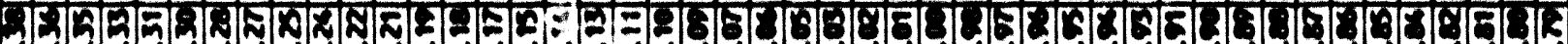

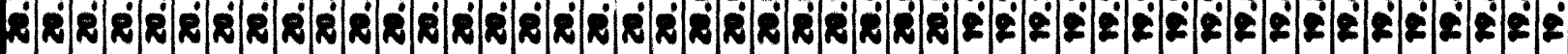

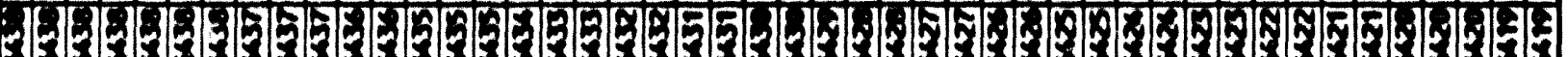
6 อ.

SI

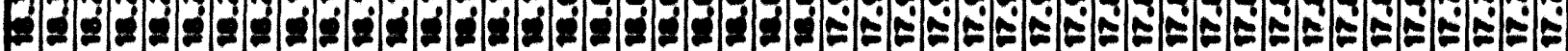

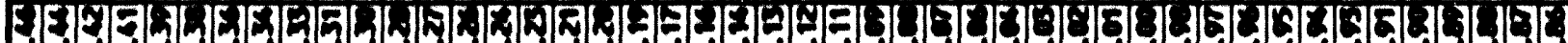

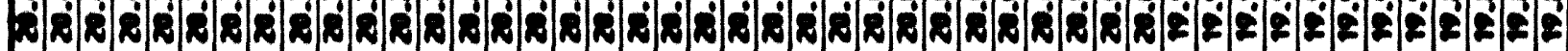

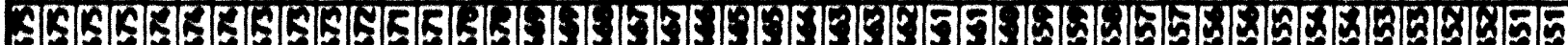

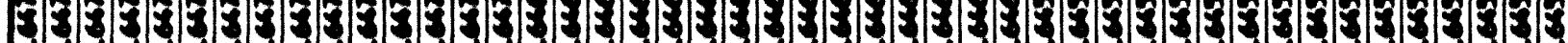

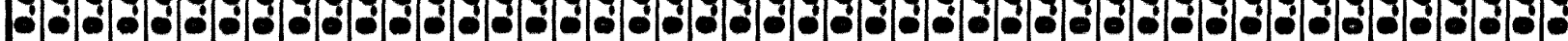

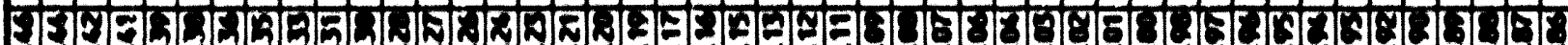

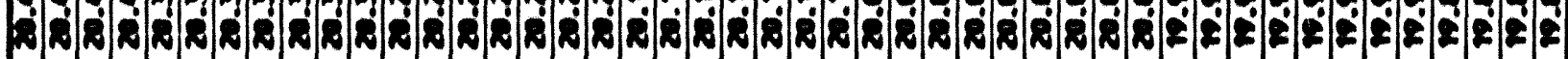

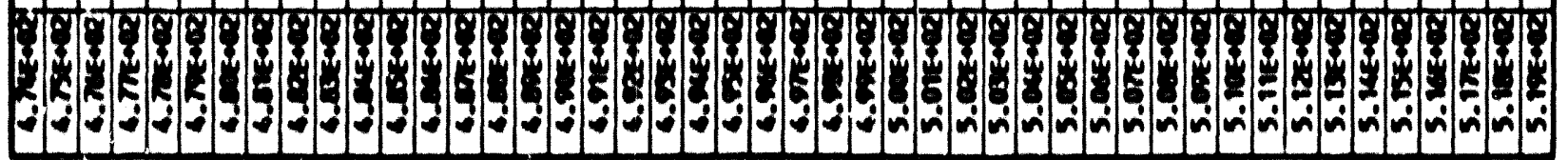




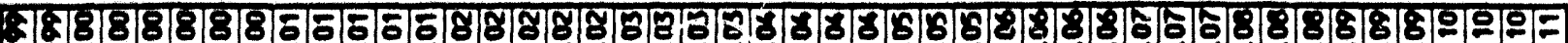

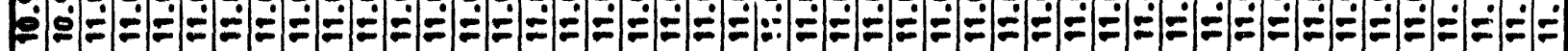

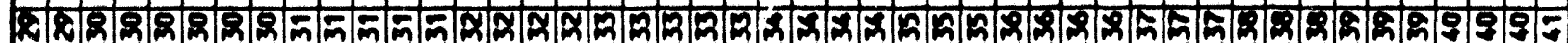

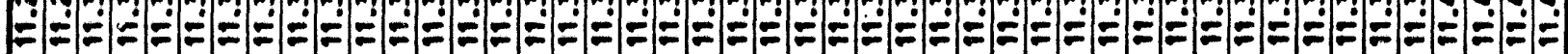

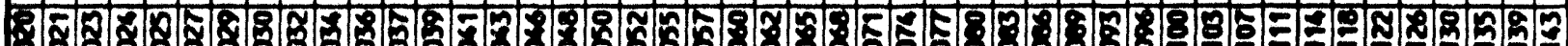
8.

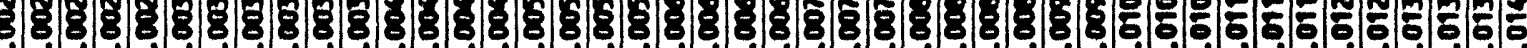

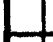

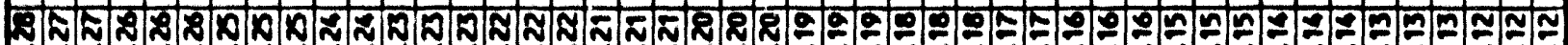

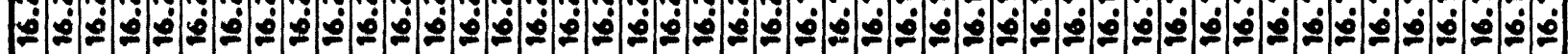

- 7 -

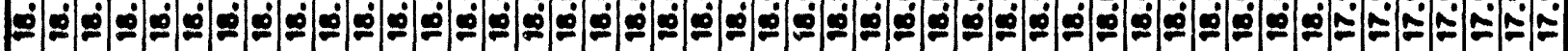

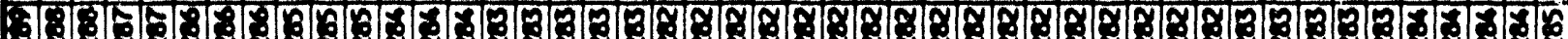

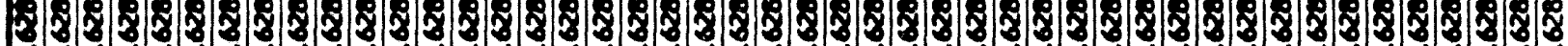

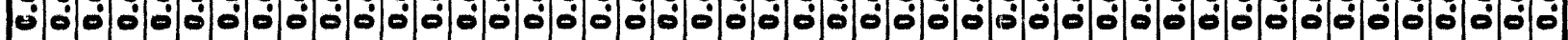

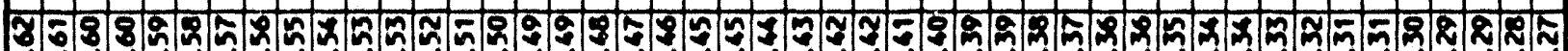

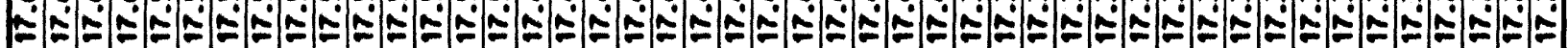

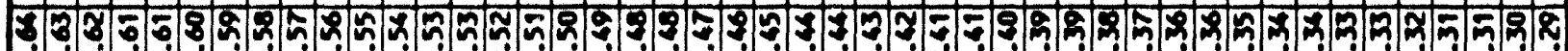

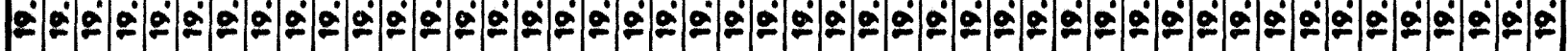

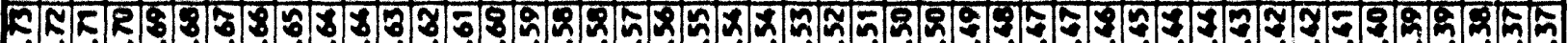

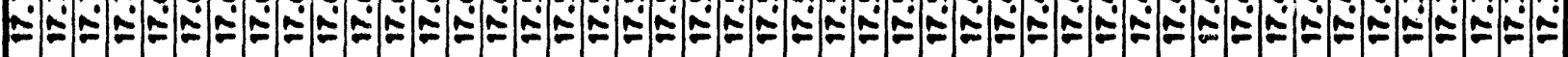

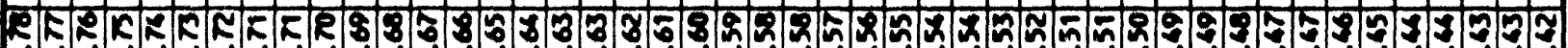

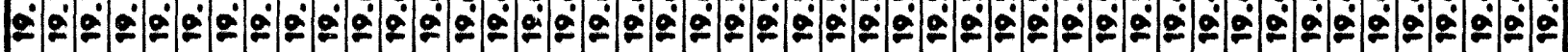

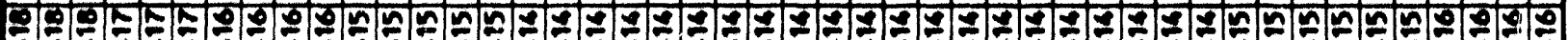

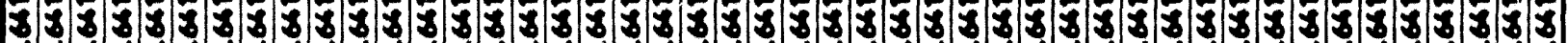

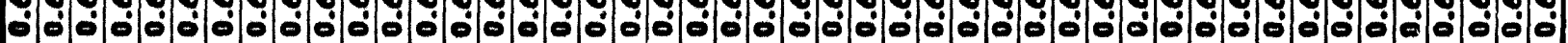

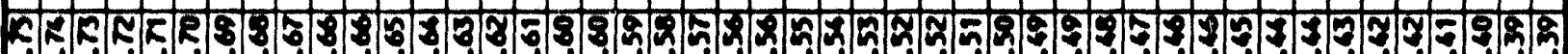

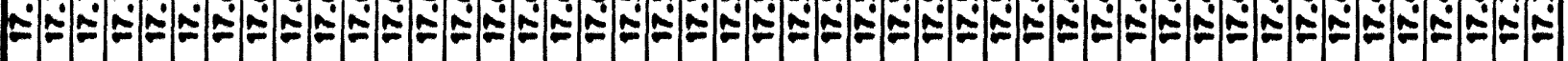

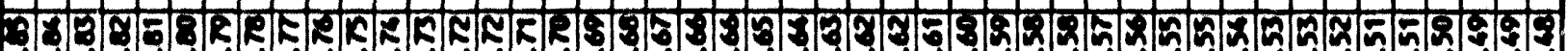

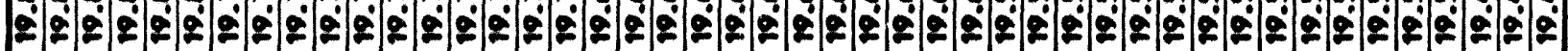

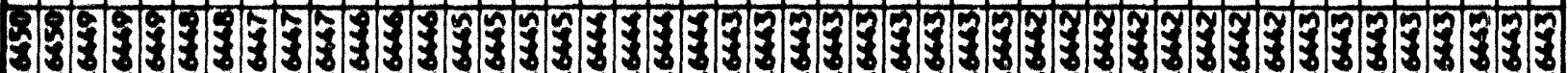
- 0 -

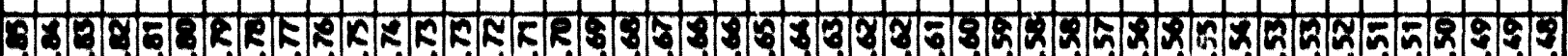

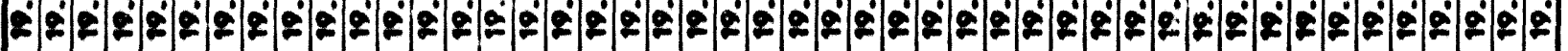

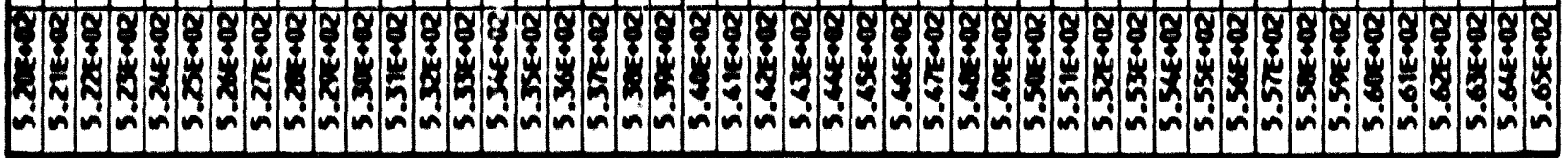




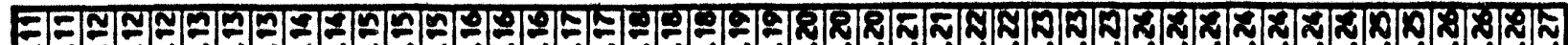

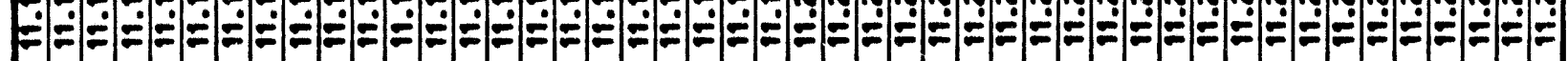

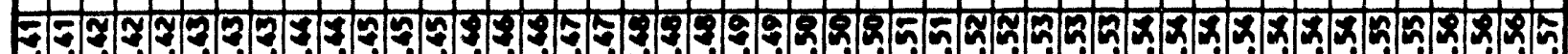

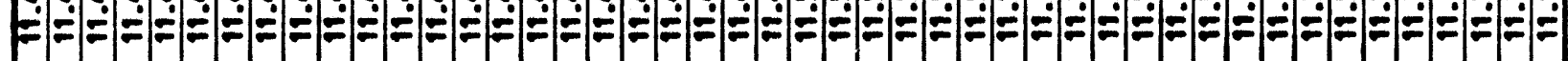

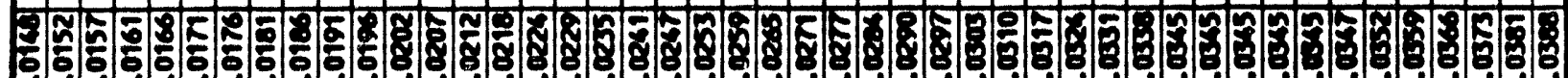

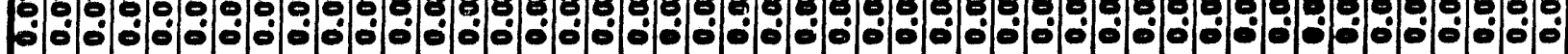

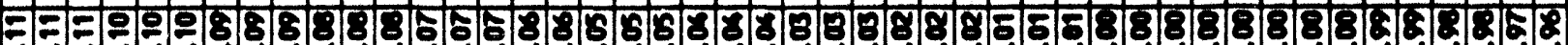

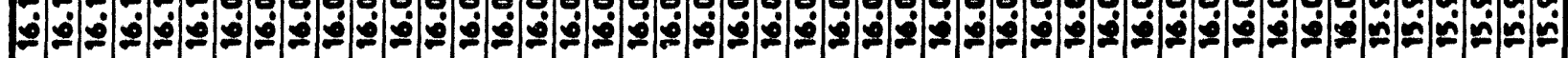

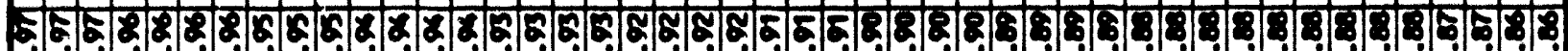

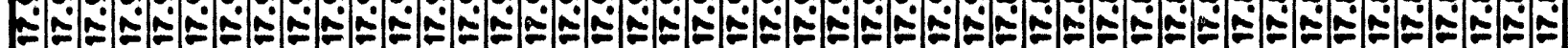

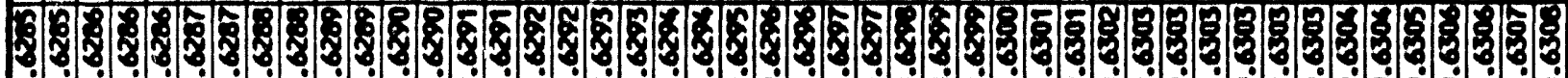

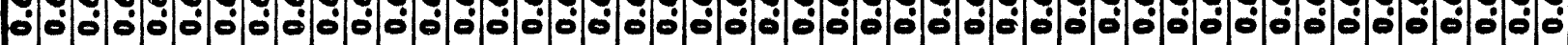

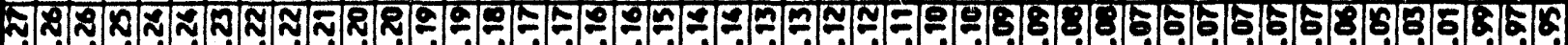

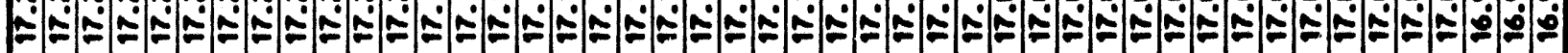

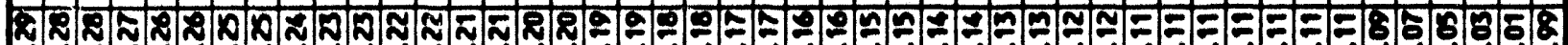

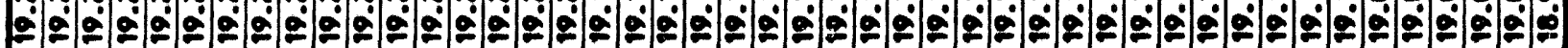

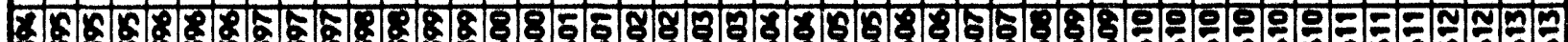

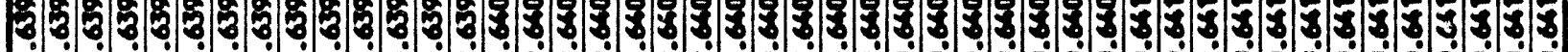

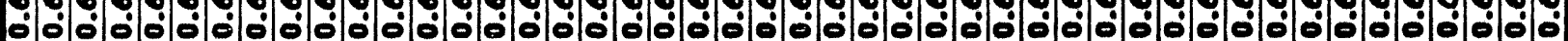

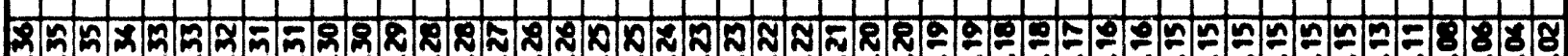

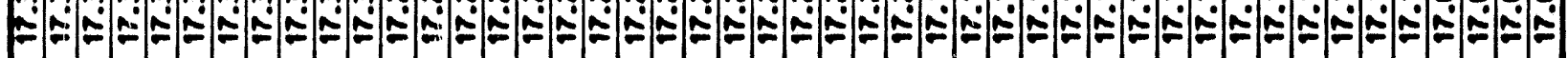
- =

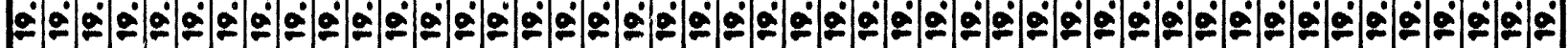

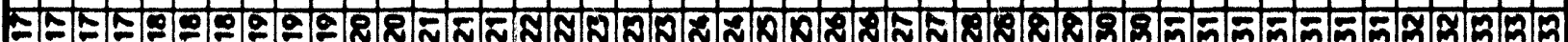

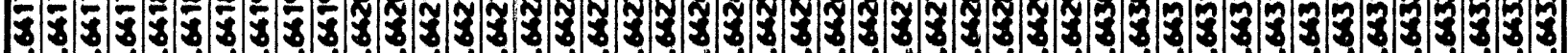

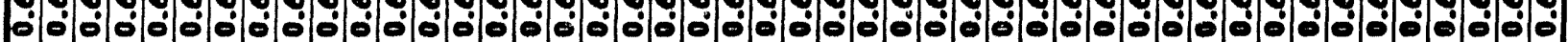

6.

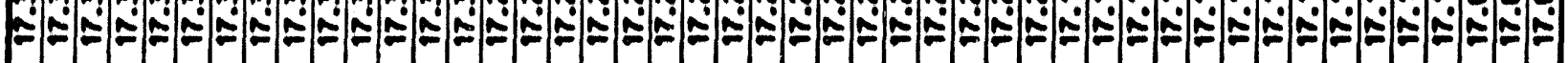

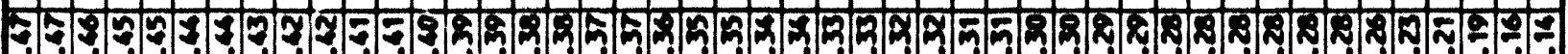

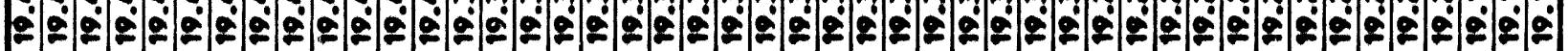

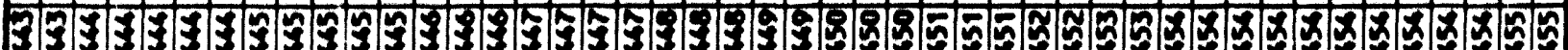

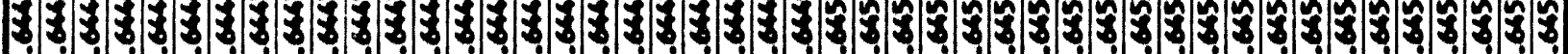
0. 0. :

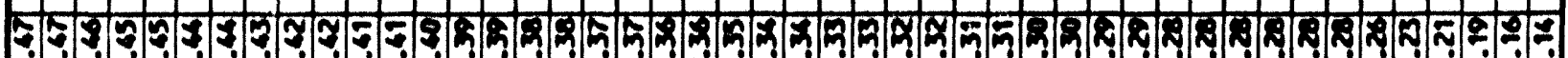

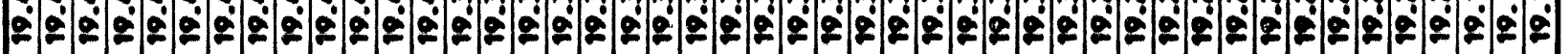

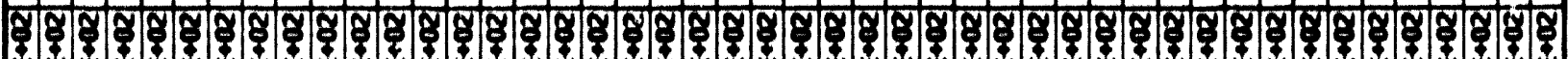

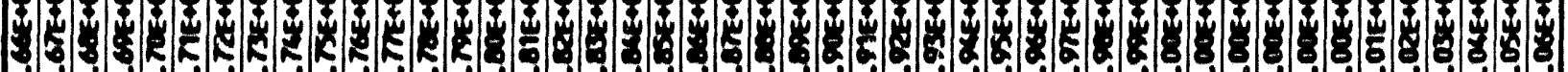

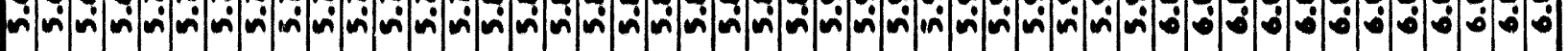




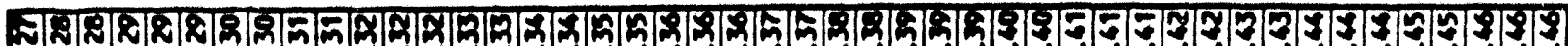

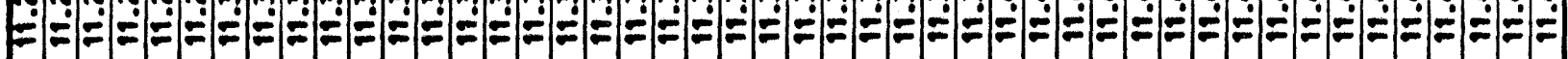

Sर

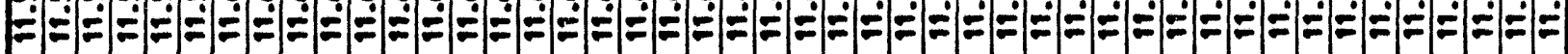

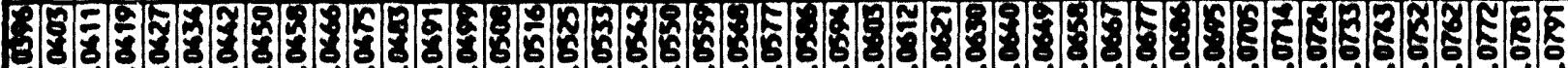
- ó

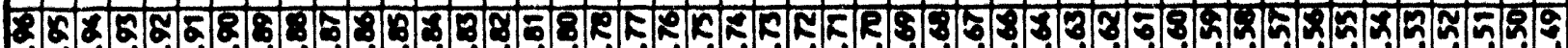

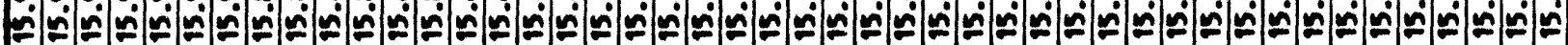

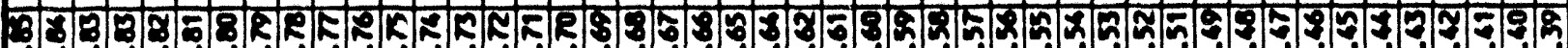

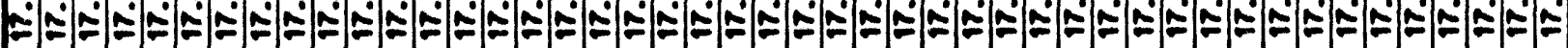

3)

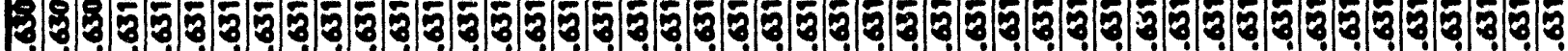

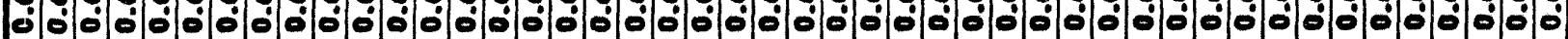

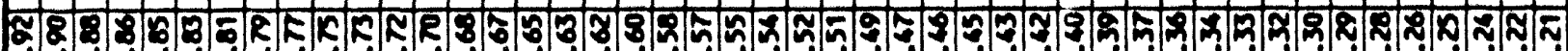

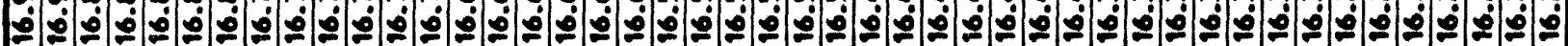

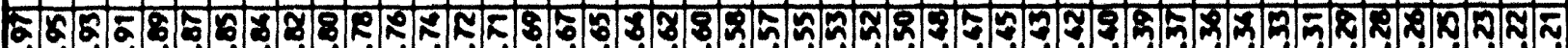

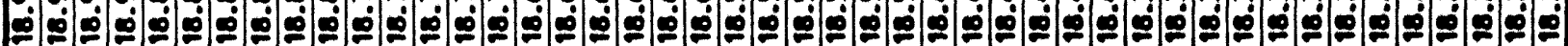

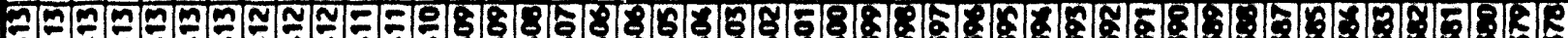

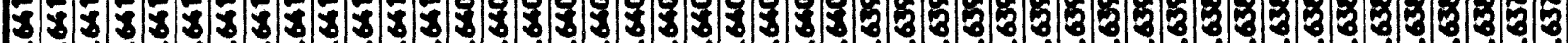

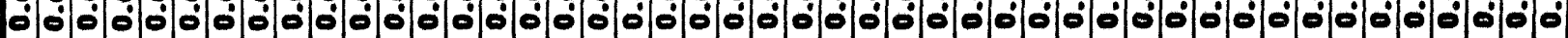

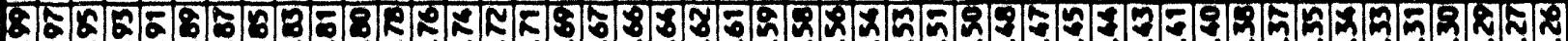

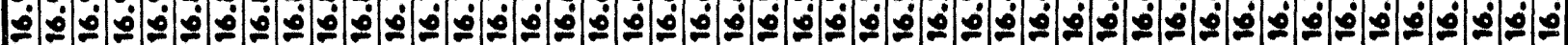

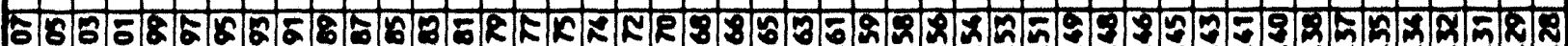

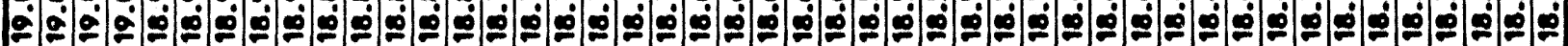

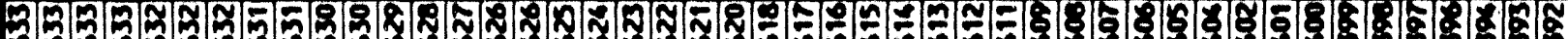

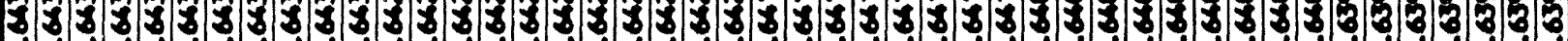

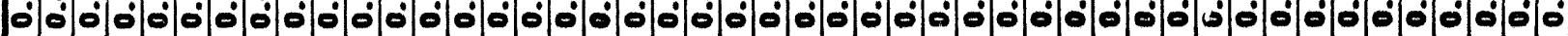

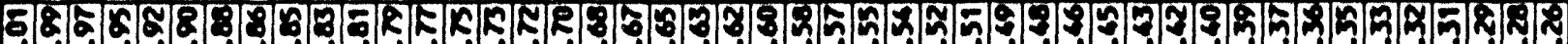

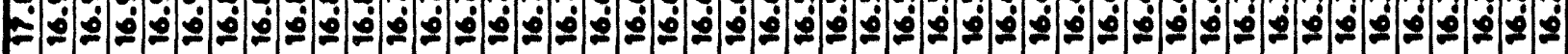

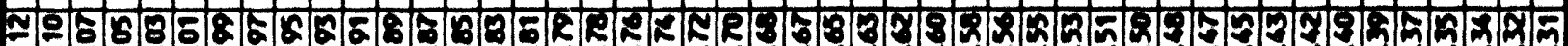

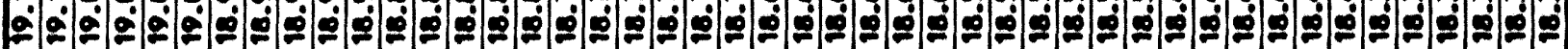

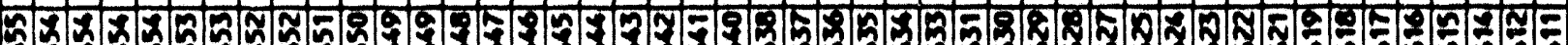
కิ

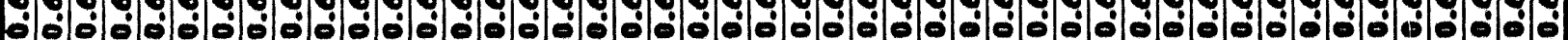

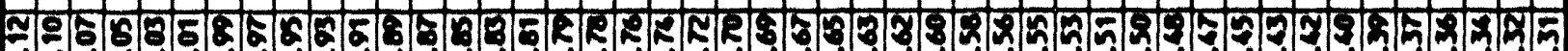

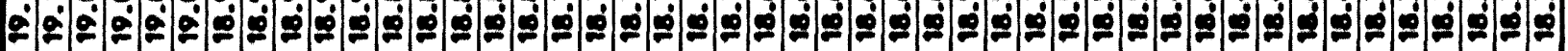

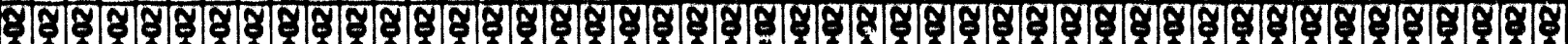

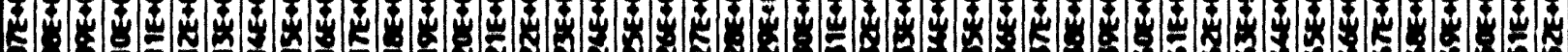

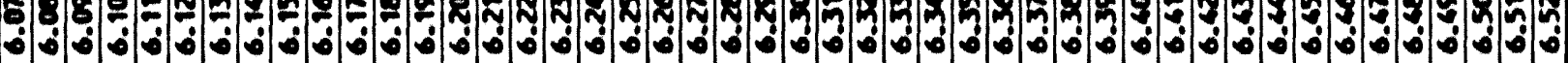


75\%? $\Rightarrow=\Rightarrow=\Rightarrow=\Rightarrow=\Rightarrow=\Rightarrow=\Rightarrow=\Rightarrow=\Rightarrow=\Rightarrow=\Rightarrow=\Rightarrow=\Rightarrow=\Rightarrow=\Rightarrow=\Rightarrow$

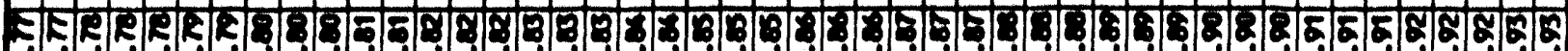

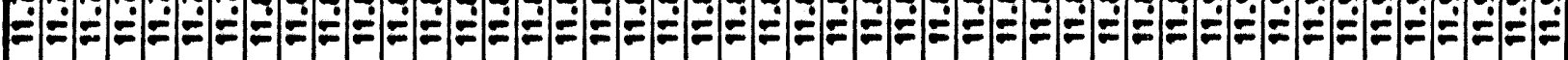
5

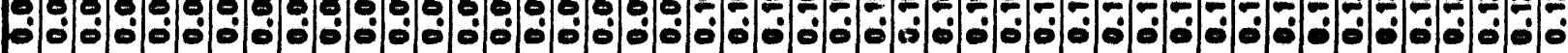

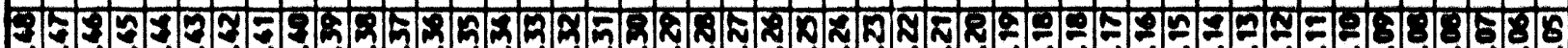

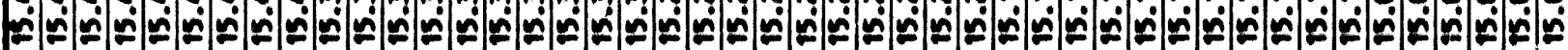

Q

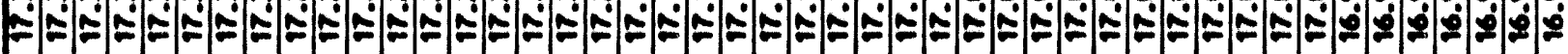
ANNNNE=

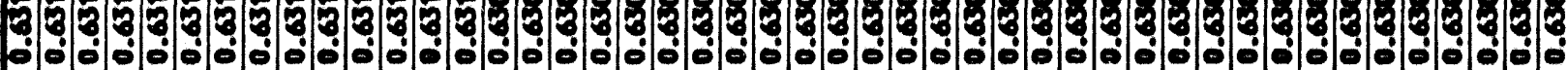

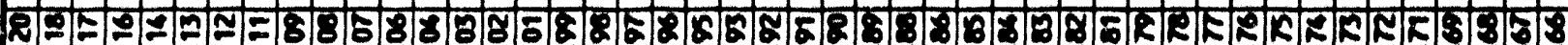

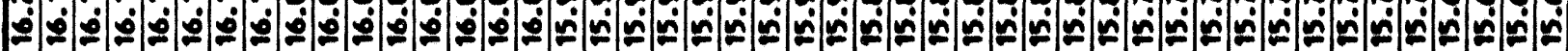

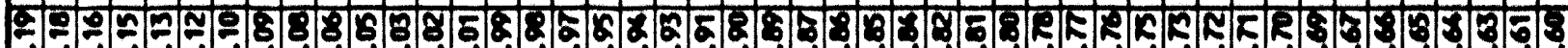

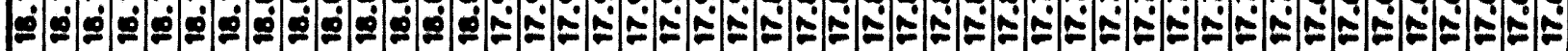

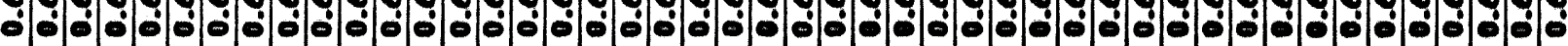

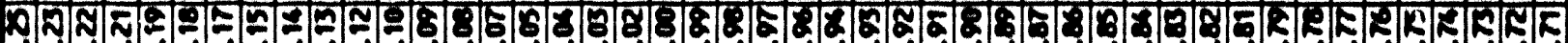

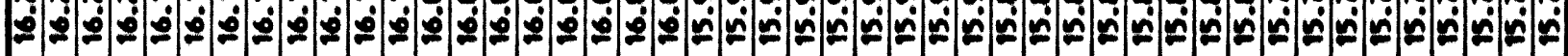

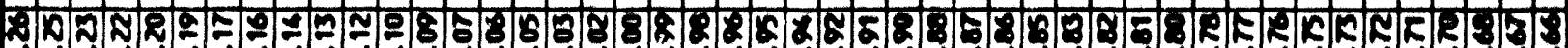

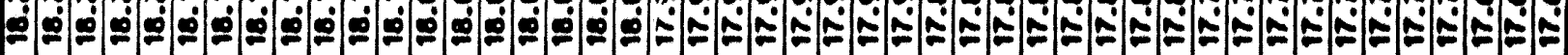

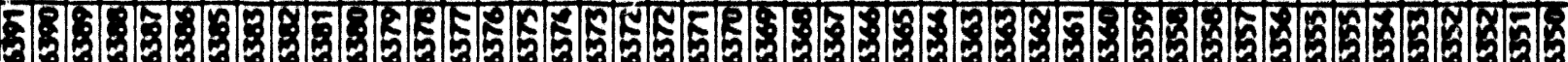

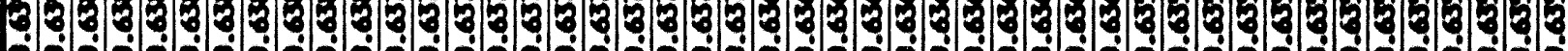

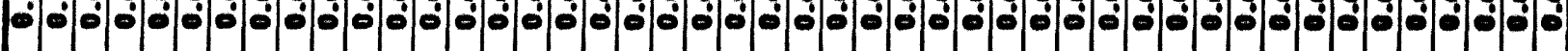

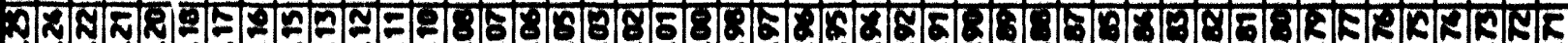

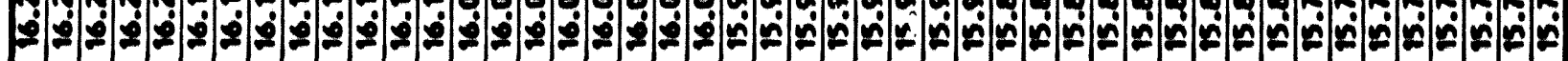
G

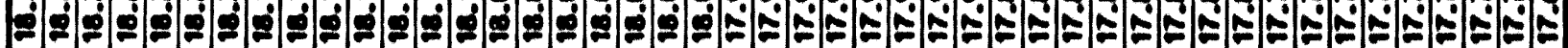

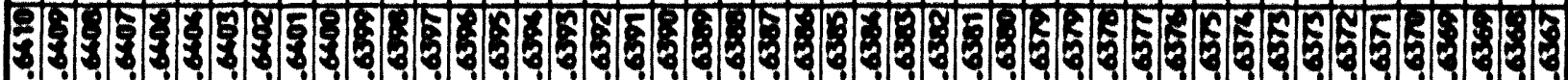

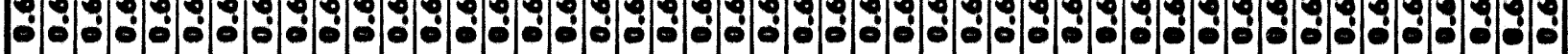

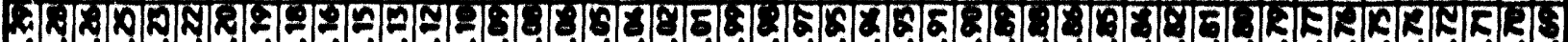

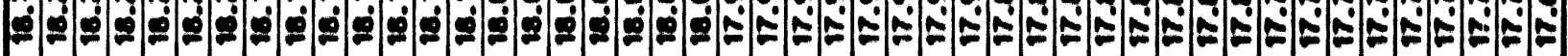

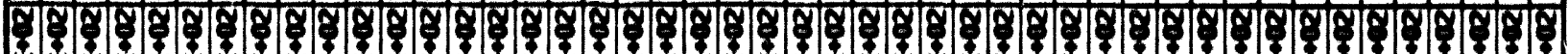

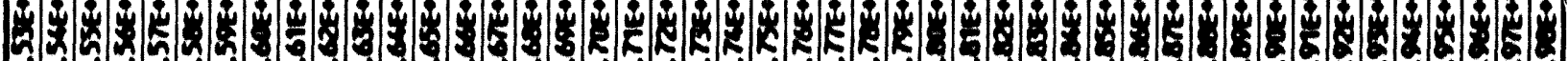
6. 


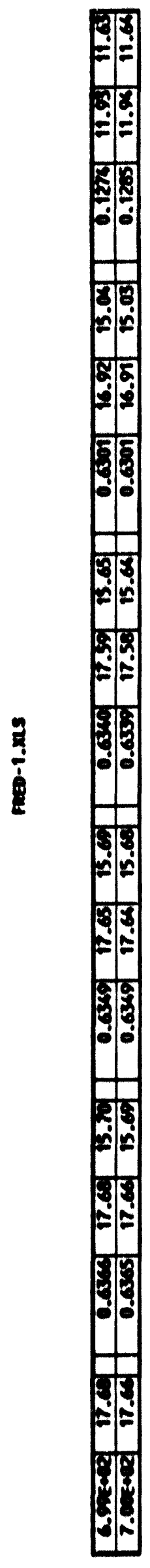


Figure 1.
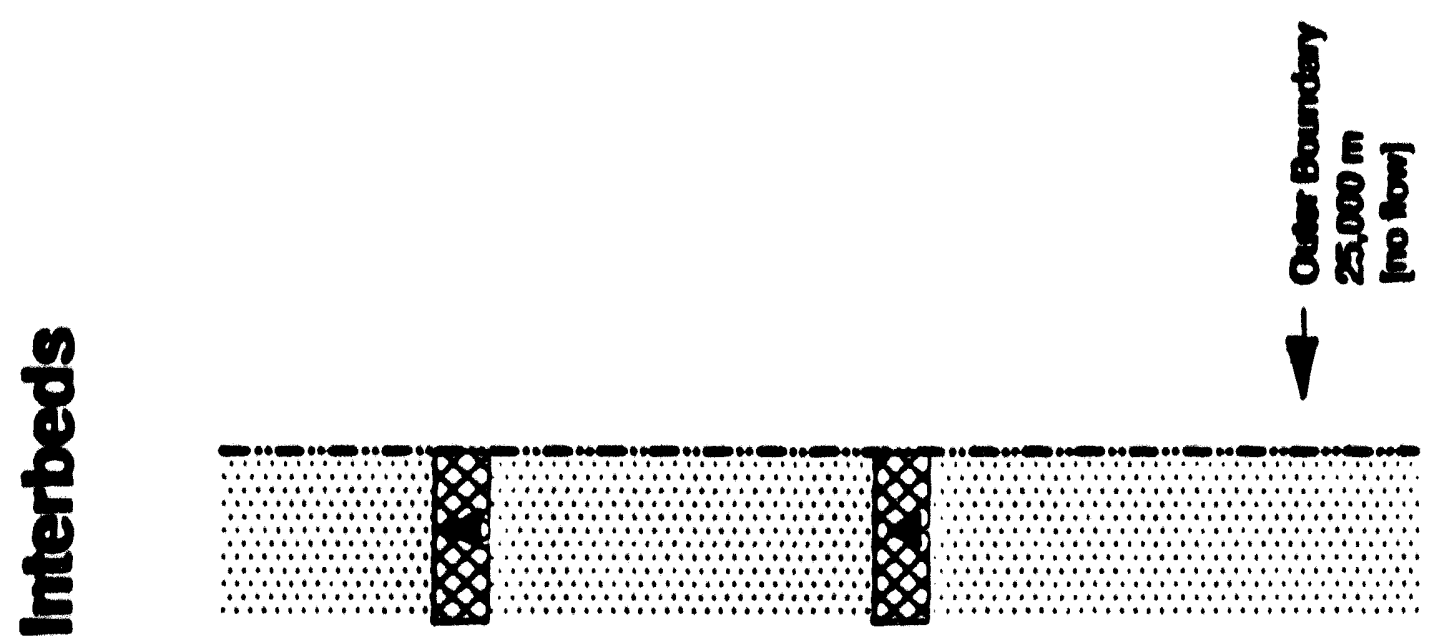

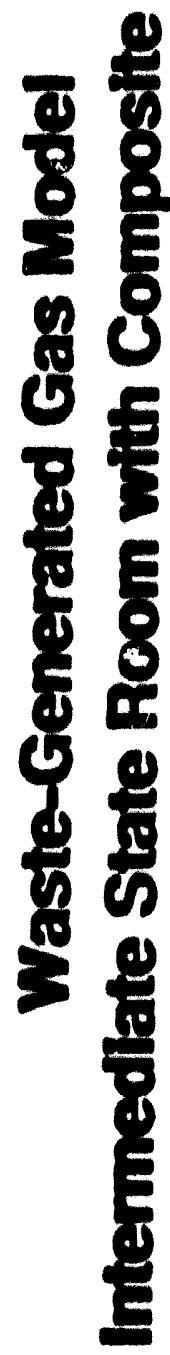

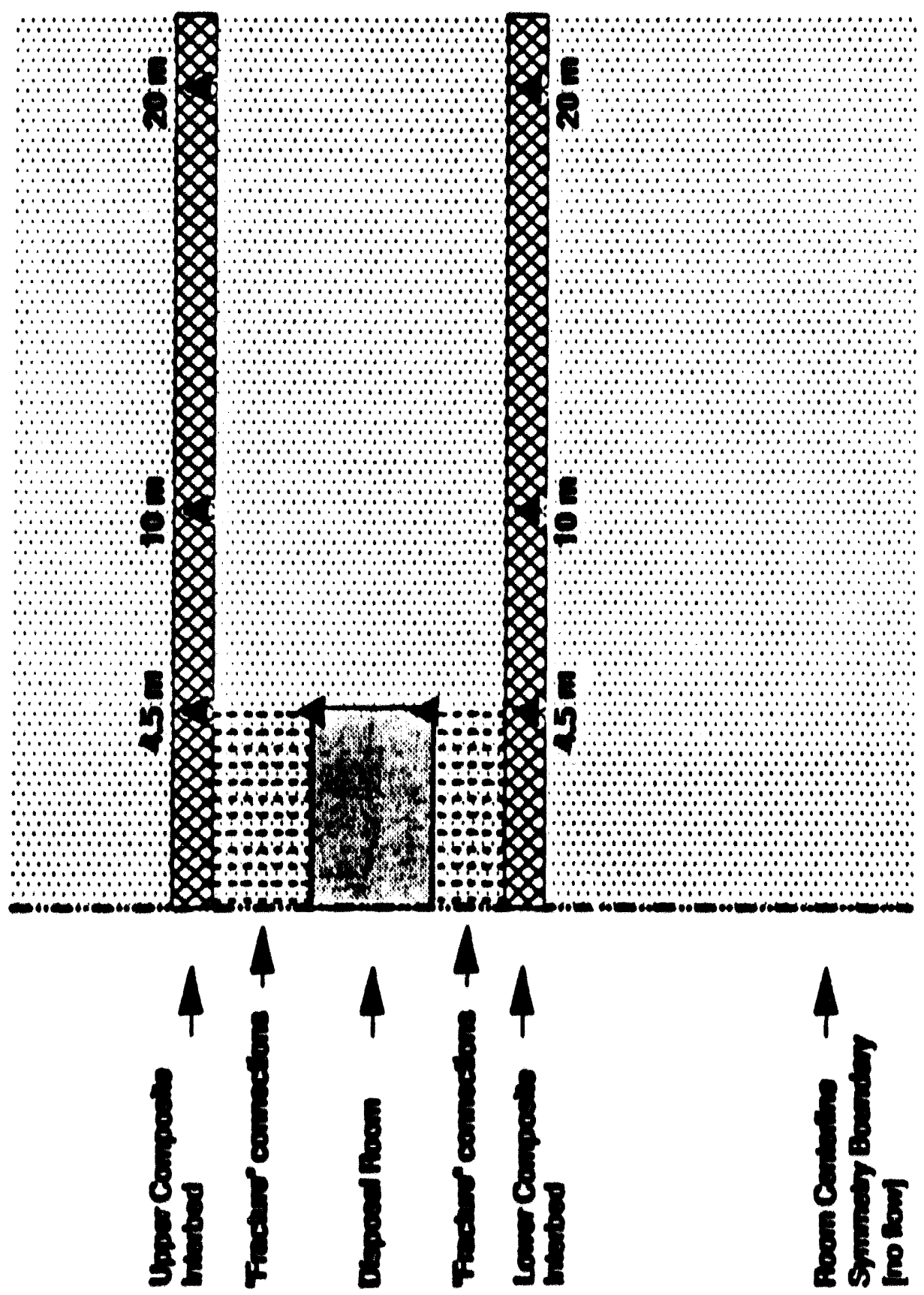

$A \cdot 64$ 
Figura 3.
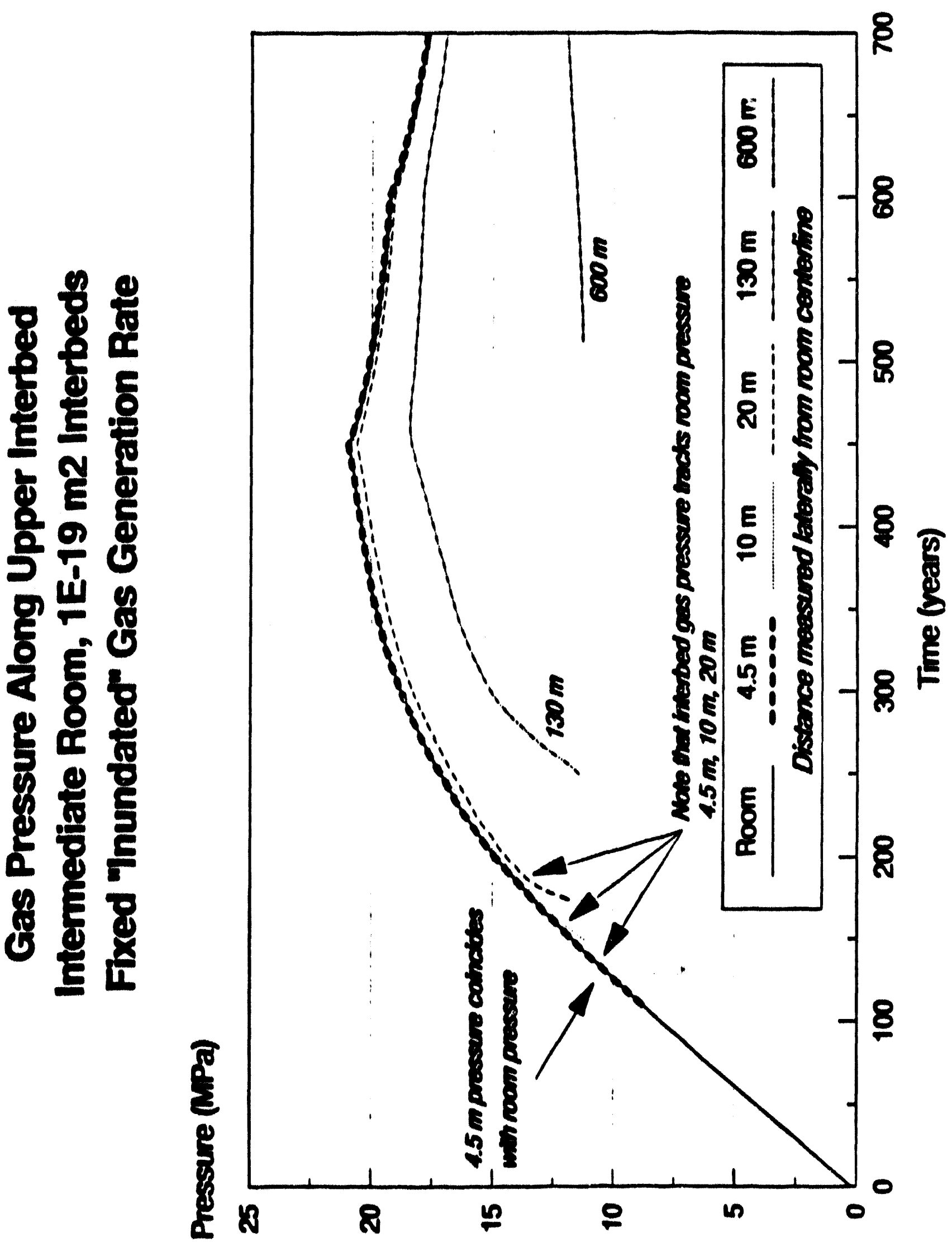
Figure 2.
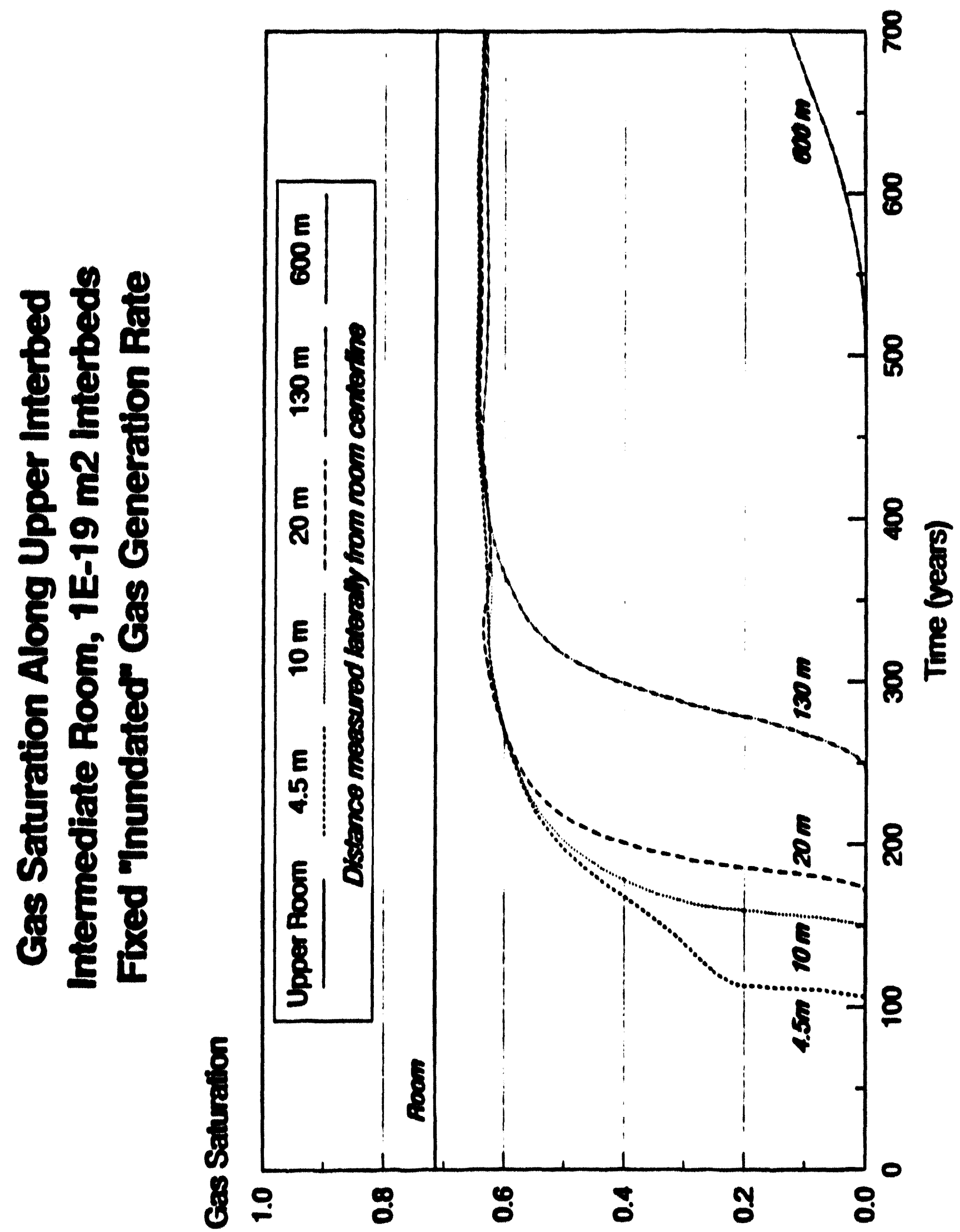
iren:

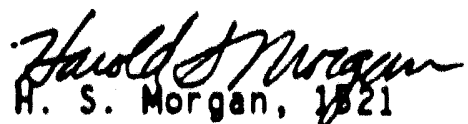

oubject: Estimate of the Time Needed for TRU Storage Rooms to Close

\section{INTRODUCTION}

An est imate of the time needed for TRU storage rooms to close is needed to answer varlous questions related to the performance assessment of the Waste Isolation Pllot Plant (WIPP). As a result, the creep response of a storage room has been computed for a simulation per lod of two hundred years. The results are presented here.

In order to obtain good est imates of the time needed for a room to close, the computed closures should agree with measured storage room response for the short time periods for which data are avallable. A recent calculation [1] showed that good agreement between computed and measured horizontal closure rates could be obtained when the WIPP reference secondary creep law is used with the reference lastic properties of halite divided by 12.5. However, using this procedure to get agreement between calculation and data leads to computed vertical closure rates which are still considerably less than the measured vertical closure rates. The WIPP reference stratigraphy was used in this calculation, and the anhydrite layers found therein were modeled as elastic layers with moduli equal to the WIPP reference values. This method of modeling the anhydrites had a significant effect on vertical closure in that the thick anhydrite layer directly beneath the floors of the storage rooms was inherently stiff, could not fail, and hence restricted the upward deformation. Thus, the elastic model produced anhydrite behavior which is inconsistent with the observed in situ behavior where the anhydrite layer in the floor falls early and provides little resistance to the movement of the surrounding salt. In order to obtain a better prediction of vertical closure for est imating the time of storage room closure, a more realistic model for the anhydrite layers was needed.

STORAGE ROOM MODEL

All anhydrite layers were replaced by salt in the model used in the analys is described here. The finite element mesh is shown in Figure 1. Most of the elements are concentrated around the room so that the large deformations expected to occur over the long time spans of interest can 
ari is 809

be captured. The small elements around the room also facilitate smooth interactions between the room surfaces. As the deformations get large, the floor. roof, and pillar will come into contact so over lap between - lements in the pillar and elements in the floor and roof must be prevented. This is accomplished by using the slide line capability in SANCHO [2], the computer code used for this and most WIPP structural calculations. The boundary conditions, loads, and initial stresses are the same as those used in the previous calculation [1] and are described in the parallel calculation report [3]. As in the previous calculation, the WIPP reference secondary creep law with the WIPP reference lastic constants divided by 12.5 are used. This adaptation of the WIPP reference creep model does not represent the resolution of the discrepancy found to exist between WIPP calculations and data but is only an expediency which can be used until the discrepancy is resolved. The storage room is empty throughout the simulation period. That is, the closure of the room is not affected by the presence of waste or backf 111 .

\section{RESULTS}

Deformations and deformation rates computed with the model described above are compared in Figures 2 - 7 to those computed in the original storage room calculation [1]. Removal of the anhydr ite layers from the model does not change the roof deformations appreciably over the fifty year time span shown in Figure 1, but the floor deformations, and hence the vertical closures in figure 4 , increase by a large amount. The pillar deformations in Figure 3 and horizontal closures in Figure 5 also increase when the all salt stratigraphy is used. Use of the all salt model produces vertical closure rates that are in good agreement with the measured vertical closure rates as shown in Figure 6 . Horizontal closure rates increase only slightly as shown in Figure 7 . Overall the deformations and deformation rates predicted with the all salt model are in better agreement with the avallable storage room data than are the deformations and deformation rates computed with the model that includes the stratigraphy. Thus, an estimate of closure based on the all salt model is probably as accurate as any that can be made at this time.

The time scale for the vertical closure histories in Figure 4 is extended to two hundred years in Figure 8 , and the history computed with the all salt stratigraphy indicates that the floor and roof will touch in approximately 195 years. (The storage room was originally $3.96 \mathrm{~m}$ $(13 \mathrm{ft}) \mathrm{high})$. The horizontal closure history is extended to 200 years in Figure 9, and the closure histories are plotted on logarithmic scales in Figures 10 and 11 . Logarithmic closure histories computed with the reference stratigraphy both with and without reduced lastic constants are also shown in Figures 10 and 11 . Deformed shapes of the storage room are shown at times of $0,10,50,100,125,150,175$, and 195 years in $F$ igures 12 - 19. These deformed shapes indicate that the volume of 
the room has been reduced significantly by 100 years. The volume reduction is quantified in Figure 20 where the volume loss of the room. expressed as a percentage of the original volume, is plotted versus time. The volume was computed from the displacements around the opening with the VOLCAV program [4]. At approximately 44 years, the volume of the storage room has been reduced by $50 \%$, and at 100 years the volume loss is about $76 \%$.

\section{CONCLUSIONS}

The use of a storage room model with no stratigraphy (all salt) and with - constitutive model for salt consisting of the WIPP reference creed model and reduced lastic properties produces both vertical and horizontal closure rates in good agreement with closure rates measured in the SPDV test panel. Based on this model, the storage rooms will close in approximately 195 years with volume reduction of $76 \%$ occurring in 100 years. These predictions are made without modeling the waste or backfill which will be placed in the rooms. If additional calculations are needed, a more complicated model which includes both the waste and backfill should be used. The model also does not account for fallures in the salt, such as slabbing and layer separations, which could affect the predictions.

\section{REFERENCES}

1. Morgan, H. S., "SPOV Test Room Calculation with Reduced Elastic Moduli," Memo to D. E. Munson, 6332. Sandia National Laboratories. Albuquerque, NM. March 25, 1987.

2. Stone. C. M., Krieg, R. D., and Beisinger, 2. E.. "SANCHO - A Finite Element Computer Program for the Quasistatic. Large Deformation. Inelastic Response of Two-Dimensional Solids, "SAND84-2618, Sandia National Laboratories. Albuquerque. NM. April 1985.

3. Munson, D. E. and Morgan, H. S., "Methodology for Performing Parallel Design Calculations (Nuelear Waste Repository Application)." SAND85-0324, Sandia National Laboratories, Albuquerque. NM, May 1986.

4. Preece, D. S.. "VOLCAV," unpublished computer program to compute cavity volume from cavity displacements. Sandia National Laboratories. May 1987.

HSM: 1521: tbh 


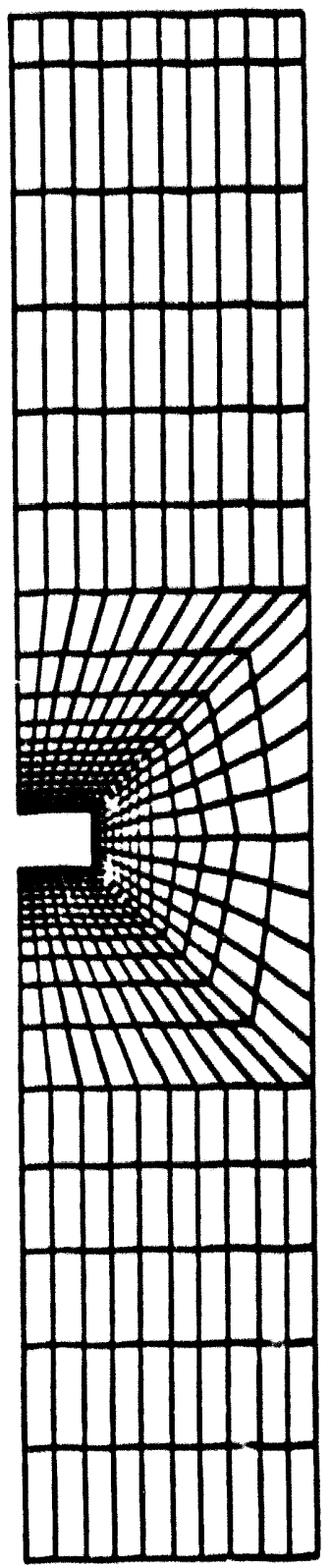

Figure 1. All Sale Mesh for TRll Storage Room 


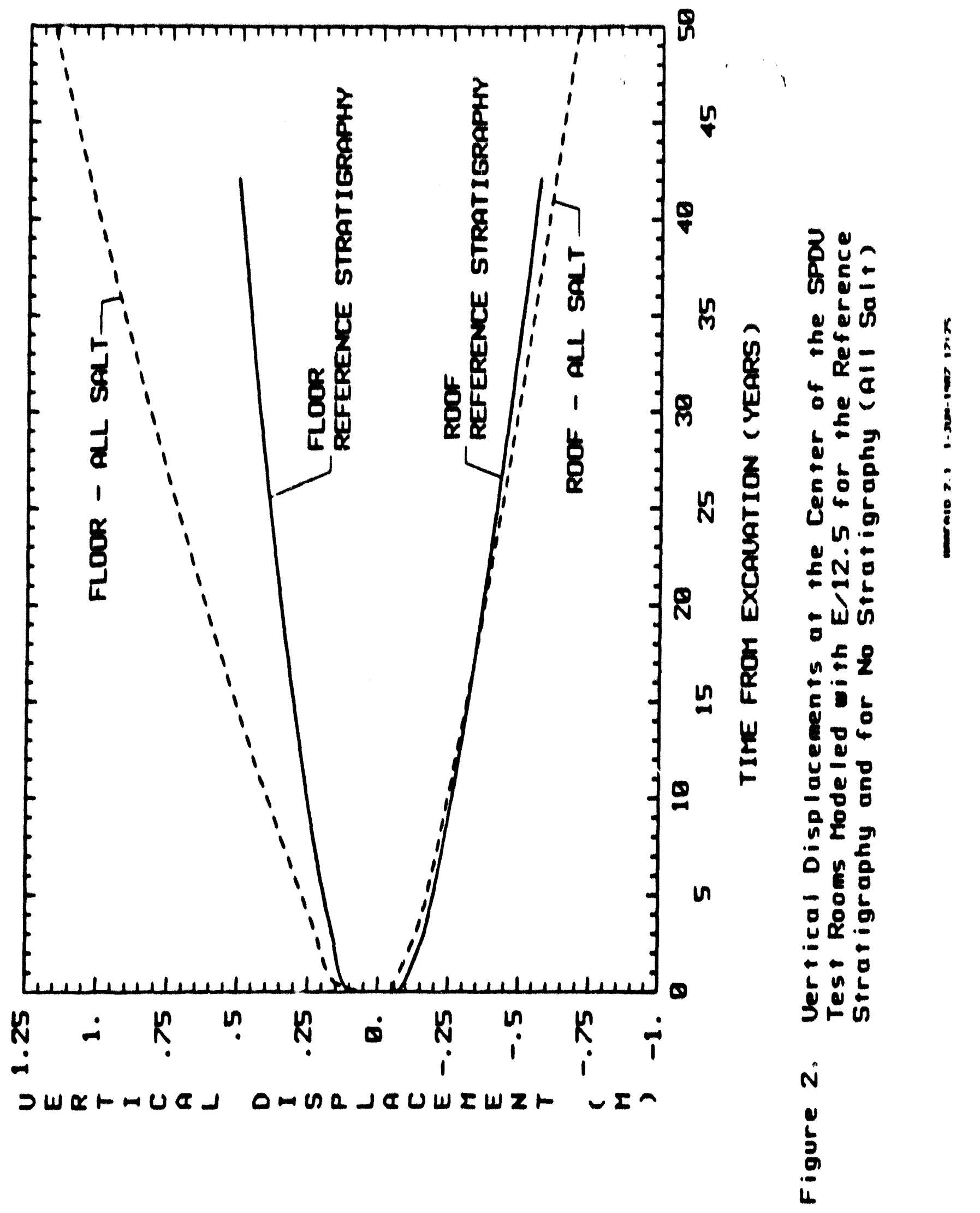




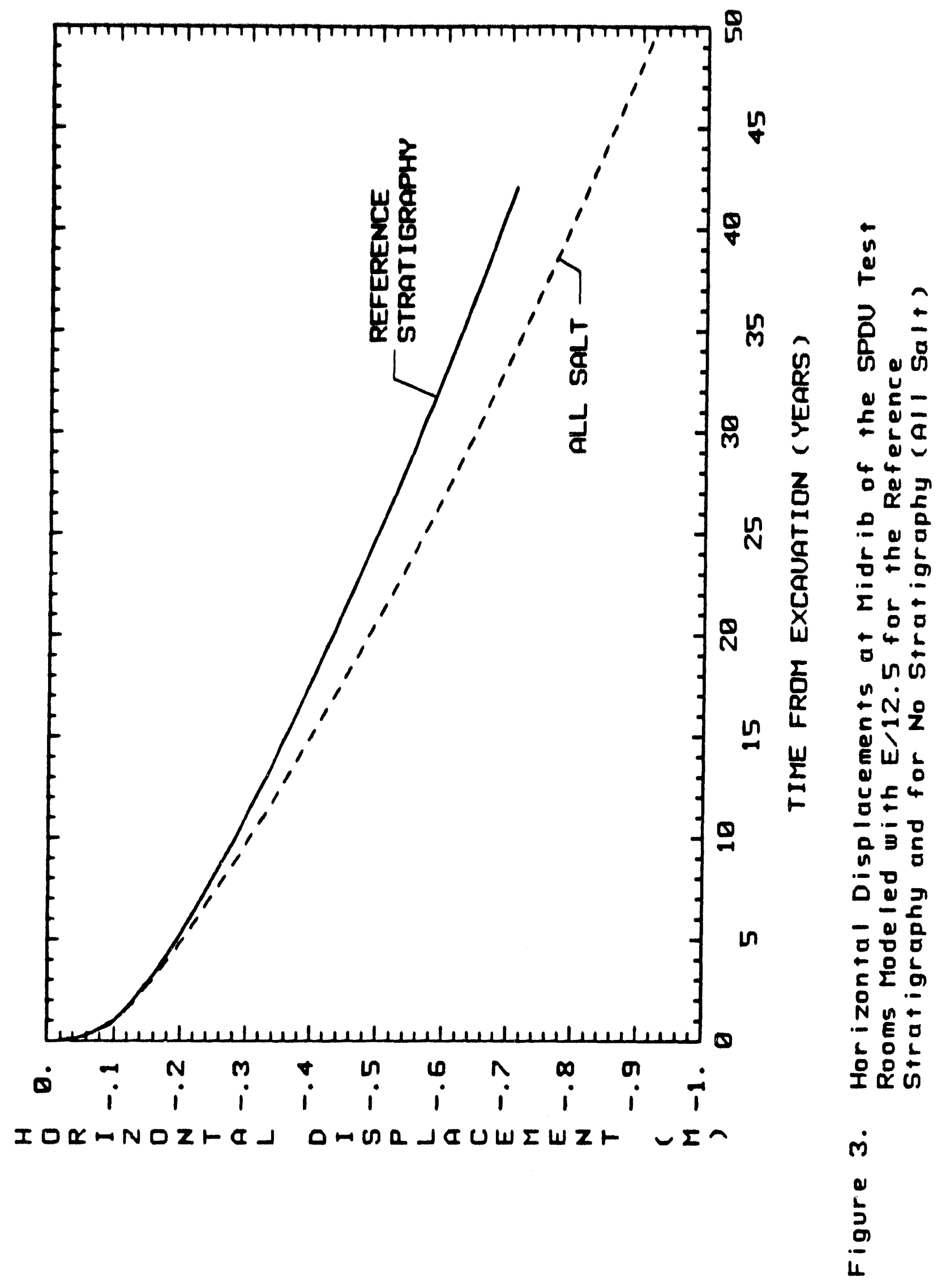




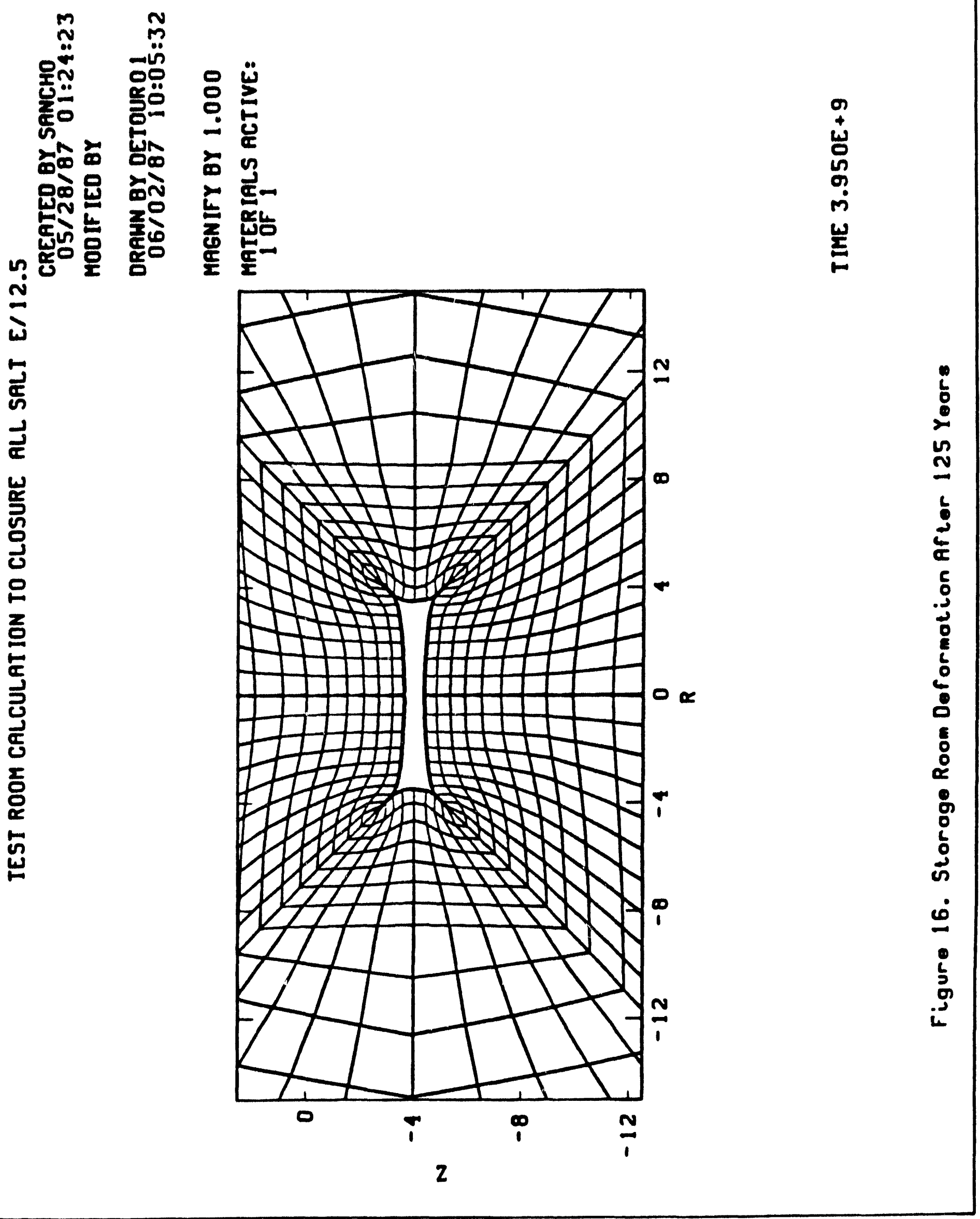




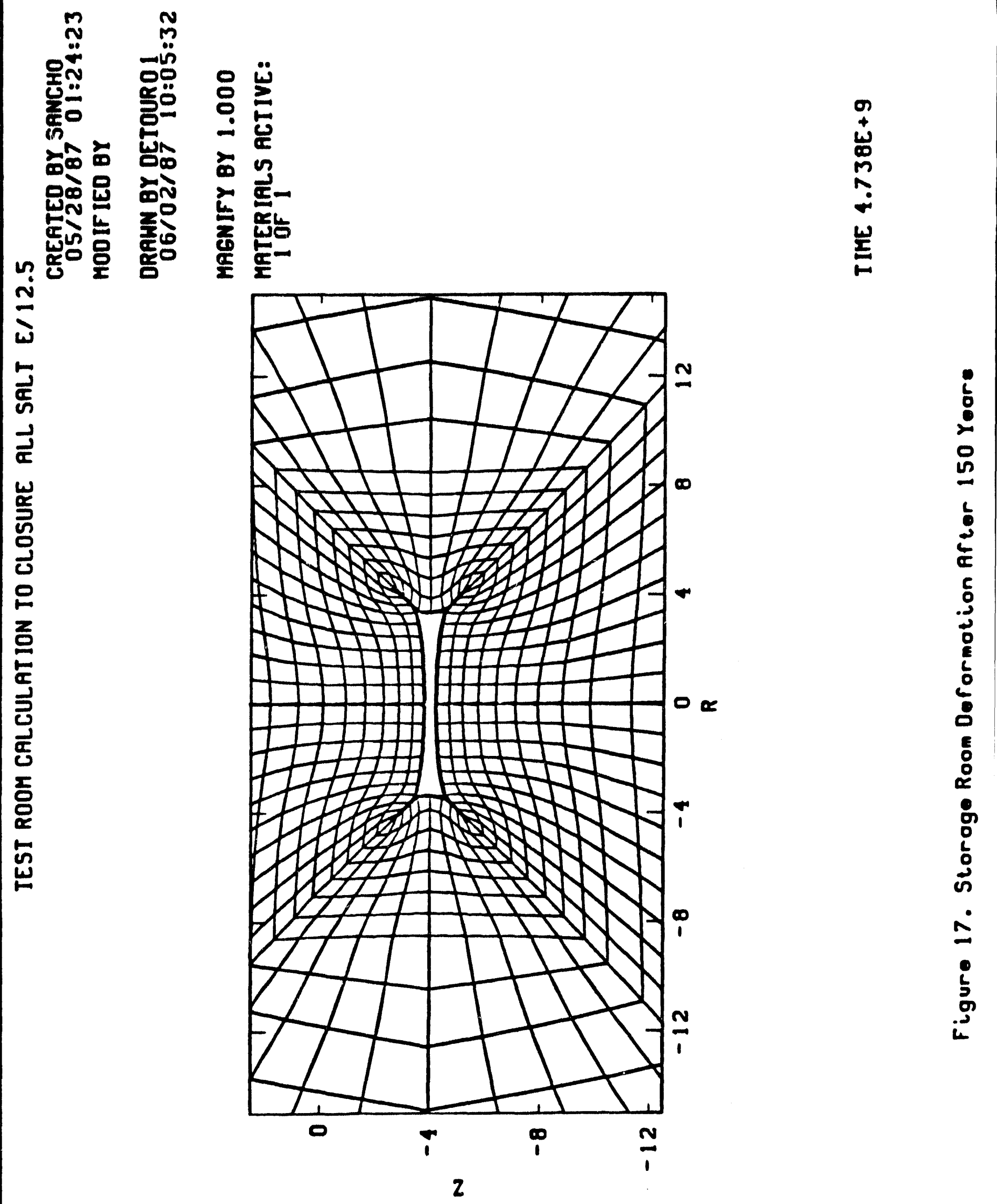



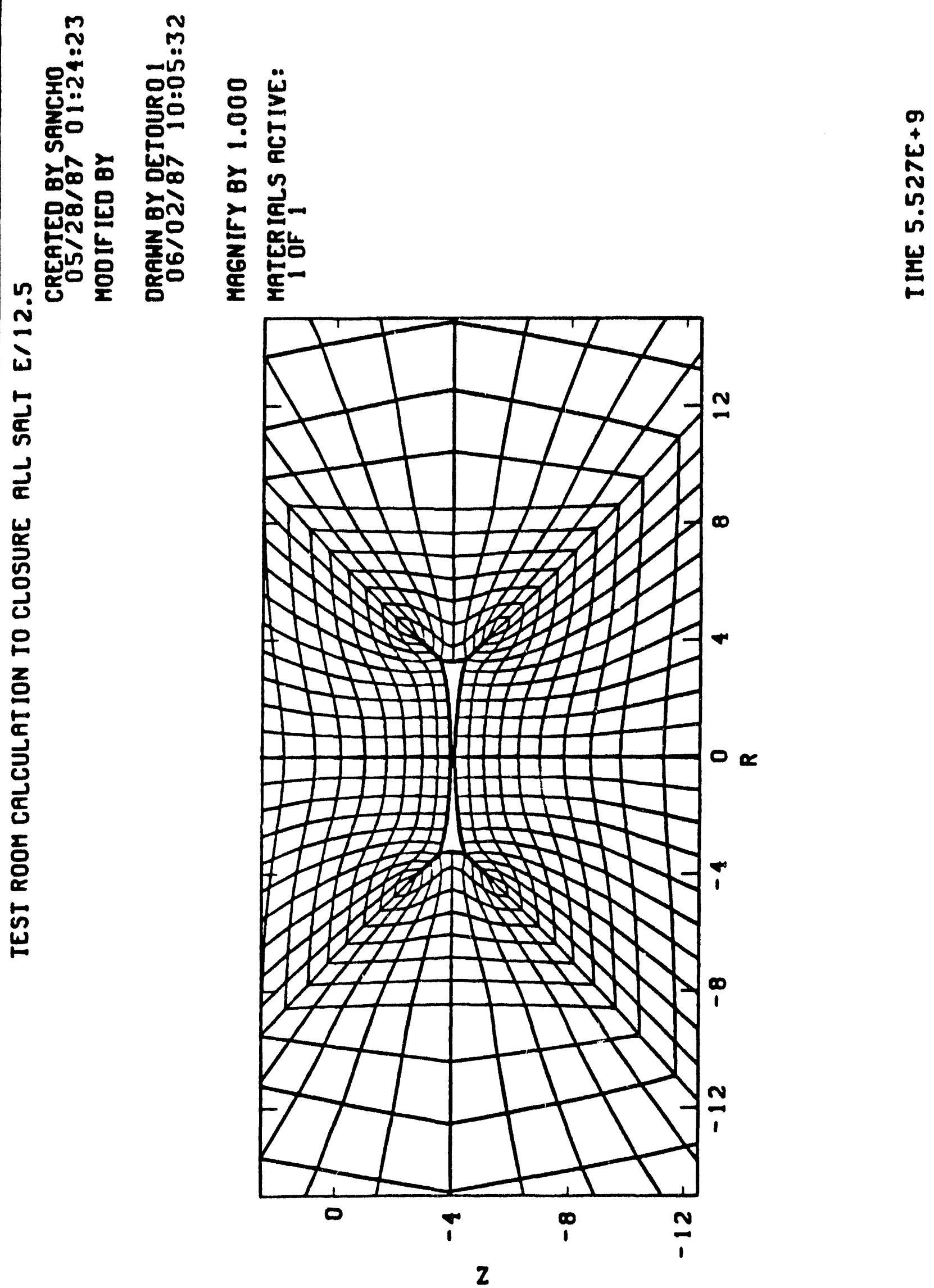

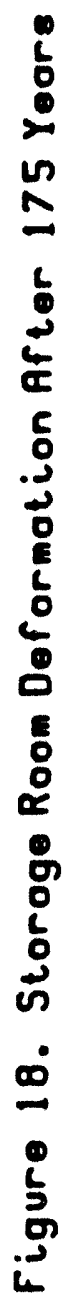




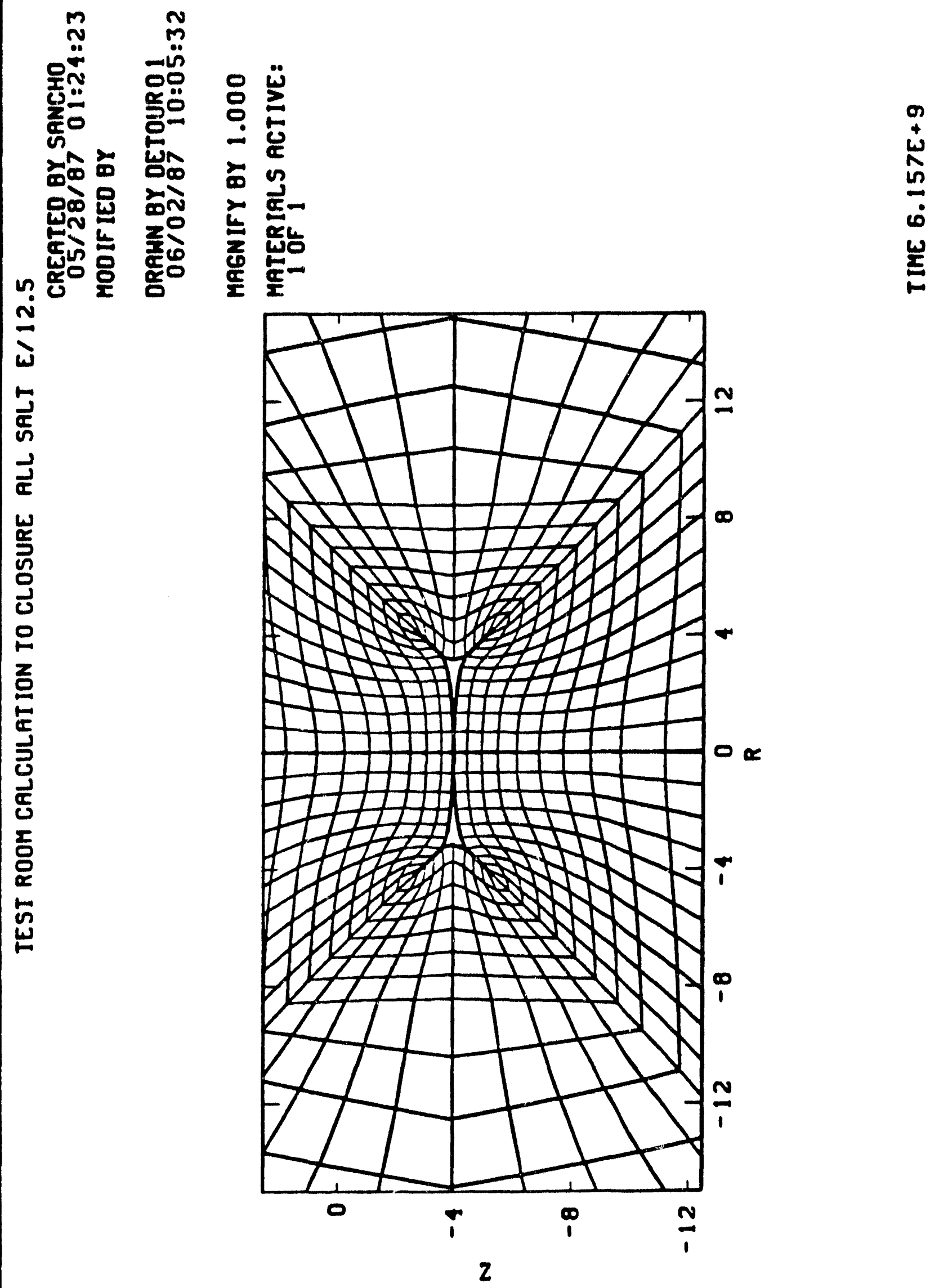

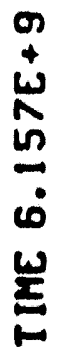




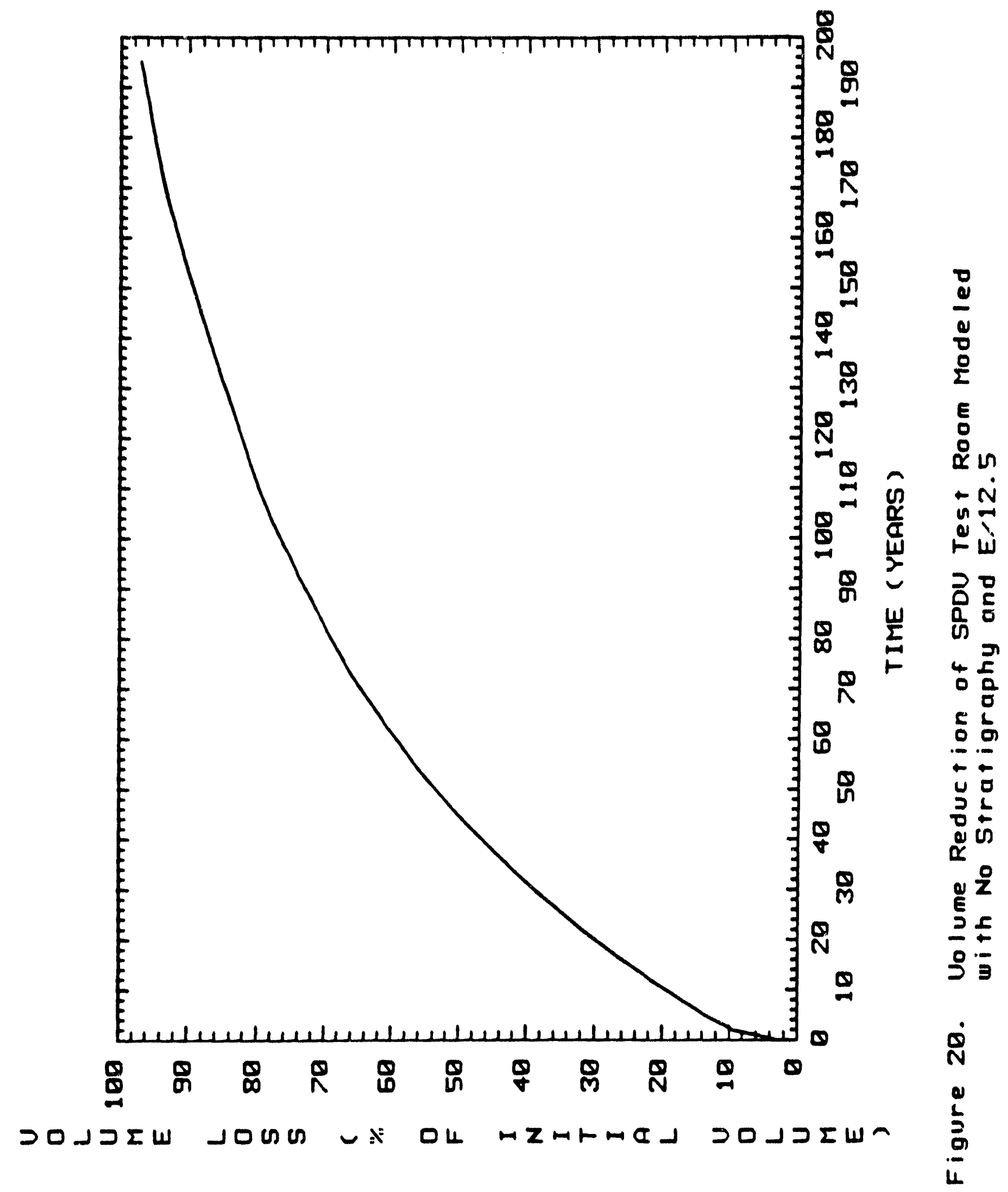




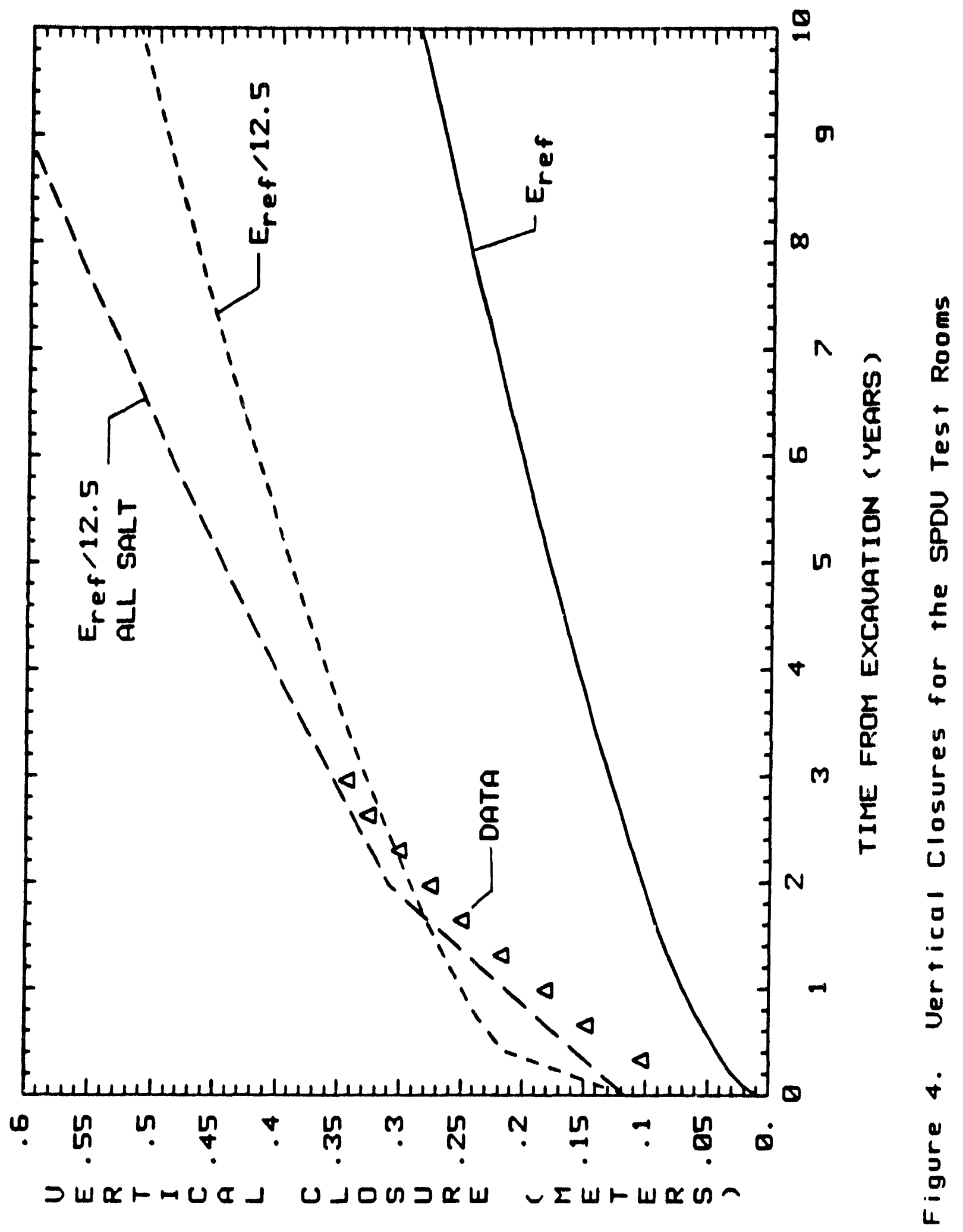




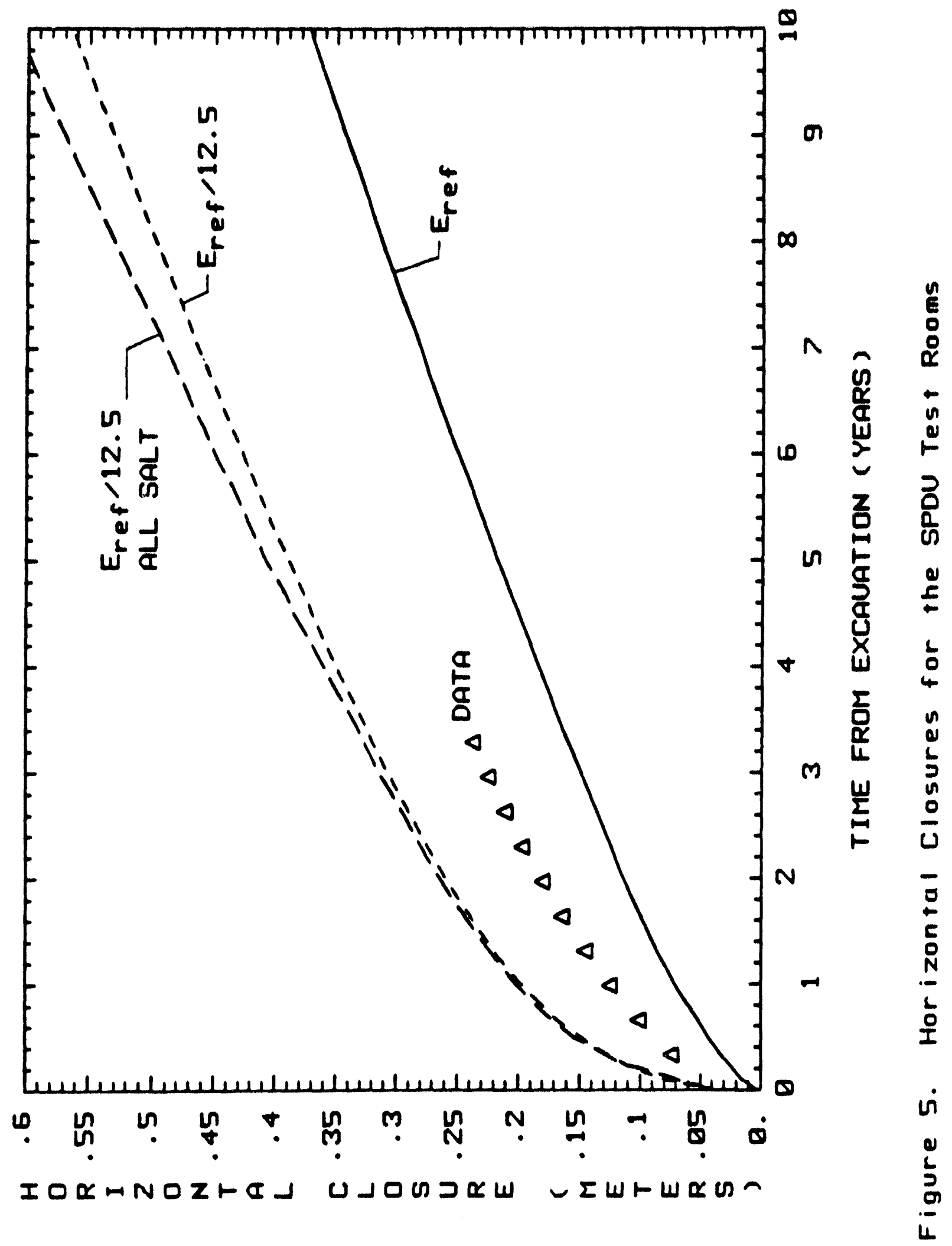




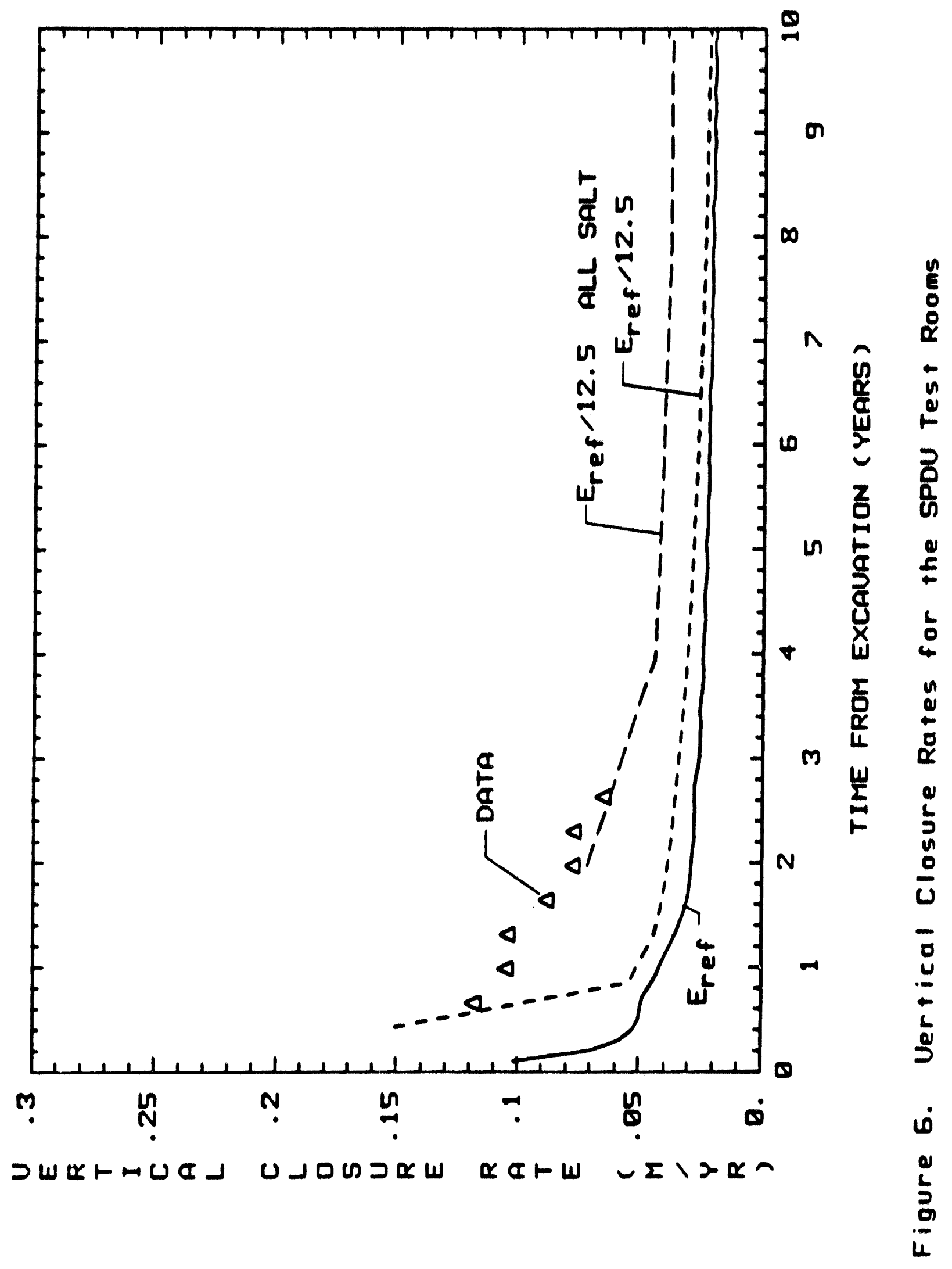




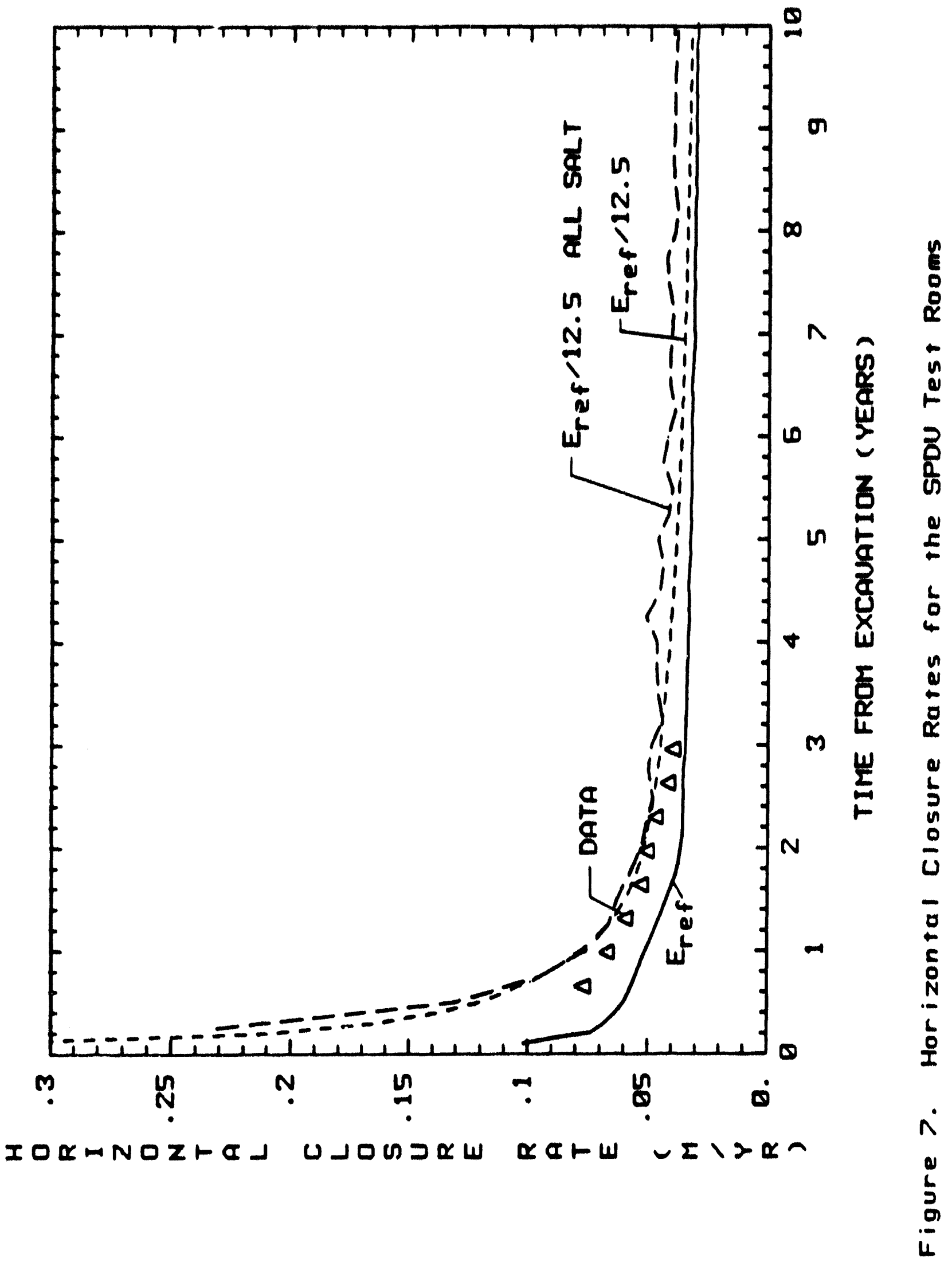




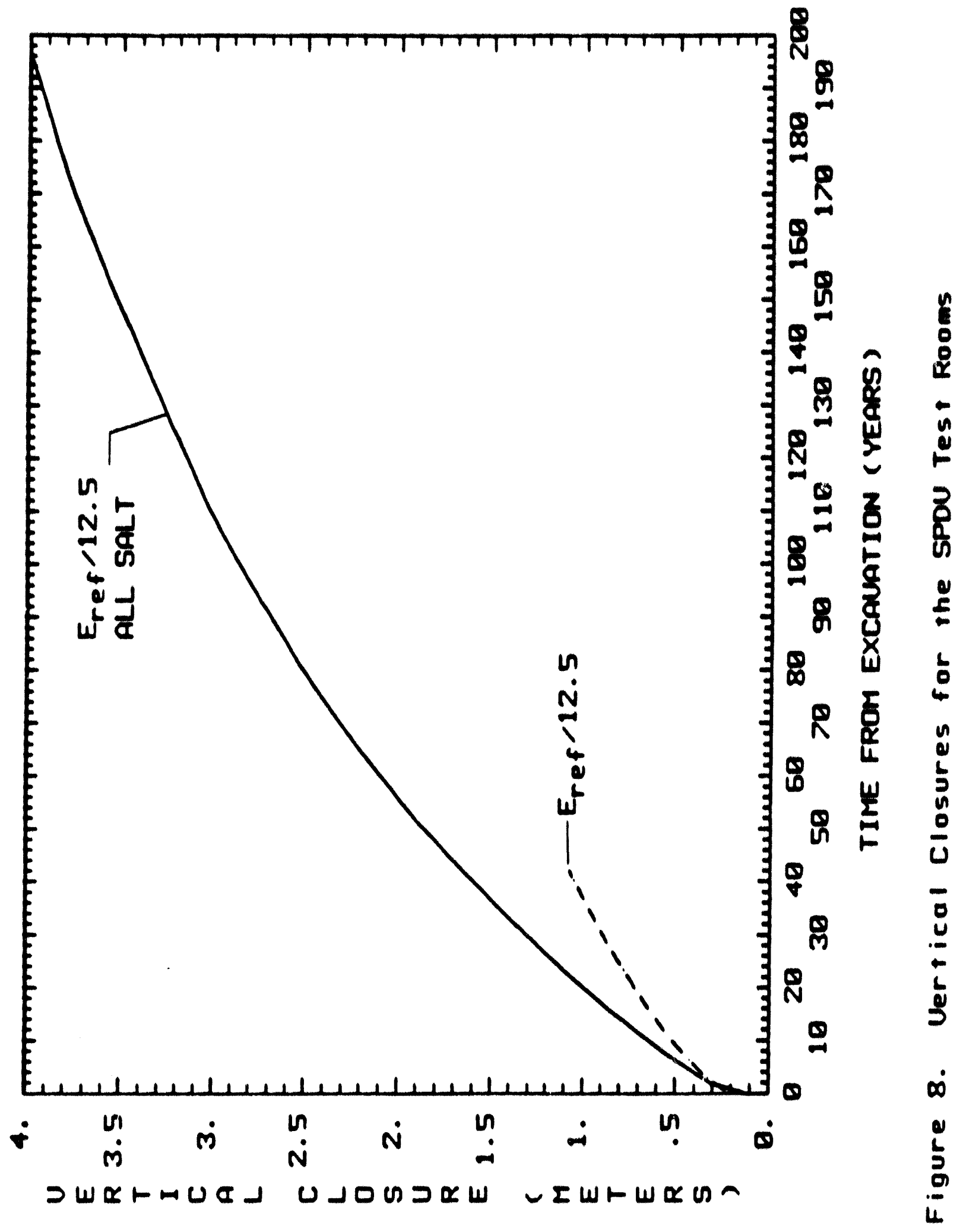




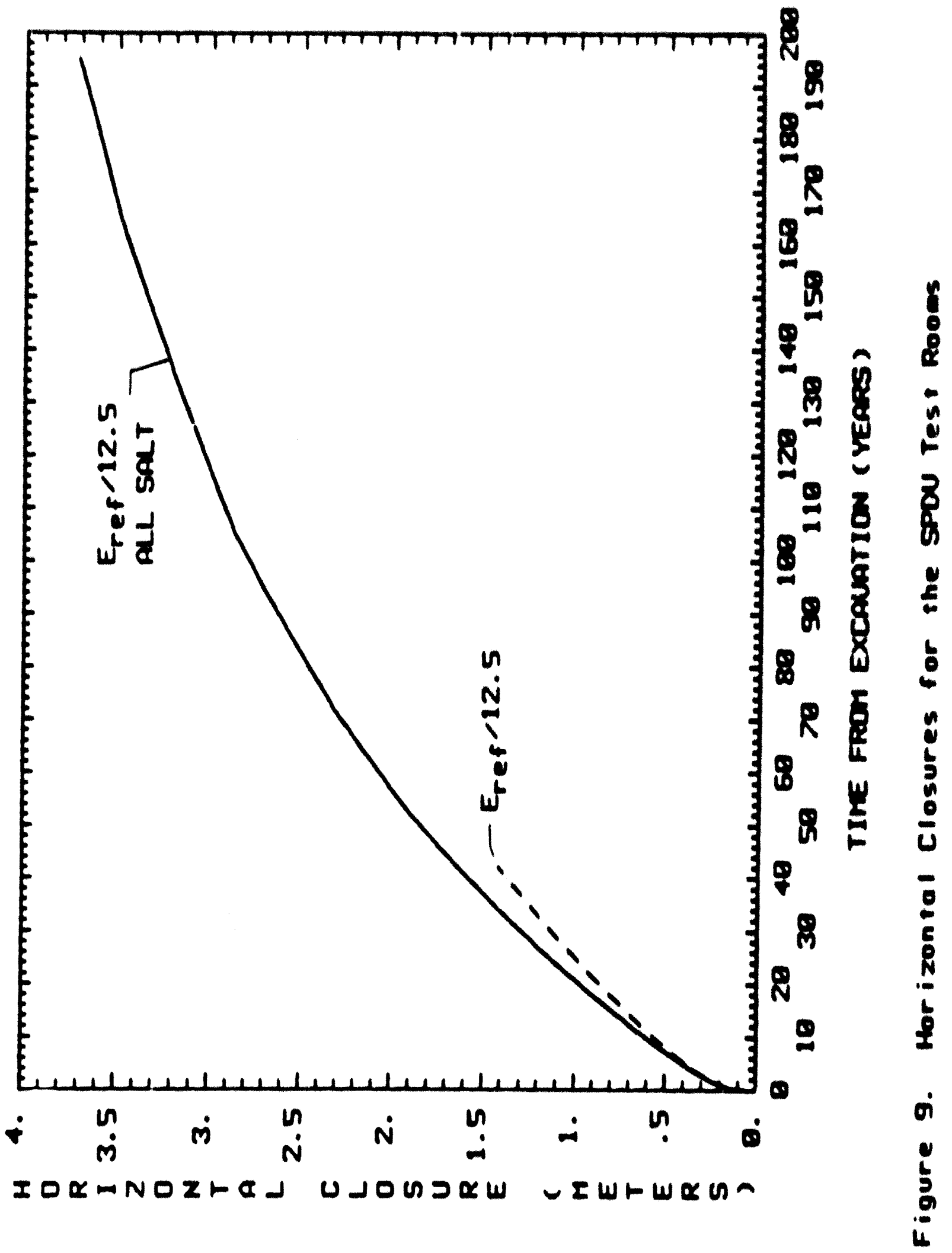




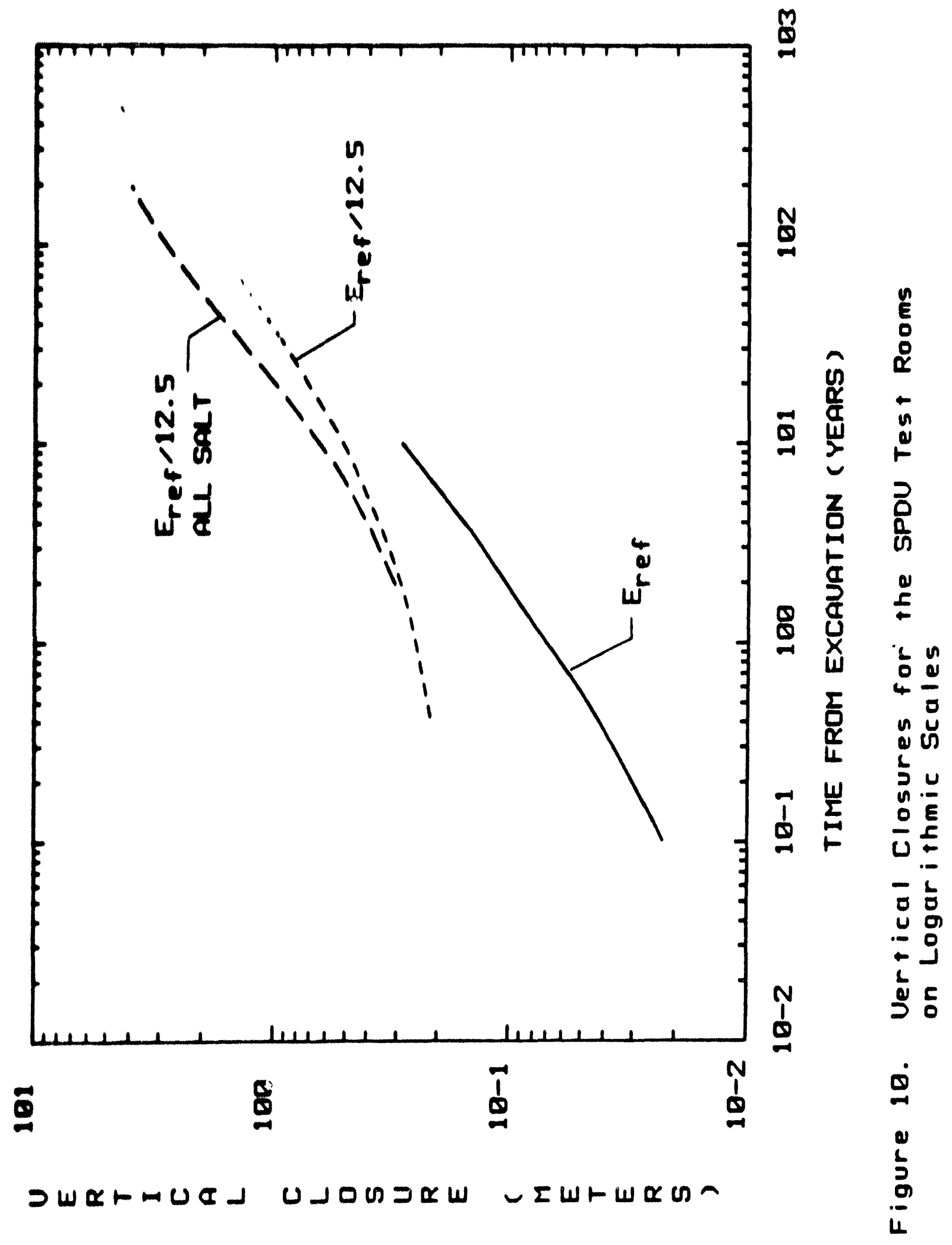




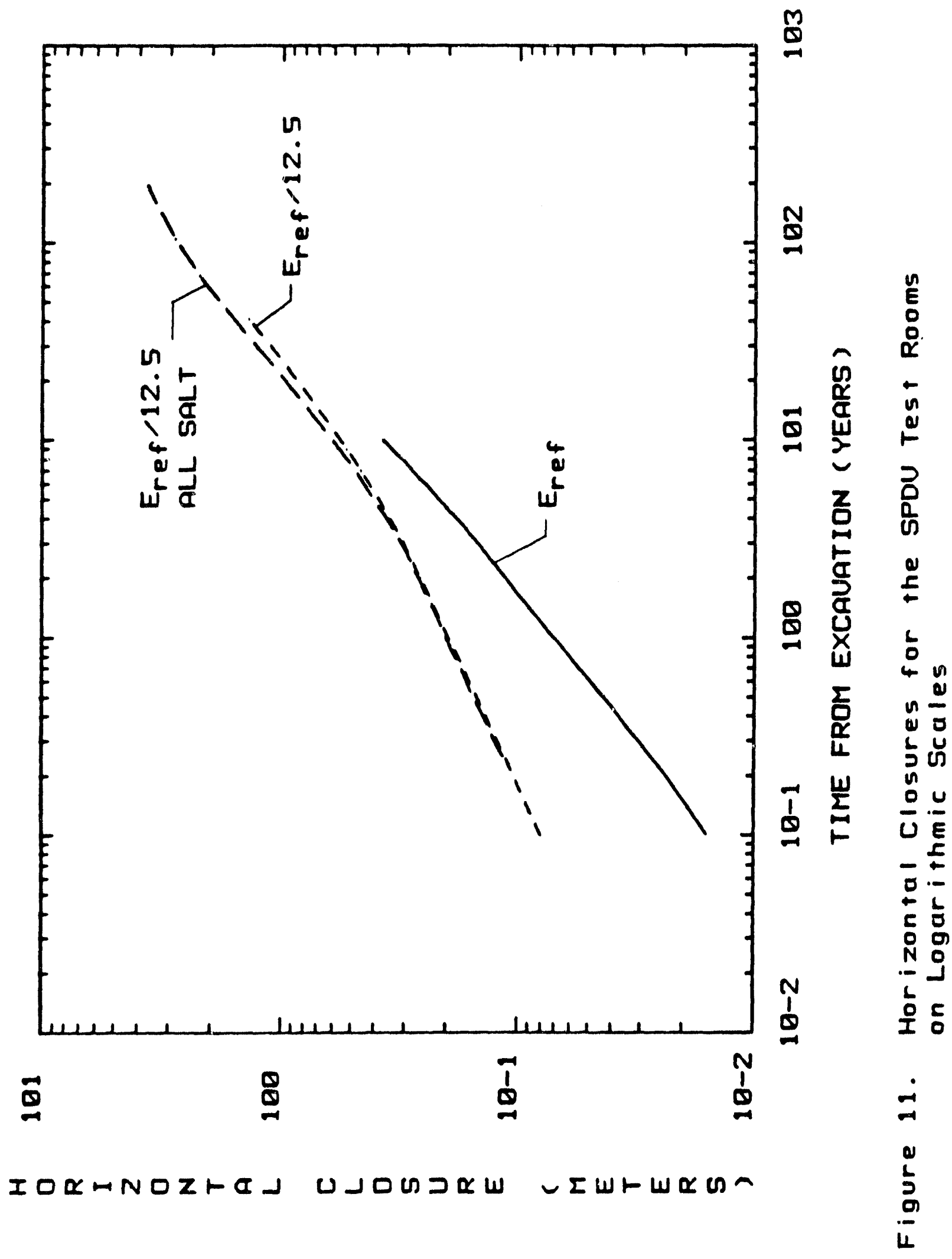




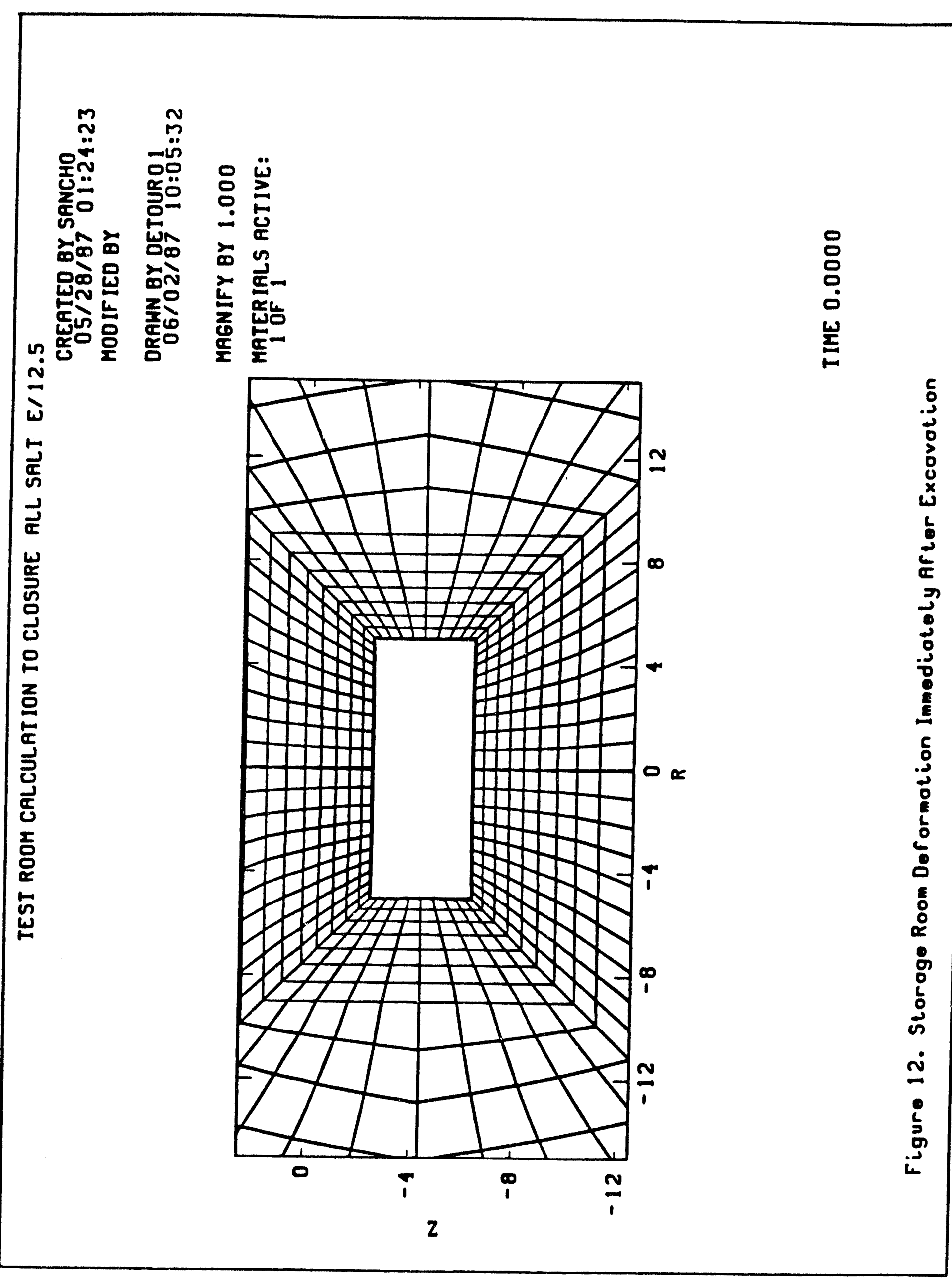



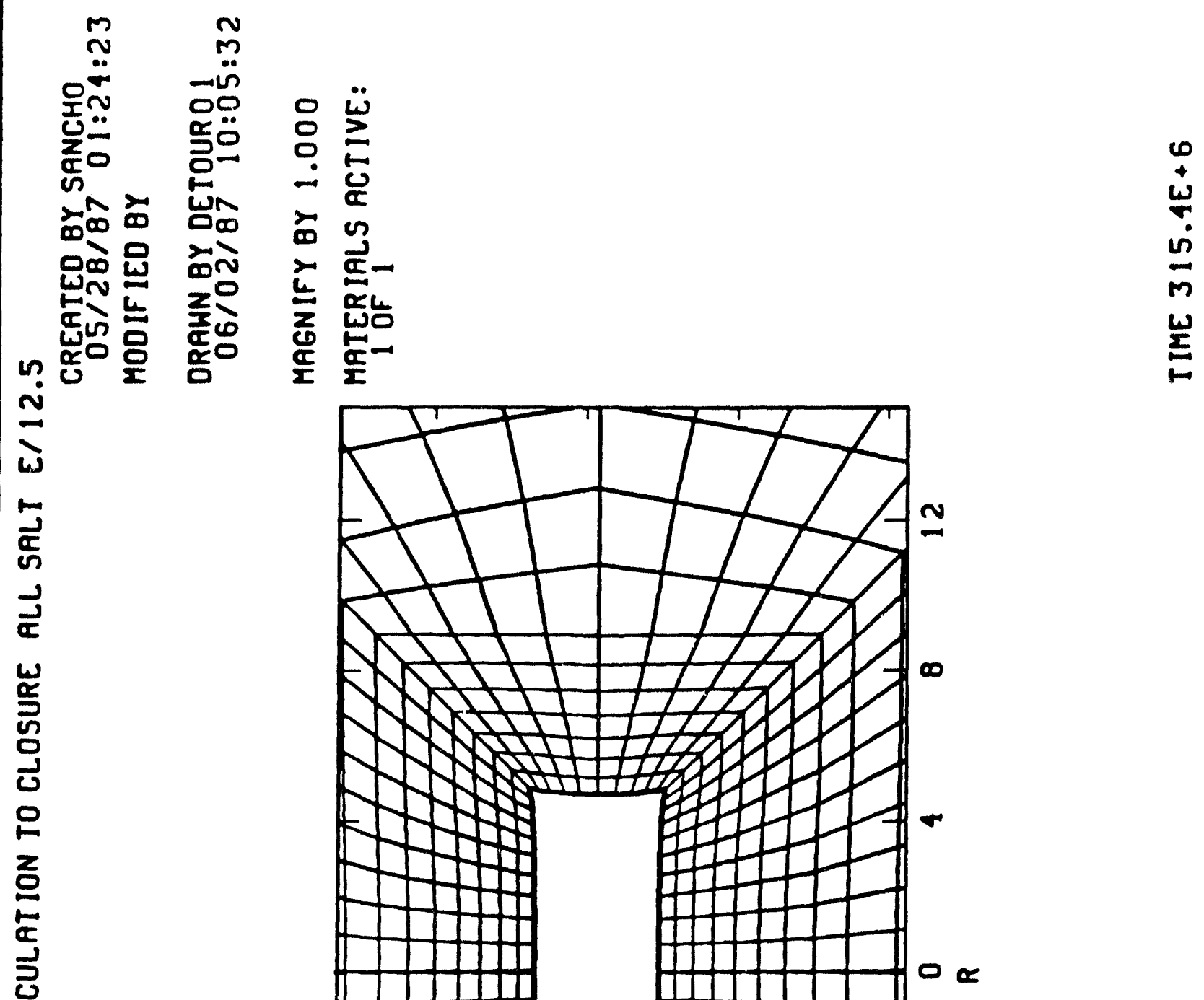

0
0
0
0
0
6
0
0
0
0
0
0
8
0
0
0
0
0
0
0
0
0
0
0
0
0
0
0
0
0
0
0
0
0
0
0 


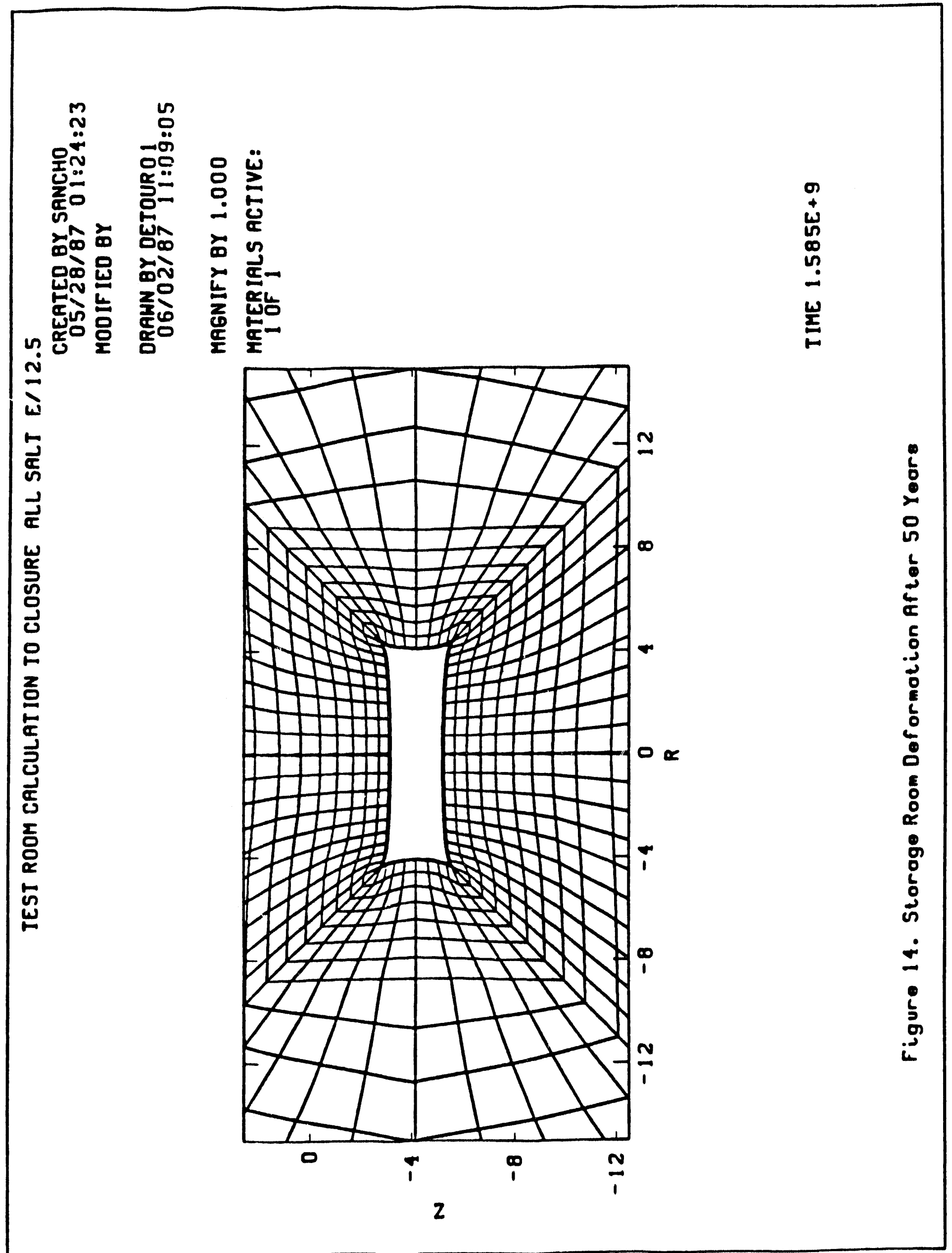




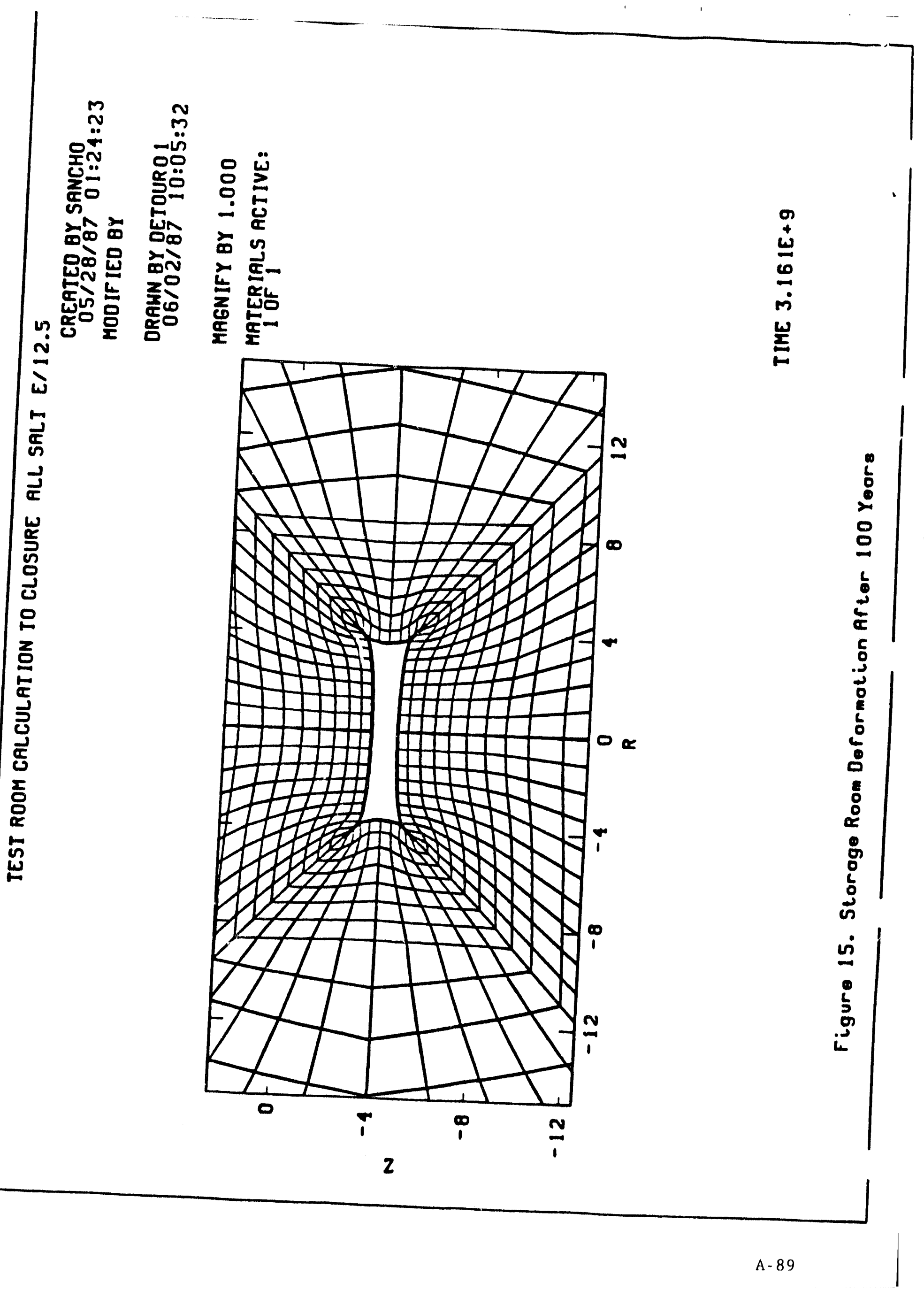


arto: December 9, 1987

so: D. E. Munson, 6332

irom:

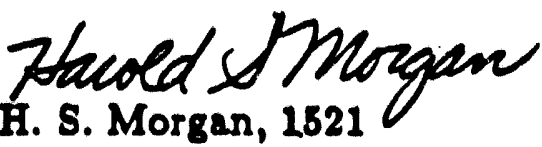

3ubjoct: TRU Storage Room Calculation with Stratigraphy

\section{INTRODUCTION}

A calculation to determine the creep response of a WIPP storage room for transuranic (TRU) wastes has been completed to a simulation time of 115 years. The stratigraphic layering around the room is included in this calculation with the anhydrite layers and the o.. polyhalite layer modeled as plastic materials.with pressure dependent deviatoric yield strengths defined by the Drucker-Prager yield criterion. The constants for the DruckerPrager model are variations of the WIPP reference values [1] and have been used for various other WIPP calculations [2-5]. The halite layers were modeled with the WIPP reference secondary creep law [1] and with the WIPP reference elastic modull divided by 12.5 .

This latest geomechanical model is the fourth to be applied to the TRU rooms in the past few years. In the first model [6], the anhydrites were treated elastically, and the WIPP reference secondary creep model, with reference values for the elastic constants, was used to characterize the halite layers. Measured TRU room closures and closure rates, both vertical and horizontal, were at least three times larger than those computed with this original model. Similar results had been found for the Snuth Drift [7], and an in-depth study of the many parameters affecting closure of the South Drift indicated that changing the elastic moduli used with the WIPP reference secondary creep law could produce large increases in both drift closures and closure rates (7). A subsequent study showed that dividing the reference Young's modulus by $\mathbf{1 2 . 5}$ would produce horizontal closures and closure rates for the South Drift that were in good agreement with measurements [8]. As a result, in the second model of the TRU room [0], the reference elastic properties for the halite layers were divided by 12.5 in an attempt to improve the agreement between measured and predicted closures. The anhydrite and polyhalite layers were still treated as elastic materials. Horizontal closures and closure rates computed with this second model were in reasonably good agreement with measured values, but agreement was not as good in the vertical direction. Observations of the anhydrite layers around the roome at the WIPP aite, most notably Marker Bed 139, indicated that the anhydrites were cracked and probably provided little reaistance to the flow of adjacent salt layers. Consequently, in the third geomechanical model of the TRU room [10), the stratigraphy was omitted, and all of the rock around the room was modeled as halite, which was characterized by 
the WIPP reference secondary creep law with the reference elastic properties divided by 12.5. This model produced both vertical and horizontal closure rates that were in good agreement with the data. This model was used to determine that a TRU room would be $76 \%$ closed in 100 years and $98 \%$ closed in 195 years. These closure predictiona represent "best" estimates in the sense that the closures and closure rates agree with data for as long a data are available. The lateat geomechanical model, described in this memo, was used to determine a more conservative bound on the time needed for a TRU room to close.

\section{MODEL DESCRIPTION}

The geometrical configuration, boundary conditions, initial conditions, and stratigraphy for the TRU room calculation described here are indentical to those used in the earlier TRU room calculations. A detailed problem description is provided in [6] and will not be repeated here. The reference secondary creep law for the halitic layers is described in [1]. The elastic modull for hallte, which were used in this analysis, are the reference values reported in [1] divided by 12.8. The Drucker-Prager constants for anhydrite and polyhalite are presented in [6]. Clay seams in the stratigraphy were treated as slide lines with coefficients of friction of 0.4 . Slide lines were also used along the floor, roof, and wall of the room to prevent these surfaces from overlapping when the deformations became large.

The calculation was terminated at a simulation time of 118 years because of computational expense. To that point the calculation had taken 46 hours on the CRAY-1S and had cost in the neighborhood of 820,000 . The earlier all-salt calculation in which the stratigraphy had been omitted was run to completion, that is, to a simulation time of 200 years, in leas than 6 hours. The increased expense of the new calculation is due primarily to the fact that small time steps had to be taken for the solution to converge. The slow convergence was caused by the large arnount of sliding that takes place along some of the clay seams. The calculation can be resumed from its termination point if necessary.

\section{RESULTS}

Floor, roof, and wall displacements computed in the new calculation are compared in Figures 1 and 2 to those computed with the earlier models of the TRU room. These results Indicate that the displacements obtained by modeling the anhydrites with a Drucker-Prager model are easentially the same as those obtained by modeling the anhydrites elastically. The reason for this is that all of the anhydrite layers are located either above or below the room where they have horizontal confinement and hence large pressures and deviatoric yield atrengths. If anhydrite layers had been located in the wall of the room, as in the cases of Room $A$ and $B$, the pressures in these anhydrites intersecting the room would have been much lower; the layern would bave yielded $[2-3]$; and the reaults obtained with the Drucker-Prager model for anhydrite would have been quite difierent from the results 
obtained with elastic anhydrites.

Vertical and horizontal closure histories are shown in Figures 3 and 4, respectively; closure rates are provided in Figures 5 and 6; and logarithmic plots of tine closures are shown in Figures 7 and 8 . As shown in Figure 5 , the vertical closure rates predicted in the new calculation with the Drucker-Prager anhydrites are much less than the measured rates. As a result, "time to closure" (the time needed for a TRU room to close completely) predictions arising from this model should be extremely conservative. For all practical purposes, the room is closed when the floor touches the roof. The earlier all-salt calculation [10] indicated that when the foor touched the roof at a simulation time of 195 yeare the room had lost $98 \%$ of its initial volume. A "time to closure" estimate based on the calculation with the Drucker-Prager model for anhydrite can be extrapolated from the logarithmic vertical closure history in Figure 7. An extrapolation, based on the assumption that the new bistory has the same shape as the closure history obtained with the all-salt model, results in a "time to closure" of between 400 and 500 years. This bounding prediction is much larger than the "best" estimate of 195 years obtained with the all-salt model.

The bounding condition does not look near as severe, hn;ever, when room volume loss after 100 years is considered. Volume loas, expressed as a percentage of the initial TRU room volume, is plotted as a function of time in Figure 9 . At 100 years, the "best" estimate obtained with the all-salt model is a volume loss of $76 \%$. The bounding value obtained with the Drucker-Prager anhydrite nodel is a volume loss of $86 \%$. This means that volume reduction of the room should be no leas than $36 \%$ in 100 years, but it could be as high as 76\%. A volume reduction in this range should be sufficient to remove all of the void space initially contained in the backfill and in the drums partially filled with TRU waste.

Deformations around the TRU room are shown in Figures 10-12 for simulation times of 10, 80, and 100 years, respectively. Also shown in each of these figures are deformations computed in an all-salt calculation with the same mesh as was used in the calculation with the Drucker-Prager anhydrites. These deformed shapes clearly show, as do the displacement histories in Figure 1, that the main difference in the two calculations is the floor response which is governed primarily by the material model assumed for the anhydrite in Marker Bed 139. Treating the anhydrite layers, especially Marker Bed 139, as homogeneous, intact layers, with either an elastic or a Drucker-Prager model, ignores the pervasive cracks observed in the anhydrite and is probably valid only in a bounding calculation such as the one presented here. Ignoring the anhydrites completely, as in the all-salt model, is not completely valid either, but the cracked anhydrites probably do provide almost no resistance to the creep of the surrounding salt.

\section{CONCLUSIONS}

An upper bound estimate of the time needed for a TRU storage room to close can be obtained from the computed TRU room response described in this memo. The anhydrite layers are treated with a Drucker-Prager plasticity model, and the WIPP reference sec- 
ondary creep model, with the reference elastic moduli divided by 12.5, is used to represent the behavior of the halite layers. Although the calculation was terminated after a simulation time of 115 years, when the room had lost $56 \%$ of its initial volume, a "time to closure" eatimate of between 400 and 800 years was obtained by extrapolating the vertical closure history. This estimate is almost as conservative as the estimate that would have reaulted by treating the anhydrites w elantic layers. It is also much larger than the earlier "beat" estimate of 195 years which is based on an all-salt model in which the "atrength" of the cracked anhydrite is modeled to be much lower. The $86 \%$ volume reduction at 100 yeare is a reasonable lower bound on the "best" eatimate of $76 \%$ obtained with the all-salt model. Better bounds can only be obtained with a better model for anhydrite behavior.

\section{REFERENCES}

1. R. D. Krieg, Reference Stratigraphy and Rock Properties for the Wrste Isolation Pilot Plant (WIPP) Project, SAND83-1908, Sandia National Laboratories, Albuquerque, NM, January 1984.

2. H. S. Morgan and C. M. Stone, Pretest Reference Calculation for the Overtest for Simulated Defense High Level Waste (WIPP Room B In Situ Experiment), SAND850213, Sandia National isaboratories, Albuquerque, NM, April 1985.

3. H. S. Morgan and C. M. Stone, Pretest Reference Calculation for the $18 \mathrm{~W} / \mathrm{m}^{2}$ Mockup for Defense High Level Waste (WIPP Room A In Situ Experiment), SAND850807, Sandia National Laboratories, Albuquerque, NM, May 1985.

4. H. S. Morgan and C. M. Stone, Preteat Reference Calculation for the 6.1 Meter ( $80 \mathrm{ft}$ ) Wide Drifts of the Geomechanical Evaluation (WIPP Room G In Situ Experiment), SAND85-0893, Sandia National Laboratories, Albuquerque, NM, June 1985.

3. H. S. Morgan and C. M. Stone, Pretest Reference Calculation for the Heated Axisym. metric Pillar (WIPP Room $H$ In Situ Experiment), SAND85-1146, Sandia National Laboratories, Albuquerque, NM, August 1985.

6. D. E. Munson and H. S. Morgan, Methodology for Performing Parallel Design Calculations, SAND85-0324, Sandia National Laboratories, Albuquerque, NM, May 1986.

7. H. 8. Morgan, C. M. Stone, and R. D. Krieg, An Evaluation of WIPP Structural Modeling Capabilities Based on Comparisons with South Drift Data, SAND85-0323, Sandia National Laboratories, Albuquerque, NM, November 1986.

8. G. D. Sjaardema and R. D. Krieg, A Constitutive Model for the Consolidation of WIPP Crushed Salt and Its Use in Analyses of Backfilled Shaft and Drift Configurations, SAND87-1977, Sandia National Laboratories, Albuquerque, NM, October 1987. 
9. H. S. Morgan, "SPDV Teat Room Calculation with Reduced Elastic Moduli," Memo to D. E. Munson, 6332, Sandia National Laboratories, Albuquerque, NM, March 25, 1087.

10. H. S. Morgan, "Estimate of Time Needed for TRU Storage Rooms to Close," Memo to D. E. Munson, 6332, Sandia National Laboratories, Albuquerque, NM, June 2, 1987.

HSM:1321:hsm 


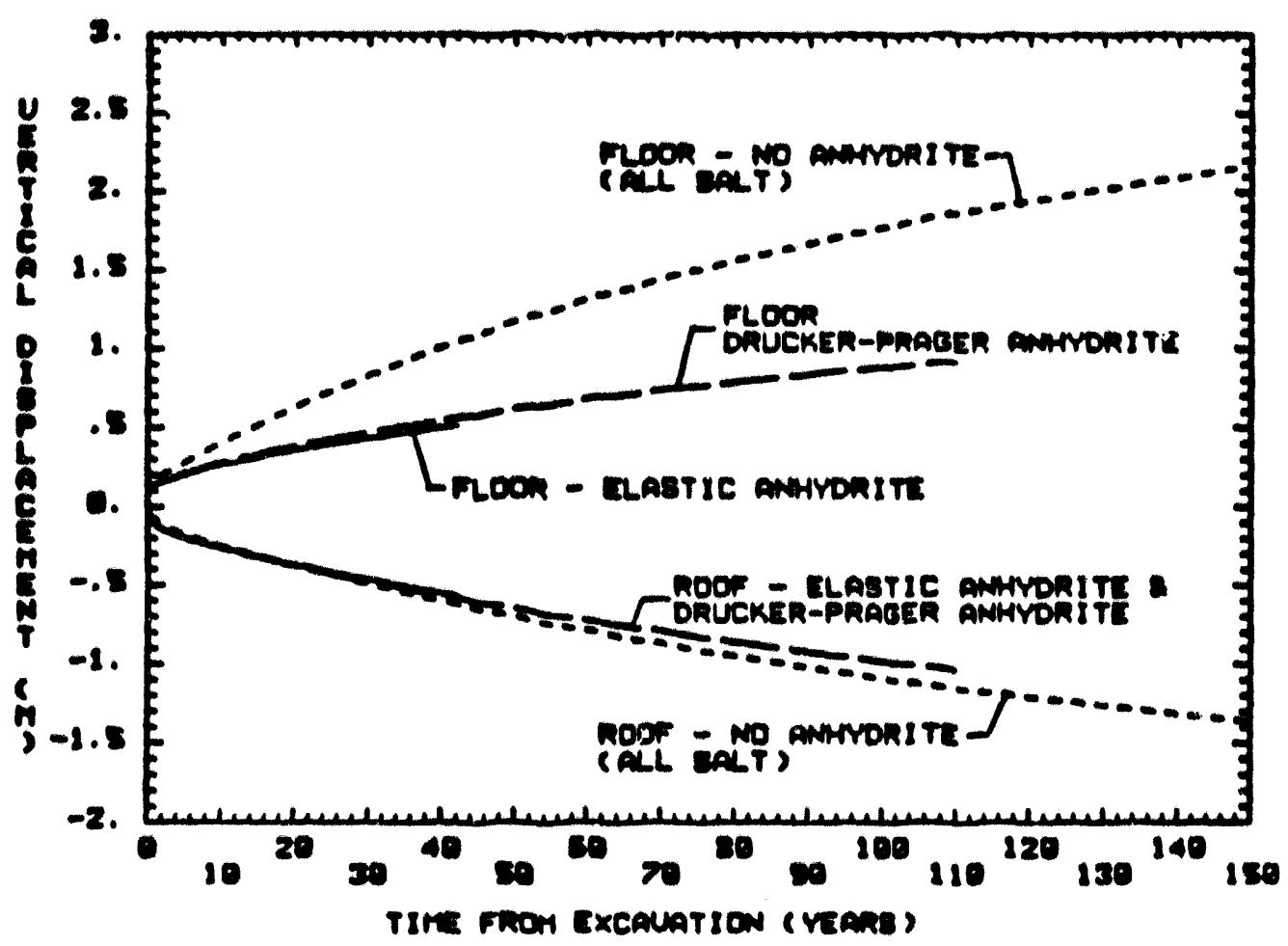

Figure 1: Vertical Displacements at the Center of a TRU Storage Room

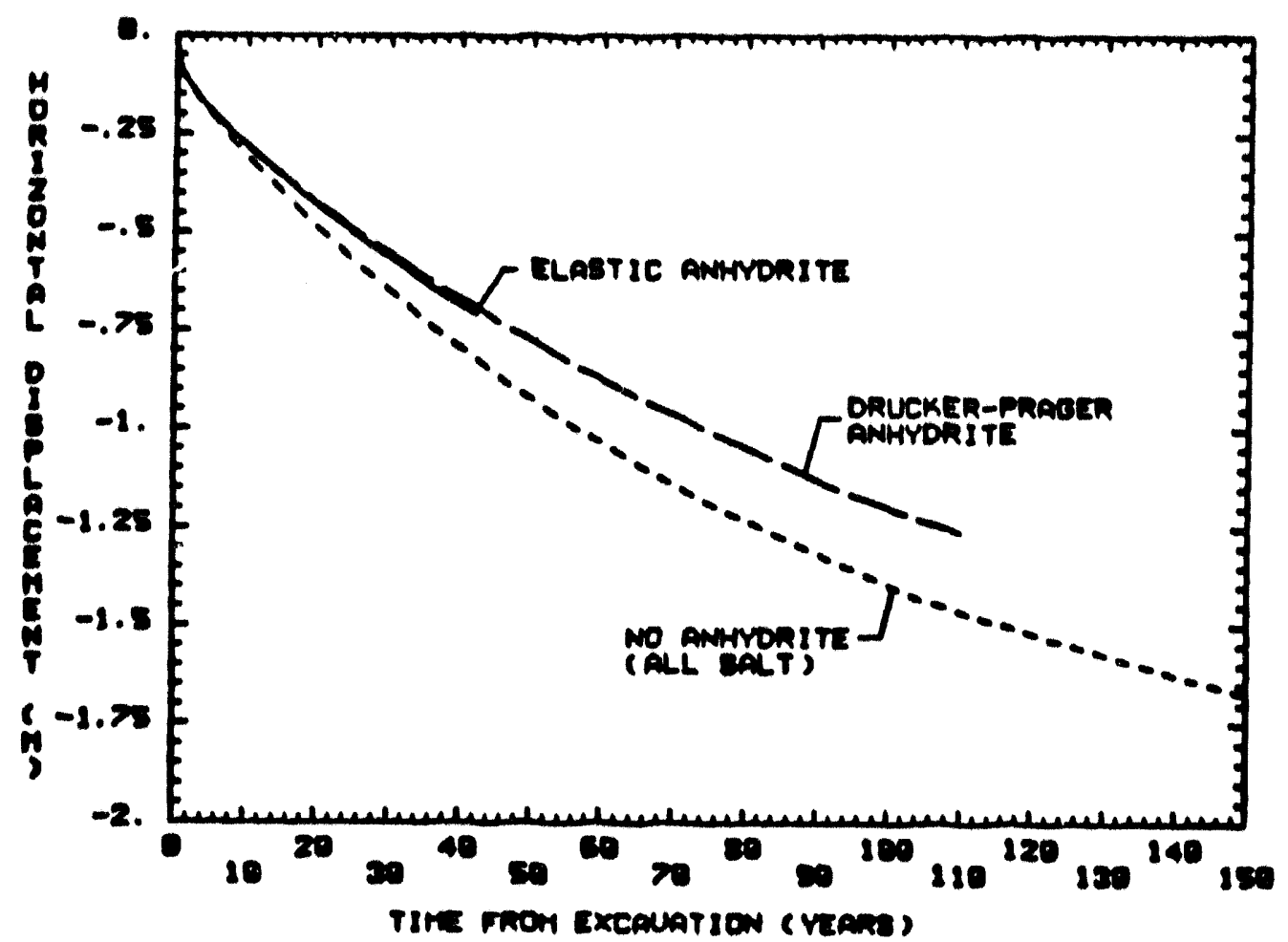

Figure 2: Horizontal Displacements at the Mid-Pillar of a TRU Storage Room 


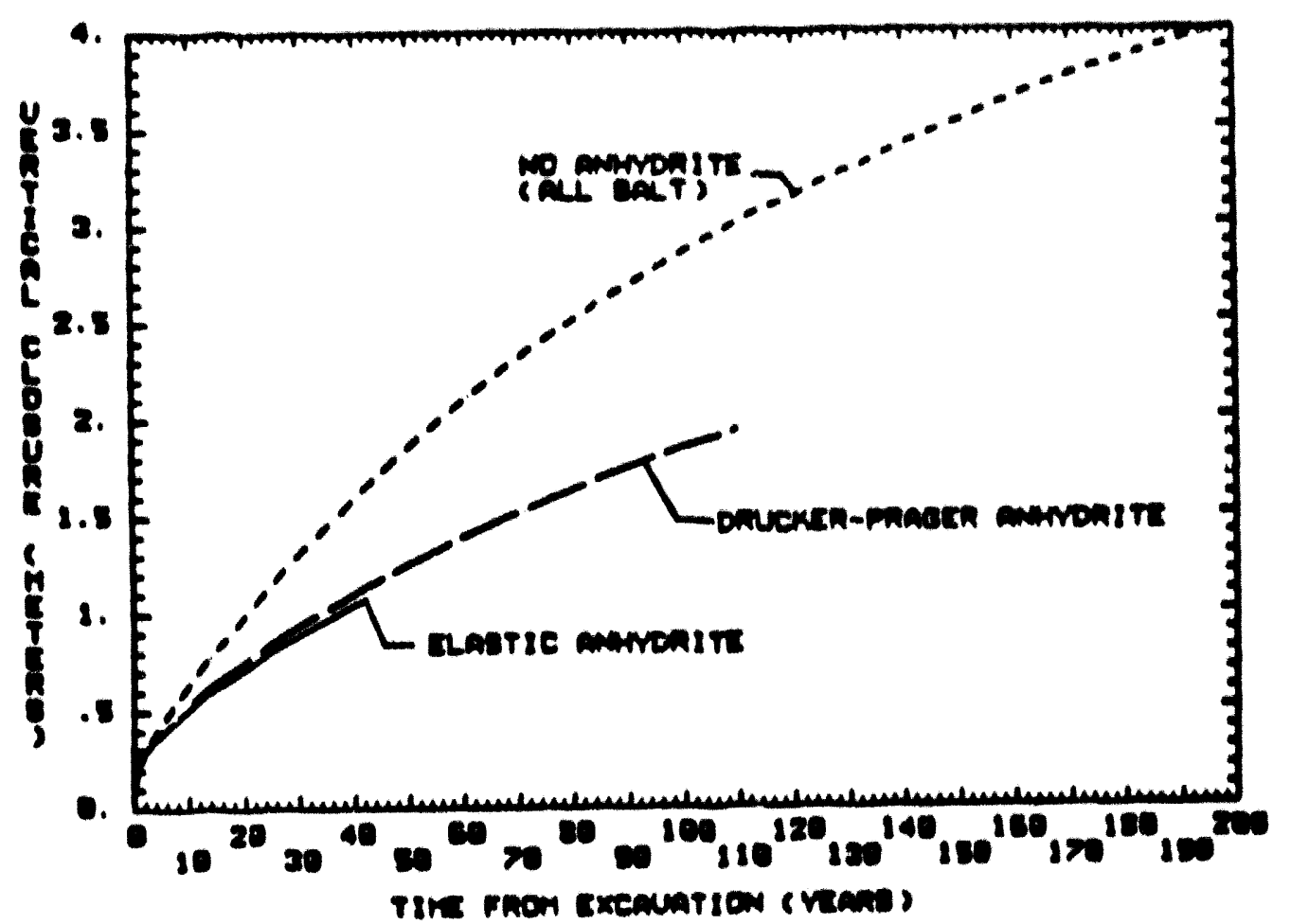

Figure 3: Vertical Closure Hibtorien for a TRU 8torage Room

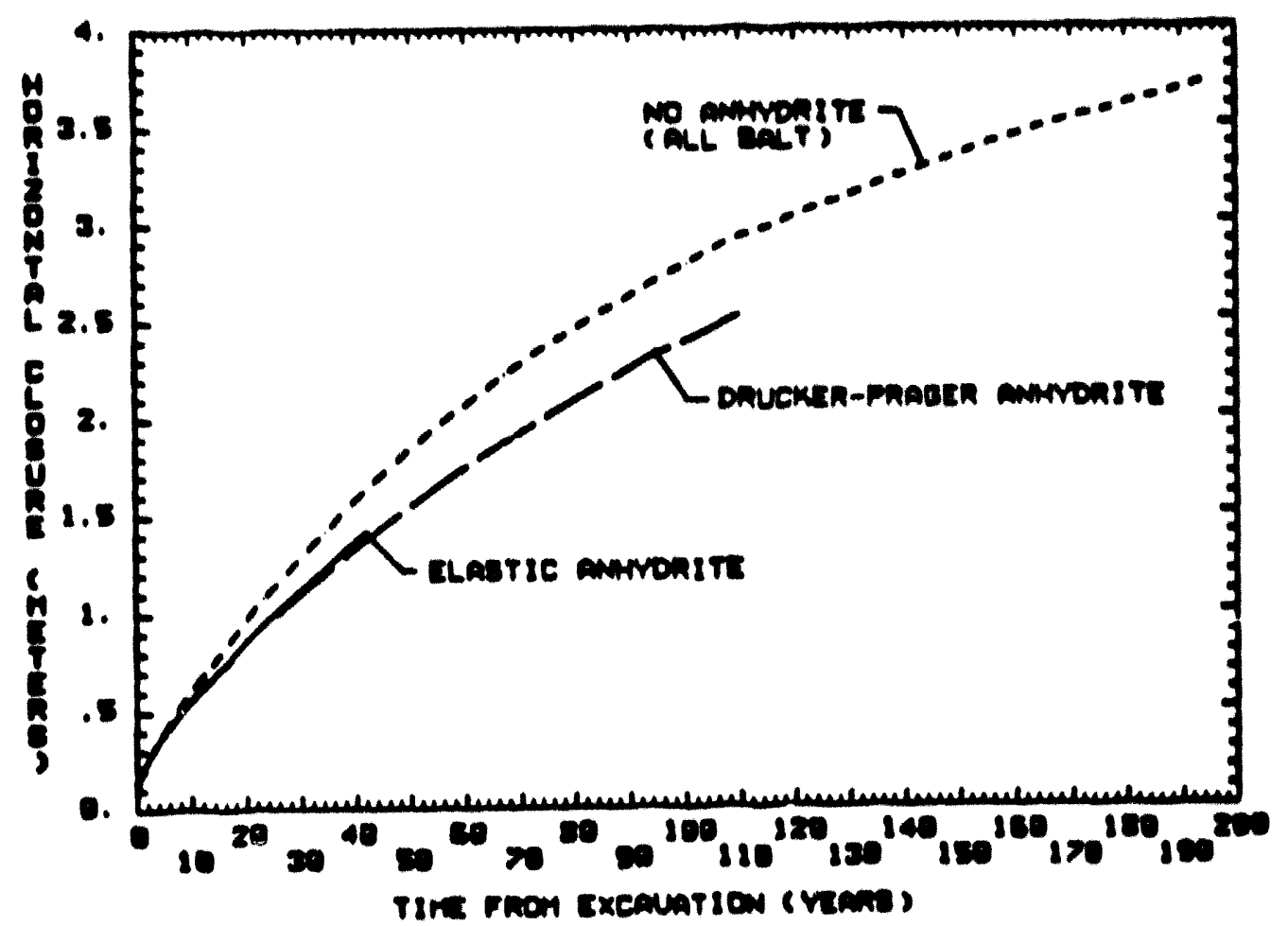

Figure 4: Horizontal Closure Bintorien for a TRU Btoraze Roam 


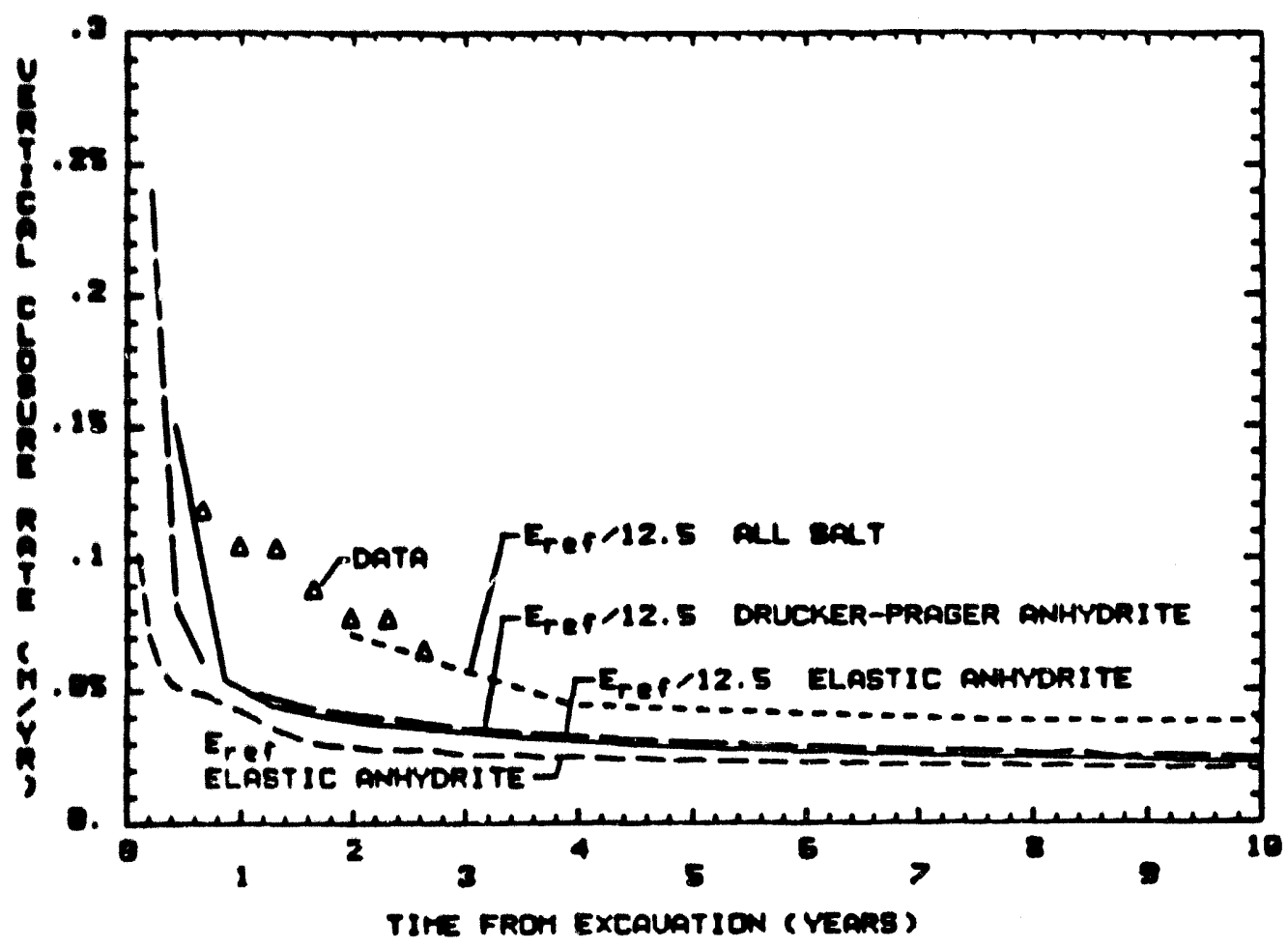

Figure 8: Vertical Closure Rate Histories for a TRU Storage Room

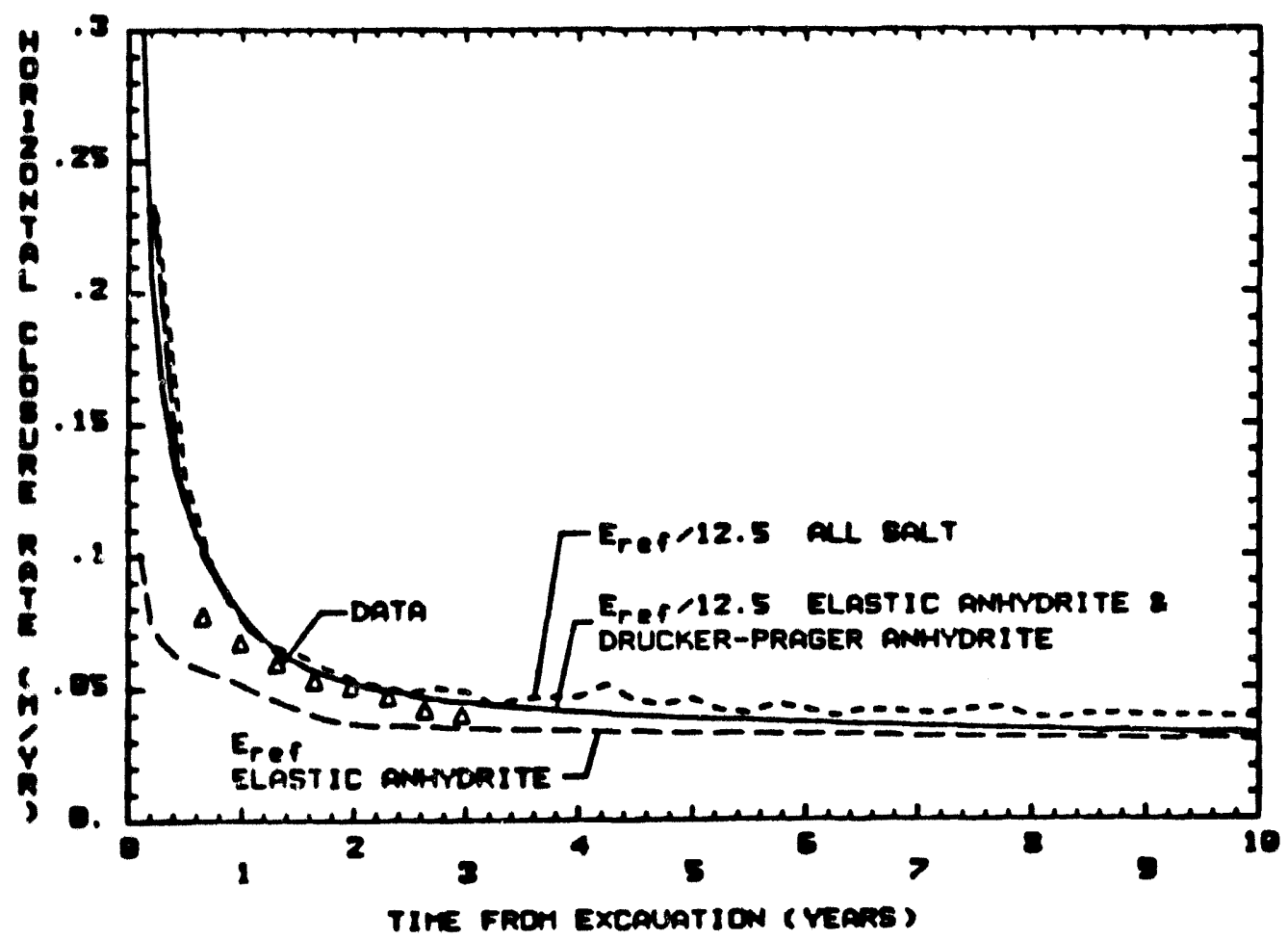

Figure 6: Horizontal Closure Rate Histories for a TRU Storage Room 


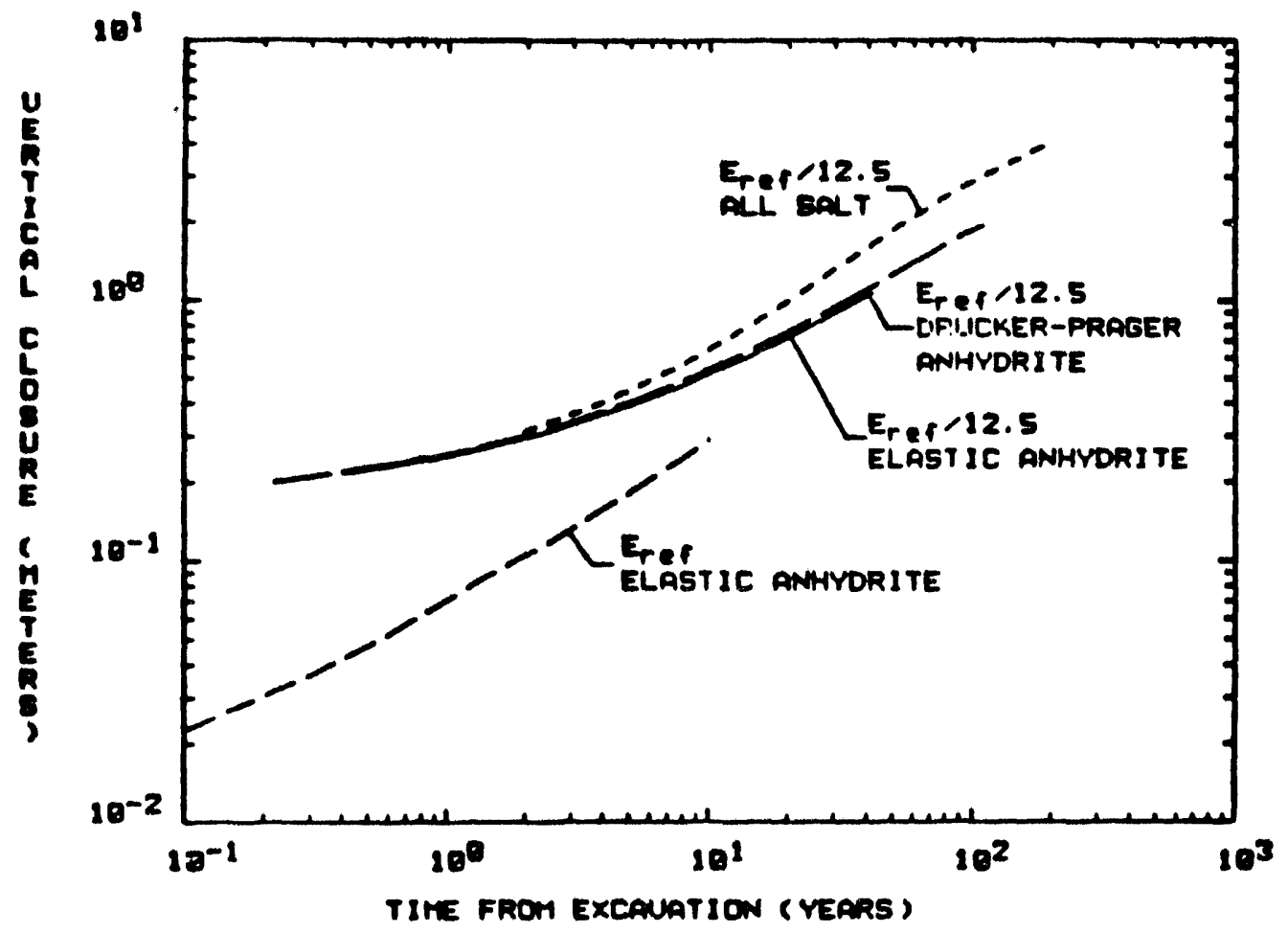

Figure 7: Logarithmic Vertical Closure Histories for a TRU Storage Room

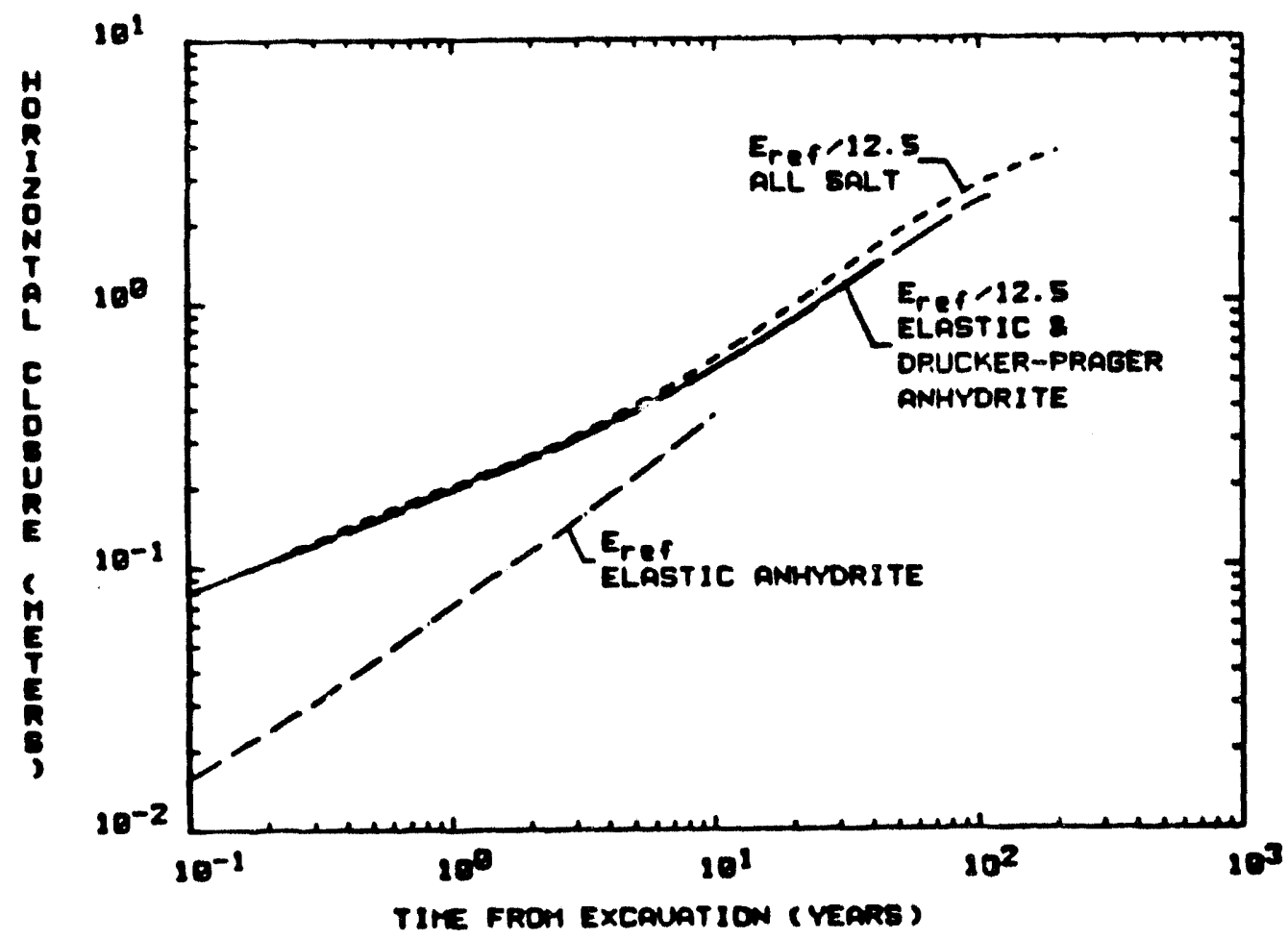

Figure 8: Logarithmic Horizontal Closure Histories for a TRU Storage Room 


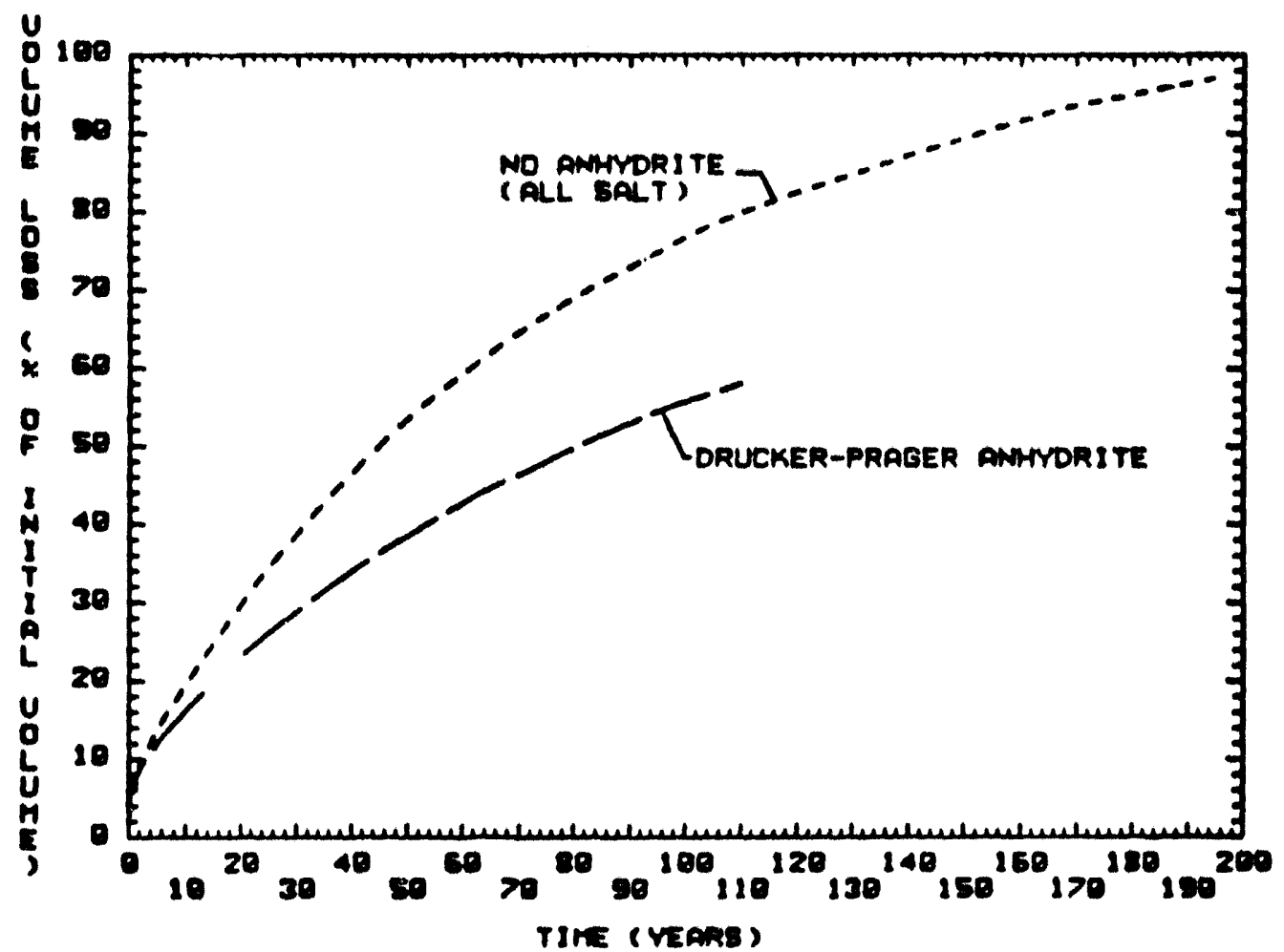

Figure 9: Volume Reduction of a TRU Storage Room 


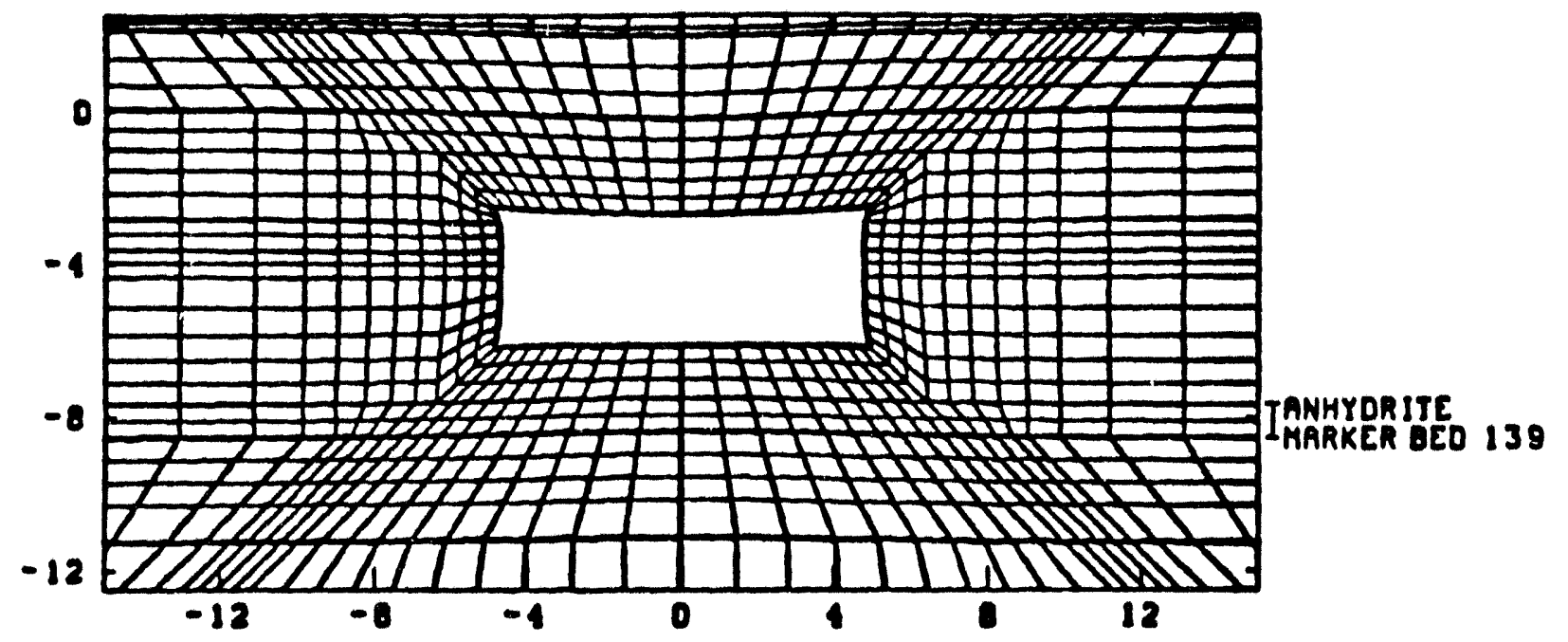

Figure 10a: TRU Storage Room Deformations at 10 Years for Drucker-Prager Anhydrites

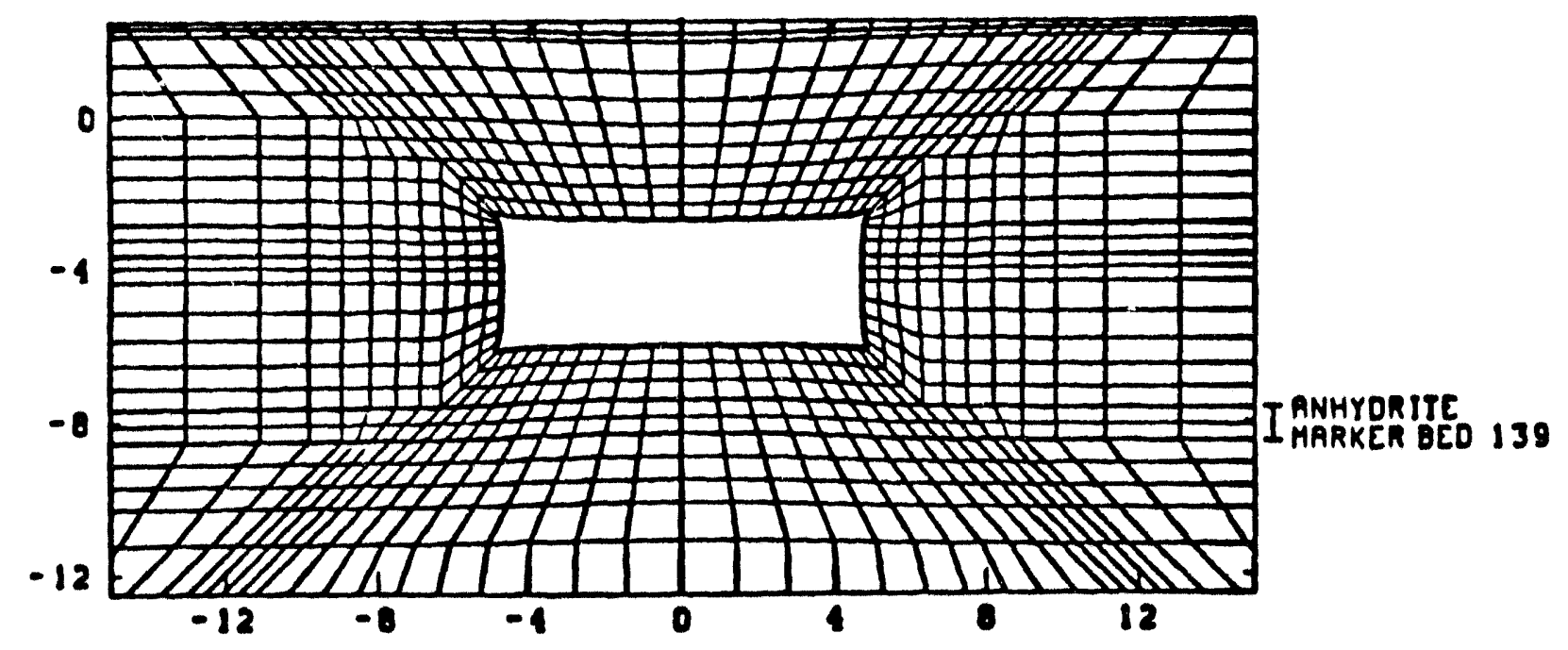

Figure 10b: TRU Storage Room Deformations at 10 Years for All Salt Model 


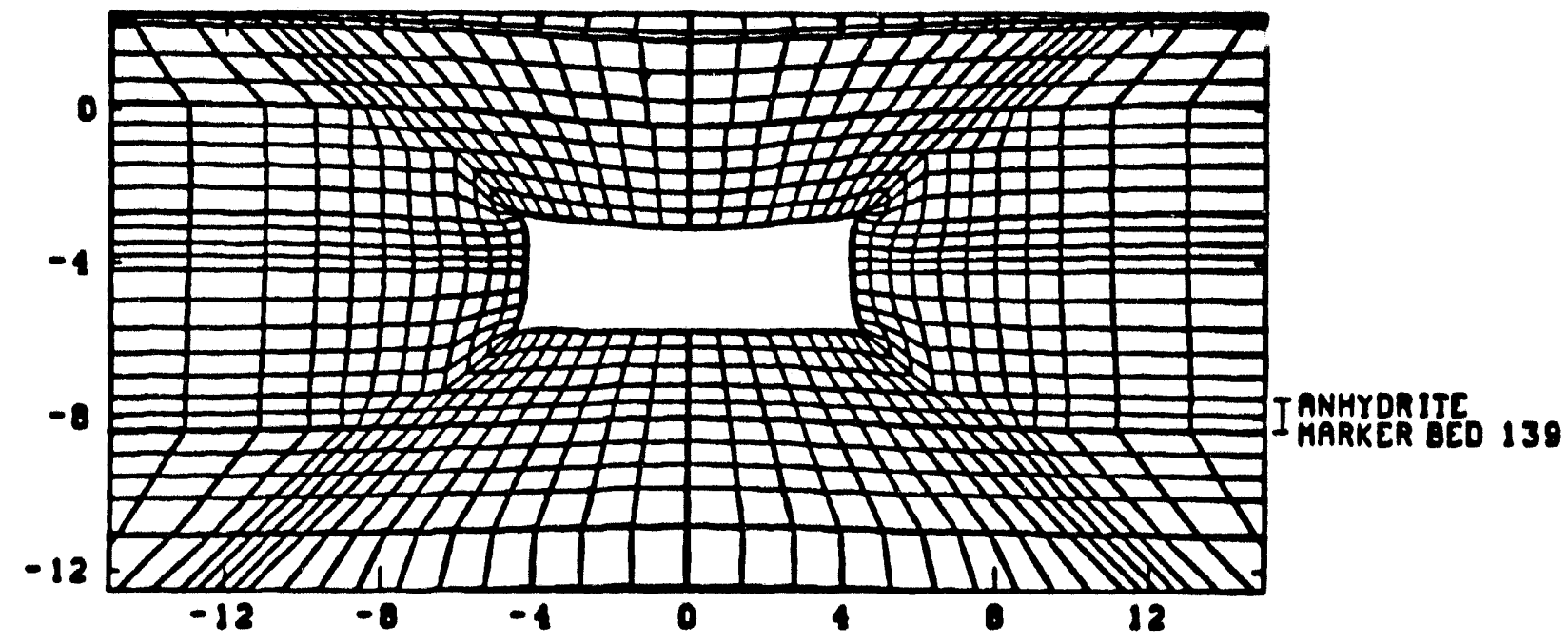

Figure 11a: TRU Storage Room Deformations at 30 Years for Drucker-Prager Anhydrites

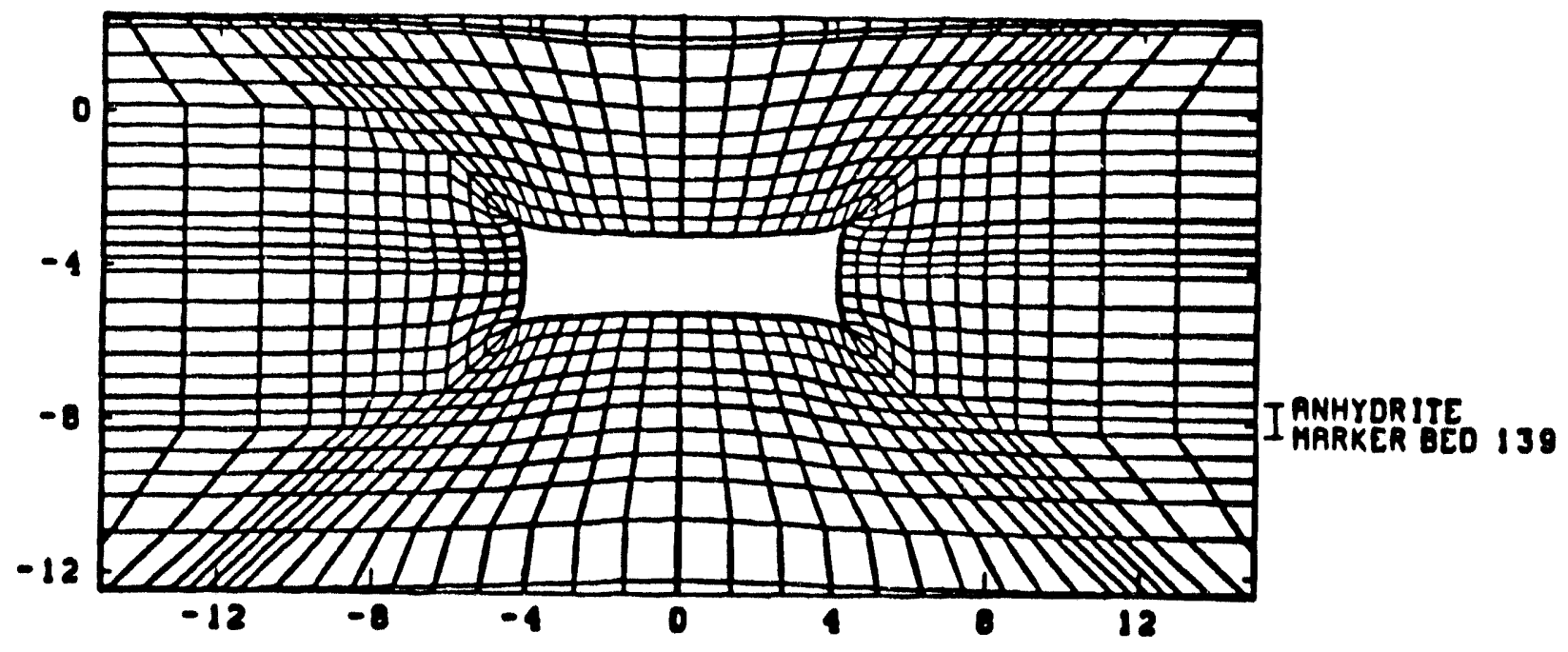

Figure 11b: TRU Storage Room Deformations at 50 Years for All Salt Model 


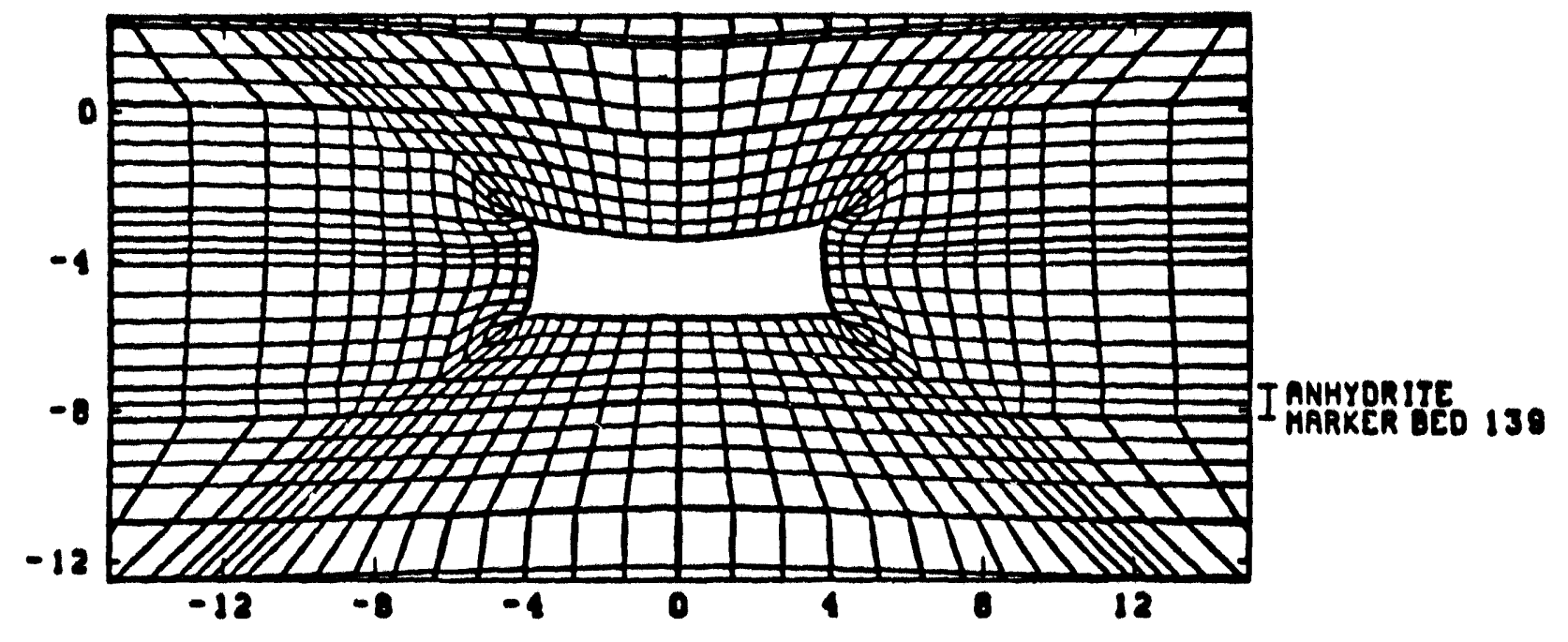

Figure 12a: TRU Storage Room Deformations at 100 Years for Drucker-Prager Anhydrites

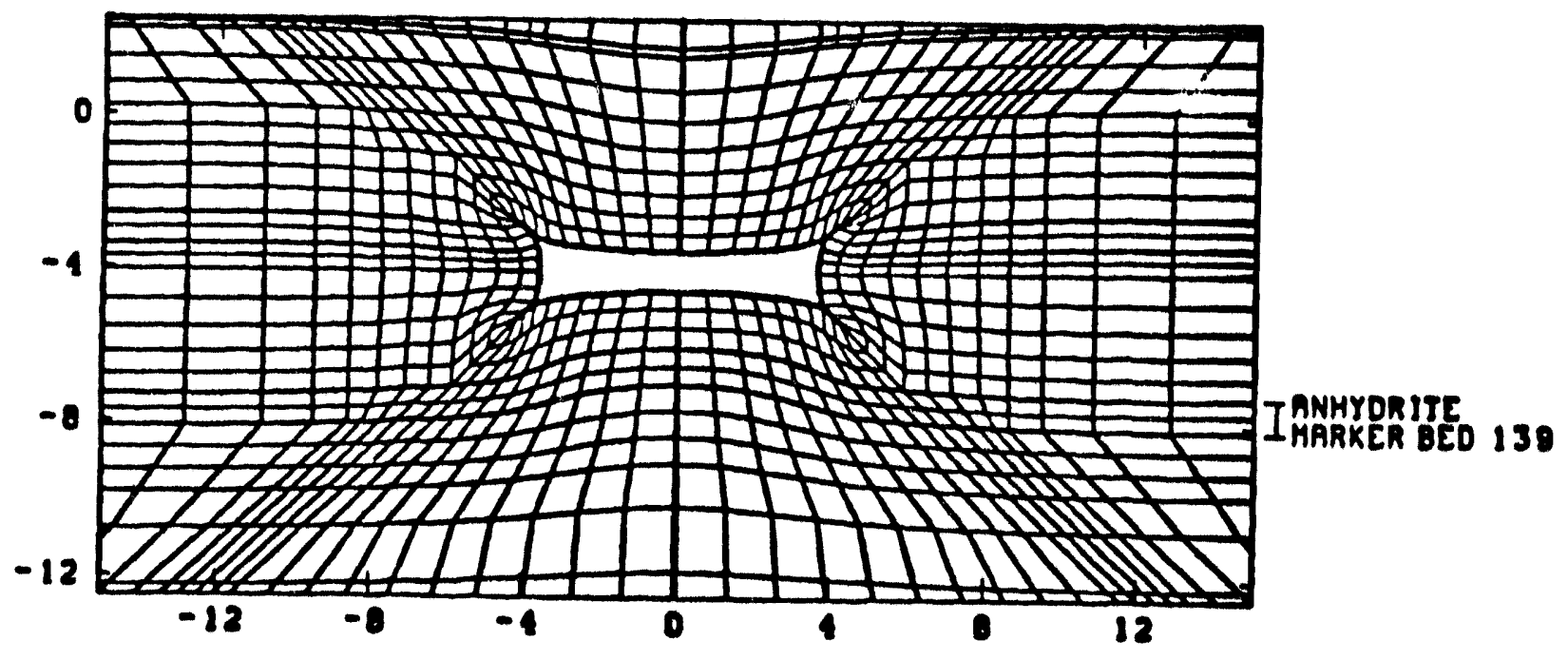

Figure 12b: TRU Storage Room Deformations at 100 Years for All Salt Model 
A -104 


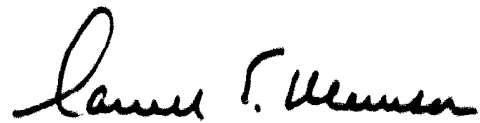

from: D. E. Munson, 6346

subject thy Reduced Modulus and similar Methode Appear to Work for Calculations of Creep Closure in salt.

As attompte to make predletions of the creep closure of underground openlngs In salt have been made over the yoars, number of Interasting calculational prodiction mothods have resulted from the use of constlutive modele based on ateady state creop, togethor with algniflcant reductions of the elestle modulus or Increases in initial olastic stralns. In genoral, the changes in modulus or otrain requited by these methode are far In excess of the permiteed values of these parameters as determined in the laboratory. At this point it le perhape worthwh1le to explain why these aethods appear to work, and aluo to denonstrate potentlal shortcoulnge.

Historlcally, the development of constlutive formulatione for the predlction of the creep response of salt have been based on laboratory observatione, but have not necesearlly included all of the observed creep bohavior. We can rofer to Figure 1 to see the customary decomposition of the conventional laboratory creep curve Into ateady etate component and - transient component. The llgure shows only the atress loading or primary transiont response: typlcally, the more general response must also Include atrose unloading transiont. In salt croep, tho unloading transient is not the negative of the loading responas. As shown, the "tranalent strain 11 itt" or ete lo the masure of the total transient -traln requlred to achleve the uteady state condition. For those conatitutive models based on eteady state, the translunt coaponent is essentially Ignored, so the creep otraln is Just given by the line of conatant slope, ${ }^{*}$, boginning at the orlgln, as ohow In riguro 1 . For more complete aodele, the tranalent responses are also Included in the conatleutive wodol, soset daes thls abounte to just the loading transiont and soactaos to both the loading and unloading translente.

Juet how wuch of the ereop bohavlor to nocesary in the conseftutive fornulation used for prediction of creep closure is the subject of conelderablo discuselon. 0 courae, what is actually necessary way bo a atter of the eleld problen bolng oolved. For oxample, In porformance prodiction for nuclear wate reposttorles in ealt, one anjor requirement 1s to deteralne the cla for complete closure of repository roome and, hence, the encapeulation of the waste. It Is known from imple extension of salt croep rates that it vould take tene, porhape hundrods, of yoars to attaln complote closure. Bocause in the laboratory tost the tranalont 
atraine are emall In comparison to possible steady state strains, it would appoar that for "long time" predictions, as in thls example, only the -teady atate creap contribution 1s lmportant. In other words, the characterlatic relaxation time for tranalent effect: la hort compared to the time frame of the problem. In more etralghtforward example, the use of eteady otate constitutive descriptlone would not, In general, be adequate for predicting early closure of repository response for design of ceal eyetens or for providing ectlantes of roon fallure for operational cafety. The steady state description would underpredict the actual creep deformatione for these altuatione where the transient atralne are clearly of the same ceneral augnttude as the steady state otrains.

Regardless of the apecifle problea, however, In using olmplifled models of actual anterlal bohavior, thare is always a danger of unknowingly elialnating sose aspect of the behavior that is of vital importance to the IInal application.

\section{The HIPP Expovelenas}

The elrat opportunlty for a comparieon betweon predlotion and WIPP in - Itu roos closure moasuremonte caused Imediate concern. Calculations of a WIPP access drift underpredicted the meceured closure and closure rate by at least a factor of three (Morgan et al., 1985). These calculations weed the Waste Ioolation Pllot Plant (WIPP) Progran reference eteady state creop law and properties (KrIos, 2984) establiohed at that two. The reforence creop law wa based on steady state creop, wlth an optlonal elret order kInetice translent response. The optlonal translent response was not used in the calculations. The reason for the discrepancy was not understood at the tine. It was thought that errors in the ereep paraneters or that a very low strese vlecous acchanden not found in laboratory teste alght be responalble. An extenalve study along these lines showed that withln the range of poselble uncertalneles in paraweter values, even axisialng the effocts of the uncertalnties, could not explain the diserepancy, and that the Inclusion of the viscous aochanlen altered the calculated closures Inapproprlately at vory late taes. As a part of a later study by Morgan ot al. (1986), It was found that a degradod alastle eodulue Inereased the calculated closure rates whlle proserving all of the Inportant characterletice observed in the wosured resulte. A roduceton of Young' modulue by a factor of 12.5 produced an excoptional "fle" between calculated and moasured elosures of the WIPP south Drift. Why thle arbltrary roduction of elastle nodulus gave such a good flt could not be explalned at that tive; however, the ouccese of the calculations led to the more-or-lese routino use of the reduced aodulus in subsequent WIPP calculations.

More recently, Morgan and Krles (1990) explored varlous hypotheses in on ettempt to explain why the roduced modulus mothod worke. Hypotheses Ineluded (1) potentelal alcrolracture dovelopant in the salt noar the room surfaces whlch vould result in a lowering of the apparent olast le vodulua

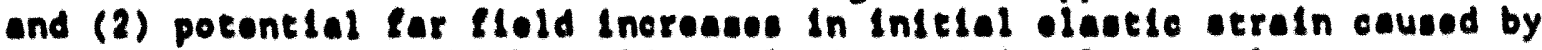
the reduced modulue whlch could result in additlonal roon closure dieplecesent. Intle not subseantlating ol thor of the intelal hypothoses. the atudy uncovered a further observation that a roduced modulus gave beter resulte in the analysels of laboratory stress rolaxation teats. 


\section{The Suropen cosA Benchmark Bxpertence}

starting In 1986, the European Communlty eponsored a erles of Important benchmark tudies Involving bench scale salt block laboratory tests (COSA I); deep borchole, with some heating, In eltu tests (COSA II): and numerical calculations (both COSA I and II) (Lowe and Knowles, 1989). The block testing was done on ASSE MIne ealt at the Energleonderzoek Contrum Nederland (ECN) In the Netherlande. The deep borehole was an ECN ("the Dutch Borehole") test flelded In the ASSE Mine renearch facllity in the Federal Republic of Germany (FRG). A oteady etate creep reference law and materlel properties based on the laboratory tests conducted at the Bundesanstalt fur Ceowlesenshaften und Rohstoffe (BGR) In the FRC was given as the base Ilne for the benchmark. Thue, the COSA reforence const tutlve law was for steady state only, using the Norton form.

However, In the actual calculatione, the partlclpants had conalderabla froedon to elect alternate constitutive models, to vary materlal propertles, and to reduce the inltlal boundary stress, whlch makes any generalization of the results every difflcult. Nevorthelese, there ware some very interiguling aspects of the results.

Among the partlclpant calculations, fow matched the moasured unheated Dutch borehole closures. There were throe exceptions. Two participants that used a complicated, two component, steady state constltutlve model wth at of non-COSA bonchnark materlal propertles obtalned good calculational agreeaent. However, the othor exceptlon was a particlpant that used the cosa reforence constlutive steady state model with a ignificant modiflcations in the acterlal properties. The modiflcation. were a factor of 2 Increase in the etructure factor and a factor of 2 decrease in the sodulus besed on a reference modulus of 24 CPA.

Many of the Intetal partlcipante, In later calculatlone, when they changed the uame two parameters could obtaln almllar agreoment to the experleental data. Interestingly, In one of these subsequent calculations, one partlelpant (Choreychl/Barest) used the COSA reforence law and proportles, but incorporated a Tresca rathor than the customary von Mises tlow condition, to obtaln very good agreoment to the moasured resulta.

The wost laportant aspect of the cosa II calculations, for our purpose, Is that calculational agreoment could be obtalned by modiflcation

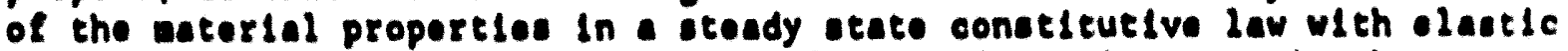
-traln. The linal modlfleation wan a factor of two lnerease In the - tructure lactor and a lactor of two decrease In the modulus. Although the process of arriving at thlo conblnation of changed paramoter values is unknown, It appears that slaple changes in one paranoter was Inaufficlant to match the borohole cloeure data. An eseontlal part of the parameter eodiflestion was the erblerary reduction in the modulus. Thls roduction. however, is analler in angnteude than that used by Morgan ot al. (1986). Based on the WIPP 31 OPa sodulus value, the European roduction 1 s a factor of 2.6 rather than 12.5. In ordor to got the creep rate hIgher, the Europoans opperently had to Increase the etructure factor: an Incroase In -tructure lactor causes comparable one-to-one Increase In oteady etate croop rate. 


\section{Berent Modifiantion}

Barest (1990) In the wake of the COSA II calculations has not continued to use the Tresca flow condition nor the arbitrary change in the COSA reference materlal constants. Rather, he has assumed in the calculation an artiflclally large elastlc component whlch has a magnitude equal to *t, can be stated as:

$$
\star_{t}(s)-\bullet_{0}(s)
$$

where o 1s the artiflclal elastlc straln whlch has the same dependence upon the otrese, S, as the tranalent otraln limlt. Typlcally ete la a wonotonle, Increasing function of the cubs of stress. Thus, Barest has replaced the etrain dopendent accunulation of transient etrain with an Instantaneous stop function elastic etraln at time zero. Th1a permits Incorporation of "tranalent" like reaponse Into the oteady etate conseltutive model without the numerlcal compllcations of a true transient constlutive model.

\section{Imollantion}

It Is now easy to establish the common thread in these mothods which use arbitrary degradations in elastle modulus or increases in elastic stralne. Morgan ot al. (1986) reduced the modulue according to

$$
\text { - }-\$ / 8
$$

where a 1s the modulus reduction factor. However, if we write the following:

$$
e *_{t}(s)-\bullet_{0}(s)-\Delta / E f(s)
$$

where $f(s)$ is some function of etress. The Implication is that a roduction in modulus or an assumption of an artificlally large elastic -traln can be almply related. Elther of the mothoda are actually stop function rapresentatione of the total transient etrain (transiont straln IImlt) requlred to achlove eteady state creep.

Flgure 2 1s the laboratory determination of the tranalent otraln lialt, o*f, for clean WIPP ealt as a function of etrese (Munson ot al. , 1989). Afthough wo do not have Bareat' data, it could be expected that hls asuumed stop function elastle straine would have to watch closely the trenslont strain linlt as a function of stress. Thls suggosts that had ho dotermined the otress dependence, it would have been nearly cuble.

Morgan ot al. $(2986)$, without any basls to do otherwlse, when thay used Eq. 2, affoctivaly oet $l(s)$ to orlo. Equation 2, wlth a roduction factor of 12.5, Is given by the approprlately marked line in Figure 2. Thla IIne 1s conatralned to a lope of one, so it can only approximate the measured translent atraln limlt data. Howover, the cholce of the modulus reduction factor would, through the backfleting procedure for a given problen, place the ruduced modulus stralns at a position to best represent the approprlate creep date. Thle appears to be at a stess of about 12 MPa, whlch could be expected to be a representation of the "average 
streas" of the in situ problew used in the backfitting. We can see however that the reduced modulus method can not give very adequate representation of the transient atrain 11 mit for all stresses. For problems with higher "average stresses", unch as an excavation at a greater depth, then the reduction in modulus would probably have to be larger to obtain an acceptable back fit.

\section{Apoliantion to calaulations}

An Interpretation of step function elastic strains to represent the transient response in calculations of in situ test results is reasonably complicated. The etep function representation insists that all of the transient strain appears instantly upon application of the etress, rather than gradually with time. If, Indeed, the otresses were applied Instantaneously, then the etep function representation would be clearly unacceptable because it would overpredict the early straln. As can be seen from Flgure 1, the overprediction decreases with time, unt1l the step function response and the correct transient strain function become qual when steady state creep is achleved. As a result, if we look at very late timas for strese loading problems, the calculated atrains would be the same for alther step function elastlc or correct transient creep etrain representations. However, thls Indlcates that how well the sep function - lastic stralne can ropresent actual measured creep otrains 1s 1 tself time dependent.

What seems the mout confusing of all is the ability of these time Independent, tep function lastic etrain mothods, combined with only a ateady state creep conetitutive model, to calculate clearly time dependent closure results for undergrisund rooms or deep borsholes. We need to examine this process careflilly. Firat, if only a steady state constlutive model 1 s used to calculate borehole convergence, the calculated convergence is not constant, but continues to decrease with time. This is caused by the stress fleld continuing to cha.ige with time because of the geometry of the problem. Consequently, it 1s clear that a timo dependent behavior can develop from non-materlal (geometrical), as well as materlal, sources. Second, any instantaneous atrain increment whlch 1s a function of stress wlll mirror, In tlme, the changes of the strese fleld. If the strese fleld changes, the instantaneous strain Increment w1ll also change. Again, the meterial response is not time dependent, rather the time dependence of the closure is the result of the changlng utress fleld, non-materlal effect. Thus, oteady sate creep model with instantanoous elastlc strain st1ll will ylold time dependent closure for problems in which the strese fleld is changing with time. Third, Introduction of a the true materlal behavior, with time dependent transient creep, together with steady atate creop, will only alter the detalls of the time dependent closure in those problems which already have a geometrical time dependence rosulting from a changing otress fiold.

The Interprotation 10 further clouded for real probloms which involve gradual stress loading or progreasive changes in the stress fleld because of the geometry of the problem. Gradual changes in loading will produce a timo dependent incremental Increase in the tep function elastic otrain whlch 1" directly proportlonal to the rate of atress Increase. Of course the came effects occur on stress unloading. As a result, for any problem In whlch the stress fleld 1s changing, such as a fleld expanding with time 
to include prevlously unstrained material, the top function elastic strain w1ll continue to add incrementally to total deformation. The lmportance of such expanding stress flelds, and hence strain flelds, to calculations of room closure was first polnted out by Munson et al. [1989]. Although the stralne in the leading edge of an expanding tress fleld may be mall, the expanding fleld encompases a large volume of material; as a result the integrated displacement at the room surface from these small otrains can be significant.

The difference between using an artificlally large elastlc strain to represent the transient creep etrain and using the true transient etrain function for a problew with changing otress fields is that deformations are forced to earlier times with the artificlally large elastic strain representation. For the ame Incremental change in stress the true transient creep strain model will develop strain more slowly than for the assumed instantaneous elastic strain model. Consequently, closures calculated using the instantaneous elastic step function to represent the transient strain w1ll have a greater amount of early curvature than those using the true transient response representation.

The difference between using an artificlally reduced elastic modulus to represent the transient croep strain and using the true transient - train function for a problem with changing stress flelds is as tated above, except for additional concern introduced because the linear relationshlp between the lastic strain and stress of Eq. 2 is in general not a good representation of the transient strain 1imit. Consequently, the best fit value of the reduction factor wust be problem dependent.

Because there appears to be no characterlstlc differences in general appearance of calculated closure curves for those cases with just time dependent geometrical effects and those with time dependent material response, we cannot discern the difference between steady state (with or without artificlally large lastic stralne) and transient creep constlutive models. What this means is that fiold data, in general, will not contaln sufficlent information to permit us to uniquely define the constitutive model. It seems therefore critical to use fully our knowledge of laboratory data, obtained under condltions of constant stress (no geometrical time dependent effects), in determining the constitutive model for creep. In such a model, wo know that the material transient, as well as the steady state, response must be included.

There remalne another lmportant area where the step function elastic stain representation may cause concern. Th1s 1s particularly true for calculations of seal component response based on returning the adjacent salt to the 11 thostatic strese condition and room reinflation because of gas generation since in both these cases the salt undergoes stress unloading and pressure reloading. If after initlal stress loading, the stress then 1s reduced, all of the step function elastic strain will "come back out" of the calculation. The Implication is that negative creep of appreclable magnitude w1ll occur. However, this is known not to happen in salt creep. Actual creep strains, once accumulated, are not recovered -lastically. 
In summary:

(1) It 1s demonetratnd that the use of arbltrarily reduced modulue and the assumption of abnormally large inltial olastlc otralne are equivalent.

(2) These methods can be considered as a etep function representation of the transient creep etrain.

(3) The abnormally large intelal etralne can be rolated and perhap: are deduced from laboratory transient creep data, opeclfically the transient otrain $11 \mathrm{mlt}$.

(4) The reduced modulus representation can, at best, be only a crude approximation to the laboratory tranolont croep data.

(5) Although step function representations w111 glve what appears to be time dependent accumulation of etrain for problome where the etreas flelds change because of the problem geometry, these representations will not necessarliy give accumulation rates comparable to the transient creep strain representation.

(6) Upon unloading, the sep function representations wlll result in negative creop, an effect not seen in actual salt creop.

\section{Becommendntion}

Although probably not always poselble, it would soow advantageous to Incorporated the time dependent accumulation of transient etrain, with different response functions for tress loading and unloading. Into all our creep calculatione, whenever to do so would not caues al gnifleant caiculational problems.

\section{Beferenae}

Barest, P. (1990), (Private Communlcation), Ecole Polytechnlque, Palalseau, France.

Lowe, M.J.S., and N.C. Knowles (1989), "CosA II - Further Benchmark Exercises to Compare Geo-mechanlcal Computer Codes for Salt," Report E0948.001, W.S. Atkins Englneerling Sclences, Surry, UK.

Munson, D.E., A.F. Fossum and P.E. Senseny (1989), "Advances in Resolution of Discrepancles between Predicted and Measured In SItu WIPP Roow Clonures," SAND88-2948, Sandie Natlonal Laboratorles, Albuquerque, NM.

Morgan, H.S., C.M. Stone and R.D. KrIeg (1986), Evaluation of WIPP Structural Modeling Capabllitles Based on Comparlione with South Drift Data, SAND85.0323, Sandla Natlonal Laboratorlea, Albuquerque, MM.

Morgan, H.S., C.M. Stone and R.D. Krieg (1985), "The Uee of Fleld Date to Evaluate and Improve Drift Reaponse Modele for the Waste Inolation Pllot Plant (WIPP)," Proc. 26th U.S. Symp. on Rock Mechantca, A.A. Balkema, Boston, MA: Pp. $769 \cdot 776$.

Krleg, R.D., [1984], Reference Strat1graphy and Rock Propert1es for the Waste Isolation Pllot Plant (WIPP) Project, SAND83-1908, Sandia Natlonal Laboratories, Albuquerque, NM. 


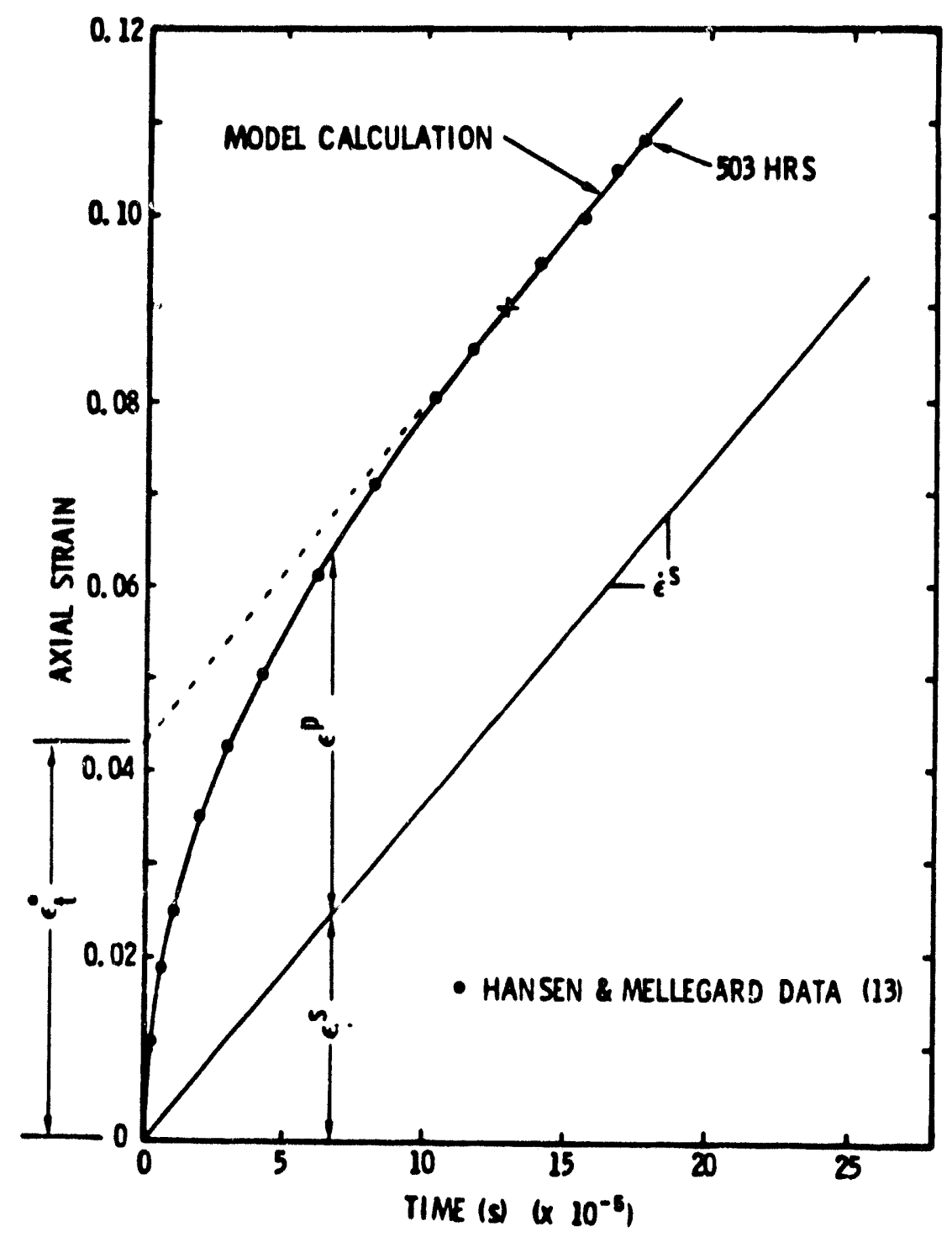

Figure 1. Steady State and Transient Components of a Typical Laboratory Creep Curve. 


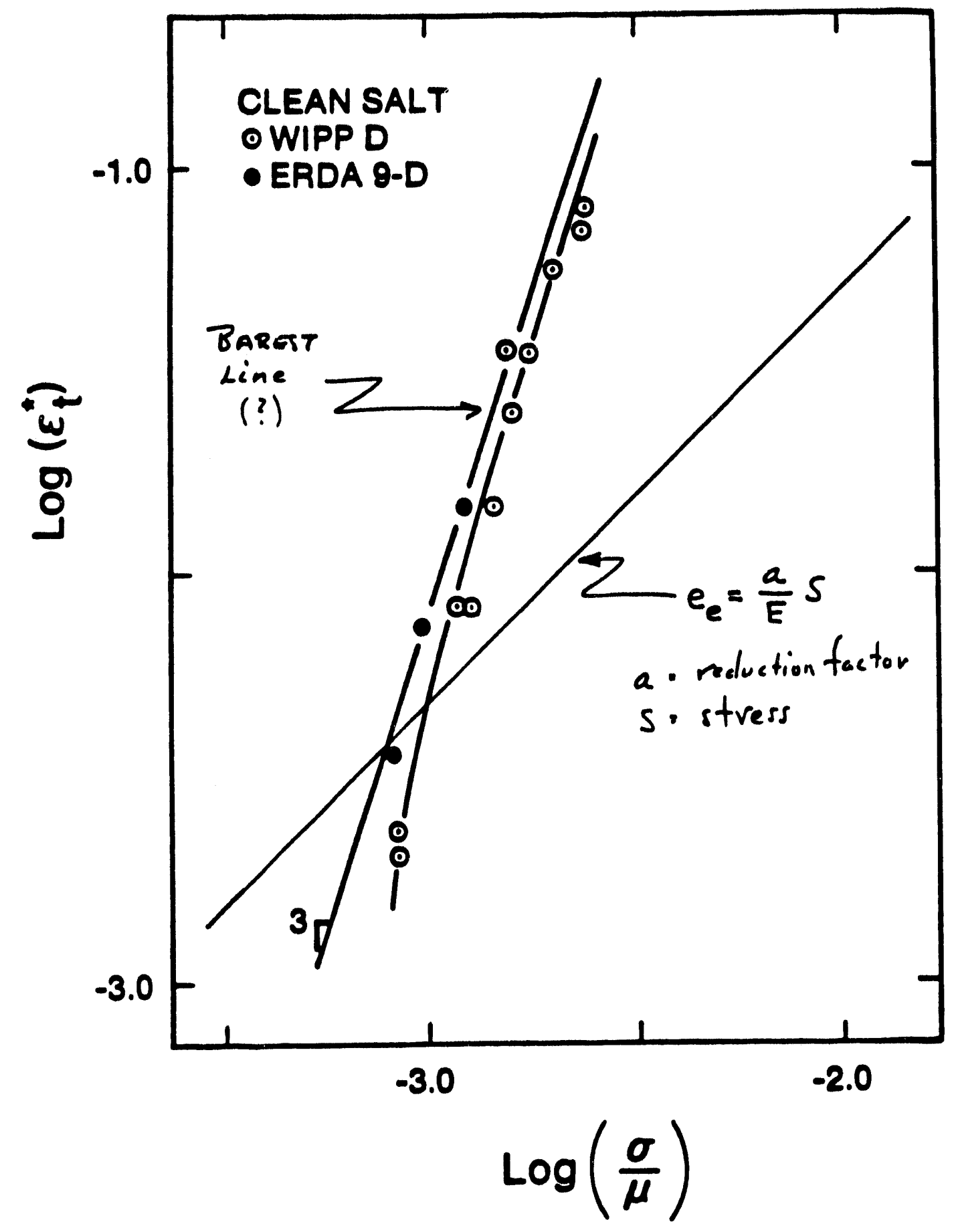

Figure 2. Laboratory Determined Transient Strain Limit, e*t, for Pure
Salt with the 12.5 Reduction of Modulus Curve Superimposed. 
A -114 
oate: November 7, 1991

to: A. R. Lappin, 6345

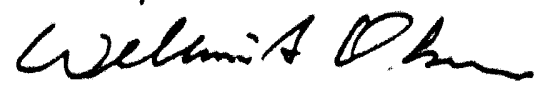

trom: W. A. Olsson, 6232

subjoct. Implications of the Experimental Consolidation of Crushed Salt/metal Mixtures and Proposed Work

\section{INTRODUCTION}

An important problem in the design of backfill materials and techniques for the WIPP is to ensure the consolidation of the backfill to a low-permeability mass encapsulating the waste. The major component of suggested backfills is crushed salt. Previous experiments have shown that when damp crushed salt is subjected to hydrostatic pressure, it has the desirable property of consolidating rapidly to high fractional densities (Holcomb and Shields, 1987) and hence to low porosities and permeabilities.

Various additives to crushed salt are being considered to achieve higher absorption of brine, radionuclides and gases. Further, shredded wastes may be mixed with crushed salt and placed into storage drums to decrease void space.

\section{BACKGROUND}

Additives to crushed salt will certainly alter the consolidation rate. At one extreme are likely to be non-deforming, inert materials, such as metals and hard plastics. At the other extreme will be the highly deformable materials, which flow easily, exemplified by betonite. It is expected that the rigid materials will have a stronger influence on consolidation rates of crushed salt. Accordingly, we have begun to investigate the effects of the presence of rigid, inert inclusions on the hydrostatic, time-dependent compaction of crushed salt. Of interest is the response of the $\mathrm{mix}$ to long-term hydrostatic pressure and the evolution of the permeability of the mix as it densifies. The tests mentioned here cover only the constitutive behavior as we did not measure the permeability of the post-test samples. We have completed 6 creep consolidation tests on salt-metal mixtures. The samples comprised mine-run crushed salt, mild steel disks, and water. The steel disks were $22.4 \mathrm{~mm}$ in diameter and $1.55 \mathrm{~mm}$ thick. Sufficient water was added to give a water content of $1.8 \%$ (exclusive of the water content of the salt). The mass fraction of inert material was about 0.44 in each sample which corresponds to an inert solid volume fraction of 0.18 . By way of comparison, 20\% is the solids content of the Engineered Alternatives Task Force (EATF) option of shredding combustibles and mixing with grout. The solids content is $12 \%$ in the EATF option of shredding metals and mixing them with salt (R. Beraun, Org. 6345). The concentrations of gas getting materials are yet to be established. Thus, the 18 volume 
per-cent of solids used in these tests is representative of the concentrations of solids that might be mixed with salt filler. At higher concentrations, the inert inclusions can begin to form a continuous framework that would be more effective in resisting consolidation, and could tend to create continuous pathways for fluid migration.

Jacketed samples of the mixture, vented to the atmosphere, were subjected to hydrostatic pressures for up 69 days. Figure 1 summarizes the time-dependent response of the six experiments. Of particular interest is how well the currently used constitutive equation, modified for the presence of inert inclusions agrees with the data. Sjaardema and Krieg (1987) develop the constitutive equation for damp crushed salt, based on experiments of Holcomb and Shields (1987), that is given by

$$
\dot{\rho}_{0}=B_{0}\left[e^{B_{10}}-1\right] e^{A \rho_{0}},
$$

where $\rho_{0}$ is the density of the crushed salt; $B_{0}|\cdots|$ describes the pressure effect; $A, B_{0}$, and $B_{1}$ are experimentally determined constants; and $\sigma$ is the consolidation pressure. Equation 1 may be modified (Callahan and DeVries, 1991) to describe a mixture of 2 non-interacting components

$$
\dot{\epsilon}=\rho_{0} \frac{m_{1}}{\rho_{?}^{2}} B_{0}\left[e^{B_{10} \sigma}-1\right] e^{A \rho}
$$

where $\rho_{0}$ is the initial density of the mixture, $\dot{\epsilon}$ is the volume strain rate (also called the compaction rate), and $m$, is the mass fraction of crushed salt. One of the components is inert and presumed to be dilute so that the inclusions do not influence each other. Thus, the volume strain rate is a function of the parameters of damp, crushed salt and the initial mixture density. It can be shown that equation 2 is also written

$$
\dot{\epsilon}_{\text {mix }}=v_{\text {salt }}^{0} \dot{\epsilon}_{\text {salt }} \text {. }
$$

Thus, the simple mixture theory approach indicates that the volume strain rate for damp, crushed salt mixed with inert inclusions can be obtained directly from the equation for damp, crushed salt weighted by the initial volume fraction of crushed salt, $v_{\text {salt. I took }}^{0}$ the following values for $A, B_{1}$, and $B_{0}$ from Callahan and DeVries (1991); these differ only in units from Sjaardema and Krieg (1987):

\begin{tabular}{lcc} 
Parameter & Value & Units \\
\hline$B_{0}$ & $1.3 \times 10^{8}$ & $\mathrm{~kg} / \mathrm{m}^{3} \mathrm{~s}$ \\
$B_{1}$ & 0.82 & $\mathrm{MPa}^{-1}$ \\
$A$ & $-1.73 \times 10^{-2}$ & $\mathrm{~m}^{3} / \mathrm{kg}$ \\
\hline
\end{tabular}

Figure 1 shows the regression line of volume strain rate plotted against the fractional density of the salt for each test. Also shown are the volume strain rates computed from Equation 2 using the data from the table and the appropriate volume fractions of salt. The numbers at the upper ends of the solid lines indicates the consolidation pressure used to make the calculation. 


\section{IMPLICATIONS}

The consolidation data are compared to the predictions of the mixture theory at differant consolidation pressures in Figure 1. The variation of the compaction rates with fractional density are fairly represented. But, the observed volume strain rates at any given frac. tional density are two orders of magnitude lower than the predicted rates at equivalent pressures. (Compaction rates of the mixture are also lower than plain crushed salt by similar amounts.) The fact that the observed volume strain rates are much lower than predicted from simple mixture theory predictions and from plain crushed salt suggests that addition of $18 \%$ by volume of inert solids can significantly increase the time required for encapsulation of the wastes.

In an attempt to determine the source of the discrepancy, the pressure effect term was recalculated for the mixture data. The term $B_{1}$ was found to be reduced from 0.82 to 0.71 , only $13 \%$. This has little effect on the difference between predicted and measured compaction rates. Next, the constitutive parameters for the salt fraction of the mixture were back-calculated and new values of $A$ and $B_{0}$ were found. The densification rate is much more sensitive to changes in $A$ and this new value, $A=-2.16 \times 10^{-2}$, was used to compute new prediction curves and these are shown in Figure 2. There is much better agreement between the predicted and measured compaction rates for the new value of $A$. Nevertheless, it is not clear how this type of adjustment can be justified because at this time we do not know how the presence of the inert inclusions influences the apparent behavior of the crushed salt. In any event, this result seems to suggest that mixture theory, as applied here, is inadequate to describe the response of the mix.

To get information on mixtures having a wider range of concentrations; and to further investigate the validity of mixture theory, which is linear in the volume fraction of salt (or equivalently $1-v_{\text {inert }}^{0}$ ); it will be necessary to compact mixtures at an additional concentration of inert inclusions.

At this time the lack of agreement between the observed and predicted response is not understood. The following items will have unknown effects:

- The concentration of inert inclusions may not satisfy the assumption of diluteness and non-interaction of inclusions.

- The shape of the inert particles was discoid, and this may alter the response because implicitly the shapes are assumed spherical when calculating the concentration.

- Some of the constitutive parameters from the crushed salt may be in error. For example, Sjaardema and Krieg (1987) calculated the pressure effect term from the results of only one pressure-stepped test. In addition, back-calculation indicates that the salt fraction of the mixture samples are better fitted by different values of $A$. 


\section{NEW WORK}

Resolution of the above issues may be approached by a more comprehensive test plan. It is clear that we need to examine the effect of concentration of inert inclusions, and also the effect of their shape. At least one more concentration needs to be studied. The best would be a value near to that of a proposed metallic drum composition, about $10 \%$ metal by volume (R. Beraun, Org. 6345). Further, the effect of inclusion shape needs to be clarified. This would be most clearly brought out by adding spherical inert particles. The test matrix required to accomplish these goals is as follows:

\section{Volume Fraction Shape Replications Pressure}

\begin{tabular}{llll}
\hline & & & \\
0.10 & disk & 2 & 3.45 \\
0.10 & disk & 2 & 6.90 \\
0.10 & disk & 2 & 10.3 \\
0.10 & sphere & 2 & 3.45 \\
0.10 & sphere & 2 & 6.90 \\
0.10 & sphere & 2 & 10.3 \\
0.20 & sphere & 2 & 3.45 \\
0.20 & sphere & 2 & 6.90 \\
0.20 & sphere & 2 & 10.3 \\
& & & \\
\hline
\end{tabular}

At recent levels of funding for similar work we were able to finish 6 consolidation tests in one year. Thus, only certain tests from this test matrix could be selected for initial work. It is best to concentrate our initial efforts on the disk shaped materials as this is probably closer to the aspect ratio of shredded metal pieces. Thus, 4 experiments on disks and 2 on spheres would be suggested.

\section{REFERENCES}

Callahan, G.D. and DeVries, K.L., Analysis of Backfilled Transuranic Wastes Disopsal Rooms, Sandia National Laboratories Report, SAND91-7052, 1991.

Holcomb, D.J. and Shields, M.F., Hydrostatic Consolidation of Crushed Salt with Added Water, Sandia National Laboratories Report, SAND87-1990, 1987.

Sjaardema, G.D. and Krieg, R.D., A Constitutive Model for the Consolidation of WIPP Crushed Salt and Its Use In Analyses of Backfilled Shaft and Drift Configurations, Sandia National Laboratories Report, SAND87-1977, 1987. 


\section{FIGURE 1}

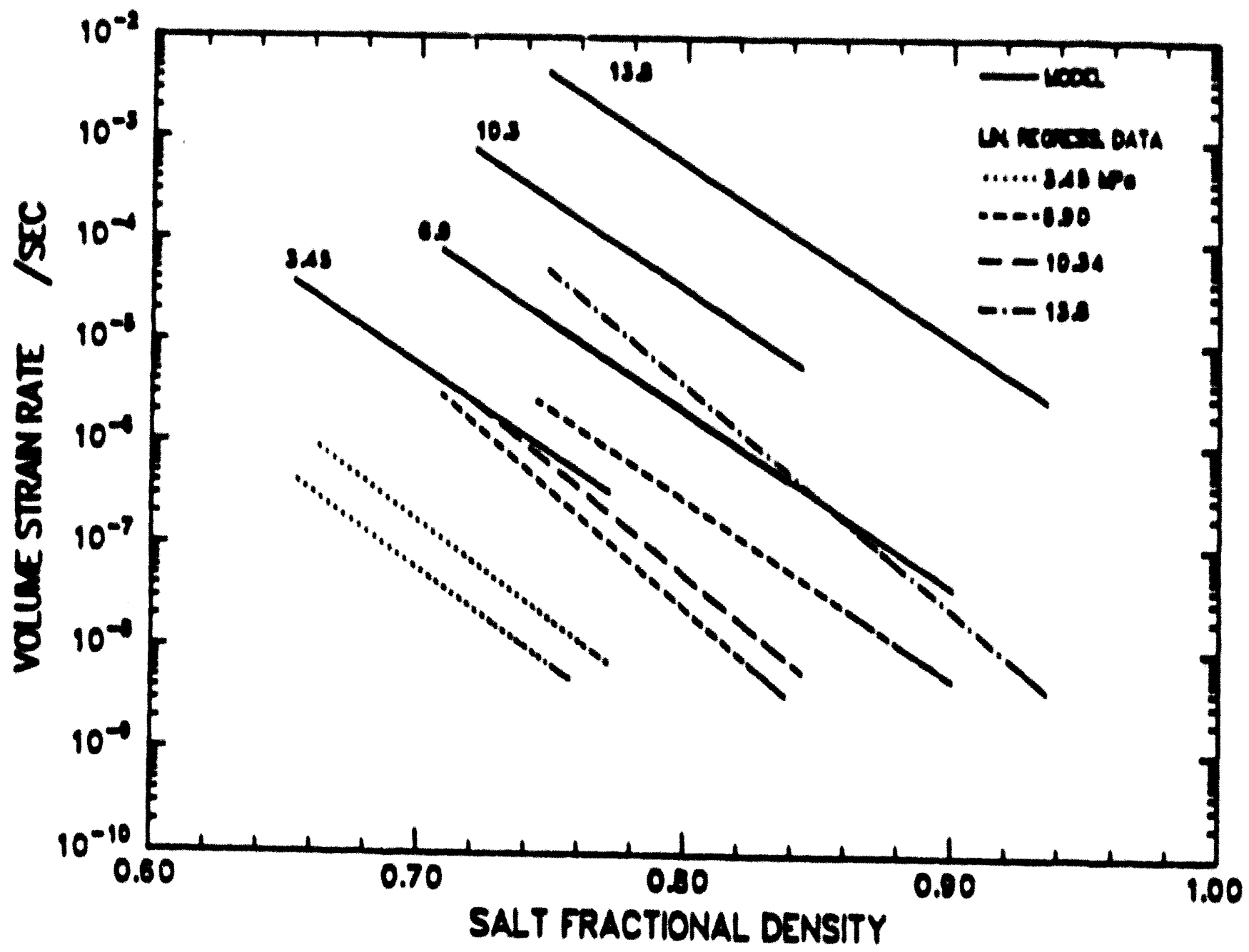

Figure d: Volume strain rate plotted againat frectional denalty. The broken lines are from experiments on crushed eale mixed with inert inclualons. The solid lines ore from mixture thoory usine $A=-1.73 \times 10^{-1}$ from plain, damp, erushed salt louts. 
FIGURE 2

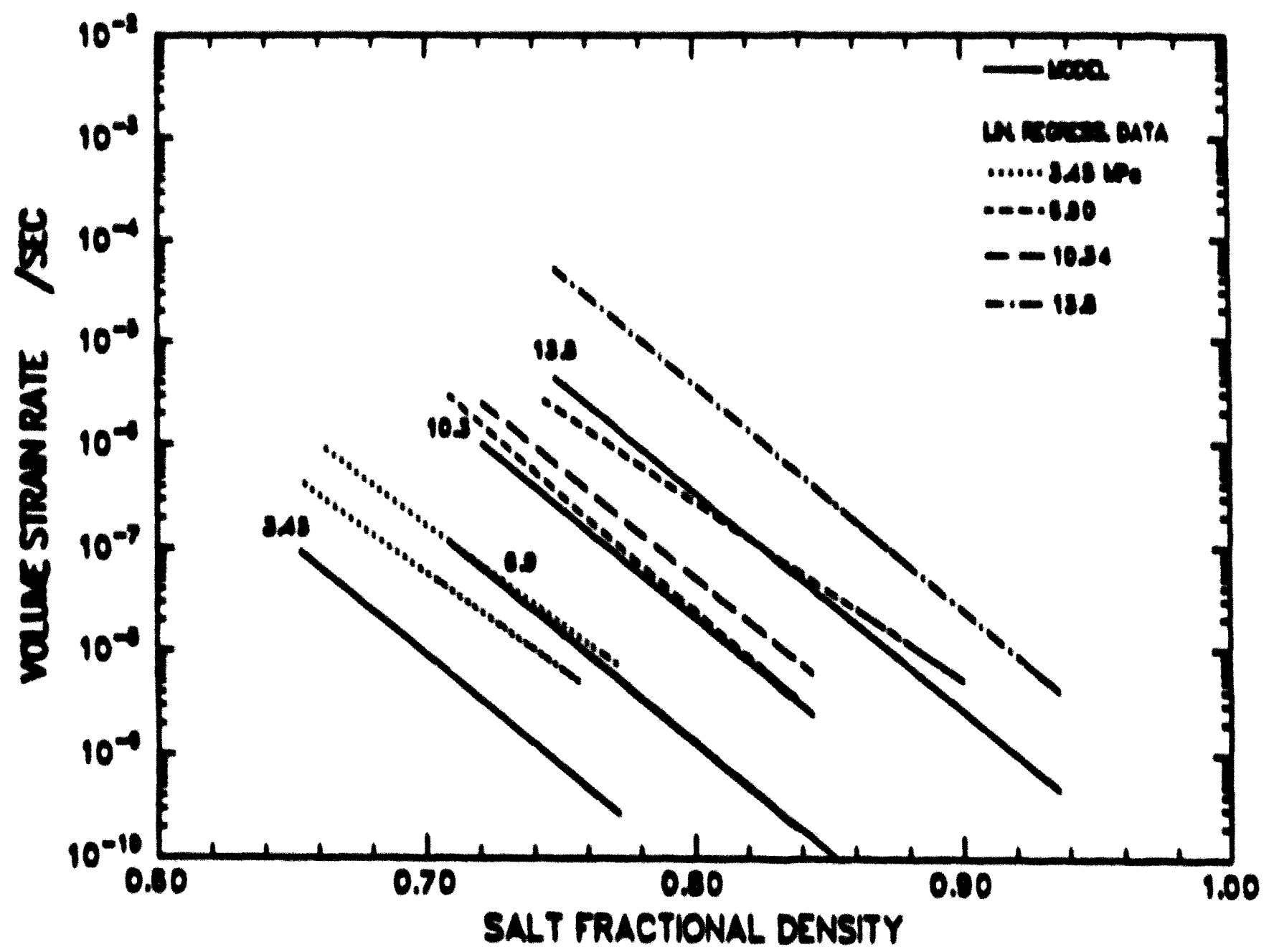

Fisure 2: Volume atrain rale plotled agalnat fractlonal denally. The broken lines are from experlments on eruahed sell anixed with Inert inclusions. The solid lines are from the mixture theory equation with $A=-2.16 \times 10^{-1}$ eseleulated from the demp, erushed acli, fraction of the mixture tosts. 


\section{Ause December 17, 1991}

M.T.Madentid:4048

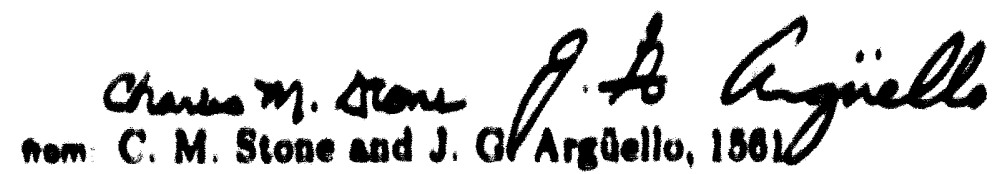

ownen Panel Scale Calculations for the Wate Inolation Pilot Plant (WIPP)

\section{INTRODUCTION}

One of the cask liated in the Diapoual Room lavealigations Work Agreement for FY 91 wa a demonatration calculation of the creep cloeure of a complete WIPP atorage panel containing neven empty dispoeal roome and two haulage ways. The ataled purpowe of this calculation is to determine the fealbility of such calculations for addreasing important inues related to repostlory performance. It is expected that large numbers of panel ecale calculations may be required to anawer questione regardiag long term repository performance. The determiantion of whether such calculatione can be performed and their probable cont are Important parts in developing future plans for the Diaponal Room Investigations program. The faice element code SANTOS(I) in to be used for these calculations aince ils computational performance is better than SANCHO|2).

A previous calculation of the creep closure of a uingle empty disposal room was performed by Morgan(3) uaing SANCHO. In thio calculation, the creep clonure of a alngle diapoual room wa determined for a period of 200 years. Thls calculation showed that creep closure reduced the room volume by 76 percent after 100 years and that the roof and foor touched al approximately 108 years. Typically, a diaponal room model utilizew room and pillar nymmetry and therefore conaider ouly one-half of a diaponal room geometry. The aingle disposal room model correaponda to the responce of a room in an Infinite array of aimilar rooms which implien that all rooms in the panel would clone at the same rate. Thun, panel seale calculations provide iaformation regardiag variationa in room closure that are needed for performance asearment. The model uned by Morgan|3| utlized approximatoly eoven hundred quadrilateral finite elements for the meah diacretization. This calculation required 200 minutes of epu time on the Cray computer.

This memo documents the firat two-dimensional analyais of the creep clonure of a complate WIPP panel. The next section dencriben the panel grometry and modeling asumptions. The third cection presents the results of the analyais followed by a wetion summarizing the results. 
Table 1. Salt Material Properties Used for the Panel Scale Calculation

\begin{tabular}{|c|c|c|c|c|}
\hline $\begin{array}{c}\text { Young's } \\
\text { Modulus } \\
\text { (GPa) }\end{array}$ & $\begin{array}{c}\text { Poloson's } \\
\text { Ratio }\end{array}$ & $\begin{array}{c}\text { Creep } \\
\text { Conatant, } \\
A\end{array}$ & $\begin{array}{c}\text { Croep } \\
\text { Exponent, } \\
n\end{array}$ & $\begin{array}{c}\text { Therma } \\
\text { Conatant, } \\
\text { Q/RT }\end{array}$ \\
\hline 2.48 & 0.25 & $8.79 \times 10^{-30}$ & 1.9 & 20.13 \\
\hline
\end{tabular}

\section{Numerical Model}

As abowa in Figure 1, a typical WIPP panel consiats of seven diaposal rooms. Panels are located Eat and Weat of four beulage ways which run North/South at the facility. The disponal rooms all bave the oume dimenalons. Each room bas a width of $10.06 \mathrm{~m}$ and a beight of $3.96 \mathrm{~m}$. The rooms are located at a borizon of $630.43 \mathrm{~m}$ below the surface, and are eeparated by a centerline-to-centerline diatance of $40.34 \mathrm{~m}$. A plane of aymmetry is asumed to exiat about a Nortb/Soutb line drawn through the center of the four baulage ways. Thus, only two haulage ways and one panel of disposal rooms form the besis for the aumerical model. The two baulage ways bave different dimenaiona from the dispowal rooms and trom each other. The first baulage way, which is neareat the line of aymmetry, bes a widtb of $7.62 \mathrm{~m}$ and a beight of $2.44 \mathrm{~m}$. The second baulage way has a width of $4.27 \mathrm{~m}$ and a beight of $3.66 \mathrm{~m}$. The centerlines of the two haulage ways are separated by diatance of $48.78 \mathrm{~m}$. The borison of the baulage ways is asumed to be the same horison as that for the diaposal rooms, $630.43 \mathrm{~m}$.

The faite element meab for the panel was generated by developing meahea for a single diaponal room and botb baulage ways and then joining them together uning a utility program called GJOIN(4). Figure 2 abowe the meab developed for a tingle disposal room. This meab was duplicated eeven times to create the baic panel meab. Figure 3 sbows the basic menh for a seven disposal room panel. Contact surfaces were defined within each room to hendle contact between the room surfaces as the creep closure becomes large. The vertical boundaries of the mesh were located a bundred meters from the room to eliminate boundary effecto. The haulage way mesbee were joined together and to the ceven diaponal room meah uaing tranaition meshes. Figure 4 abows the two baulage ways and the transition meab. An additional transition meah of $200 \mathrm{~m}$ was added to the basic panel meab to create a farfield horizontal boundary. The complete meab is shown in Figure 5. The model containa over 23,000 quadrilateral finite elements. This model is over 30 times larger than the model uned by Morgan/3) in bis calculation.

The etratigraphy wes asumed to be composed of all alt with Young's modulus divided by the unual factor of 12.8. The properties used in the analyala are abown in Table 1. The creep model uned is the power-law elatic/secondary creep model implemented in SAN. TOS. An applied overburden preasure of $12.23 \mathrm{MPa}$ was applied along the top boundary in conjunction with a gravity body force load in the salt. The combination of overbur. 
den and gravity load is equilibrated by a pressure of $17.52 \mathrm{MPa}$ applied along the lower boundary of the mesh. A horizontal symmetry boundary condition was applied along the left side of the mesh. A no horizontal displacement boundary condition representing the effects of a farfield boundary was applied to the rigbt boundary of the mesh. The applied boundary conditions are shown schematically in Figure 6.

\section{Analysis Results}

This calculation contains several important areas of interest for the Disposal Room Investigations Program. The firat is the variation in vertical room closure within a panel. The accond is a comparison of panel acale room closure with the closure from a single diaposal room calculation. The room numbering scheme used in discussing the results is shown in Figure 6. The vertical room closure is defined as the sum of the floor and ceiling vertical diuplacements at the room centerline. Contact between the floor and roof correspondo to a closure of $3.96 \mathrm{~m}$. Figure 7 shows the vertical closure history for Rooms 1. 4, and Figure 8 shows the closure history for Rooms 4 - 7. The curves of vertical closure show that Rooms 1 and 2 are the only rooms that do not experience floor and roof contact within the 200 year analysis period. After studying the results, it appears that the closures are aimilar for Rlooms 2 and 6 and Rooms 3 and 5 . Rooms 1 and 7 were expected to have similar closure histories but the presence of the haulage ways results in - faster closure for Room 7. Comparison of the closures for Rooms 1 and 7, Rooms 2 and 6 , in Rooms 3 and 3 are shown in Figures 9 - 11, respectively. The results show that the fastest closure (153 years) occurs at the center room (Room 4). The time to achieve room closure increases with distance from Room 4. The two baulage ways (Rooms 8 and 9) have closure biatories, Figure 12, different from the other rooms due to their size differences and their location near the plane of aymmetry. Room 9 experiences contact between the floor and roof within the 200 year analyais period; however, Room 8 exhibits a very slow closure during the 200 year period due to ito initially square shape. Individuals concerned with the closure and the degree of backfill compaction in the underground rooms will be interested in the response of Room 8 because it reaches only 50 percent closure in 200 years.

The closure of Rnom 4 can be compared to the closure for a single disposal room config. uration similar to the one analyzed by Morgan[3]. The single disposal room calculation presented here was performed using the finite element code, SANTOS, with a mesh discretization utilizing approximately 1200 quadrilateral finite elements. The closure comparison between Room 4 response and the single disposal room model is shown in Figure 13. The single disposal room model shows a slightly faster closure (140 years) than Room 4. This result is not unexpected aince the single room corresponds to a larger underground extraction ratio. The single disposal room calculation provides a benchmark for relating single room analyses to probable panel scale responses.

The execution time for the panel scale calculation needs to be compared to other dis. 
posal room calculations in order to assess the cost of performing such large scale calcula. tions. The panel scale calculation performed for a 200-year study period using SANTOS required 55 minutes of cpu time on the Cray YMP computer. This time should be compared to the 200-minute cpu time required for the single disposal room model an. alyzed by Morgan[3] using SANCHO. The single disposal room model required $16 \mathrm{cpu}$ minutes using SANTOS which clearly demonstrates the faster execution times available with SANTOS. Based upon the $55 \mathrm{cpu}$ minutes required for the panel scale calculation, performing panel acale calculations on a regular basis seems to be reasonable both from the perapective of cost and time.

\section{Summary}

Several important observations can be made based on the panel scale calculation. First, the panel acale calculation can provide the details necessary to determine the room to room variation in vertical closure. This is important since three different room geometries exist within a single panel. Room response within the panel varies from complete closure of a disposal room to 30 percent closure of a baulage way in 200 years. Second, the fastest closure of a panel room is within 10 percent of the closure computed by a single disposal room model. Finally, the cost in both analysis time and money of performing a panel scale calculation is within bounds which should be acceptable to the Disposal Room Investigations program. Variations in the problem definition requiring different boundary conditions or constitutive models will probably increase the cpu time required from the values reported here.

\section{References}

1. Stone, C. M., "SANTOS - A Two-Dimensional Finite Element Program for the Quasistatic, Large Deformation, Inelastic Response of Solids," SAND90-0543, Sandia National Laboratories, nibuquerque, NM, in preparation.

2. Stone, C. M., R. D. Krieg, and Z. E. Beisinger, "SANCHO - A Finite Element Code for the Quasistatic, Large Deformation, Inelastic Response of Two-Dimensional Solids," SAND84-2618, Sandia National Laboratories, Albuquerque, NM, April, 1985.

3. Morgan, H. S., "Estimate of the time needed for TRU storage rooms to close," Memorandum to D. E. Munson, 6332, Sandia National Laboratories, 1987.

4. Sjaardema, G. D., "GJOIN: A Program for Merging Two or More GENESIS Databases," Memorandum to Distribution, Sandia National Laboratories, Albuquerque, NM, June 19, 1991.

CMS:1561 
Copy to:

1425 J. H. Biffle

1434 D. R. Martinez

1510 J. C. Cummings

1550 C. W. Peterson

1561 H. S. Morgan

1561 J. G. Argüello

1561 C. M. Stone

1561 J. R. Weatberby

1562 R. K. Thomes, acting

6345 B. M. Butcher 


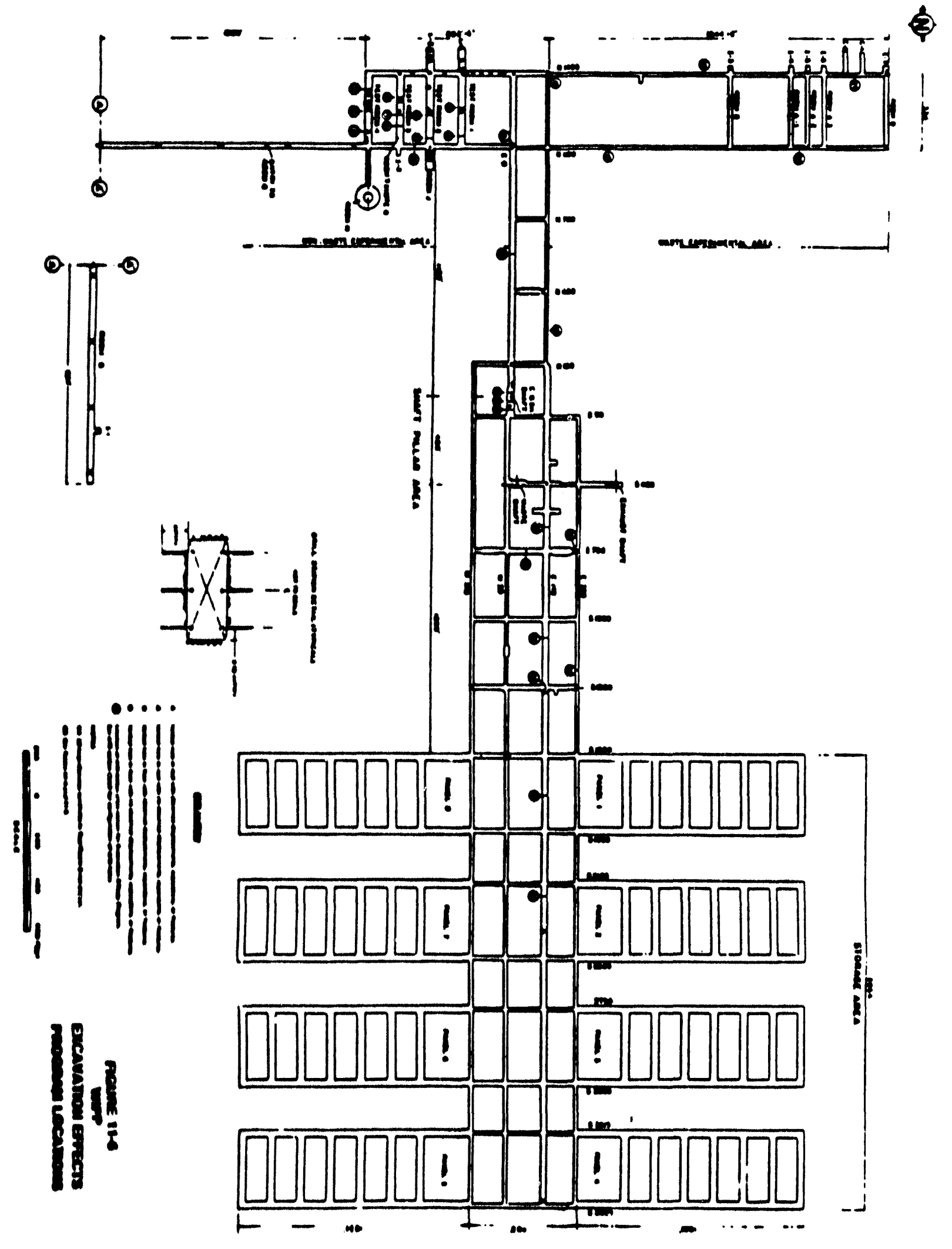

Figure 1. Plan View of the WIPP Underground Facility Showing the Disposal Rooms and the Haulage Ways. 


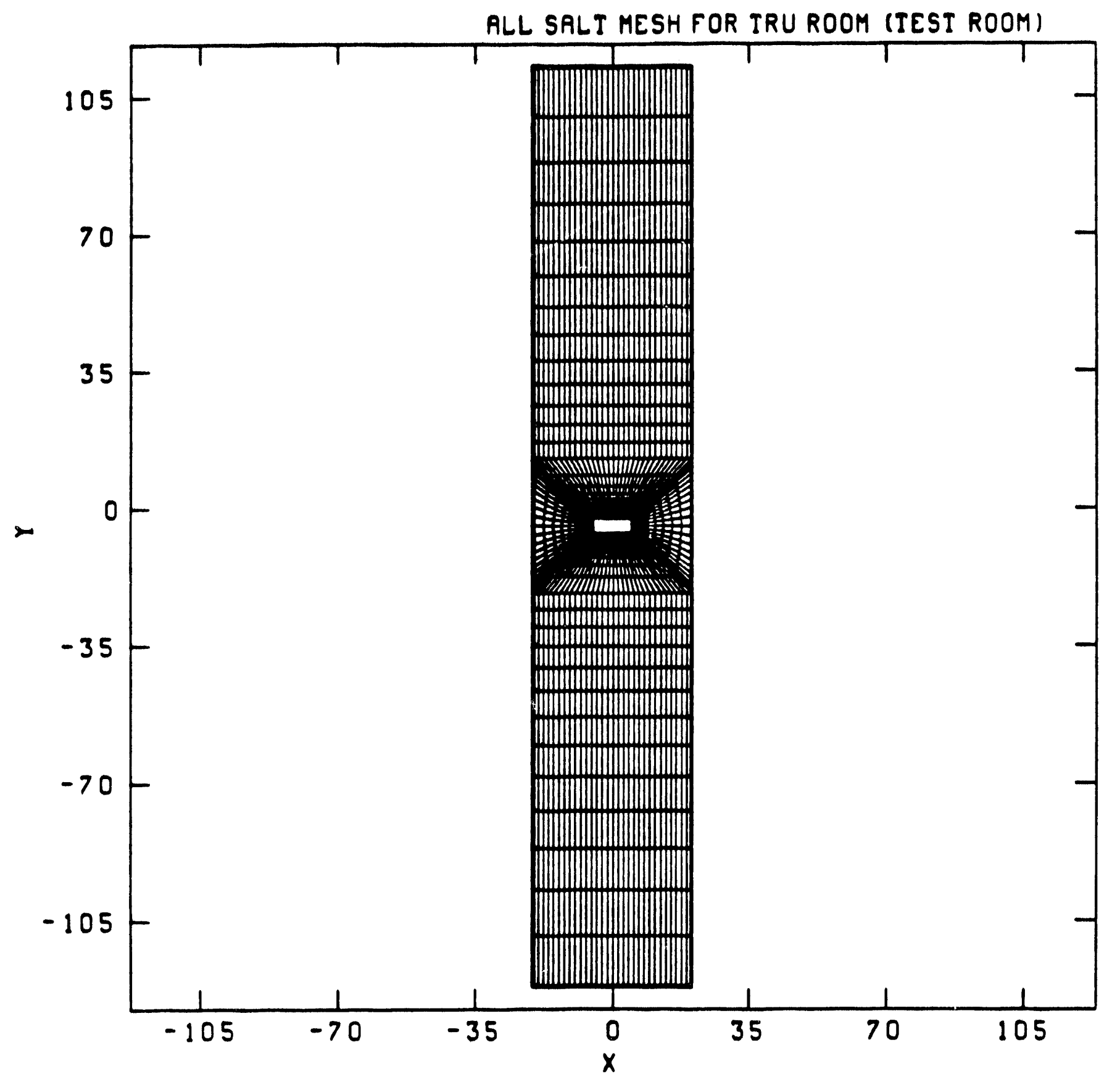

Figure 2. Mesh Discretization Developed for Use With the Single Disposal Room Model. 
ALL SALT MESH FOR TRU ROOM (TEST ROOM)

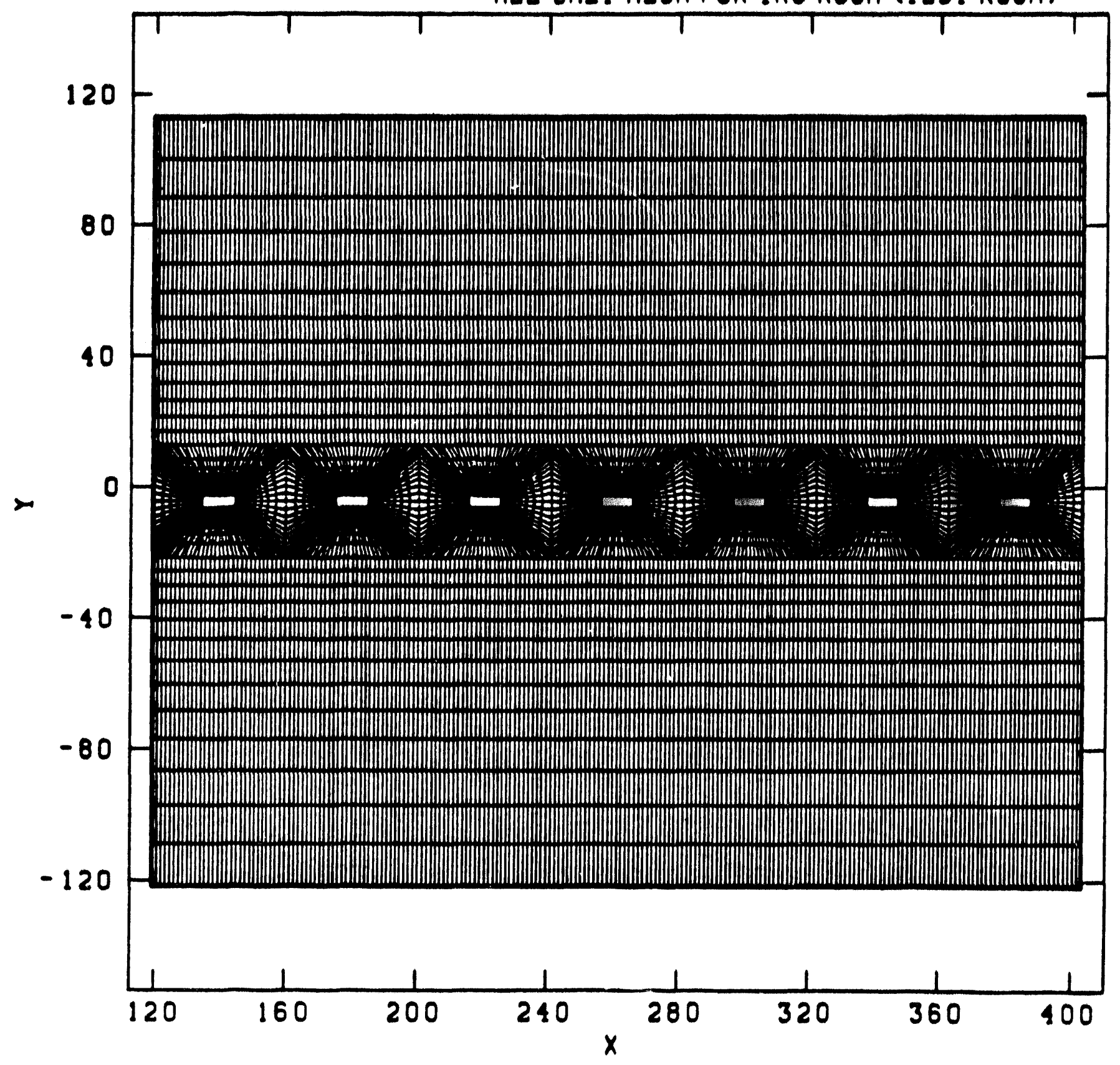

Figure 3. Mesh Discretization Used for the Basic Seven Room Panel. 


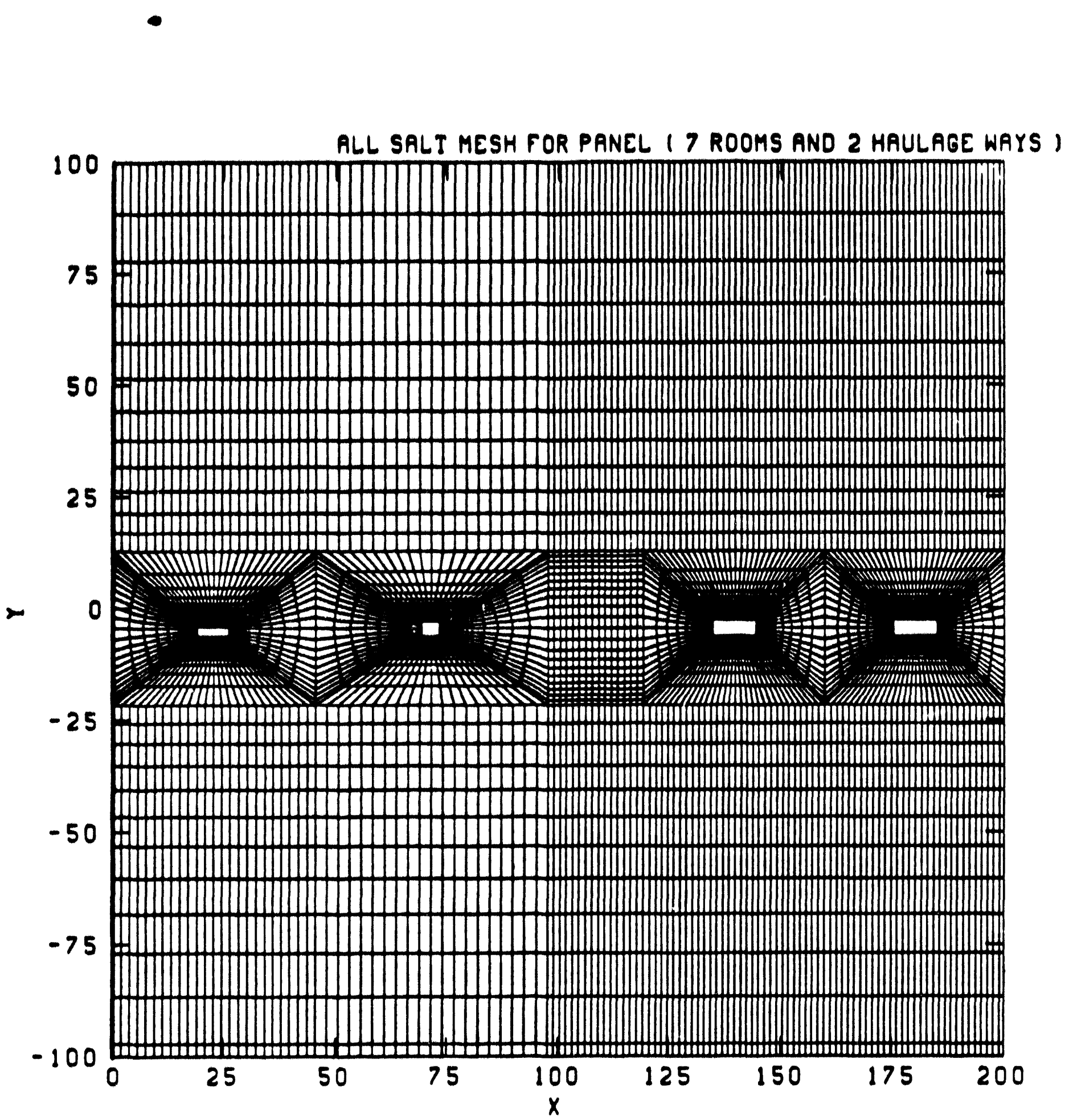

Figure 4. Mesh Discretization Used for the Two Haulage Ways and Transition Mesh. 


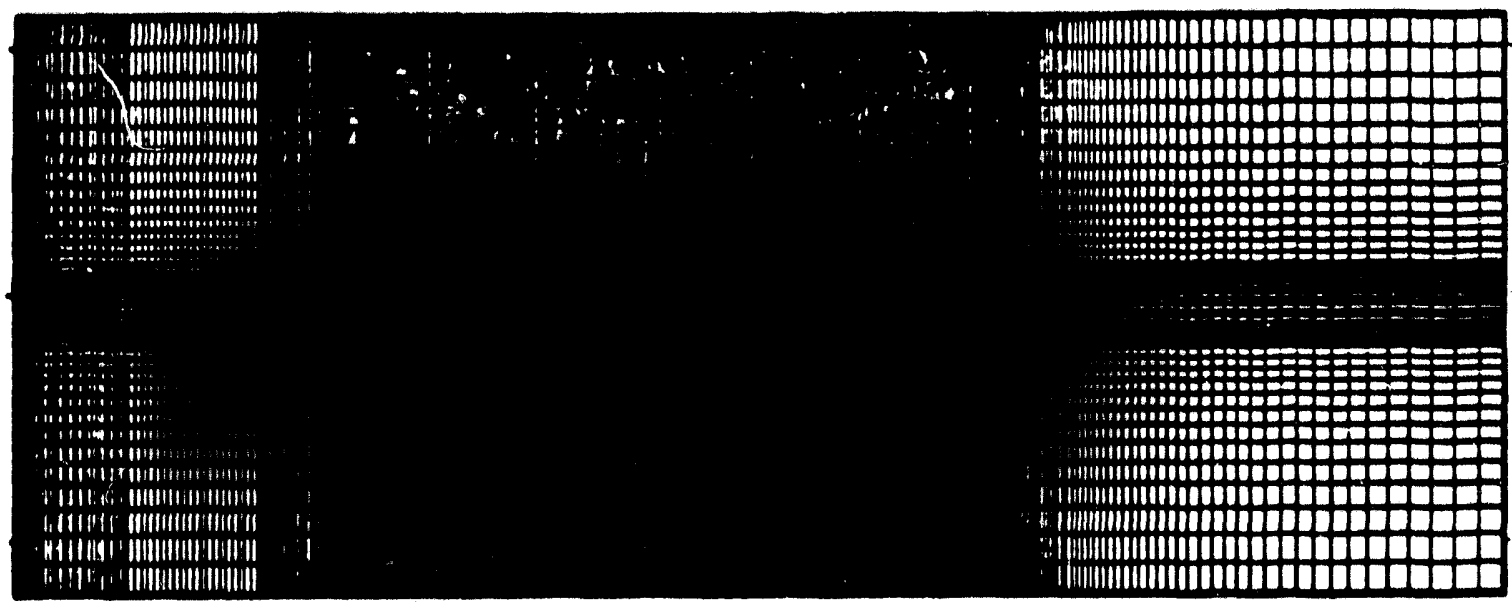

Figure 5. Mesh Discretization Used for the Complete Panel Scale Analysis.

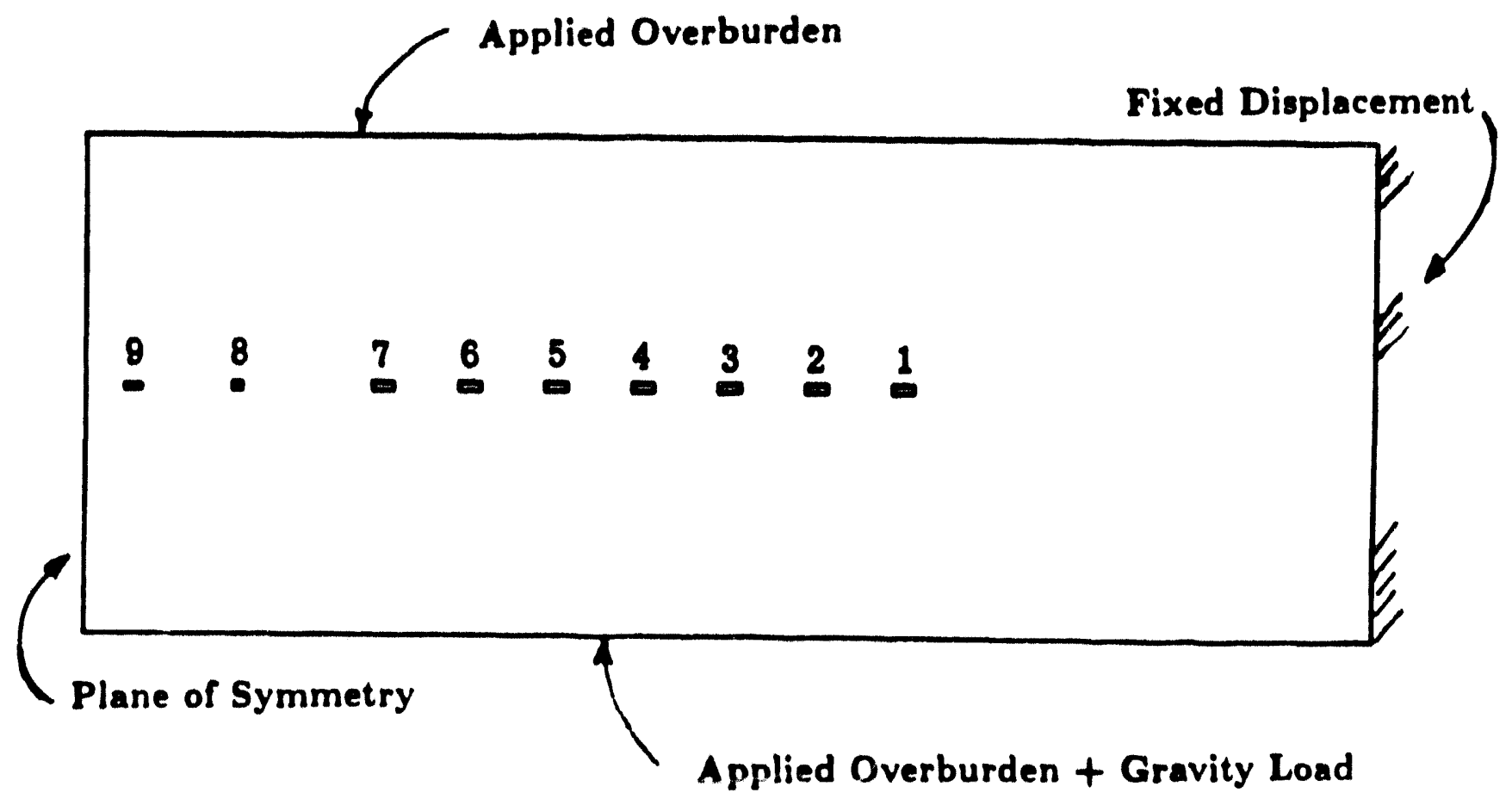

Figure 6. Schematic of the Boundary Conditions Applied to the Panel Scale Analysis. The Room Numbering Scheme Used in the Discussion is Also Shown. 
F. T. Mendenball, 6345

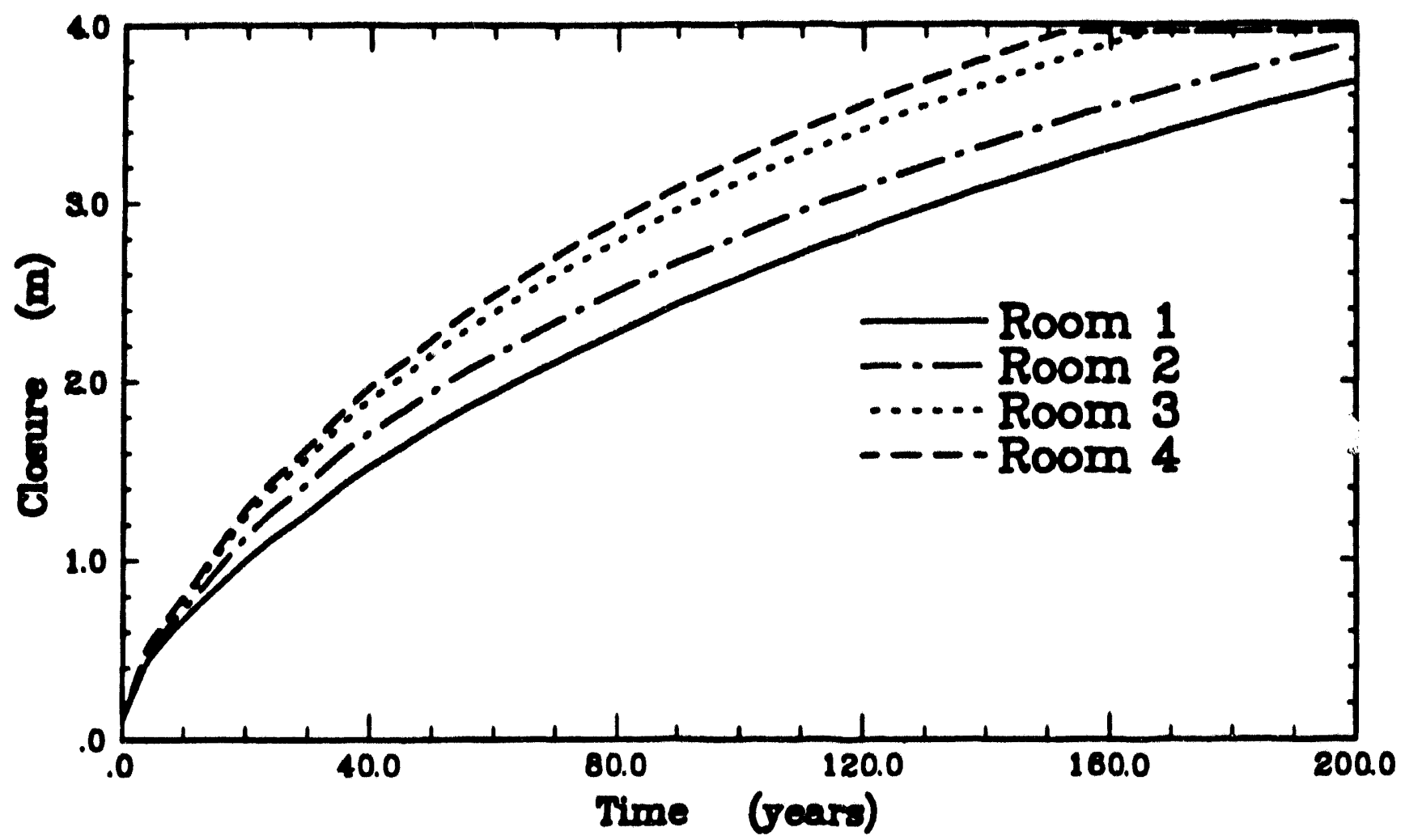

Figure 7. Vertical Closure History for Rooms 1 Through 4.

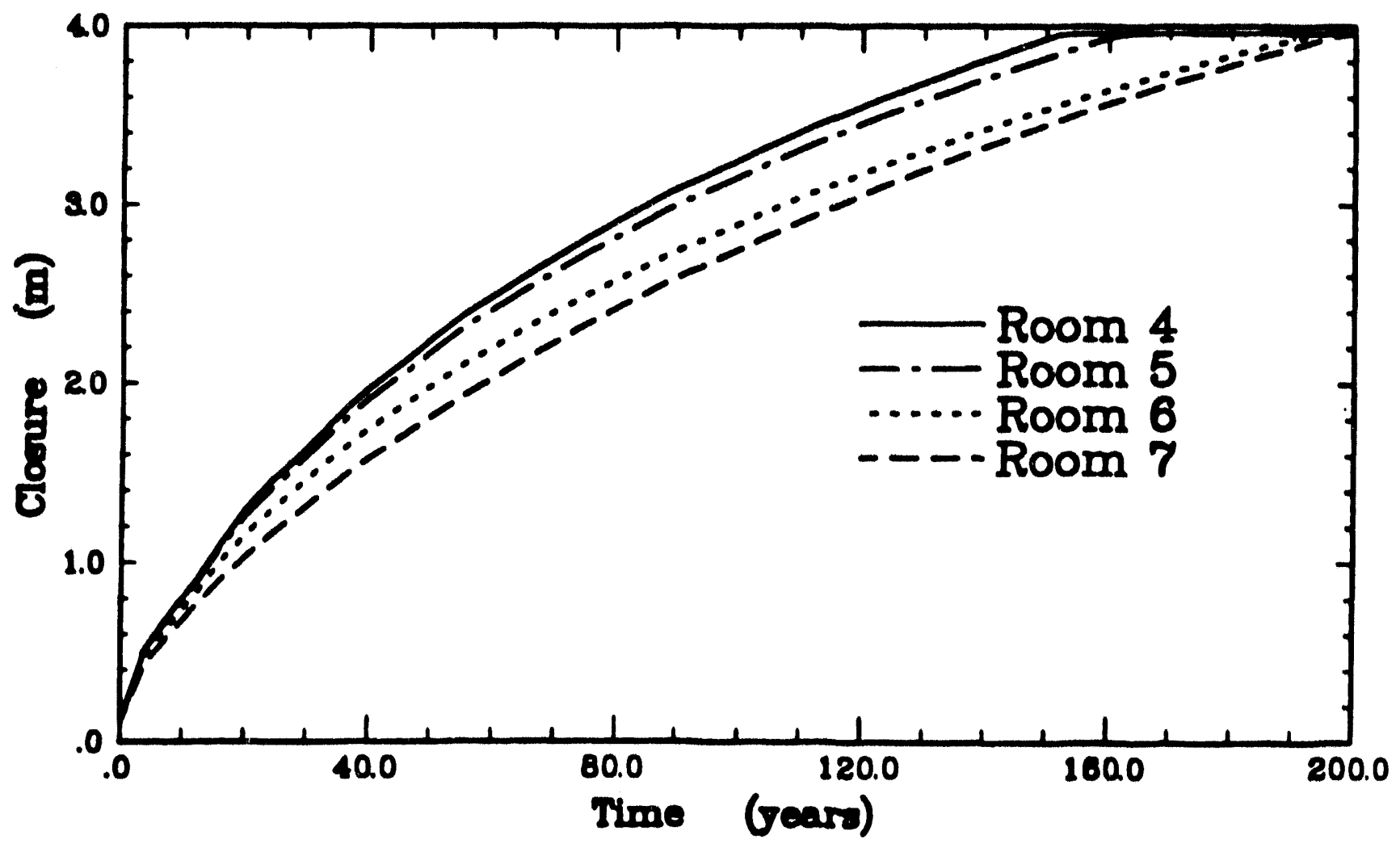

Figure 8. Vertical Closure History for Rooms 4 Through 7. 


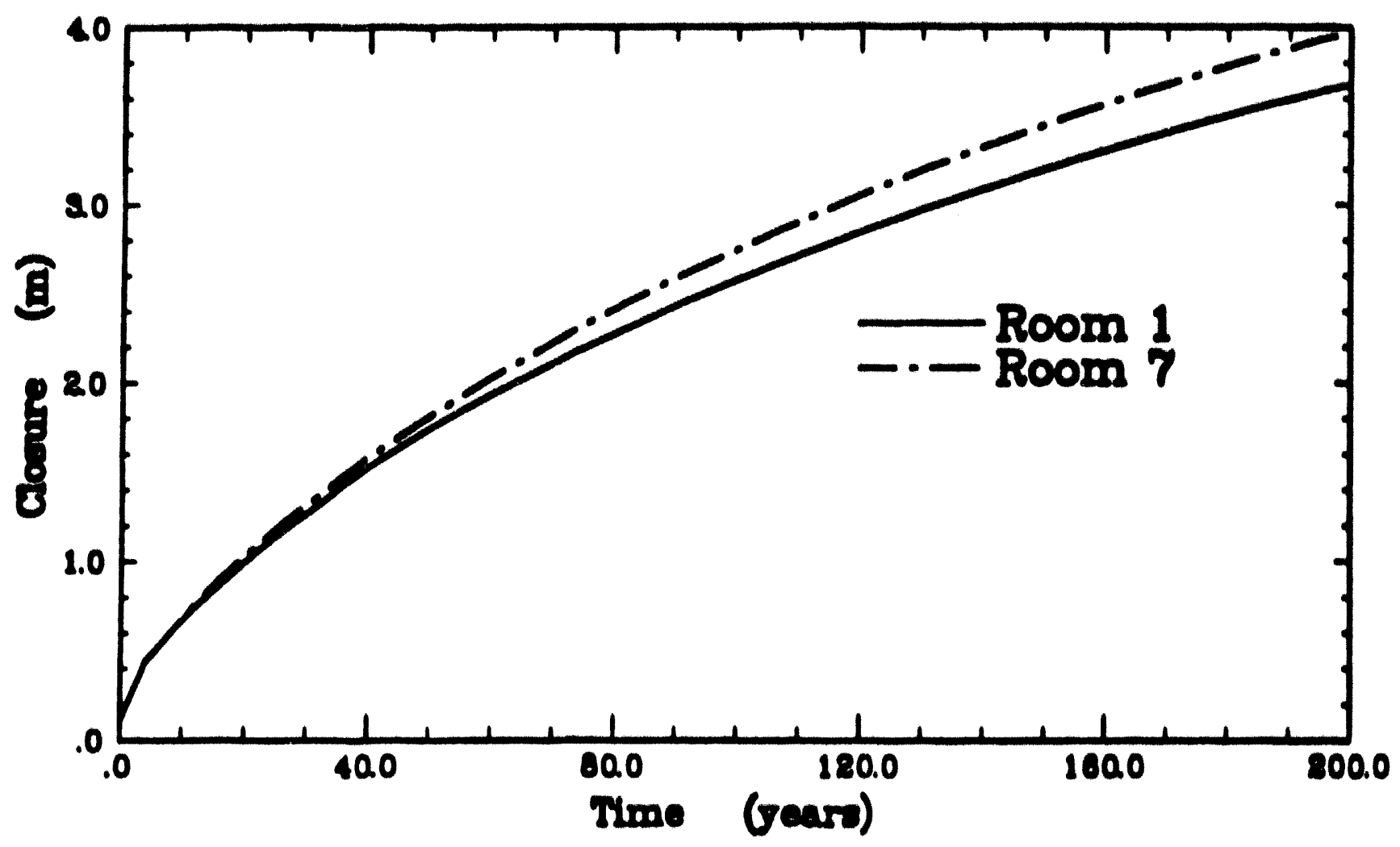

Figure 9. Comparison of Vertiral Closure for Rooms 1 and 7.

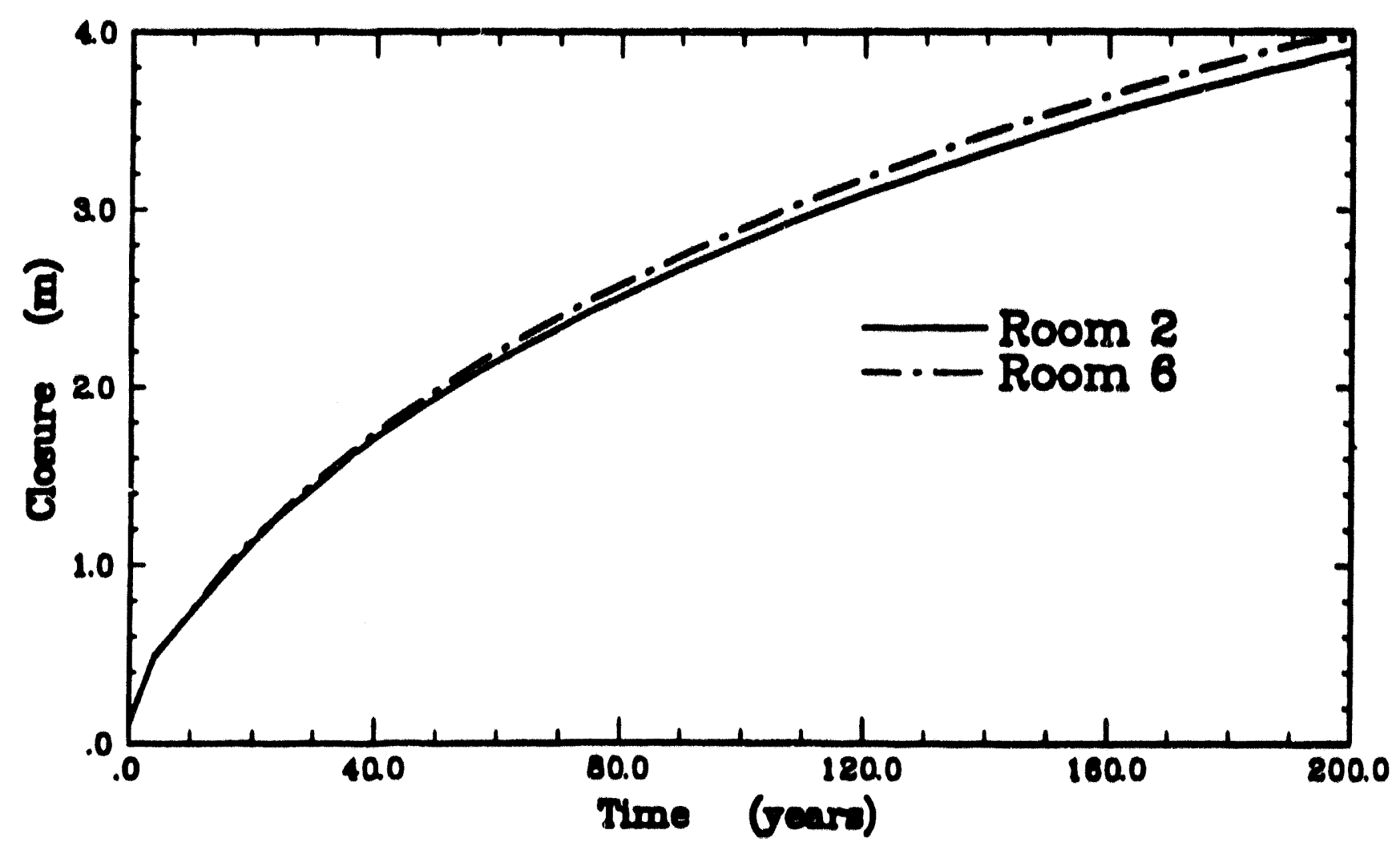

Figure 10. Comparison of Vertical Closure for Rooms 2 and 6. 
F. T. Mendenball, 6345

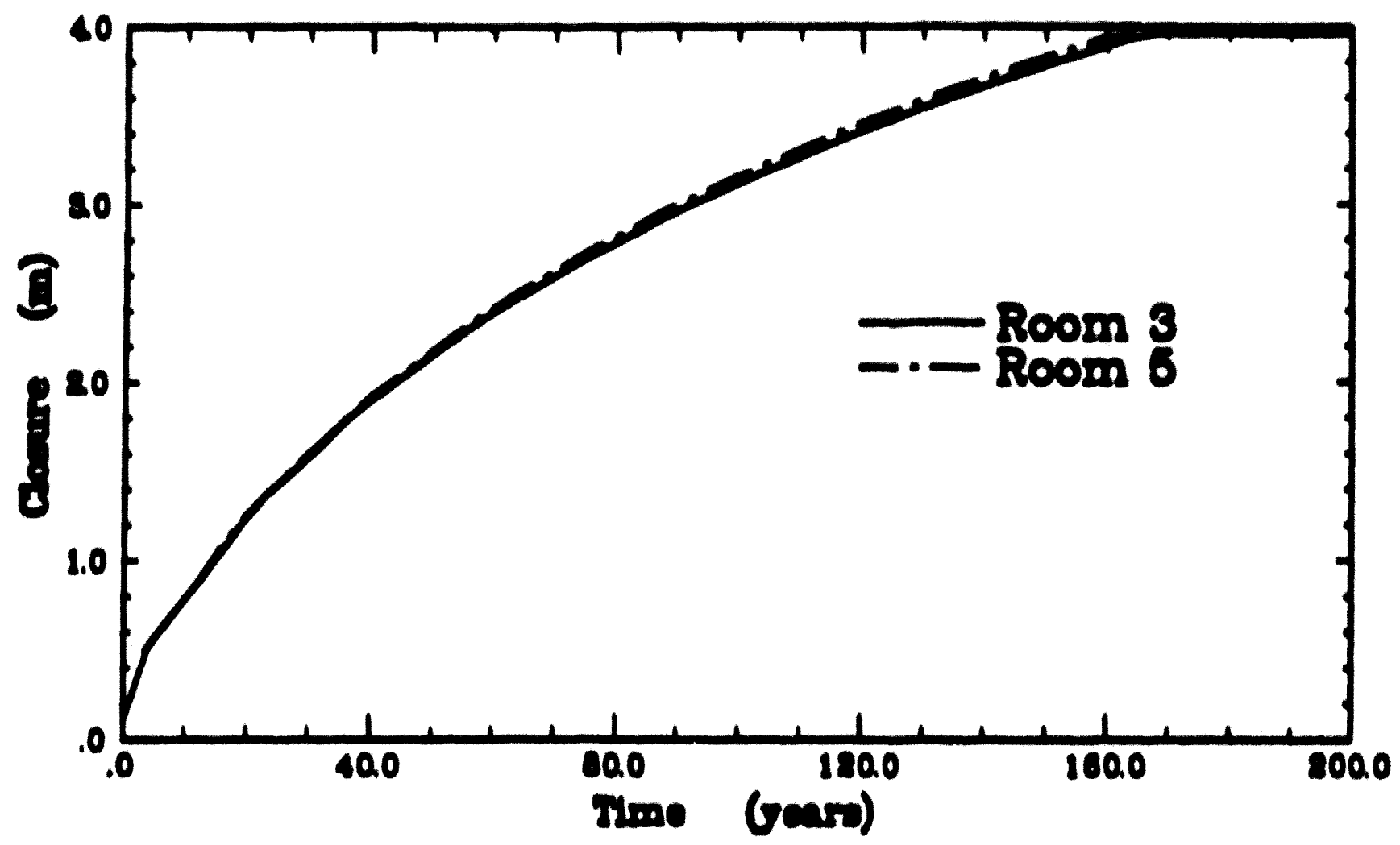

Figure 11. Comparison of Vertiral Clonure for Reomis 3 anil ;

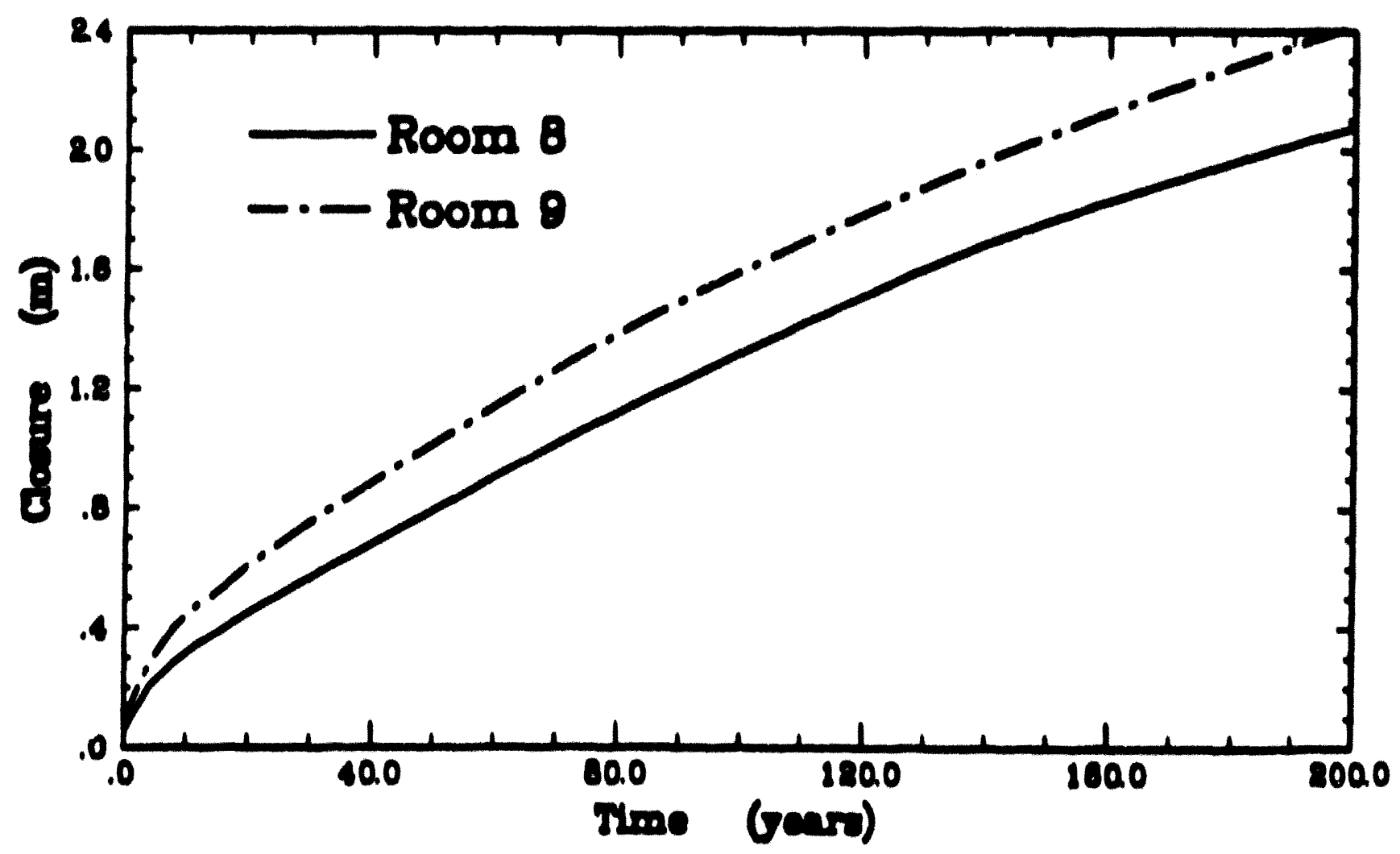

Figure 12. Comparioon of Vertical Clonure for Haulage Way Rooms 8 and 9. 


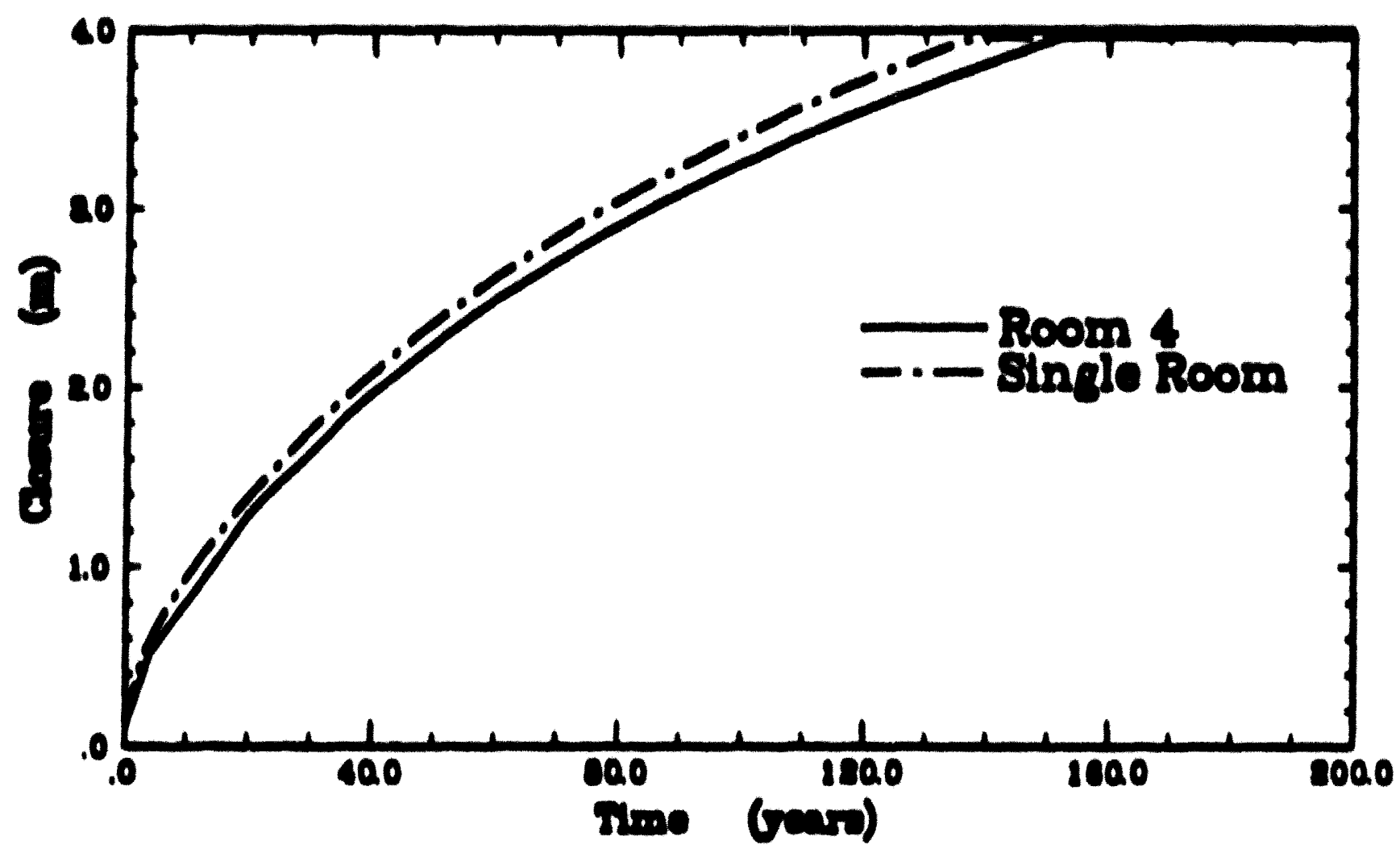

Figure 18. Comparison of Vertical Closure Results for Room 4 and for a Single Disposal Room. 
from: C. M. Stune and J. O. Aruduello, 1561

subject: Resolution of the Discrepancy in Predicted Closure of an Emply Disposal Room

\section{Introduatlon}

Recently, there has been some concern expressed over the differing results obtained for the computed creep closure of empty disposul rooms in sult. The calculations $\mid 2-31$ were to be used as a first estimate of the vold fraction of compacted wase within the disposal rooms. The calculations performed with iwo different finite element codes, SANCHO $|6|$ and SPECTROM-32 11), showed Ierge differences. greater than a factor of 2.5, In the time to completely close the room. The analysis performed with SPECTROM-32 showed a fuster closure rate than the calculation performed with SANCHO. We belleve these differences to be aunciated with the different kinemate formulations used in the codes, apecifleally, the difference between imall deformation and large deformation formulations. In addition. we belleve the leck of contact surfeces applied to the roof, foor, and $\mathrm{rb}$ of the SPECTROM-32 disposal room model also contributed to these differences.

The necesaity of Including large deformation kinematics in analyzing the response of WIPP dispoual rooms is not new. As early a 1986, the WIPP project sponsored a parallel calculation exercise (4.5) between these ame codes. This exercise involved computing the closure of a heated disposal room for a period of five yean. The ventical closure results differed by more than 25 percent for the time period atudied. The major factor contributing to this difference was identifled during the discrepancy resolution process as the difference between large deformation behavior (SANCHO) and amall deformation (SPECTROM: 32). The magnitude of the vertical closure in the parallel calculation was computed to be approximately $1.0 \mathrm{~m}$ a five years. This amount of closure is amall in comparison to the $3.96 \mathrm{~m}$ closure required for the floor and roof in the calculadon of current interest so the probability of experiencing large deformation behavior is high. This memonandum will present the results of a numerical study performed to document the cause of these differences. The following section will describe the physical problem and the numerical model used for the stc dy. The third section will describe the numerical codes and point out their differences. The final section will present the resulis of the atudy and discuss the reasons for the apparent discrepancy. 


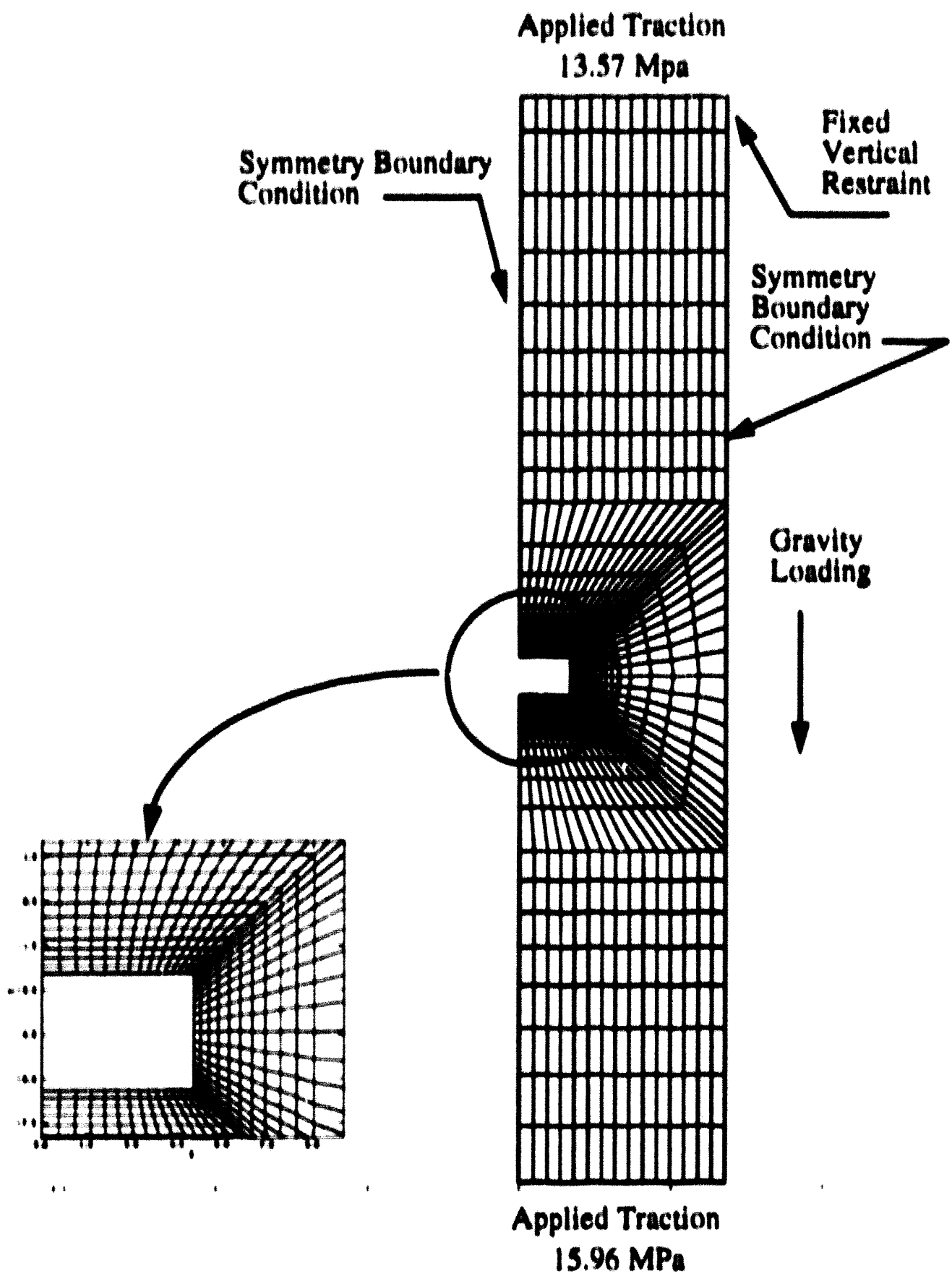

Figure 1. Geometry, Boundary Condidions und Mesh Discretization Used for the Analysis of the Creep Closure of the Empty Disposal Room

\section{Problem Dasarintlon}

The problem of interest is the computati in of the time-dependent closure of an empty WIPP disposal room. We will study a model problem involving the closure of an empty disposal room in an all sale stratigraphy. The room is one of an infinite array of disposal rooms, $40.54 \mathrm{~m}$ on center, so symmetry boundary conditions are used to simplify the problem. The disposal room size is $10.06 \mathrm{~m}$ wide by $3.96 \mathrm{~m} \mathrm{high}$. The room is located at 
the repository horizon which is $650.43 \mathrm{~m}$ below the surface. The vertical extent of the problem is approximately $50 \mathrm{~m}$ above and below the room horizon. A traction boundary condition is applied to the problem at the top boundary to represent the load associated with the salt overburden. Gravity loads are applied to the salt and a vertically varying initial stress state is applied based on the density of the overburden and depth below the surface. The lower boundary traction reacts the applied overburden and gravity loads. The problem geometry and applied boundary conditions are shown on the generated mesh in Figure 1. The mesh contains approximately 1100 uniform strain quadrilateral elements. The constitutive model used for the salt is the old reference elastic-secondary creep model with reduced lastic constants, E/12.5. The constants used are shown in Table 1.

Table 1: Salt Material Properties Used for Disposal Room Calculations

\begin{tabular}{|c|c|c|c|c|}
\hline $\begin{array}{c}\text { Young's } \\
\text { Modulus } \\
(\mathrm{Pa})\end{array}$ & $\begin{array}{c}\text { Poisson's } \\
\text { Ratio }\end{array}$ & $\begin{array}{c}\text { Creep } \\
\text { Constant, A }\end{array}$ & $\begin{array}{c}\text { Stress } \\
\text { Exponent, } n\end{array}$ & $\begin{array}{c}\text { Thermal } \\
\text { Constant } \\
\text { Q/RT }\end{array}$ \\
\hline $24.8 \times 10^{8}$ & 0.25 & $5.79 \times 10^{-36}$ & 4.9 & 20.13 \\
\hline
\end{tabular}

\section{Code Descrintions}

The two structural codes, SANCHO [6] and SPECTROM-32 [1], used for the calculations are quite different. SANCHO is a special purpose finite element program which has a single element type. The element is a four-node quadrilateral with the numerical integration performed at the $2 \times 2$ gauss points for the deviatoric part of the stress and at the element centroid for the bulk pressure part. The code uses an adaptive dynamic relaxation technique to achieve an equilibrium solution to the applied loads. The code is based on a large displacement formulation which means that the current deformed geometry of an element is used in computing the element stiffness, body forces, and traction boundary conditions. This can be a very important effect when large deformations occur such as in the problem discussed in this memo. SANCHO also has a master-slave contact surface algorithm which can be used to model arbitrary contact of surfaces such as contact between the roof, floor and ribs of a disposal room. SPECTROM-32 is a general purpose, finite element code with several different elements in its element library. It has the four-node quadrilateral element which is found in SANCHO. SPECTROM-32 is based on a small deformation formulation, which means that the element stiffness is based on the original element geometry. Changes in geometry will not be reflected in updates to the element stiffness or to the applied loads that should result when an element's shape changes. SPECTROM-32 also does not have the capability for modeling arbitrary surface contact. However, it does have the capability to model sliding surfaces using a thin, special element.

The SANTOS [7] code, a new code based on the original SANCHO solution algorithm, has been used for the present work. It is based on a large deformation formulation. However, it was modified for this study so that it would also generate small deformation solutions for comparison to the large deformation solutions. The code contains a single element type 
which is a uniform strain, four-node quadrilateral element. The code uses a self-adaptive dynamic relaxation scheme to reach the equilibrium solution. An arbitrary contact surface algorithm based on the master-slave concept is also implemented. The element type and code architecture are based on current state-of-the-art finite element technology. It should be noted here that a finite element code with a large displacement formulation can generate small displacement solutions if the displacements are small. However, a small displacement code cannot produce a large displacement solution..

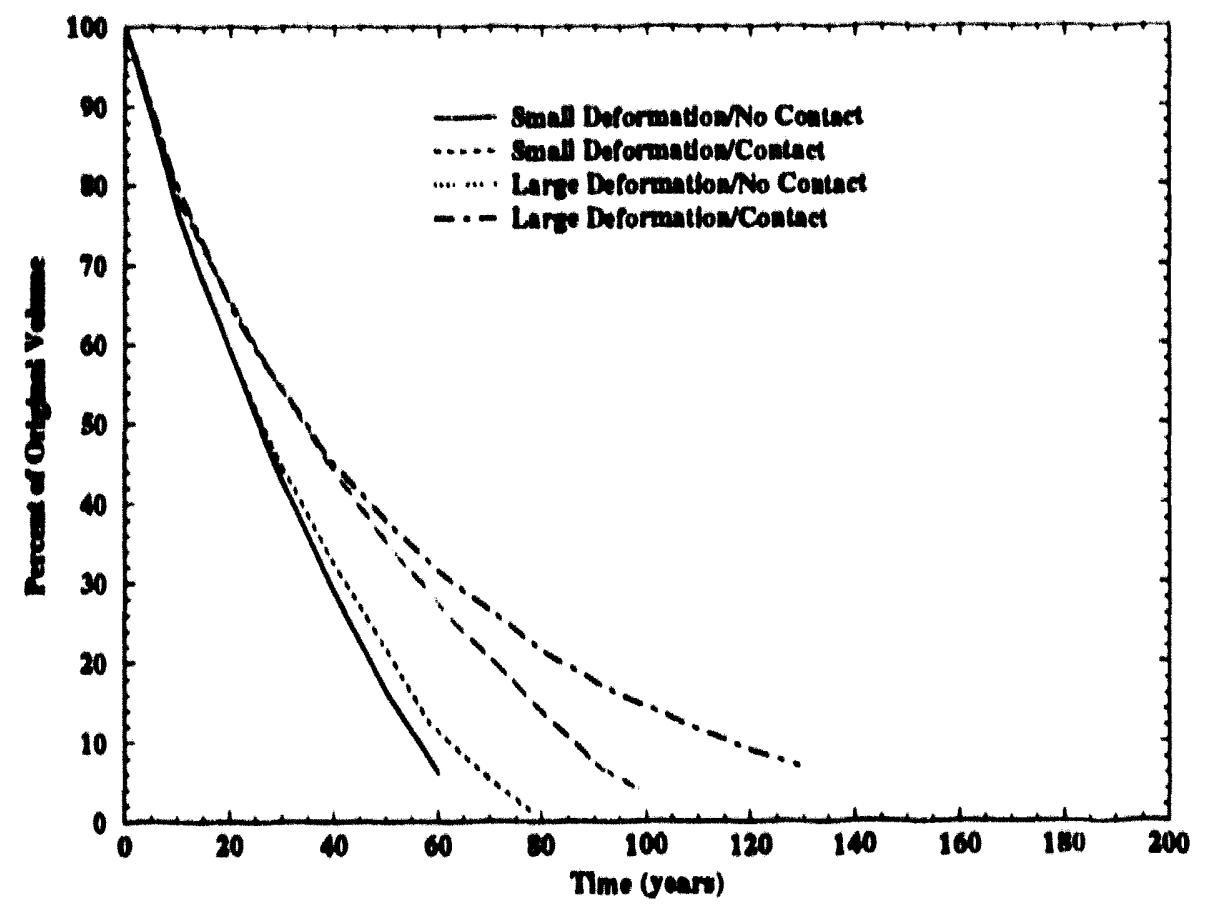

Figure 2. Comparison of Analysis Assumptions Used for Calculation of the Disposal Room to Closure

\section{Disposal Room Calculations}

Several calculations were performed to investigate the effects on closure of large deformation and of including contact surfaces between the roof, floor, and rib of the disposal room. Figure 2 shows the percent of original room volume as a function of time for the four different analyses performed in this study. The first analysis involved the assumption of small deformation behavior without the presence of contact surfaces. This calculation, designated as Small Deformation/No Contact in Figure 2, represents the results possible with SPECTROM-32. The volume of the disposal room is shown to decrease almost linearly with tume. This same linear behavior is seen in the percent volume-time results reported in [2]. The final room deformed shape is shown in Figure 3. Note that the comers of the room have overlapped during the calculation. This means that after overlap has occurred the results no longer have any physical meaning. The deformed shape in Figure 3 also illustrates the difficulties in determining the correct room volume for this comparison. In this study, the reported volume at any particular time was computed on the basis of the non-overlapped area. 
The second analysis again used the small deformation assumption but included the effects of contact surfaces along the roof, floor, and rib. The percent of original volume results are shown in Figure 2, as Small Deformation/ Contact. The rate of room closure is slowed somewhat by the propping action of the rib but the overall effect of the contact surface is small. The deformed shape at the final analysis time is shown in Figure 4.

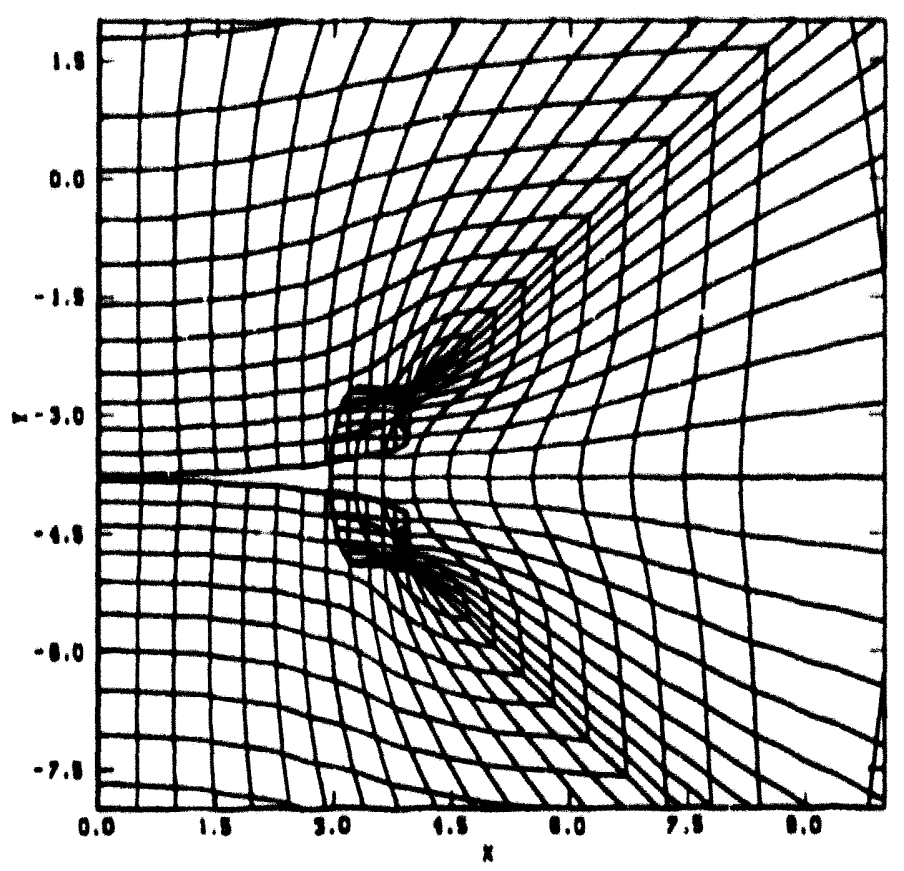

Figure 3. Final Deformed Shape of the Disposal Room for the Case of Small Deformation Analysis With No Contact Surfaces

Our analyses will now include the effects of large deformation. Large deformation will account for increases in pillar width and other geometry changes to compute the actual stress state experienced by the salt. The first calculation does not include the use of contact surfaces. The room volume calculation, labeled Large Deformation/No Contact in Figure 2 , shows a large reduction in room closure rate in comparison to the small deformation cases. There is almost a factor of two difference betwein the Small Deformation/No Contact and Large Deformation/No Contact analyses. This difference is much greater than the effect of including contact surfaces for the small deformation analyses. It must be remembered, however, that the results of the Large Deformation/No Contact analysis are invalid after overlap has occurred. The final deformed shape is shown in Figure 5. The last analysis is the large deformation analysis with contact surfaces included. The results, shown in Figure 2 as Large Deformation/Contact, are very similar to those obtained in [3]. The rate of room closure is slowed even further when large deformation effects and room propping occur. The final room configuration is shown in Figure 6 . Note the difference in deformed shape occurring near the cormers when compared to the small deformation analysis with contact surfaces.

A final observation about the results shown in Figure 2 is that the differences between small deformation and large deformation are seen to occur at early times. Differences in the 
curves can be observed at times less than 10 years. The effects of the contact surfaces can be observed as branching along the two curves associated with small and large deformation solutions. The contact surfaces do not apnear to be a factor until approximately 25 years for the small deformation analyses and 40 years for the large deformation analyses.

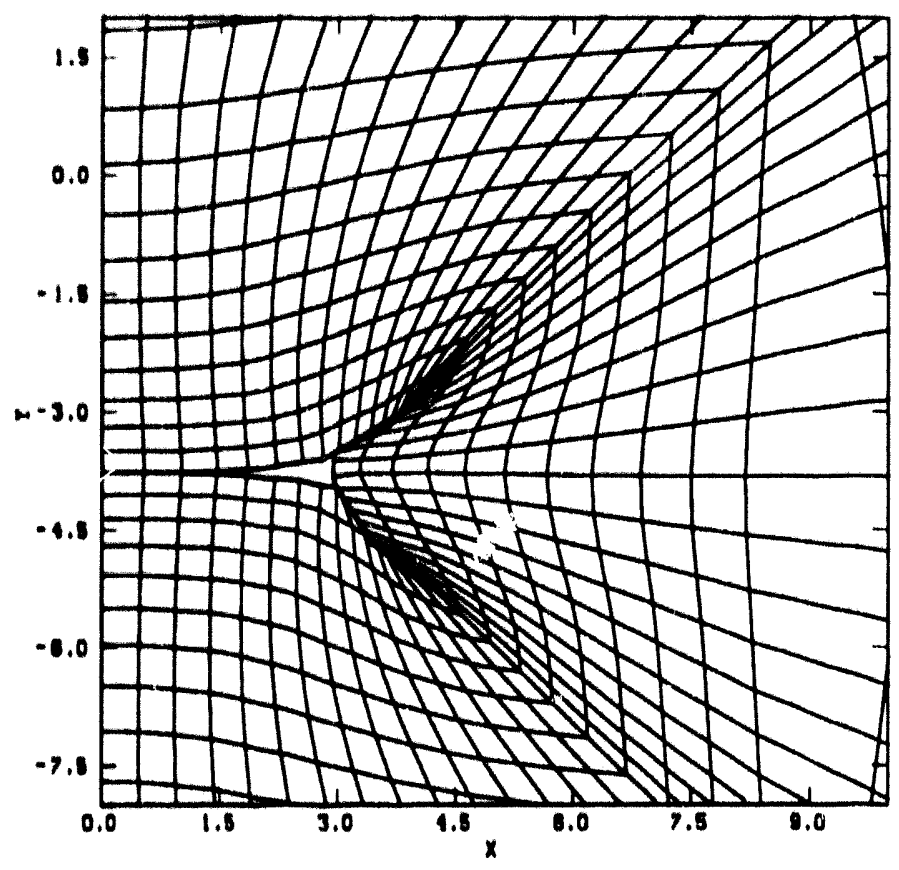

Figure 4. Final Deformed Shape of the Disposal Room for the Case of Small Deformations With Contact Surfaces.

\section{Summary}

Computational analyses of a disposal room in an all salt stratigraphy have been performed to determine the cause of the significant differences in room closure calculations reported in [2-3]. The analyses investigated the effects of large deformation kinematics and the use of contact surfaces for the roof, floor, and rib of the disposal room model. The results show that the largest difference between the calculations reported was due to the ability or inability by the finite element codes to model large deformation behavior. The effect of including contact surfaces was important but not to the same extent as the large deformation assumption. It is important to mention here again that the results for the analyses which did not include contact surfaces are not valid after the surfaces of the room have overlapped. Therefore, caution should be exercised with regard to reporting these results. Differences between the small and large deformation solutions were shown to occur within the first ten years of the analyses. 


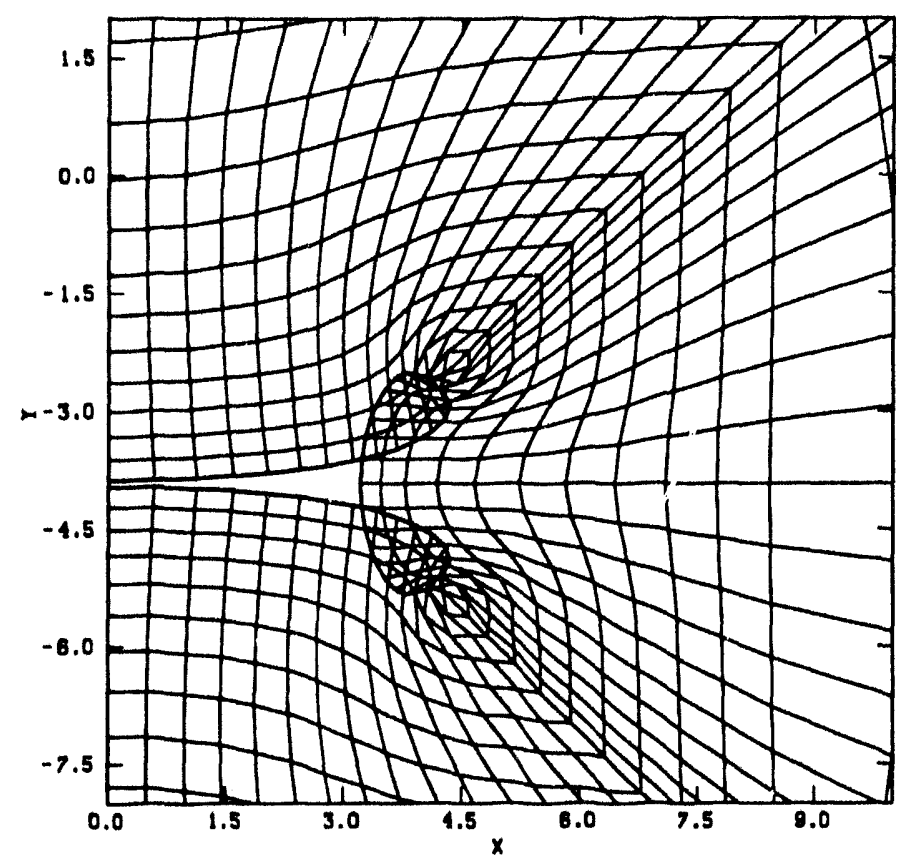

Figure 5. Final Deformed Shape of the Disposal Room for the Case of Large Deformation With No Contact Surfaces.

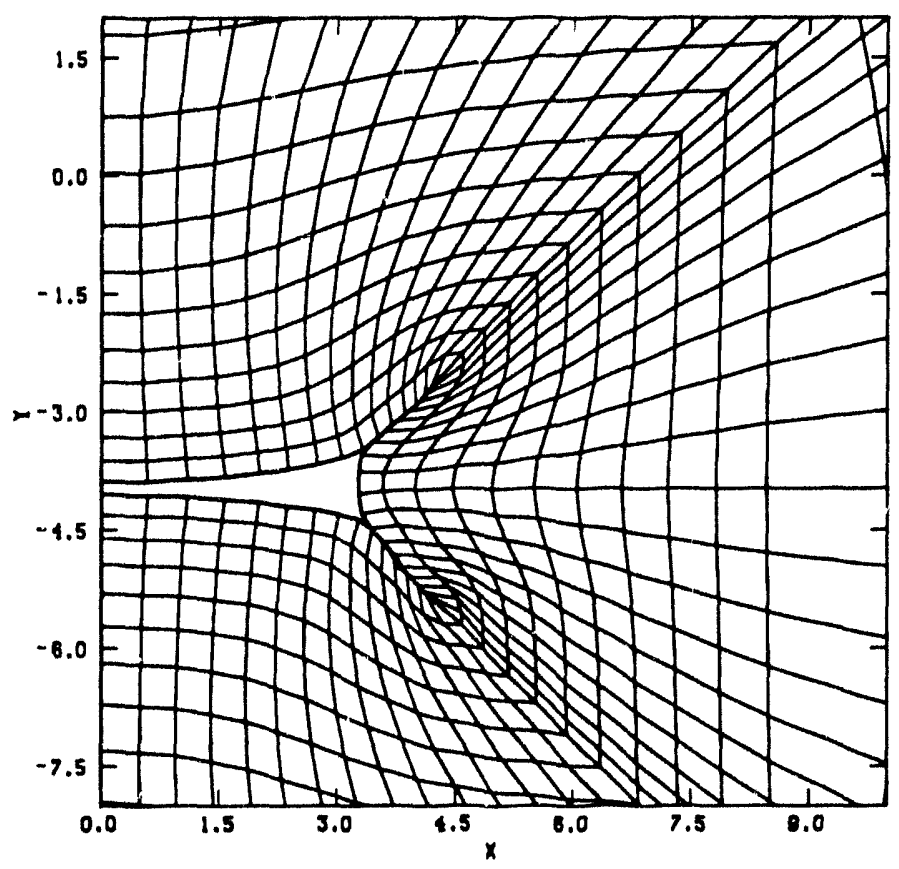

Figure 6. Final Deformed Shape of the Disposal Room for the Case of Large Deformation With Contact Surfaces. 
A- 142 


\section{Sandia National Laboratories}

Albuquerque, New Mexico 87185

dato: May 17, 1989

10: B. M. Butcher, 6332

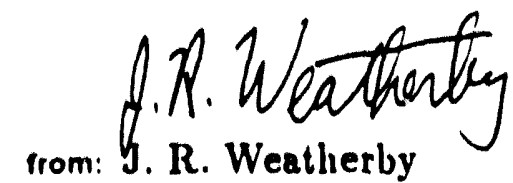

subject: Finite Element Analysis of TRU Storage Rooms Filled With Waste and Crushed Salt

\section{Introduction}

The transuranic waste that will eventually be stored at the WIPP site has a very high void content. Based on the results of a sampling program [1], it is estimated that approximately $70 \%$ of the volume inside of a typical waste container will be void. Under the present plan, these waste containers will be placed in the storage rooms in their present state (i.e., without additional compaction). Crushed salt will be used to fill the empty space between containers and between the container stacks and the walls of the rooms. Closure of the rooms due to creep in the surrounding salt formation will gradually consolidate the waste and backfill into a dense state, impeding the diffusion of waste and flow of fluids. Because of the large void volume in both the waste stack and the crushed salt, the hydraulic conductivity of the storage rooms will be much greater than that of the salt formation. Since the void decreases as the rooms close, the hydraulic conductivity of the rooms will also decrease with time. A storage room has been analyzed using the finite element code SANCHO to provide estimates of the void volume in the room as a function of time. Such information will eventually be used to estimate the permeability of the room contents. These permeability estimates will, in turn, be used to evaluate the consequences of hypothetical scenarios where the repository is breaclied.

Previous estimates of the void volume in storage rooms have primarily been derived from the results of an empty room analysis reported in [2]. These estimates were based on the assumption that the stresses that develop inside of the room will be sufficiently small so that they will have little effect on the closure of the room until the consolidation of the waste and backfill is nearly complete. Results from the analysis of a storage room filled with wet crushed salt [3] supported this assumption.

The purpose of the present investigation was to determine the amount and distribution of void volume in a storage room containing both waste and backfill. In the room that was analyzed, $70 \%$ of the volume in the waste stack was void while $35 \%$ of the volume in the backfill region was void. These void fractions are typical of what is anticipated in the waste and backfill that will eventually be emplaced at the WIPP site. These initial void fractions are much higher than those considered in a previous analysis of a storage room filled with 
waste and backfill [4], and a considerable amount of development work was required before this problem could be properly analyzed.

The results of the present moded ohow that the total roid rolume in the rante-filled room is expected to decrease to $5 \%$ of the total room volume 80 years after the waste is emplaced. The analysis also shows that the crushed salt backfill consolidates much faster than the waste. Because these results are based on mechanical properties derived from a very limited number of experiments, they should be regarded as tentative. As more experimental results become available for the crushed salt and waste, the room will be reanalyzed using updated mechanical properties.

\section{Overview of Analytical Approach}

Early efforts to obtain a solution to the room closure problem with waste and backfill were unsuccessful. After an extensive study, it was determined that several factors were responsible for the difficulties. These factors were:

1. The material inside the storage room is initially very soft compared to the intact salt in the surrounding formation.

2. The crushed salt rapidly stiffens as it consolidates.

3. The elastic/plastic model used to represent the deviatoric response of the crushed salt gives unrealistically high stresses in the backfill.

4. The use of slide lines between the crushed salt and the intact salt makes it difficult for the dynamic relaxation algorithm to converge to the equilibrium state.

To circumvent these problems, the following measures were taken:

1. A new error measure was developed and implemented to better evaluate when the analytical solution is sufficiently close to equilibrium (i.e., when the nodal forces balance).

2. The fictitious mass matrix defined for the dynamic relaxation algorithm was updated after each iteration based on the effective stiffness of the material (this updating was made for the crushed salt only).

3. The planticity model used to describe the deviatoric behevior of the cruohed salt was replaced with an elestic-necondary creep model.

4. All slide lines were eliminated from the mesh.

The discussion which follows describes in more detail the problems encountered in the analysis of room closure and the actions that were taken to circumvent the problems. 
The large difference between the initial stiffness of the room contents and the stiffness of the intact salt made it difficult to determine when an "acceptable" solution had been obtained to the force equilibrium equations at the nodes. In SANCHO, iterations are made within each time step until the nodal forces resulting from the stress within the elements approximately balance the externally applied forces at each node. To determine when this condition is satisfied, an "error measure" is computed at the end of each iteration and compared against a specified tolerance. The error measure used in SANCHO and in many other finite element codes gives a single value based on the sum of the magnitudes of the force imbalance at all nodes in the mesh. This is referred to as alobal error measure. For the room closure problem, the force imbalances in the waste and backfill regions have very little effect on the global error measure because the materials are initially very soft and occupy only a small part of the total mesh. As a result, when an error tolerance of $0.5 \%$ is used (this is the default value in SANCHO), the iterative process is terminated before a good solution is obtained in the waste and backfill. Unfortunately, when a much tighter tolerance is uned with the global error measure, the convergence requirement is too atringent for the force residuals at nodes located in the intact salt region, and the program continues with equilibrium iterations long after a good stress solution has been obtained throughout the model. Because of this, the global error measure does not provide an acceptable basis for determining convergence in this particular problem.

To circumvent this problem, a new error measure was defined for the force imbalances. This new error measure is evaluated at each node in the mesh and, as a result, is referred to as a local error measure. The local error measure compares the magnitude of the force imbalances at each node to the magnitudes of the internal and external forces acting on the node. The solution is accepted when the value of the local error measure at all of the nodes is amaller than a specified tolerance. A more detailed description of both the local and global error measures is given in Appendix A.

The second unusual feature of the room closure problem is that the stiffness of the crushed salt increases as it consolidates. This has an adverse effect on the dynamic relaxation algorithm that is used in SANCHO. In the method of dynarnic relaxation, the static solution is obtained by finding the steady-state solution to a damped dynamics problem. In the room closure problem, the critical time step size for the crushed salt elements decreases as the stiffness of the crushed salt increases. In the standard version of SANCHO, the time step size is not modified to account for changes in the atiffness of the material, and, w a result, the dynamic relaxation algorithm becomes unatable and does not converge to the equilibrium solution. To fix this problem, the masses associated with nodes in the crushed salt region were modified after each iteration based on the effective stiffness of the crushed salt. The effective stiffness was computed from the stress and strain increments.

At the present time, consolidation tests on the crushed salt have been limited to hydrostatic loading only. Information is not yet available on the deviatoric stress-strain behavior of the backfill material. In previous analyses $[3,4]$, an ad hoc plasticity model was used to compute the deviatoric stresses in the crushed salt. When this conotitutive model was used in the present analysis of a room containing waste and backfill, the stresses in the backfill became 
unrealiatically high. To avoid this problem, the deviatoric plasticity model was replaced with an elastic-secondary creep model. This model is described in Appendix B. Other millor clianges were made to the crushed salt model to account for finite volume strains. These changes are also described in Appendix B.

\section{Finite Element Results}

A series of three finite element analyses were conducted for a TRU storage room. The first analysis was made on an empty room, the second analysis was made on a room filled with crushed salt, and the third analysis was made on a room filled with a combination of crushed salt and waste.

The same finite element mesh was used to model the surrounding salt formation in all three cases. Figure 1 shows the dimensions of the mesh and the boundary conditions that were assumed in the models. The room has a height of 4 meters and a width of 10 meters. A symmetry houndary condition is specified along the right edge of the mesh to simulate conditions that would exist around a typical room located near the middle of a periodic array of ronms spaced at 411.5 meters on centers in the horizontal direction. This mesh differs from ones used in previous analyses in that:

1. The deformation is assumed to be symmetric about a horizontal plane that passes through the center of the rib.

2. The distance from the center of the room to the upper edge of the mesh is approximately half of the distance used in previous analyses.

3. The corners of the room are assumed to be round $(0.23 \mathrm{~m}$ radius $)$ instead of square.

The first two modeling assumptions in this list were made to reduce the CPU time and actual clock time of conducting the analyses during the development phase of this project. Future computations will be made with a larger mesh. The rounded corners match those in the actual rooms at the WIPP site.

An elastic-secondary creep constitutive model was used to represent the stress-strain behavior of the intact salt. Following [2], the reference elastic constants were divided by 12.5 , and the stratigraphy was assumed to be composed completely of salt. These approximations have been shown to produce room closure histories which match those in empty roums at the WIPP site.

\subsection{Analysis of an Empty Room}

An empty room was analyzed to evaluate the performance of the new error measure and to evaluate the performance of the mesh shown in Figure 1. These evaluations were made by coinparing the room closure results to those obtained from a previous analysis [2] made with 
the mesh shown in Figure 2. In the following discussion, the model shown in Figure 2 will be referred to as Model 1, and the model ahown in Figure 1 will be referred to a Model 2.

Figures 3 and 4 show how the room closed a function of time in the Model 2 analysis. After approximately 25 years, the rib began to penetrate the roof and floor. Slide lines were not used in this calculation so the results obtained from the Model 2 allalyois are not meaningful after 25 years of clonure.

In Figures 5 through 7, the Model 2 results for the vertical closure, the horizontal closure, and the volume change in the room are compared againat the corresponding values calculated with Model 1. These comparioone show that Model 2 clowes somewhat falter than Model 1. After 25 years of closure, the change in room volume in Model 2 is $12 \%$ more than the change in room volume obtained from Model 1 . The more rapid clonure is mont likely due to the shorler distance between the center of the room and the upper boundary of the meah in Model 2 as compased to Model 1. Again, this distance was made shorter to reduce the computation time.

\subsection{Analysis of Room Filled With Crushed Salt}

The closure of a room filled with crushed salt was analyzed using the mesh shown in Figure 8. This model will be referred to as Model 3 . The crushed salt was given an initial density of $1400 \mathrm{~kg} / \mathrm{m}^{3}$ which corresponds to a void fraction of approximately $35 \%$. The material constants used with the crushed salt model are listed in Table 2.

The closure of the room at the midheight of the rib and the room closure at the center of the roof and floor are plotted in Figures 9 and 10 as functions of time. These figures also show the pressure at the center of the room and the corresponding closures oblained from the Model 2 analysis of the empty room. Initially the results from Model 3 closely track the empty room results; however, the closure of the backfilled room alowe rignificantly before the pressure at the center of the room reaches $1 \mathrm{MPa}$. The ratio of the horizontal closure to the vestical closure for Model 3 is shown in Figure 11.

The void fraction in any element or region inside the room can be computed from the relationship:

$$
v \equiv \frac{V_{\text {old }}}{V}=\frac{V_{\text {old }}^{0}+\Delta V}{V}
$$

where $v$ is the void fraction in the element or region, $V_{\text {vold }}$ is the void volume in the element or region, $V$ is the current volume of the element or region, $\Delta V$ is the difference between the current volume and the initial volume inside the element or region, and $V_{\text {vold }}^{0}$ is the initial void volume inside the element or region. Figure 12 shows how the total void fraction in the room changes as a function of time. The estimated void fraction based on the empty room analysis (Model 2) is also shown in this figure. In the Model 3 analysis, the void in the backfill decreases to $5 \%$ of the total room volume approximately 30 years after emplacement of the backfill. Based on the empty room calculation, the void fraction in the room decreases to $5 \%$ after 20 years 
Figure 13 shows the diatributlon of void in the backfll s years, 10 years, 28 years, and 80 years after emplacement of the backfll. These results show that the backfill conoulidated moat rapidly near the cornefs of the room. This is in contrat to the results obtained in $|3|$ where the cornert were observed to be the oilea with the greated void fraction. Drbe cource

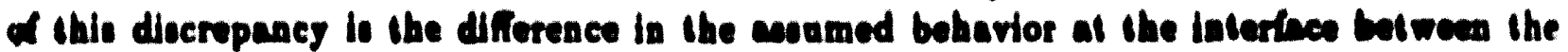
arubed oalt and the walle of the room. In the current model, the crushed oalt was rigidly attaclied to the boundaries of the room, while $f|B|$ the bectall was allowed to aljde freely relative to the walls.

The real consolidation rale in the corners probably lies comewhere between the results ob. inined with frictionleas aliding interfaces at the wallo and the results oblained with interfaces that are rigidly tied logether. In reality, the cruahed aalt granules at the boundary of the room probably bond to the intact ant as readily as they bond to other granules of cruahed oalt. It is posaible that, near the walls, high ohearing otrains will develop in a very thin band of crualied salt. Slide lines call be used at the boundary of the rooin to model the ouclden diaplacement change acrose this thin layer of backfill. The use of aliding interface would be unnecensary if the ohear behavior of the crualied oalt were well characterised and if an extremely fine mesh were used near the boundasies of the room. Unfortunately, the menll used in this analyois is too coarse to caplure the shear band phestomenon if does exiot. At the present time, solutione cannot be obtained with oliding interfaces when the initia backfill denaity is a low a $1400 \mathrm{~kg} / \mathrm{m}^{3}$. Hence, the results from the Model 3 analyois may overestimate the concolidation rate in the corners of the room.

Figures 14 and 18 contain contour plots of the vertical and horisontal atresses in the backfill at four different times. The highest otreases are found in the corners of the room. Away from the corners, the otresses are rewonably uniform.

\section{3.s Analysis of a Room Filled With Waste and Backfill}

A rontn containing combination of crushed salt and waste was analyzed after it was verified that the two previous closure problemo could be solved with the updated veroion of SANCHO. The finite element mesh used to model this problem is shown in Figure 16. As in the two previous analyses, the geometry and loading were assumed to be aymmetric abuut a horizontal plane that cut through the midheight of the rib. At a consequence, the waste in the finite element model was located at the center of the room and was surrounded on all sides by a layer of crushed salt. In the actual configuration, the waste will rest directly on the floor of the room and will be in contact with the backfill on three sides only. The distance between the waste at ack and the rib is the same in the finile element model and in the actual configuration. To make the total volume of crushed salt the same as that called for in the actual configuration, the distance in the finite element model between the top of the waste stack and the roof of the room was made half as large as that in the aclual configuration. In future analyses the lower half of the room will be modeled instead of assuming vertical symmetry at the rib midheight.

The backfill material and the intact salt were modeled with the same constitutive models and 
with the oame material conatants used in the Model 3 analyois. The volumetric plasticity model described in the SANCllO Uners Manual $|8|$ wa used to model the waste. The curve defining the relationahip between the presuure and the volumetric atrain for the wate is plolled in Figure 17. Thio curve was derived from the results of cruah leals conducted on ilx.pack arrangements of eniply drumo that were ourrounded by crushed aall and compressed perpendiculat to the axio of the drum (see Figures 18 and 20 in |6|). Axial crushing teols conducted on emply drume show that once an emply drum begina to collapee it continues to collapae with very little increase in the applied load (oee Figure 3 in |6|). In the aboence of other information, il wa asumed that drumo filled with waste wuald collapae like einply drums until all of the void $(\approx 70 \%)$ was eliminaled from the drum.

In Figure 18, the horisontal closure at the rib midheight is plotled as a function of time. Figure 10 containa a aimilar plot of the vertical closure between the roof and floor at the vertical centerline of the room. Aner 28 yearn of closure, the horisonid and rertical clonures for the room Alled with wate and backfll were roughly $20 \%$ lew than dimiles clonures compuled in the Model 2 amply room andyait. The ratio of horisontal closure to vertical closure io plotled in Figure 20. Although tlie vertical closure after 200 years io only $6 \%$ larger thatl the horizontal closure, the final vertical closure represents $60 \%$ of the initial height of the room while the horizontal closure accounts for only $23 \%$ of the initial width of the room.

In these calculations, the initial void fraction of the cruohed oalt was asoumed to be $34.6 \%$ and the initial void fraction of the waste was assumed to be $71.3 \%$. The volume atrain $E_{v}$ at the point where the pressure-volumetric atrain curve begins to atifien was chosen 10 that

$$
c_{v}=\log \left(1-v^{0}\right)
$$

where $v^{0}$ is llie initial void fraction of the waste. The vold fraction in the waste, the void fraction in the backfill, and the void fraction in the room as a whole are plotled in Figure 21 an functions of tione. The void volume in the backfill decreased to $3 \%$ of the total volume ill the backfill region after approximately $\mathbf{4 0}$ years of closure, while 100 years of closure are required before the void volume in the wate was reduced to $5 \%$ of the total volume of the waste region.

In Figure 22, the void fraction computed in the Model 4 analyois is compared againat the void inferred from the eanpty otorage room calculations (Mudels 1 and 2). Based ont the rate of closure from the Model 1 analyais, the void fraction in the room would be reduced to less than $1 \%$ after 60 years if the backatress did not alow the rate of room closure. The results obtained from the Model 4 assalysis suggest that closure is olowed considerably by the presence of the room contents. With the backutress acting on the walls, 130 years of closure were required before the void fraction in the room was reduced to less than $1 \%$.

The contour plots in Figures 23 and 24 show how the void volume is distributed in the waste and backfill regions at four different points in time. In both regions, the void fraction is lowest in the corners. As mentioned previously, this result appears to be sensitive to the method used to model the interfaces between the various regions (i.e., whether the two inaterials at an interface are connected rigidly or are connected with sliding interfaces). At 
this time it is not postible to entablish whether the void volume will be higher or lower in the corners of waste and backfill regions.

Contour plots of the vertical and horizontal atresses in the waste and backfill are shown in Figures 28 through 28. These figures show that the atreases initially rise much faster in the cruatied oult region than in the waste region. Furthermore, the otress otate in the backfill io not hydroutatic while the cruehed oalt is consolidating. This is further confirmed by the plots in Figures 29 throught 31 which show the vertical, horizontal, and out.of.plane atress a a function of time al three locations in the waste and backfill. Since the conotilutive model for the crushed onlt was derived from conoolidation teats that were conducted under hydroalatic loading conditions, the accuracy of the conotilutive model is unknown for the type of loading observed in the alorage room.

\section{Conclusion}

An approach ha been developed to analyze the closure of rooms filled with waste and crushed oalt which contain a large amount of void volume. Two of the key fealures in this approach are:

1. new error measure was used to determine when equilibrium was satiofied in the finite eleinent model (see Appendix A), and

2. the mass matrix defined for the dynamic relaxation algorithm wa updated after each iteration based on the effective stiffness of the crushed alt.

Three different roome were analyzed uning the new approach. The cases included an analysis of all emply storage room, an analysis of a storage room filled with crushed oalt, and an analyois of astorage room filled with a combination of waste and cruslied ealt. These analyses indicate that the consolidation of the material in atorage room can be significantly alowed by the backetress exerted on the walls of the room by the room contents. In the analyois of the storage room filled with waste and backfill, 135 years of closure were required befure the void in the room was completely eliminated. By comparison, approximalely 60 years would be required to reach complete compaction if the backstress were not present (see Figure 22).

In contrast to the results found in previous analyses $[3,4]$, the present set of analyses indicute that the backfill consolidates faster in the cornern of the room than at other locations. The rale of compaction in the corners of the room appear to depend on the assumed beliavior at the interface (aliding interfaces as compared to fixed interfaces). At the present time it is not possible to determine if the backfill does in fact consolidate faster at these locations.

When the waste stack is present, the deviatoric component of stress in the backfill is large while the crushed salt is consolidating. In the past, consolidation tests of the cruslied salt have only been conducted under hydrostatic loading conditions. The results from the present investigation emphasize the need for conducting consolidation experiments on crushed salt 
under loading conditions where the principal otresses are unequal.

As a final nole, it should be atressed that the results of the present in vestigation are based on a very limited amount of experimental data with regard to the properties of the waste and crualied aalt. Teats that are presently underway will provide new information concerning the properties of these materials. Future analyses based oll updated properties of the room contents could yield results that are different from those reported here.

\section{( References}

1. T. L. Clementa, Jr. and D. E. Kutera, "TRU Wasle Sampling Program: Volume 1 Waste Cliaracterization," EGG.WM-0303.Vol.1, September 1985.

2. H. S. Morgan, "Eutimate of the Time Needed for TRU Storage Rooms to Close," memo to D. E. Mullon, 6332 , June 2, 1887.

3. G. D. Sjaardema and R. D. Krieg, "A Constitutive Model for the Consolidation of WIPP Crushed Salt and Its Use in Analyses of Backfilled Shaft and Drift Configura. tions," SAND87.1977, October 1987.

4. J. R. Weatherby, H. S. Morgan, and G. D. Sjeardema, "Calculation, of TRU Room Closure with Waste and Backfill," memo to L. D. Tyler, 6332, February 26, 1988.

5. C. M. Stone, R. D. Krieg, and Z. E. Beisinger, "SANCHO - A Finite Element Com. puter Program for the Quasistatic, Large Deformation, Inelastic Response of Two. Dimensional Solids," SAND84.2618, April 1885.

6. J. VandeKraatr, "Quarter.Scale Modeling of Room Convergence Effects on CH.TRU Drum Waste Emplacements Using WIPP Reference Design Geometries," DOE/WIPP 87. 012, November 1987. 


\section{A Error Measures for Force Equilibrium in Finite Element Prob- lems}

When an iteralive method is used to solve system of equations, some criterion must be used to determine when the estimated solution is sufficiently close to the actual solution. In - finite element problem, the aystem of equatious that must be solved can be written as:

$$
R=\mathbf{F}_{\mathbf{x t}}-\mathbf{r}_{\operatorname{lnt}}=\mathbf{0}
$$

where $\mathbf{R}$ is referred to as the "residual" force vector, $\mathbf{F}_{\mathbf{x x t}}$ is the "external" force vector which is composed of the nodal forces from surface tractions and body forces, and $F_{\text {int }}$ is the "internal" force vector which is composed of the nodal forces that arise from stresses inside the elements. The length of the residual force vector is equal to the number of degrees of freedom in the problem. For example, in a two-dimenaional problem, the length of the residual force vector will be equal to twice the number of nodes less the number of constrained degrees of freedom. The ith entry in the internal force vector is coniputed from the summation of the forces from each element containing the ith degree-of-freedom. The internal force vector can be expressed as:

$$
F_{\text {Int }}^{i}=\sum_{j=1}^{N} f_{i}^{j}
$$

where $N$ is the number of degrees of freedom, and $f_{i}^{j}$ is the force on degree-of-freedom $i$ due to the stresses in element $j$.

One metliod used to measure the relative size of the error in the approximate solution of Equation 3 is to compare the magnitude of the residual force vector to the magnitude of the external force vector, namely:

$$
e=\frac{\left(\sum_{j=1}^{N} R_{j}^{2}\right)^{1 / 2}}{\left(\sum_{j=1}^{N}\left(F_{(x)}^{j}\right)^{2}\right)^{1 / 2}}
$$

When $e$ is less than apecified tolerance (a typical tolerance is $0.5 \%$ ), the aystem of equations in Equation 3 is assumed to be satiofied, the ilerative procedure is terminated, and the next time slep is initialed.

Unfortunately, the external and internal nodal forces have almost no physical meaning in the struclural problem, but instead are artifacts of the finite element method. The magnitude of the internal force acting at a node is a function of the size of the elements that are connected to the node as well as the stresses exioting in adjoining elements. The magnitude of the external force acting at a node is also function of element size. For axisym netric problems, both the internal and external forces at a node are functions of the radial coordinate of the node. 
Since the primary variables of intereat are the otresses and displacements in the structure, a good convergence criterion should be satisfied if and only if further reductions in the renidual forces will produce only slight changes in the atresses and displacements throughout the mesh. Unfortunately, what is an "acceptable" force residual in one portion of llie iliesh may be unacceptable at another location in the mesh. For example, further reduction of a set of force residuals acting in a region of the mesli where the elements are large and stiff may produce virtually no change in the element atresses in this region. If, however, the same set of force residuals were present at a different location where the element sizes were much inaller and the material was much more flexible, further reduction of the residuals could produce a large change in the element stresses.

The error measure given in Equation 5 suffers from three major deficiencies. First, all force residuals are weighted equally regardless of the element size or radial location. Second, because this error measure is based on the magnitude of a vector that cuntains all of the residual forces, it is possible that the value of the error measure can be very small evell when the residuals are large in amall portion of the mesh. Third, the error measure defined in Equation 5 compares the residual forces to the externally applied forces. This is a disadvantage in thermal stress or initial atress problems where no external loads are applied to the atructure.

In the WIPP room closure problems, the uncompacted waste and backfill initially have very low stresses conipared to the large lithostatic stresses in the surrounding salt formation. In addition, the room contents account for only amall fraction of the total ares that is modeled. The error measure is really not suitable for this type of problem. When the tolerance for was set to $0.5 \%$ in the room closure analyses, the equilibrium ilerations were often terminated before good solution was achieved in the room. When the tolerance for a was reduced, the number of iterations required to attiofy the tolerance became too large.

To circumvent these problems, a new error measure, $\dot{e}$, was defined. The new error measure is defilled as

$$
\dot{e} \equiv \max _{i}\left(\dot{e}_{i}^{\prime}\right), \quad i=1, N
$$

where $N$ is the number of degrees of freedom, and $\hat{e}_{i}^{\prime}$ is defined as

$$
\dot{e}_{i}^{\prime} \equiv \frac{\left|R_{i}\right|}{\left|F_{i x i}^{i}\right|+\sum_{j=1}^{M_{1}}\left|f_{i}^{j}\right|}
$$

In Equation 7, $F_{e x i}^{i}$ is the external force acting on degree-of-freedom $i, f_{i}^{j}$ is the force acting on degree-of. freedom $i$ due to the stresses in element $j, M_{i}$ is the number of elements containing degree-of freedom $i$, and $|x|$ denotes the absolute value of $x$. Equation 6 stales liat $\dot{e}$ is equal to the largent value of $\dot{e}_{i}^{\prime}$ existing at any degree-of-freedom.

To ensure that the denominator in Equation 7 is never zero and to prevent elements witl negligible stresses from controlling the problem, a ininimum value is established for $\left|f_{i}^{j}\right|$. This minimum is established in the following way. First, the nodal forces are computed for the element based on the element stresses. These forces will be denoled by $f_{i}^{j}$. Next, second 
set of nodal forces are computed for the element assuming that a small hydrostalic pressure of $p_{\min }$ exists inside the element. These forces will be referred to as $f_{i}^{j}$. The pressure $p_{\min }$ is defined so that it is smaller than the smallest pressure of interest in the problem (i.e., $p_{\min }$ essentially defines zero stress). The value of $f_{i}^{j}$ used in Equation 7 is the larger of $\left|f_{i}^{j}\right|$ and $\left|f_{i}^{j}\right|$. In the room closure problems, a tolerance of $10 \%$ was used for $\hat{e}$, and $p_{\min }$ was set equal to $2 \times 10^{-4} \mathrm{MPa}$.

The advanlages of using $\hat{e}$ as an error measure in place of $\bar{\varepsilon}$ are:

1. In $\hat{e}$, the magnitude of the force imbalance (residual) at each degree-of-freedom is compared to the magnitude of the forces acting on that degree-of-freedom whereas all residuals are given equal weighting in $e$.

2. The error measure $\hat{e}$ is based on the most severe residual force in the mesh. Thus, when the error tolerance for $\hat{e}$ is satisfied, it is known that the residual forces at every location in the inesh satisfy a known size restriction. The error measure $\bar{e}$ is equally sensitive to both the case where small force residuals are evenly distributed throughout a large region in the inesh and a case where large force residuals are concentrated in a small region of the mesh.

3. The error messure ê lass meaning in problems where there are no external forces (eg., thermal stress problems) while $\bar{e}$ is undefined for such problems. 


\section{B Constitutive Model for the Crushed Salt}

\section{B.1 Volumetric Response}

A few minor adjustments were made to the volumetric creep model described in [3] to account for a finite change in the volumetric strain within a time step. These adjustments were related to the assumed relationship between the density at the beginning of the time step $\rho_{0}$, the density at the end of the time step $\rho^{t+\Delta t}$, and the change in volumetric strain during the time step $\Delta e_{v} . \ln [3]$, this relationship was assumed to be:

$$
\rho^{t+\Delta t}=\rho_{0} /\left(1-\Delta e_{v}\right)
$$

Equation 8 holds for small increments in the volumetric strain; however, if the volumetric strain increment is large, then the exact relationship should be used. From the continuity equation, the volumetric strain rate $\dot{e}_{v}$ is related to the density through:

$$
\dot{e}_{v}=\frac{\dot{\rho}}{\rho}
$$

which, when integrated over a time slep yields:

$$
\rho^{t+\Delta t}=\rho_{0} \exp \left(\Delta e_{v}\right)
$$

When Equation 10 is substituted in Equation 3.3.7 of $[3]$, the result can be rearranged into the form of Equation 3.3.12 of [3]. The expressions for the constants $\hat{K}, \hat{B}$, and $\beta$ remain the same; however, the expression for $\alpha$ is slightly different. The new expression for $\alpha$ is given by:

$$
\alpha=\left(K_{1} \rho_{0}+A \rho_{0}-1\right) \dot{e}_{v}
$$

One other modification was made to the volumetric creep model to handle the case where the crushed salt reaches the fully compacted state in the middle of a time step. In this case, volumetric creep occurs during the first part of the time step $\Delta t_{\text {creep }}$, while the volumetric response is elastic during the latter part of the time step. The increment in the pressure, $\Delta p$, is calculated from the relationship:

$$
\Delta p=\Delta p_{\text {creep }}+K_{\text {intact }} \dot{e}_{v}\left(\Delta t-\Delta t_{\text {ereep }}\right)
$$

where $K_{\text {intect }}$ is the density of the intact salt, $\Delta t_{\text {ereep }}$ is the part of the time step over which volumetric creep occurs, and $\Delta p_{\text {creep }}$ is the increment in pressure that is calculaled from the volumetric creep equations over the time increment $\Delta t_{\text {creep. }}$. The length of time over which the volumetric creep equations hold can be calculated from: 


$$
\Delta t_{\text {creep }}=\frac{1}{\dot{e}_{\mathrm{v}}} \log \left(\frac{\rho_{\text {intoct }}}{\rho_{0}}\right)
$$

If $\Delta t_{\text {creep }}$ as computed in Equation 13 is larger than $\Delta t$, then volumetric creep occurs over the entire time step.

\section{B.2 Deviatoric Model}

In the analyses described in $[3,4]$, an elastic-plastic model was used to represent the devialoric response of the crushed salt. This model is described in Section 3.4.2 of [3]. When this model was constructed, no information was available for determining the yield stress since all experimental data had been obtained from tests where the loading was hydrostatic. As a result, the yield stress was computed to obtain the stiffest possible deviatoric response.

The following method was used to compute the yield stress in $[3,4]$ for plane strain analyses. First, Hooke's law was used to compute trial stresses from the strain increments. If the out-of-plane stress was found to be compressive, the trial stresses were accepted as the final stresses. If the out-of-plane stress was found to be tensile, a yield stress was compuled so that the out-of-plane stress was zero. The radial return method was used to return the trial stresses to the yield surface.

When the above method was used in analyzing room closure for a storage room containing both waste and backfill, the deviatoric stresses in the crushed salt became unrealistically high as compared to the deviatoric stresses in the intact salt. It was decided at this point to use the elastic-creeping model described in Section 3.4.3 of [3]. The stresses in this model are found by integrating the following equation:

$$
\dot{s}_{i j}=2 G_{p}\left[\dot{e}_{i j}^{\prime}-A\left(\rho_{\text {intect }} / \rho\right)^{N} \exp (Q / R T)\left(s_{k l} s_{k l}\right)^{(N-1) / 2} s_{i j}\right]
$$

where $s_{i j}$ is the deviatoric stress, $G_{p}$ is the shear modulus of the crushed salt, $\dot{e}_{i j}^{\prime}$ is the deviatoric strain rate, $T$ is the temperature, and $A, Q, R$, and $N$ are the material constants for the intact salt.

Equation 14 is integrated with the same semi-analytical method used to integrate the secondary creep model for the intact salt. Both the shear modulus and the density change during the time step; however, constant values must be used for $G_{\rho}$ and $\rho$ in order to apply the semi-analytical integration scheme. To satisfy this restriction, the average value of $\rho$ during the time step and the value of $G_{p}$ corresponding to the average value of $\rho$ are computed and then used in the integration algorithm.

After the deviatoric stresses are computed from the elastic-creeping model they are combined with the mean atress to form the trial stresses. The trial stresses are accepted as the final stresses if: 
1. The mean stress is tensile, or

2. The out-of-plane trial stress is compressive, or

3. The mean stress is compressive and the out-of-plane trial stress is tensile but is less than $10 \%$ of the absolute value of the mean stress.

If these conditions are not met, then the deviatoric stresses are scaled back so that the out-of-plane stress is equal to $10 \%$ of the absolute value of the mean atress. 
Table 1. Material Constants Used With the Elastic/Secondary Creep Model for the Intact Salt.

\begin{tabular}{|c|c|}
\hline PARAMETER & VALUE \\
\hline $\bar{G}$ & $1.242 \times 10^{9} \quad \mathrm{~Pa}$ \\
\hline $\boldsymbol{K}$ & $1.656 \times 10^{\circ} \mathrm{Pa}$ \\
\hline$A$ & $5.79 \times 10^{-36} \quad \mathrm{~Pa}^{-4.9} /(\mathrm{sec})$. \\
\hline$N$ & 4.9 \\
\hline$Q / R T$ & 20.13 \\
\hline \multicolumn{2}{|c|}{$\dot{s}_{i j}=2 G\left[\dot{e}_{i j}^{\prime}-A \exp (Q / R T)\left(s_{h l} s_{h l}\right)^{(N-1) / 2} s_{i j}\right.$} \\
\hline
\end{tabular}

Table 2. Material Constants Used With the Crushed Salt Backfill Model.

\begin{tabular}{|c|c|}
\hline PARAMETER & VALUE \\
\hline$\overline{G_{0}}$ & 864. $\mathrm{Pa}$ \\
\hline$G_{1}$ & $6.53 \times 10^{-3} \mathrm{~m}^{3} / \mathrm{kg}$ \\
\hline$K_{0}$ & $1.41 \times 10^{3} \mathrm{~Pa}$ \\
\hline$K_{1}$ & $6.53 \times 10^{-3} \mathrm{~m}^{3} / \mathrm{kg}$ \\
\hline$A_{\mathrm{c}}$ & $5.79 \times 10^{-36} \quad \mathrm{~Pa}^{-4.0} /$ (sec. $)$ \\
\hline$N$ & 4.8 \\
\hline$Q / R T$ & 20.13 \\
\hline $\boldsymbol{A}$ & $-17.3 \times 10^{-3} \quad \mathrm{in}^{3} / \mathrm{kg}$ \\
\hline$B_{0}$ & $1.3 \times 10^{8} \mathrm{~kg} /\left(\mathrm{m}^{3} \mathrm{nec}\right)$ \\
\hline$B_{1}$ & $.82 \times 10^{-6} \mathrm{~Pa}^{-1}$ \\
\hline \multicolumn{2}{|c|}{ 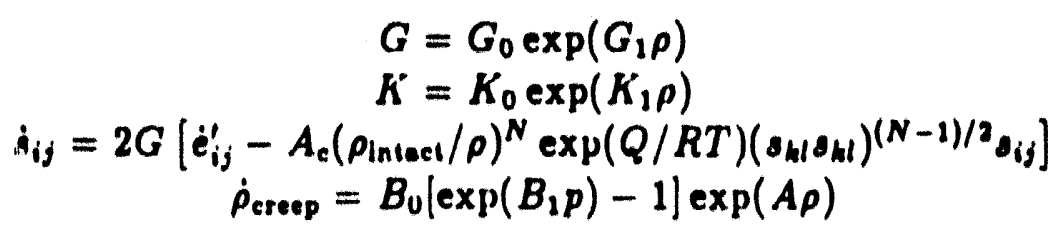 } \\
\hline
\end{tabular}


Table 3. Pressure-Volumetric Strain Data Used in the Volumetric Plasticity Model for the Waste Drums.

\begin{tabular}{||c|c||}
\hline $\begin{array}{c}\text { PRESSURE } \\
(\mathrm{MPa})\end{array}$ & $\ln \left(\rho / \rho_{0}\right)$ \\
\hline 0.16 & $7.2 \times 10^{-1}$ \\
0.21 & 1.04 \\
0.307 & 1.11 \\
0.893 & 1.21 \\
2.89 & 1.25 \\
14.0 & 1.30 \\
\hline
\end{tabular}

Table 4. Other Material Constants Used With the Volumetric Plasticity Model for the Drums.

\begin{tabular}{||c|c||}
\hline PARAMETER & VALUE \\
\hline \hline$\mu$ & $333 \mathrm{MPa}$. \\
$K_{0}$ & $222 \mathrm{MPa}$. \\
$a_{0}$ & 0 \\
$a_{1}$ & 0 \\
$a_{2}$ & 3 \\
\hline$\mu, K_{0}=$ & elastic shear and bulk mod- \\
& ulus, respectively \\
$a_{0}, a_{1}, a_{2}=$ & constants for yield function \\
\hline
\end{tabular}


14.3 MPA.

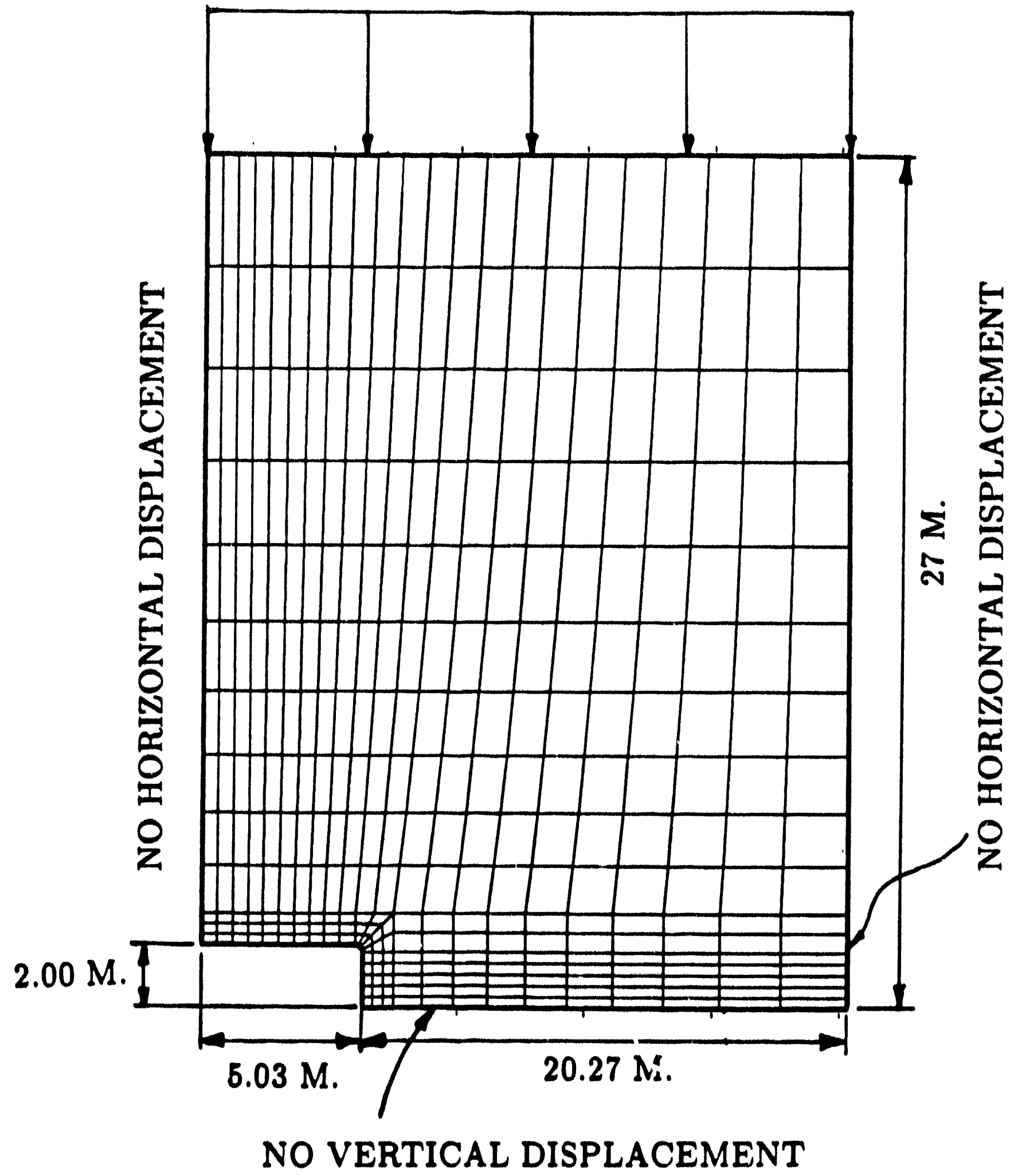

Figure 1. Finite Element Mesh Used to Analyze Empty Room (Model 2). 


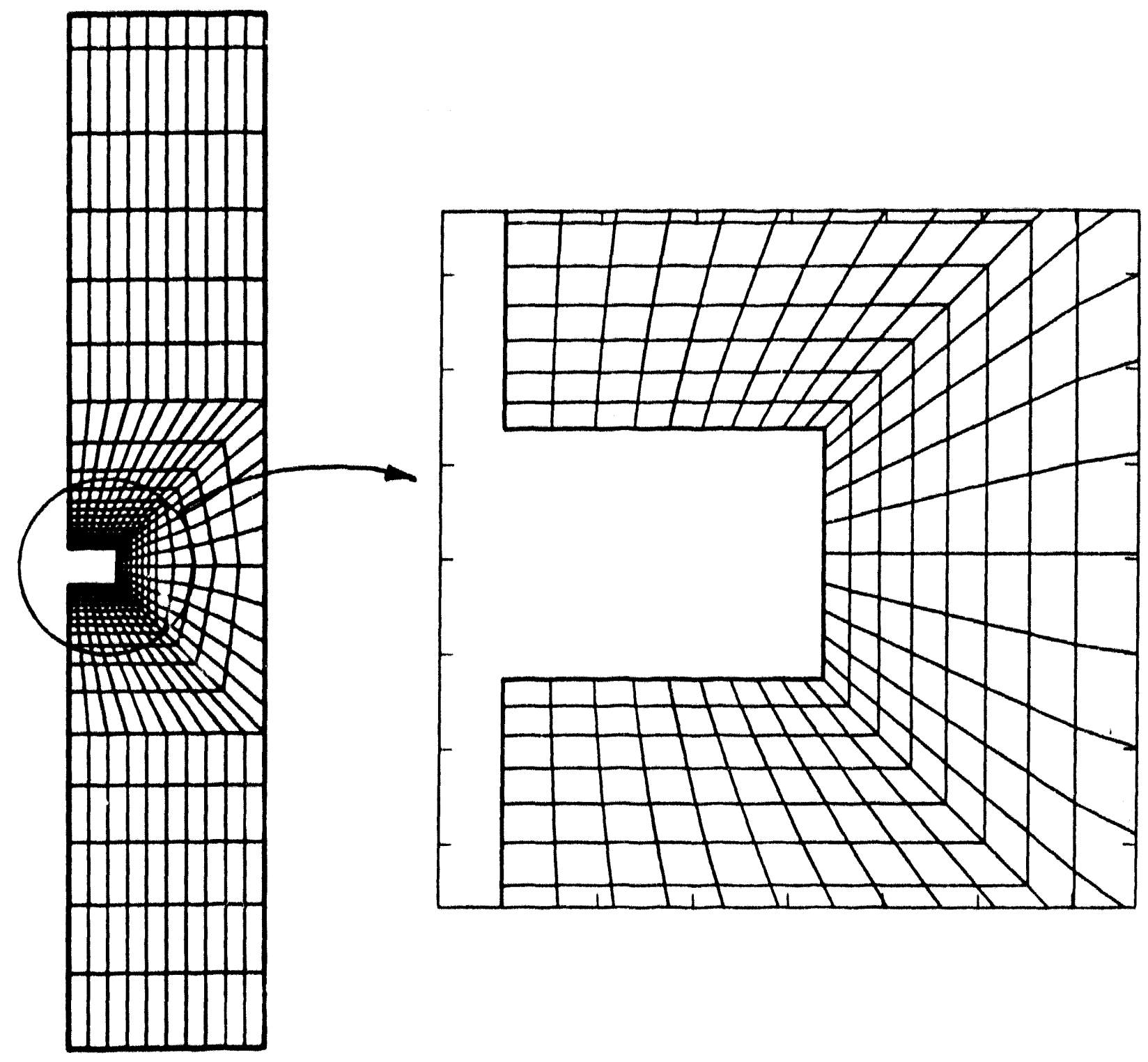

Figure 2. Finite Element Mesh Used in [2] to Analyze Empty Room (Model 1). 

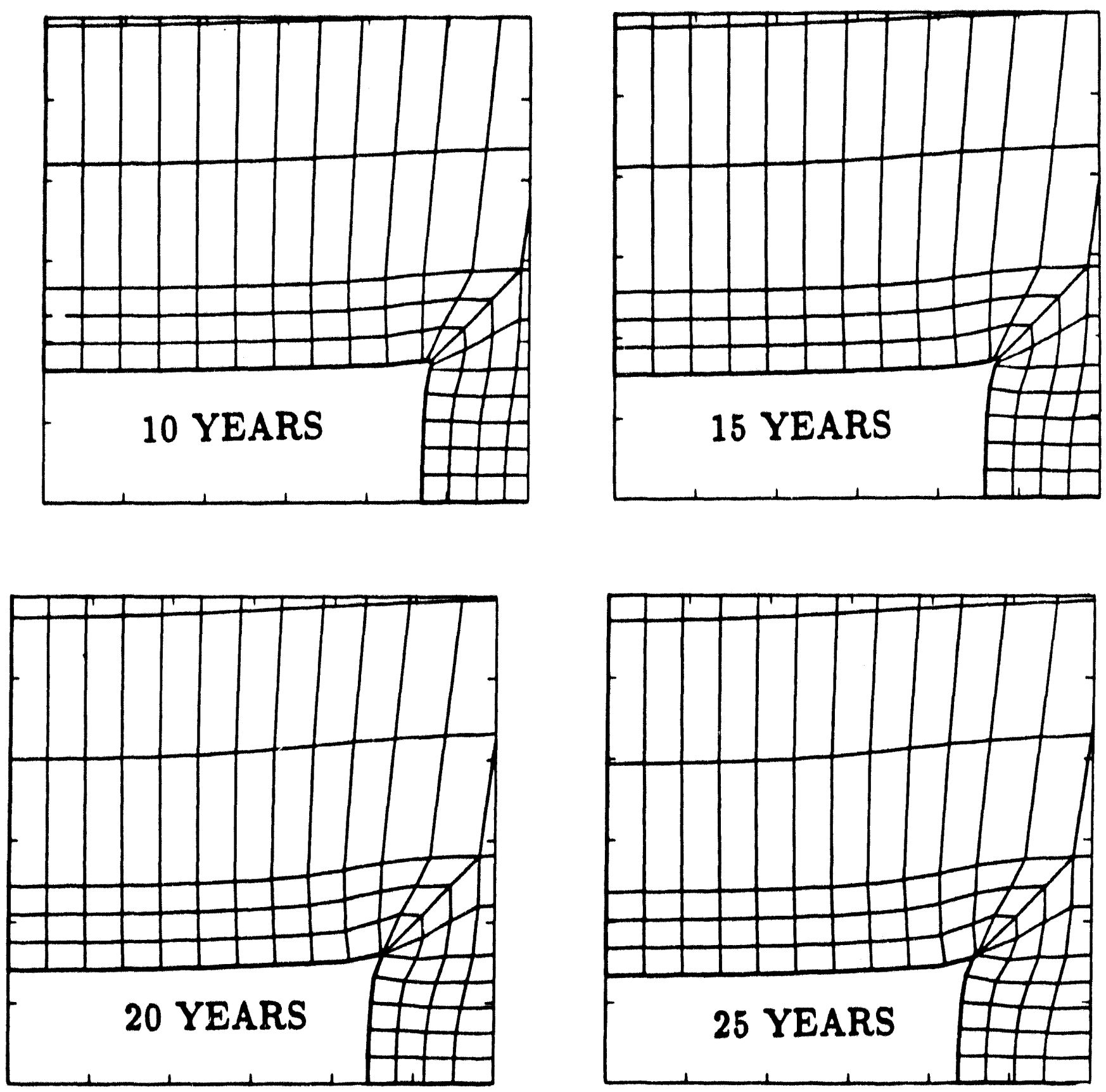

Figure 3. Profile of the Empty Room At 10, 15, 20, and 25 years (Model 2 Results). 

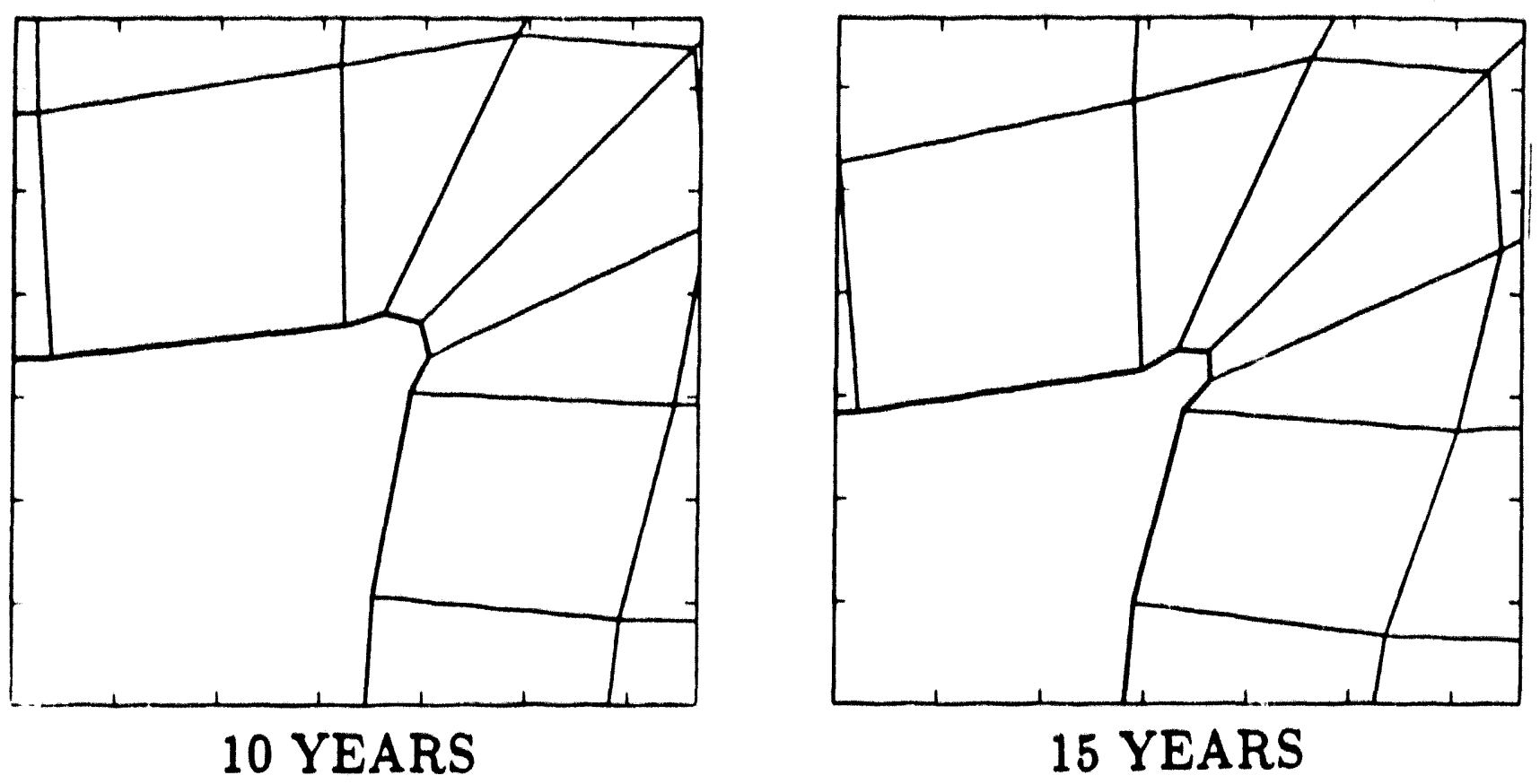

15 YEARS

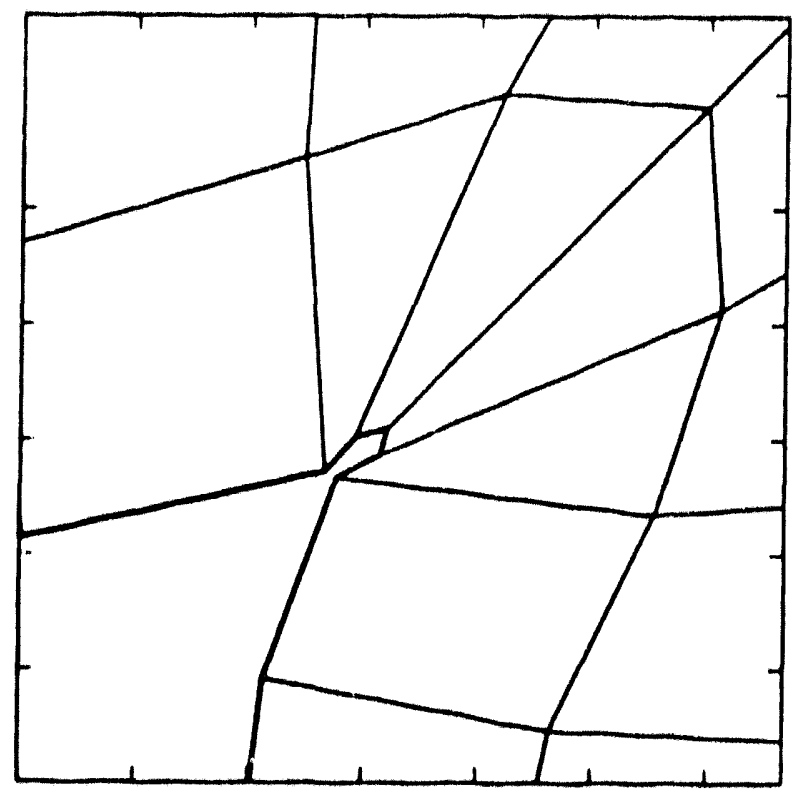

20 YEARS

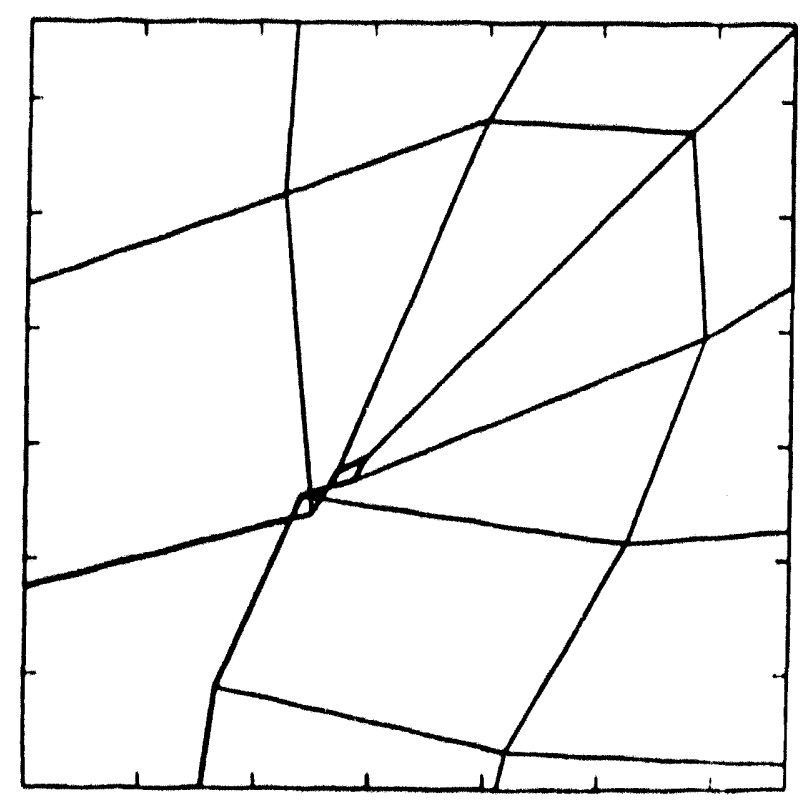

25 YEARS

Figure 4. Closure of the Corner in an Empty Room (Model 2 Results). 


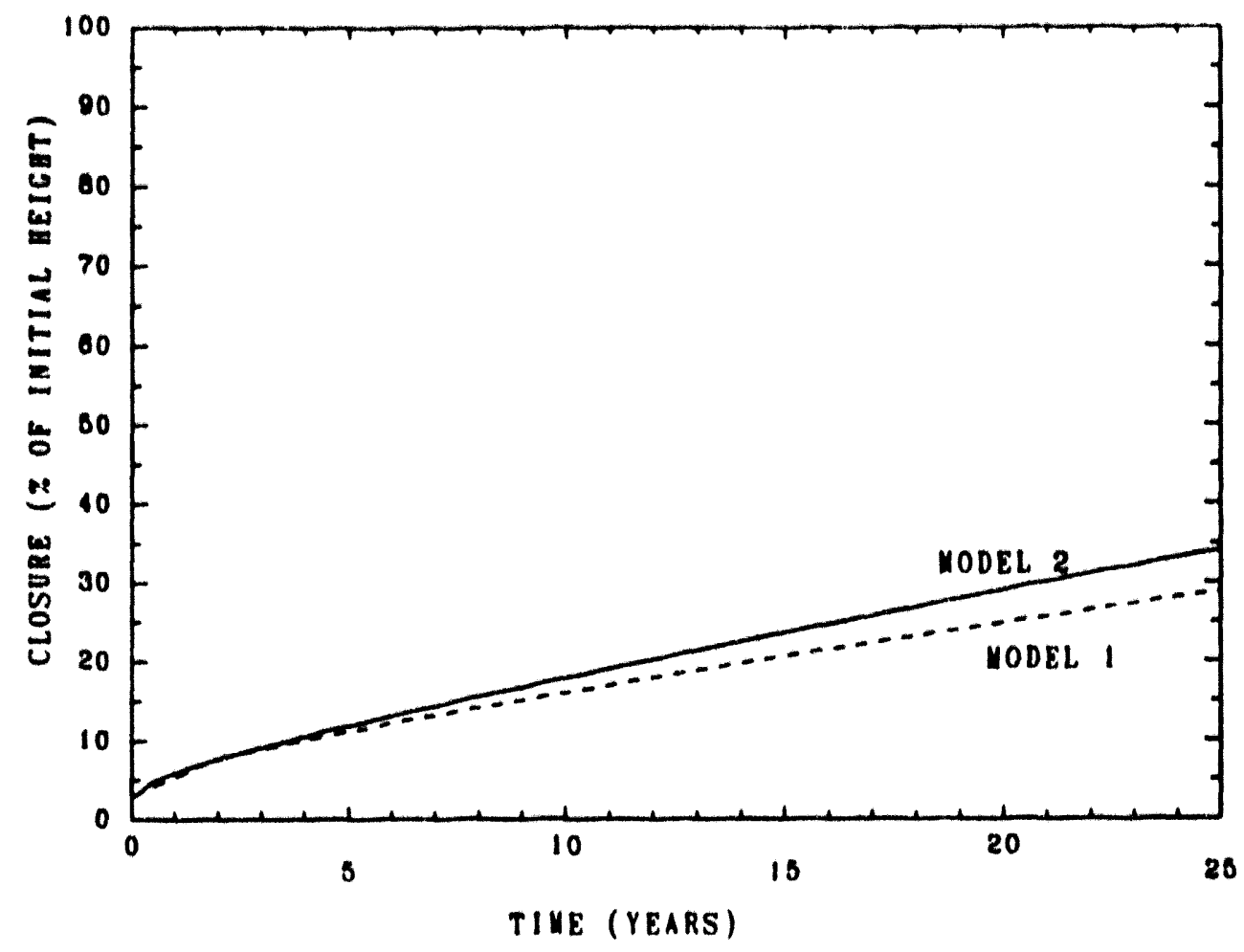

Figure 5. Comparison of Model 1 and Model 2 Results for Vertical Closure in an Empty Room at the Center of the Roof and Floor.

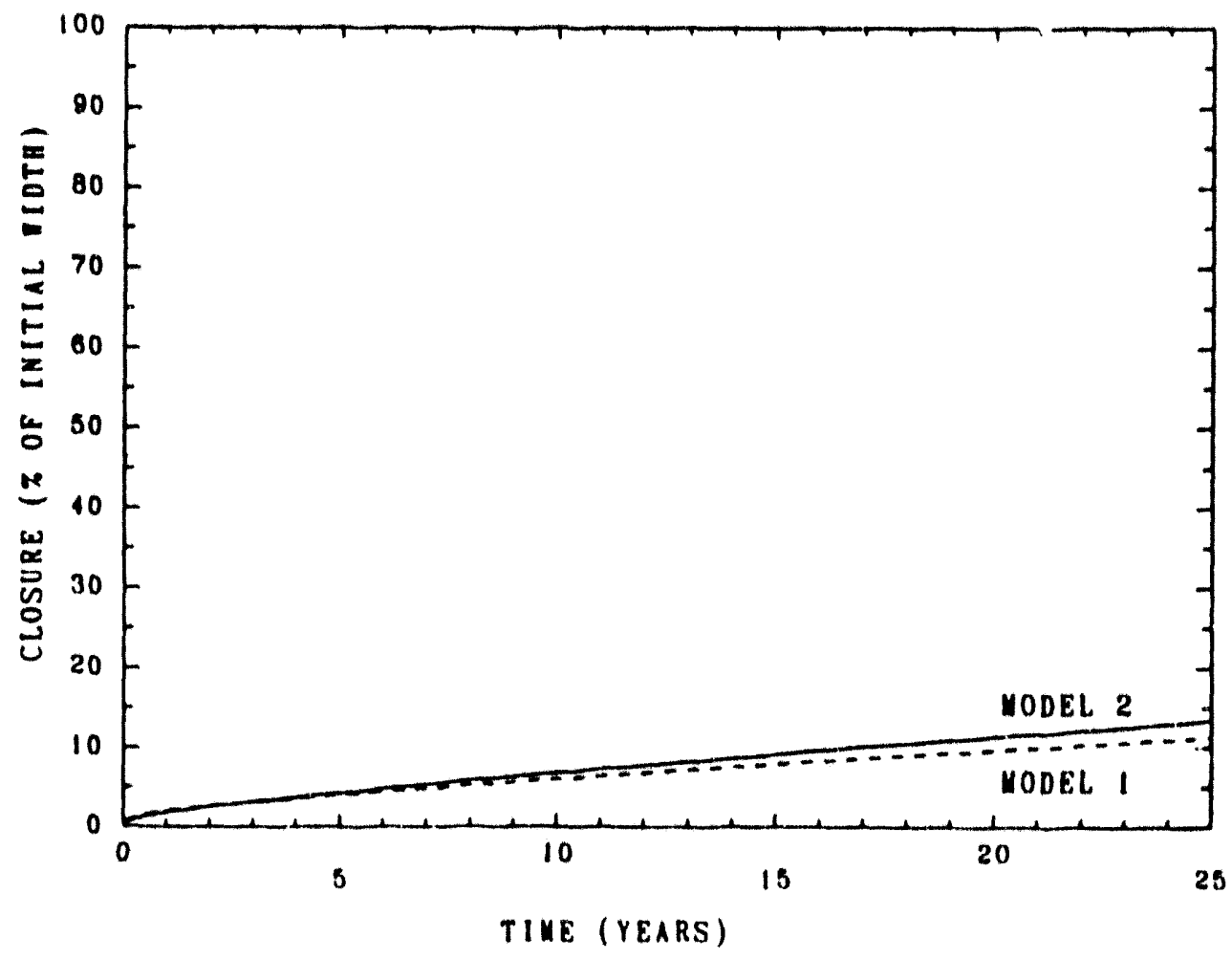

Figure 6. Comparison of Model 1 and Model 2 Results for Horizontal Closure in an Empty Room at the Midheight of the Rit. 


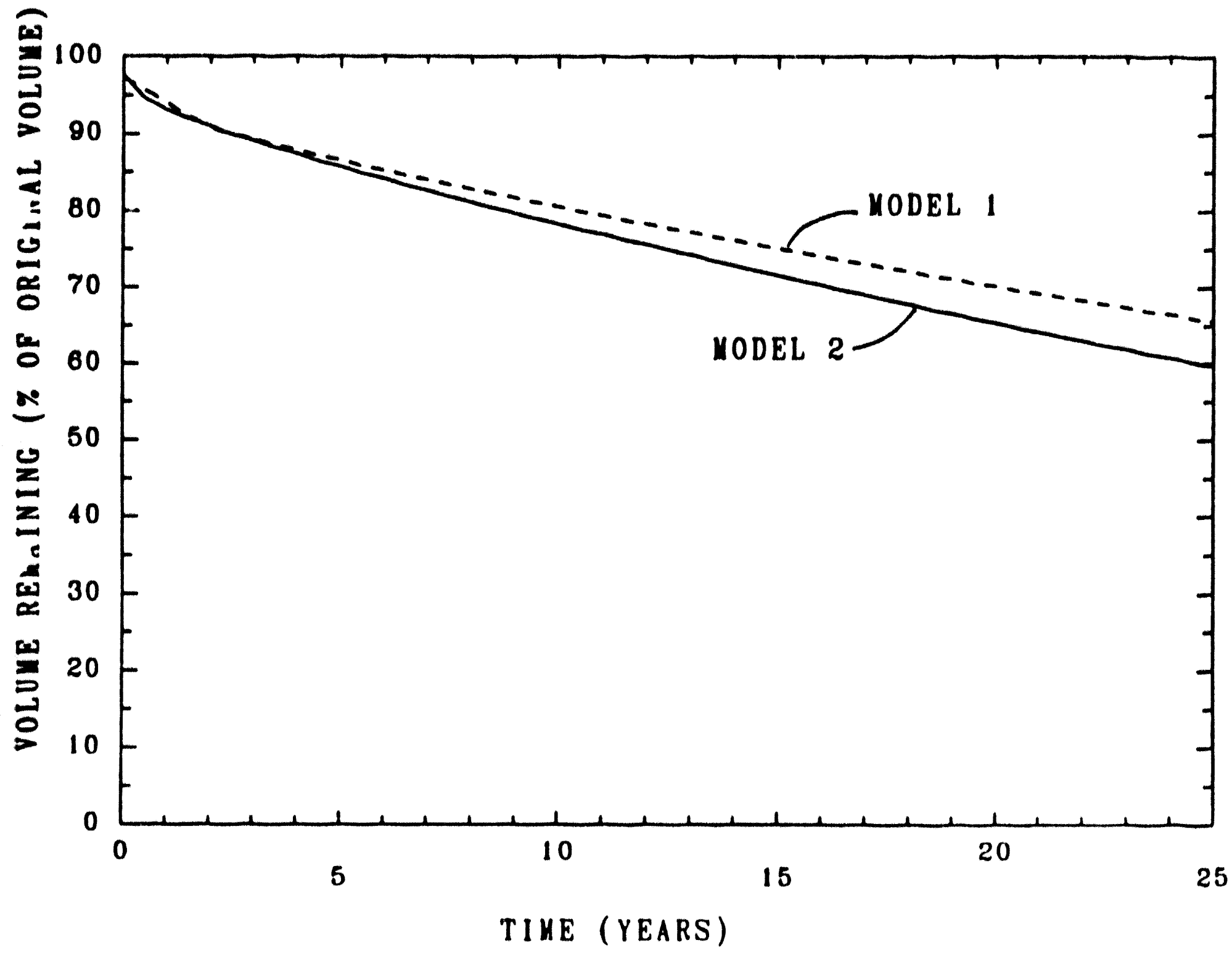

Figure 7. Comparison of Model 1 and Model 2 Results for Volume Remaining in an Empty Room. 


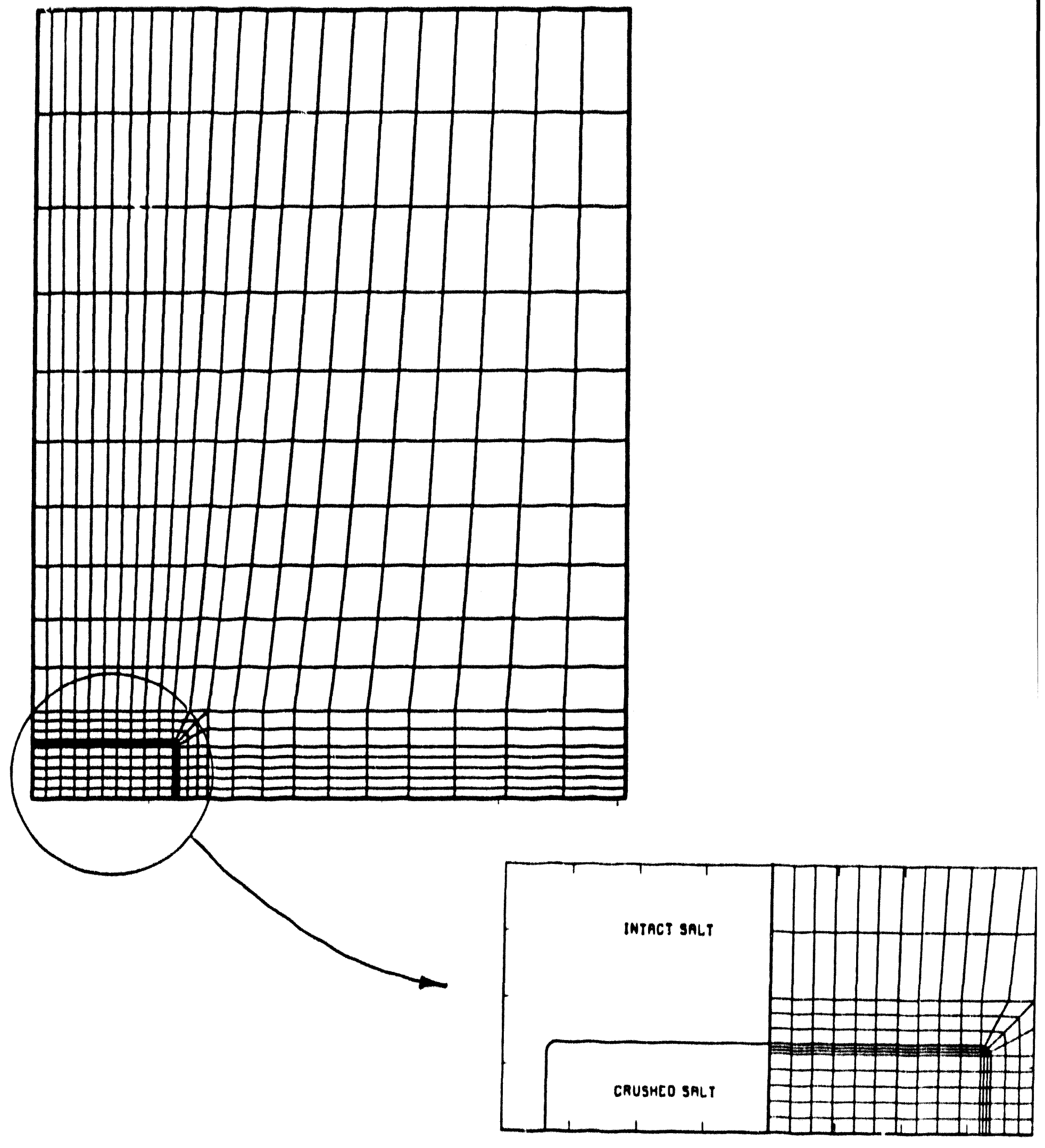

Figure 8. Finite Element Mesh Used to A nalyze a Storage Room Filled With Crushed Salt (Model 3). 


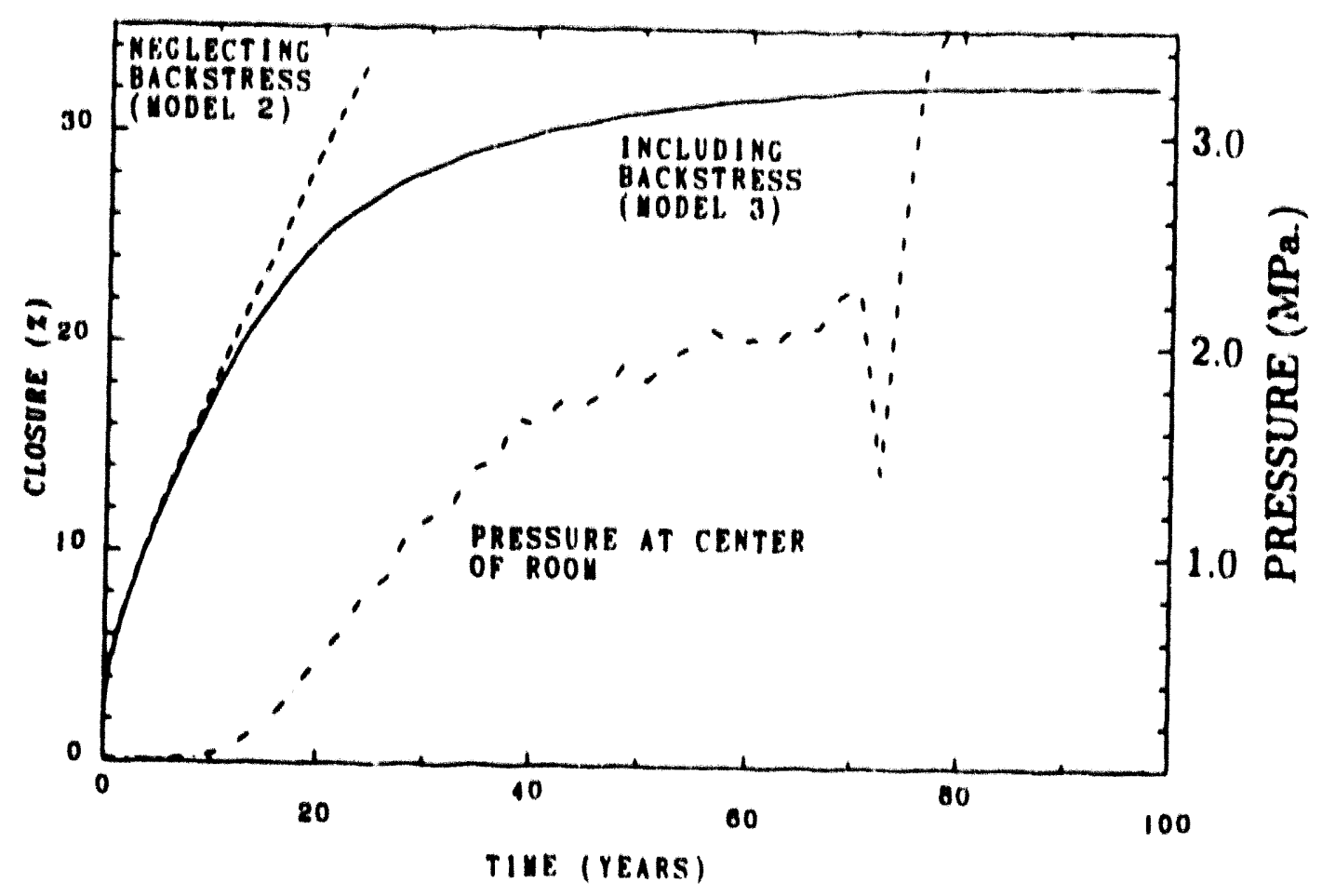

Figure 9. Vertical Closure Between the Center of the Roof and Floor in a Room Filled With Crushed Salt and in an Empty Room. Closure is Expressed as a Percentage of the Initial Height of the Room.

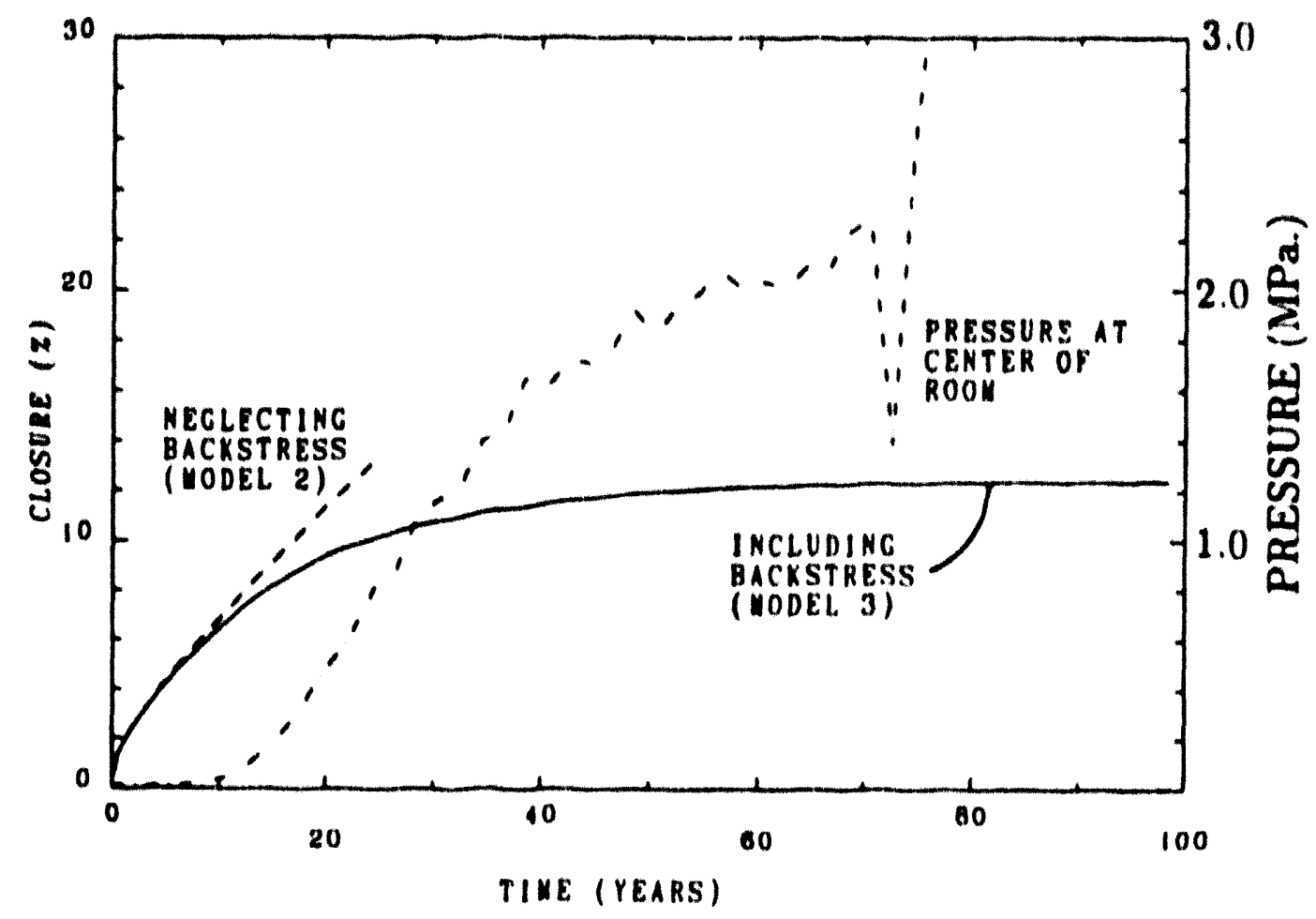

Figure 10. Horizontal Closure at the Midheight of the Rib in a Room Filled With Crushed Salt and in an Empty Room. Closure is Expressed as a Percentage of the Initial Width of the Room. 


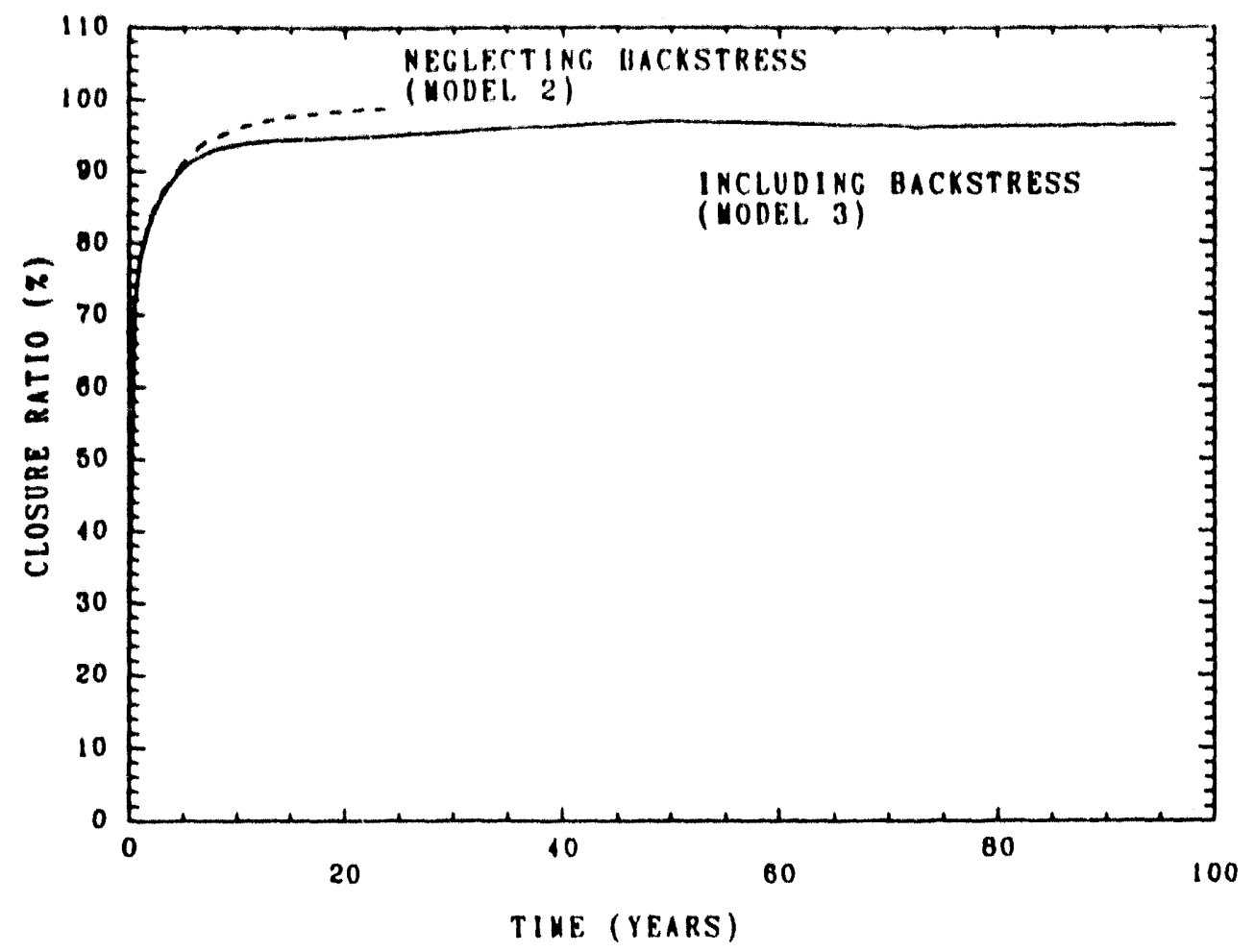

Figure 11. Ratio of the Horizontal Closure to the Vertical Closure in a Room Filled With Crushed Salt and in an Empty Room.

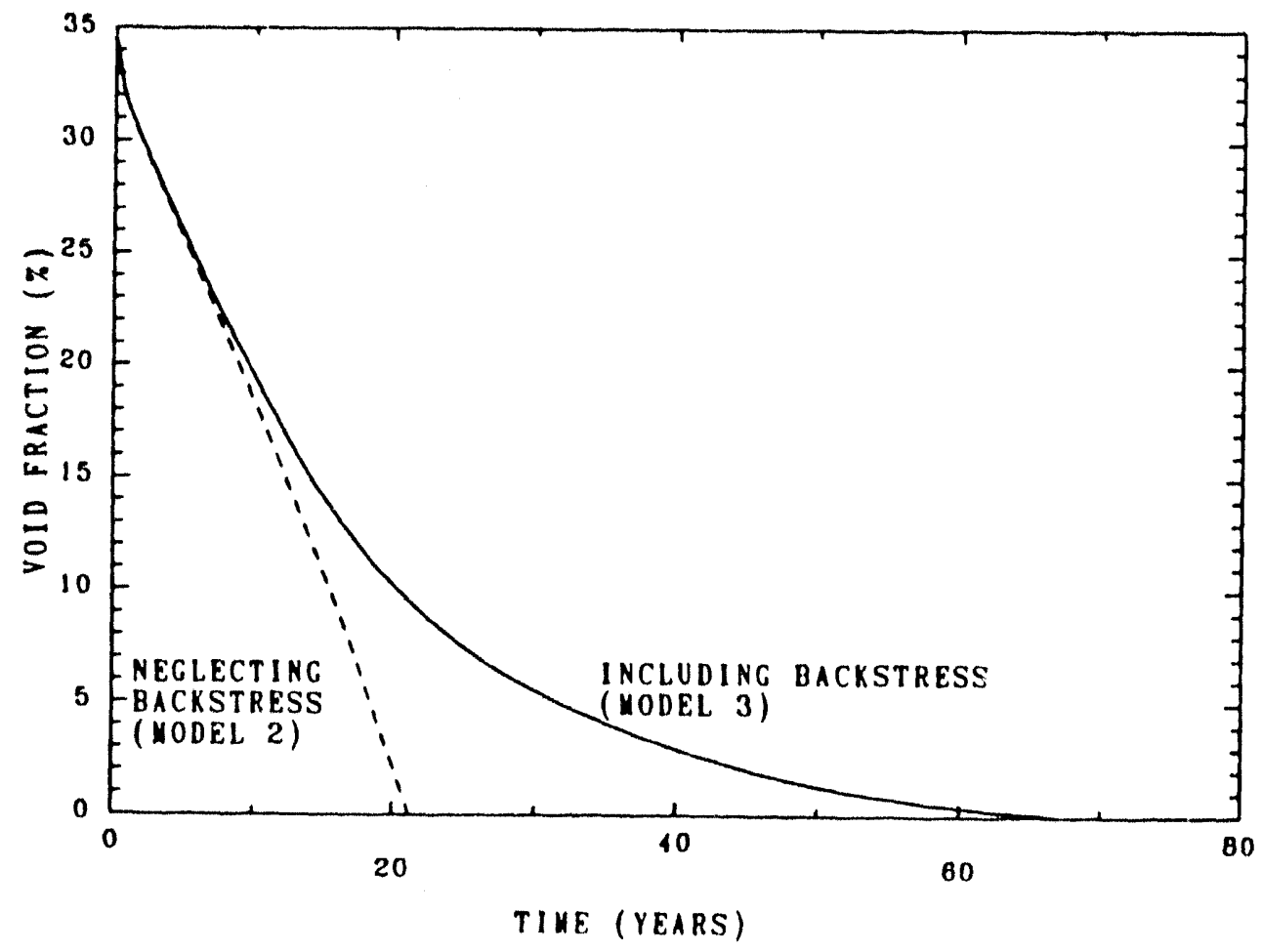

Figure 12. Remaining Void Fraction in a Room Filled With Crushed Salt, Comparison Between Results With and Without Backstress from the Room Contents. The Void Fraction is Defined in Equation 1. 


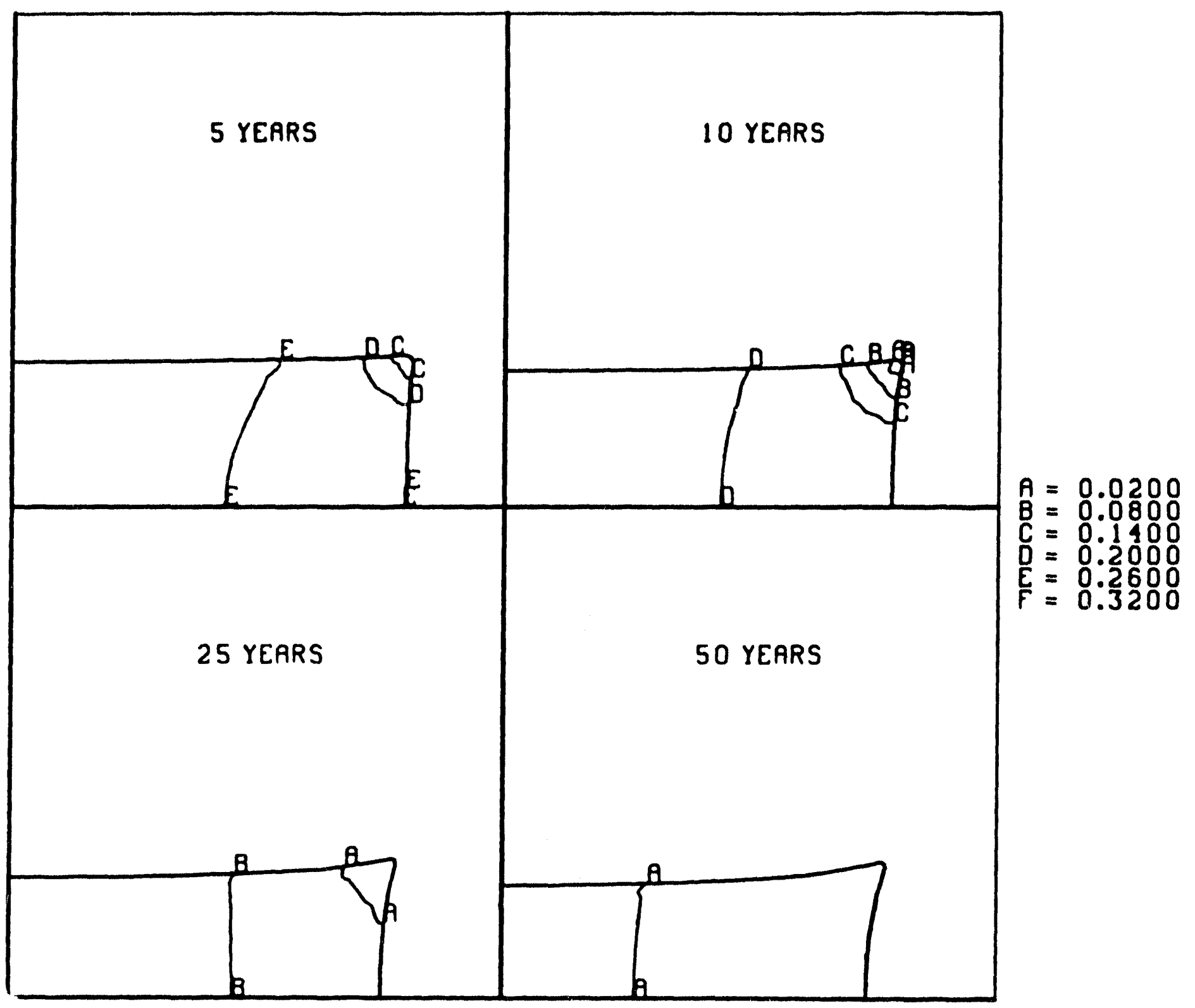

Figure 13. Distribution of the Void Fraction in a Room Filled With Crushed Salt (Model 3 Results). The Void Fraction is Defined in Equation 1. 

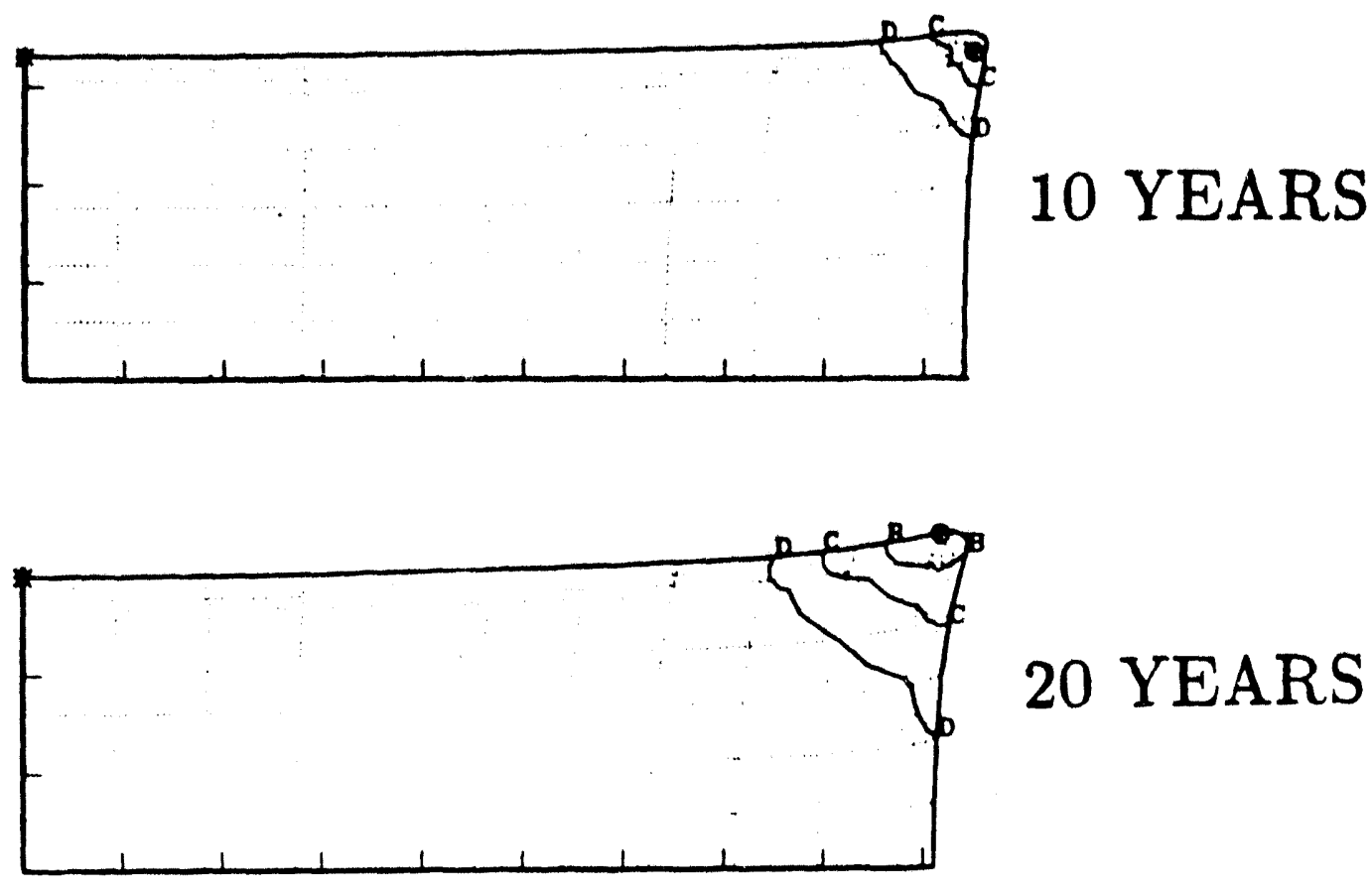

20 YEARS
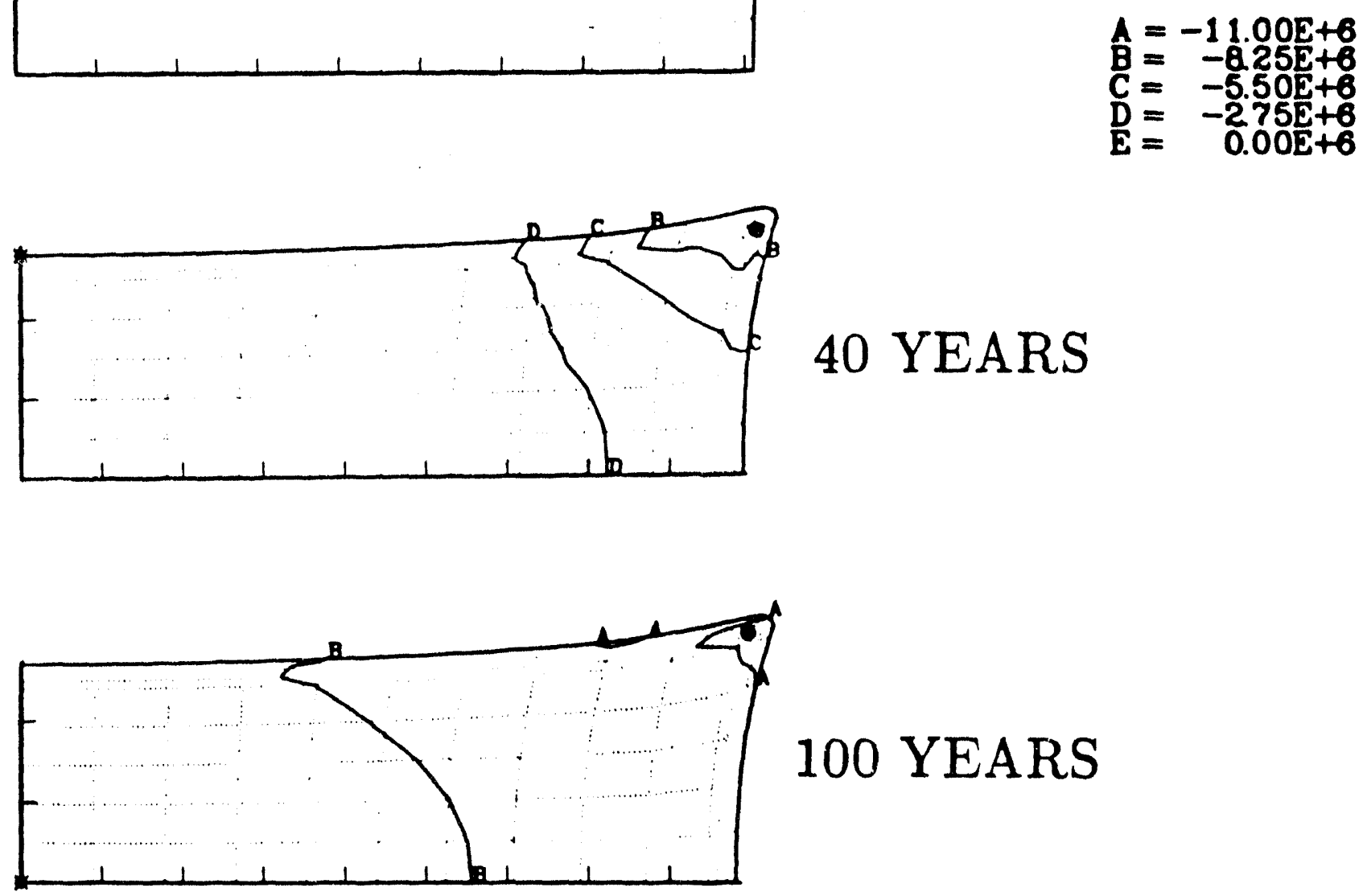

Figure 14. Horizontal Stress Inside of a Room Filled With Crushed Salt (Stress is in Pascals). 


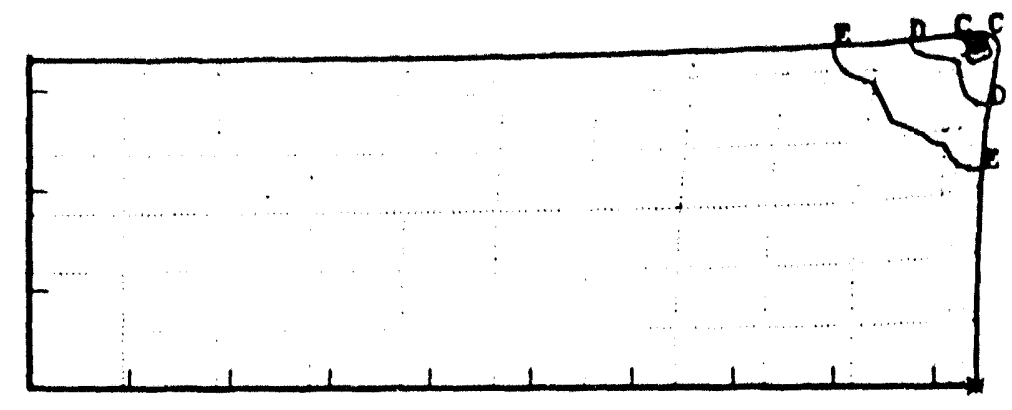

\section{YEARS}

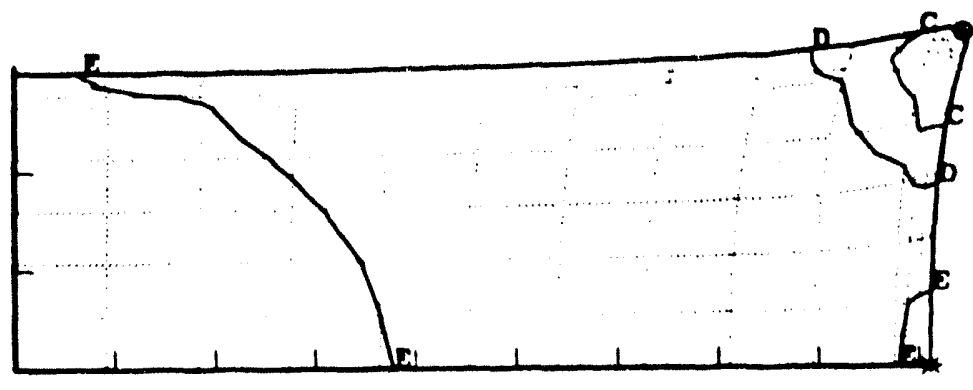

\section{YEARS}

$\mathrm{A}=-13.50 \mathrm{E}+6$
$\mathrm{~B}=-10.50 \mathrm{D}+6$
$\mathrm{C}=-7.50 \mathrm{E}+6$
$\mathrm{D}=-4.50 \mathrm{E}+6$
$\mathrm{E}=-1.50 \mathrm{E}+6$

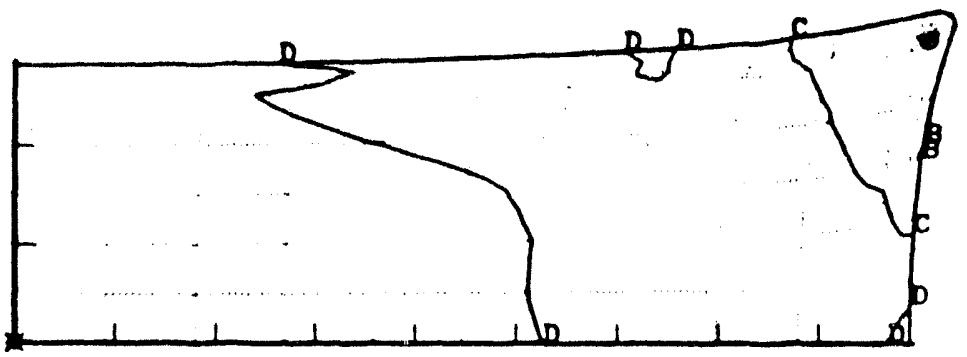

\section{YEARS}

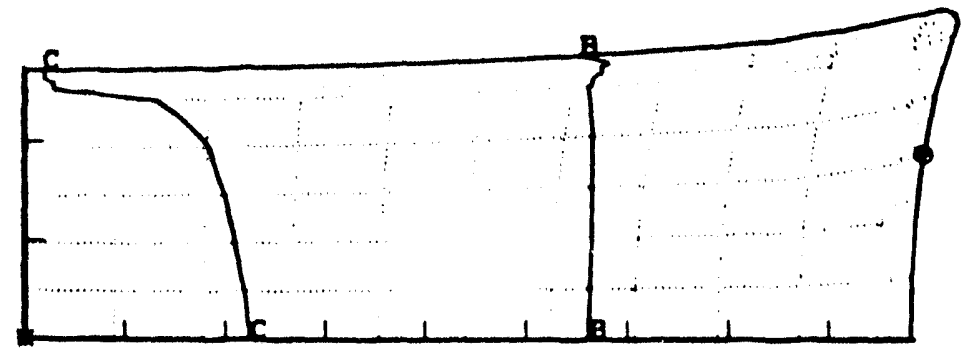

100 YEARS

Figure 15. Vertical Stress Inside of a Room Filled With Crushed Salt (Stress is in Pascals). 


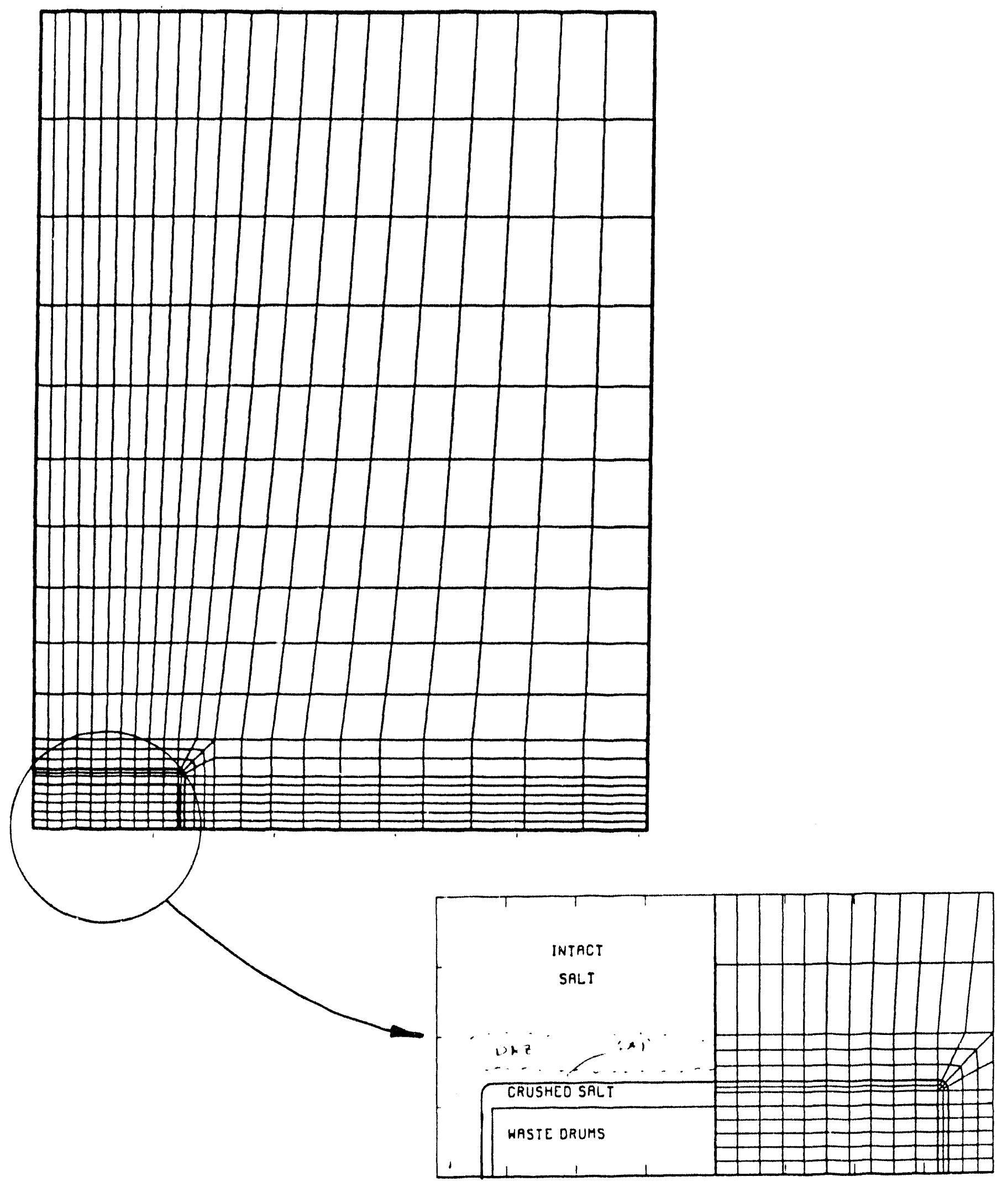

Figure 16. Finite Element Mesh Used to Analyze a Storage Room Filled With Waste and Crushed Salt (Model 4). 


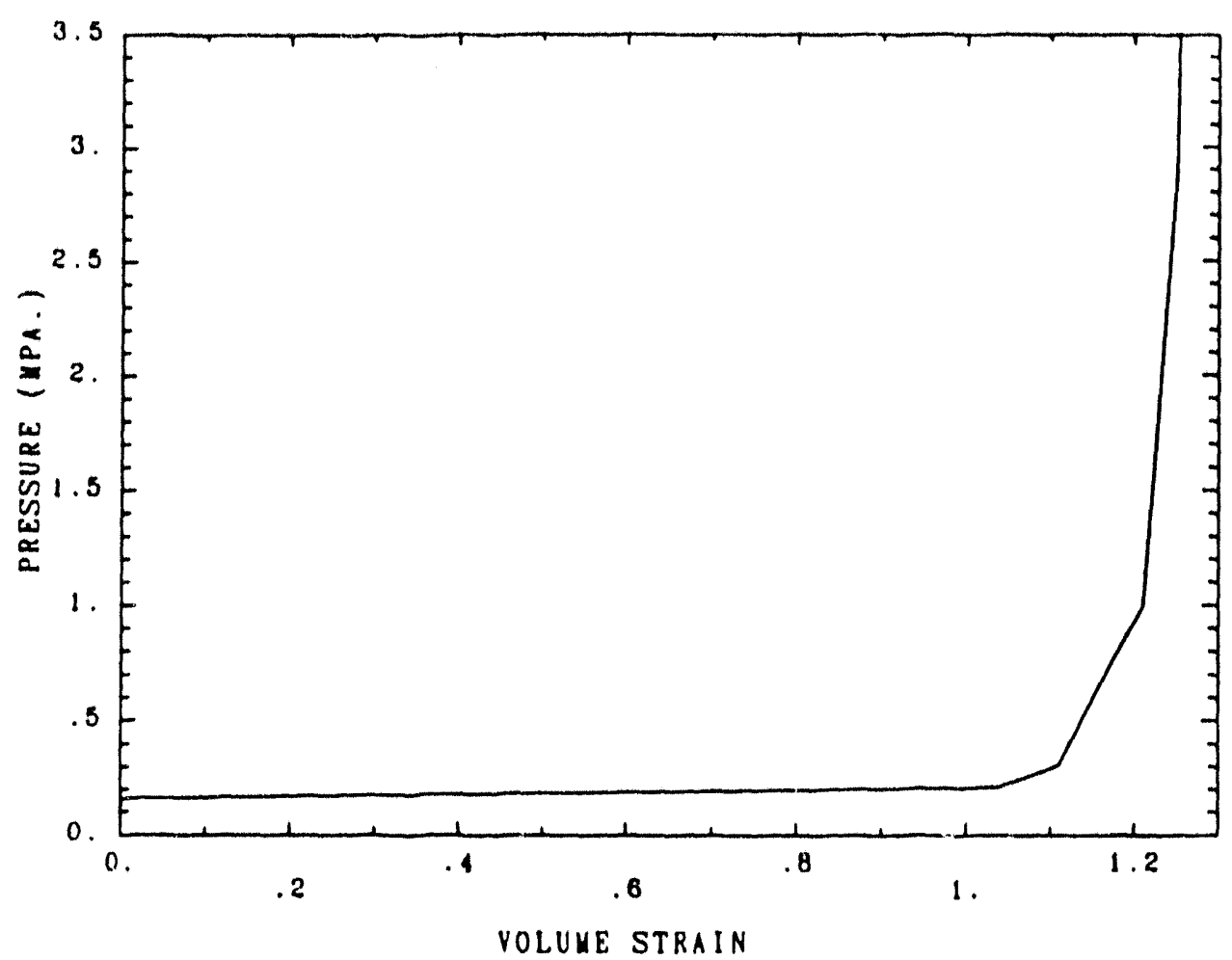

Figric 17. Relationship Between Pressure and Volumetric Strain For the Waste Model.

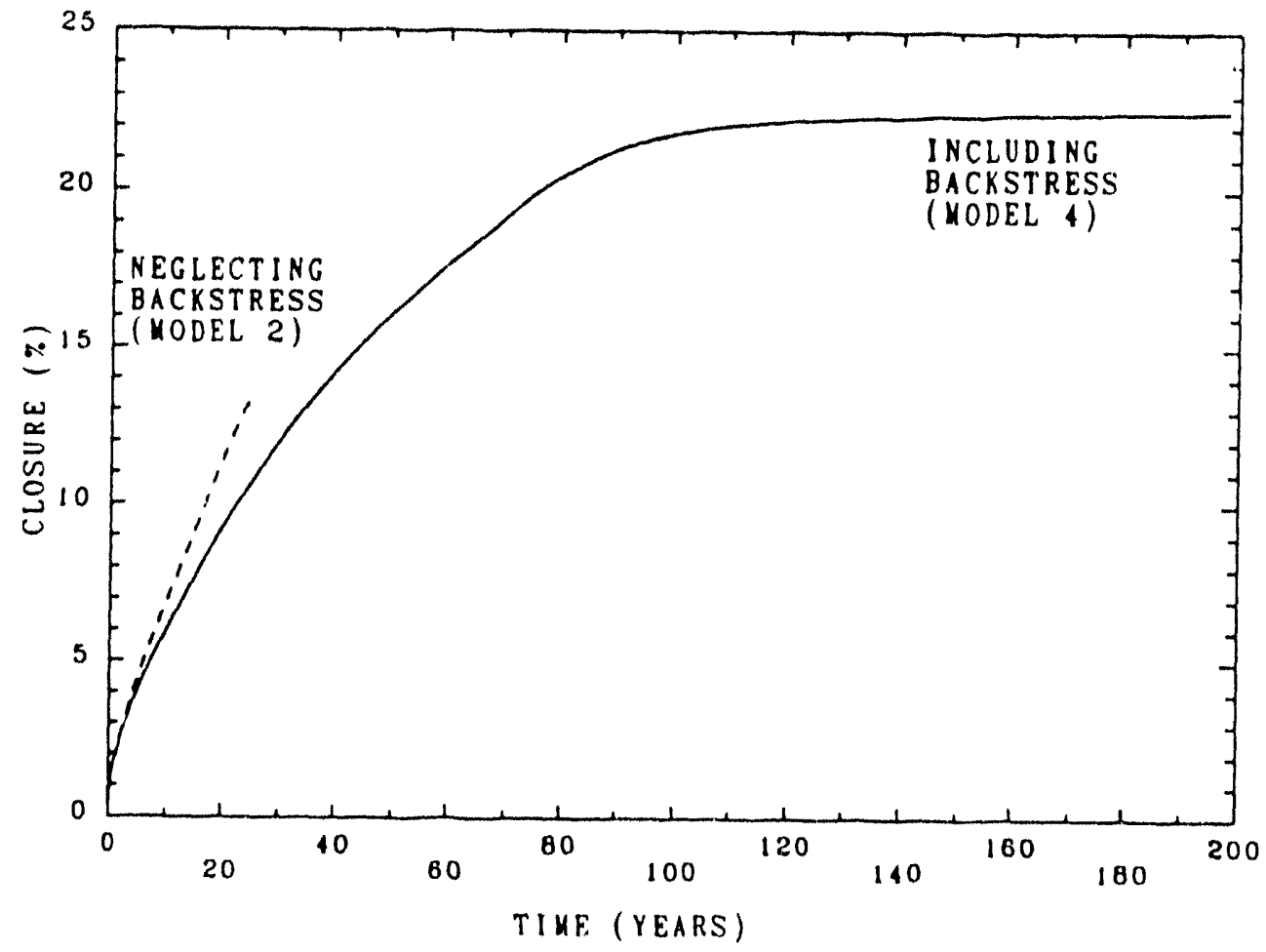

Figure 18. Horizontal Closure at the Midheight of the Rib in a Room Filled With Waste and Crushed Salt and in an Empty Room. Closure is Expressed as a Percentage of the Initial Width of the Room. 


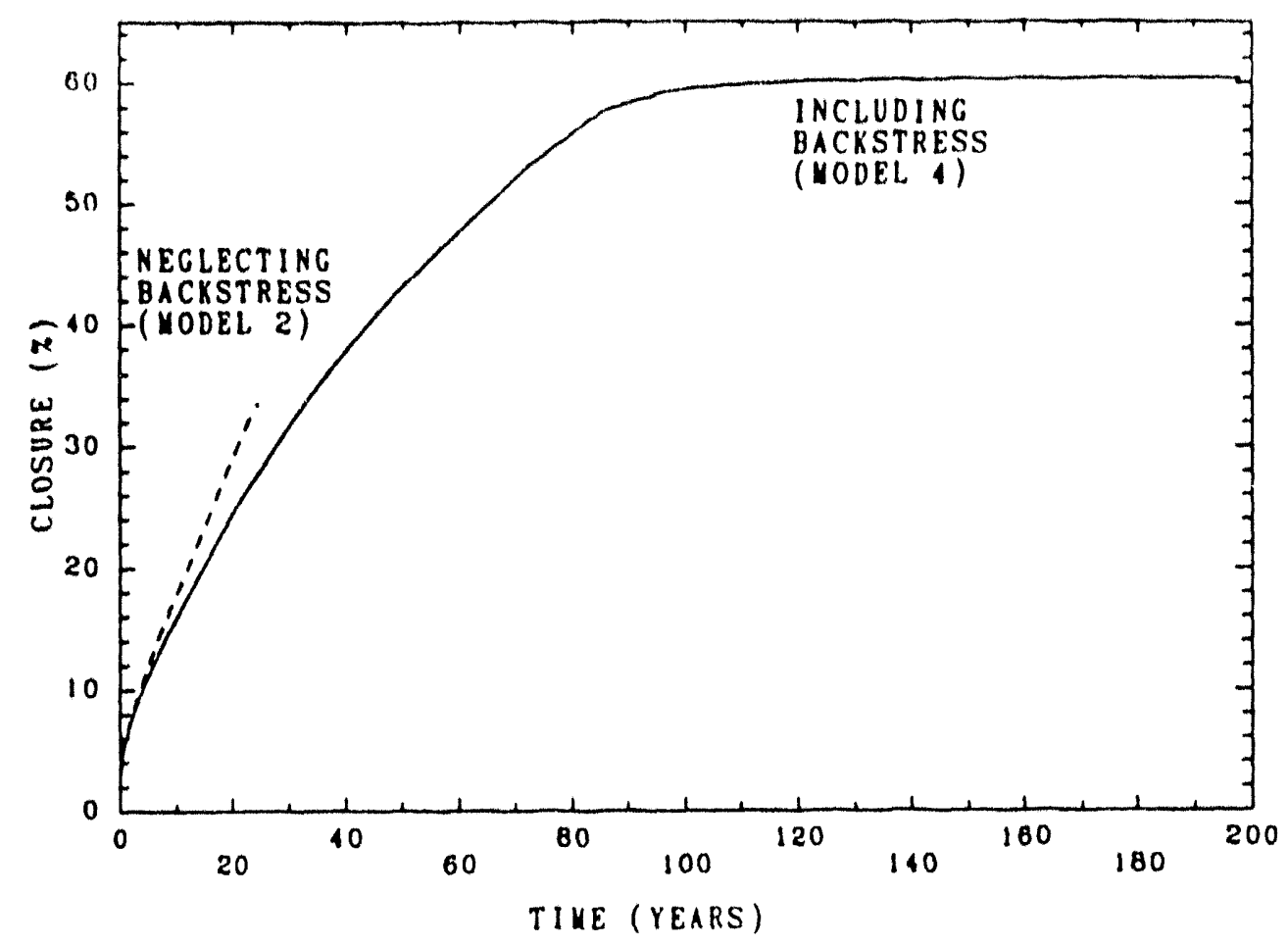

Figure 19. Vertical Closure Between the Center of the Roof and Floor for a Room Filled With Waste and Crushed Salt and for an Empty Room. Closure is Expressed as a Percentage of the Initial Height of the Room.

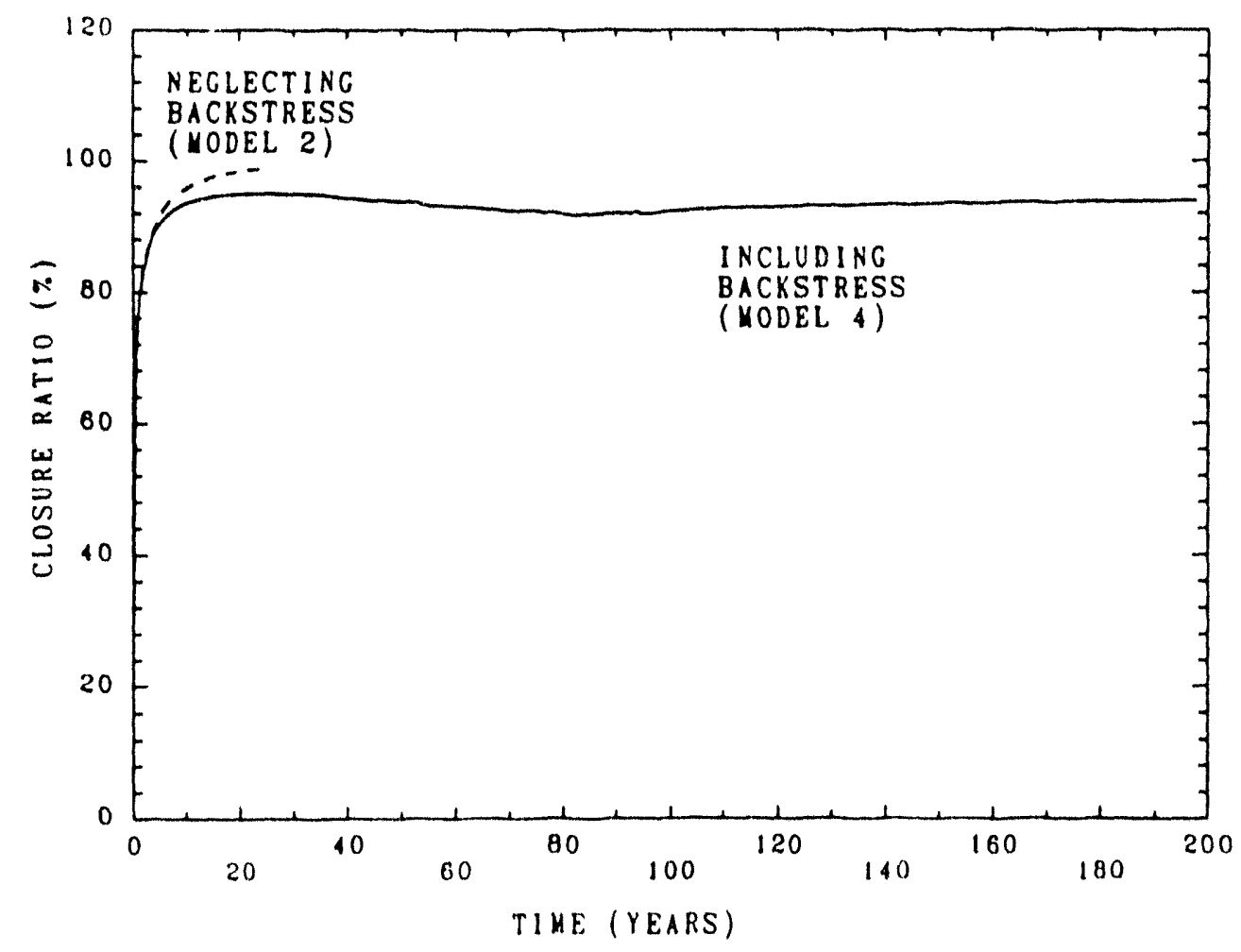

Figure 20. Katio of the Horizontal Closure to the Vertical Closure in a Room Filled With Waste and Crushed Salt and in an Empty Room. 


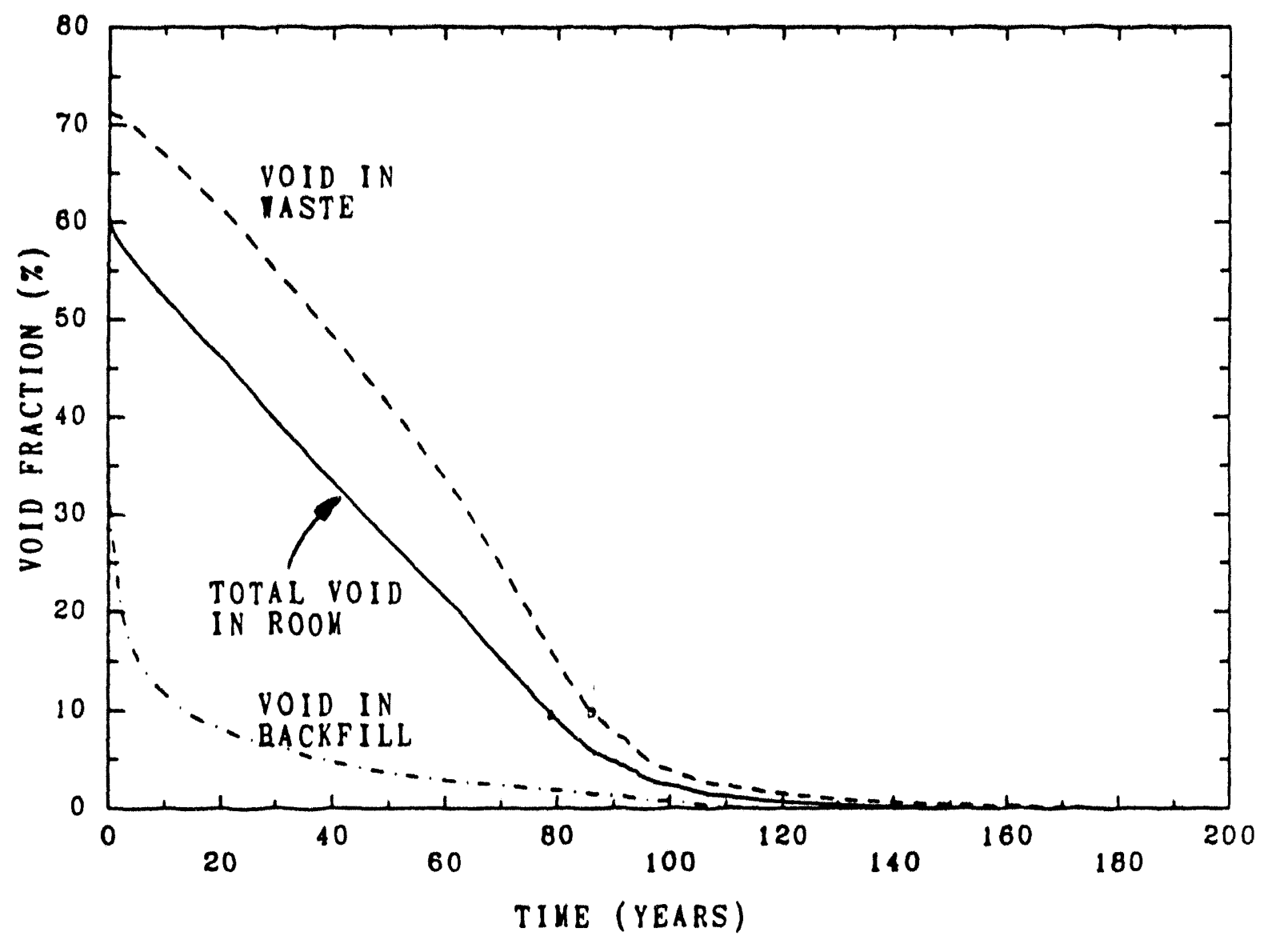

Figure 21. Remaining Void Fraction in the Waste Region, in the Backfill, and in the Room as a Whole. The Void Fraction is Defined as the Ratio of the Void Volume to the Current Volume (See Equation 1). 


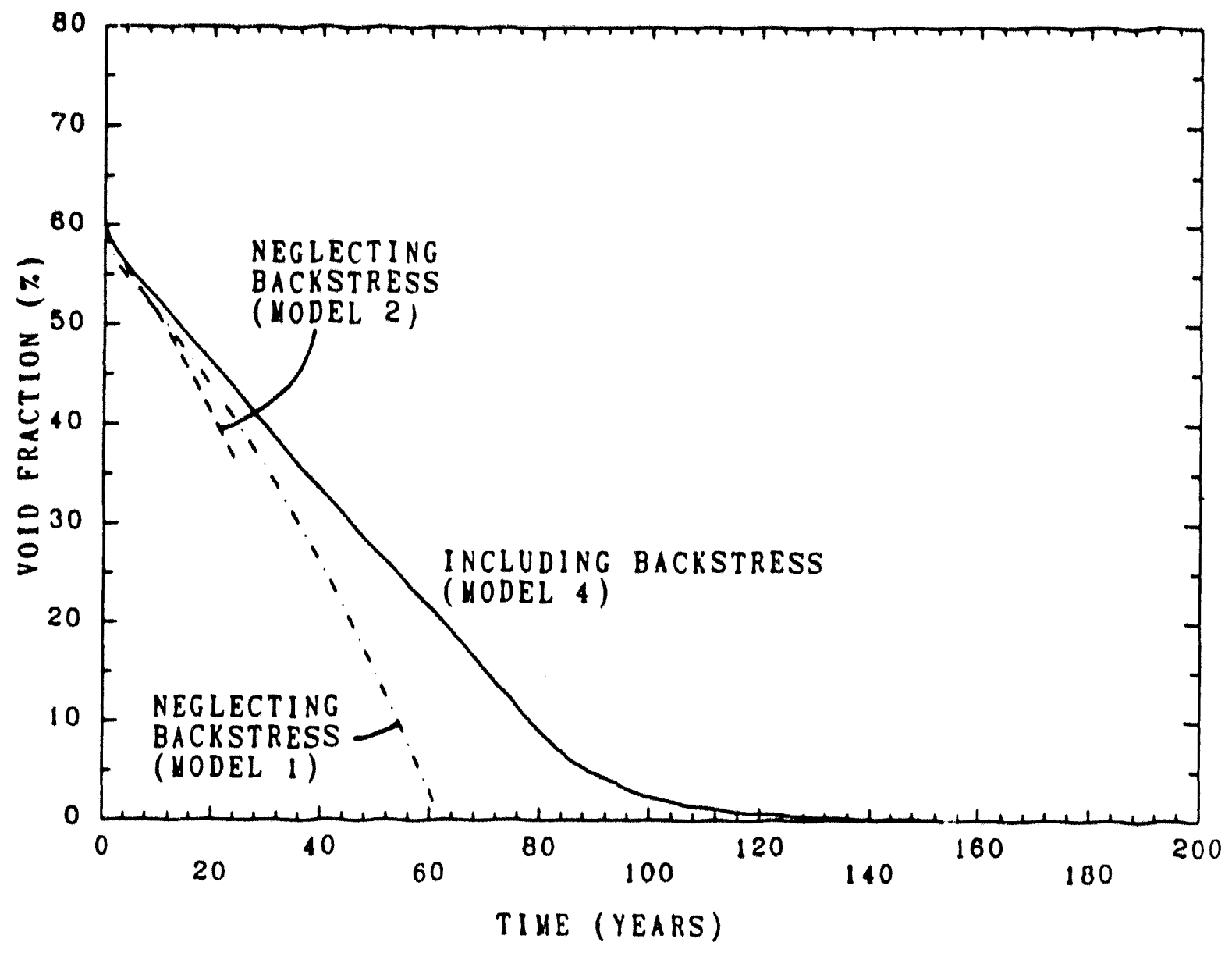

Figure 22. Remaining Void Fraction in a Room Filled With Waste and Crushed Salt Comparison of Results With and Without Backstress from the Room Contents. 


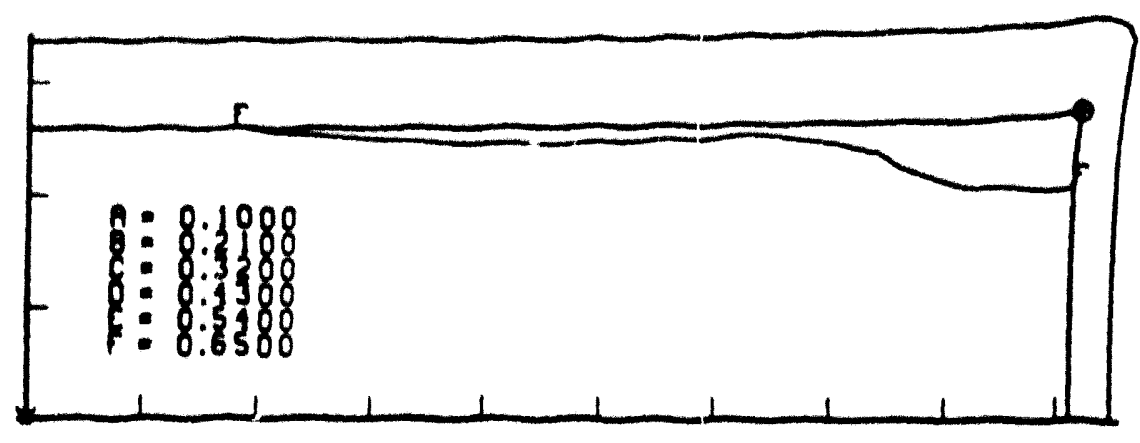

\section{YEARS}

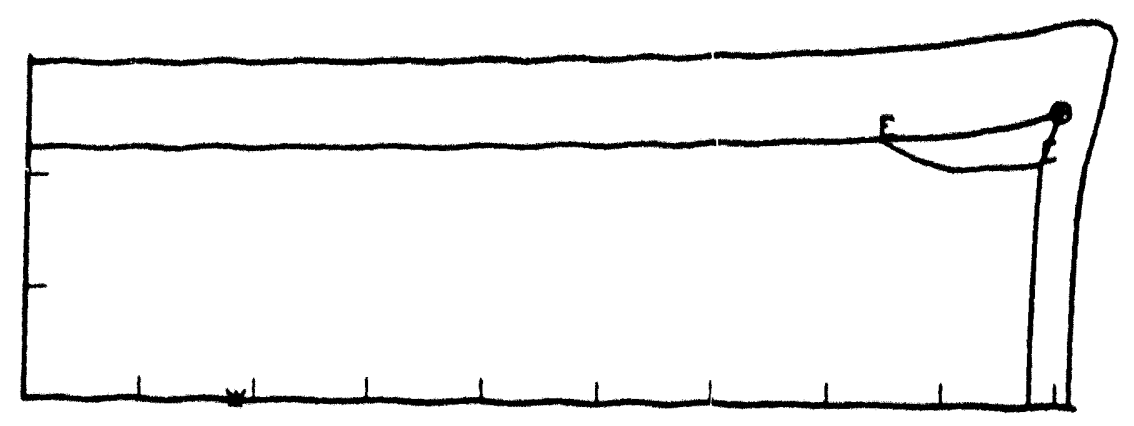

20 YEARS

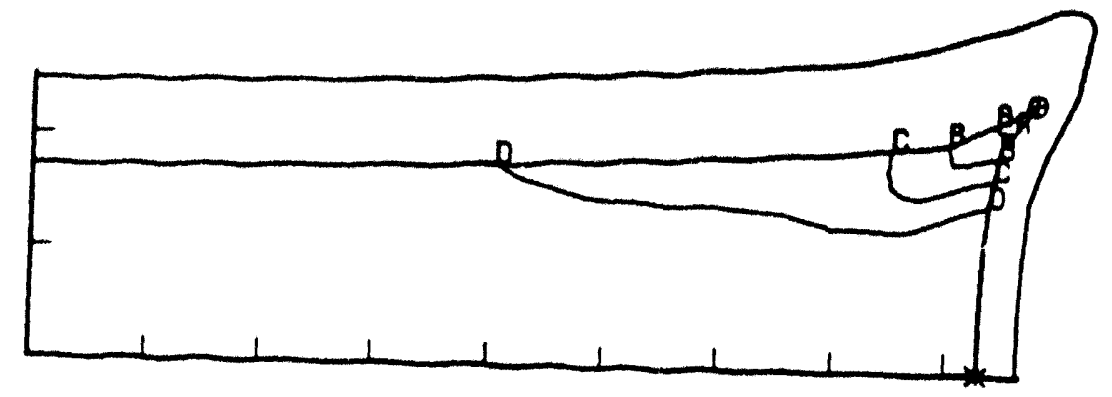

\section{YEARS}

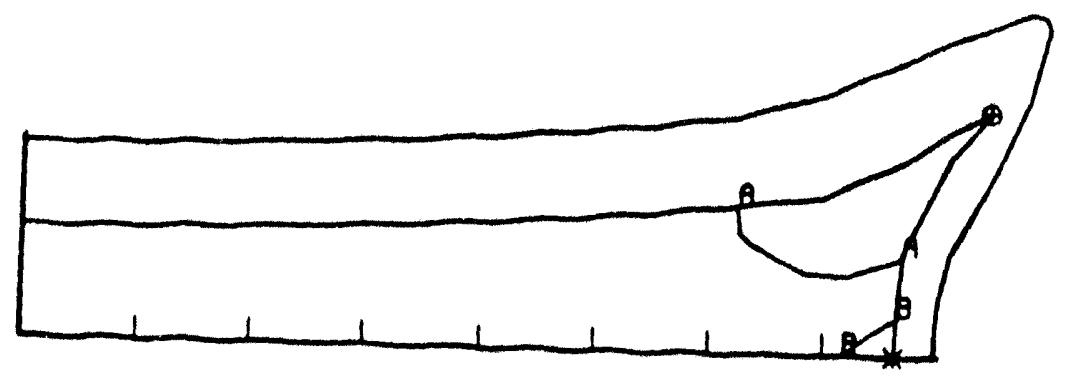

83 YEARS

Figure 23. Distribution of the Void Fraction in the Waste Region (Model 4 Results). The Void Fraction is Defined in Equation 1. 

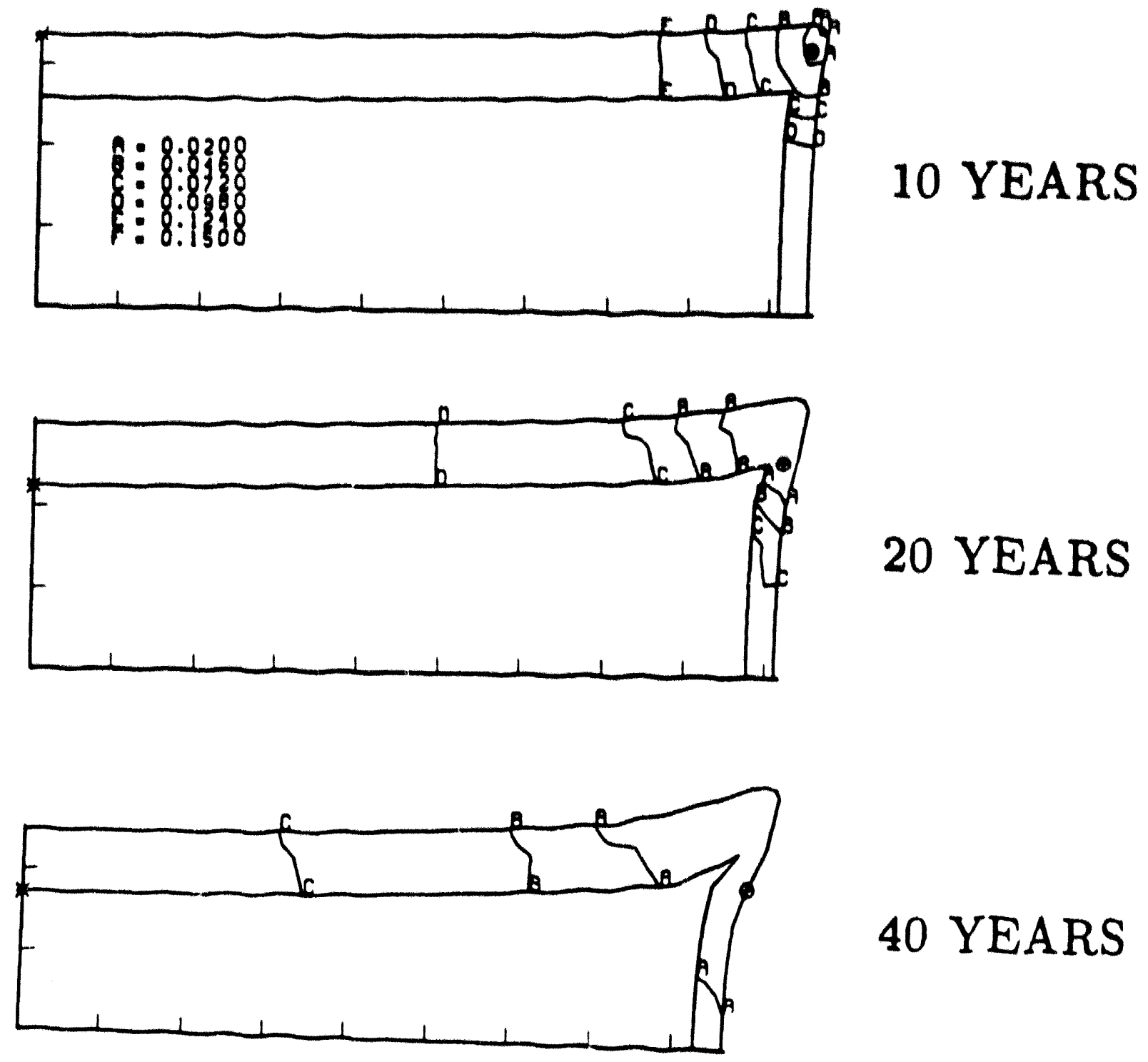

40 YEARS

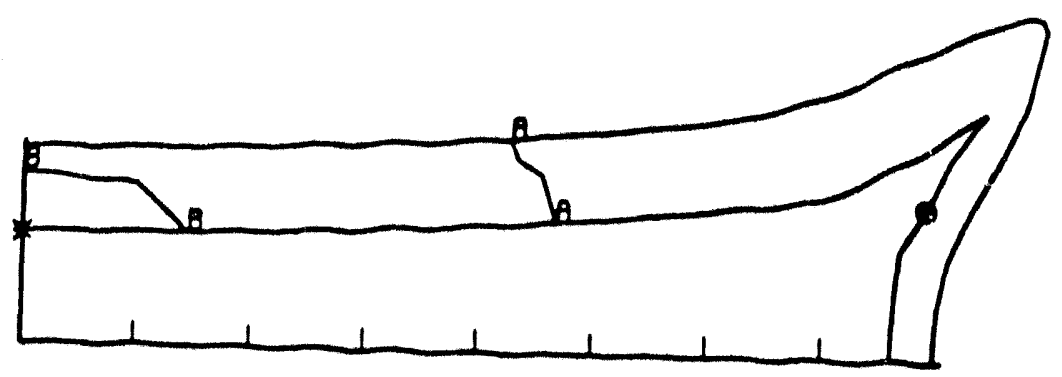

83 YEARS

Figure 24. Distribution of the Void Fraction in the Backfill Region (Model 4 Results). The Void Fraction is Defined in Equation 1. 


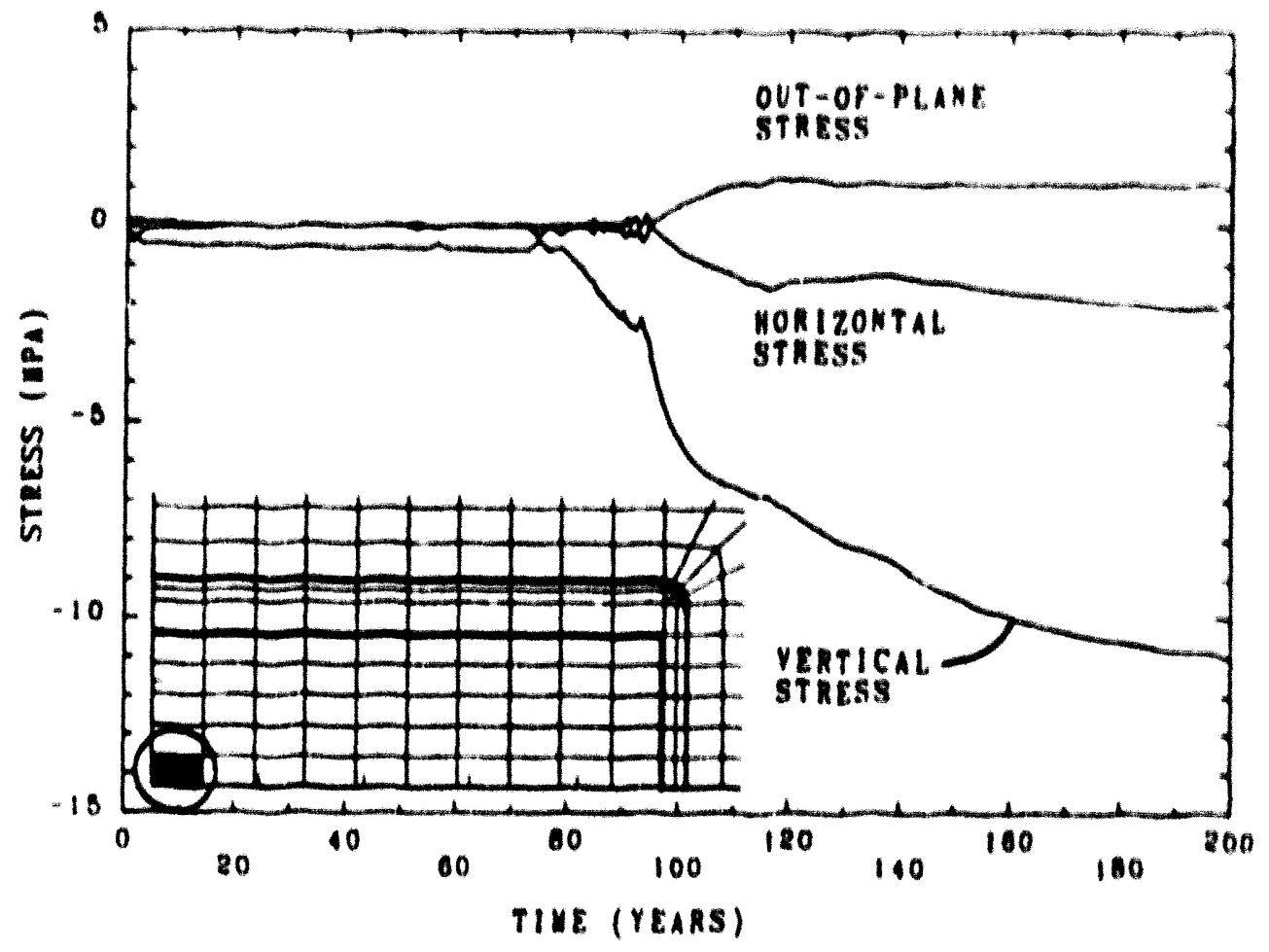

Figure 25. Stress History at the Center of the Waste Region (Model 4 Resulis).

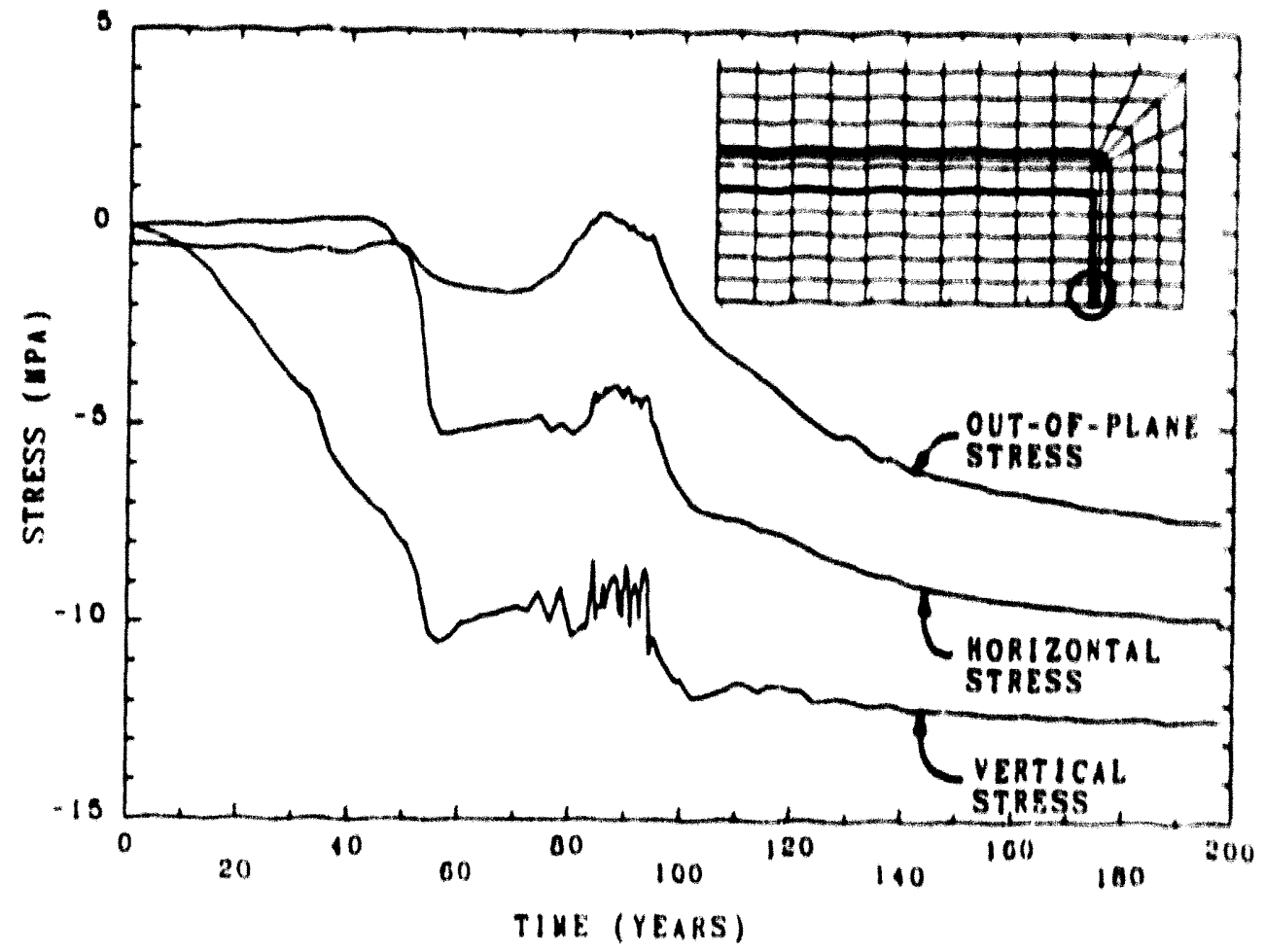

Figure 26. Stress History in the Backfill Near the Midheight of the Rib (Model a Results). 


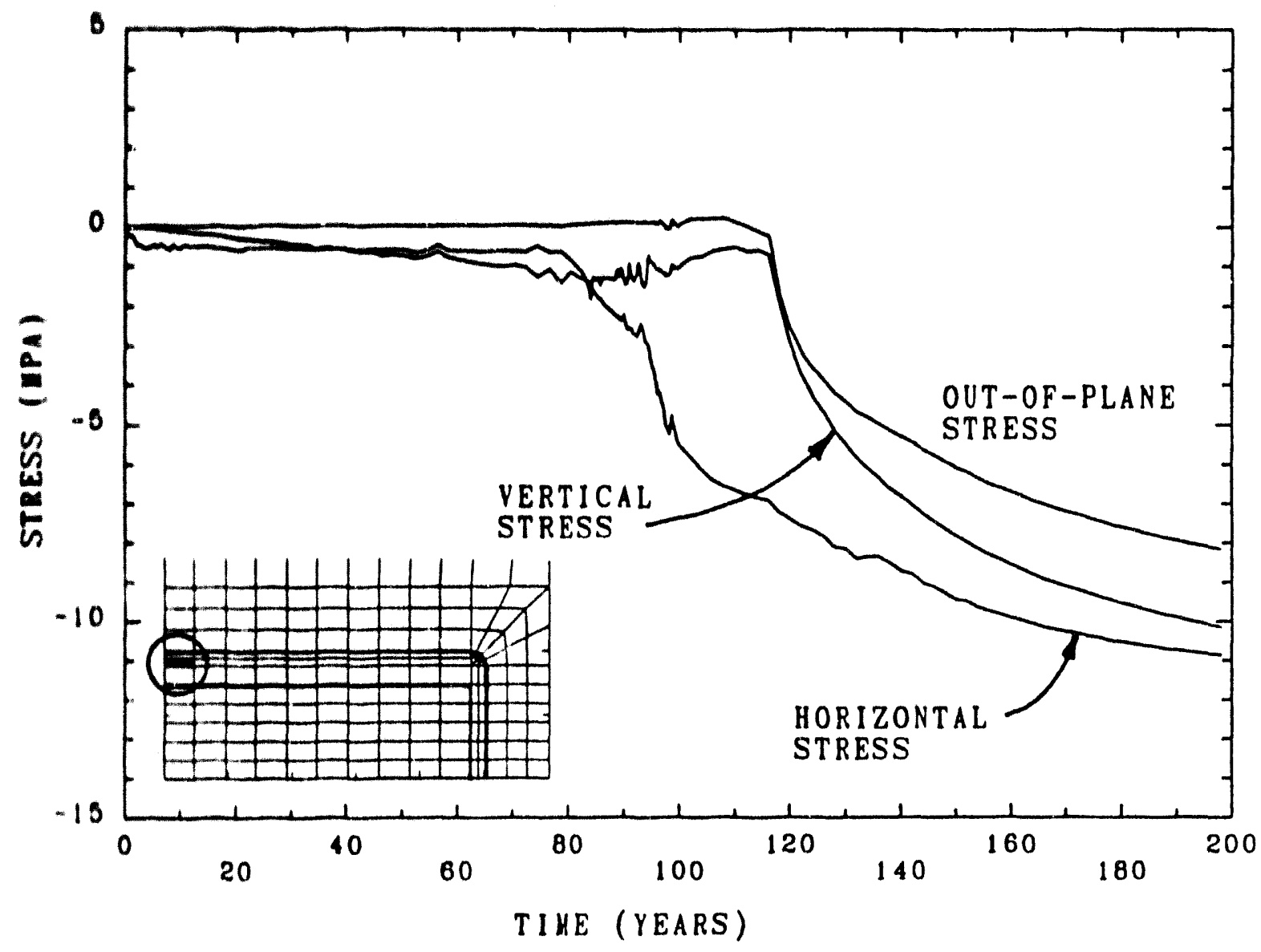

Figure 27. Stress History in the Backfill Near the Center of the Room. 


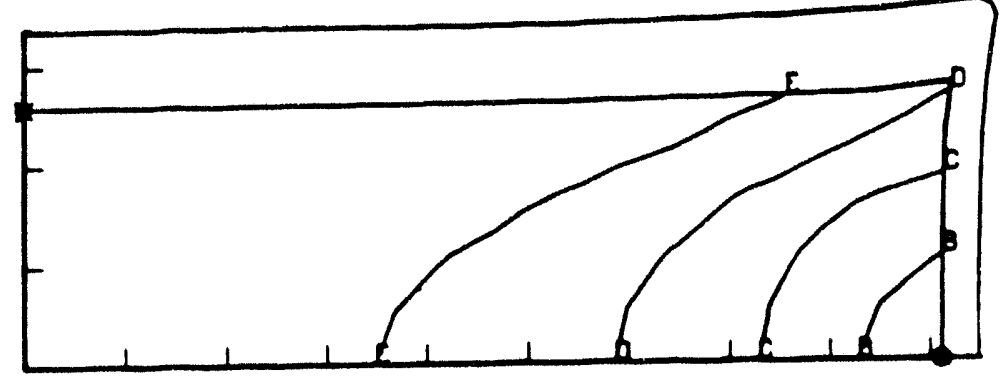

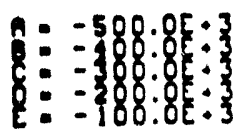

Q: $--451: 5 E: 3$

10 YEARS

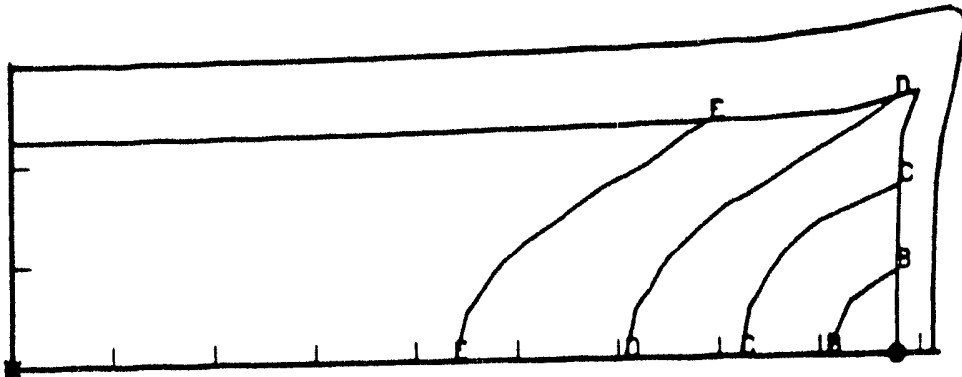

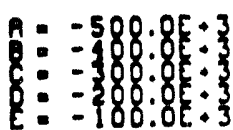

20 YEARS

e: $-451: 3 E: 3$

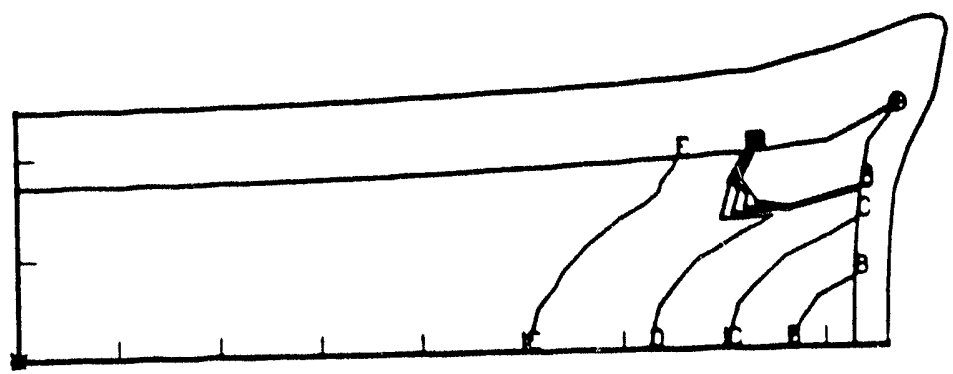

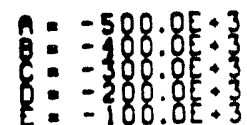

e:- $-5.803 \xi \div 6$

40 YEARS

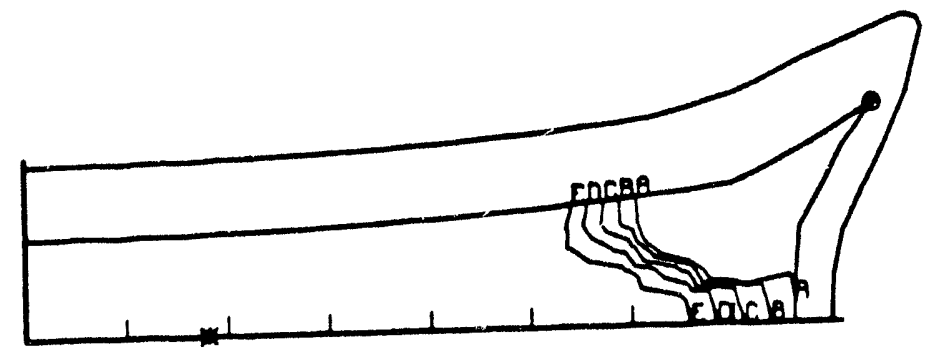

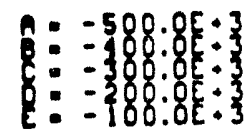

:-5.1195.6

83 YEARS
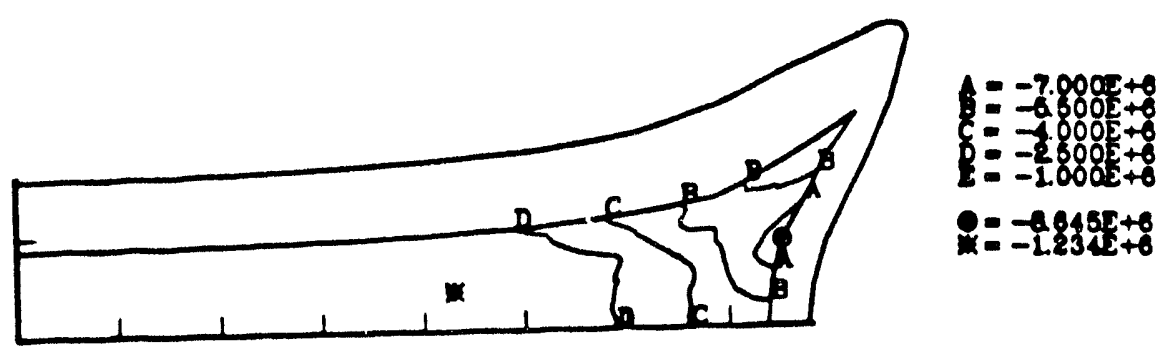

200 YEARS

Figure 28. Horizontal Stress in the Waste Region (Model 4 Results, Stress is in Pascals). 


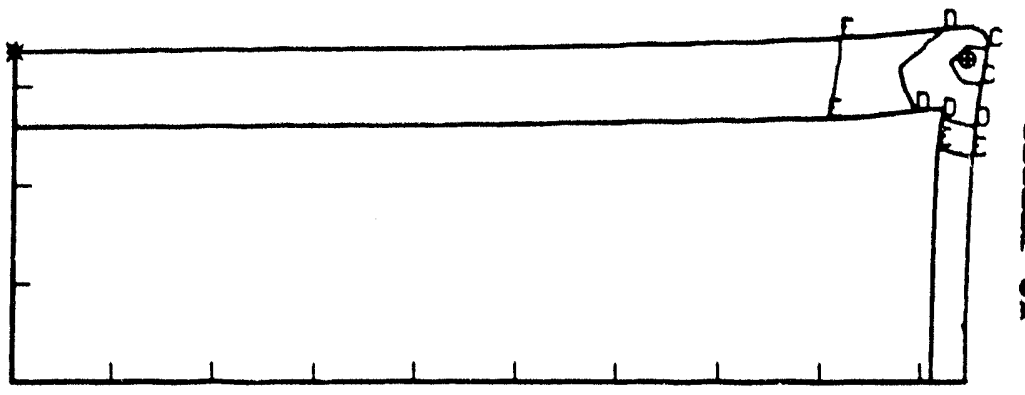

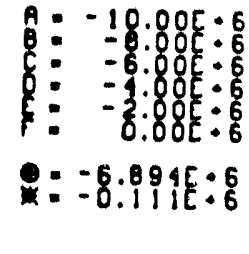

10 YEARS

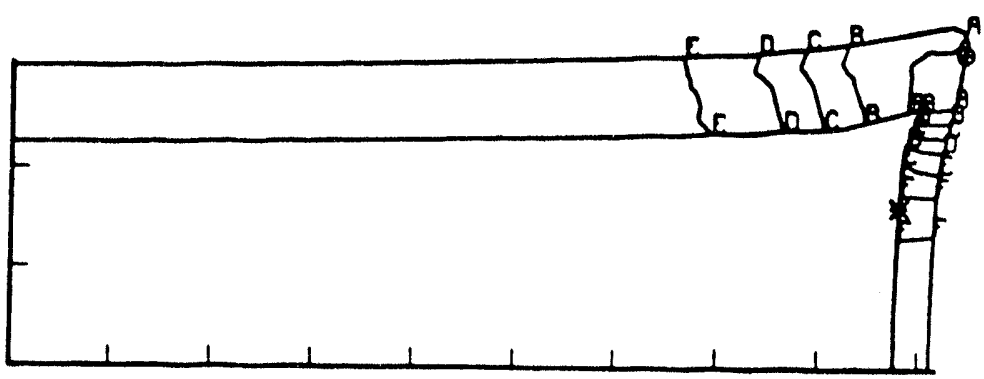

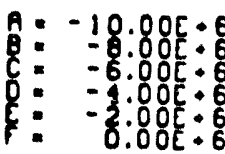

20 YEARS

Q. $=-10.855 \cdot 6$

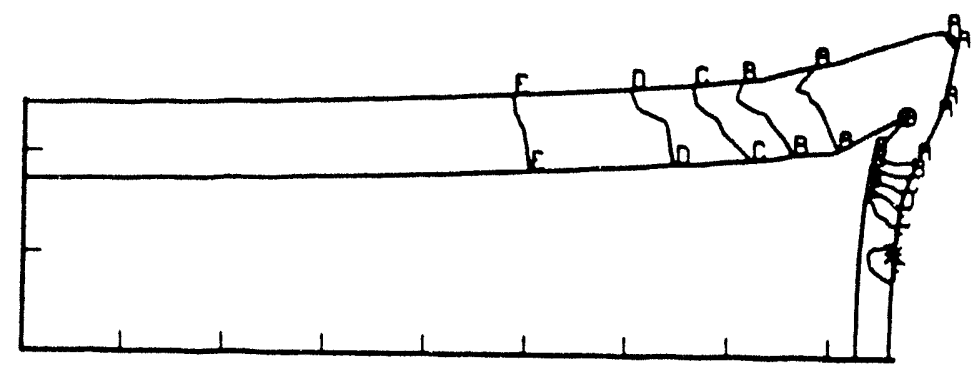

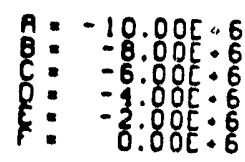

Q. $=-12.446 \cdot 6$

40 YEARS

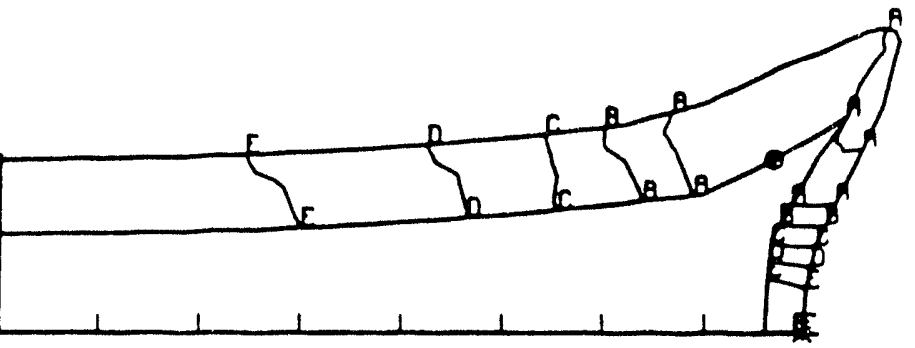

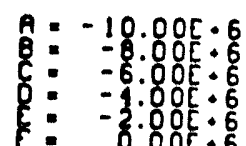

83 YEARS

Q. - - $12.18 E \div 6$

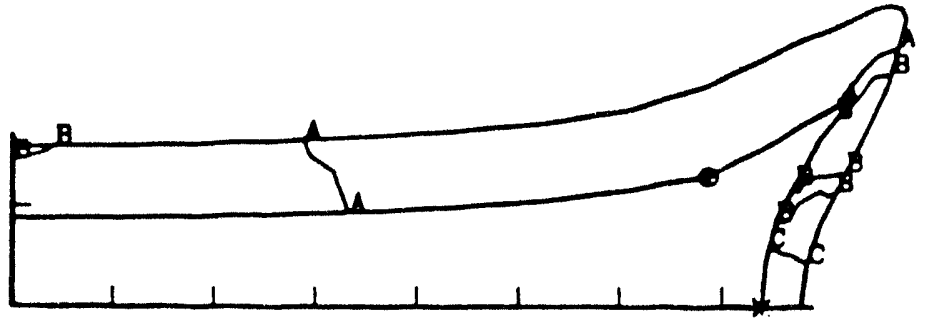

$6=-11.00 E+6$
$-10.00 E+6$
$-0.00 E+6$

$=-13325+6$

200 YEARS

Figure 29. Horizontal Stress in the Backfill Region (Model 4 Results, Stress is in Pascals). 


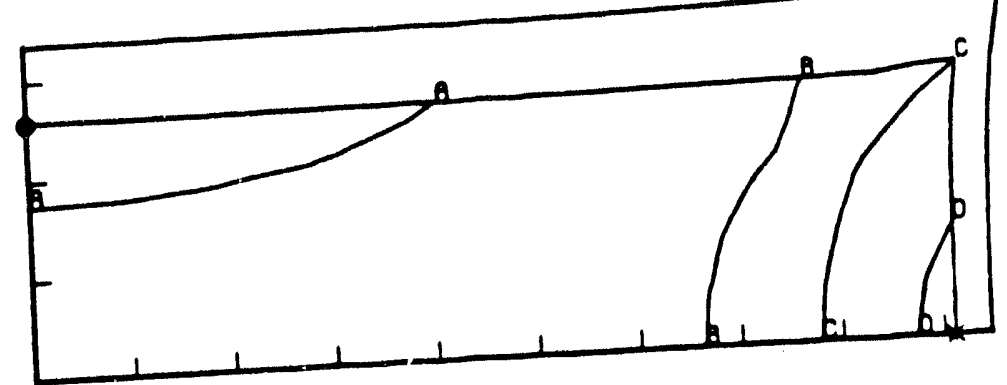

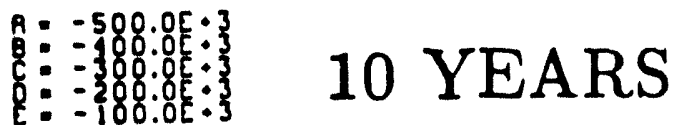

$0:-507: 68: 3$

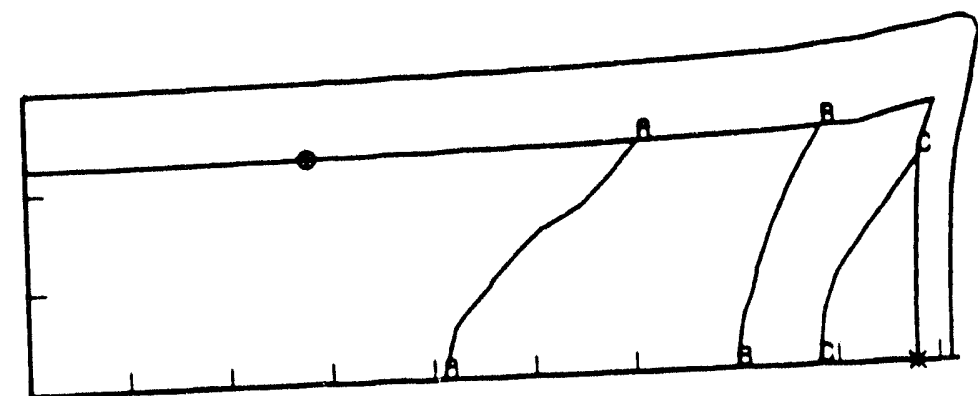

A. $-500.05 \cdot 3$

照:

$Q=-530.2 E: 3$

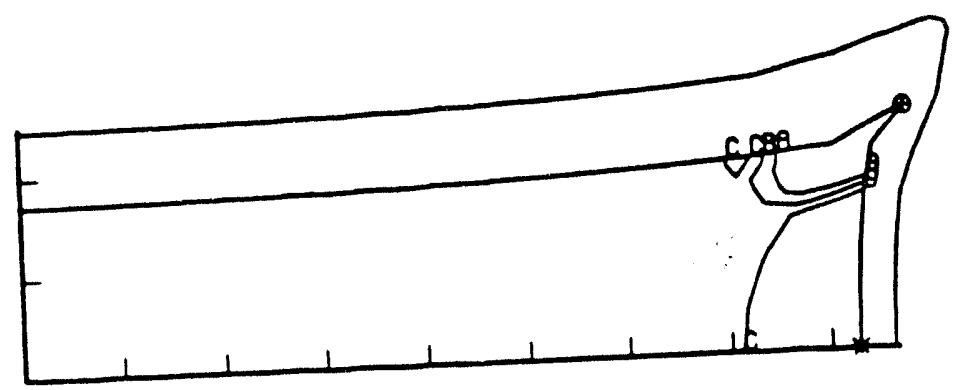

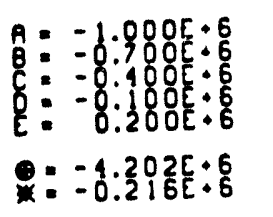

40 YEARS

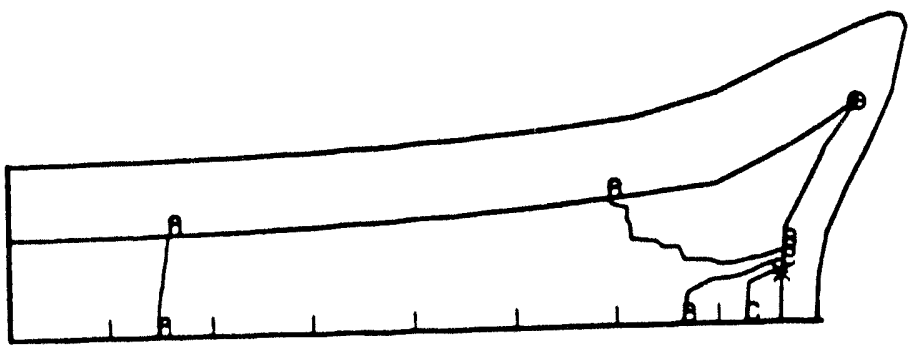

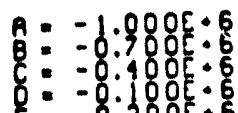

83 YEARS

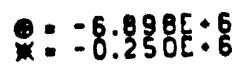

200 YEARS
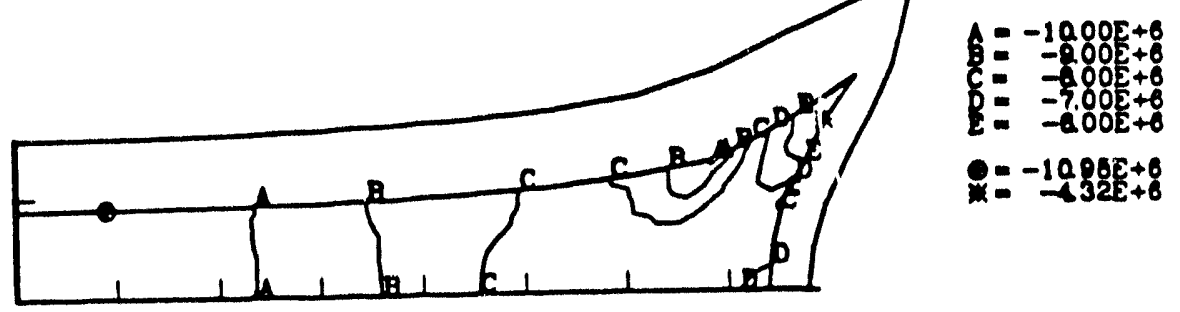

Figure 30. Vertical Stress in the Waste Region (Model 4 Results, Stress is in Pascals). 


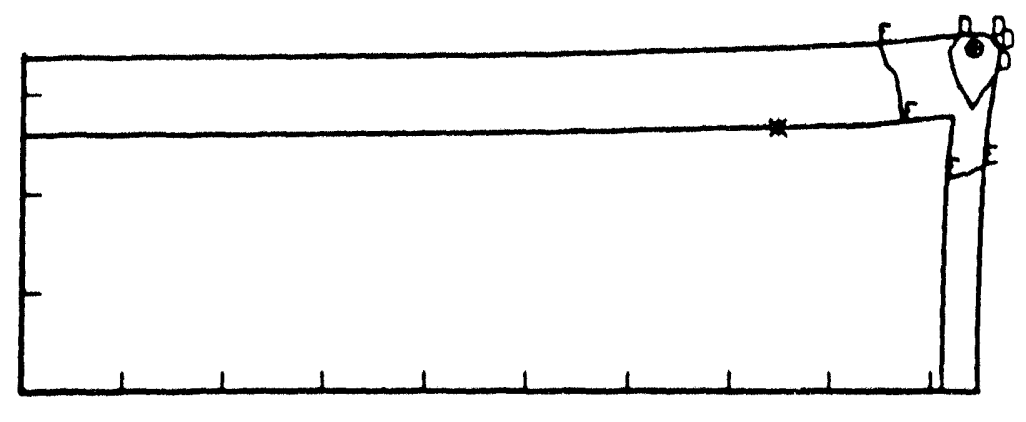

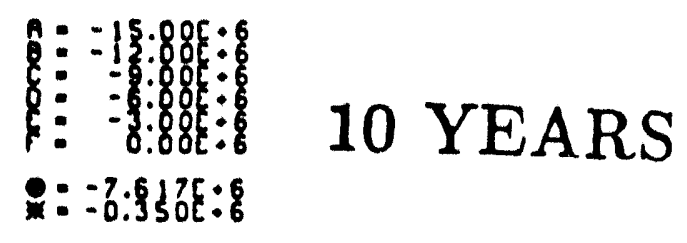

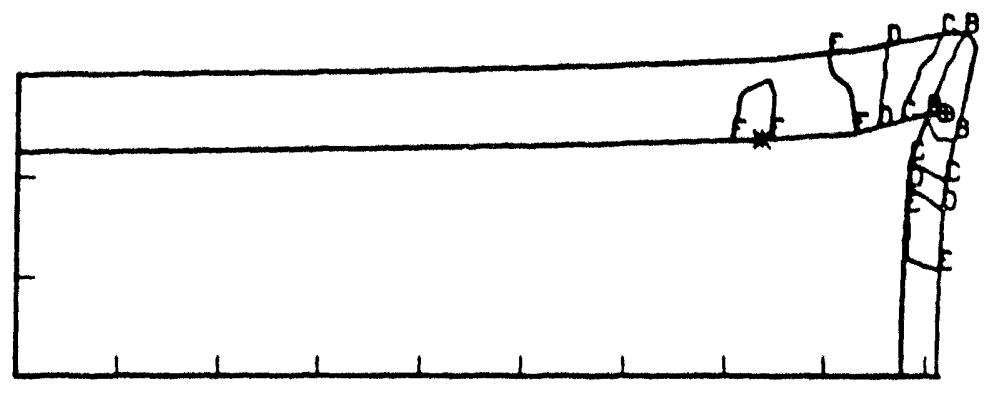

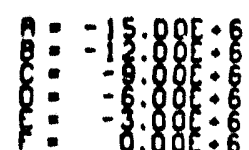

20 YEARS

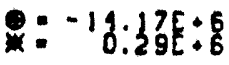

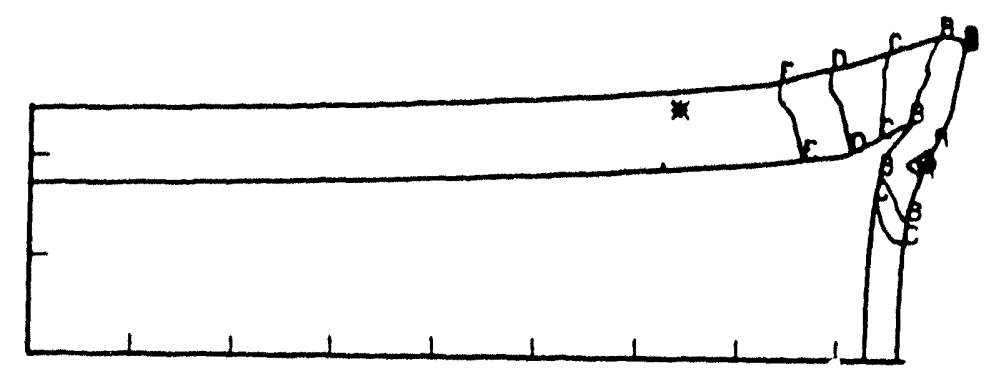

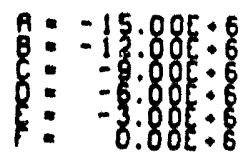

40 YEARS

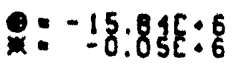
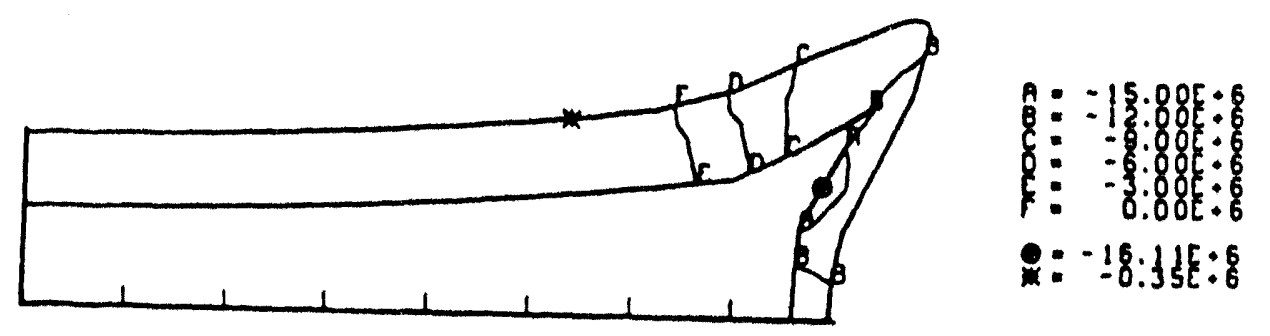

83 YEARS
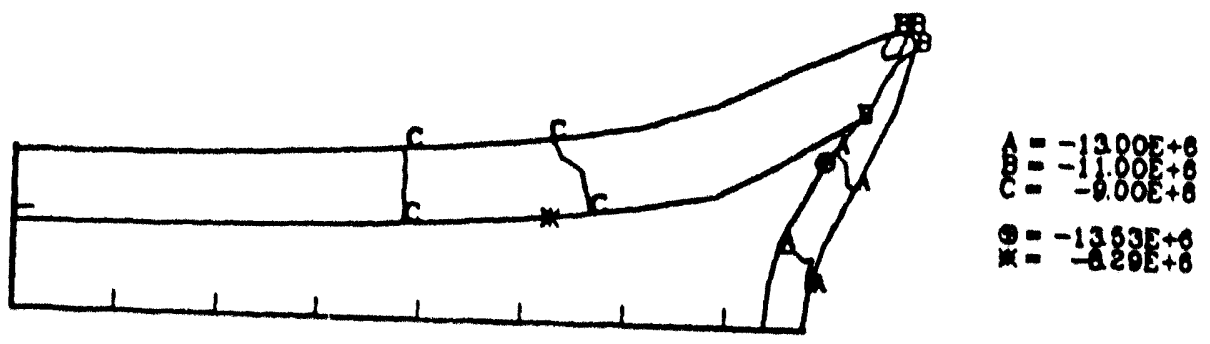

200 YEARS

Figure 31. Vertical Stress in the Backfill Region (Model 4 Results, Stress is in Pascals). 
Sandia National Laboratories

Albuquerque, New Mexico 87185

date: November 14, 1989

w: B. M. Butcher, 6345 and D. E. Munson, 6346

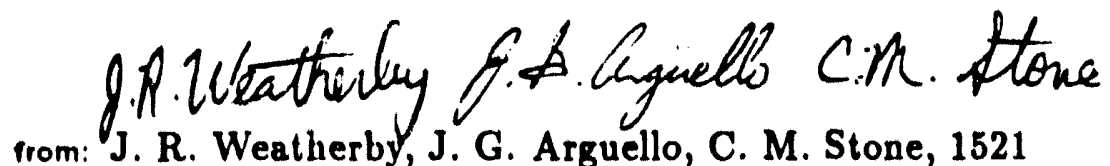

subject: The Effect of Gas Generation on the Performance of CH-TRU Disposal Rooms

\section{Introduction}

Gases will be generated as a by-product of the corrosion and decomposition of CH-TRU waste and waste containers that will be stored at the WIPP site. Microbial degradation of the waste will produce carbon dioxide as well as potentially significant quantities of nitrogen, hydrogen sulfide and methane. Anoxic corrosion of iron and iron-based alloys in the waste will produce hydrogen gas. The WIPP project is presently investigating how the generation of gas will affect the performance of the waste repository.

At the present time, it is believed that the salt formation will be essentially impermeable to the gases that are produced. As a result, gas generation will lead to a slow buildup of pressure inside of the disposal rooms. One concern is that pressures inside the rooms could become large enough to produce fractures that propagate outward from the repository. If such fractures form, they could provide an escape path for the radionuclides stored in the repository.

To inveshigate this problem, typical storage room filled with waste and backfill was analyzed with a two-dimensional finite element model. The results of this analysis show that, while current estimates of the gas generation rate give rise to room pressures that exceed the lithostatic stress level at the repository horizon, the principal stresses at all locations in the salt formation will remain compressive. Such a condition implies that fracture of the salt is unlikely. These results should be viewed with caution, however, since the effects of clay seams and preexisting fractures in the salt have not yet been considered.

Following completion of the two-dimensional storage room analysis, one-dimensional problem of a pressurized borehole was analyzed. The purpose of this study was to investigale how the rate of pressurization affects the stress state in the creeping salt. Four different pressurization rates were considered. The analyses suggest that if the pressurization rate is sufficiently slow, tensile stresses will not develop in the salt, even when the borehole pressure is three times as large as the lithostatic pressure. 


\section{Storage Room Analysis With Gas Generation}

\subsection{The Finite Element Model}

Figure 1 shows the finite element mesh that was used in the plane strain analysis. The waste, crushed-salt backfill, and intact salt were all included in the model. The boundary conditions that were applied in the analysis approximate the conditions that exist around a deeply buried storage room located near the middle of a long string of equally spaced storage rooms. Both the left and right edges of the mesh were fixed against horizontal motion. The lower edge of the mesh was fixed against vertical motion. A pressure of $15 \mathrm{MPa}$ was applied along the upper boundary of the mesh. The stratigraphy was assumed to be all-salt (i.e., clays seams and anhydrite beds were not included in the model). The intact salt was given an initial stress field of $\sigma_{z}=\sigma_{y}=\sigma_{z}=15 \mathrm{MPa}$, where $\sigma_{z}, \sigma_{y}$, and $\sigma_{z}$ are the stresses in the horizontal, vertical, and out-of-plane directions. This corresponds to the stress state that is believed to exist at the repository horizon before excavation.

The elastic-secondary creep constitutive model described in Reference 1 was used for the intact salt. The shear and bulk moduli of the intact salt were given values equal to the values listed in Reference 1 divided by factor of 12.5; while the creep constants were given values equal to those listed in Reference 1 . This set of constants has been found to produce good agreement between the measured closure rates of empty storage rooms and the closure rates calculated in finite element analyses [2]. The material models and properties used for the waste and backfill were identical to those used in Reference 3.

\subsection{Gas Generation Rates and Calculation of Gas Pressure}

Gas generation rates assumed in the structural analysis were taken from information presented in Reference 3 . Each storage room was assumed to contain 6804 drums. During the first 500 years both microbial decomposition and anoxic corrosion contribute to gas production. The gas generation rate during this period was assumed to be constant and equal to $2.66 \mathrm{~g}-\mathrm{moles} / \mathrm{drum} / \mathrm{yr}$. After the first 500 years, the gas production due to corrosion was assumed to stop. During the time period between $500 \mathrm{yr}$ and $700 \mathrm{yr}$, gas generation due to microbial decomposition was assumed to continue at a rale of $0.86 \mathrm{~g}$-moles $/$ drum $/ \mathrm{yr}$. Gas production was assumed to cease completely $700 \mathrm{yr}$ after excavation.

The finite element code SANCHO was modified to compule the room pressure and to apply the resulting nodal forces to nodes on the room boundary. The gas pressure was computed from the ideal gas law based on the current "void" volume in the room (i.e., the volume not occupied by solids) and the total anount of gas in the room. Specifically, 
the gas pressure $p_{g}$ was computed from the relationship:

$$
\begin{aligned}
& \qquad p_{g}=\frac{N R T}{V-V_{S}} \\
& N=\text { mass of gas in } \mathrm{g} \text {-moles } \\
& R=8.23\left(\mathrm{~m}^{3} \mathrm{~Pa}\right) /(\mathrm{g} \text {-mole } \mathrm{K}) \\
& T=300 \mathrm{~K} \\
& V=\text { current volume of the storage room in } \mathrm{m}^{3} \\
& V_{S}=\text { volume of solids in the storage room }=1330 \mathrm{~m}^{3}
\end{aligned}
$$

Table 1 shows how the volume of solids $V_{S}$ was delermined. After each iteration in the analysis, the current room volume $V$ was calculated based on the locations of the nodes on the houndary of the storage room. Next, the gas pressure was calculated according to Equation 1. Finally, the resulting nodal forces were computed and added to the external force vector.

\subsection{Results}

The storage room problem was analyzed for the first $3,000 \mathrm{yr}$ following excavation. It was assumed in the analysis that the room was filled and sealed immediately after excavation. The problem was defined so that the elastic closure of the room at the time of excavation did not produce stresses in the waste or backfill. Time was advanced in increments of 0.025 years.

Figure 2 shows how the room pressure increased with time. A peak room pressure of $20.8 \mathrm{MPa}$ was reached at 700 years. Gas generation ceased after 700 years, and the pressure decreased as the room continued to expand. The pressure in the room will decay to the lithostatic pressure $(15 \mathrm{MPa})$ in the limit as time goes to infinity.

The void volume in the room is plotted as a function of time in Figure 3 and as a function of pressure in Figure 4. These figures show that the void volume decreased to minimum of $973 \mathrm{~m}^{3}$ after 200 years and then began to increase when the room pressure reached 9.2 $\mathrm{MPa}$. In the long-term, the void volume will approach $1675 \mathrm{~m}^{3}$ as the room pressure decays to $15 \mathrm{MPa}$. ('This can be calculated directly from the ideal gas law.)

Figure 5 and Figure 6 show the maximum principal stress and minimum principal stress in the intact salt 700 years after excavation when the room pressure reached its inaxinum value. These two figures clearly show that all stresses remained compressive even though the room pressure was larger than the lithostatic pressure at the depth of the repository. Although this result may seem to contradict the results obtained from hydro-frac experiments described in Reference 5, one must keep in mind that the hydro-frac experiments were conducled at pressurization rates that were many orders of magnitude larger than those encountered in the gas generation problem analyzed here. 


\section{Analysis of a Pressurized Borehole}

The one-dimensional problem of an internally-pressurized borehole in rock salt was analyzed to qualitatively determine how the rate of pressurization would affect the stress field near a storage room. Figure 7 shows the geometry and boundary conditions for the problem. The inner radius of the hole was selected so that the cross-sectional area of the hole was equal to the cross-sectional area of the storage room considered in the previous section. All elements were given an initial stress state of $\sigma_{r}=\sigma_{0}=\sigma_{z}=15 \mathrm{MPa}$. A pressure of $15 \mathrm{MPa}$ was applied at the outer radius. The pressure at the inner radius was held at zero for the first 10 years of the analysis and was chen increased at a constant rate to a final pressure of $45 \mathrm{MPa}$. Four different pressurization rates were considered: $10 \mathrm{MPa} / \mathrm{yr}, 20 \mathrm{MPa} / \mathrm{yr}, 40 \mathrm{MPa} / \mathrm{yr}$, and $80 \mathrm{MPa} / \mathrm{yr}$.

The finite element code SANTOS was used for the borehole calculations. The material model and material constants for the salt were the same as those used in the storage room calculation described in the previous section. Figure 8 shows how the hoop stress at the edge of the borehole changed as a function of time for each of the four pressurization rates. In all four cases, the hoop stress next to the hole initially increased to a peak value, and then began to decrease (become more compressive) with increasing borehole pressure. For the two lowest pressurization rates $(10 \mathrm{MPa} / \mathrm{yr}$ and $20 \mathrm{MPa} / \mathrm{yr}$ ), the hoop stress next to the borehole remained compressive at all times. For the two highest pressurization rates, the hoop stress next to the borehole became tensile when the borehole pressure reached approximately $12 \mathrm{MPa}$. Results similar to those obtained here have also been reported in Reference 6. Figure 9 shows how the hoop stress varied as a function of distance from the centerline of the hole for the $80 \mathrm{MPa} / \mathrm{yr}$ pressurization rate. Curves are plotted for four different values of the internal pressure. Note that the peak hoop stress was initially located next to the edge of the borehole, but moved radially outward from the hole with time.

The results from the one-dimensional calculations indicate that, if the pressurization rate is sufficiently slow, a compressive stress field will exist around a pressurized borehole even when the borehole pressure is three times as large as the lithostatic pressure. For a borehole located at the same depth as the WIPP repository, the minimum pressurization rale required to produce tension in the salt is approximately $20 \mathrm{MPa} / \mathrm{yr}$. This is between two and three orders of magnitude larger than the rate of pressurization in the storage room analysis described in the previous section. 


\section{Conclusion}

The results of the pressurized storage room analysis and the pressurized borehole analyses suggest that gas production is unlikely to initiate fractures in the surrounding salt furmation. This conclusion is based on two observations:

1. In the two-dimensional storage room analysis, the salt was never in tension.

2. In the one-dimensional borehole analyses, the pressurization rates required to produce tension in the salt were between two and three orders of maguitude larger than the pressurization rate anticipated for the storage room.

Analytical models are presently being developed to det-rmine if pressure resulting from gas production can cause separation along clay seams us lead to the extension of preexisting cracks.

\section{References}

1. R. D. Krieg, "Reference Stratigraphy and Rock Properties for the Waste Isolation Pilot Plant (WIPP) Project," SAND83-1908, January 1884.

2. H. S. Morgan, "Estimate of the Time Needed for TRU Storage Rooms to Close," memo to D. E. Munsou, June 1987.

3. J. R. Weatherby, "Finite Element Analysis of TRU Storage Rooms Filled With Waste and Crushed Salt," memo to B. M. Butcher, 6345, May 17, 1989.

4. "Systems Analysis, Long-Term Radionuclide Transport, and Dose Assessments, Waste Isolation Pilot Plant, Southeastern New Mexico, March 1989," A. Lappin and R. Bunter (editors), SAND89-0462, April 1989.

5. W. R. Wawersik and C. M. Stone, "Application of Hydraulic Fracturing to Determine Virgin In Situ Stress State Around Waste Isolation Pilot Plant - In Situ Measurements," SAND85-1776, October 1985.

6. M. Wallner, "Frac-Pressure for Cavities in Rock Salt," in Proceedings of the Second Conference on the Mechanical Behavior of Salt, Hanover, lederal Republic of Germany, September 24-28, 1984, pp. 645-658. 
Table 1. Total Volume and Void Fraction of Each Region in the Storage Room.

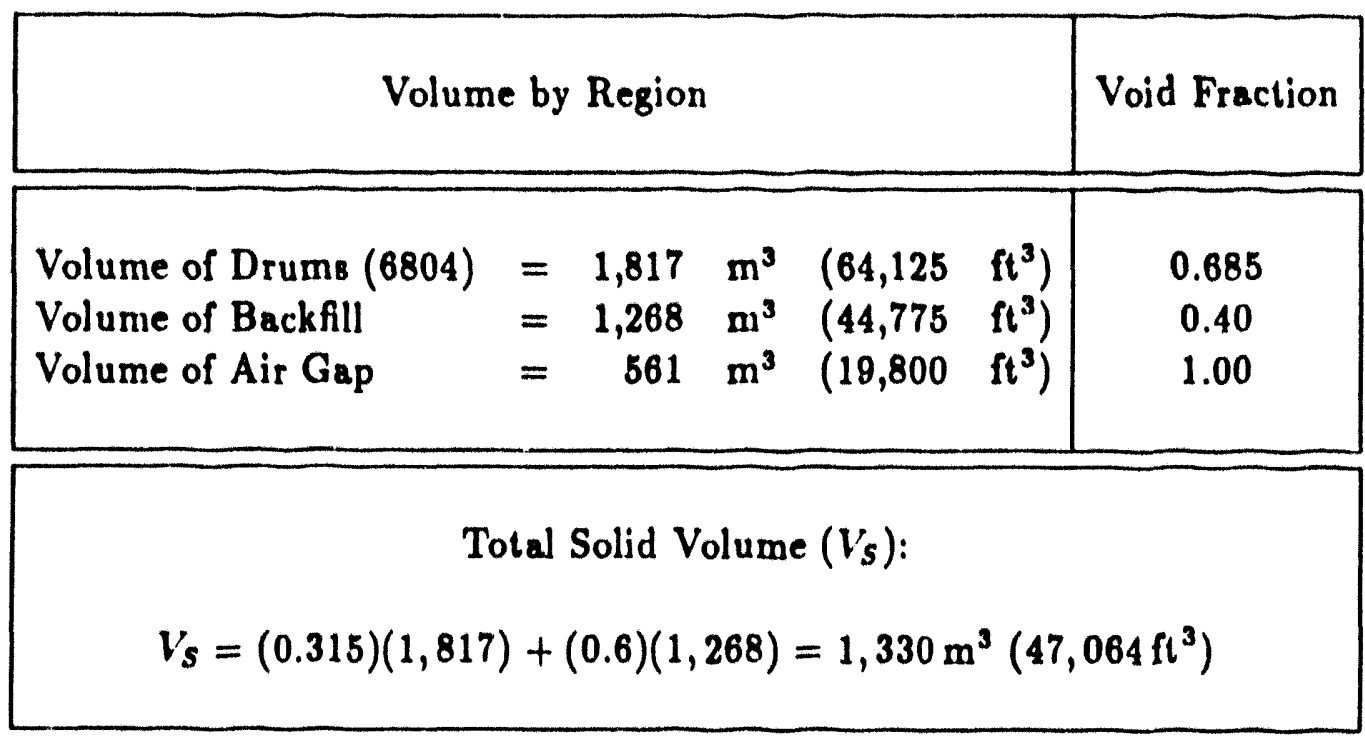


Copy to:

1510 J. W. Nunzialo 1511 D. F. McTigue 1520 L. W. Davison 1521 L. W. Davison (actg.) 1521 J. G. Arguello 1521 H. S. Morgan 1521 G. D. Sjardema 1521 C. M. Stune 1521 J. R. Weatherby 1522 R. C. Reuter 1523 J. H. Biffle 1524 D. R. Martinez 1530 D. B. Hayes 1550 C. W. Peterson 6232 W. R. Waversik 6232 D. H. Zeuch 6340 W. D. Weart 6342 D. R. Anderson 6344 E. Gorham-Bergeron 6345 A. R. Lappin 6345 M. A. Molecke 6345 L. H. Brush 6346 J. R. Tillerson 6346 B. L. Ehgartner 


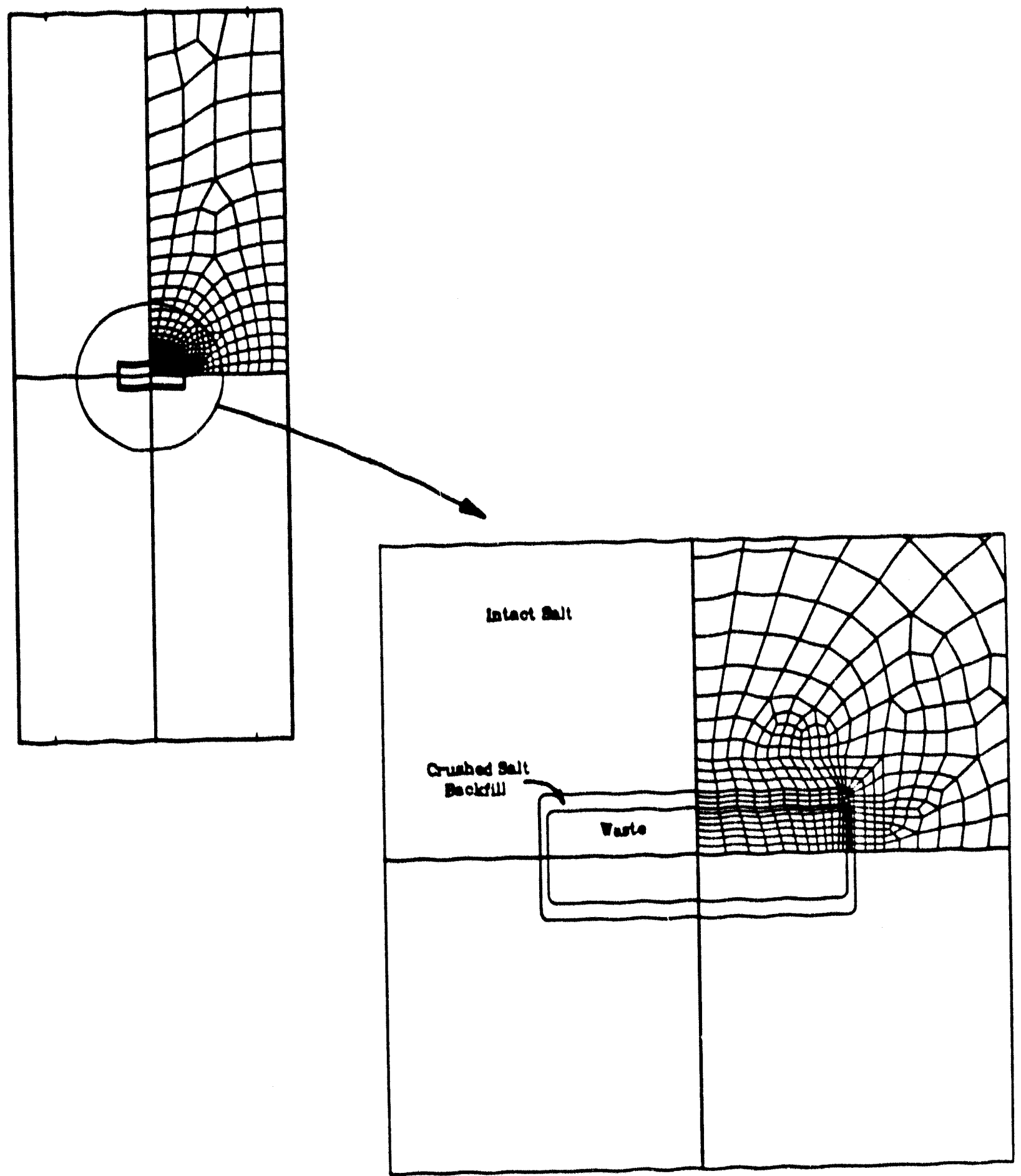

Figure 1. Plane Strain Finite Element Model of a TRU Storage Room. 


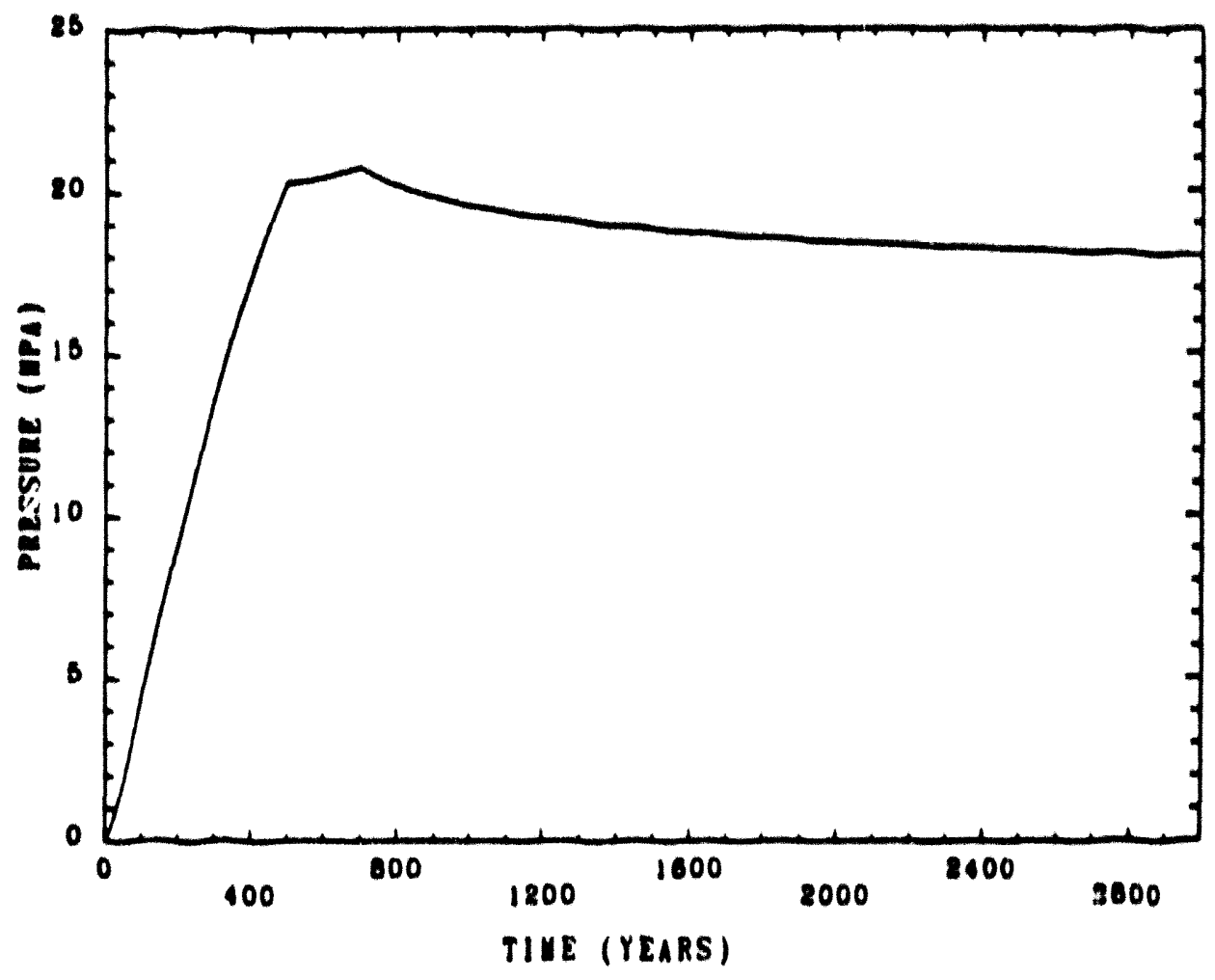

Figure 2. Pressurc in llie Storage Roum as a Function of Time. 


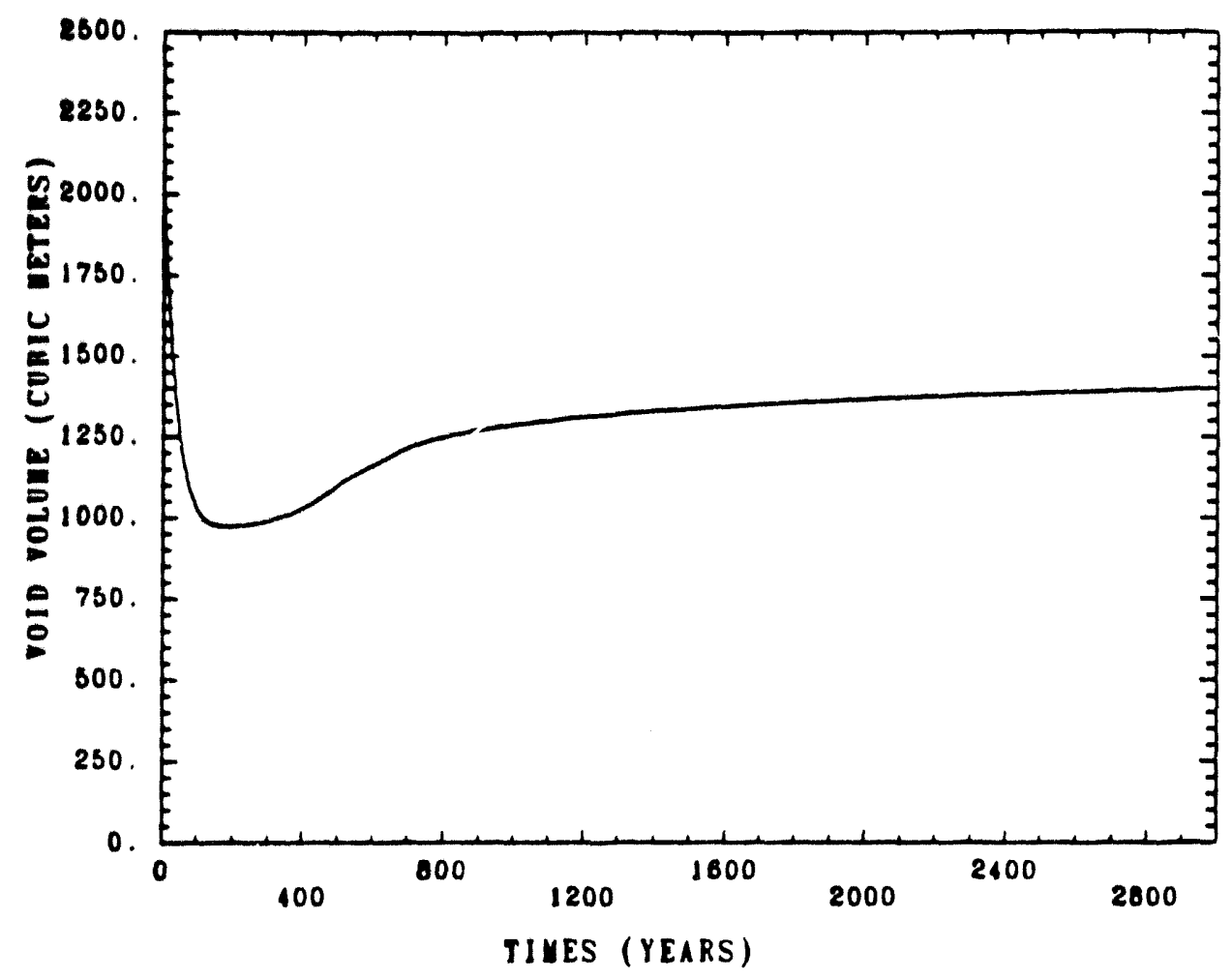

Figure 3. Void Volume in the Storage Room as a Function of Time.

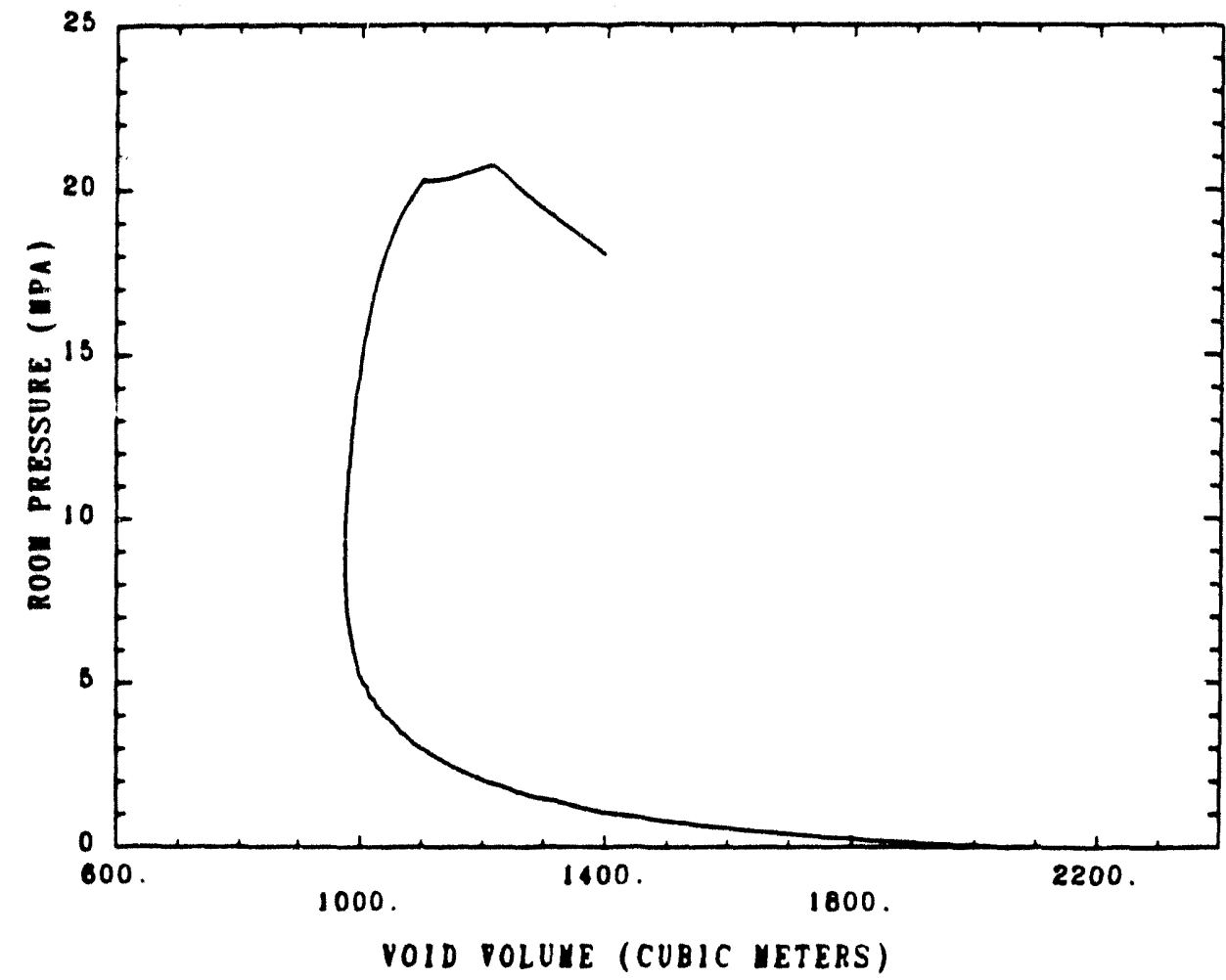

Figure 4. Void Volume in the Storage Room as a Function of Gas Pressure. 
B. M. Butcher, 6345

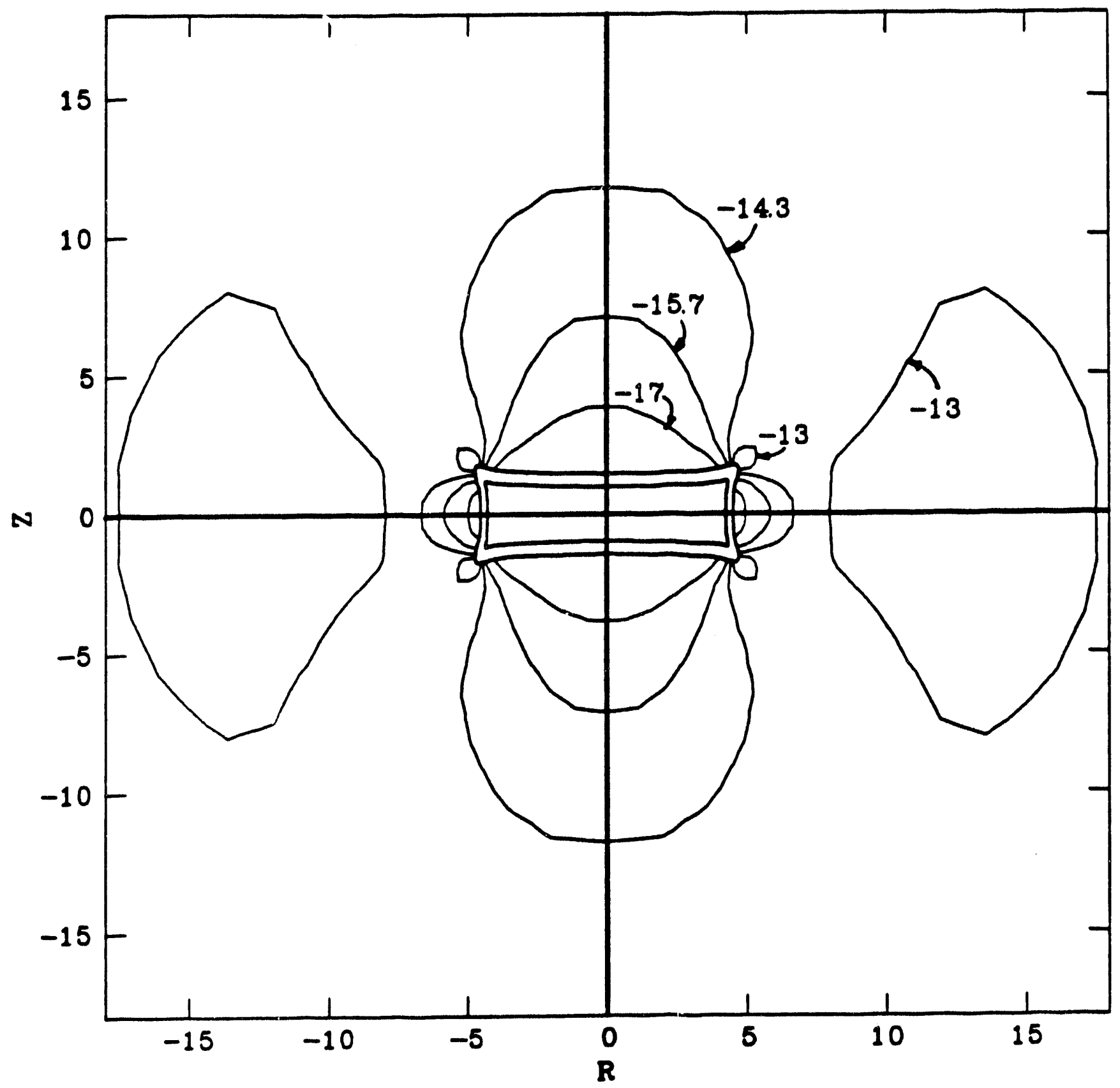

Figure 5. Maximum Principal Stress in the Salt Formation at 700 Years. (Stress in $\mathrm{MPa})$. 


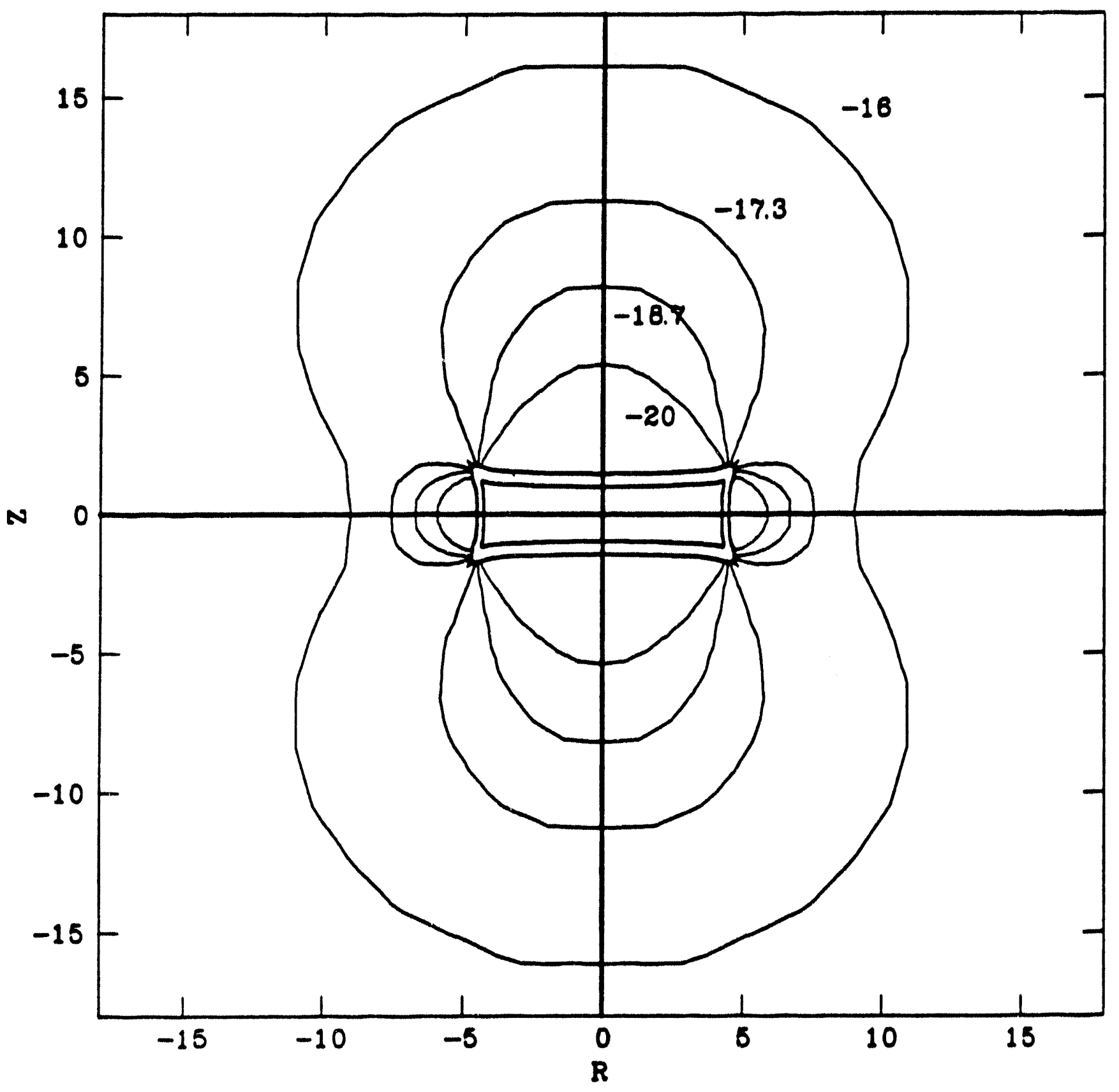

Figure 6. Minimum Principal Stress in the Salt Formation at 700 Years. (Stress in $\mathrm{MPa})$. 


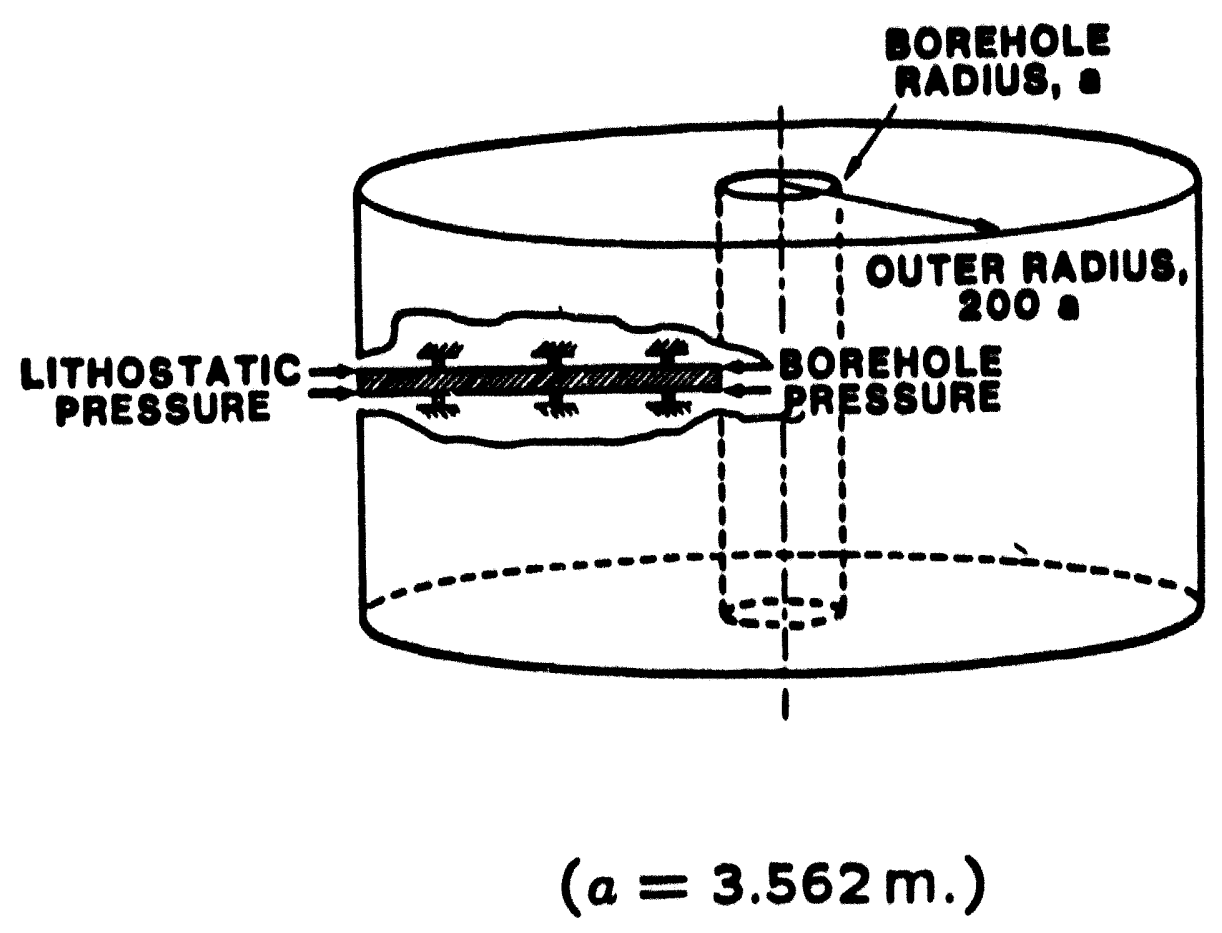

Figure 7. Geonetry and Boundary Conditions for the Pressurized Borehole Problem. 
B. M. Butcher, 6345

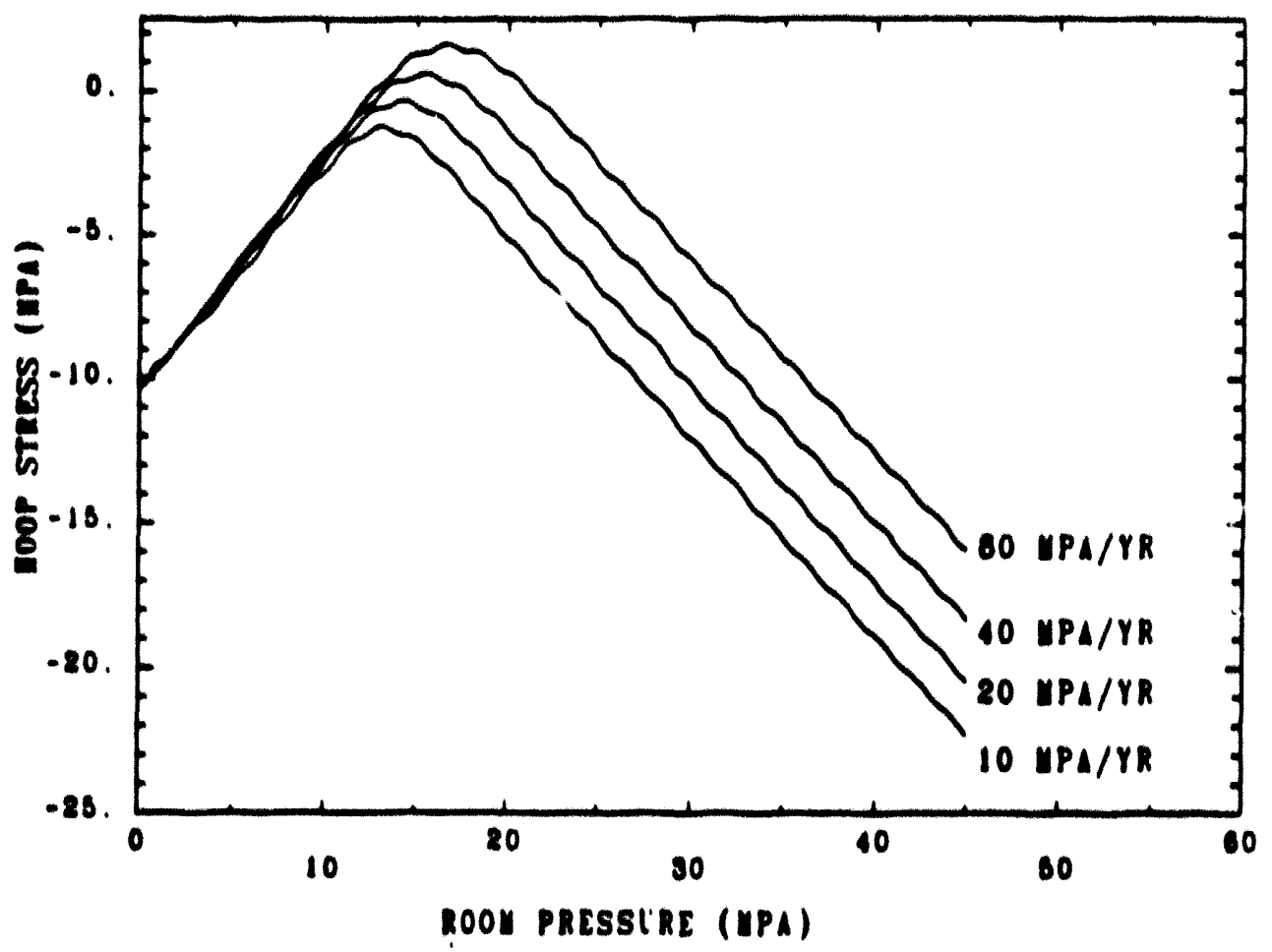

Figure 8. Hoop Streas at the Edge of the Borehole as a Function of Tizne.

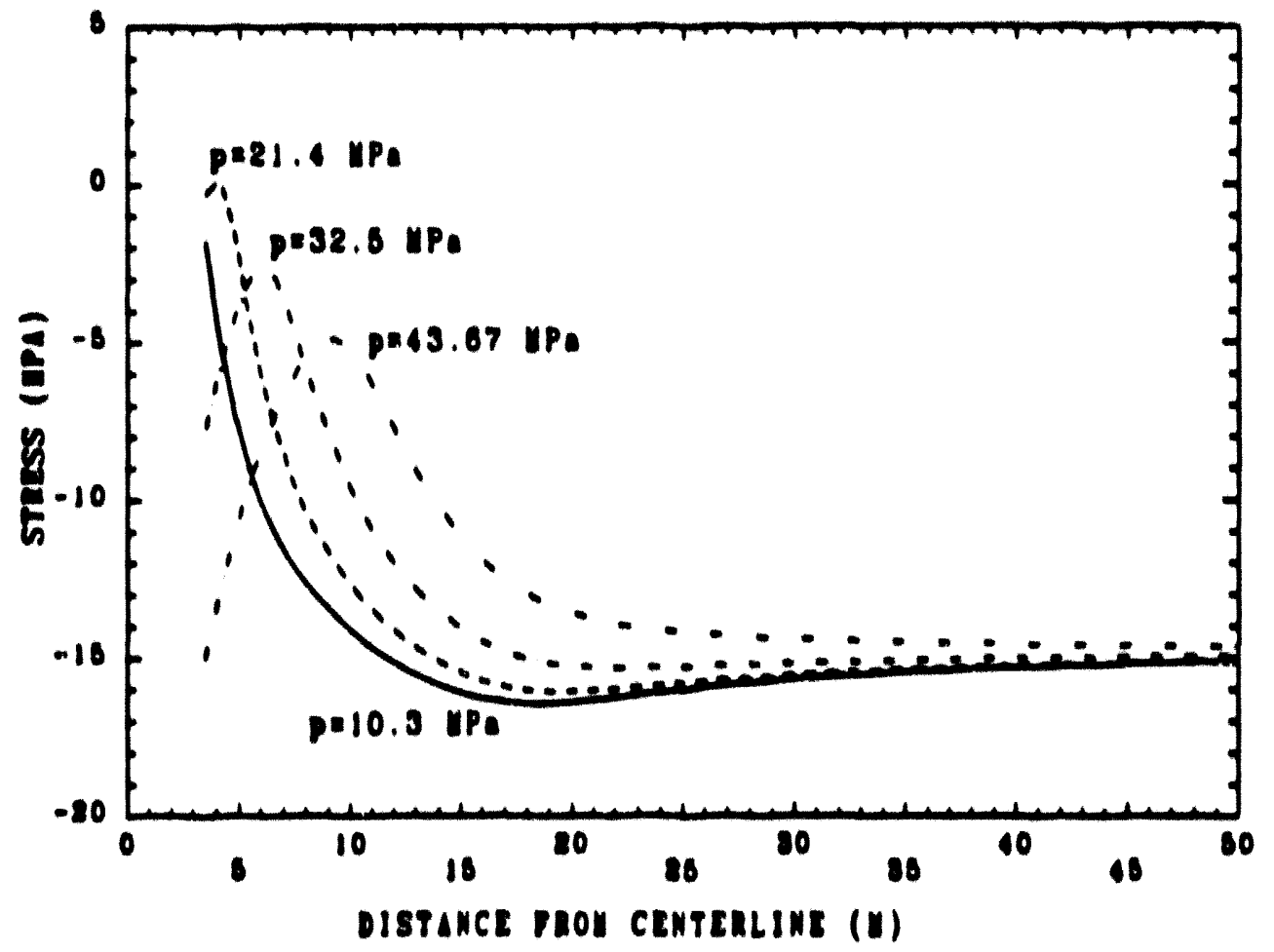

Tigure 0. Hoop Streas a a Puaction of Dialance From the Center of llie Boreliule fur the $80 \mathrm{MPa} / \mathrm{yr}$ Prensurisation Rale. 
dote: April 30, 1890

(0: B. M. Butcher, 6345

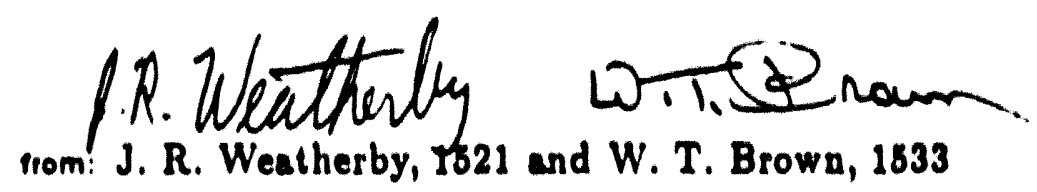

oubject: Closure of a Disposal Room Backfilled With a Salt/Bentonite Mix

\section{Introduction}

II order to minimize the hydraulic conductivity of the WIPP repository, empty opace in the disposal roomo, accessways, and ohafts will be backfilled after the operational phase of the reponitory is complete. A leading candidate for the backfill material is the cruolied ialt mined during excavation of the rooms. Although the permeabillty of the crushed alt will initially be much larger than that of the intact oal in the bout formation, tlie crushed ant reconsolidates as the roome close due to flow of the oalt in the surrounding formation.

Samples of crualied aalt have been leated under hydrostatic loading conditions $|1,2|$. In these tests, the consolidation of the samples was mesured a a function of time at different levels of hydrostatic pressure. These leats bave shown that the addition of a imall amount of water ( $0.5 \%$ by weight) greatly increases the rate of coneolidation of the cruahed oalt. Sjaardema and Krieg $|3|$ have conatructed an empirical conatitutive model for crushed alt based on experimental dala from the consolidation teats on wet crushed ollt. In Reference $|4|$, this model bas beell used to andyze dioposal rooms which are: 1) completely filled with the backfill, and 2) filled with a combination of waste and backfill. The model hes also been used in analyeing olorage rooms pressurised by gases produced by corrosion and decomposition of the waste $(5,6)$. A primary objective in these analyses lias been to eatimate the degree of compaction in the backfill as a function of time.

A mixture of cruahed ant and bentonite has aloo been conoldered for use as a backfill inalerial. Beillonile is a desirable additive because it can absorb brine and radionuclides. Unfortunately, consolidation teats show that adt/beatonite asmples compact at mucli slower rates than samples condioting of crushed aalt alone (7). This rajoes the concersi that the permeability of the oalt-bentonite backfll in the dinpoial rooms could remain large relative to that of the intact oalt for long time pertods extending well beyond 100 years. To investigate this problem, a dioposal rooin backfilled with a mixture of crualied anlt and bentonite has been analyzed with the otructural finite element code SANCHO. 


\section{Constitutive Model For the Backfll}

The constitutive model for the backfill is described in Reference 3. The constitutive equations in the third chapter of Reference 3 are based on the assumption that the change in density between the current configuration and the reference configuration is very small relative to the density in the reference configuration. In the original implementation of the backfill model in SANCHO, the reference configuration was taken to be the configuration existing at the end of the previous time step. Hence, the original formulation of the conotitulive relations is valid if the volume change within a time otep is "sufficiently imall".

The backfill model was reformulated so that the conotitutive relatione remain valid for arbitrarily large changes in density. In order to deacribe the reformulaled constilutive relations, several quantities must firat be defined. The Cauchy otress tensor is denoted by $\sigma_{i j}$, and the velocity vector is denoted by $v_{i}$. The atretching tensor $d_{i j}$ and the apin tenoor $w_{i j}$ are defined as:

$$
\begin{aligned}
& d_{i j}=\frac{1}{2}\left(v_{i, j}+v_{j, i}\right) \\
& w_{i j}=\frac{1}{2}\left(v_{i, j}-v_{j, i}\right)
\end{aligned}
$$

Both the Cauchy atress tensor and the atretching tensor can be decomposed into opherical and devialoric parts through:

$$
\begin{aligned}
& \sigma_{i j}=o_{i j}-\delta_{i j p} \\
& d_{i j}=d_{i j}^{\prime}-\frac{1}{j} \delta_{i j} \dot{e}_{v}
\end{aligned}
$$

where,

$$
\begin{aligned}
& p=-\frac{1}{j} \sigma_{h t} \\
& d_{v}=-d_{h t}
\end{aligned}
$$

The corotational flux of the Cauchy stress tensor is defined to be:

$$
Z_{i j}=\sigma_{i j}-w_{i h} \sigma_{h j}+\sigma_{i h} w_{h j}
$$

The following relationahip between the corolational flux of the Cauchy atress, llie devia. toric otrens $\alpha_{1}$, and the hydroutatic pressure $p$ can be shown to hold:

$$
g_{1 j}=\gamma_{1 j}-\delta_{1, p}
$$

In the backfill conatitutive model, the rate of dilatation $e_{2}$ ls assumed to depend on the hydrostatic pressure through:

$$
d_{v}=\dot{p} / K+\frac{1}{\rho} B_{0}\left(e^{B_{1}} p-1\right) e^{A \rho}
$$


where $B_{0}, B_{1}$, and $A$ are the material constants obtained from the creep consolidation experiments, $K$ is the elastic bulk modulus, and $\rho$ is the density of the backfill. The density $\rho$ is compuled from the relationship:

$$
\rho=\rho_{0} \exp \left(\int_{t_{0}}^{t} \dot{e}_{v} d t\right)
$$

where $\rho_{0}$ is the density at time $t_{0}$. In the formulation presented in $|3|$, the following relationships were asoumed in place of Equations $\theta$ and 10:

$$
\begin{aligned}
\dot{e}_{v} & =\dot{p} / K+\frac{\rho_{0}}{\rho^{2}} B_{0}\left[e^{B_{1} p}-1\right] e^{A p} \\
p & =\rho_{0} /\left[1-\dot{e}_{v}\left(t-t_{0}\right)\right]
\end{aligned}
$$

The deviatoric part of the atretching tensor is asoumed to depend on the deviatoric stress and corolational fux of the deviatoric atress through the relationahip (3):

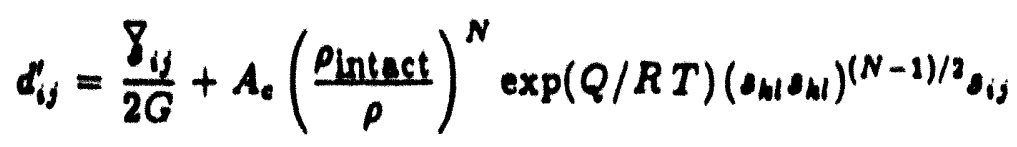

where $A_{e}, N$, and $Q$ are material conolants obtained from uniaxial creep experiments on the intact adt, $R$ is the ideal gas conotant, $T$ is the absolute temperature, $G$ is the elastic ohear modulus, and Pintact is the denoity of the intact salt.

The eleatic moduli are asoumed to depend on the density of the backfill throught rela. tionahips of the form:

$$
\begin{aligned}
& K=K_{0} \exp \left(K_{1} \rho\right) \\
& G=G_{0} \exp \left(G_{1} \rho\right)
\end{aligned}
$$

The empirical equation for the eleatic bulk modulue $K$ is based on data from a hydrootatic test on dey crualied oalt (1). In this experiment, the bulk modulus was deternilied by performing unload.reload cycles on the same specimen at different degrees of consolidation. Conotents $K_{0}$ and $K_{1}$ were determined in $|3|$ by using the least aquares method to fit the modulus data to the function in Equation 14. In the fit, the function was constrained so that the bulk modulus of the cruahed salt was equal to the bulk modulus of the intact aslt when the cruslied ant was fully compacted. No experiments have been conducted to delermine how the thear modulus varies with density, so the ahear modulus was asoumed to vary according to the same exponential form as the bulk modulus. The constant $G_{n}$ was selected oo that the oliear modulus for the crushed alt was equal to that of the intact oalt when the crualied ialt was fully consolidated, and the consiant $G_{1}$ was anoumed to be the anme as $K_{1}$. 


\section{Creep Consolidation Properties of the Salt-Bentonite Back- fll}

Three samples of a salt-bentonite mixture were tested under constant bydrostatic loading by RE/SPEC Inc. [7]. All three oamples were composed of $70 \%$ by dry weight crushed salt and $30 \%$ by dry weight bentonite. Two of the samples contained $5 \%$ moisture by weight, and the third sample contained $10 \%$ moisture by weight. The loading history for each specimen consisted of aeries of constant-pressure stages. The lowest pressure applied in any otage was $3 \mathrm{MPa}$, and the highest pressure applied in any stage was $14 \mathrm{MPa}$. Specimen volume was monitored continuously during each test. The specimens were oubjected to load for approximately 300 days.

From Equations $\theta$ and 10, the consolidation rate for constant pressure can be expressed as:

$$
\dot{\rho}=B_{0}\left(e^{B_{1 p}}-1\right) e^{A p}
$$

where $p$ is the hydrostatic pressure (compression gives a positive value of $p$ ), $\rho$ is the dry density of the mixture, and $A, B_{0}$, and $B_{1}$ are material constants. Integration of Equation 16 for the case of constant bydrostatic pressure gives:

$$
\rho=-\frac{1}{A} \log \left[e^{-A p 0}-A B_{0}\left(e^{B_{1} p}-1\right) t\right]
$$

where $t$ is the elapsed time, and $\rho_{0}$ is the density at $t=0$. In [7], the constants $A$, $B_{0}$, and $B_{1}$ were determined by fitting measurements of density as a function of time to Equation 17 using the method of least-squares. Table 1 lints the values of the constants that were obtained from creep tests on each of the three teat specimens. The parameters referred to as "Selected Values" were determined by Callahan [11] to be representative of the parameters obtained from the three tests of the salt/bentonite mixture. Values of the parameters for pure "wet" crushed salt are also provided.

\section{Modification of the Backfill Constitutive Subroutine}

In SANCHO the volumetric part of the backfill conatitutive model, given by Equation $\theta$, is integrated using a semi-analytical approach. In this method, oeveral approximations are made to oblain an approximale analytical expression for the final pressure at the end of the time step based on $\dot{e}_{v}$ and the length of the time step $\Delta t$. For the integration to be accurate, the value of $\Delta t$ muat be small. When $\Delta t$ is too large, the time slep is divided into omaller segments within the constitutive routine so that:

$$
\Delta t=\sum_{i=1}^{n} \Delta t_{i}
$$


Table 1. Creep Consolidation Constants for Salt/Bentonite Backfill [7] and All-Salt Backfill [3].

\begin{tabular}{l}
\begin{tabular}{|l|c|c|c|c|c|}
\hline $\begin{array}{l}\text { Test } \\
\text { 1.D. }\end{array}$ & $\begin{array}{c}\text { Moisture } \\
\text { Content } \\
(\%)\end{array}$ & $\begin{array}{c}\text { Applied } \\
\text { Pressure } \\
(\mathrm{MPa})\end{array}$ & $\begin{array}{c}\text { P } \\
\left(\mathrm{m}^{3} / \mathrm{kg}\right)\end{array}$ & $\begin{array}{c}B_{0} \\
\left(\mathrm{~kg} /\left(\mathrm{m}^{-3} \cdot \mathrm{s}\right)\right)\end{array}$ & $\begin{array}{c}B_{1} \\
\left(\mathrm{MPa}^{-1}\right)\end{array}$ \\
\cline { 4 - 6 } CS1 & 5.3 & 3.45 to 14 & $-33.9 \times 10^{-3}$ & $0.972 \times 10^{21}$ & 0.693 \\
CS3 & 9.97 & 3.45 to 7 & $-30.3 \times 10^{-3}$ & $0.970 \times 10^{21}$ & 0.084 \\
CS4 & 5.24 & 3.45 to 14 & $-34.5 \times 10^{-3}$ & $1.021 \times 10^{21}$ & 0.509 \\
\hline Selected Values for & $-34.5 \times 10^{-3}$ & $1.0 \times 10^{21}$ & 0.6 \\
Salt/Bentonite Mixture $[11]$ & & & 0.820 \\
\hline
\end{tabular} \\
\hline
\end{tabular}

where,

$$
\begin{aligned}
\Delta t_{i} & =\min \left(\delta / \alpha, \delta / \beta, \delta / \dot{e}_{v}\right) \\
\alpha & =\left(K_{1} \rho_{0}+A \rho_{0}-1\right) \dot{e}_{v} \\
\beta & =K_{1} \rho_{0} \dot{e}_{v} \\
\rho_{0} & =\text { density at the beginning of the subdivision. }
\end{aligned}
$$

The variable $\delta$ appearing in Equation 19 determines the accuracy of the integration. In the original implementation of the constitutive model, a value of 0.1 was used for $\delta$. For the crushed salt backfill, the use of $\delta=0.1$ produced acceptable results; however, smaller values of $\delta$ were required to obtain the desired accuracy for the salt/bentonite mixture. For this reason, $\delta$ was made an input variable which can be specified by the user through the input data file to SANCHO.

\section{Analysis of Hydrostatic Creep Experiments}

Equation 17 gives the density of the backfill as a function of time for a constant hydrostatic pressure. Because an analytical solution for this loading condition exists, it was used as a check on the implementation of the backfill constitutive model in SANCHO using the "Selected Values" from Table 1. Figures 1 and 2 show how the variable $\delta$ affects the accuracy of the integration for a constant hydrostatic pressure of $0.05 \mathrm{MPa}$ for the 
pure crushed salt and the salt/bentonite mixture, respectively. The solid line in these figures represents the analytical solution from Equation 17. Based on this comparison, it was decided that the accuracy provided by $\delta=0.0025$ was oufficient for inlegrating the constitutive equations with the salt/bentonite properties. For pure crushed salt, all values of $\delta$ are within $0.1 \%$ of the analytical solution for density, although $\delta=0.0005$ provides results which are about an order of magnitude more accurate than the others. For the salt/bentonite mixture, $\delta=0.1$ fails to capture the initial part of the density history with the desired accuracy, but is only $0.6 \%$ less than the exact solution at the end of 0.10 years. The solutions for $\delta=0.005$ and $\delta=0.0005$ track the exact solution accurately and are off by less than $0.2 \%$ over the entire simulation period.

In each hydrostatic creep test described in Reference 7 the specimens wee subjected to 3.4 different levels of hydrostatic pressure. Within each stage, the pressure was held constant. To evaluate the performance of the constitutive model under a time-varying load, SANCHO was used to track the volumetric response of the speciment to the loading history applied in test CS1. In the experiment, the apecimen was first conditioned by rapidly loading it to hydrostatic stress of $3.45 \mathrm{MPa}$. During conditioning, the density increased from an initial value of $1448 \mathrm{~kg} / \mathrm{m}^{3}$ to value of $1685 \mathrm{~kg} / \mathrm{m}^{3}$. The conditioning phase was followed by three additional loading stages where the hydrostatic pressure was held at $3.45 \mathrm{MPa}, 7 \mathrm{MPa}$, and $14 \mathrm{MPa}$. Between each loading stage, the specimen was again conditioned by rapidly loading the sample to the hydrostatic pressure level in the next stage. The change in density during conditioning was negligible between Stages 1 and 2 and between Stages 2 and 3.

For comparison purposes, the density from the SANCHO analysis was converted to volumetric "engineering" strain through the following relationship:

$$
\left(V_{0}-V\right) / V_{0}=1-\frac{\rho_{i}}{\rho}
$$

where $V_{0}$ is the initial volume of the sample, $V$ is the current volume, $\rho$ is the density, and $\rho_{i}$ is the density of the mixture before the initial conditioning $\left(1449 \mathrm{~kg} / \mathrm{m}^{3}\right)$. In the SANCHO calculation, the starting density of the specimen was set equal to the density after the initial conditioning slage $\left(1685 \mathrm{~kg} / \mathrm{m}^{3}\right)$. The creep consolidation constants listed in Table 1 for test CS1 were used in the analysis. Figure 3 shows the volumetric strain history measured in experiment CS1 compared to the volumetric strain calculated with the SANCHO model. The agreement is good between the volume strains calculated with SANCHO and the volume strains measured in the test. The constitutive model, however, cannot accurately track the rapid consolidation which occurs during the initial conditioning of the specimen where the density increases from $1449 \mathrm{~kg} / \mathrm{m}^{3}$ to $1685 \mathrm{~kg} / \mathrm{m}^{3}$ over a period of a few seconds or minutes. 


\section{Finite Element Analyses of Consolidation in Backfilled Dis- posal Rooms}

SANCHO was used to analyze the closure of a disposal room that is backfilled immediately following excavation. The two main objectives of this investigation were to:

1. Compare the void reduction in a room backfilled with crushed salt to the void reduction in room backfilled with $70 / 30$ mixture of crushed salt and bentonite.

2. Determine the sensitivity of room closure to the elastic moduli of backfill.

Five different cases were considered, and, in each case, a different set of constants was used in the backfill constitutive model. The deviatoric creep behavior of the backfill was assumed to be the same in all cases; only the constants defining the elastic moduli and the creep consolidation behavior were varied. The cases considered were:

- Creep consolidation properties for crushed salt with elastic moduli of crushed salt

- Creep consolidation properties for crushed salt with elastic moduli of crushed salt scaled by a factor of $1 / 12.5$

- Creep consolidation properties for a 70/30 salt/bentonite mixture with elastic mod. uli of crushed salt

- Creep consolidation properties for a $70 / 30 \mathrm{salt} /$ bentonite mixture with elastic moduli of crushed salt scaled by a factor of $1 / 12.5$

- Creep consolidation properties for a 70/30 salt/bentonite mixture with elastic mod. uli estimated with a mixtures approximation.

The scaling factor of $1 / 12.5$ is an issue because the elastic moduli of the intact salt have been scaled by this factor. This scaling has produced analytical results which are in good agreement with measurements of room closure at the WIPP site [0]. It is natural to ask whether it is appropriate to scale the elastic constants of the backfill by the same factor.

\subsection{Finite Element Model and Boundary Conditions}

The finite element model shown in Figure 4 was used in the five analyses of the disposal room. The nodes along the left and right edges of the mesh were fixed against horizontal motion to approximate the conditions that exist around a room that is located near the center of a very long panel of rooms. A pressure of $14.8 \mathrm{MPa}$ was applied along the upper boundary of the mesh to represent the overburden at the depth of the repository. The initial stress state in the undeformed configuration was assumed to be constant 
in the salt formation. The normal stresses in the horizontal, vertical, and out-of-plane directions were set equal to $-14.8 \mathrm{MPa}$ in all elements in the intact salt. The initial otresses for elements representing the backfill were set equal to zero. No gravitational forces were applied in the problem as it has been assumed that closure of the disposal rooms is not greatly affected by the gravitational forces acting on the malerial in the immediate vicinity of the room [8]. Following [9], the stratigraphy was also ignored, and the formation was considered to be all-salt. The temperature in the room and surrounding salt was assumed to be constant at $300 \mathrm{~K}$.

\subsection{Constitutive Model Parameters}

The WIPP reference elastic-secondary creep model described in Reference 10 was used to represent the mechanical behavior of the intact salt. Following [0], the reference elastic constants were divided by 12.5 to produce closures similar in magnitude to those measured at the WIPP. Table 2 lists the material constants that were uned with the elastic-secondary creep model.

Two sets of constants were used to define the creep behavior of the backfill: one set for analyses involving the all-salt backfill, and one set for analyses involving the salt/bentonite mixture. Table 3 lists the values of the creep constants for both backfill compositions. The consolidation constants $A, B_{0}$, and $B_{1}$ are those recommended by RE/SPEC, Inc. in [11). The initial density of the all-oalt backfill was taken to be $1400 \mathrm{~kg} / \mathrm{m}^{3}$, and the fully. compacted density was taken to be $2140 \mathrm{~kg} / \mathrm{m}^{3}$. The iujtial density of the salt/bentonite backfill was taken to be $1480 \mathrm{~kg} / \mathrm{m}^{3}$, and the fully-compacted density was taken to be $2260 \mathrm{~kg} / \mathrm{m}^{3}$.

In analyses of the backfilled disposal room, three sets of constants were used to describe the variation in the elastic moduli. These constants are listed in Table 4. The set of constants labeled "unscaled" is the set derived in Reference 3. The "scaled" constants are equal to the unscaled constants divided by 12.5 . Since bentonite has mechanical properties which are different from those of salt, the moduli for the salt/bentonite mixture will be different from that of the crushed salt alone. To estimate the elastic moduli of the mixture, a crude mixture approximation was used to estimate the elastic moduli of the salt/bentonite backfill. The mixture approximation is based on an incomplete set of experimental data and an unverified assumption about how the bentonite is dispersed within the mixture. In Table 4, the set of constants labeled "mixture" corresponds to the constants derived from the mixture approximation. Details of the procedure that was used to obtain the constants for the mixture can be found in the appendix. While the mixture approximation is useful in a parametric study, it should not be used in place of experimental measurements to determine how the modulus varies with density. 
Table 2. Material Constants Used With the Elastic/Secondary Creep Model for the Intact Salt.

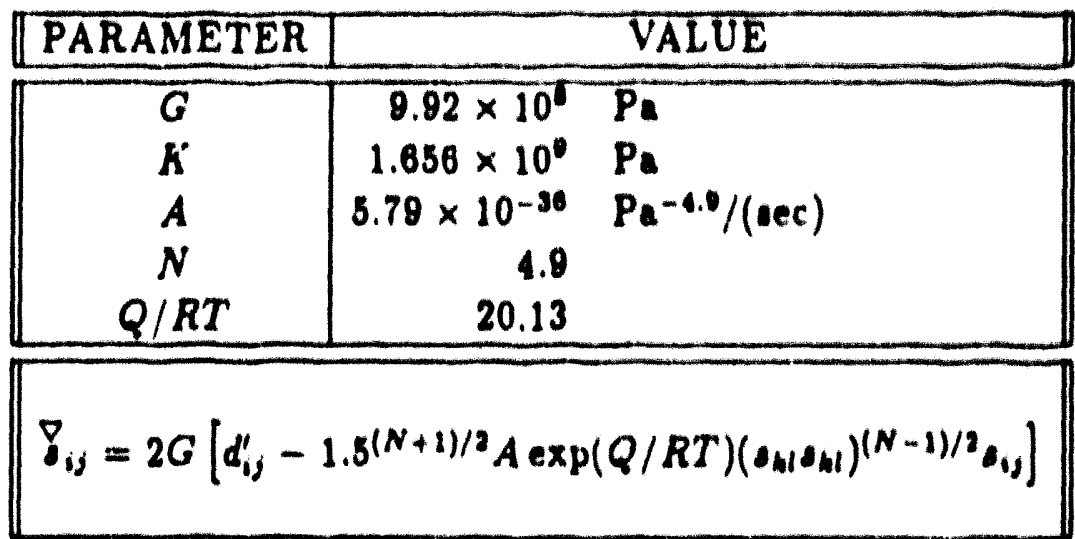

Table 3. Creep Constants Used With the Backfill Model for the All.Salt and Salt/Bentonite Backfills.

\begin{tabular}{|c|c|c|}
\hline \multirow[t]{2}{*}{ Parameter } & \multicolumn{2}{|c|}{ Value } \\
\hline & All.Salt & Sal/Bentonite \\
\hline $\begin{array}{c}A_{c}\left(\mathrm{~Pa}_{\mathrm{a}}^{-40} /\left(\mathrm{sec}_{\mathrm{c}}\right)\right) \\
N \\
Q / R T \\
A\left(\mathrm{~m}^{3} / \mathrm{kg}\right) \\
\left.B_{0}\left(\mathrm{~kg}^{2} / \mathrm{m}^{3} \mathrm{sec}\right)\right) \\
B_{1}\left(\mathrm{~Pa}^{-1}\right) \\
\end{array}$ & $\begin{array}{c}3.79 \times 10^{-30} \\
4.9 \\
20.13 \\
-17.3 \times 10^{-3} \\
1.3 \times 10^{6} \\
0.82 \times 10^{-6} \\
\end{array}$ & $\begin{array}{c}5.70 \times 10^{-30} \\
4.9 \\
20.13 \\
-34.8 \times 10^{-3} \\
1 \times 10^{21} \\
0.6 \times 10^{-1} \\
\end{array}$ \\
\hline
\end{tabular}

Table 4. Three Sets of Constants Used to Describe the Elastic Moduli of the Backfill.

\begin{tabular}{|c|c|c|c|c|}
\hline $\begin{array}{c}\text { Set } \\
\text { Label }\end{array}$ & $\begin{array}{c}K_{0} \\
(\mathrm{~Pa})\end{array}$ & $\begin{array}{c}K_{1} \\
\left(\mathrm{~m}^{3} / \mathrm{kg}\right)\end{array}$ & $\begin{array}{c}G_{0} \\
(\mathrm{~Pa})\end{array}$ & $\begin{array}{c}G_{1} \\
\left(\mathrm{~m}^{3} / \mathrm{kg}\right)\end{array}$ \\
\hline \hline $\begin{array}{c}\text { Unscaled } \\
\text { Scaled }\end{array}$ & $\begin{array}{c}17600 . \\
1408 .\end{array}$ & 0.00653 & 10600. & 0.00633 \\
Mixture & 351900. & 0.00433 & 848. & 0.00653 \\
\hline
\end{tabular}




\subsection{Finite Element Results}

The horizontal and vertical closures of the disposal room are shown in Figures 5 and 6 for each of the five cases. In these figures, the vertical closure is the change in height of the room at the center of the roof and floor expressed as a percentage of the original room height. The horizontal closure is the change in the width at the midheight of the room expressed as a percentage of the original room width.

The void volume in the disposal room expressed as a percentage of the total room volume is shown in Figure 7 as a function of time for each of the five cases analyzed. The void fraction is computed from the relationship:

$$
v \equiv \frac{V_{\text {void }}}{V}=\frac{\left(1-\rho_{i} / \rho_{\text {intact }}\right) V_{0}+\Delta V}{V}
$$

where $\rho_{i}$ is the initial density of the backfill when it is placed in the room, $V_{0}$ is the initial volume of the room, $V$ is the current volume of the room, and $\Delta V$ is the change in room volume from the undeformed state.

Changing the elastic moduli of the backfill had virtually no effect on the closure of the storage room. The creep consolidation properties, however, had a significant effect on room closure. With the all-salt backfill, zero void volume was reached in the room 70 years after excavation; whereas, for the salt/bentonite backfill, $7.5 \%$ of the room volume was void 200 years after excavation.

Contour plots showing the distribution of void volume in the room are sliown in Figure 8 for the salt/bentonite backfill. As found in previous calculations with all-salt backfill [4], the salt/bentonite backfill consolidates most rapidly in the corners and shows the slowest rate of consolidation in the center of the disposal room.

The mean stress $\left(\sigma_{\text {mean }}=\frac{1}{3} \sigma_{i i}\right)$ at the center of the disposal room is shown in Figure 9 for the all-salt backfill and for the salt/bentonite backfill. The all-salt backfill reaches complete consolidation at 70 years when the mean stress is $-2 \mathrm{MPa}$ at the center of the room. At 70 years, the mean stress in the salt/bentonite mixture is approximately $-4 \mathrm{MPa}$ at the center of the room. The higher stresses in the salt/bentonite backfill slow closure of the room and are responsible for the slower rate of compaction of the backfill.

\section{Conclusion}

The algorithm used to integrate the backfill constitutive model has been fine-tuned so that it can be used to accurately integrate the constitutive equations with material constants derived for a $70 / 30$ mixture of crushed salt and bentonite. The integration algorithm was tested on a single-element problem where a constant hydrostatic load was applied. The constilutive model was shown to match experimental results from a creep consolidation experiment where the hydrostatic pressure was increased in a sequence of three steps. 
Analyses of a backfilled disposal room show that the salt/bentonite backfill is much slower to consolidate than the all-salt backfill. After 200 years of closure, the salt/bentonite backfill still contained $7.5 \%$ void by volume. In contrast, all void was eliminated from the all-salt backfill 70 years after excavation. The analyses also indicate that predictions of room closure and void volume are insensitive to the elastic modulus of the backfill.

\section{References}

1. D. J. Holcomb and D. W. Hannum, "Consolidation of Crushed Salt Backfill Under Conditions Appropriate to the WIPP Facility," SAND82-0630, November 1982.

2. D. J. Holcomb and M. Shields, "Hydrostatic Creep Consolidation of Crushed Salt With Added Water," SAND87-1990, October 1987.

3. G. D. Sjaardema and R. D. Krieg, "A Constitutive Model for the Consolidation of WIPP Crushed Salt and Its Use in Analyses of Backfilled Shaft and Drift Configurations," SAND87-1977, October 1987.

4. J. R. Weatherby, "Finite Element Analysis of TRU Storage Rooms Filled With Waste and Crushed Salt," memo to B. M. Butcher, 6345, May 17, 1989.

5. J. R. Weatherby, J. G. Arguello, C. M. Stone, "The Effect of Gas Generation on the Performance of CH-TRU Disposal Rooms," memo to B. M. Butcher, 6345, and D. E. Munson, 6346, November 14, 1989.

6. J. R. Weatherby and J. G. Arguello, "The Effect of Gas Generation on the Extension of Preexisting Fractures Around WIPP Disposal Rooms," memo to B. M. Butcher, 6345, and D. E. Munson, 6346, March 28, 1990.

7. T. W. Pfeifle, "Consolidation, Permeability, and Strength of Crushed Salt/Bentonite Mixtures with Application to the WIPP," Topical Report RSI-0357, RE/SPEC Inc., September 1989.

8. J. G. Arguello, "Use of Midheight Symmetry Plane to Compute Disposal Room Closure," memo to Distribution, January 17, 1990.

9. H. S. Morgan, "Estimate of the Time Needed for TRU Storage Rooms to Close," memo to D. E. Munson, 6346, June 1987.

10. R. D. Krieg, "Reference Stratigraphy and Rock Properties for the Waste Isolation Pilot Plant (WIPP) Project," SAND83-1908, January 1984.

11. G. D. Callahan, "Presentation and Discussion of Properties for Crushed Salt/ Bentonite Backfill Material," RE/SPEC, Inc. external memorandum to B. M. Butclier, 6345, January 18, 1990. 
12. T. W. Pfeifle, "Mechanical Properties and Consolidation of Potential DHLW Back. fill Materials: Crushed Salt and 70/30 Bentonite/Sand," SAND85.7208, July 1987.

13. W. H. Gerstle and A. K. Jones, "Mechanical Properties of Crust ad Salt/Bentonite Blocks," SAND86-0707, August 1986. 
B. M. Butcher, 6345

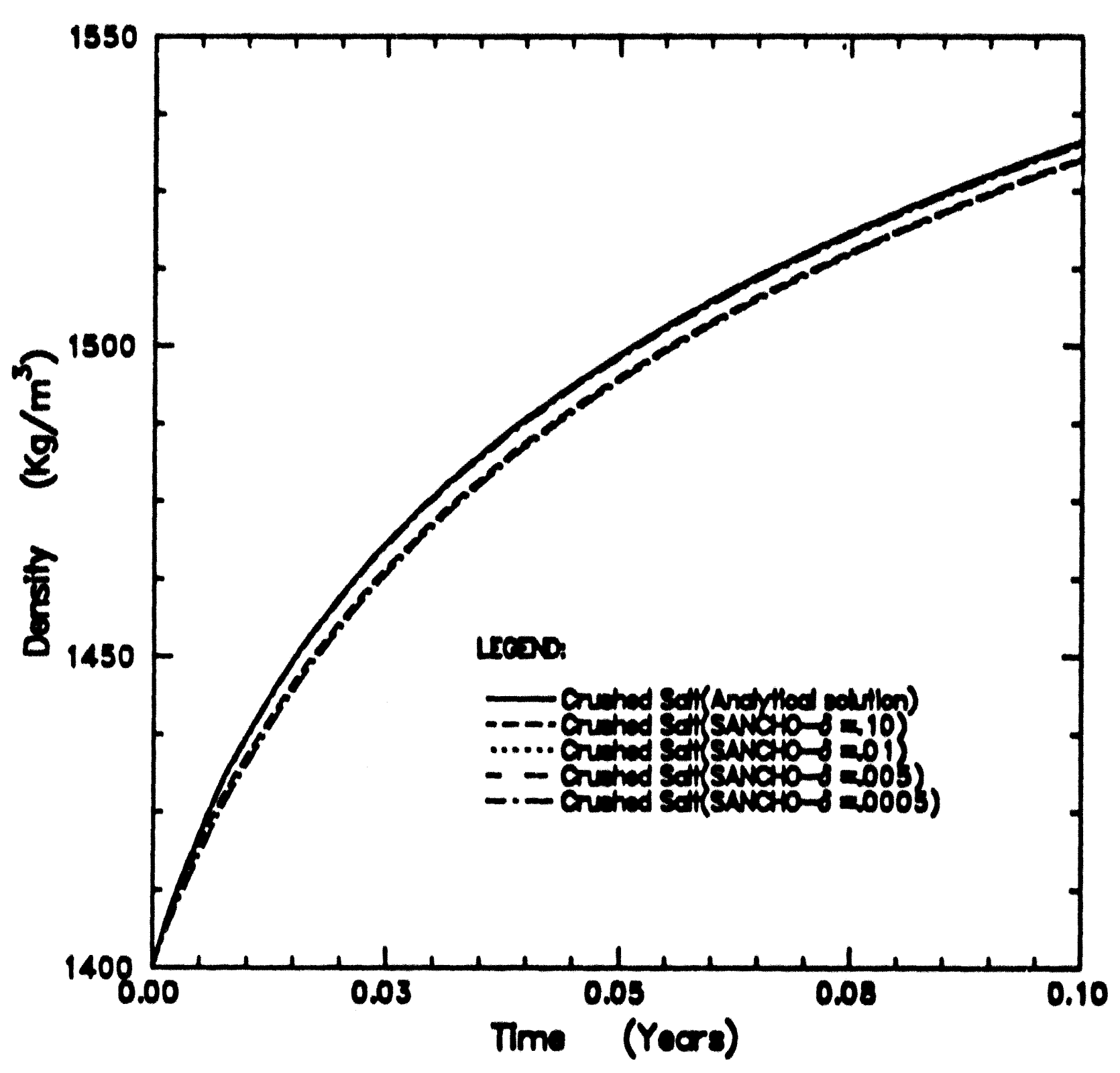

Figure 1. Density as a Function of 'Time for the Backfill Under a Consant Hydrostatic Pressure of 0.05 MPa (/ll.Salt Properties). 
B. M. Dulcher, 6545

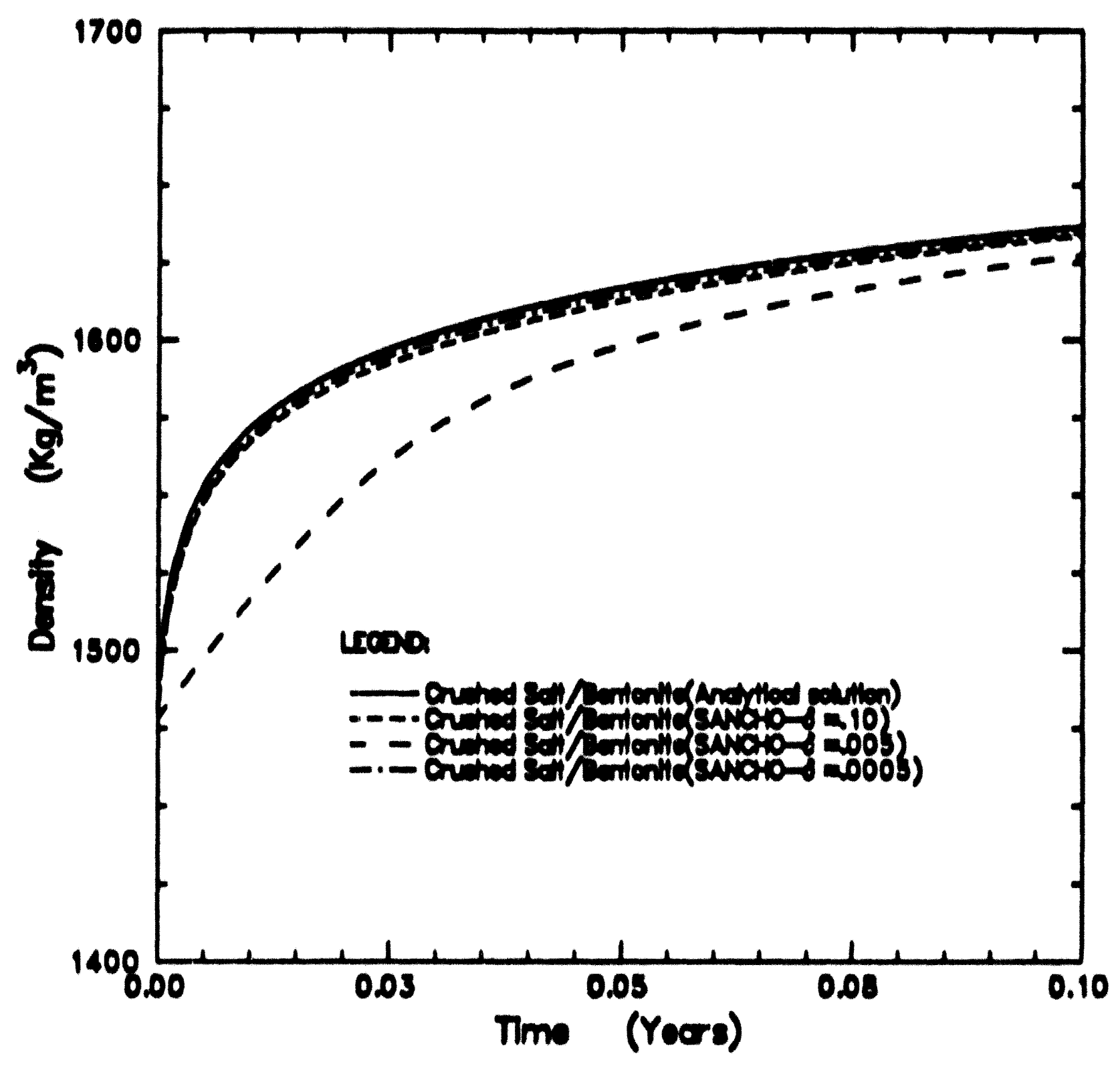

Figure 2. Denoity an a Function of Time for llie Backfill Under a Comalnuil Hydrustatic Pressure of U.03 MIP. (Sall/Benlonite Properties) 
D. M. Butcher, 034s

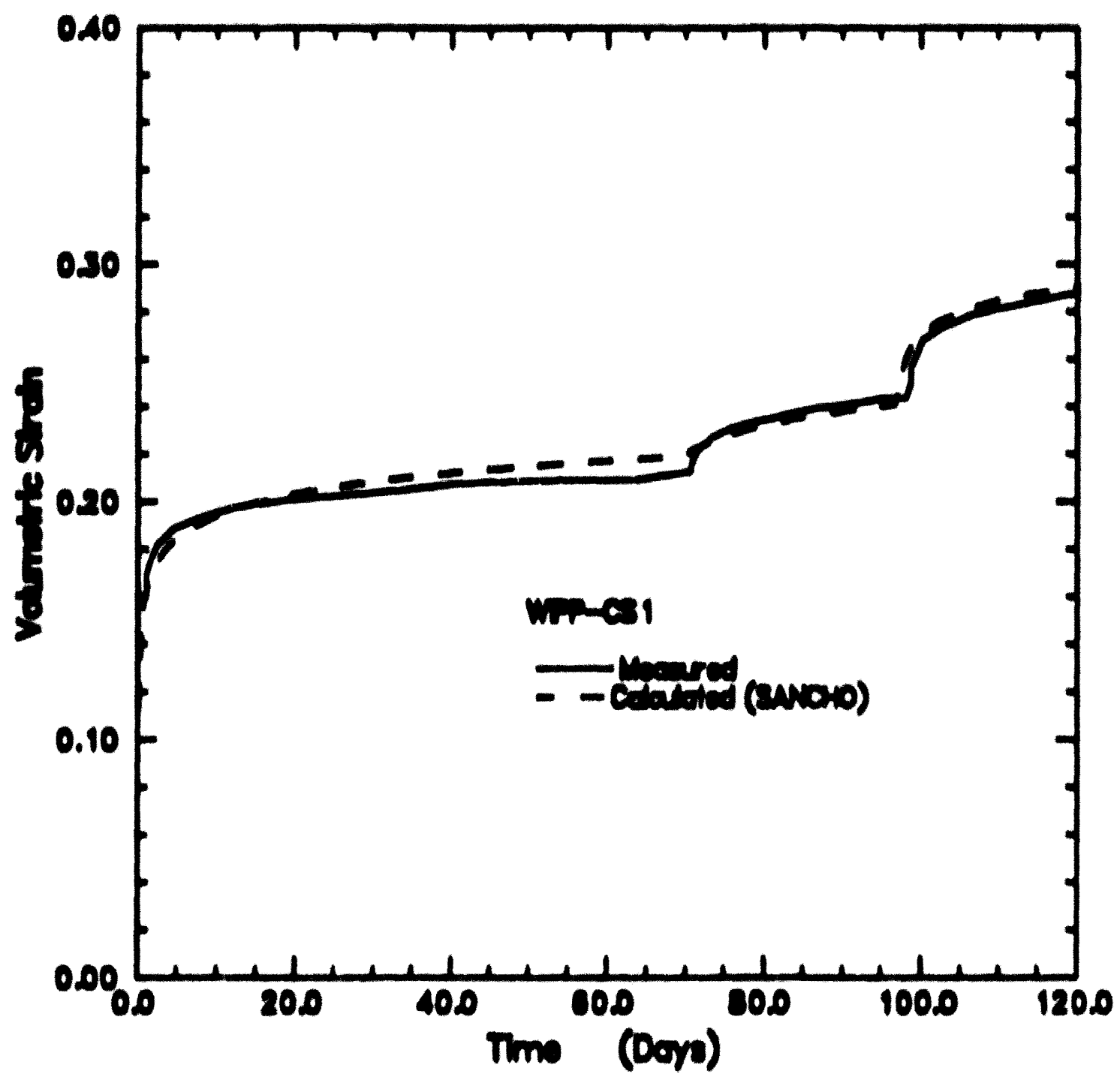

Figure 8. Volumetric Strall as a Function of Time For Toul CSI of Reference? 
B. Ml Bulcher, 6345
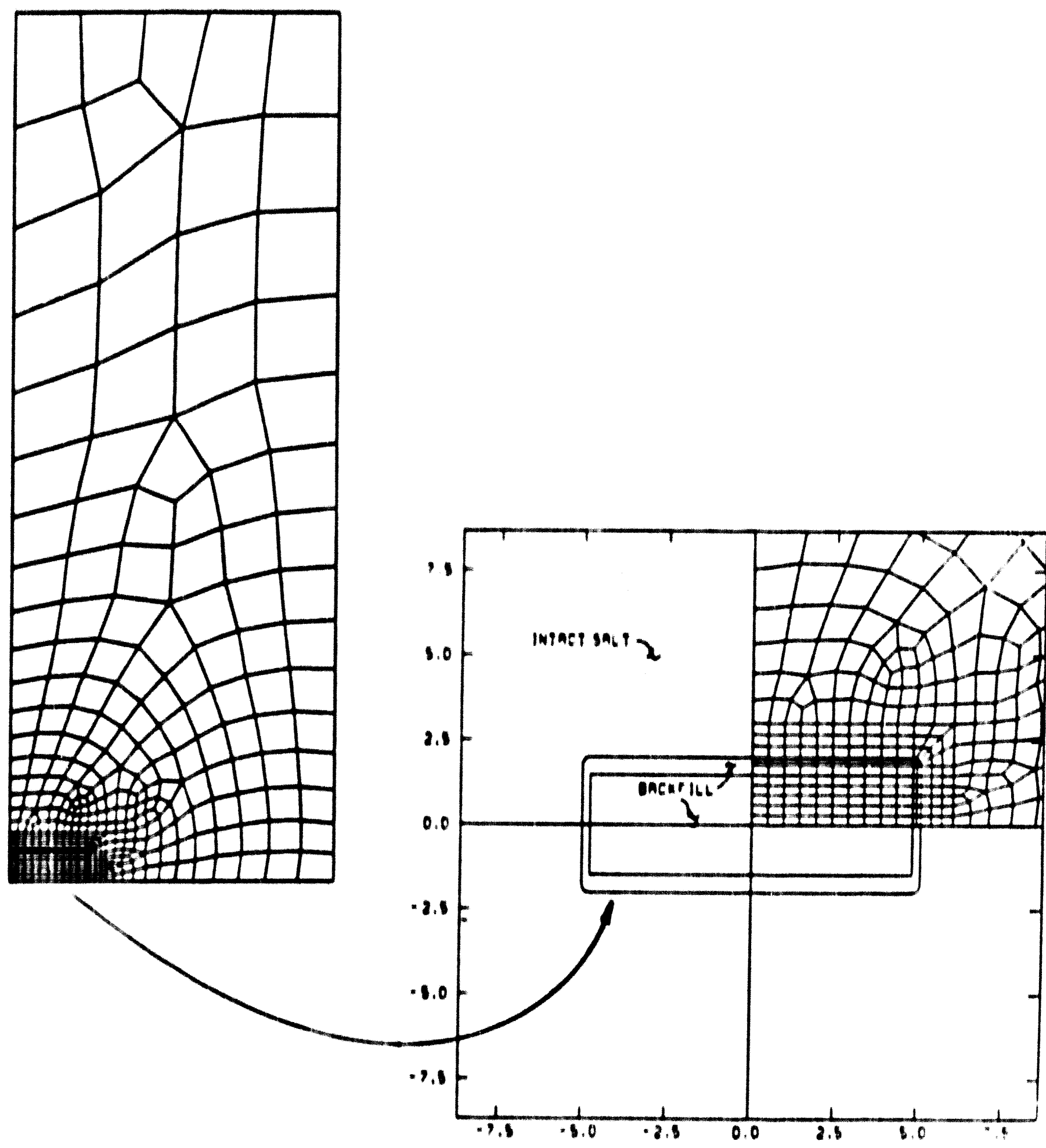

Figure 4. Finite Element Mesh Used in llie Allalyolis of Backnlled Rooms. 
B. Al. Butcher, 6345

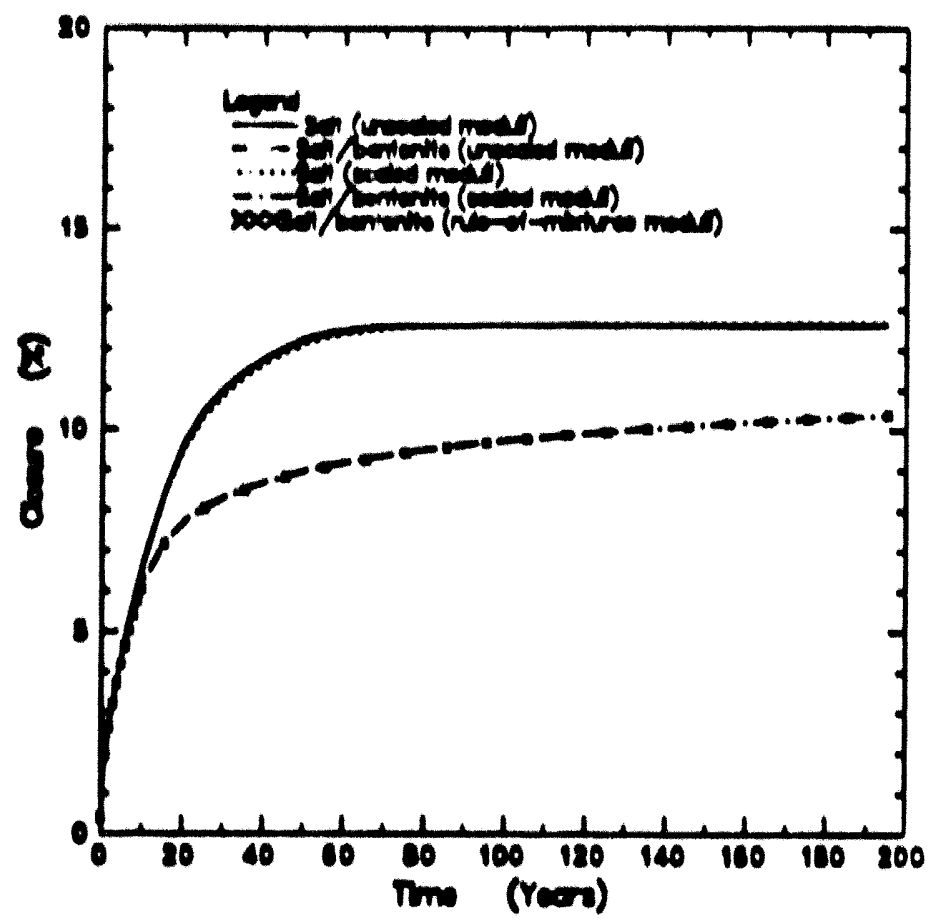

Figure 8. Horizontal Closure at the Midlieiglit of the Disposal Room as a Function of Time Expressed as a Percentage of the Original Room Width.

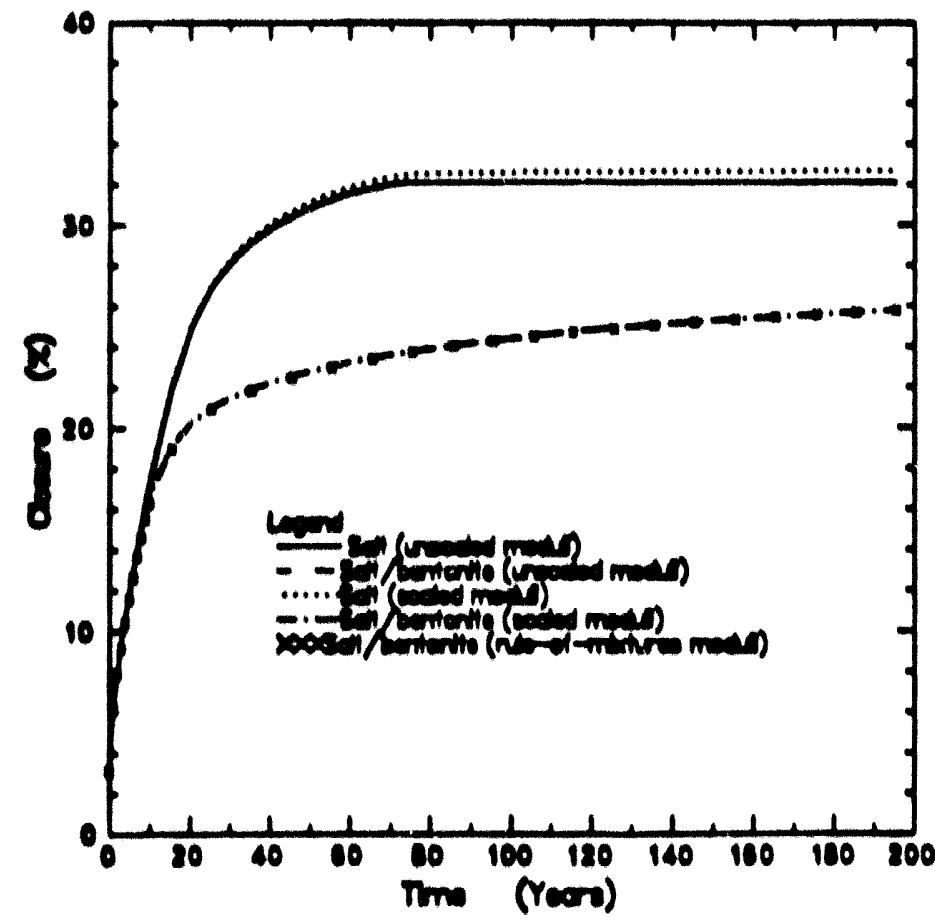

Figure 0. Vertical Closure at the Center of the Disposal Room as a Function of Time Expressed as a Percentage of the Original Ruom Height. 
B. M. Butcher, 6345

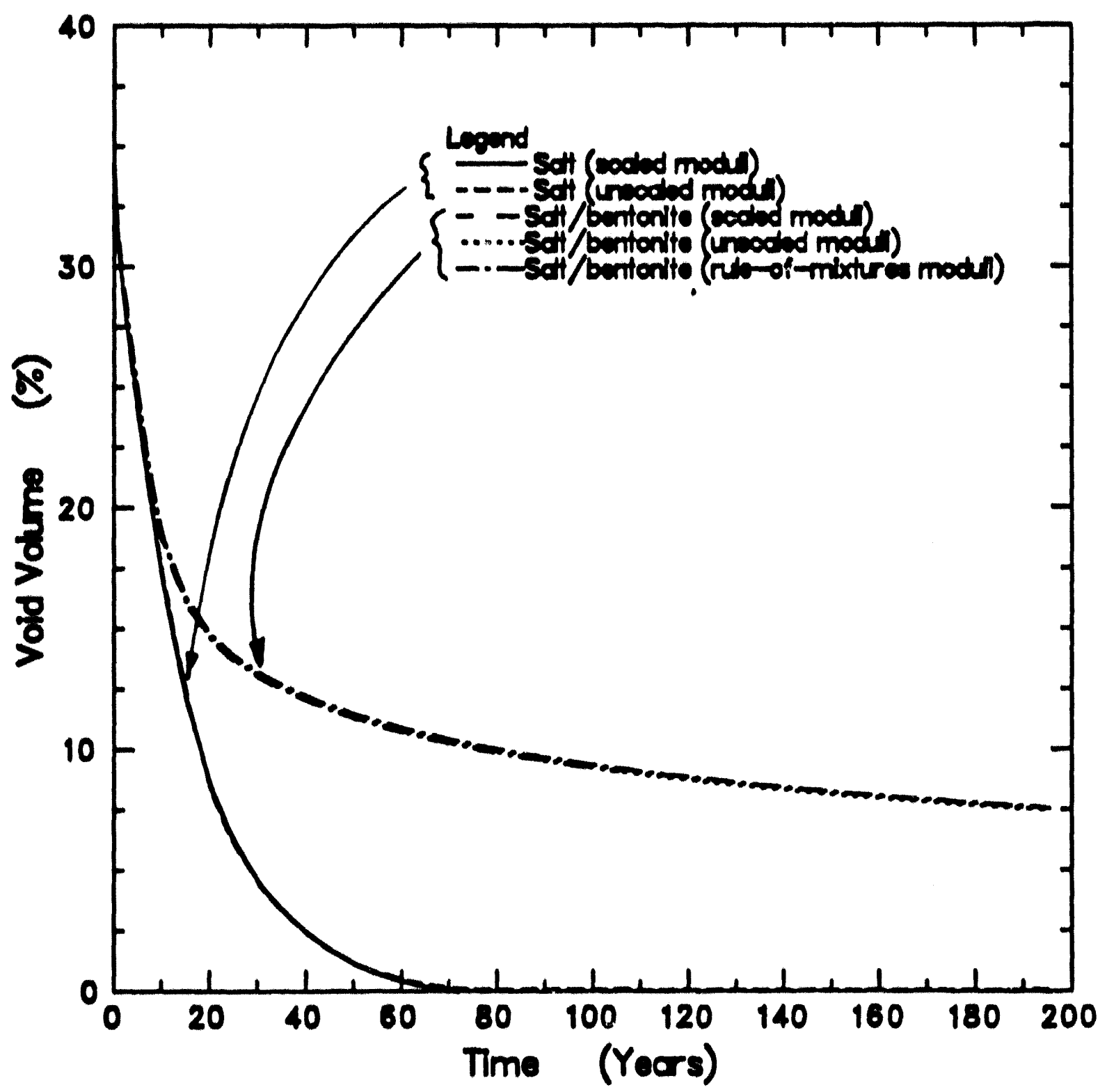

Figure 7. Void Volume in the Disposal Room as a Function of Time. Vuid Volume is Expressed as a Percentage of the Total Room Volume. 


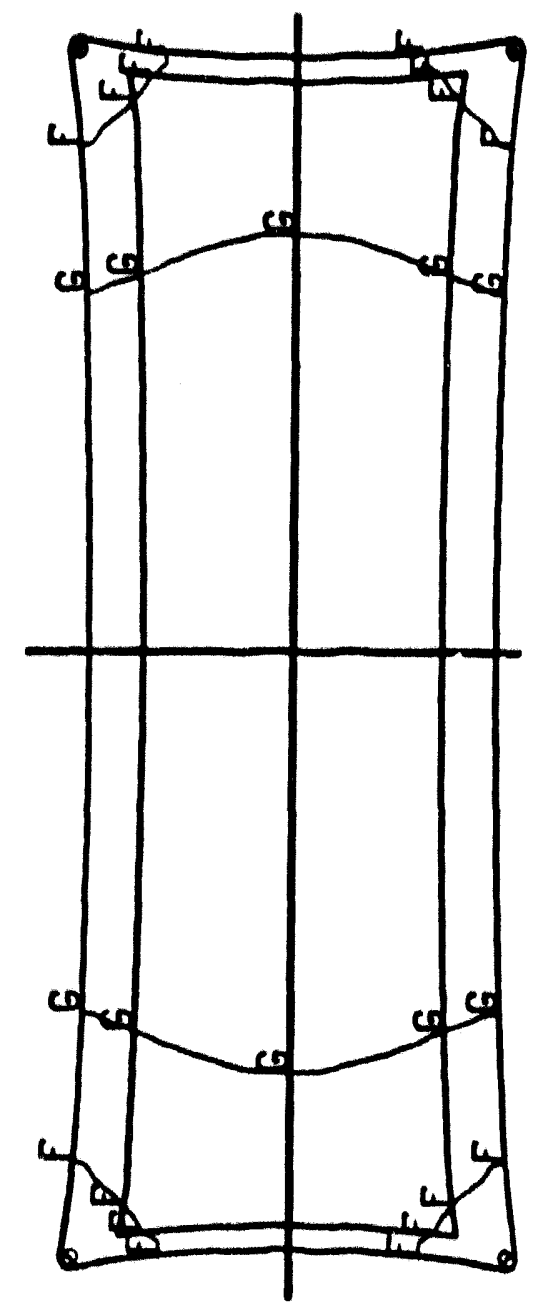

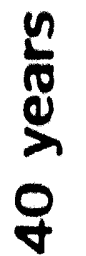

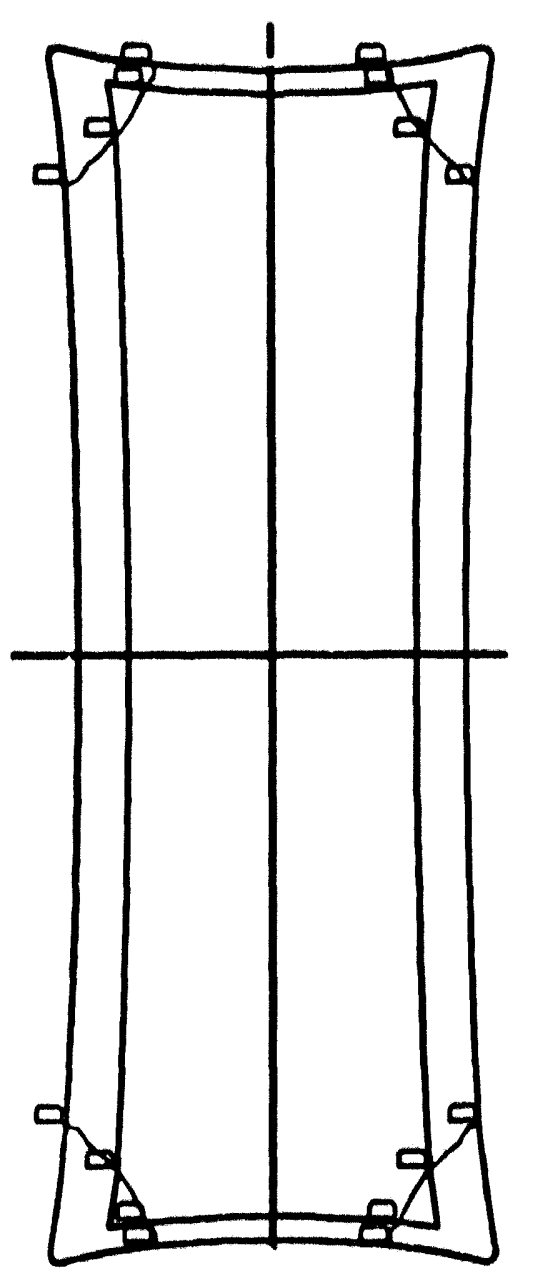

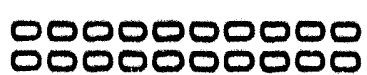

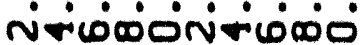
$m-m-N$

$\sum_{n}^{n}$

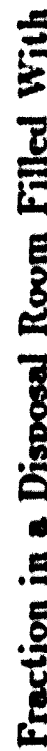
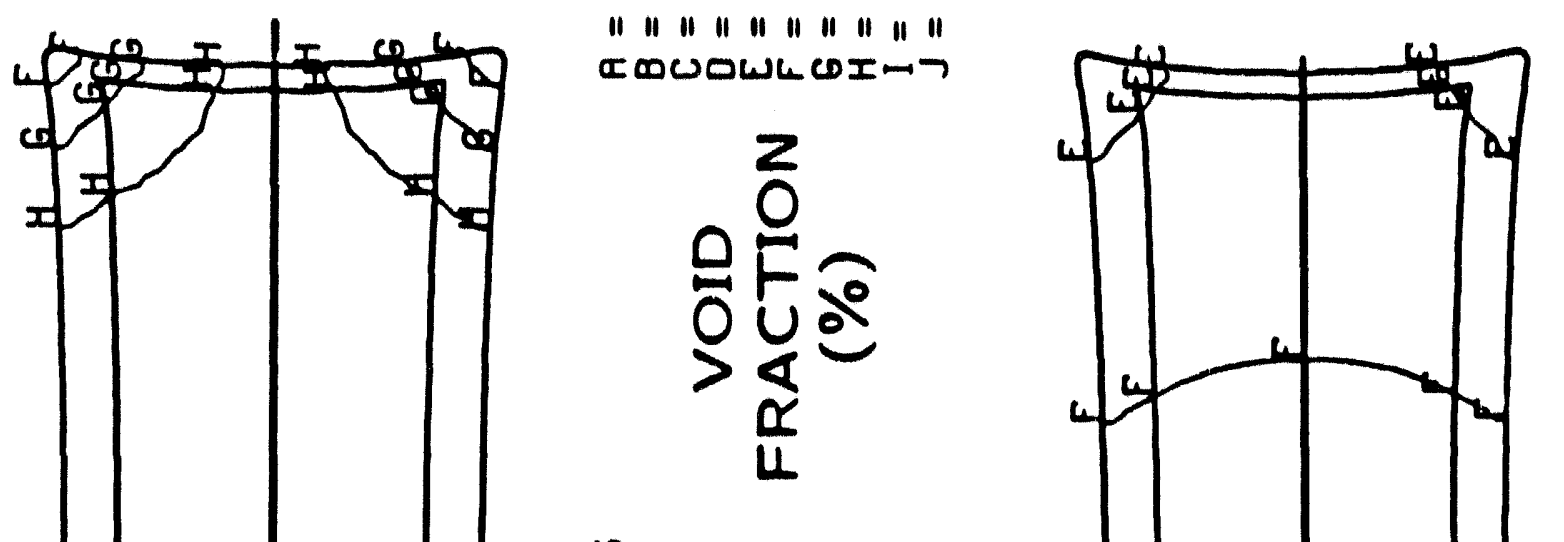

$\frac{n}{0}$
8
8
$N$

$\begin{array}{ll}n & 0 \\ 8 & 0 \\ 0 & 0\end{array}$ 
B. M. Bulcher, 6345

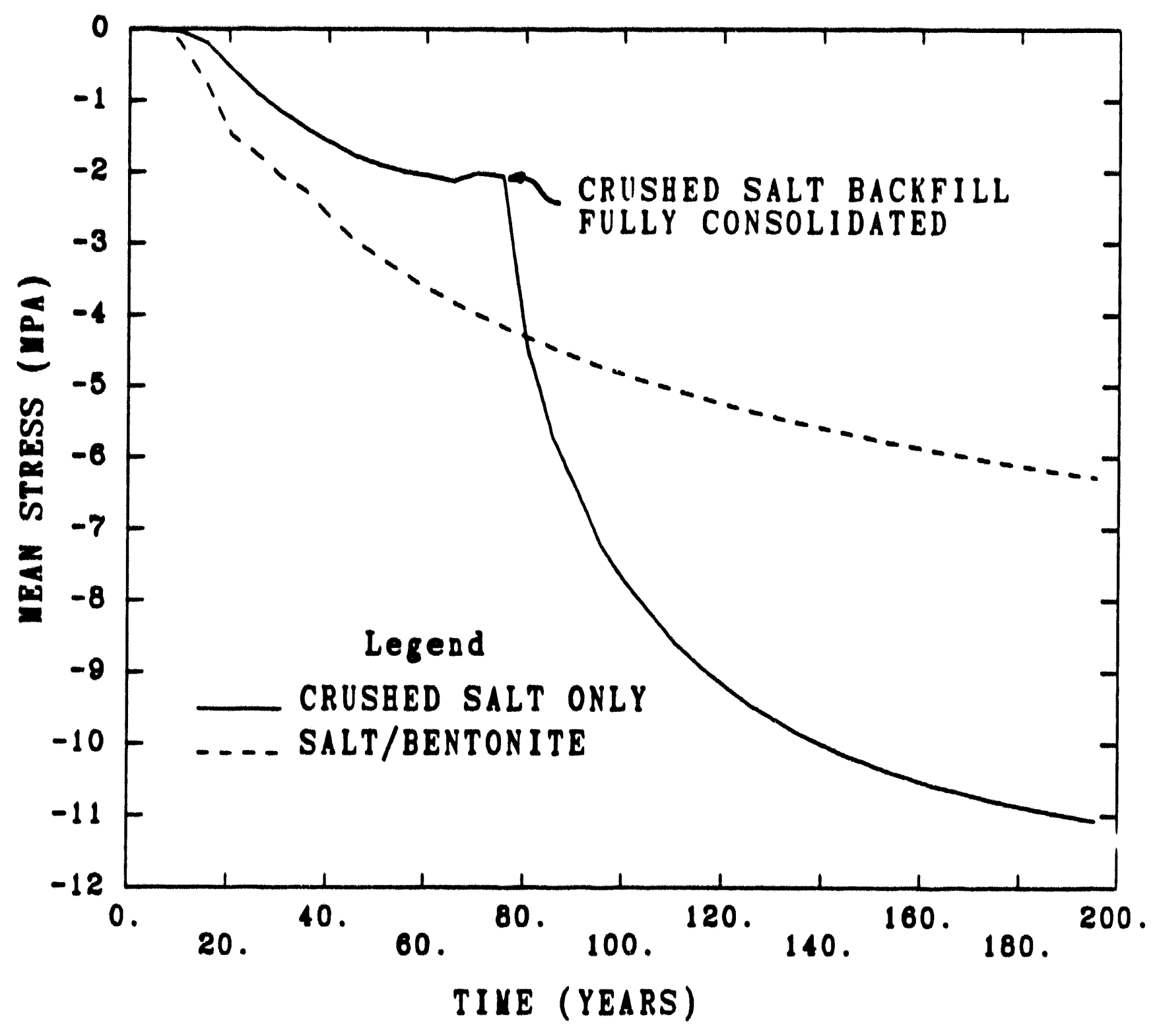

Figure 9. Mean Stress in the Backfill at the Center of the Disposal Ruom as a Function of Time. 


\section{Appendix A. Estimation of the Bulk Modulus of a Salt/Bentonite Mixture}

No experimental data are currently available for the density variation of the elastic moduli of a crushed salt/bentonite mixture. However, ouch data are avalable for dry crushed salt [1] and a sand/bentonite mixture [12]. These properties along with an unverified assumption about the nalure of the mixture were used to obtain crude estimale of how the moduli of the alt $/$ bentonite might vary with density. This estimate was used in a parameter atudy to determine the sensitivity of room closure calculalions to the constants appearing in Equations 14 and 15.

If the salt/bentonite is assumed to be non-interacting mixture where the bentonile is collected into small pockets which are uniformly distributed within a crushed salt matrix, then the moduli of the mixture can be eatimated with a simple mixture rule. For a mass fraction $\lambda$ of bentonite, the apecific volume of the mixture can be written as:

$$
V=(1-\lambda) V_{s}+\lambda V_{B}
$$

where $V_{S}$ and $V_{B}$ are the opecific volumes of salt and bentonite, respectively, and $V=1 / \rho$ where $\rho$ is the mass density.

The compressibility of the mixture is then estimated as:

$$
\beta V=(1-\lambda) \beta_{S} V_{S}+\lambda \beta_{B} V_{B} .
$$

where $\beta$ is the inverse of the bulk modulus. This equation allows for the calculation of the compressibility of the mixture if the volume and compressibility of each phase is known.

The bulk modulus as a function of peak pressure in crushed salt has been measured in tests reported in [1]. These same data have been converted to bulk modulus as a function of density by Sjaardema and Krieg [3]. Deusity of the crushed salt as a function of pressure has the form:

$$
\rho=1.5425+18.092 p
$$

where the density has units of $\mathrm{g} / \mathrm{cm}^{3}$ and pressure is in MPa. The corresponding bulk modulus has the form:

$$
K_{S}=1.76 \times 10^{-2} e^{0.63 p}
$$

where the units of $K$ are MPa. Thus, at a given pressure the density can be determined as a function of pressure; and the corresponding modulus is then calculated as a function of density.

The only data available for bentonite at various densities were obtained for a belltonite/sand mixture which is $30 \%$ sand by weight [12]. The data were used in lieu of other data sources. For this material, the pressure as a function of the volumetric strain $e_{v}$ was determined to be:

$$
p=2.61 \exp \left(8.10 e_{v}\right)-1 .(\mathrm{MPa} .)
$$


This equation can be inverted to give $e_{v}(p)$, and the density is calculaled from $\rho=$ $\rho_{0} /\left(1-e_{v}\right)$. The corresponding modulus has the form

$$
K_{B}=1.2 \rho-1836.0 \text { (MPa.) }
$$

where the density is in $\mathrm{kg} / \mathrm{m}^{3}$.

The final modulue of the mixture as a function of density is then calculated from Equation A.2 above. The result is shown in Figure A.1. The expression for the bulk modulus of the mixture was fit to function of the form $K=K_{0} \exp \left(K_{1} \rho\right)$ for use in the backfill constitutive model. This model also requires an expression for the variation of the shear modulus with density. Since no test data was available for determining this function, the shear modulus was computed from the bulk modulus assuming that Poisson's ratio of the nixture remains conotant during consolidation. Based on the results presented in Reference 13, value of 0.12 was used for Poisson's ratio of the mixture. 


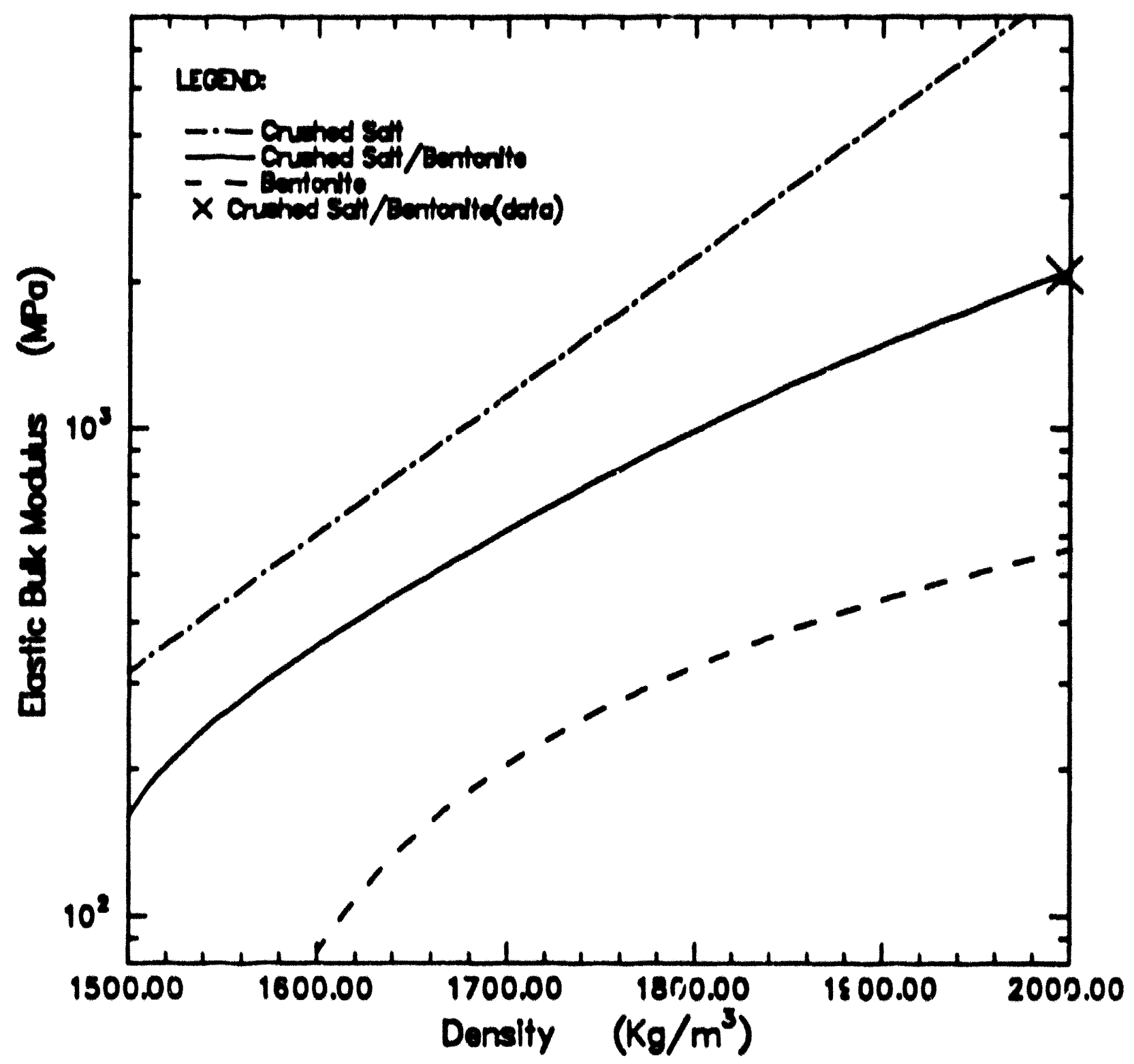

Figure A.1. Bulk Modulus as a Function of Density for the Dry Cruslied Salt, fur llie 70/30 Sand/Bentonite Mixture, and for the Mixtures Approximation. 
APPENDIX B: CONSTITUTIVE EQUATIONS FOR BACKFILL CONSOLIDATION IN SANCHO AND IN SPECTROM-32

B-1 


$$
\text { B-2 }
$$

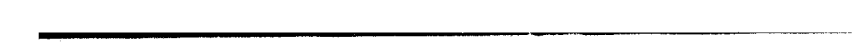

$+$

B-2

(1) 


\section{Appendix B: Constitutlve Equations For Backill Consolldation In SANCHO And In BPECTROM-32}

\section{Constltutlve Equations in SANCHO}

Backfill consolidation is defined in SANCHO by the following equations (Sjaardema and Krieg, 1987). Decomposing the current values of the stress $o_{i j}$ and the total strain rate (de, $/$ di) into volumetric and deviatoric parts (using the summation convention):

$$
\begin{aligned}
& P=-1 / 3 \sigma_{k k}, \\
& S_{i j}=\sigma_{i j}=1 / 3 \sigma_{k k}, \\
& \left(d e_{v} / d t\right)=-\left(d c_{k k} / d t\right), \\
& \left(d e_{i j} / d t\right)=\left(d c_{i j} / d t\right)-1 / 3\left(d c_{k k} / d t\right) .
\end{aligned}
$$

where: $\quad P$ is the pressure (positive in compression),

$S_{i j}$ is the deviatoric stress,

(de $/ \mathrm{dt}$ ) is the volumetric strain rate (positive for decreasing volume), and $\left(d e_{i j} / d t\right)$ is the deviatoric strain rate.

The density $\rho$ is computed from

$$
\rho=\rho_{0} \exp \left(\int_{t_{0}}^{t}\left(d c_{v} / d t\right) d t\right. \text {. }
$$

where $\rho_{0}$ is the density of the backfill at time $t_{0}$.

\section{ELASTIC CONSTANTS}

The elastic response of the consolidating material was defined by Sjaardema and Krieg in terms of the shear modulus $\mathrm{G}$ and bulk modulus $\mathrm{K}$ as

$$
\begin{aligned}
& K=K_{0} \bullet \exp \left(K_{1} \bullet \rho\right), \\
& G=G_{0} \bullet \exp \left(G_{1} \bullet \rho\right),
\end{aligned}
$$

where $K_{0}, K_{1}, G_{0}$, and $G_{1}$ are constants (Table 3.2.1). These relationships are also used in SPECTROM- 32 .

\section{SANCHO CREEP MODEL}

The volumetric constitutive model for pure crushed salt is (e.8., Sjaardema and Krieg, 1987. Equation 3.3.7)

$$
\begin{aligned}
\{d p / d t\} & =K_{0} \exp \left(K_{1} \rho_{0}\left(1+e_{v}\right)\right)\left[\left(d e_{v} / d t\right\}\right. \\
& \left.-\left(1-e_{v}\right)^{2} / \rho_{0} \bullet B_{0}\left(\exp \left(B_{1} P\right)-1\right) \bullet \exp \left(A \bullet \rho_{0} \bullet\left(1+e_{v}\right)\right)\right]
\end{aligned}
$$


For creep under deviatoric stresses, Sjaardema and Krieg used a relationship for the deviatoric stress rate that depends upon the sccondary creen model for intact salt. This assumnlion is important because it represents a major difference between the constifutive model used in the finite-element code SANCIIO and the finite clement code SPICTROM 12 . The continuum model for the rate of the deviatoric stres of sruahed salt in SANC11O is 1e B. Sjandema and Krieg. Equation 1.4.6)

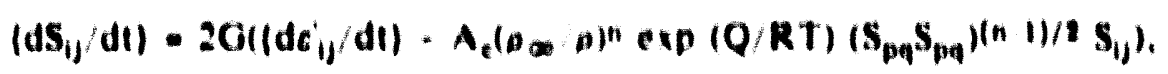

where material constanis $A_{e} . Q_{\text {. and }} n$ refer to the values for intact sall (Table 3.2.1). The densify Po is the solid density of halite.

The volumetric creep equation (Lquation 1,1.4) and the deviatoric slress tate (tiquation B-3) are then integrated to determine the total stress at any time, given by

$$
a_{1 j} \cdot s_{1 j} \cdot \partial_{1 j} \bullet p
$$

where $d_{13}$ is the Kronecker delia. Both $S_{1,}$ and $P$ contain elastic response terms.

\section{Conotltutlve Equations in 8PECTROM-32}

\section{SPECTROM.32 DEVIATORIC CAEEP MODEL}

Instend of summing stresses. the continuum model for the rate of consolidation of crushed salt in SPECTROM-32 sums strain tates (0.9. Callahan and DeVries. 1991):

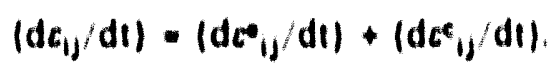

Elastic response is given by the $1 \mathrm{erm}\left(\mathrm{dc} c_{i j} / \mathrm{dl}\right)$, and the creep response is given by the lerm $\left(d c^{e}, j / d\right)$. The creep strain is, in turn, decomposed into a volumetric component and a shear component. The invariant strain-rate measure of the shear component. $c^{6}$ oq2. is then assumed to be a scalar multiple of the invariant measure of the volumetric strain rate (dev dI) (the volumetric strnin is defined in SPECTROM .32 as $c_{v}-\frac{2 u}{p}=1$

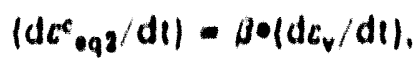

and the invariant stress is assumed to be a scalar multiple of the octahedral shear stress.

$$
0 .=\left(3 \cdot J_{2}\right)^{08}
$$

where $J_{2}$ is the second invariant of the stress deviator $\left(I_{2}=1 / 2 \cdot S_{1 j} S_{1 j}\right)$, so that the total creep strain rate (including both volumetric and shear cumponents) becomes

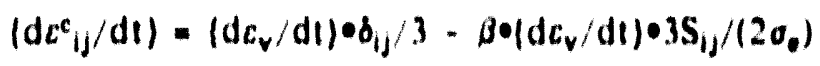

To apply this equation, a value of $2 / 3$ is assumed for the constant $\beta$, which causes the lateral components of the total creep strain, $d c^{c}{ } j / d t$, in a uniaxial test to equal zero. To 
understand the implications of this assumption. considet a short colindereal sample of ctushed matesial with a uniform sompressive load distrihuted weer each end the sides of the sample are unrestrained. Hecause the mechanism for the reduction of volume of the sample during crees coneolidation is the deformation of material into enternal voids. tather than llow of material laterally, lateral inelastic erpansion of this sample would appear unlikely thus, using the condition of be checked by esperimenis. Iqualion 1211 then becomes

$$
\left(d c_{i j} d t\right)=\left(d c_{v} d t\right) \cdot\left(\theta_{i},-S_{i j} \theta_{e}\right)
$$

and the full constitutive relation is

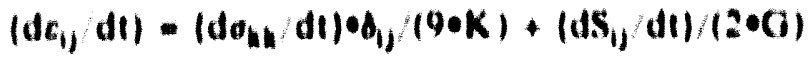

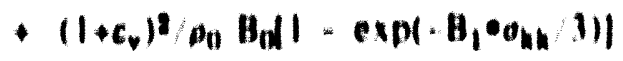

$$
\begin{aligned}
& \exp \left(A \cdot \theta _ { 0 } ( 1 + c _ { v } ) \left(\left(\theta_{1 j} / 3=S_{i j} / \sigma_{e}\right) .\right.\right.
\end{aligned}
$$

with constanis $B_{0}, B_{1}$, and $A$ listed in Table 1.21 .

While fquation $\mathbf{B}$ : 11 is corres for the inilial model that was incorporated into SPECTROM.12, the model was subseguenily expanded 60 include the deviatorle response of the Munson-Dawson model (Callahan and DeVries, 1991) much the same as lquation H-A defines the deviatoric portion of the secondary creep model This expanded model is expected lo give a smooth eransifion from erushed salt behavior in infact sull behavior as the vold fraction approaches tefo 


\section{Roferences For Appendix B}

Callahan, G.D., and K.L. DeVries. 1991. Allaly'ses of Backfilled Tramsuranic Wastes Disposal Raums. SAND91-7052. Albuquerque, NM: Sandia National Laboratories.

Sjaardema, G.D., and R.D. Krieg. 1987. Comstimutive Model for the Comsolidation of WIPP Crushed Salt and IIs Use in Allalyses of Bachfilled Shaft and Drift Configurations. SAND87-1977. Albuquerque. NM: Sandia National Laboratories. 
APPENDIX C: SANCHO AND RE/SPEC WASTE MODELS

Ci 


\section{Appendix C: SANCHO And RE/SPEC Waste Models}

\section{SANCHO Waste Model}

The stress-strain behavior of waste is represented in SANCHO by a volumetric plasticity model with a piecewise linear function defining the relationship between the mean stress $p_{m}$ and the volume strain $e_{v}$ (Weatherby et al., 1991) (See Appendix B, Equations B-la, B-Ic). Compaction experiments on simulated waste were used to develop this relationship, given in Table $\mathrm{C}-\mathrm{I}$. Although the deviatoric response of the waste has not been characterized, compaction experiments on 55-gallon drums of simulated combustible, metallic, and sludge waste showed that the drums do not undergo significant lateral expansion until most of the void space inside the drums has been eliminated (Butcher et al., 1991) (i.e., the thin steel walls of the drums have sufficient strength to elastically confine the waste). Therefore, the constants in the volumetric plasticity model were defined to capture this anticipated characteristic. The deviatoric yield function $F$ has the form

$$
F=o_{d}-3 p=0
$$

where $\sigma_{d}=\left(3 / 2 S_{i j} S_{i j}\right)^{1 / 2}$ (See Appendix B, Equation B-lb). The deviatoric response was assumed to be elastic-perfectly plastic. With this assumption, the yield surface of the waste is a surface of revolution around the mean stress axis in three-dimensional principal stress space. The curve traced by its intersection with any $\pi$ plane is a circle, with all stress states within the circles elastic. A value of $222 \mathrm{MPa}$ was assumed for the elastic shear modulus, and the elastic bulk modulus was $333 \mathrm{MPa}$ (Weatherby et al., 1991).

Table C-1. Assumed relationship between the mean stress and the volume strain for $\mathrm{CH}-\mathrm{TRU}$ waste used in SANCHO disposal room closure analyses (new SANCHO compaction curve)

\begin{tabular}{cc}
$\begin{array}{c}\text { Mean Stress, } p \\
(\mathrm{MPa})\end{array}$ & $\begin{array}{c}\text { Volume Strain, } e_{\mathrm{v}} \\
\left(\log \left(\rho / \rho_{0}\right)\right)\end{array}$ \\
0.028 & 0.032 \\
0.733 & 0.741 \\
1.133 & 0.898 \\
1.667 & 1.029 \\
2.800 & 1.180 \\
10.17 & 1.536 \\
\hline
\end{tabular}

\section{RE/SPEC Waste Model}

The stress-strain behavior of waste is represented in SPECTROM-32 by a nonlinear elastic model based on the assumption that the axial stress is proportional to the mean stress*

*The definition of the mean stress differs from the definition used for SANCHO; for SPECTROM-32, $\sigma_{m}=\frac{\sigma_{k k}}{3}$, for SANCHO, $p=-\frac{\sigma_{k k}}{3}$. 
(Figure 3.-3) (Callahan and DeVries, 1991). This assumption is related to the compaction tests of 55-gallon drums of simulated combustible, metallic, and sludge waste that were laterally unconfined: for zero lateral stresses, the definition of mean stress becomes

$$
\sigma_{m}=\left(\sigma_{r}+\sigma_{\theta}+\sigma_{a}\right) / 3, \text { with } \sigma_{r}=\sigma_{\theta}=0, \text { so that } \sigma_{a}=3 \sigma_{m} \text {. }
$$

In contrast, if the assumption is made that the waste is actually under a hydrostatic state of stress, then $\sigma_{\mathrm{r}}$ and $\sigma_{\theta}$ would equal $\sigma_{\mathrm{a}}$, and $\sigma_{\mathrm{a}}=\sigma_{\mathrm{m}}$. Callahan and DeVries recognized that neither assumption was correct and initially chose the $\sigma_{\mathrm{a}}=3 \sigma_{\mathrm{m}}$ assumption because it provided the less stiff representation of the TRU waste. They felt that the less stiff representation was more conservative because it provided less resistance to room closure and lower back pressure on the surrounding backfill and increased the time required to obtain lower porosities. In later calculations, closure calculations were repeated using the $\sigma_{a}=\sigma_{m}$ assumption (Callahan, March 13, 1992 memo in Appendix A). These results described in Section 5.1.3, were found to be much closure to the expected response.

The functional form used for compaction was

$$
\sigma_{\mathrm{a}}=\ln \left(\phi / \phi_{0}\right) / \kappa, \text { with } \sigma_{\mathrm{a}}=3 \sigma_{\mathrm{m}}
$$

where $\phi$ is the porosity, $\phi_{0}$ is the initial porosity, and $\alpha$ is a material parameter (Table C-2). To use the stiffer representation of the TRU waste (i.e $\sigma_{n}=\sigma_{m}$, the material parameters $K$ was replaced by $K / 3$. The elastic tangent bulk modulus $K$ was calculated directly from the relationships in Equation ( $C-3)$, and the elastic shear modulus $G$ was assumed to be $G=3 K / 5$, corresponding to a value for Poisson's ratio of 0.25 .

Table C-2. Composite CH-TRU waste consolidation model constants used in the SPECTROM-32 disposal room closure analyses

\begin{tabular}{lllll}
\hline Model & $\begin{array}{l}\text { Solid } \\
\text { Density } \\
\rho_{f}\left(\mathrm{~kg} / \mathrm{m}^{3}\right)\end{array}$ & $\begin{array}{c}\text { Initial } \\
\text { Porosity } \\
\phi_{0}\end{array}$ & $\begin{array}{c}\kappa \\
\left(\mathrm{MPa}^{2}\right)^{-1}\end{array}$ & $\begin{array}{c}\text { Initial } \\
\text { Density } \\
\rho_{\mathrm{f}}\left(\mathrm{kg} / \mathrm{m}^{3}\right)\end{array}$ \\
\hline Series & 2,790 & 0.650 & 0.068 & 978 \\
Parallel & 2,790 & 0.637 & 0.169 & 1,010 \\
\hline
\end{tabular}




\section{References For Appendix C}

Butche!, B.M., T.W. Thompson, R.G. Van Buskirk, and N.C. Patti. 1991. M/echantical Compaction of Waste Isolation Pilot Plant Simulated Waste. SAND90-1206. Albuquerque, NM: Sandia National Laboratories. See also: Luker, R.S., T.W. Thompson, and B.M. Butcher. 1991. "Compaction and Permeability of Simulated Waste," Roch Mechanics as a Mulfidisciplinary Science. Proceedings of the 32nd U.S. Symposium. Universuly of Oklahoma. Norman. OK. July 10-12,1991. Ed. J-C.Roegiers. SAND90-2368C. Brookfield, VT: A.A. Balkena. 693-702.

Callahan, G.D., and K.L. DeVries. 1991. Allalyses of Backfilled Transurantic Waste's Disposal Rooms. SAND91-7052. Albuquerque, NM: Sandia National Laboratories.

Weatherby, J.R., W r. Brown, and B.M. Butcher. 1991. "The Closure of WIPP Disposal Rooms Filled with Various Waste and Backfill Combinations," Rock Mechanics as a Multidisciplinary Srience. Proceedings of the 32nd U.S. Symposium. Universul! of Oklahoma. Norman. OK. July. 10-12. 1991. Ed. J-C. Roegiers. Brookfield, VT: A. A. Balkema. 919-928. 
APPENDIX D: DISPOSAL ROOM CALCULATIONS

D-1 
D- 2

\section{1) -2}




\section{Appendix D: Disposal Room Calculations}

Development of the closure part of the disposal room model was accomplished in a series of stages (Arguello et al. 1991), that became increasir, ly more detailed as better material response models and mathematical methods became available. Even though the final results of closure sludies as of October 1991 are reported in several key documents, other references provide additional detail of the methods for their acquisition. Summaries of key references are listed in this appendix in roughly the chronological order that the studies were completed, to provide insight into the steps leading to the final results.

\section{Calculation 1: An Empty Room}

\section{REFERENCE}

Ehgariner, B. 1990. Geomechanical Alnalyses in Support of the Haste Isolation Pilot Plant (WIPP). SAND90-0285. Albuquerque, NM: Sandia National Laboratories. Chapter 9.

CODE

SPECTROM - 32

\section{ROOM DIMENSIONS AND BOUNDARY CONDITIONS}

An cmply room in two-dimensional (2-D) plane strain was modeled as an infinite series of $33 \mathrm{ft}$ wide by $13 \mathrm{ft}$ high, infinitely long rooms separated by $100 \mathrm{ft}$ pillars. The finite element mesh was symmetric around the vertical centerline of the room with dimensions of $66.5 \mathrm{ft}$ by 342.2 $\mathrm{fl}$. Boundary conditions were roller or unidirectional displacements along each side of the mesh. except at the top where a unidirectional overburden pressure of $13.65 \mathrm{MPa}$ was applied. The vertical in situ stress gradient from the top of the model was internally calculated from the densily and thickness of the layers.

\section{STRATIGRAPHY}

Layers of pure halits, argillaceous halite, and anhydrite were separated by clay seams. Clay seams more than 33 it away from the room were not included.

\section{CONSTITUTIVE MODELS}

Salt: Munson-Dawson model and properties were used (Munson et al., 1989a; Munson et al., 1989b), except that the transient strain limit constant for argillaceous halite $u$ as varied from that of the reference set.

Anhydrite: An elastic material with a reduced modulus to account for the presence of fractures was used. 
Clay: A friction coefficient of 0.2 was assumed.

\section{RESULTS}

The room volume as a function of time was normalized to the room volume predicted elastically immediately after room excavation.

\section{Calculation 2: An Empty Room}

\section{REFERENCE}

Morgan, H.S. 1987. "Estimate of the Time Needed for TRU Storage Rooms to Close," June 2, 1987 memo to D.E. Munson. Albuquerque, NM: Sandia National Laboratories. (Memo in Appendix A).

\section{Code}

\section{SANCHO}

\section{ROOM DIMENSIONS AND BOUNDARY CONDITIONS}

An empty room in two-dimensional (2-D) plane strain was modeled as one in an infinite series of $33 \mathrm{ft}$ wide by $13 \mathrm{ft}$ high, infinitely long rooms separated by $100 \mathrm{ft}$ pillars. The finite element mesh was symmetric around the vertical centerline of the room with dimensions of 66.5 $\mathrm{ft}$. by $351.3 \mathrm{ft}$. Boundary conditions were roller or unidirectional displacements along each side of the mesh, except at the top where a unidirectional overburden pressure of $13.57 \mathrm{MPa}$ was applied. The vertical in situ stress gradient from the top of the model was calculated from the density and thickness of the layers.

\section{STRATIGRAPHY}

The formation was assumed to be completely salt.

\section{CONSTITUTIVE MODELS}

Salt: $\quad$ WIPP reference secondary creep law was used, with the reference elastic properties of halite divided by 12.5 (Krieg, 1984).

\section{RESULTS}

The room volume was normalized to the original room volume. Good agreement was observed with the closure rates measured in the SPDV test panel. 
An iteration of this was completed by Weatherby (May 17, 1989 memo in Appendix A) in which he compared the results of a calculation with the finite element mesh symmetric around the vertical centerline of the room with a quarter panel model. After 25 years of closure, the quarter panel representation closes $12 \%$ more than the half panel, largely because the quarter panel has a shorter distance between the center of the room and the upper boundary of the mesh.

\section{Calculation 3: An Empty Room}

\section{REFERENCE}

Morgan, H.S. 1987. "TRU Storage Room Calculation with Stratigraphy," December 9, 1987 memo to D.E. Munson. Albuquerque, NM: Sandia National Laboratories. (Memo in Appendix A).

CODE

\section{SANCHO}

\section{ROOM DIMENSIONS AND BOUNDARY CONDITIONS}

An empty room in two-dimensional (2-D) plane strain was modeled as one in an infinite series of $33 \mathrm{ft}$ wide by $13 \mathrm{ft}$ high, infinitely long rooms separated by $100 \mathrm{ft}$ pillars. The finite element mesh was symmetric around the vertical centerline of the room with dimensions of $66.5 \mathrm{ft}$ by $351.3 \mathrm{ft}$. Boundary conditions were roller or unidirectional displacements along each side of the mesh, except at the top where a unidirectional overburden pressure of $13.57 \mathrm{MPa}$ was applied. The vertical in situ stress gradient from the top of the model was calculated from the density and thickness of the layers.

\section{STRATIGRAPHY}

The stratigraphy is described by Munson and Morgan (1986).

\section{CONSTITUTIVE MODEL}

Salt: WIPP reference secondary creep law was used with the reference elastic properties of halite divided by 12.5 (Krieg, 1984).

Anhydrite layers and a single polyhalite layer:

These materials were modeled as plastic materials with pressure dependent deviatoric yield strengths defined by the Drucker-Prager yield criterion. The Drucker-Prager constants were variations of the WIPP reference values (Krieg, 1984). 
Room volumes were normalized to the original room volume. The closure estimate was almost the same as when the anhydrite layers were assumed elastic (without elastic constant reduction) and predicted $56 \%$ volume reduction in 100 years, as opposed to $76 \%$ reduction obtained with the all-salt model.

\section{Calculation 4: Rooms Completely Filled With Salt Backfill or Waste and Salt Backfill}

\section{REFERENCE}

Weatherby, J.R. 1989. "Finite Element Analysis of TRU Storage Rooms Filled with Waste and Crushed Salt," May 17, 1989 memo to B.M. Butcher. Albuquerque, NM: Sandia National Laboratories. (Memo in Appendix A).

CODE

SANCHO

\section{ROOM DIMENSIONS AND BOUNDARY CONDITIONS}

A room filled with waste and backfill in two-dimensional (2-D) plane strain was modeled as one in an infinite series of $33 \mathrm{ft}$ wide by $13 \mathrm{ft}$ high, infinitely long rooms with rounded corners separated by $100 \mathrm{ft}$ pillars. The finite element mesh was a quarter panel room with dimensions 20 $\mathrm{m}(66.5 \mathrm{ft})$ by $27 \mathrm{~m}(88.5 \mathrm{ft})$. Thus, in addition to symmetry about a vertical plane, the disposal room contents were approximated as symmetric around a horizontal plane passing through the center of the disposal room. Boundary conditions were roller or unidirectional displacements along each side of the mesh (based on symmetry conditions), except at the top where a unidirectional overburden pressure of $14.3 \mathrm{MPa}$ was applied. All locations within the salt formation had this initial stress state with all three principal stresses set equal to $14.3 \mathrm{MPa}$. Room contents were $100 \%$ backfill (crushed salt) or backfill plus waste. This configuration did not include the $2 \mathrm{ft}$ air gap normally considered part of the disposal room contents. The volume assumed for the waste was $1817 \mathrm{~m}^{3}$, and the volume assumed for the backfill was $1829 \mathrm{~m}^{3}$. An empty room calculation was also performed to determine the effect of reducing the distance from the center of the room to the upper edge of the mesh by half. This reduction was made in order to reduce CPU time.

\section{STRATIGRAPHY}

The formation was assumed to be completely salt. 
Salt: WIPP reference secondary creep law was used, with the reference elastic properties of halite divided by 12.5 (Krieg, 1984).

Backfill Crushed salt - The Sjaardema and Krieg constitutive relations were used with slight modifications, mostly concerned with how the deviatoric stresses are defined, as described in Appendix $B .2$ of this memo. The initial density of the crushed salt was $1400 \mathrm{~kg} / \mathrm{m}^{3}$.

Waste: A volumetric plasticity model with a piecewise linear function defining the relationship between the mean stress and the volumetric strain was used. Since no data was available to define this relationship. the model parameters were estimated. Deviatoric response assumed that the waste would not undergo significant lateral expansion, with the constants in the volumetric plasticity model defined to capture this anticipated characteristic. A deviatoric yield function was defined, and a non-associative flow rule was used. This relationship is shown as the "old" Sancho model in Figure 3.3-2 of the current report.

RESULTS

Void-fraction versus time curves are obtained at a given time by dividing the void volume in the region by the current volume of the region. Contrary to previous assumptions, in lieu of specific information, these results are the first to demonstrate that collapse rates of backfill and waste differ, and therefore the void fraction within the waste may be quite different than the void fraction within the backfill at a given time. Consolidation of the backfill occurs much more rapidly than consolidation of the waste. A concern with regard to the results of these calculations is that, contrary to expectations, the waste is shown to consolidate to almost zero void fraction. This computed response was a direct consequence of the parameter values assumed for the waste consolidation model, which were revised later as experimental data became available.

\section{Calculation 5: Rooms Completely Filled With Crushed Salt or Crushed Salt/Bentonite Backfill}

\section{REFERENCE}

Weatherby, J.R., and W.T. Brown. 1990. "Closure of a Disposal Room Backfilled With a Salt/Bentonite Mix," April 30, 1990 memo to B.M. Butcher. Albuquerque, NM: Sandia National Laboratories. (Memo in Appendix A).

CODE

SANCHO 


\section{ROOM DIMENSIONS AND BOUNDARY CONDITIONS}

A room filled with backfill in two-dimensional (2-D) plane strain was modeled as one in an infinite series of $33 \mathrm{ft}$ wide by $13 \mathrm{ft}$ high, infinitely long rooms with rounded corners separated by $100 \mathrm{ft}$ pillars. The finite element mesh was a quarter panel rnom with dimensions of $20 \mathrm{~m}$ $(66.5 \mathrm{ft})$ by $27 \mathrm{~m}(88.5 \mathrm{ft})$. Boundary conditions were roller or unidirectional displacements along each side of the mesh (based on symmetry conditions), except at the top where a unidirectional overburden pressure of $14.8 \mathrm{MPa}$ was applied. All locations within the salt formation had this initial stress state with all three principal stresses set equal to $14.8 \mathrm{MPa}$.

\section{STRATIGRAPHY}

The formation was assumed to be completely salt.

\section{CONSTITUTIVE MODELS}

Salt: WIPP reference secundary creep law was used, with the reference elastic properties of halite divided by 12.5 (Krieg, 1984).

Backfill Crushed salt - The Sjaardema and Krieg constitutive relations were used with two different sets of elastic moduli. The initial density of the crushed salt was $1400 \mathrm{~kg} / \mathrm{m}^{3}$.

Crushed salt and bentonite - The Sjaardema and Krieg constitutive relations were used with parameters defined by Callahan and DeVries (1991). These calculations also were for two different sets of elastic moduli. The initial density of the crushed salt was $1480 \mathrm{~kg} / \mathrm{m}^{3}$.

\section{RESULTS}

Void-fraction versus time curves are obtained at a given time by dividing the void volume in the region by the current volume of the region. These calculations represent the current state of the art with regard to incorporation of the backfill into disposal ronm closure analysis.

\section{Calculation 6: Rooms Completely Filled With Different Backfllis and Waste}

\section{REFERENCE}

Weatherby, J.R., W.T. Brown, and B.M. Butcher. 1991. "The Closure of WIPP Disposal Rooms Filled with Various Waste and Backfill Combinations," Rock Mochanics as a Mullidisciplinary Science. Proceedings of the 32nd U.S. Symposium. University of Oklahoma. Norman. OK, July 10-12,1991. Ed. J-C. Roegiers. Brookfield, VT: A.A. Balkema. 919-928. 


\section{SANCHO}

\section{ROOM DIMENSIONS AND BOUNDARY CONDITIONS}

A room filled with waste and backfill in two-dimensional (2-D) plane strain was modeled as one in an infinite series of $33 \mathrm{ft}$ wide by $13 \mathrm{ft}$ high, infinitely long rooms separated by $100 \mathrm{ft}$ pillars. The finite element mesh was a quarter panel room with dimensions of $20 \mathrm{~m}(66.5 \mathrm{ft})$ by $54 \mathrm{~m}(177 \mathrm{ft})$. A quarter panel requires the approximation that the disposal room contents are symmetric around a horizontal plane passing through the midpoint of the disposal room. Boundary conditions were roller or unidirectional displacements along each side of the mesh (based on symmetry conditions), except at the top where a unidirectional overburden pressure of $14.8 \mathrm{MPa}$ was applied. Initially all three principal stresses were set equal to $14.8 \mathrm{MPa}$ at all locations within the salt formation to define the initial stress state. The boundary conditions simulate conditions that would exist around a deeply buried disposal room located near the middle of a long panel of equally spaced disposal rooms. Room contents were either 100\% backfill (crushed salt or 70 wt $\%$ crushed salt and $30 \mathrm{wt}(\%)$ bentonite) or backfill plus waste. This configuration did not include the $2 \mathrm{ft}$ air gap normally considered part of the disposal room contents. The volume assumed for the waste was $1817 \mathrm{~m}^{3}$ and the volume assumed for the backfill was $1829 \mathrm{~m}^{3}$.

\section{STRATIGRAPHY}

The formation was assumed to be completely salt.

\section{CONSTITUTIVE MODELS}

Salt: WIPP reference secondary creep law was used, with the reference elastic properties of halite divided by 12.5 (Krieg, 1984).

Backfill: $\quad$ Crushed salt - The Sjaardema and Krieg constitutive relations were used with two different sets of elastic moduli. The initial density of the crushed salt was $1400 \mathrm{~kg} / \mathrm{m}^{3}$.

Crushed salt and bentonite - The Sjaardema and Krieg constitutive relations were used with parameters defined by Callahan and DeVries (1991). These calculations also were for two different sets of elastic moduli. The initial density of the crushed salt was $1480 \mathrm{~kg} / \mathrm{m}^{3}$.

Waste: A volumetric plasticity model with a piecewise linear function defining the relationship between the mean stress and the volumetric strain was used. Compaction experiments on simulated waste were used to develop this relationship, which is defined in the paper and in Appendix $C$ of this report. 


\section{RESULTS}

Void-fraction versus time curves are obtained at a given time by dividing the void volume in the region by the current volume of the region. Results show that the slower consolidation of the salt-bentonite backfill (cf. Butcher et al., 1991) does not significantly affect the rate of waste compaction.

\section{Calculation 7: Rooms Completely Filled With Different Backfills and Waste}

\section{REFERENCE}

Callahan, G.D., and K.L. DeVries. 1991. Analyses of Backfilled Transuranic Wastes Disposal Rooms. SAND91-7052. Albuquerque, NM: Sandia National Laboratories.

CODE

SPECTROM-32

\section{ROOM DIMENSIONS AND BOUNDARY CONDITIONS}

A room filled with waste and backfill in two-dimensional $(2-D)$ plane strain was modeled as one in an infinite series of $33 \mathrm{ft}$ wide by $13 \mathrm{ft}$ high, infinitely long rooms separated by $100 \mathrm{ft}$ pillars. The finite element mesh was a quarter panel room with dimensions of $20 \mathrm{~m}(66.5 \mathrm{ft})$ by $27 \mathrm{~m}(89 \mathrm{ft})$. Boundary conditions were roller or unidirectional displacements along each side of the mesh (based on symmetry conditions), except at the top where a unidirectional overburden pressure of $14.3 \mathrm{MPa}$ was applied. Initially all three principal stresses were set equal to $14.3 \mathrm{MPa}$ at all locations within the salt formation to define the initial stress state. Room contents were either 100\% backfill (crushed salt or $70 \mathrm{wt} \%$ crushed salt and $30 \mathrm{wt} \%$ bentonite) or backfill plus waste. This configuration did not include the $2 \mathrm{ft}$ air gap normally considered part of the disposal room contents.

\section{STRATIGRAPHY}

The formation was assumed to be completely argillaceous salt in order to facilitate comparison of results with SANCHO calculations.

\section{CONSTITUTIVE MODELS}

Salt: The modified Munson-Dawson material model was used (Munson et al., 1989a).

Backfill Crushed salt - The Sjaardema and Krieg constitutive relations were used. The initial density of the crushed salt was $1400 \mathrm{~kg} / \mathrm{m}^{3}$. 
Crushed salt and bentonite - The Sjardema and Krieg constitutive relations were used with parameters defined by Callahan and DeVries (1991). The initial density of the crushed salt was $1480 \mathrm{~kg} / \mathrm{m}^{3}$.

Waste: The stress-strain behavior of waste is represented in SPECTROM-32 by a nonlinear elastic model based on the assumption that the axial stress is always equal to three times the mean stress. The same compaction data were used to develop this relationship as the data used for the SANCHO calculations (Weatherby et al., 1991), as shown in Figure 3.3-2.

\section{RESULTS}

Void-fraction versus time curves are obtained at a given time by dividing the void volume in the region by the initial volume of the region. Differences between analyses with SPECTROM32 and SANCHO are discussed in Appendix B of the report. These calculations were difficult because excessive CPU lime was required for their completion. A comparison of the results for rooms filled completely with backfill with SANCHO results showed that in all cases the SPECTROM-32 results predicted more rapid consolidation. Another major difference was that the waste compaction model in the SPECTROM-32 calculations caused the waste 10 compact to much lower porosities than expected.

\section{Calculation 8: Effect of Gas Generation on Room Closure}

\section{REFERENCE}

Weatherby, J.R., J.G. Arguello, and C.M. Stone, 1989. "The Effect of Gas Generation on the Performance of CH-TRU Disposal Rooms," November 14, 1989 memo to B.M. Butcher and D.E. Munson. Albuquerque, NM: Sandia National Laboratories. (Memo in Appendix A).

CODE

SANCHO

\section{ROOM DIMENSIONS AND BOUNDARY CONDITIONS}

A room filled with waste and backfill in two-dimensional (2-D) plane strain was modeled as one in an infinite series of $33 \mathrm{ft}$ wide by $13 \mathrm{ft}$ high, infinitely long rooms with rounded corners separated by $100 \mathrm{ft}$ pillars. The finite element mesh was a quarter panel room with dimensions of $20 \mathrm{~m}(66.5 \mathrm{ft})$ by $27 \mathrm{~m}(88.5 \mathrm{rt})$. In addition to symmetry about a vertical plane, the disposal room contents were approximated as symmetric around a horizontal plane passing through the center of the disposal room. Boundary conditions were roller or unidirectional displacements along each side of the mesh (based on symmetry conditions), except at the top where a unidirectional overburden pressure of $15 \mathrm{MPa}$ was applied. All locations within the salt formation had this initial stress state with all three principal stresses set equal to $15 \mathrm{MPa}$. Room conten were backfill plus waste. This configuration did not include the $2 \mathrm{ft}$ air gap normally considered part of the disposal room contents. However, an inconsistency of the calculation was that the $2 \mathrm{ft}$ air gap 
volume was included in determination of the void volume for the gas, as described below. The volume assumed for the waste was $1817 \mathrm{~m}^{3}$, and the volume assumed for the backfill was $1829 \mathrm{~m}^{3}$.

\section{STRATIGRAPHY}

The formation was assumed to be completely salt.

\section{CONSTITUTIVE MODELS}

Salt: WIPP reference secondary creep law was used, with the reference elastic properties of halite divided by 12.5 (Krieg, 1984).

Backfill: $\quad$ Crushed salt - The Sjaardema and Krieg constitutive relations were used with slight modifications, mostly concerned with how the deviatoric stresses are defined, as described in Appendix B of this memo. The initial density of the crushed salt was $1400 \mathrm{~kg} / \mathrm{m}^{3}$.

Waste: $\quad$ A volumetric plasticity model with a piecewise lincar function defining the relationship between the mean stress and the volumetric strain was used. Since no data was available to define this relationship, the model parameters were estimated. Deviatoric response assumed that the waste would not undergo significant lateral expansion, with the constants in the volumetric plasticity model defined 10 capture this anticipated characteristic. A deviatoric yield function was defined, and a non-associative flow rule was used. This relationship is shown as the "old" Sancho model in Figure 3.3-2 of the current report.

Gas: The ideal gas law was used to compute gas pressures. The gas generation rate was $2.66 \mathrm{~g}$-moles $/ \mathrm{drum} / \mathrm{yr}$ for the first 500 years, after which gas production due to corrosion was assumed to stop. The gas generation rate between 500 years and 700 years was $0.86 \mathrm{~g}$-mole/drum/year, due to microbial action, and after 700 years all gas generation was assumed to stop. The room was assumed to contain 6804 drums of waste, the maximum physically possible.

For the gas calculation, the code SANCHO was modified to compute the room pressure and to apply the resulting nodal forces to the room boundary. Volume available for the gas was determined by subtracting the volume of the solids in the disposal room from the current room volume. Solid volume was determined from the sum of the assumed initial solid fraction of the waste $(0.315)$ multiplied by its initial volume $\left(1,817 \mathrm{~m}^{3}\right)$, added to the assumed initial solid fraction of the backfill $(0.6)$ multiplied by an initial volume of $1,268 \mathrm{~m}^{3}$. However, the total solid volume of $1,330 \mathrm{~m}^{3}$ computed in this manner was inconsistent with other assumptions for the calculation because it was based on the assumption that an air gap was present. For the quarter panel configuration used for the analysis, the air gap was assumed filled with backfill to overcome computational difficulties, so that the total initial backfill volume was actually $1829 \mathrm{~m}^{3}$. Thus the backfill volume used for estimation of the total solid volume should have been $1829 \mathrm{~m}^{3}$ rather than $1,268 \mathrm{~m}^{3}$, allowing less room for gas. These two values differ by the volume of the air gap, which is $561 \mathrm{~m}^{3}$. 


\section{RESULTS}

Void-fraction versus time curves are obtained at a given time by dividing the void volume in the region by the current volume of the region. Gas generation was observed to stop closure when the backfill and waste were still highly porous and the internal gas pressures were well below lithostatic. Although these results were based on the "old" waste compaction model, the consequences of its use were not as severe as when it was used for estimates of closure without gas generation. The reason for this conclusion is that the "old" waste compaction model is considered to be in error when the waste is compacted 10 low states of porosity and backstress becomes important. In the gas generation calculation described in this section, the room contents never became dense enough 10 generate much backstress. In fact, little effect on the results is anticipated had the more recent compaction model been used in this calculation.

\section{Calculation 9: Effect of Gas Generation on Room Closure}

\section{REFERENCE}

Brown, W.T., and J.R. Weatherby. 1990. "Influence of Gas Generation Potential and Gas Generation Rate on the Performance of CH-TRU Disposal Rooms," September 17, 1990 memo to B.M. Butcher and F.T. Mendenhall. Albuquerque, NM: Sandia National Laboratories. (Memo in Appendix A).

CODE

\section{SANCHO}

\section{ROOM DIMENSIONS AND BOUNDARY CONDITIONS}

A room filled with waste and backlill in two-dimensional (2-D) plane strain was modeled as one in an infinite series of $33 \mathrm{ft}$ wide by $13 \mathrm{ft}$ high, infinitely long rooms with rounded corners separated by $100 \mathrm{ft}$ pillars. The finite element mesh was a quarter panel room with dimensions of $20 \mathrm{~m}(66.5 \mathrm{ft})$ by $27 \mathrm{~m}(88.5 \mathrm{ft})$. In addition to symmetry about a vertical plane, the disposal room contents were approximated as symmetric around a horizontal plane passing through the center of the disposal room. Boundary conditions were roller or unidirectional displacements along each side of the mesh (based on symmetry conditions), except at the top where a unidirectional overburden pressure of $14.8 \mathrm{MPa}$ was applied. All locations within the salt formation had this initial stress state, with all three principal stresses set equal to $14.8 \mathrm{MPa}$. Room contents were backfill plus waste. This configuration did not include the 2 it air gap normally considered part of the disposal room contents. The volume assumed for the waste was $1817 \mathrm{~m}^{3}$, and the volume assumed for the backfill was $1829 \mathrm{~m}^{3}$.

\section{STRATIGRAPHY}

The formation was assumed to be completely salt. 


\section{CONSTITUTIVE MODELS}

Salt: WIPP reference secondary creep law was used, with the reference elastic properties of halite divided by 12.5 (Krieg, 1984).

Backfill: Crushed salt - The Sjaardema and Krieg constitulive relations were used with slight modifications, mostly concerned with how the deviatoric stresses are defined, as described in Appendix $B$ of this memo. The initial density of the crushed salt was $1400 \mathrm{~kg} / \mathrm{m}^{3}$.

Waste: A volumetric plasticity model with a piecewise linear function defining the relationship between the mean stress and the volumetric strain was used. Since no data was available to define this relationship, the model parameters were estimated. Deviatoric response assumed that the waste would not undergo significant lateral expansion, with the constants in the volumetric plasticity model defined to capture this anticipated characteristic. A deviatoric yield function was defined, and a non-associative flow rule was used. This relationship is shown as the "old" Sancho model in Figure 3.3-2 of the current report.

Gas: The ideal gas law was used to compute gas pressures. The gas generation rate was $2.66 \mathrm{~g}$-moles $/ \mathrm{drum} / \mathrm{yr}$ for the first 500 years, after which gas production due to corrosion was assumed to stop. The gas generation rate between 500 years and 700 years was $0.86 \mathrm{~g}$-mole/drum/year due to microbial action, and after 700 years all gas generation was assumed to stop. Both the gas potential and the time for gas to be generated was varied in this calculation by changing the number of drums in the room and the gas generation rates.

For the gas calculation, the code SANCHO was modified to compute the room pressure and to apply the resulting nodal forces to the room boundary. Volume available for the gas was determined by subtracting the volume of the solids in the disposal room from the current room volume. Solid volume was determined from the sum of the assumed initial solid fraction of the waste $(0.3 ! 5)$ multiplied by its initial volume $\left(1,817 \mathrm{~m}^{3}\right)$, added to the assumed initial solid fraction of the backfill (0.6) multiplied by an initial volume of $1,829 \mathrm{~m}^{3}$. Unlike previous calculations, the total solid volume of $1.670 \mathrm{~m}^{3}$ computed in this manner was completely consistent with other assumptions for the the calculations.

\section{RESULTS}

Void-fraction versus time curves are obtained at a given time by dividing the void volume in the region by the current volume of the region. For maximum gas generation, closure was observed to cease when the backfill and waste were still highly porous and the internal gas pressures were well below lithostatic. In contrast, reductions in gas generation caused more closure before reversal, and closure for the least amount of gas was greater (a smaller void fraction) than expected. The reason the closure results for minimum gas generation are considered unrealistic is that these calculations were based on the "old" waste compaction model. For a given value of backstress, the "old" waste compaction model preseribed greater compaction (lower porosity) than is obtained from the more recent model. 


\section{References For Appendix D}

Arguello, J.G., J.R. Weatherby, C.M. Stone, H.S. Morgan, and W.R. Wawersik, 1991. "Room Response Analyses to Investigate Waste Disposal lssues," Procecdings of the 7th International Congress on Roch Mechanics, Aachen. FRG. September 10-20, 1991. Vol. 3, 1867-1871.

Butcher, B.M., C.F. Novak, and M. Jercinovic. 1991. The Advamtages of a Sall/Bimlomile Backlill for Waste lsolation Pilot Plamt Disposal Rooms. SAND90-3074. Albuquerque, NM: Sandia National Laboratories.

Callahan, G.D., and K.L. DeVries. 1991. Allalyses of Backfilled Transuranic Wasles Disposal Rooms. SAND91-7052. Albuquerque, NM: Sandia National Laboratories.

Ehgartner, B. 1990. Geomechanical Analyses in Support of the Waste Isolation Pilot Plant (WIPP). SAND90-0285. Albuquerque, NM: Sandia National Laboratories.

Krieg, R.D. 1984. Reference Stratigraphy and Rock Properties for the Waste Isolation Pilot Plant (WIPP) Project. SAND83-1908. Albuquerque, NM: Sandia National Laboratories.

Munson, D.E., and H.S. Morgan. 1986. Methodology for Performing Parallel Design Calculations (Nuclear Wastc Repository' Application). SAND85-0324. Albuquerque, NM: Sandia National Laboratories.

Munson, D.E., A.F. Fossum, and P.E. Senseny. 1989a. Advances in Resolution of Discrepancies Be'tu'e'n Predicled and Measured In Silu WIPP Room Closure's. SAND88-2948. Albuquerque, NM: Sandia National Laboratories. See also: Munson, D.E., and K.L. Devries. 1991. "Development and Validation of a Predictive Technology for Creep Closure of Underground Rooms in Salt," Proccedings of the 7th International Congress on Rock Mechanics. Aachen. FRG. September 16-20, 1991. Ed. W. Wittke. SAND90-1147, Vol. 7, 127-134.

Munson, D.E., A.F. Fossum, and P.E. Senseny. 1989b. Approach (1) First Principles Model Prediclion of Measured WIPP In Silu Room Closure in Sall. SAND88-2535. Albuquerque, NM: Sandia National Laboratories.

Sjaardema, G.D., and R.D. Krieg. 1987. A Comstilutive Model for the Consolidation of WIPP Crushed Salt and IIs Use in Alnalyses of Backfilled Shaft and Drift Configurations. SAND87-1977. Albuquerque, NM: Sandia National Laboratories.

Weatherby, J.R., W.T. Brown, and B.M. Butcher, 1991. "The Closure of WIPP Disposal Rooms Filled with Various Waste and Backfill Combinations," Rock Mechanics as a Multidisciplinary Science, Procecedings of the 32nd U.S. Simposium. Universily of OKlahoma, Norman. OK. July" 10-12, 1991. Ed. J-C. Roegiers. Broofield, VT: A. A. Balkema. 919-928. 


\section{DISTRIBUTION}

\section{Federal Agencies}

US Department of Energy (6)

Offlce of CIvillan Radloactive Waste Management

Attn: Deputy Director, RW-2

Associate DIrector, RW. $10 / 50$

offlce of Program and

Resources Management

Offlce of Contract Business

Management

Director, Analys is and

Veriflcation DIVlsion, RW-22

Assoclate DIrector, RW- 30

Office of systems and

Comp 11 ance

Assoclate DIrector, RW- 40

Office of Storage and

Transportatinn

Director, $R W-4 / 5$

office of strategic planning and International Programs

Offlce of External Relations

Forrestal Bullding

Washington, DC 20585

US Department of Energy

Albuquerque Operations offlce

Attri: National Atomic Museum Library PO Box 5400

Albuquerque, NM 87185.5400

US Department of Energy (4)

WIPP Project Integration of fice

Attn: W.J. Arthur 111

L.W. Gage

P.J. HiggIns

D.A. 01 ona

PO Box 5400

Albuquerque, NM 87115.5400

US Department of Energy (2)

WIPP Project Integration Satellite

Office

Aten:

R. Batra

R. Becker

L. Truj 1110

PO Box 3090

Carlsbad, NM 88221-3090

US Department of Energy (3)

WIPP Project Site Offlce (Carlsbal)

Attn: V. Daub

J. LIppis

J.A. Mewhinney

PO Box 3090

Carlsbad, NM 88221.3090
US Department of Energy

Research \& Waste Management DIvision

Attin: DIrector

PO Box E

Oak RIdge, TN 37831

US Department of Energy

Attn: E. Young

Room E- 178

GAO/RCED/GTN

WashIngton, DC 20545

US Departinent of Energy

office of Environmental Restoration and Waste Management

Attr: J. Lyt1e, EM-30

(Trevion II)

Washington, DC 20585.0002

US Department of Energy (3)

office of Environmental Restoration and Waste Management

Attn: M. Fral, EM-34

(Trevion 11)

Washington, DC 20585.0002

US Department of Energy

Office of Enviromental Restoration and Wast Management

Attn: S. Schnelder, EM- 342

(Trevion 11)

Washington, DC 20585.0002

US Department of Energy (2)

offlce of Enviromment. Safety and Health

Attn: C. Borgstrom, EH.25

R. Pelletier, EH. 231

Washington, DC 20585

US Department of Energy (2)

I daho Operations office

Fuel Processing and Waste

Management DIVIsIon

785 DOE Place

Idaho Falls, ID 83402

US Environmental Protection Agency (2)

Radiation Protection Programs

Attn: $M$. Oge

ANR -460

Washlngton, DC 20460 
US Geologlcal Survey (2)

Water Resources DIviston

Attn: $R$. Livingston

4501 Indlan School NE

sulte 200

Albuquerque, NM 87110

US Nuclear Regulatory Commission Division of Waste Management

Attn: H. Marson

Mall Stop 4.H.3

Washington, DC 20555

\section{Boards}

Defense Nuclear Facilit les Safety Board

Attn: D. WInters

625 Indlana Ave. NW, Sulte 700

Washington, DC 20004

Nuclear Waste Technlcal Review Board (2)

Attn: Chalrman

$$
\text { S.J.S, Parry }
$$

1100 Wllson Blvd. Sulte 910

Ar1Ington, VA 22209.2297

Advlsory Commlttee on Nuclear

Waste

Nuclear Regulatory Commission

Attn: R. Major

7920 Norfolk Ave.

Bethesda, MD 20814

\section{State Agencles}

Environmental Evaluation Group (3)

Attn: Library

7007 Wyom Ing NE

Sulte $F=2$

Albuquerque, NM 87109

NM Bureau of Mines and MIneral Resources

Socorro, NM 87801

NM Energy, MInerals, and Natural Resources Department

Aten: Library

$2040 \mathrm{~S}$. Pacheco

Santa Fe. NM 87505

NM Environment Department (3)

Secretary of the Environment

Attn: J. Esplnosa

$1190 \mathrm{St}$. Francls Drive

Santa Fo, NM 87503.0968
NM Environment Department

WIPP Project Site

Aten: P. McCasland

PO Box 3090

Carlsbad, NM 88221

\section{Laboratorles/Corporations}

Battelle Pacific Northwest

Laboratorles

Attn: R.E. Westerman, MSIN P8-44

Battelle Blud.

Richland, WA 99352

INTERA Inc.

Attn: J.F. Plckens

6850 Aust In Center B1vd.

Sulte 300

Austin, TX 78731

INTERA Inc.

Attn: W. Stensrud

PO Box 2123

Carlsbad, NM 88221

IT Corporation (2)

Aten: R.F. Mckinney

M. Abashlan

Reglonal office

5301 Central NE, Sulte 700

Albuquerqua, NM 87108

Los Alamos National Laboratory

Attn: B. Erdal, CNC.11

PO Box 1663

Los Alamos, NM 87544

$\mathrm{RE} / \mathrm{SPEC}$, Ine. (3)

Attn: W. Coons

D.A. Labreche

S. Key

4775 Indlan School NE

Sulte 300

Albuquerque, NM 87110.3927

$\mathrm{RE} / \mathrm{SPEC}$, Ine. (5)

Attn: J.L. Ratlgan

N.S. Brodsky

G.D. Callahan

K.L. DeVries

T.W. Pfolfle

Po Box 725

Rapld Clty, SD 57709 
Southwest Research Institute (2)

Center for Nuclear Waste

Regulatory Analysis

Attn: P.K. Nair

6220 Culebra Road

San Antonio, TX 78228-0510

SAIC

Attn: D.C. Royer

101 Convention Center Dr.

Las Vegas, NV 89109

SAIC

Attn: H.R. Pratt

10260 Campus Point Dr.

San Diego, CA 92121

SAIC (2)

Attn: M. Davis

J. Tollison

2109 Air Park Rd. SE

Albuquerque, NM 87106

SAIC

Attn: T'W. Thompson

14062 Denver West Parkway

Sulte 255

Golden, CO 80401

Tech Reps Inc. (3)

Attn: J. Chapinan

C. Crawford

T. Peterson

5000 Marble NE, Suite 222

Albuquerque, NM 87110

TRW Environmental Safety Systems

At (1): L. Wildinan

2650 Park Tower Dr. Suite 1300

Vienna, VA 22180.7306

Westinghouse Electrle Corporation (5)

Atu: Library

C. Cox

L. Fitch

B. A. Howard

R. Kelurmant

Po Bor 20\%8

Carlsbal, NM 88?21

Wost loghouse Savamah RIver

Technology center (a)

Attin: N. Bibler

J.R. Harbour

M.J. Plodinec

G. G. Wicks

Alken, se: 2980 ?

\section{National Academy of Sciences,} WIPP Panel

Howard Adler

Oak Ridge Associated Universities

Medical Sciences Division

PO Box 117

Oak Ridge, TN 37831-0117

Ina Alterman

Board on Radioactive

Waste Management, GF456

2101 Constitution Ave.

Washington, DC 20418

Fred M. Ernsberger

250 Old M111 Road

Pittsburgh, PA 15238

John D. Bredehoeft

Western Region Hydrologist

Water Resources Division

US Geological Survey (M/S 439)

345 Middlefield Road

Menlo Park, CA 94025

Rodney C. Ewing

Department of Geology

University of New Mexico

Albuquerque, NM 87131

Charles Fairhurst, Chairman

Department of Civil and

Mineral Engineering

University of Minnesota

500 Pillsbury Dr. SE

Minneapolis, MN 55455-0220

B. John Garrick

PLG Incorporated

4590 MacArthur Blvd., Suite 400

Newport Beach, CA 92660-2027

Leonard $F$. Konikow

US Geological Survey

431 National Center

Reston, VA 22092

Peter B. Myers

National Academy of sclences

Board on Radioactive

Waste Management

2101 Coust f tution Ave.

Washingt on, DC 20418 
Jeremiah O'Driscoll

Jody Incorporated

505 Valley Hill Drive

Atlanta, GA 30350

Christopher G. Whipple

Clement International.

160 Spear St., Suite 1380

San Francisco, CA 94105

\section{Performance Assessment Peer Review Panel}

G. Ross Heath

College of Ocean and Fishery

Sciences, $\mathrm{HN}-15$

583 Henderson Hall

University of Washington

Seattle, WA 98195

Thomas H. Pigford

Departinent of Nuclear Engineering

4159 Etcheverry Hall

University of California

Berkeley, CA 94720

Thoinas A. Cotton

JK Research Associates, Inc.

4429 Butterworth Place NW

Washington, DC 20016

Robert J. Budnitz

President, Future Resources

Associates, Inc.

2000 Center street, Suite 418

Berkeley, CA 94704

C. John Mann

Department of Geology

245 Natural History Bldg.

1301 West Green Street

University of Illinois

Urbana, IL 61801

Frank W. Schwartz

Departinent of Grology and Mineralogy

The Ohio State University

Scott Hall

1090 Carmack Rd.

columbus, $\mathrm{OH} 43210$

\section{Individuals}

P. Drez

8816 Cherry HI1Is Rd. NE

Albuquerque, NM 87111
D.W. Powers
Star Route Box 87
Anthony, TX 79821

Universities

University of New Mexico

Geology Department

Attn: Library

Albuquerque, NM 87131

University of Washington

College of Ocean

and Fishery Sciences

Attn: G.R.Heath

583 Henders on Hall

Seattle, WA 98195

\section{Libraries}

Thomas Brannigan Library

Attn: D. Dresp

$106 \mathrm{~W}$. Hadley St.

Las Cruces, NM 88001

Government Publications Department

Zimmerman Library

University of New Mexico

Albuquerque, NM 87131

Hobbs Public Library

Attn: M. Lewis

$509 \mathrm{~N}$. Ship St.

Hobbs, NM 88248

New Mexico Junior College

Pannell Library

Attn: R, Hill

Lovington Highway

Hobbs, NM 88240

New Mexico State Library

Attn: N. McCallan

325 Don Gaspar

Santa Fe, NM 87503

New Mexico Tech

Martin Speere Memorial Library

Campus Street

Socorro, NM 87810

WIPP Public Reading Room

Carlsbad Public Library

Attn: Director

$101 \mathrm{~S}$. Halagueno St.

Carlsbad, NM 88220 


\section{Foreign Addresses}

Studiecentrum Voor Kernenergie Centre D'Energie Nucleaire

Attn: A. Bonne

SCK/CEN Boeretang 200

B-2400 Mo1, BELGIUM

Atomic Energy of Canada, Ltd. (3)

Whiteshe11 Research Estab.

Attn: B. Goodwin
M. Stevens
D. Wushke

Pinewa, Manitoha, CANADA ROE 1 LO

Francois Chenevier (2)

ANDRA

Route du Panorama Robert. Schumann

B.P. 38

92266 Fontenay-aux-Roses, Cedex

FRANCE

Jean-Pierre Olivier

OECD Nuclear Energy Agency

Division of Radiation Protection and Waste Management

38, Boulevard Suchet

75016 Paris, FRANCE

Claude Sombret

Centre D'Etudes Nucleaires

De La Vallee Rhone

CEN/VALRHO

S.D.H.A. B.P. 171

30205 Bagnols-Sur-Ceze, FRANCE

Gesellschaft fur Reaktorsicherheit

(GRS) (2)

Attn: B. Baltes

W. Muller

Schwertnergasse 1

D.5000 Cologne, GERMANY

Bundesanstalt fur Cieowissenschaften und Rohst offe

Attn: $M$. Langer

Postfach 510153

3000 Hanover 51, GERMANY

Bundesministerium fur Forschung und Technologie

Postfach 200706

5300 Bonn 2, CERMANY
Institut fur Tieflagerung (2)

Attn: K. Kuhn

Theodor-Heuss-Strasse 4

D-3300 Braunschweig, GERMANY

Physikalisch-Technische Bundesanstalt

Attn: P. Brenneke

Postfach 3345

D-3300 Braunschweig, GERMANY

Shingo Tashiro

Japan Atomic Energy Research Inst.

Toka1-Mura, Ibaraki-Ken, 319-11.

JAPAN

Netherlands Energy Research

Foundation ECN

Attn: L.H. Vons

3 Westerduinweg

PO Box 1

1755 ZG Petten, THE NETHERLANDS

Svensk Karnbransleforsorfning $A B$

Attn: F. Karlsson

Project KBS

Karnbranslesakerhet

Box 5864

10248 Stockholm, SWEDEN

Nationale Genossenschaft für die

Lagerung radioaktiver Abfalle (2)

Attn: S. Vomvoris

P. Zuidema

Hardstrasse 73

$\mathrm{CH}-5430$ wettingen, SWITZERLAND

AEA Technology

Attn: J.H. Rees

D5W/29 Culham Laboratory

Abington, Oxfordshire OX14 3DB

UNITED KINGDOM

AEA Technology

Attn: W.R. Rodwe 11

044/A31 Winfrith Technical Centre

Dorchester, Dorset DT2 8DH

UNITED KINGDOM

AEA Technology

Attn: J.E. Tinson

B4244 Harwell Laboratory

Didcot, Oxfordshire OX11 ORA

UNITED KINGDOM 
D.R. Knowles

British Nuclear Fuels, plc

Risley, Warrington, Cheshire WA3 6AS

1002607 UNITED KINGDOM

\section{Internal}

\begin{tabular}{|c|c|}
\hline 1502 & J.C. Cummings \\
\hline 1561 & H.S. Morgan \\
\hline 1561 & $J, R$. Weatherby \\
\hline 1561 & J.G. Arguello \\
\hline 1.561 & C.M. Stone \\
\hline 6000 & D.L. Hartley \\
\hline 6115 & P.B. Davles \\
\hline 6115 & G. Freeze (INTERA) \\
\hline 6115 & K. Larson (INTERA) \\
\hline 6116 & D.J. Borns \\
\hline 6117 & W.R. Wawersik \\
\hline 6119 & E.D. Gorhain \\
\hline 6119 & Staff $(14)$ \\
\hline 6121 & J.R. Tillerson \\
\hline 6121 & Staft $(7)$ \\
\hline 6300 & D.E. El11s \\
\hline 6302 & L.E. Shephard \\
\hline 6303 & S.Y. Pickering \\
\hline 6303 & W.D. Weart \\
\hline 6305 & S.A. Goldstein \\
\hline 6306 & A.L. Stevens \\
\hline 6342 & D.R. Anderson \\
\hline 6342 & Staff $(20)$ \\
\hline 6343 & V. Harper-slaboszewicz \\
\hline 6343 & Staff (2) \\
\hline 6345 & R.C. Lincoln \\
\hline 6345 & B.M. Butcher (20) \\
\hline 6345 & Staft (9) \\
\hline 6347 & D.R. Schafer \\
\hline 6348 & J, T'. Holmes \\
\hline 6348 & Staff $\left(4_{4}\right)$ \\
\hline 6351 & R.E. Thompson \\
\hline 6352 & D.P. Gatber \\
\hline 6352 & S.E. Sharpton \\
\hline 6352 & WIPP Central Flles (10) \\
\hline 7141 & Technical Library (5) \\
\hline 7151 & Technical Publications \\
\hline $7613 \cdot 2$ & $\begin{array}{l}\text { Document Processing for } \\
\text { Dok/OS'l' }(10)\end{array}$ \\
\hline 8523.2 & Central fechnical \\
\hline
\end{tabular}


